\title{
Checklist of British and Irish Hymenoptera - Ichneumonidae
}

\author{
Gavin R. Broad ‡ \\ ‡ The Natural History Museum, London, United Kingdom
}

Corresponding author: Gavin R. Broad (g.broad@nhm.ac.uk)

Academic editor: Pavel Stoev

Received: 28 Apr 2016 | Accepted: 16 Jun 2016 | Published: 05 Jul 2016

Citation: Broad G (2016) Checklist of British and Irish Hymenoptera - Ichneumonidae. Biodiversity Data Journal 4: e9042. doi: 10.3897/BDJ.4.e9042

\section{Abstract \\ Background}

The checklist of British and Irish Ichneumonidae is revised, based in large part on the collections of the Natural History Museum, London and the National Museums of Scotland, Edinburgh. Distribution records are provided at the country level.

\section{New information}

Of the 2,447 species regarded as valid and certainly identified, 214 are here recorded for the first time from the British Isles. Neorhacodinae is considered to be a separate subfamily rather than a synonym of Tersilochinae. Echthrini is treated as a junior synonym of the tribe Cryptini, not Hemigastrini. Echthrus Gravenhorst and Helcostizus Förster are classified in Cryptini rather than, respectively, Hemigastrini and Phygadeuontini.

\section{Keywords}

Britain, Ireland, fauna, Ichneumonoidea 


\section{Introduction}

The Ichneumonidae is one of two families of the superfamily Ichneumonoidea, along with the Braconidae. Given the size of each family in Britain (over 1,300 braconids and nearly 2,500 ichneumonids) we are publishing the two checklists separately. This is one part of a series of papers revising the British and Irish list of Hymenoptera, that started with Broad (2014), Broad and Livermore (2014b), Broad and Livermore (2014a) and Liston et al. (2014). For the background and rationale behind these British Hymenoptera checklists see Broad (2014). The bases for the ichneumonid taxonomy are Yu and Horstmann (1997) and Taxapad (Yu et al. 2012) (ichneumonid data for both compiled by Klaus Horstmann). I have not relied on Yu et al. (2012) for data on species occurrence in Britain. Rather, I have relied mostly on the primary literature and collections, especially NMS and BMNH. Indeed, large parts of the checklist rely on original work, identifying specimens in these collections. The collections of the NMS have been invaluable in updating this checklist as Mark Shaw has very actively encouraged taxonomists to use the collections, which contain a large amount of recently collected, often reared, material. The late J.F. Perkins also did a huge amount to improve the BMNH collection of Ichneumonidae that was not really reflected in his published output. I have made great use of Perkins's identifications as well as Horstmann's, Andrey Khalaim's and many other workers, including my own. Many Irish records are taken from O'Connor et al. (2007).

All additions to and deletions from the British list since Fitton (1978) are recorded as well as country-level distribution within the British Isles (but regarding the Republic of Ireland and Northern Ireland as one geographical unit), i.e. England, Scotland, Wales, Ireland, Isle of Man. The current numbers of valid, certainly identified ichneumonid species are listed per subfamily and by country in Table 1. This highlights the lack of data from countries other than England. Because the ichneumonid literature is extensive and scattered I also provide many taxonomic references. Some changes to classification are employed here, following molecular phylogenetic studies (Laurenne et al. 2006, Quicke et al. 2009). Two genera that are usually classified in Hemigastrini and Phygadeuontini - Echthrus Gravenhorst and Helcostizus Förster - are transferred to Cryptini (as originally suggested by Laurenne et al. 2006), which means that Echthrini Narayanan \& Lal, 1958, becomes a junior synonym of Cryptini Kirby, 1837. And, contrary to Quicke et al. (2009), Neorhacodinae is again regarded as a valid subfamily, not as a synonym of Tersilochinae, following phylogenetic work by A.M.R. Bennett et al. (in prep.) and reflecting differences in host use.

Table 1.

Numbers of confirmed British and Irish Ichneumonidae broken down by subfamily and country. Totals do not include manuscript names or uncertain identifications.

\begin{tabular}{|l|l|l|l|l|l|l|}
\hline subfamily & total valid species & England & Scotland & Wales & Ireland & Isle of Man \\
\hline Acaenitinae & 6 & 3 & 2 & & & \\
\hline Adelognathinae & 19 & 18 & 8 & 4 & 11 & 2 \\
\hline
\end{tabular}




\begin{tabular}{|c|c|c|c|c|c|c|}
\hline Agriotypinae & 1 & 1 & 1 & 1 & & \\
\hline Alomyinae & 2 & 2 & 1 & & 1 & 1 \\
\hline Anomaloninae & 38 & 36 & 20 & 14 & 13 & 3 \\
\hline Banchinae & 132 & 122 & 65 & 21 & 55 & 5 \\
\hline Campopleginae & 361 & 284 & 169 & 41 & 122 & 22 \\
\hline Collyriinae & 2 & 2 & & & & \\
\hline Cremastinae & 15 & 15 & 1 & 2 & 7 & \\
\hline Cryptinae & 529 & 404 & 245 & 123 & 140 & 40 \\
\hline Ctenopelmatinae & 295 & 244 & 147 & 35 & 30 & 19 \\
\hline Cylloceriinae & 5 & 5 & 3 & & 4 & \\
\hline Diacritinae & 1 & 1 & 1 & & 1 & \\
\hline Diplazontinae & 60 & 57 & 45 & 35 & 40 & 22 \\
\hline Eucerotinae & 3 & 3 & 2 & & 2 & \\
\hline Hybrizontinae & 2 & 2 & & 1 & & \\
\hline Ichneumoninae & 383 & 334 & 181 & 81 & 176 & 28 \\
\hline Lycorininae & 1 & 1 & 1 & & 1 & \\
\hline Mesochorinae & 86 & 73 & 25 & 4 & 11 & 3 \\
\hline Metopiinae & 76 & 67 & 42 & 10 & 14 & 1 \\
\hline Microleptinae & 3 & 3 & 3 & 2 & 2 & \\
\hline Neorhacodinae & 1 & 1 & 1 & & 1 & \\
\hline Ophioninae & 27 & 26 & 19 & 15 & 10 & 1 \\
\hline Orthopelmatinae & 2 & 2 & 1 & 2 & 1 & \\
\hline Oxytorinae & 2 & 2 & 2 & 2 & & \\
\hline Pimplinae & 109 & 103 & 64 & 52 & 56 & 20 \\
\hline Poemeniinae & 6 & 6 & 1 & 2 & 2 & \\
\hline Rhyssinae & 2 & 2 & 2 & 1 & 1 & 1 \\
\hline Stilbopinae & 5 & 4 & 4 & 1 & 3 & \\
\hline Tersilochinae & 81 & 73 & 27 & 9 & 46 & 1 \\
\hline Tryphoninae & 179 & 153 & 108 & 37 & 53 & 20 \\
\hline \multirow[t]{2}{*}{ Xoridinae } & 13 & 13 & 4 & 3 & 4 & \\
\hline & 2447 & 2062 & 1195 & 498 & 807 & 189 \\
\hline
\end{tabular}

Figs 1, 2, 3, 4, 5 illustrate a tiny part of the diversity of Ichneumonidae. A handbook to the British fauna (Broad, Shaw \& Fitton, in prep.) provides an introduction to the biology, classification and identification of this family of parasitoid wasps and will be published soon. 


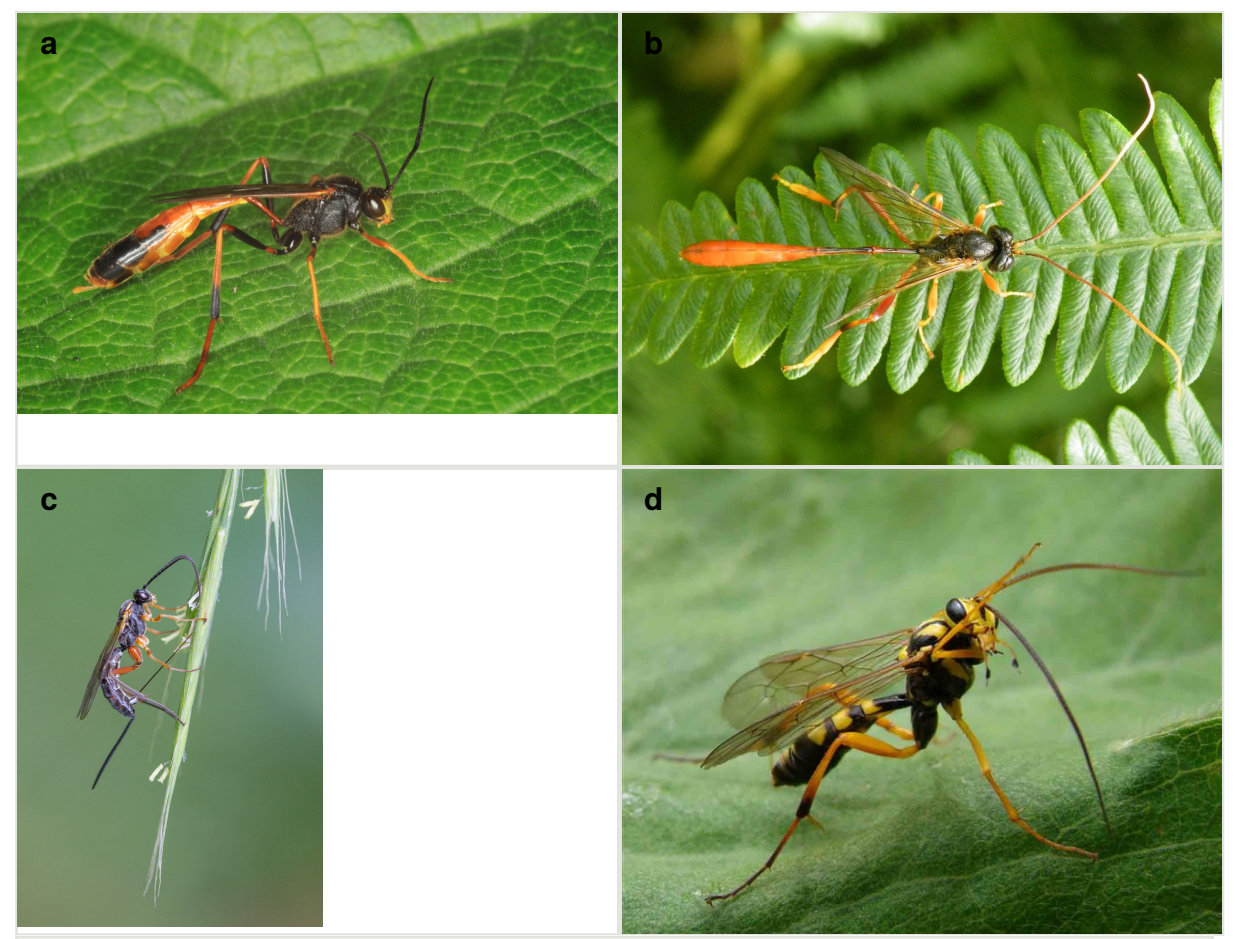

Figure 1.

Representative British Ichneumonidae

a: Anomaloninae: Erigorgus cerinops (Gravenhorst) female (courtesy of B. Formstone)

b: Anomaloninae: Heteropelma amictum (Fabricius) male (courtesy of I. Middlebrook)

c: Banchinae: Lissonota lineolaris (Gmelin) female probing for Apamea (Lepidoptera: Noctuidae) larva (courtesy of P. Adams)

d: Banchinae: Banchus volutatorius (Linnaeus) male (courtesy of A. Watson Featherstone)

\section{Materials and methods}

For a more detailed description of the background and rationale to the Hymenoptera checklist, see Broad (2014). I provide rather extensive Palaearctic synonymy and citations because if you do not have access to the Taxapad database (Yu et al. 2012) it can be very difficult to trace the fate of names in the voluminous and scattered literature, although some of the functionality of the catalogue is available online. Conventions and abbreviations are listed below. 

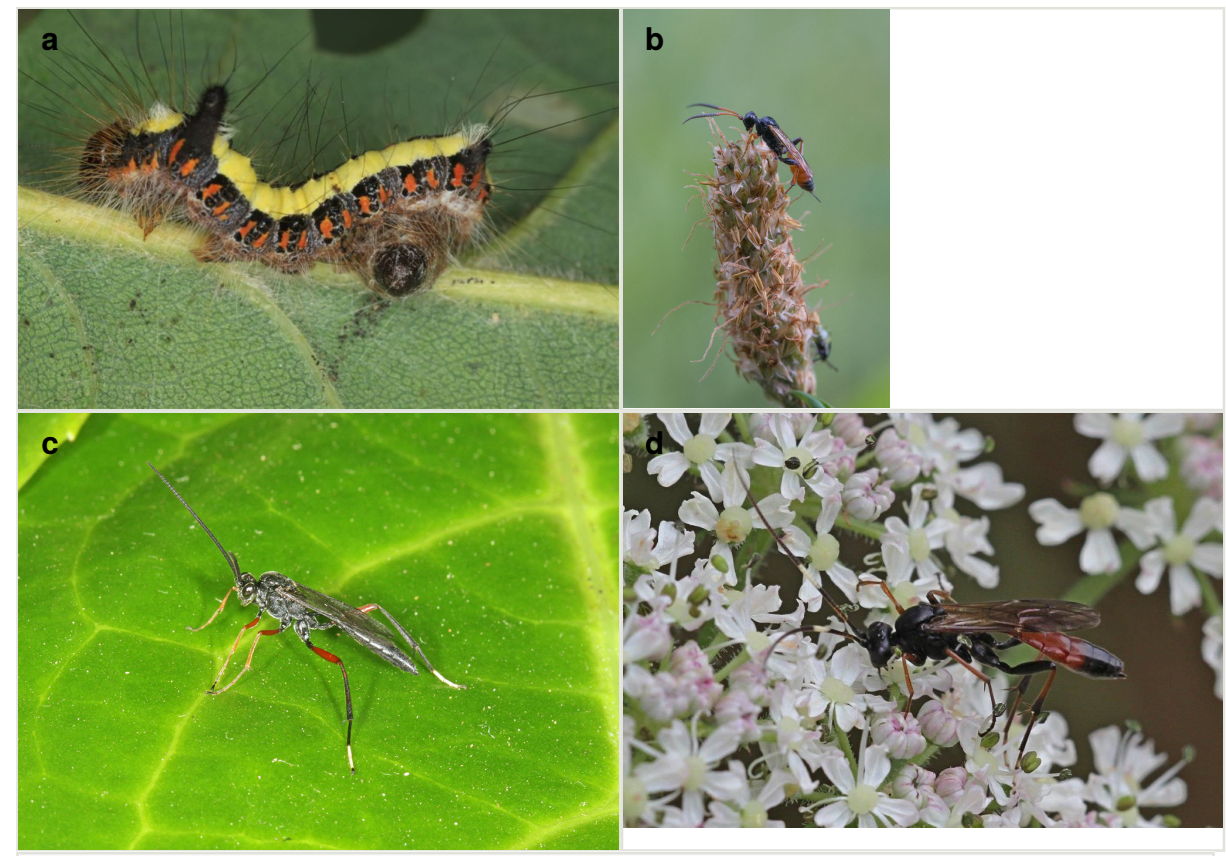

Figure 2.

Representative British Ichneumonidae

a: Campopleginae: cocoon of Hyposoter sp. ex Acronicta psi (Linnaeus) (Lepidoptera: Noctuidae) (courtesy of B. Formstone)

b: Cryptinae: Colocnema rufina (Gravenhorst) female

c: Cryptinae: Polytribax perspicillator (Gravenhorst) male (courtesy of J. Early)

d: Ctenopelmatinae: Euryproctus sp. female (courtesy of D. Bateson)

[species] taxon deleted from the British and Irish list

BMNH Natural History Museum, London

NMS National Museums of Scotland, Edinburgh

UM Ulster Museum [J. Brock collection]

\# known introductions occurring only under artificial conditions 


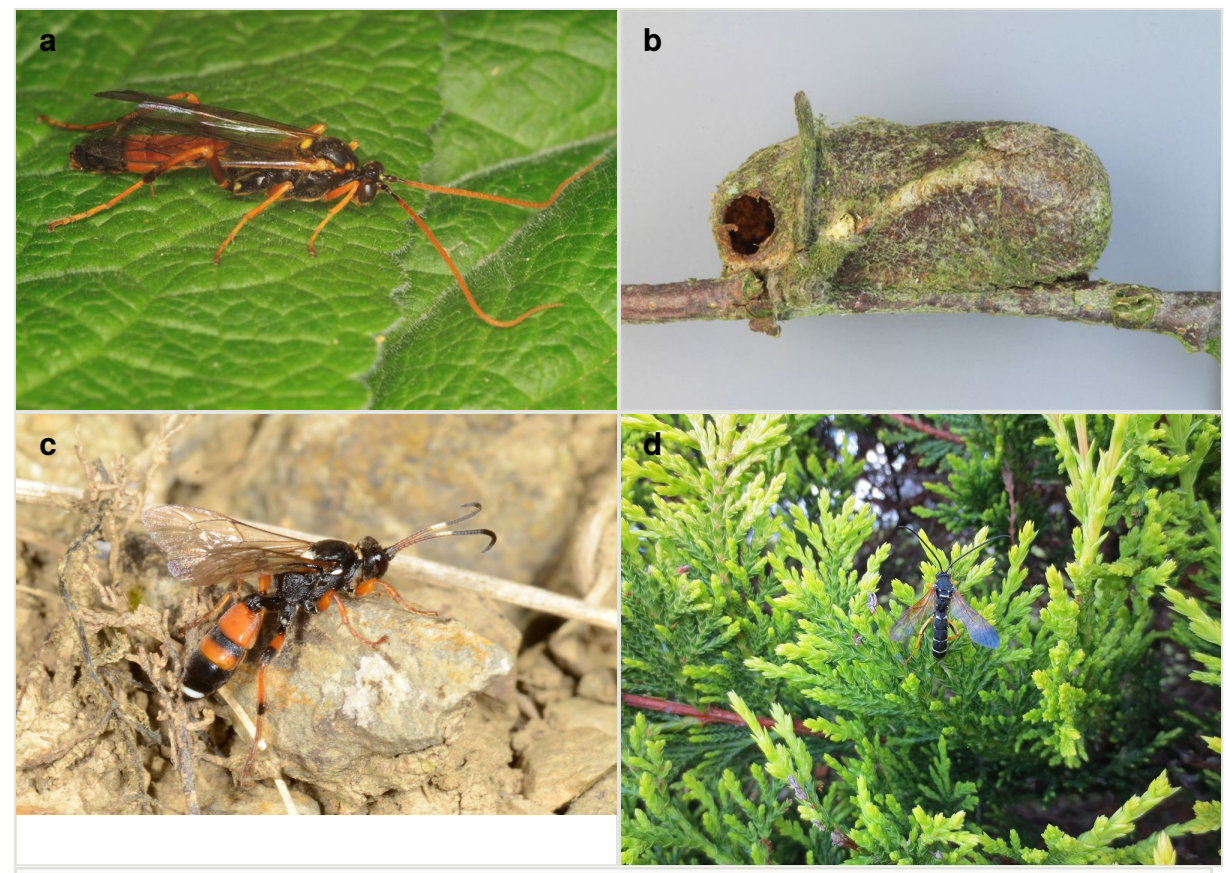

Figure 3.

Representative British Ichneumonidae

a: Ctenopelmatinae: Protarchus melanurus (Thomson) female (courtesy of B. Formstone)

b: Ctenopelmatinae: Cimbex (Hymenoptera: Cimbicidae) cocoon with Protarchus melanurus (Thomson) emergence hole (courtesy of B. Formstone)

c: Ichneumoninae: Ichneumon sarcitorius Linnaeus female (courtesy of J. Bingham)

d: Metopiinae: Metopius dentatus (Fabricius) ex Lasiocampa quercus callunae Palmer (Lepidoptera: Lasiocampidae) pupa (courtesy of F. Stark)

? status (including uncertain synonymy) or identification in the British Isles uncertain

misident. has been misidentified as this name

nom. dub. nomen dubium, a name of doubtful status

nom. ob. nomen oblitum, 'forgotten name', does not have priority over a younger name

nom. nov. nomen novum, a replacement name

nom. nud. nomen nudum, an unavailable name, with no type specimen

preocc. name preoccupied (junior homonym) 

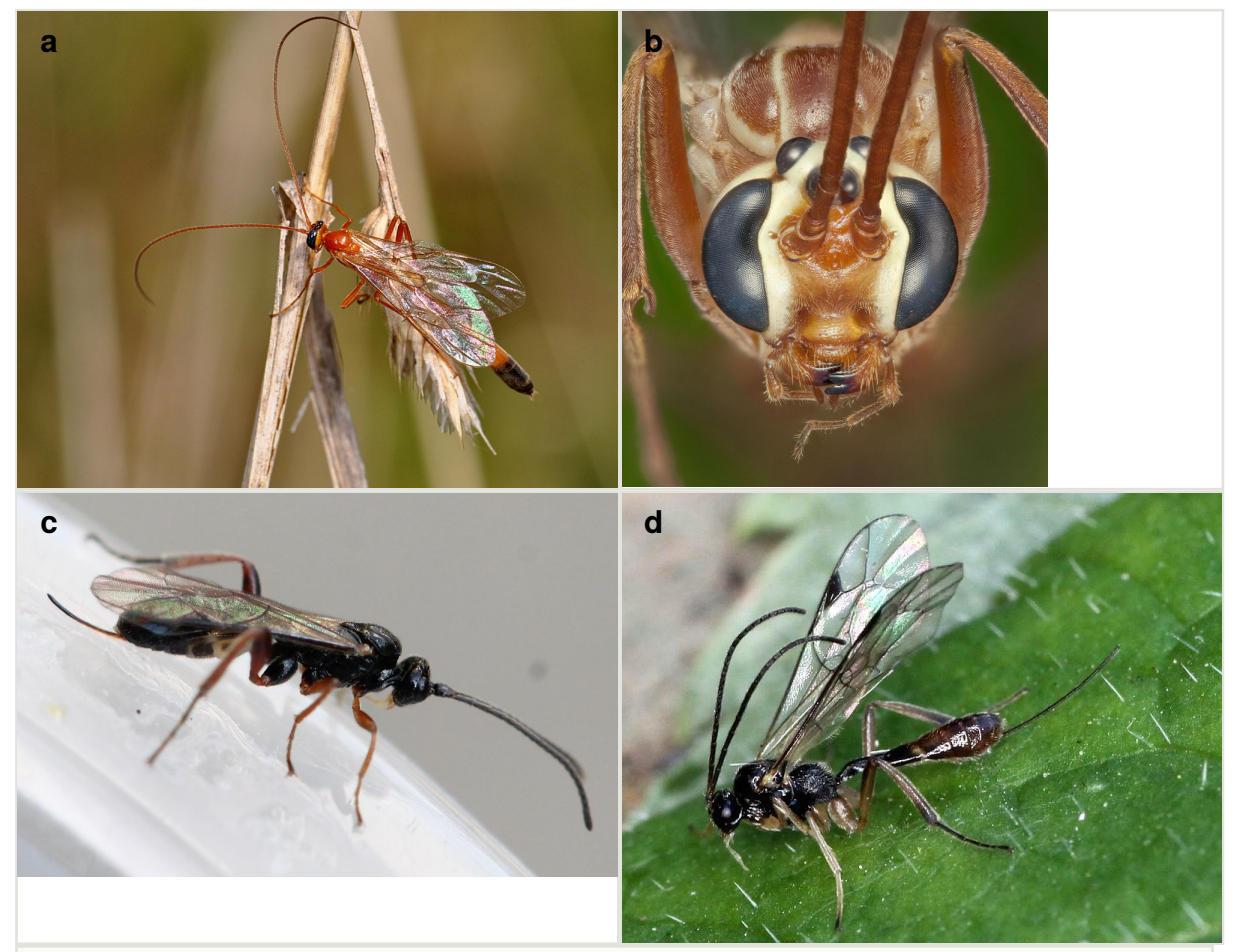

Figure 4.

Representative British Ichneumonidae

a: Ophioninae: Enicospilus ramidulus (Linnaeus) (courtesy of P. Brock)

b: Ophioninae: Ophion obscuratus Fabricius (courtesy of $\mathrm{H}$. Taylor, BMNH)

c: Orthocentrinae: Entypoma sp. (courtesy of T. LeGrand)

d: Orthocentrinae: Plectiscidea sp. (courtesy of M. Stemmer)

stat. rev. status revocatus, revived status (e.g., raised from synonymy)

unavailable name unavailable under provisions of the ICZN code

var. variety, only available as a valid name under certain provisions of the ICZN code

When there are no countries listed for a species, there are two explanations. First, the species has been carried over from the previous checklist (Fitton 1978) and, although I have not seen any British specimens I have no reason to doubt the original identification. Second, specimens in collections are labelled with imprecise locality data, such as 'Great Britain'. This is the case with many of the older collections. 


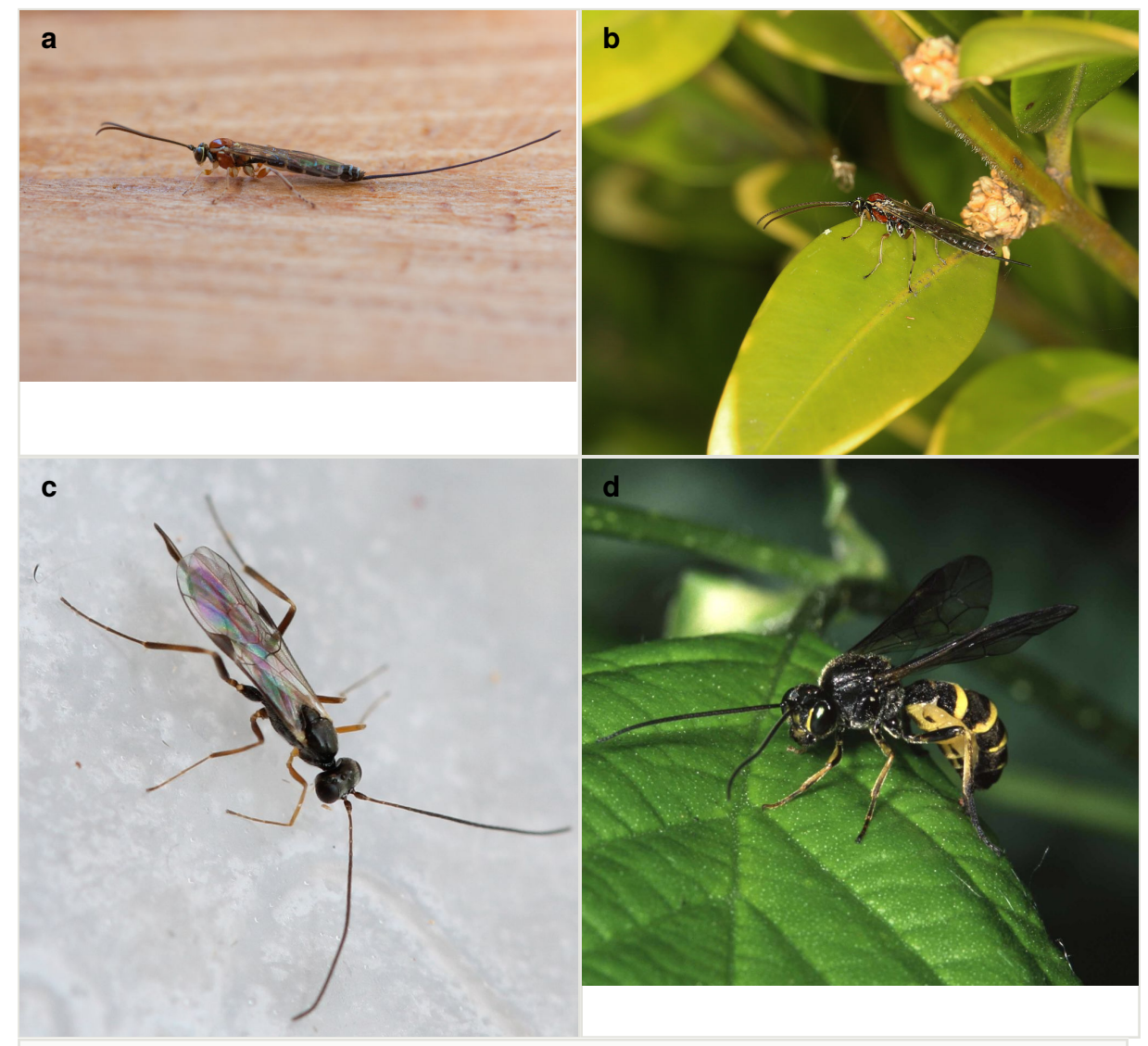

Figure 5.

Representative British Ichneumonidae

a: Pimplinae: Perithous scurra (Panzer) female (courtesy of P. Adams)

b: Pimplinae: Tromatobia lineatoria (Villers) female (courtesy of J. Davison)

c: Tryphoninae: Thymaris niger (Taschenberg) female (courtesy of T. LeGrand)

d: Tryphoninae: Excavarus sp. (details unfortunately lost)

Alternative versions of the checklist can be downloaded here as a Word document or Excel spreadsheet under supplementary materials: Suppl. materials 1, 2. The British and Irish ichneumonid checklist, together with the entire Hymenoptera checklist, will be kept up to date in a Scratchpad, Hymenoptera of the British Isles. 


\section{Acaenitinae}

\section{Subfamily ACAENITINAE Förster, 1869}

Notes: Tribes within Acaenitinae (formerly Acaenitini and Coleocentrini) were abandoned by Wahl and Gauld (1998). Distribution data from Fitton (1981) and Shaw and Wahl (1989), with further references given.

Genus Acaenitus Latreille, 1809

Nomenclature:

ACOENITES Latreille, 1810

ACOENITUS Griffith, 1832

Acaenitus dubitator (Panzer, 1800)

Nomenclature:

Ichneumon dubitator Panzer, 1800

Distribution: Scotland

Genus Arotes Gravenhorst, 1829

Nomenclature:

ASTHENOMERIS Förster, 1869

SPHALERUS Kriechbaumer, 1878

Notes: annulicornis synonymised by Varga (2013)

Arotes albicinctus Gravenhorst, 1829

Nomenclature:

bifasciatus (Kriechbaumer, 1878, Sphalerus)

annulicornis Kriechbaumer, 1894

Distribution: England

Genus Coleocentrus Gravenhorst, 1829

Nomenclature:

MACROCOLEUS Desvignes, 1850 
Coleocentrus croceicornis (Gravenhorst, 1829)

Nomenclature:

Macrus croceicornis Gravenhorst, 1829

Distribution: England

Notes: Coleocentrus soleatus (Gravenhorst, 1829, Macrus) was removed from synonymy by Kasparyan and Khalaim (2007a).

\section{Coleocentrus excitator (Poda, 1761)}

Nomenclature:

Ichneumon excitator Poda, 1761

segmentator (Fabricius, 1793, Ichneumon)

gigantor (Thunberg, 1824, Ichneumon)

longiventris (Gravenhorst, 1829, Macrus)

segmentatrix (Schulz, 1906, Lissonota)

Distribution: Scotland

Notes: added by Shaw (1986)

Genus Leptacoenites Strobl, 1902

\section{Leptacoenites notabilis (Desvignes, 1856)}

Nomenclature:

Lampronota notabilis Desvignes, 1856

frauenfeldi (Tschek, 1869, Lissonota)

marginatus (Kriechbaumer, 1899, Heterolabis)

petiolaris (Kriechbaumer, 1899, Heterolabis)

tscheki (Strobl, 1902, Procinetus)

\section{Genus Phaenolobus Förster, 1869}

Nomenclature:

CHORISCHIZUS Förster, 1869

MOLDACOENITUS Constantineanu \& Constantineanu, 1968 
Phaenolobus terebrator (Scopoli, 1763)

Nomenclature:

Ichneumon terebrator Scopoli, 1763

arator (Rossi, 1790, Ichneumon)

Distribution: England

\section{Adelognathinae}

\section{Subfamily ADELOGNATHINAE Thomson, 1888}

Notes: Distribution data from Fitton et al. (1982)supplemented by the collections of NMS, with further references given. Note that, according to the molecular phylogenetic results of Laurenne et al. (2006), Adelognathus may be an aberrant genus of Cryptinae.

Genus Adelognathus Holmgren, 1857

Nomenclature:

PAMMICRA Förster, 1869

NOTOMERIS Förster, 1869

CNEMISCHYS Förster, 1869

EPITROPUS Rossem, 1990

Notes: Epitropus synonymised by Broad (2004)

Adelognathus acantholydae Kasparyan, 1986

Distribution: Scotland

Notes: NMS, det. Shaw, added here

Adelognathus brevicornis Holmgren, 1857

Nomenclature:

limbatus Thomson, 1888

montivagator Aubert, 1976

Distribution: England, Scotland, Ireland 
Notes: The chrysopygus (Grav.) referred to by Fitton et al. (1982) actually refers to punctulatus Thoms.; chrysopygus is the correct name for the species referred to as granulatus Perkins by Fitton et al. (1982) (Kasparyan 1986). Some distribution data from Bennett et al. (2002).

Adelognathus britannicus Perkins, 1943

Distribution: England

Adelognathus chrysopygus (Gravenhorst, 1829)

Nomenclature:

Hemiteles chrysopygus Gravenhorst, 1829

granulatus Perkins, 1943

Distribution: England, Scotland, Ireland, Isle of Man

Adelognathus difformis Holmgren, 1857

Distribution: England

Notes: NMS, det. Shaw, added here

Adelognathus dorsalis (Gravenhorst, 1829)

Nomenclature:

Hemiteles dorsalis Gravenhorst, 1829

melanius Roman, 1918

insolitus (Rossem, 1990, Epitropus)

Distribution: England, Scotland, Wales, Ireland, Isle of Man

Notes: insolitus synonymised by Broad (2004)

Adelognathus laevicollis Thomson, 1883

Distribution: England, Ireland

Adelognathus leucotrochi Shaw \& Wahl, 2014

Distribution: England, Scotland

Notes: added by Shaw and Wahl (2014) 
Adelognathus nigriceps Thomson, 1888

Distribution: England, Wales

Notes: some distribution data from Askew (2000)

Adelognathus nigrifrons Holmgren, 1857

Distribution: England, Ireland

Notes: added by Fitton et al. (1982)

Adelognathus obscurus Kasparyan, 1986

Distribution: England, Scotland

Notes: added by Kasparyan (1990)

Adelognathus pallipes (Gravenhorst, 1829)

Nomenclature:

Plectiscus pallipes Gravenhorst, 1829

ruthei Holmgren, 1857

pallidipes (Marshall, 1872, Plectiscus)

Distribution: England, Scotland, Ireland

Adelognathus pilosus Thomson, 1888

Distribution: England

Adelognathus punctulatus Thomson, 1883

Nomenclature:

chrysopygus misident.

pallipes Holmgren, 1857 preocc.

dimidiatus Thomson, 1888

pallidipes Dalla Torre, 1901

Distribution: England, Ireland

Adelognathus pusillus Holmgren, 1857

Distribution: England, Wales, Ireland 
Adelognathus stelfoxi Fitton, Gauld \& Shaw, 1982

Distribution: England, Scotland, Wales, Ireland

Notes: added by Fitton et al. (1982); Ely (2001)

Adelognathus tenthredinarum (Giraud, 1872)

Nomenclature:

Plectiscus tenthredinarum Giraud, 1872

nigricornis Thomson, 1888

Distribution: England

Adelognathus tetratinctorius (Thunberg, 1824)

Nomenclature:

Ichneumon tetratinctorius Thunberg, 1824

fasciatus Thomson, 1883

scabriculus Thomson, 1883

Distribution: England, Ireland

Adelognathus thomsoni Schmiedeknecht, 1911

Nomenclature:

thuringiacus Schmiedeknecht, 1911

Distribution: England, Ireland

\section{Agriotypinae}

Subfamily AGRIOTYPINAE Haliday, 1838

Notes: Distribution data from Bennett (2001), Howe and Howe (2005) and the collections of the NMS.

Genus Agriotypus Curtis, 1832

Nomenclature:

CROTOPUS Holmgren, 1859

ATOPOTYPUS Chao, 1992 
Notes: Atopotypus synonymised by Bennett (2001)

\section{Agriotypus armatus Curtis, 1832}

Nomenclature:

abnormis (Holmgren, 1859, Crotopus)

Distribution: England, Scotland, Wales

\section{Alomyinae}

\section{Subfamily ALOMYINAE Förster, 1869}

Notes: Alomya has frequently been placed in its own subfamily, Alomyinae (e.g. Perkins 1959), or in a separate tribe within the Ichneumoninae (Diller 1981, Selfa and Diller 1994) but a close relationship with Colpognathus and Centeterus was proposed by Wahl and Mason (1995), with the result that the ichneumonine tribe here called Phaeogenini took the name Alomyini. Based on the molecular phylogenetic results of Laurenne et al. (2006)Alomya and Megalomya Uchida are considered here to comprise a separate subfamily.

\section{Genus Alomya Panzer, 1806}

\section{Nomenclature:}

ALOMYIA misspelling

HALOMYA Billberg, 1820

Notes: Distribution data from Perkins (1952), Perkins (1959) and the NMS.

\section{Alomya debellator (Fabricius, 1775)}

\section{Nomenclature:}

Ichneumon debellator Fabricius, 1775

fischeri (Schrank, 1776, Ichneumon)

trituberculata (Gmelin, 1790, Ichneumon)

ovator (Fabricius, 1793, Ichneumon)

victor Curtis, 1826

nigra Gravenhorst, 1829

debellatrix Schulz, 1906

victrix Schulz, 1906 
silvicola Ulbricht, 1909 unavailable

Distribution: England, Scotland, Ireland, Isle of Man

Alomya semiflava Stephens, 1835

Nomenclature:

minor Ulbricht, 1909 unvailable

minor Ulbricht, 1911 preocc.

Distribution: England

\section{Anomaloninae}

Subfamily ANOMALONINAE Viereck, 1918

Nomenclature:

ANOMALINAE misspelling

Notes: Distribution data mostly taken from the collections of the NMS and BMNH, supplemented by Gauld and Mitchell (1977a), unless noted otherwise. Most of the NMS and some of the BMNH material has been identified recently by $\mathrm{H}$. Schnee.

\section{Tribe ANOMALONINI Viereck, 1918}

Nomenclature:

ANOMALINI misspelling

\section{Genus Anomalon Panzer, 1804}

Nomenclature:

TRACHYNOTUS Gravenhorst, 1829 preocc.

OCHLERUS Gistel, 1848 preocc.

NOTOTRACHYS Marshall, 1872

ANOMALUM Schulz, 1906

TRACHYOPTERUS Morley, 1912

PSEUDONOTOTRACHYS Meyer, 1930

MICROCREMASTUS Hedwig, 1961 


\section{Anomalon cruentatum (Geoffroy, 1785)}

\section{Nomenclature:}

Ichneumon cruentatus Geoffroy, 1785

petiolatum (Geoffroy, 1785, Ichneumon)

foliator (Fabricius, 1798, Ophion)

cruentatum Panzer, 1804

humerale (Brullé, 1832, Trachynotus)

epiphanii Izquierdo, 1977

Distribution: England

\section{Tribe GRAVENHORSTIINI Enderlein, 1912}

\section{Nomenclature:}

THERIONINI Viereck, 1918

Notes: Species of Gravenhorstiini excluded from the British and Irish list:

[PERISPHINCTER Townes, 1961 brevicollis (Wesmael, 1849, Anomalon)] Gauld and Mitchell (1977a) recorded one specimen of brevicollis from Killiecrankie, Scotland, as a species of Agrypon. As this species is now included in Perisphincter (Schnee 1978), a genus which Gauld knew at the time, and the specimen is lost (M.R. Shaw, pers. comm.), this is considered here to be a misidentification.

\section{Genus Agrypon Förster, 1860}

\section{Nomenclature:}

AGRYPUM Schulz, 1906

Notes: much synonymy from Schnee (2008)

species of Agrypon excluded from the British and Irish list:

[interruptus (Desvignes, 1856, Anomalon)] Listed as a doubtfully placed species of Agrypon by Fitton (1978), but Gauld and Mitchell (1977b) had established that this species is actually an exotic cremastine. Currently listed in Yu and Horstmann (1997) as a doubtfully placed species of Cremastus.

\section{Agrypon anomelas (Gravenhorst, 1829)}

\section{Nomenclature:}

Anomalon anomelas Gravenhorst, 1829

anomalas misspelling 
furtivum Förster, 1860

trochanteratum (Holmgren, 1860, Anomalon)

rufipes Kiss, 1926

Distribution: England

Notes: George (1978) provides information on its occurrence in Britain

Agrypon anxium (Wesmael, 1849)

Nomenclature:

Anomalon anxium Wesmael, 1849

pictum Kiss, 1924

Distribution: England, Scotland, Wales, Ireland

\section{Agrypon batis (Ratzeburg, 1855)}

Nomenclature:

Anomalon batis Ratzeburg, 1855

serpentinum Förster, 1860

stenostigma (Thomson, 1892, Anomalon)

segne (Tosquinet, 1896, Anomalon)

Distribution: England, Scotland, Ireland

Notes: NMS, BMNH, det. Schnee, added here

Agrypon brachycerum Hellén, 1950

Distribution: England, Scotland

Notes: NMS, BMNH, det. Schnee, added here. Listed as a synonym of anxium in Yu and Horstmann (1997).

\section{Agrypon canaliculatum (Ratzeburg, 1844)}

Nomenclature:

Anomalon canaliculatum Ratzeburg, 1844

Distribution: England, Scotland

Notes: NMS, BMNH, det. Schnee, added here. Misidentified canaliculatum of authors is Habronyx nigricornis (Wesm.). 
Agrypon clandestinum (Gravenhorst, 1829)

Nomenclature:

Anomalon clandestinum Gravenhorst, 1829

delarvatum misident.

capillosum (Hartig, 1838, Anomalon)

affine (Holmgren, 1857, Anomalon)

brachypterum Förster, 1860

clandestinum Förster, 1860 preocc.

ruficoxis (Szépligeti, 1899, Labrorychus)

flavopunctatum (Kiss, 1933, Blaptocampus)

Distribution: England, Scotland, Ireland, Isle of Man

Agrypon flaveolatum (Gravenhorst, 1807)

Nomenclature:

Ophion flaveolatum Gravenhorst, 1807

cribrator (Thunberg, 1824, Ichneumon)

laedator (Thunberg, 1824, Ichneumon)

arquatum (Gravenhorst, 1829, Anomalon)

septentrionale (Holmgren, 1857, Anomalon)

aggressorium Förster, 1860

confusum Förster, 1860

elegantulum Förster, 1860

rubricatum Förster, 1860

rubricatum (Förster, 1878, Atrometus) preocc.

Distribution: England, Scotland, Wales, Ireland

\section{Agrypon flexorium (Thunberg, 1824)}

Nomenclature:

Ichneumon flexorium Thunberg, 1824

tenuicorne (Gravenhorst, 1829, Anomalon)

subclavatum Förster, 1860

anaitidis (Szépligeti, 1899, Labrorychus)

sibiricum (Shestakov, 1923, Labrorychus) 
Distribution: England, Scotland, Wales, Ireland

Notes: Agrypon polyxenae (Szépligeti, 1899, Labrorychus) removed from synonymy by Schnee (2008).

\section{Agrypon gracilipes (Curtis, 1839)}

Nomenclature:

Therion gracilipes Curtis, 1839

debile (Wesmael, 1849, Anomalon)

meridionator Aubert, 1964 preocc.

Distribution: England, Wales

\section{Agrypon interstitiale Schnee, 1989}

Distribution: England, Scotland

Notes: NMS, BMNH, det. Schnee, added here

\section{Agrypon minutum (Bridgman \& Fitch, 1884)}

Nomenclature:

Anomalon minutum Bridgman \& Fitch, 1884

minutum (Bridgman, 1884, Anomalon) preocc.

Distribution: England, Scotland, Wales

Notes: Listed as a synonym of anxium (Wesm.) in Yu and Horstmann (1997), treated by Schnee as a valid species, but unpublished.

\section{Agrypon rugifer (Thomson, 1894)}

Nomenclature:

Anomalon rugifer Thomson, 1894

Distribution: Wales

Notes: NMS, det. Schnee, added here

\section{Agrypon varitarsum (Wesmael, 1849)}

Nomenclature:

Anomalon varitarsum Wesmael, 1849

cognatum Förster, 1860 
nigripes (Bridgman, 1887, Anomalon)

variitarsum Dalla Torre, 1901

Distribution: England, Scotland, Ireland

\section{Agrypon sp. H}

Distribution: England, Wales

Notes: NMS, BMNH, det. Schnee, added here

\section{Genus Aphanistes Förster, 1869}

Nomenclature:

ANOCHILACRUM Enderlein, 1921

\section{Aphanistes bellicosus (Wesmael, 1849)}

Nomenclature:

Anomalon bellicosum Wesmael, 1849

Distribution: England

Notes: NMS, det. Schnee, added here

\section{Aphanistes gliscens (Hartig, 1838)}

Nomenclature:

Anomalon gliscens Hartig, 1838

bellicosus misident.

xanthopus misident.

armatus (Wesmael, 1849, Anomalon)

Distribution: England, Scotland, Wales

Aphanistes ruficornis (Gravenhorst, 1829)

Nomenclature:

Anomalon ruficorne Gravenhorst, 1829

excavatus (Ratzeburg, 1848, Anomalon)

wesmaeli (Holmgren, 1856, Anomalon)

Distribution: England, Scotland, Wales, Ireland 


\title{
Genus Atrometus Förster, 1869
}

Notes: synonymy follows Schnee (2008)

\section{Atrometus ? insignis Förster, 1878}

Nomenclature:

\author{
rubricatus Förster, 1878 \\ trachynotus (Brauns, 1895, Anomalon) \\ melanosoma Szépligeti, 1899 \\ pulchellator Aubert, 1971
}

Notes: No British specimen could be located in the depositories cited by Gauld and Mitchell (1977b) (M.R. Shaw, pers comm.).

\section{Genus Barylypa Förster, 1869}

\section{Nomenclature:}

LAPHYCTES Förster, 1869

SARNTHEINIA Dalla Torre, 1901

HADROMANUS Szépligeti, 1905

MAGNIBUCCA Morley, 1913

TROCHISCOMERUS Meyer, 1931

Notes: some synonymy from Schnee (2008)

\section{Barylypa delictor (Thunberg, 1824)}

Nomenclature:

$$
\begin{aligned}
& \text { Ichneumon delictor Thunberg, } 1824 \\
& \text { perspicillator (Gravenhorst, 1829, Anomalon) } \\
& \text { affinis (Lucas, 1849, Anomalon) } \\
& \text { menyanthidis (Boie, 1855, Anomalon) } \\
& \text { mesozona (Förster, 1878, Laphyctes) } \\
& \text { genalis (Thomson, 1892, Anomalon) } \\
& \text { frisiaca Habermehl, } 1922 \\
& \text { temporalis Meyer, } 1935
\end{aligned}
$$

Distribution: England, Scotland, Wales 


\section{Barylypa propugnator (Förster, 1855)}

Nomenclature:

Anomalon propugnator Förster, 1855

insidiator (Förster, 1878, Laphyctes)

carinata (Brischke, 1880, Anomalon)

cylindrica (Bridgman \& Fitch, 1884, Anomalon)

cylindrica (Bridgman, 1884, Anomalon) preocc.

rufa (Habermehl, 1920, Anomalon) preocc.

Distribution: England, Wales, Ireland

Notes: Listed as a species of Erigorgus in Yu and Horstmann (1997), transferred to Barylypa by Schnee (2008).

\section{Barylypa rubricator (Szépligeti, 1899)}

\section{Nomenclature:}

Laphyctes rubricator Szépligeti, 1899

rubricatrix (Schulz, 1906, Sarntheinia)

rossica Meyer, 1935

Distribution: England

Notes: BMNH, Hunterian, det. Schnee, added here

\section{Barylypa ? uniguttata (Gravenhorst, 1829)}

\section{Nomenclature:}

Anomalon uniguttatum Gravenhorst, 1829

\section{Distribution: England}

Notes: Although Gauld and Mitchell (1977b) record uniguttata as British on the basis of one specimen from Norfolk, no British specimen could be located in the depositories cited by Gauld \& Mitchell (M.R. Shaw, pers comm.); Gauld was not aware at the time of the presence of rubricator in Britain.

\section{Genus Erigorgus Förster, 1869}

\section{Nomenclature:}


Notes: Although Gauld (1976b), Gauld (1997) regarded Erigorgus as a subgenus of Gravenhorstia, most recent works regard it as a separate genus, a position followed by Yu and Horstmann (1997). As the relative merits of genus versus subgenus are rather subjective I have followed the majority opinion.

\section{Erigorgus cerinops (Gravenhorst, 1829)}

\section{Nomenclature:}

Anomalon cerinops Gravenhorst, 1829

flavifrons (Gravenhorst, 1807, Ophion) preocc.

xantha (Boie, 1855, Anomalon)

facialis (Boie, 1857, Campoplex)

lapponicus (Thomson, 1892, Anomalon)

rufofemoralis (Schmiedeknecht, 1936, Anomalon)

Distribution: England, Scotland, Wales, Ireland

\section{Erigorgus fibulator (Gravenhorst, 1829)}

Nomenclature:

Anomalon fibulator Gravenhorst, 1829

claripennis (Thomson, 1892, Anomalon)

Distribution: England, Wales

Notes: BMNH, det. Schnee, added here

\section{Erigorgus foersteri (Mocsáry, 1897)}

\section{Nomenclature:}

Anomalon foersteri Mocsáry, 1897

melanops misident.

brevicorne (Förster, 1855, Anomalon) preocc.

Notes: NMS, det. Schnee, added here; reared by M.R. Shaw: Bradwell Village, Burford, Oxon. The name foersteri was overlooked by Yu and Horstmann (1997) and other authors (Schnee 2008).

\section{Erigorgus melanops (Förster, 1855)}

\section{Nomenclature:}


melanobata misident.
varians (Brauns, 1895, Anomalon)
flavimana (Szépligeti, 1899, Erigorgus)
interstitialis (Szépligeti, 1899, Erigorgus)
similis (Szépligeti, 1899, Erigorgus)
purpuratae (Kriechbaumer, 1900, Erigorgus)

Distribution: England, Scotland

\section{Erigorgus procerus (Gravenhorst, 1829)}

Nomenclature:

Anomalon procerum Gravenhorst, 1829

Distribution: England

Notes: BMNH, det. Schnee, added here; various authors included procerus as a British

species but Gauld and Mitchell (1977b) did not see any British or Irish material and there were no such specimens in BMNH or NMS at that time.

\section{Erigorgus varicornis (Thomson, 1894)}

Nomenclature:

Anomalon varicorne Thomson, 1894

Distribution: England, Scotland

Notes: BMNH, det Schnee, added here. Listed as a synonym of propugnator (Förster) in Yu and Horstmann (1997).

\section{Genus Gravenhorstia Boie, 1836}

Nomenclature:

ODONTOPSIS Förster, 1869

\section{Gravenhorstia picta Boie, 1836}

Nomenclature:

fasciata (Giraud, 1857, Anomalon)

fasciata (Marshall, 1873, Anomalon) preocc.

septemfasciata (Taschenberg, 1875, Ophion)

picta (Rudow, 1882, Anomalon) 
Distribution: England

Genus Habrocampulum Gauld, 1976

Habrocampulum biguttatum (Gravenhorst, 1829)

Nomenclature:

Anomalon biguttatum Gravenhorst, 1829

Distribution: England

Genus Habronyx Förster, 1869

Subgenus Camposcopus Förster, 1869

Nomenclature:

LABRORYCHUS Förster, 1869

BLAPTOCAMPUS Thomson, 1892

Notes: species of Habronyx (Camposcopus) deleted from the British and Irish list:

[perspicuus (Wesmael, 1849, Anomalon)] Recorded by Gauld and Mitchell (1977b) but there are no British specimens in BMNH or NMS and the identification must be regarded as doubtful.

Habronyx (Camposcopus) nigricornis (Wesmael, 1849)

Nomenclature:

Anomalon nigricorne Wesmael, 1849

canaliculatus misident.

melanomerus (Förster, 1860, Agrypon)

maidan (Shestakov, 1923, Blaptocampus)

Distribution: England, Scotland, Wales, Ireland

Subgenus Habronyx Förster, 1869

Nomenclature:

ACANTHOSTOMA Kriechbaumer, 1895

MACROSTEMMA Shestakov, 1923 
Habronyx (Habronyx) heros (Wesmael, 1849)

Nomenclature:

Anomalon heros Wesmael, 1849

mirabilis (Desvignes, 1856, Anomalon)

gravenhorstii Förster, 1860

gigas (Kriechbaumer, 1880, Anomalon)

oti (Kriechbaumer, 1895, Anomalon)

Distribution: England

Genus Heteropelma Wesmael, 1849

Nomenclature:

SCHIZOLOMA Wesmael, 1849

SCHIZOPOMA Förster, 1869

Heteropelma amictum (Fabricius, 1775)

Nomenclature:

Ichneumon amictus Fabricius, 1775

xanthopus (Schrank, 1781, Ichneumon)

amictor (Thunberg, 1824, Ichneumon)

xanthopor (Thunberg, 1824, Ichneumon)

capitatum (Desvignes, 1856, Anomalon)

bucephalum (Vollenhoven, 1858, Anomalon)

bucephalum (Brauns, 1898, Schizoloma) preocc.

Distribution: England, Scotland, Ireland, Isle of Man

Notes: distribution data from Gauld (1976a)

Heteropelma megarthrum (Ratzeburg, 1848)

Nomenclature:

Anomalon megarthrum Ratzeburg, 1848

calcator Wesmael, 1849

scabridum (Boie, 1855, Anomalon)

megalarthrum (Schulz, 1906, Anomalon)

nigriscutum (Fahringer, 1941, Anomalon) 
Distribution: England, Scotland, Ireland

\section{Genus Parania Morley, 1913}

\section{Parania geniculata (Holmgren, 1857)}

Nomenclature:

Anomalon geniculatum Holmgren, 1857

Distribution: England

Genus Therion Curtis, 1829

Nomenclature:

THERIUM Agassiz, 1846

EXOCHILUM Wesmael, 1849

\section{Therion circumflexum (Linnaeus, 1758)}

Nomenclature:

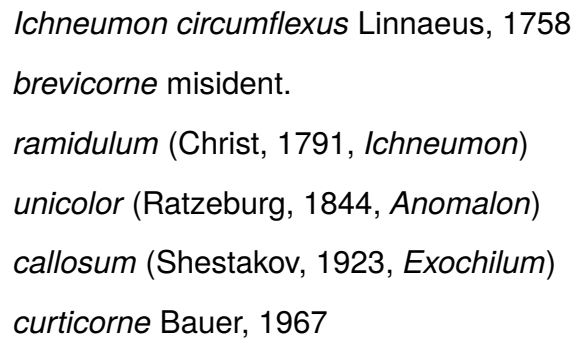

Distribution: England, Scotland, Wales, Ireland

Notes: Specimens identified as brevicorne (Gauld and Mitchell 1977b) are misidentifications of circumflexum (, and pers. comm. to M.R. Shaw).

\section{Genus Trichomma Wesmael, 1849}

Nomenclature:

TRICHOMELLA Szépligeti, 1910

\section{Trichomma enecator (Rossi, 1790)}

Nomenclature:

Ichneumon enecator Rossi, 1790

ruficoxis Förster, 1860 
Distribution: England, Isle of Man

Trichomma fulvidens Wesmael, 1849

Nomenclature:

bituberculatum Schmiedeknecht, 1902

Distribution: England

Trichomma intermedium Krieger, 1904

Distribution: England

Trichomma occisor Habermehl, 1909

Distribution: England

\section{Banchinae}

Subfamily BANCHINAE Wesmael, 1845

Nomenclature:

LISSONOTINAE Förster, 1869

Notes: Taxonomy and distribution data follow Brock's (in prep.) handbook to the British Banchinae, with some additions from NMS, BMNH, Aubert (1978) and Fitton (1976). Additional references are given.

Tribe ATROPHINI Seyrig, 1932

Nomenclature:

LISSONOTINI Förster, 1869

Genus Alloplasta Förster, 1869

Nomenclature:

ASYMMICTUS Förster, 1869

TRYSICAMPE Förster, 1869

Alloplasta piceator (Thunberg, 1824)

Nomenclature:

Ichneumon piceator Thunberg, 1824 


$$
\begin{aligned}
& \text { creditor (Thunberg, 1824, Ichneumon) } \\
& \text { albitarsus (Gravenhorst, 1829, Exetastes) } \\
& \text { lata (Gravenhorst, 1829, Exetastes) } \\
& \text { murina (Gravenhorst, 1829, Lissonota) } \\
& \text { murina (Gravenhorst, 1829, Tryphon) } \\
& \text { albitarsoria (Zetterstedt, 1838, Tryphon) } \\
& \text { genucincta (Rudow, 1886, Cryptus) } \\
& \text { variipes (Szépligeti, 1899, Meniscus) }
\end{aligned}
$$

Distribution: England, Scotland, Ireland

\section{Alloplasta plantaria (Gravenhorst, 1829)}

Nomenclature:

Phytodietus plantarius Gravenhorst, 1829

Distribution: England, Wales

Genus Arenetra Holmgren, 1859

Nomenclature:

LASIOPS Holmgren, 1856 preocc.

\section{Arenetra pilosella (Gravenhorst, 1829)}

Nomenclature:

Tryphon pilosellus Gravenhorst, 1829

Distribution: England, Scotland

\section{Genus Cryptopimpla Taschenberg, 1863}

Nomenclature:

APHANODON Förster, 1869

XENACIS Förster, 1869

XENOCORNIA Schmiedeknecht, 1900

\section{Cryptopimpla altipes (Holmgren, 1860)}

\section{Nomenclature:}

Lissonota altipes Holmgren, 1860 
Distribution: England, Scotland

Notes: added by Brock (in prep.). Removed from synomymy with quadrilineata by Brock (in prep.).

\section{Cryptopimpla anomala (Holmgren, 1860)}

Nomenclature:

Lissonota anomala Holmgren, 1860

Distribution: England, Scotland, Wales

Notes: added by Brock (in prep.)

\section{Cryptopimpla arvicola (Gravenhorst, 1829)}

Nomenclature:

Lissonota arvicola Gravenhorst, 1829

brachycentra (Gravenhorst, 1829, Lissonota)

kaisdii (Kiss, 1929, Arenetra)

Distribution: England

Cryptopimpla calceolata (Gravenhorst, 1829)

Nomenclature:

Phytodietus calceolatus Gravenhorst, 1829

leptogaster (Holmgren, 1860, Lissonota)

Distribution: England, Scotland

Cryptopimpla caligata (Gravenhorst, 1829)

Nomenclature:

Lissonota caligata Gravenhorst, 1829

Distribution: England, Ireland

Cryptopimpla errabunda (Gravenhorst, 1829)

Nomenclature:

Phytodietus errabundus Gravenhorst, 1829

Distribution: England, Ireland 
Cryptopimpla hertrichi Heinrich, 1952

Distribution: England, Scotland

Notes: added by Brock (in prep.)

Cryptopimpla quadrilineata (Gravenhorst, 1829)

Nomenclature:

Tryphon quadrilineatus Gravenhorst, 1829

blanda (Gravenhorst, 1829, Phytodietus)

hungarica (Szépligeti, 1899, Xenacis)

vaga (Szépligeti, 1899, Lissonota)

Distribution: England, Wales

Genus Lissonota Gravenhorst, 1829

Nomenclature:

LAMPRONOTA Curtis, 1832

STILBONOTA Stephens, 1835

MENISCUS Schiødte, 1839

ASYNIDA Gistel, 1848

AMERSIBIA Förster, 1869

BATHYCETES Förster, 1869

BOTHYNOPHRYS Förster, 1869

ENSIMUS Förster, 1869

OPISORHYSSA Kriechbaumer, 1890

ANARTHRONOTA Schmiedeknecht, 1900

CAMPOCINETA Schmiedeknecht, 1900

ECHTHRODOCA Schmiedeknecht, 1900

PIMPLOPTERUS Ashmead, 1900

ADELOPIMPLA Schulz, 1906

LOPHANTIUM Clément, 1925

GIBBONOTA Heinrich, 1937

LOXONOTA Aubert, 1993 
Notes: Contra Brock (in prep.), subgenera are not employed as there is little justification for their use and some 'subgenera' seem to be of use only in Europe, whereas there is a great variety of species in the wider world referred to Lissonota ( Lissonota) by default.

species of Lissonota excluded from British and Irish list:

[bilineata Gravenhorst, 1829] J.P. Brock (pers. comm.) has not seen any authentic British material of this species.

[funebris Habermehl, 1923] Only known in Britain from L. Carr material (supposedly from Lichfield) and hence should have been excluded from the previous checklist (J.P. Brock, pers. comm.). See Perkins (1953) and Shaw (2003) for discussions on the inadmissability of species to the British list that are only represented by Carr's material.

[impressor Gravenhorst, 1829; syn. basalis Brischke, 1865] Identified by Morley (1908) and Aubert (1978) as impressor Grav., based on Morley's interpretation of Thomson's interpretation; the species involved is apparently undescribed (J.P. Brock, pers. comm.).

\section{Lissonota accusator (Fabricius, 1793)}

\section{Nomenclature:}

Ichneumon accusator Fabricius, 1793

segmentator misident.

rusticator (Thunberg, 1824, Ichneumon)

humeralis (Zetterstedt, 1838, Tryphon) preocc.

unicincta Holmgren, 1860

thomsoni Schmiedeknecht, 1900

nigricoxa Strobl, 1902

accusatrix Schulz, 1906

segmentellator Aubert, 1967

Distribution: England, Ireland

Notes: Brock (in prep.) does not explain his use of rusticator as the valid name for the species Horstmann (2001a) refers to as accusator, so the latter is followed here. The name accusator has often been applied to Cylloceria melancholica (Gravenhorst).

\section{Lissonota admontensis Strobl, 1902}

\section{Nomenclature:}

alpina Strobl, 1902 
praebellator Aubert, 1967

Distribution: England, Scotland

Notes: added by Brock (in prep.)

Lissonota anomala Holmgren, 1860

Distribution: Ireland

Lissonota antennalis Thomson, 1877

Distribution: England, Wales

Notes: added by Brock (in prep.)

Lissonota argiola Gravenhorst, 1829

Nomenclature:

eximia Habermehl, 1918

Distribution: England, Scotland, Wales, Ireland

Lissonota biguttata Holmgren, 1860

Nomenclature:

femorata Holmgren, 1860

crassipes Thomson, 1877

Distribution: England, Ireland

\section{Lissonota buccator (Thunberg, 1824)}

Nomenclature:

Ichneumon buccator Thunberg, 1824

varicoxa Thomson, 1887

iridipennis Kriechbaumer, 1900

Distribution: England, Scotland

Notes: There is some doubt about the correct name of this speceis as it is not certain which species Thunberg's type belongs to within the buccator group of species (Brock, in prep.); distribution data from Horstmann (2003b); Brock (in prep.) states that Irish records require confirmation. 
Lissonota canaliculata (Szépligeti, 1899)

Nomenclature:

Meniscus canaliculatus Szépligeti, 1899

pimplator misident.

flavipes Lucas, 1849

Distribution: England

Notes: added by Brock (in prep.)

Lissonota carbonaria Holmgren, 1860

Nomenclature:

melania Holmgren, 1860

artemisiae Tschek, 1871

Distribution: England, Scotland

Notes: Brock (in prep.) states that Irish records require confirmation.

Lissonota clypealis Thomson, 1877

Nomenclature:

albobarbata Strobl, 1902

Distribution: England, Scotland, Ireland

Lissonota clypeator (Gravenhorst, 1820)

Nomenclature:

Ichneumon clypeator Gravenhorst, 1820

cylindrator misident.

?coccinea (Christ, 1791, Ichneumon) preocc.

unicornis Strobl, 1902

nigrescens Constantineanu, 1929

spectabilis Schmiedeknecht, 1935

magna Heinrich, 1952

Distribution: England, Scotland, Ireland 
Lissonota coracina (Gmelin, 1790)

Nomenclature:

Ichneumon coracinus Gmelin, 1790

bellator (Gravenhorst, 1807, Ichneumon) preocc.

tricoloria (Thunberg, 1824, Ichneumon)

irrigua Thomson, 1888

bellatrix Schulz, 1906

meridionalis Seyrig, 1928

Distribution: England, Scotland, Ireland

Lissonota cruentator (Panzer, 1809)

Nomenclature:

Alomya cruentator Panzer, 1809

insignita Gravenhorst, 1829

verberans Gravenhorst, 1829

cruentatrix (Schulz, 1906, Alomya)

rufifemur Kiss, 1926

szepligeti Kiss, 1926

Distribution: England, Ireland

Lissonota culiciformis Gravenhorst, 1829

Nomenclature:

lateralis Gravenhorst, 1829

cruenta Vollenhoven, 1858

assimilis Brischke, 1880

sziladyi Kiss, 1926

Distribution: England, Wales

Notes: added by Brock (in prep.)

\section{Lissonota deversor Gravenhorst, 1829}

Distribution: England 
Lissonota digestor (Thunberg, 1824)

Nomenclature:

Ichneumon digestor Thunberg, 1824

vocator (Thunberg, 1824, Ichneumon)

hians Thomson, 1877

Distribution: England, Scotland

Lissonota distincta Bridgman, 1889

Distribution: England

Lissonota dubia Holmgren, 1856

Nomenclature:

jugorum (Strobl, 1903, Mesoleius)

duplanae (Heinrich, 1937, Gibbonota)

Distribution: England, Scotland

Notes: Brock (in prep.) states that Irish records require confirmation.

Lissonota erythrina Holmgren, 1860

Nomenclature:

pusilla Habermehl, 1918

Distribution: Scotland

Notes: added by Brock (in prep.)

Lissonota fletcheri Bridgman, 1882

Distribution: England

Notes: Brock (in prep.) states that Irish records require confirmation.

Lissonota folii Thomson, 1877

Nomenclature:

transversa Bridgman, 1889

areolata (Kiss, 1924, Clistopyga) preocc.

Distribution: England, Ireland 


\section{Lissonota freyi (Hellén, 1915)}

Nomenclature:

Meniscus freyi Hellén, 1915

tuberculata (Hellén, 1915, Meniscus)

sesiae Habermehl, 1918

Distribution: England

Notes: added by Brock (in prep.)

\section{Lissonota frontalis (Desvignes, 1856)}

Nomenclature:

Lampronota frontalis Desvignes, 1856

canaliculata misident.

sulcator (Morley, 1908, Meniscus)

Distribution: England, Scotland

\section{Lissonota fulvipes (Desvignes, 1856)}

Nomenclature:

Lampronota fulvipes Desvignes, 1856

piffardi (Morley, 1908, Meniscus)

Distribution: England

\section{Lissonota fundator (Thunberg, 1824)}

\section{Nomenclature:}

Ichneumon fundator Thunberg, 1824

sulphurifera Gravenhorst, 1829

rimator Thomson, 1877

affinis (Szépligeti, 1899, Meniscus) preocc.

caudata (Szépligeti, 1899, Meniscus)

ruficoxis Schmiedeknecht, 1900

nigricoxis Pfankuch, 1920 preocc., unavailable

Distribution: England, Scotland, Ireland 
Lissonota genator Aubert, 1972

Distribution: Scotland

Notes: added by Brock (in prep.)

Lissonota gracilenta Holmgren, 1860

Distribution: England, Scotland

Notes: added by Brock (in prep.)

Lissonota gracilipes Thomson, 1877

Distribution: England

Notes: added by Brock (in prep.)

Lissonota halidayi Holmgren, 1860

Distribution: England

Lissonota histrio (Fabricius, 1798)

Nomenclature:

Banchus histrio Fabricius, 1798

marginator (Fabricius, 1804, Bassus) synonymy by Horstmann (2001a)

parallela Gravenhorst, 1829

dioszeghyi (Kiss, 1924, Syzeuctus)

nigrobasalis Constantineanu \& Pisica, 1960

Distribution: England, Scotland, Ireland

\section{Lissonota impressor Gravenhorst, 1829}

Nomenclature:

basalis Brischke, 1865

nigricoxis Ulbricht, 1913 preocc.

humerella Habermehl, 1918 preocc.

Distribution: England

Notes: added by Brock (in prep.) 
Lissonota linearis Gravenhorst, 1829

Nomenclature:

varicornis (Schmiedeknecht, 1900, Campocineta)

incerta Habermehl, 1918

Distribution: England

Notes: Brock (in prep.) states that Irish records require confirmation.

\section{Lissonota lineata Gravenhorst, 1829}

Nomenclature:

flavovariegatus (Lucas, 1849, Mesoleptus) synonymy by Horstmann (1997)

Distribution: England, Wales

\section{Lissonota lineolaris (Gmelin, 1790)}

Nomenclature:

Ichneumon lineolaris Gmelin, 1790

catenator (Panzer, 1804, Ichneumon)

gladiator (Thunberg, 1824, Ichneumon) preocc.

mammillator (Thunberg, 1824, Ichneumon)

signator (Thunberg, 1824, Ichneumon)

excavator (Zetterstedt, 1838, Tryphon)

facialis (Desvignes, 1862, Ephialtes)

Distribution: England, Scotland, Ireland, Isle of Man

\section{Lissonota luffiator Aubert, 1969}

Distribution: England

Notes: added by Brock (in prep.)

\section{Lissonota maculata Brischke, 1865}

Nomenclature:

affinis Brischke, 1865

Distribution: England

Notes: Brock (in prep.) states that Irish records require confirmation. 
Lissonota magdalenae Pfankuch, 1921

Nomenclature:

vernalis Roman, 1925

Distribution: England, Scotland, Ireland

Notes: added by Stelfox (1932); recorded originally from Ireland and therefore not listed in Fitton (1978), then from Scotland by Ashmole et al. (1983).

\section{Lissonota mutator Aubert, 1969}

Distribution: England

Notes: distribution data from Shaw (1999)

Lissonota nigridens Thomson, 1889

Distribution: England, Scotland, Ireland

Lissonota nitida Gravenhorst, 1829

Nomenclature:

agnata Gravenhorst, 1829

rhenana Ulbricht, 1916 unavailable

lissonotoides (Habermehl, 1917, Meniscus)

Distribution: England

Lissonota obsoleta Bridgman, 1889

Distribution: England

Lissonota palpalis Thomson, 1889

Nomenclature:

oudemansi Smits

exareolata (Habermehl, 1923, Meniscus)

inareolata (Kiss, 1824, Meniscus) preocc.

Distribution: England, Ireland 
Lissonota palpator Aubert, 1969

Nomenclature:

parasitellae Horstmann, 2003 synonymy by Brock (in prep.)

errabunda misident. (Horstmann 2003b)

Distribution: England, Scotland

Notes: added by Horstmann (2003b)

Lissonota picticoxis Schmiedeknecht, 1900

Distribution: England, Scotland

Notes: added by Brock (in prep.)

Lissonota pimplator (Zetterstedt, 1838)

Nomenclature:

Tryphon pimplator Zetterstedt, 1838

Distribution: Scotland

Notes: added by Aubert (1978); Morley's (Morley 1908) pimplator refers to canaliculata (Brock in prep.).

Lissonota pleuralis Brischke, 1880

Nomenclature:

strigifrons Schmiedeknecht, 1900

Distribution: England

Notes: added by Aubert (1978)

Lissonota proxima Fonscolombe, 1854

Nomenclature:

varipes (Desvignes, 1856, Lampronota)

commixta Holmgren, 1860

lapponica Holmgren, 1860

opacula Szépligeti, 1899

variipes Dalla Torre, 1901

Distribution: England, Ireland 
Lissonota punctiventrator Aubert, 1977

Nomenclature:

punctiventris misident.

Distribution: England, Scotland, Wales

Notes: added by Aubert (1978)

Lissonota punctiventris Thomson, 1877

Nomenclature:

?errabunda Holmgren

Distribution: England, Scotland

Notes: added by Brock (in prep.)

Lissonota quadrinotata Gravenhorst, 1829

Nomenclature:

leucogona Gravenhorst, 1829

carinifrons Thomson, 1877

Distribution: England, Wales

Lissonota saturator (Thunberg, 1824)

Nomenclature:

Ichneumon saturator Thunberg, 1824

pubescens (Zetterstedt, 1838, Bassus)

vicina Holmgren, 1860

basalis Thomson, 1889 preocc.

mutanda Schmiedeknecht, 1900

Distribution: England, Scotland, Wales

Notes: Brock (in prep.) states that Irish records require confirmation.

\section{Lissonota semirufa (Desvignes, 1856)}

Nomenclature:

Lampronota semirufa Desvignes, 1856

Distribution: England, Scotland 
Notes: some distribution data from Horstmann (2004c)

\section{Lissonota setosa (Geoffroy, 1785)}

Nomenclature:

Ichneumon setosus Geoffroy, 1785

enervator (Fabricius, 1793, Ichneumon) preocc.

cryptator (Thunberg, 1824, Ichneumon)

renovator (Thunberg, 1824, Ichneumon)

nigra (Szépligeti, 1914, Odinophora)

Distribution: England

\section{Lissonota silvatica Habermehl, 1918}

Nomenclature:

palpator Aubert, 1969

Distribution: England

Notes: added by Aubert (1978)

\section{Lissonota stigmator Aubert, 1972}

Distribution: England, Scotland

\section{Lissonota subaciculata Bridgman, 1886}

Nomenclature:

nitida Bridgman, 1886 preocc.

Distribution: England

Notes: Brock (in prep.) states that Irish records require confirmation.

\section{Lissonota tenerrima Thomson, 1877}

\section{Nomenclature:}

variabilis Holmgren, 1860 synonymy by Brock (in prep.)

fracta Taschenberg, 1863

rufomedia Bridgman, 1886

trochanterata Bridgman, 1889 preocc.

trochanteralis Dalla Torre, 1901 
procera Pfeffer, 1913

bimaculata Constantineanu \& Ciochia, 1968 preocc.

Distribution: England, Scotland, Ireland

Lissonota trochanterator Aubert, 1972

Distribution: England

Lissonota versicolor Holmgren, 1860

Nomenclature:

formosa Bridgman, 1888

coxata Smits

rufithorax Habermehl, 1918

Distribution: England, Ireland

Lissonota sp. A

Distribution: England

Notes: added by Brock (in prep.)

Lissonota sp. C

Distribution: England

Notes: added by Brock (in prep.)

Lissonota sp. D

Distribution: England, Scotland, Wales

Notes: added by Brock (in prep.)

Lissonota sp. P

Distribution: Scotland

Notes: added by Brock (in prep.)

\section{Lissonota sp. S}

Distribution: England 
Notes: added by Brock (in prep.); impressor misident.

\section{Lissonota sp. SI}

Distribution: England

Notes: added by Brock (in prep.)

\section{Lissonota sp. V}

Nomenclature:

basalis Brischke, 1865]

Distribution: England

Notes: added by Brock (in prep.)

\section{Genus Syzeuctus Förster, 1869}

Nomenclature:

DICERATOPS Förster, 1869

SYZEUCTA Thomson, 1889

Notes: Species of Syzeuctus excluded from the British and Irish list:

[irrisorius (Rossius, 1794, Ichneumon)] Brock (in prep.) found no British specimens of irrisorius.

Syzeuctus bicornis (Gravenhorst, 1829)

Nomenclature:

Lissonta bicornis Gravenhorst, 1829

Distribution: England

Syzeuctus fuscator (Panzer, 1809)

Nomenclature:

Ophion fuscator Panzer, 1809

maculatorius (Fabricius, 1787, Ichneumon) preocc.

bicolor Szépligeti, 1899

rufipes Kiss, 1933

Distribution: England, Wales 
Tribe BANCHINI Wesmael, 1845

Genus Banchus Fabricius, 1798

Notes: Distribution data from Fitton (1985a) and the NMS.

\section{Banchus crefeldensis Ulbricht, 1916}

Nomenclature:

croaticus Hensch, 1928

Distribution: Scotland, Ireland

\section{Banchus dilatatorius (Thunberg, 1824)}

Nomenclature:

Ichneumon dilatatorius Thunberg, 1824

variegator misident.

acuminator (Fabricius, 1787, Ichneumon)

compressus (Fabricius, 1787, Ichneumon)

sibiricus Meyer, 1927

Distribution: England, Scotland

\section{Banchus falcatorius (Fabricius, 1775)}

\section{Nomenclature:}

Ichneumon falcatorius Fabricius, 1775

variegator (Fabricius, 1775, Ichneumon)

tricolor (Schrank, 1776, Ichneumon)

intersectus (Geoffroy, 1785, Ichneumon)

aries (Christ, 1791, Ichneumon)

notatorius (Olivier, 1792, Ichneumon) preocc.

histrio (Schrank, 1802, Ichneumon) preocc.

labiatus (Schrank, 1802, Ichneumon)

falcator Fabricius, 1804

luteofasciatus Ulbricht, 1911 unavailable

nobilitator Morley, 1915

sanguinator Meyer, 1922 
lavrovi Meyer, 1927

nigromarginatus Constantineanu \& Pisica, 1960

propitius Kuslitzky, 1979

Distribution: England

Banchus hastator (Fabricius, 1793)

Nomenclature:

Ichneumon hastator Fabricius, 1793

pungitor (Thunberg, 1824, Ichneumon)

reticulator (Thunberg, 1824, Ichneumon)

femoralis Thomson, 1897

kolosovi Meyer, 1925

Distribution: England, Scotland, Ireland

Banchus moppiti Fitton, 1985

Notes: added by Fitton (1985a); known in Britain only from an unlabelled specimen from Desvignes's collection.

Banchus palpalis Ruthe, 1859

Nomenclature:

monileatus misident.

groenlandicus Aurivilius, 1890

Distribution: England, Scotland, Ireland

Notes: added by Fitton (1985a)

\section{Banchus pictus Fabricius, 1798}

Nomenclature:

cultratus (Gmelin, 1790, Ichneumon) preocc.

mutillatus (Christ, 1791, Ichneumon) preocc.

bipunctatus Hensch, 1928

zagoriensis Hensch, 1928

Distribution: England, Wales 
Banchus volutatorius (Linnaeus, 1758)

Nomenclature:

Ichneumon volutatorius Linnaeus, 1758

venator (Linnaeus, 1758, Ichneumon)

umbellatarum (Schrank, 1786, Ichneumon)

certator (Thunberg, 1824, Ichneumon)

monileatus Gravenhorst, 1829

farrani Curtis, 1836

moniliatus Marshall, 1872

alticola Schmiedeknecht, 1910

calcaratus Szépligeti, 1910

obscurus Meyer, 1926

Distribution: England, Scotland, Wales, Ireland

\section{Genus Exetastes Gravenhorst, 1829}

Nomenclature:

LEPTOBATUS Gravenhorst, 1829

RHIMPHALEA Förster, 1869

SEMNOPHRYS Förster, 1869

ALLEXETASTES Kokujev, 1904

\section{Exetastes adpressorius (Thunberg, 1824)}

Nomenclature:

Ichneumon adpressorius Thunberg, 1824

guttatorius Gravenhorst, 1829

tristis Gravenhorst, 1829

procera Kriechbaumer, 1894 unavailable

guttifer Thomson, 1897

medianus Szépligeti, 1898

albopictus Aubert, 1959

albopictor Aubert, 1972

Distribution: England, Scotland, Ireland 


\section{Exetastes atrator (Forster, 1771)}

Nomenclature:

$$
\begin{aligned}
& \text { Ichneumon atrator Forster, } 1771 \\
& \text { cinctipes (Retzius, 1783, Ichneumon) } \\
& \text { junci (Geoffroy, 1785, Ichneumon) } \\
& \text { osculatorius (Fabricius, 1787, Ichneumon) } \\
& \text { obscurator (Gmelin, 1790, Ichneumon) } \\
& \text { clavator (Fabricius, 1793, Ichneumon) preocc. } \\
& \text { tarsator (Fabricius, 1804, Ophion) } \\
& \text { sinuatorius (Thunberg, 1824, Ichneumon) }
\end{aligned}
$$

Distribution: England, Scotland, Ireland

\section{Exetastes calobatus Gravenhorst, 1829}

Nomenclature:

calobates Dalla Torre, 1901

Distribution: England

\section{Exetastes femorator Desvignes, 1856}

Distribution: England

Notes: Brock (in prep.) states that Irish records require confirmation.

\section{Exetastes fornicator (Fabricius, 1781)}

\section{Nomenclature:}

Ichneumon fornicator Fabricius, 1781

exapansor (Thunberg, 1824, Ichneumon)

punctulatus Kokujev, 1905

Distribution: England, Ireland

\section{Exetastes illusor Gravenhorst, 1829}

Nomenclature:

minor Szépligeti, 1901

annulatus Habermehl, 1927

?geniculosus Holmgren, 1860 
Distribution: England, Scotland, Ireland, Isle of Man

Exetastes illyricus Strobl, 1904

Distribution: England

Notes: added by Brock (in prep.)

Exetastes laevigator (Villers, 1789)

Nomenclature:

Ichneumon laevigator Villers, 1789

cothurnatus (Gravenhorst, 1807, Ichneumon) preocc.

incurvator (Thunberg, 1824, Ichneumon)

alpinus Kriechbaumer, 1888

puberulus (Szépligeti, 1898,)

levigator Dalla Torre, 1901

similis Kokujev, 1905

nigriventris Meyer, 1927

Distribution: England

\section{Exetastes maurus Desvignes, 1856}

Nomenclature:

facialis Desvignes, 1856

benoisti Seyrig, 1926

melanopus Meyer, 1927

croaticus Hensch, 1928

Distribution: England

Exetastes nigripes Gravenhorst, 1829

Distribution: England, Scotland, Wales

Notes: Brock (in prep.) states that Irish records require confirmation.

Exetastes tibialis Pfankuch, 1921

Distribution: England

Notes: added by Brock (in prep.) 
Genus Rynchobanchus Kriechbaumer, 1894

Rynchobanchus flavopictus Heinrich, 1937

Distribution: England

Notes: added by Fitton (1987)

Tribe GLYPTINI Cushman \& Rohwer, 1920

Genus Apophua Morley, 1913

Apophua bipunctoria (Thunberg, 1824)

Nomenclature:

Ichneumon bipunctorius Thunberg, 1824

cubitoria (Thunberg, 1824, Ichneumon)

flavolineata (Gravenhorst, 1829, Glypta)

baltica (Habermehl, 1926, Glypta)

Distribution: England, Scotland, Wales, Ireland, Isle of Man

Apophua cicatricosa (Ratzeburg, 1848)

Nomenclature:

Glypta cicatricosa Ratzeburg, 1848

crenulata (Thomson, 1889, Glypta)

Apophua evanescens (Ratzeburg, 1848)

Nomenclature:

Glypta evanescens Ratzeburg, 1848

albifrons (Holmgren, 1856, Glypta)

Distribution: England, Scotland, Ireland

Apophua genalis (Möller, 1883)

Nomenclature:

Glypta genalis Möller, 1883

superba (Hellén, 1915, Glypta)

Distribution: Ireland 


\section{Genus Diblastomorpha Förster, 1869}

Notes: Reinstated as a valid genus, from synonymy under Glypta, by Watanabe and Maeto (2013).

\section{Diblastomorpha cylindrator (Fabricius, 1787)}

Nomenclature:

Ichneumon cylindrator Fabricius, 1787

erythrogaster Lucas, 1849

bicornis Boie, 1850

bicornis Desvignes, 1856 preocc.

corniculata Brischke, 1865

elegans Vollenhoven, 1873

ephippigera Kriechbaumer, 1895

ruficornis Szépligeti, 1898 preocc.

paleanae Kriechbaumer, 1900

szepligetii Dalla Torre, 1901

cylindatrix (Schulz, 1906, Lissonota)

abundans (Schmiedeknecht, 1934, Diblastomorpha)

Distribution: England, Scotland, Wales, Ireland

Diblastomorpha rostrata Holmgren, 1860

Distribution: England, Ireland, Isle of Man

Notes: Treated as a valid species, rather than a synonym of cylindrator, by Brock (in prep.).

Genus Glypta Gravenhorst, 1829

Nomenclature:

CONOBLASTA Förster, 1869

FOVEOGLYPTA Hellén, 1915

Notes: Species of Glypta excluded from the British and Irish list: 
[schneideri Krieger, 1897] Recorded from Ireland by Johnson (1921) (and not listed by Fitton 1978 as not known from Britain), but J.P. Brock has seen no authentic British or Irish material.

[scalaris Gravenhorst, 1829] Misidentified scalaris are described by Brock (in prep.) as Glypa sp. PU.

[teres Gravenhorst, 1829] British specimens identified as teres have proved to be misidentified bifoveolata (Brock, in prep.).

\section{Glypta bifoveolata Gravenhorst, 1829}

Nomenclature:

setosa Roman, 1909

Distribution: England, Scotland, Wales, Ireland

\section{Glypta ceratites Gravenhorst, 1829}

Distribution: England, Scotland, Ireland, Isle of Man

\section{Glypta consimilis Holmgren, 1860}

Nomenclature:

brevicornis Rudow, 1883

parvicornuta Bridgman, 1886

xanthognatha Thomson, 1889

berolinae (Strand, 1918, Conoblasta)

Distribution: England, Scotland, Ireland

Glypta elongata Holmgren, 1860

Distribution: England, Scotland, Wales, Ireland

\section{Glypta extincta Ratzeburg, 1852}

\section{Nomenclature:}

nigriventris Thomson, 1889

Distribution: England, Scotland 


\section{Glypta femorator Desvignes, 1856}

Nomenclature:

filicornis Thomson, 1889

femoratrix Schulz, 1906

elegantula Hellén, 1915

obscurata Kiss, 1929

pellucida Schmiedeknecht, 1935

triangularis Schmiedeknecht, 1935

curvicoxa Kuslitzky, 1977

Distribution: England, Scotland, Ireland

Glypta fronticornis Gravenhorst, 1829

Nomenclature:

dispar Schiødte, 1839 synonymy by Horstmann (2004a)

Distribution: England, Scotland, Ireland

\section{Glypta haesitator Gravenhorst, 1829}

Nomenclature:

haesitatrix Schulz, 1906

australis (Hedwig, 1959, Lycorina)

Distribution: England, Scotland, Ireland

\section{Glypta incisa Gravenhorst, 1829}

Distribution: England, Wales

\section{Glypta lapponica Holmgren, 1860}

Nomenclature:

annulata Bridgman, 1890

areolaris Hellén, 1915

nigricoxa (Kokujev, 1927, Conoblasta)

alpina (Heinrich, 1949, Conoblasta)

Distribution: England, Scotland, Ireland 
Glypta lineata Desvignes, 1856

Distribution: England

Glypta longicauda Hartig, 1838

Nomenclature:

nigrotrochanterata Strobl, 1902

Distribution: Ireland

Glypta longispinis (Gmelin, 1790)

Nomenclature:

Ichneumon longispinis Gmelin, 1790

provincialis Fonscolombe, 1854

rubicunda Bridgman, 1890

algerica Habemehl, 1917

zangezurica Kuslitzky, 1974

Distribution: England

Glypta mensurator (Fabricius, 1775)

Nomenclature:

Ichneumon mensurator Fabricius, 1775

lugubrina Holmgren, 1860

macropyga Hellén, 1915

heydeni Habemehl, 1917

jaroslavensis Shestakov, 1927

Distribution: England, Scotland, Wales, Ireland

Glypta microcera Thomson, 1899

Nomenclature:

segrex Kokujev, 1913

Distribution: England

Notes: added by Aubert (1978) 
Glypta monoceros Gravenhorst, 1829

Distribution: England, Scotland, Wales, Ireland

Glypta nigricornis Thomson, 1899

Nomenclature:

rufipes Brischke, 1865 preocc.

brischkei Dalla Torre, 1901 preocc.

papyri Speiser, 1908

Distribution: England, Scotland, Ireland

Notes: added by Aubert (1978)

\section{Glypta nigrina Desvignes, 1856}

Nomenclature:

flavipes Desvignes, 1856

ruficeps Desvignes, 1856

fractigena Thomson, 1889

obscura Pfankuch, 1924 unavailable

clypeodentata Bauer, 1959

habermani Ozols, 1959

Distribution: England, Scotland, Ireland

Glypta nigrotrochanterator Strobl, 1902

Nomenclature:

mensurator misident.

longicauda misident.

Distribution: England, Scotland, Ireland

Notes: added by Brock (in prep.)

Glypta parvicaudata Bridgman, 1889

Distribution: England, Scotland, Ireland 
Glypta pedata Desvignes, 1856

Distribution: England

Glypta pictipes Taschenberg, 1863

Distribution: England, Scotland, Ireland

Notes: added by Aubert (1978)

Glypta punctifrons Bridgman, 1890

Distribution: Scotland

Notes: Synonymised under scalaris by Aubert (1978) but this synonymy rejected by Brock (in prep.). Known only from the male type specimen, punctifrons is essentially an unknown species within Glypta.

Glypta resinanae Hartig, 1838

Nomenclature:

arreptans Hellén, 1915

summimontis Heinrich, 1953

Distribution: England

Glypta rufata Bridgman, 1887

Distribution: England, Ireland

Glypta sculpturata Gravenhorst, 1829

Nomenclature:

macrura Habermehl, 1918

rufoclypeata Kiss, 1924

Distribution: England, Ireland

Glypta scutellaris Thomson, 1899

Distribution: England, Scotland

Notes: added by Brock (in prep.) 
Glypta similis Bridgman, 1886

Nomenclature:

rufipes Thomson, 1889 preocc.

thomsonii Dalla Torre, 1901

thomsoni Strobl, 1902 preocc.

Distribution: England, Ireland

Glypta tenuicornis Thomson, 1889

Nomenclature:

pygmaea Shestakov, 1927

Distribution: England

Glypta trochanterata Bridgman, 1886

Distribution: England, Scotland, Ireland

Glypta ulbrichti Habermehl, 1926

Distribution: England

Notes: added by Brock (in prep.)

Glypta vulnerator Gravenhorst, 1829

Nomenclature:

vulneratrix Schulz, 1906

monstrosa Hellén, 1915

Distribution: England, Scotland, Ireland

Glypta woerzi (Hedwig, 1952)

Nomenclature:

Conoblasta woerzi Hedwig, 1952

Distribution: England

Notes: added by Brock (in prep.) 


\section{Glypta sp. PA}

Distribution: England

Notes: added by Brock (in prep.)

\section{Glypta sp. PU}

Nomenclature:

scalaris misident.

Distribution: Ireland

Notes: added by Brock (in prep.)

\section{Genus Teleutaea Förster, 1869}

Nomenclature:

HOPLITOPHRYS Förster, 1869

TELEUTEA Thomson, 1889

Teleutaea brischkei (Holmgren, 1860)

Nomenclature:

Glypta brischkei Holmgren, 1860

Distribution: England

Notes: added by Aubert (1978); specimens also in NMS and Horniman Museum

\section{Campopleginae}

\section{Subfamily CAMPOPLEGINAE Förster, 1869}

Notes: Many of the species new to the fauna have been identified by K. Horstmann from material in NMS and BMNH. Additional references are given.

Genus Alcima Förster, 1869

\section{Alcima orbitale (Gravenhorst, 1829)}

Nomenclature:

Campoplex orbitalis Gravenhorst, 1829

alboscutellare (Thomson, 1887, Casinaria) 
carinata (Kriechbaumer, 1898, Casinaria)

Distribution: England, Wales

Notes: Listed as Casinaria orbitalis by Fitton (1978).

Genus Bathyplectes Förster, 1869

Nomenclature:

CANIDIA Holmgren, 1860

RHEXINEURA Förster, 1869

BIOLYSIA Schmiedeknecht, 1907

BATHYPIESTA Aubert, 1979

Bathyplectes anurus (Thomson, 1887)

Nomenclature:

Canidia anura Thomson, 1887

Distribution: England, Ireland

Bathyplectes balteatus (Thomson, 1887)

Nomenclature:

Canidia balteata Thomson, 1887

trisculptus (Habermehl, 1926, Canidia)

Distribution: England

Notes: NMS, det. Horstmann, added here

Bathyplectes curculionis (Thomson, 1887)

Nomenclature:

Canidia curculionis Thomson, 1887

carthaginiensis (Smits van Burgst, 1913, Canidia) synonymy by Horstmann (2009d)

Distribution: England, Ireland

Notes: added by Horstmann (1974); omitted by Fitton (1978) 
Bathyplectes exiguus (Gravenhorst, 1829)

Nomenclature:

Campoplex exiguus Gravenhorst, 1829

subcinctus (Gravenhorst, 1829, Campoplex)

Distribution: England, Scotland, Ireland

Bathyplectes immolator (Gravenhorst, 1829)

Nomenclature:

Campoplex immolator Gravenhorst, 1829

marginellus (Thomson, 1887, Nepiesta)

Distribution: Ireland

Bathyplectes infernalis (Gravenhorst, 1820)

Nomenclature:

Ichneumon infernalis Gravenhorst, 1820

infernalis (Gravenhorst, 1829, Mesoleptus) preocc.

tristis (Gravenhorst, 1829, Campoplex)

trochantellus (Thomson, 1887, Canidia)

Distribution: England, Ireland

Bathyplectes quinqueangularis (Ratzeburg, 1852)

Nomenclature:

Campoplex quinqueangularis Ratzeburg, 1852

Distribution: Ireland

Notes: added by Horstmann (1974); omitted by Fitton (1978)

Bathyplectes rostratus (Thomson, 1887)

Nomenclature:

Canidia rostrata Thomson, 1887

Distribution: Scotland, Ireland 
Bathyplectes rufipes Horstmann, 1974

Distribution: England

Notes: NMS, det. Horstmann, added here

Bathyplectes tibiator (Gravenhorst, 1820)

Nomenclature:

Ichneumon tibiator Gravenhorst, 1820

corvinus (Thomson, 1887, Canidia)

Distribution: England

Notes: $\mathrm{BMNH}$, added here

\section{Genus Callidora Förster, 1869}

Nomenclature:

PANTROPA Förster, 1869

NEOCALLIDORA Ozols, 1966

Callidora analis (Gravenhorst, 1829)

Nomenclature:

Campoplex analis Gravenhorst, 1829

Distribution: England

Notes: NMS, det. Horstmann, added here

Genus Campoletis Förster, 1869

Nomenclature:

ANILASTUS Förster, 1869

ECPHORA Förster, 1869 preocc.

ANILASTA Thomson, 1877

ECPHOROPSIS Ashmead, 1900

Campoletis agilis (Holmgren, 1860)

Nomenclature:

Sagaritis agilis Holmgren, 1860

Distribution: Ireland 


\section{Campoletis annulata (Gravenhorst, 1829)}

Nomenclature:

Campoplex annulatus Gravenhorst, 1829

maculipes (Tschek, 1871, Sagaritis)

trochanterata (Kriechbaumer, 1894, Sagaritis)

nigripes (Seyrig, 1928, Sagaritis) preocc.

Distribution: England, Scotland, Ireland

\section{Campoletis cognata (Tschek, 1871)}

Nomenclature:

Sagaritis cognata Tschek, 1871

Campoletis crassicornis (Tschek, 1871)

Nomenclature:

Sagaritis crassicornis Tschek, 1871

brachycera (Thomson, 1877, Sagaritis)

Distribution: England, Scotland, Ireland

\section{Campoletis dilatator (Thunberg, 1824)}

Nomenclature:

Ichneumon dilatator Thunberg, 1824

mediator (Zetterstedt, 1838, Porizon)

Distribution: Ireland

\section{Campoletis ensator (Gravenhorst, 1829)}

Nomenclature:

Campoplex ensator Gravenhorst, 1829

holmgreni (Tschek, 1871, Sagaritis) synonymy by Horstmann (2000e)

Distribution: England, Scotland, Ireland

\section{Campoletis fasciata (Bridgman, 1888)}

Nomenclature:

Sagaritis fasciata Bridgman, 1888 
Distribution: England

Campoletis femoralis (Gravenhorst, 1829)

Nomenclature:

Campoplex femoralis Gravenhorst, 1829

laticollis (Holmgren, 1860, Sagaritis)

Distribution: Ireland

Campoletis fuscipes (Holmgren, 1856)

Nomenclature:

Campoplex fuscipes Holmgren, 1856

semirufa (Szépligeti, 1916, Omorgus)

Distribution: England, Ireland

Campoletis incisa (Bridgman, 1883)

Nomenclature:

Sagaritis incisa Bridgman, 1883

Distribution: Ireland

\section{Campoletis latrator (Gravenhorst, 1829)}

Nomenclature:

Campoplex latrator Gravenhorst, 1829

assimilis (Gravenhorst, 1829, Campoplex) synonymy by Horstmann (2000e)

mitis (Holmgren, 1860, Sagaritis)

latratrix (Schulz, 1906, Sagaritis)

bicingulata (Szépligeti, 1916, Omorgus)

Distribution: England, Scotland, Ireland, Isle of Man

Notes: Horstmann (2000e) shows that this is not a junior homonym (treated as such in Yu and Horstmann 1997).

Campoletis postica (Bridgman \& Fitch, 1885)

Nomenclature:

Sagaritis postica Bridgman \& Fitch, 1885 
postica (Bridgman, 1886, Sagaritis) preocc.

Distribution: England, Scotland, Ireland

Campoletis punctata (Bridgman, 1886)

Nomenclature:

Sagaritis punctata Bridgman, 1886

Distribution: England, Scotland, Ireland

\section{Campoletis rapax (Gravenhorst, 1829)}

Nomenclature:

Campoplex rapax Gravenhorst, 1829

erythropus (Thomson, 1887, Sagaritis) synonymy by Horstmann (2000e)

curticaudis (Szépligeti, 1916, Omorgus)

Distribution: England, Scotland, Wales, Ireland

Notes: J.F. Perkins, in his curation of the BMNH collection, applied the name rapax Grav. to a species of Hyposoter; Campoletis specimens were under erythropus (Thomson).

\section{Campoletis raptor (Zetterstedt, 1838)}

Nomenclature:

Porizon raptor Zetterstedt, 1838

raptrix (Schulz, 1906, Sagaritis)

dubiosa (Szépligeti, 1916, Omorgus)

rufator Aubert, 1960

\section{Campoletis thomsoni (Roman, 1915)}

Nomenclature:

Sagaritis thomsoni Roman, 1915

Distribution: England, Scotland

Notes: NMS, det. Horstmann, added here 


\section{Campoletis trichoptili (Bauer, 1936)}

Nomenclature:

Sagaritis trichoptili Bauer, 1936

Distribution: England

Notes: NMS, det. Horstmann, added here

\section{Campoletis varians (Thomson, 1887)}

Nomenclature:

Sagaritis varians Thomson, 1887

completa (Szépligeti, 1916, Omorgus)

Distribution: England, Scotland, Wales, Ireland

Notes: added by Johnson (1929); only known originally from Ireland so omitted by Fitton (1978); also specimens in NMS and BMNH, det. K. Horstmann.

\section{Campoletis viennensis (Gravenhorst, 1829)}

Nomenclature:

Campoplex viennensis Gravenhorst, 1829

annulator (Zetterstedt, 1838, Porizon)

vexans (Holmgren, 1860, Limneria)

maculipes (Strobl, 1904, Anilasta)

subdentata (Hellén, 1949, Sagaritopsis)

Distribution: England, Ireland

\section{Campoletis vimmeri (Gregor, 1935)}

Nomenclature:

Sagaritis vimmeri Gregor, 1935

Distribution: Scotland

Notes: NMS, det. Riedel, added here

\section{Campoletis zonata (Gravenhorst, 1829)}

Nomenclature:

Campoplex zonatus Gravenhorst, 1829 
Distribution: England, Scotland, Ireland

\section{Genus Campoplex Gravenhorst, 1829}

Nomenclature:

DIORATICA Förster, 1869

OMORGUS Förster, 1869

OMORGA Förster, 1869

Notes: The Campoplex material in BMNH was sorted and determined by J.F. Perkins and more recently identified by K. Horstmann.

doubtfully placed species of Campoplex:

[arvensis Gravenhorst, 1829 nom. dub.] Listed as Sinophorus arvensis by Fitton (1978), K. Horstmann (pers. comm.) has seen only the type specimen of this species, which remains uninterpreted at present

species of Campoplex excluded from the British and Irish list:

[borealis (Zetterstedt, 1838, Porizon)] All British specimens in NMS were reidentified as C. caloptiliae by Horstmann

[melanostoma (Strobl, 1904, Limneria); syn. anterior Aubert, 1960] On earlier versions of this checklist, C. melanostoma was included based on a reared specimen in NMS, but this was based on a misidentification of $C$. punctulatus.

\section{Campoplex abbreviatus (Brischke, 1880)}

Nomenclature:

Limneria abbreviata Brischke, 1880

\section{Campoplex alticolellae Horstmann, 1980}

Distribution: England, Scotland, Wales

Notes: NMS, det. Horstmann, added here

\section{Campoplex bilobus (Thomson, 1887)}

Nomenclature:

Omorga biloba Thomson, 1887

Distribution: England 
Campoplex brevicornis (Szépligeti, 1916)

Nomenclature:

Omorgus brevicornis Szépligeti, 1916

flavocinctus (Seyrig, 1928, Sagaritis)

Distribution: England

Notes: NMS, det. Horstmann, added here

Campoplex caloptiliae Horstmann, 2013

Distribution: England, Scotland

Notes: added by Horstmann (2013)

Campoplex cingulatus (Brischke, 1880)

Nomenclature:

Limneria cingulata Brischke, 1880

Campoplex continuus (Thomson, 1887)

Nomenclature:

Omorga continua Thomson, 1887

Distribution: England

Notes: The true continuus has been identified by K. Horstmann (specimen in NMS; see note under pyraustae).

Campoplex coracinus (Thomson, 1887)

Nomenclature:

Omorga coracina Thomson, 1887

submarginatus (Bridgman, 1899, Limneria)

Distribution: England

Campoplex crassus Horstmann, 1980

Distribution: England

Notes: NMS, det. Horstmann, added here 


\section{Campoplex cursitans (Holmgren, 1860)}

Nomenclature:

Limneria cursitans Holmgren, 1860

Distribution: England, Ireland

\section{Campoplex deficiens Gravenhorst, 1829}

Nomenclature:

algerica (Habermehl, 1922, Omorga)

Distribution: England

Notes: Listed as Venturia deficiens by Yu and Horstmann (1997) but treated as a species of Campoplex by Horstmann (2000e). Has been misidentified as Campoplex difformis.

\section{Campoplex difformis (Gmelin, 1790)}

Nomenclature:

Ichneumon difformis Gmelin, 1790

lineolatus Ratzeburg, 1844

mutabilis (Holmgren, 1860, Limneria)

Distribution: England, Ireland

Campoplex eudoniae Horstmann \& Yu, 1999

Nomenclature:

rufipes (Bridgman, 1883, Nemeritis) preocc.

ruficoxa (Thomson, 1887, Omorga) preocc.

Distribution: England, Scotland, Ireland

Notes: some distribution data from Horstmann and Yu (1999)

\section{Campoplex faunus Gravenhorst, 1829}

Nomenclature:

xanthocarpus (Szépligeti, 1916, Omorgus)

Distribution: England, Ireland 
Campoplex formosanae Horstmann, 2012

Distribution: England

Notes: NMS, det. Horstmann, added here

Campoplex fusciplica (Thomson, 1887)

Nomenclature:

Omorga fusciplica Thomson, 1887

Campoplex hadrocerus (Thomson, 1887)

Nomenclature:

Omorga hadrocera Thomson, 1887

fasciatus (Bridgman, 1889, Limneria)

Distribution: England, Ireland

Campoplex interruptus Horstmann, 1993

Distribution: England, Scotland

Notes: NMS, det. Horstmann, added here

Campoplex investigator (Habermehl, 1923)

Nomenclature:

Omorga investigator Habermehl, 1923

Distribution: England

Notes: NMS, det. Horstmann, added here

Campoplex jaeckhi (Bauer, 1936)

Nomenclature:

Dioctes jaeckhi Bauer, 1936

Distribution: England

Notes: added by Horstmann (2012a)

Campoplex lugubrinus (Holmgren, 1860)

Nomenclature:

Limneria lugubrina Holmgren, 1860 
pusillus (Szépligeti, 1916, Angitia)

Distribution: England, Scotland, Ireland

Campoplex lyratus (Thomson, 1887)

Nomenclature:

Omorga lyrata Thomson, 1887

Distribution: England, Scotland, Wales, Ireland, Isle of Man

Notes: added by Shaw (1984)

Campoplex melanostictus Gravenhorst, 1829

Distribution: England, Scotland

Campoplex molestus Gravenhorst, 1829

Distribution: England

Campoplex multicinctus Gravenhorst, 1829

Nomenclature:

excentricus (Bauer, 1937, Omorgus) synonymy by Horstmann (2000d)

Distribution: England, Ireland

Campoplex ovatus (Brischke, 1880)

Nomenclature:

Limneria ovata Brischke, 1880

Distribution: England, Scotland

Campoplex procerus (Brischke, 1880)

Nomenclature:

Limneria procera Brischke, 1880

Campoplex psammae (Morley, 1915)

Nomenclature:

Omorga psammae Morley, 1915

Distribution: England, Scotland, Ireland 
Notes: Described as a variety of Omorga (=Campoplex) ensator (Grav., 1829) and listed as Campoplex psammae by Fitton (1978) but not not listed in Yu and Horstmann (1997) and treated, without justification, as a subspecies of ensator by Yu et al. (2012). Horstmann (2012a) regarded this as a valid species.

\section{Campoplex punctipleuris Horstmann, 1980}

Nomenclature:

alhpictus (Pfankuch, 1924, Omorga) unavailable

albipictus Horstmann, 1986 unavailable

Distribution: England, Scotland, Wales

Notes: added by Horstmann (1980a)

\section{Campoplex punctulatus (Szépligeti, 1916)}

\section{Nomenclature:}

Omorgus punctulatus Szépligeti, 1916

Distribution: England, Scotland, Ireland

Notes: BMNH, NMS, det. Horstmann, added here

\section{Campoplex pyraustae Smith, 1931}

\section{Nomenclature:}

continuus misident.

Distribution: England, Scotland, Wales, Ireland, Isle of Man

Notes: Added by Shaw and Aeschliman (1994); Campoplex pyraustae has sometimes been misidentified as continuus (K. Horstmann, pers. comm.).

Campoplex ramidulus (Brischke, 1880)

Nomenclature:

Limneria ramidula Brischke, 1880

Distribution: England

Campoplex raschkiellae Horstmann, 1980

Distribution: England, Scotland

Notes: added by Horstmann (1980a) 
Campoplex restrictor Aubert, 1960

Distribution: England

Notes: added by Shaw (1981)

Campoplex rothii (Holmgren, 1860)

Nomenclature:

Limneria rothii Holmgren, 1860

Distribution: England, Scotland

Campoplex rufipes Gravenhorst, 1829

Nomenclature:

angulatus (Thomson, 1887, Omorga) synonymy by Horstmann (2000d)

Distribution: England, Scotland, Ireland

Campoplex striatus Horstmann, 1985

Distribution: England

Notes: BMNH, det. Horstmann, added here

\section{Campoplex sulcatus Horstmann, 1985}

Distribution: England, Scotland, Isle of Man

Notes: added by Horstmann (1985)

\section{Campoplex tibialis (Szépligeti, 1916)}

Nomenclature:

Nemeritis tibialis Szépligeti, 1916

dioszeghyi (Kiss, 1929, Omorgus)

corsicator Aubert, 1960

Distribution: England, Wales

Notes: BMNH, det. Horstmann, added here; also some Scottish specimens in NMS, tentatively identified as this species by $\mathrm{K}$. Horstmann. 
Campoplex tumidulus Gravenhorst, 1829

Nomenclature:

ensator misident.

nigrifemur (Seyrig, 1928, Omorgus)

rufinator Aubert, 1971

Distribution: England, Scotland, Wales, Ireland, Isle of Man

Notes: Recorded as Campoplex rufinator by Shaw (1984) and Shaw and Aeschliman (1994).

Campoplex tussilaginis Horstmann, 2013

Distribution: England

Notes: added by Horstmann (2013)

Campoplex unicingulatus (Schmiedeknecht, 1909)

Nomenclature:

Omorgus unicingulatus Schmiedeknecht, 1909

Distribution: England, Scotland, Isle of Man

Notes: added by Horstmann (1985)

Campoplex variabilis (Bridgman, 1886)

Nomenclature:

Limneria variabilis Bridgman, 1886

Distribution: England

Campoplex volubilis (Holmgren, 1860)

Nomenclature:

Limneria volubilis Holmgren, 1860

anterior Aubert, 1960]

Distribution: Scotland

Notes: NMS, det. Horstmann, added here 
Genus Casinaria Holmgren, 1859

Nomenclature:

AMORPHOTA Förster, 1869

ANEMPHERES Förster, 1869

CAMPOTREPHUS Förster, 1869

HOROGENES Förster, 1869

NOTHANOMALON Szépligeti, 1905

TROPHOCAMPA Schmiedeknecht, 1907

CASINARIODES Aubert, 1960

Casinaria affinis Tschek, 1871

Distribution: Ireland

Casinaria albipalpis (Gravenhorst, 1829)

Nomenclature:

Campoplex albipalpis Gravenhorst, 1829

Distribution: Ireland

Casinaria ischnogaster Thomson, 1887

Distribution: England, Ireland

Casinaria moesta (Gravenhorst, 1829)

Nomenclature:

Campoplex moestus Gravenhorst, 1829

maesta Dalla Torre, 1901 preocc.

Distribution: England

Notes: added by Horstmann (2000d)

Casinaria morionella Holmgren, 1860

Distribution: England 
Casinaria pallipes Brischke, 1880

Nomenclature:

pallidipes Dalla Torre, 1901

Distribution: England, Ireland

\section{Casinaria petiolaris (Gravenhorst, 1829)}

Nomenclature:

Mesoleptus petiolaris Gravenhorst, 1829

claviventris Holmgren, 1860

Distribution: England

\section{Casinaria tenuiventris (Gravenhorst, 1829)}

Nomenclature:

Campoplex tenuiventris Gravenhorst, 1829

conica (Ratzeburg, 1844, Campoplex)

latifrons Holmgren, 1860

protensa Thomson, 1887

Distribution: England

Genus Charops Holmgren, 1859

\section{Charops cantator (DeGeer, 1778)}

Nomenclature:

Ichneumon cantator DeGeer, 1778

decipiens (Gravenhorst, 1829, Campoplex)

tenuitarsus (Gravenhorst, 1829, Anomalon)

nigropetiolatus Strobl, 1904

nigrifacies (Kiss, 1924, Anomalon)

Distribution: England, Wales 


\section{Genus Clypeoplex Horstmann, 1987}

Clypeoplex cerophagus (Gravenhorst, 1829)

Nomenclature:

Campoplex cerophagus Gravenhorst, 1829

picticrus (Thomson, 1887, Omorga)

Distribution: England, Scotland

Notes: BMNH, NMS, UM, added here

\section{Genus Cymodusa Holmgren, 1859}

Nomenclature:

SAGARITIS Holmgren, 1859 preocc.

THERSITIA Schmiedeknecht, 1907

SAGARITOPSIS Hincks, 1944

Cymodusa antennator Holmgren, 1860

Nomenclature:

flavipes Brischke, 1880

anntenatrix Schulz, 1906

Distribution: England, Scotland, Ireland

Cymodusa cruentata (Gravenhorst, 1829)

Nomenclature:

Campoplex cruentata Gravenhorst, 1829

marginella (Zetterstedt, 1838, Porizon)

longicalcar Thomson, 1887

Distribution: Scotland, Ireland

\section{Cymodusa declinator (Gravenhorst, 1829)}

Nomenclature:

Campoplex declinator Gravenhorst, 1829

fasciata (Bridgman \& Fitch, 1885, Thymaris)

fasciata (Bridgman, 1886, Thymaris) preocc. 
declinatrix (Schulz, 1906, Sagaritis)

Distribution: England, Scotland

Notes: The name declinator Grav. was applied to a species of Campoletis by J.F. Perkins in his curation of the BMNH collection.

\section{Cymodusa exilis Holmgren, 1860}

Nomenclature:

petulans Holmgren, 1860

convergens (Thomson, 1887, Nemeritis)

Distribution: England, Ireland

\section{Cymodusa leucocera Holmgren, 1859}

Nomenclature:

pulchricornis Szépligeti, 1901

egregia (Schmiedeknecht, 1907, Thersitia)

Distribution: England, Scotland, Ireland

\section{Genus Diadegma Förster, 1869}

Nomenclature:

ANGITIA Holmgren, 1859

NYTHOBIA Förster, 1869

PECTINELLA Morley, 1915

NEOANGITIA Horstmann, 1969

AUMA Dbar, 1984

Notes: Distribution data for some species (the 'Nythobia' group) taken from Shaw and Horstmann (1997).

Diadegma aculeatum (Bridgman, 1889)

Nomenclature:

Limneria aculeata Bridgman, 1889

atrum (Kokujev, 1915, Angitia)

politor (Aubert, 1960, Horogenes)

Distribution: England, Wales 
Diadegma agile (Brischke, 1880)

Nomenclature:

Limneria agilis Brischke, 1880

Diadegma angitiaeforma Horstmann, 1969

Distribution: England, Scotland

Notes: NMS, det. Horstmann, added here

Diadegma angulator (Aubert, 1963)

Nomenclature:

Horogenes angulator Aubert, 1963

Distribution: England, Ireland

Notes: NMS, det. Horstmann, added here

Diadegma annulicrus (Thomson, 1887)

Nomenclature:

Angitia annulicrus Thomson, 1887

Diadegma anurum (Thomson, 1887)

Nomenclature:

Angitia anura Thomson, 1887

Distribution: England, Scotland

Notes: added by Shaw and Horstmann (1997)

Diadegma areolare (Holmgren, 1860)

Nomenclature:

Limneria areolaris Holmgren, 1860

Distribution: Ireland

Notes: Added by O'Connor et al. (2007) det. J.F. Perkins, also recorded from the Isle of Man by Walker (1872) but this may not be reliable.

Diadegma argentellae Horstmann, 2004

Distribution: Scotland 
Notes: added by Horstmann (2004b); described in the subgenus Nythobia but subgenera have not been used here.

Diadegma armillatum (Gravenhorst, 1829)

Nomenclature:

Campoplex armillatus Gravenhorst, 1829

tibiale (Gravenhorst, 1829, Campoplex)

pseudocombinatum (Szépligeti, 1916, Angitia)

Distribution: England, Scotland, Wales, Ireland

Notes: Diadegma monospilum (Thomson, 1887, Angitia) was removed from synonymy by Horstmann (2006a).

Diadegma berberatae Horstmann, 2013

Distribution: England

Notes: added by Horstmann (2013)

Diadegma brevipetiolatum Horstmann, 1969

Distribution: England

Notes: NMS, det. Horstmann, added here

Diadegma brevivalve (Thomson, 1887)

Nomenclature:

Angitia brevivalvis Thomson, 1887

Distribution: England

Notes: NMS, det. Horstmann, added here

Diadegma callisto Horstmann, 1993

Distribution: Scotland

Notes: NMS, det. Horstmann, added here 
Diadegma chrysostictos (Gmelin, 1790)

Nomenclature:

Ichneumon chrysostictos Gmelin, 1790

corsicator (Aubert, 1961, Horogenes)

orientator Aubert, 1965

Distribution: England, Scotland, Ireland

Notes: distribution data from Horstmann and Shaw (1984)

Diadegma claripenne (Thomson, 1887)

Nomenclature:

Angitia claripennis Thomson, 1887

Distribution: Ireland

Notes: added by O'Connor et al. (2007); also a specimen lacking locality data in $\mathrm{BMNH}$, det. J.F. Perkins.

Diadegma clavicorne (Brischke, 1880)

Nomenclature:

Limneria clavicornis Brischke, 1880

Diadegma coleophorarum (Ratzeburg, 1852)

Nomenclature:

Campoplex coleophorarum Ratzeburg, 1852

Distribution: England, Scotland

Diadegma combinatum (Holmgren, 1860)

Nomenclature:

Limneria combinata Holmgren, 1860

alpinator Aubert, 1970

Distribution: England, Scotland, Ireland

Diadegma compunctellae Horstmann, 2013

Distribution: Scotland

Notes: added by Horstmann (2013) 
Diadegma consumtor (Gravenhorst, 1829)

Nomenclature:

Campoplex consumtor Gravenhorst, 1829

varians (Brischke, 1880, Limneria)

Diadegma crassicorne (Gravenhorst, 1829)

Nomenclature:

Campoplex crassicornis Gravenhorst, 1829

carnifex (Gravenhorst, 1829, Campoplex)

brevicorne (Holmgren, 1860, Limneria)

normannicum (Rudow, 1883, Limneria)

Distribution: England, Scotland, Wales, Ireland

Notes: Listed as a species of Meloboris in Fitton (1978); some distribution data from Horstmann (2000d).

Diadegma crassiseta (Thomson, 1887)

Nomenclature:

Angitia crassiseta Thomson, 1887

Distribution: England

Notes: BMNH, det. Perkins, added here

Diadegma crassum (Bridgman, 1889)

Nomenclature:

Limneria crassa Bridgman, 1889

Distribution: England, Scotland

Diadegma crataegi Horstmann, 1980

Distribution: England, Scotland

Notes: added by Horstmann (1980a) 
Diadegma cylindricum (Brischke, 1880)

Nomenclature:

Limneria cylindrica Brischke, 1880

Distribution: England, Ireland

Notes: Noted as occurring in England and Ireland by various authors but not listed by Fitton (1978).

Diadegma duplicatum Horstmann, 1980

Distribution: England, Scotland

Notes: added by Shaw and Horstmann (1997)

Diadegma elishae (Bridgman, 1884)

Nomenclature:

Limneria elishae Bridgman, 1884

Distribution: England, Scotland, Ireland

Diadegma ericinellae Horstmann, 2013

Distribution: England

Notes: added by Horstmann (2013)

Diadegma erucator (Zetterstedt, 1838)

Nomenclature:

Porizon erucator Zetterstedt, 1838

rufipes misident.

fumipennis (Holmgren, 1856, Campoplex)

Distribution: England, Scotland, Ireland

Diadegma exareolator Aubert, 1964

Distribution: England, Scotland, Ireland

Notes: added by Shaw and Horstmann (1997) 
Diadegma fabricianae Horstmann \& Shaw, 1984

Distribution: England, Scotland, Wales

Notes: added by Horstmann and Shaw (1984)

Diadegma fenestrale (Holmgren, 1860)

Nomenclature:

Limneria fenestralis Holmgren, 1860

?gracile (Gravenhorst, 1829, Campoplex)

Distribution: England, Scotland, Wales, Ireland, Isle of Man

Notes: distribution data from Azidah et al. (2000) and NMS

Diadegma flexum Horstmann, 1973

Distribution: England

Notes: NMS, det. Horstmann, added here

Diadegma fungicola Horstmann, 2008

Distribution: England

Notes: added by Horstmann (2008b)

Diadegma grisescens (Gravenhorst, 1829)

Nomenclature:

Mesoleptus grisescens Gravenhorst, 1829

rufiventris (Gravenhorst, 1829, Campoplex)

hydropota (Holmgren, 1860, Limneria)

Distribution: England, Scotland, Ireland

Diadegma holopygum (Thomson, 1887)

Nomenclature:

Angitia holopyga Thomson, 1887

Distribution: England, Scotland, Ireland 
Diadegma hygrobium (Thomson, 1887)

Nomenclature:

Meloboris hygrobia Thomson, 1887

ischnocerum (Thomson, 1887, Meloboris)

pechlaneri (Hedwig, 1957, Angitia) unavailable

Distribution: England, Scotland, Wales, Ireland

Diadegma incompletum Horstmann, 1973

Distribution: Scotland

Notes: NMS, det. Horstmann, added here

Diadegma insectator (Schrank, 1781)

Nomenclature:

Ichneumon insectator Schrank, 1781

insectatrix (Schulz, 1906, Angitia)

Distribution: England, Ireland

Diadegma ? kyffhusanae Horstmann, 1973

Distribution: England

Notes: Tentative identification.

Diadegma laricinellum (Strobl, 1904)

Nomenclature:

Angitia laricinella Strobl, 1904

Distribution: Scotland

Notes: added by Shaw and Horstmann (1997)

Diadegma ? laterale (Gravenhorst, 1829)

Nomenclature:

Campoplex lateralis Gravenhorst, 1829

Distribution: England, Ireland 
Notes: There is one specimen labelled as '?lateralis' by K. Horstmann in NMS, from the Isle of Coll. and specimens in NMI det. A.W. Stelfox (O'Connor et al. 2007).

Diadegma latungulum (Thomson, 1887)

Nomenclature:

Angitia latungula Thomson, 1887

deletum (Morley, 1915, Pectinella)

Distribution: England, Scotland, Wales, Ireland

Diadegma ledicola Horstmann, 1969

Distribution: England, Wales

Notes: added by Shaw and Horstmann (1997)

Diadegma lithocolletis Horstmann, 1969

Distribution: England, Scotland

Notes: added by Shaw and Horstmann (1997)

Diadegma litorale (Holmgren, 1856)

Nomenclature:

Campoplex litoralis Holmgren, 1856

Distribution: England, Ireland

Diadegma majale (Gravenhorst, 1829)

Nomenclature:

Campoplex majalis Gravenhorst, 1829

Distribution: England, Scotland, Ireland

Diadegma melanium (Thomson, 1887)

Nomenclature:

Angitia melania Thomson, 1887

Distribution: England 
Diadegma monospilum (Thomson, 1887)

Nomenclature:

Angitia monospila Thomson, 1887

Distribution: England, Scotland, Wales

Notes: NMS, det. Horstmann, added here

Diadegma nanus (Gravenhorst, 1829)

Nomenclature:

Campoplex nanus Gravenhorst, 1829

Distribution: England, Scotland

Diadegma naryciae Horstmann, 2008

Nomenclature:

narcyiae misspelling

Distribution: England, Scotland

Notes: NMS, det. Horstmann, added here; originally, incorrectly, spelt narcyiae, emended by Horstmann (2012a).

Diadegma neocerophagum Horstmann, 1969

Nomenclature:

cerophaga misident.

Diadegma neomajale Horstmann, 1969

Distribution: England

Notes: BMNH, det. Horstmann, added here

Diadegma pusio (Holmgren, 1860)

Nomenclature:

Meloboris pusio Holmgren, 1860

annulipes (Bridgman, 1889, Limneria)

Distribution: England, Scotland 
Diadegma rufatum (Bridgman, 1884)

Nomenclature:

Limneria rufata Bridgman, 1884

Distribution: England, Scotland

Diadegma ruficeps (Holmgren, 1860)

Nomenclature:

Limneria ruficeps Holmgren, 1860

gracile (Ratzeburg, 1848, Campoplex) preocc.

rimator (Thomson, 1887, Angitia)

rimatrix (Schulz, 1906, Angitia)

Distribution: England, Scotland

Notes: BMNH, NMS, det. Horstmann, added here; there is also a specimen in NMS, from Co. Clare, Ireland, labelled ?ruficeps by K. Horstmann.

Diadegma scotiae (Bridgman, 1889)

Nomenclature:

Limneria scotiae Bridgman, 1889

Distribution: Scotland

Diadegma semiclausum (Hellén, 1949)

Nomenclature:

Limneria semiclausa Hellén, 1949

tibialis misident.

cerophaga misident.

eucerophagum Horstmann, 1969

Distribution: England, Scotland, Ireland, Isle of Man

Notes: distribution data from Azidah et al. (2000)

Diadegma sordipes (Thomson, 1887)

Nomenclature:

Angitia sordipes Thomson, 1887

Distribution: England, Scotland, Wales 
Diadegma stagnale (Holmgren, 1856)

Nomenclature:

Campoplex stagnalis Holmgren, 1856

Distribution: England, Ireland

Diadegma stigmatellae Horstmann, 1980

Distribution: England, Scotland

Notes: added by Horstmann (1980a)

Diadegma tenuipes (Thomson, 1887)

Nomenclature:

Angitia tenuipes Thomson, 1887

Distribution: England, Scotland, Ireland

Diadegma tripunctatum (Bridgman, 1886)

Nomenclature:

Limneria tripunctata Bridgman, 1886

Distribution: England, Ireland

Diadegma trochanteratum (Thomson, 1887)

Nomenclature:

Angitia trochanterata Thomson, 1887

Distribution: England, Ireland

Diadegma truncatum (Thomson, 1887)

Nomenclature:

Campoplex truncata Thomson, 1887

subbuccatum (Thomson, 1887, Angitia)

Distribution: England, Wales, Ireland 


\section{Genus Dolophron Förster, 1869}

Dolophron pedellum (Holmgren, 1860)

Nomenclature:

Limneria pedella Holmgren, 1860

albicoxis (Schmiedeknecht, 1909, Synetaeris)

Distribution: England, Scotland, Isle of Man

Genus Dusona Cameron, 1901

Nomenclature:

DELOPIA Cameron, 1903

Notes: Most distribution data from Horstmann (2011a), based on R. Hinz's and K. Horstmann's identifications of specimens in NMS and BMNH; older determinations of material in BMNH cannot be relied upon. Some species have been carried over from the 1978 checklist but no recent specimens have been seen, these are indicated by a '?'. Synonymy follows Hinz and Horstmann (2004) and Horstmann (2009c).

Species of Dusona excluded from the British and Irish list:

[limnobia (Thomson, 1887, Campoplex)] K. Horstmann had identified specimens in NMS as limnobia, which appeared on earlier versions of this checklist, but, following the redescription of the species (Horstmann 2009c), older identifications need to be checked (Horstmann 2011a).

[vidua (Gravenhorst, 1829, Campoplex)] Specimens identified in BMNH as Dusona vidua are actually Hyposoter tricolor (det. K. Horstmann); Shaw (2008) also notes that Irish records almost certainly refer to $H$. tricolor.

\section{Dusona admontina (Speiser, 1908)}

Nomenclature:

Campoplex admontinus Speiser, 1908

rufiventris (Strobl, 1904, Campoplex) preocc.

Distribution: England, Scotland

Notes: added by Horstmann (2011a)

\section{Dusona aemula (Förster, 1868)}

\section{Nomenclature:}




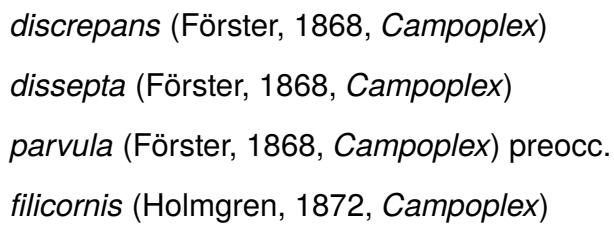

Distribution: England, Scotland, Wales

Notes: added by Horstmann (2011a)

\section{Dusona ? alpigena Hinz, 1972}

Distribution: Scotland

Notes: Tentative identification of a specimen in NMS by K. Horstmann.

\section{Dusona anceps (Holmgren, 1860)}

Nomenclature:

Campoplex anceps Holmgren, 1860

auriculata (Förster, 1868, Campoplex)

disparilis (Förster, 1868, Campoplex)

costulata (Bridgman \& Fitch, 1885, Campoplex)

costulata (Bridgman, 1886, Campoplex)

libauensis (Strand, 1918, Campoplex)

Distribution: England

\section{Dusona angustata (Thomson, 1887)}

Nomenclature:

Campoplex angustatus Thomson, 1887

Distribution: England, Scotland

\section{Dusona angustifrons (Förster, 1868)}

Nomenclature:

Campoplex angustifrons Förster, 1868

?obreptans (Förster, 1868, Campoplex)

zonella (Förster, 1868, Campoplex)

cornella (Teunissen, 1947, Campoplex)

Distribution: England, Scotland, Wales 


\section{Dusona annexa (Förster, 1868)}

Nomenclature:

Campoplex annexus Förster, 1868

limnobia (Thomson, 1887, Campoplex)

facialis (Holmgren, 1872, Campoplex) preocc.

americana (Ashmead, 1890, Casinaria)

mariae (Dalla Torre, 1901, Campoplex)

oyamadai Hinz, 1994

Distribution: England, Scotland, Ireland, Isle of Man

\section{Dusona aurita (Kriechbaumer, 1883)}

Nomenclature:

Campoplex auritus Kriechbaumer, 1883

Distribution: England

Notes: added by Horstmann (2011a)

\section{Dusona aversa (Förster, 1868)}

Nomenclature:

Campoplex aversus Förster, 1868

dubiosa (Förster, 1868, Campoplex)

tschekii (Holmgren, 1872, Campoplex)

crassipes (Thomson, 1887, Campoplex)

Distribution: England, Scotland

\section{Dusona bellipes (Holmgren, 1872)}

Nomenclature:

Campoplex bellipes Holmgren, 1872

vernalis Hinz, 1957

Distribution: England

Notes: added by Horstmann (2011a) 


\section{Dusona bicoloripes (Ashmead, 1906)}

Nomenclature:

Campoplex bicoloripes Ashmead, 1906

pugillator misident.

foersteri (Roman, 1942, Campoplex)

Distribution: England, Scotland

Dusona blanda (Förster, 1868)

Nomenclature:

Campoplex blandus Förster, 1868

remota (Förster, 1868, Campoplex)

forsselli (Holmgren, 1872, Campoplex)

punctiventris (Woldstedt, 1877, Casinaria)

Distribution: England, Scotland, Isle of Man

\section{Dusona bucculenta (Holmgren, 1860)}

Nomenclature:

Campoplex bucculentus Holmgren, 1860

melampus (Förster, 1868, Campoplex)

Distribution: England, Scotland

\section{Dusona carinifrons (Holmgren, 1860)}

Nomenclature:

Campoplex carinifrons Holmgren, 1860

minax (Förster, 1868, Campoplex)

geometrae (Rudow, 1883, Campoplex) synonymy by Horstmann (1999a)

Distribution: England

\section{Dusona carpathica (Szépligeti, 1916)}

Nomenclature:

Casinaria carpathica Szépligeti, 1916

zonella misident.

adriaansei (Teunissen, 1947, Campoplex) 
Distribution: England, Scotland

Notes: added by Horstmann (2011a)

Dusona circumcinctus (Förster, 1868)

Nomenclature:

Campoplex circumcinctus Förster, 1868

subcinctus (Förster, 1868, Campoplex) preocc.

Distribution: England

Notes: added by Horstmann (2011a)

Dusona circumspectans (Förster, 1868)

Nomenclature:

Campoplex circumspectans Förster, 1868

vagula (Förster, 1868, Campoplex)

subsulcata (Holmgren, 1872, Campoplex)

Distribution: England, Scotland

Notes: added by Horstmann (2011a)

Dusona confusa (Förster, 1868)

Nomenclature:

Campoplex confusus Förster, 1868

lacunosa (Kriechbaumer, 1883, Campoplex)

consimilis (Schmiedeknecht, 1908, Campoplex)

Distribution: England, Scotland, Wales, Isle of Man

Dusona cultrator (Gravenhorst, 1829)

Nomenclature:

Campoplex cultrator Gravenhorst, 1829

Distribution: England 


\section{Dusona disclusa (Förster, 1868)}

Nomenclature:

Campoplex disclusus Förster, 1868

Distribution: England, Scotland

Notes: added by Horstmann (2011a)

\section{Dusona dubitor Hinz, 1977}

Nomenclature:

oxyacanthae misident.

Distribution: England, Scotland

Notes: Added by Horstmann (2011a); frequently misidentified as oxyacanthae (K. Horstmann, pers. comm.)

\section{Dusona erythrogaster (Förster, 1868)}

Nomenclature:

Campoplex erythrogaster Förster, 1868 indefessa (Förster, 1868, Campoplex)

Distribution: England, Scotland

\section{Dusona falcator (Fabricius, 1775)}

Nomenclature:

Ichneumon falcator Fabricius, 1775

Distribution: England

\section{Dusona flagellator (Fabricius, 1793)}

Nomenclature:

Ichneumon flagellator Fabricius, 1793

debilis (Förster, 1868, Campoplex) synonymy by Horstmann (1999a)

heterocera (Förster, 1868, Campoplex) synonymy by Horstmann (2001a)

Distribution: England

Notes: added by Horstmann (2011a) 
Dusona ? holmgrenii (Dalla Torre, 1901)

Nomenclature:

Campoplex ?holmgrenii Dalla Torre, 1901

unicincta (Holmgren, 1872, Campoplex) preocc.

Dusona humilis (Förster, 1868)

Nomenclature:

Campoplex humilis Förster, 1868

eurynotus (Holmgren, 1872, Campoplex)

Distribution: England, Scotland

Notes: added by Horstmann (2011a)

Dusona incompleta (Bridgman, 1889)

Nomenclature:

Campoplex incompletus Bridgman, 1889

Distribution: England, Scotland

Dusona inermis (Förster, 1868)

Nomenclature:

Campoplex inermis Förster, 1868

Distribution: England, Scotland

Notes: added by Horstmann (2011a)

Dusona infesta (Förster, 1868)

Nomenclature:

Campoplex infestus Förster, 1868

terrifica (Förster, 1868, Campoplex)

Distribution: Scotland, Wales

Dusona insignita (Förster, 1868)

Nomenclature:

Campoplex insignitus Förster, 1868

bistrigosa (Holmgren, 1872, Campoplex) 
Distribution: England, Scotland

Dusona juvenilis (Förster, 1868)

Nomenclature:

Campoplex juvenilis Förster, 1868

victor (Thunberg, 1824, Ichneumon) preocc.

monozona (Förster, 1868, Campoplex)

Distribution: England, Wales

Dusona leptogaster (Holmgren, 1860)

Nomenclature:

Campoplex leptogaster Holmgren, 1860

macrostylus (Förster, 1868, Campoplex)

sylvicola (Habermehl, 1922, Campoplex)

Distribution: England, Scotland

\section{Dusona libertatis (Teunissen, 1947)}

Nomenclature:

Campoplex libertatis Teunissen, 1947

Distribution: England

Notes: added by Horstmann (2011a)

Dusona mercator (Fabricius, 1793)

Nomenclature:

Ichneumon mercator Fabricius, 1793

venditor (Thunberg, 1824, Ichneumon)

oxyacanthae (Boie, 1855,)

mesoxantha (Förster, 1868, Campoplex)

Distribution: England, Scotland

Dusona minor (Provancher, 1879)

Nomenclature:

Campoplex minor Provancher, 1879 
Distribution: England

Notes: added by Horstmann (2011a)

Dusona montana (Roman, 1929)

Nomenclature:

Campoplex montanus Roman, 1929

carinifer (Teunissen, 1947, Campoplex)

Distribution: England

Notes: added by Horstmann (2011a)

Dusona myrtilla (Desvignes, 1856)

Nomenclature:

Campoplex myrtillus Desvignes, 1856

tenthredinum (Tschek, 1871, Campoplex)

nobilitata (Holmgren, 1872, Campoplex)

Distribution: England

Dusona nidulator (Fabricius, 1804)

Nomenclature:

Ophion nidulator Fabricius, 1804

nitidulator (Holmgren, 1856, Campoplex)

circumscripta (Förster, 1868, Campoplex)

martialis (Förster, 1868, Campoplex)

vindex (Förster, 1868, Campoplex)

bifida (Thomson, 1887, Campoplex)

obscura (Kiss, 1926, Campoplex)

Distribution: England, Scotland

Dusona notabilis (Förster, 1868)

Nomenclature:

Campoplex notabilis Förster, 1868

scolator misident.

Distribution: England, Scotland 


\section{Dusona obliterata (Holmgren, 1872)}

Nomenclature:

Campoplex obliteratus Holmgren, 1872

limniventris (Kriechbaumer, 1883, Campoplex)

Distribution: England

Notes: added by Horstmann (2011a)

Dusona ? opaca (Thomson, 1887)

Nomenclature:

Campoplex opacus Thomson, 1887

Dusona petiolator (Fabricius, 1804)

Nomenclature:

Ophion petiolator Fabricius, 1804

lapponica (Holmgren, 1860, Campoplex)

callizona (Förster, 1868, Campoplex)

punctata (Bridgman \& Fitch, 1885, Campoplex)

punctata (Bridgman, 1886, Campoplex) preocc.

nigra (Kiss, 1924, Campoplex)

Distribution: England, Scotland

Dusona pineticola (Holmgren, 1872)

Nomenclature:

Campoplex pineticola Holmgren, 1872

litigiosa (Habermehl, 1922, Campoplex)

sibirica Hinz, 1985

Distribution: England, Scotland

Notes: added by Horstmann (2011a) 


\section{Dusona polita (Förster, 1868)}

\section{Nomenclature:}

Campoplex politus Förster, 1868

flavipalpis (Förster, 1868, Campoplex)

mediana (Förster, 1868, Campoplex)

spoliator (Förster, 1868, Campoplex)

trisculpta (Holmgren, 1872, Campoplex)

femorator (Bridgman \& Fitch, 1885, Campoplex)

femorator (Bridgman, 1886, Campoplex) preocc.

latungula (Thomson, 1887, Campoplex)

splendens (Thomson, 1887, Campoplex)

Distribution: England, Scotland

\section{Dusona prominula (Förster, 1868)}

Nomenclature:

Campoplex prominulus Förster, 1868

contumax (Förster, 1868, Campoplex)

foveolata (Förster, 1868, Campoplex)

Distribution: England, Scotland

\section{Dusona pugillator (Linnaeus, 1758)}

Nomenclature:

Ichneumon pugillator Linnaeus, 1758

canaliculata (Förster, 1868, Campoplex) preocc.

Distribution: England, Scotland

\section{Dusona pulchripes (Holmgren, 1872)}

Nomenclature:

Campoplex pulchripes Holmgren, 1872

praecox (Teunissen, 1947, Campoplex)

Distribution: England, Ireland

Notes: added by Horstmann (2011a) 


\section{Dusona recta (Thomson, 1887)}

Nomenclature:

Campoplex rectus Thomson, 1887

Distribution: England

Notes: added by Horstmann (2011a)

Dusona rubidatae Horstmann, 2009

Distribution: Scotland

Notes: added by Horstmann (2011a)

\section{Dusona rugifer (Förster, 1868)}

Nomenclature:

Campoplex rugifer Förster, 1868

subaequalis (Förster, 1868, Campoplex)

puncta (Kriechbaumer, 1883, Campoplex)

Distribution: England, Scotland

Notes: English record from W.A. Ely (pers. comm.).

\section{Dusona ? rugulosa (Förster, 1868)}

Nomenclature:

Campoplex rugulosus Förster, 1868

\section{Dusona semiflava (Costa, 1883)}

\section{Nomenclature:}

Campoplex semiflavus Costa, 1883

flaviscapus (Thomson, 1887, Campoplex)

Distribution: England

Notes: added by Horstmann (2011a)

Dusona sobolicida (Förster, 1868)

Nomenclature:

Campoplex sobolicida Förster, 1868 
ulcerata (Holmgren, 1872, Campoplex)

Distribution: England, Scotland, Wales

Dusona spinipes (Thomson, 1887)

Nomenclature:

Campoplex spinipes Thomson, 1887

Distribution: England

Notes: added by Horstmann (2011a)

Dusona stenogaster (Förster, 1868)

Nomenclature:

Campoplex stenogaster Förster, 1868

monticola (Habermehl, 1922, Campoplex)

Distribution: England

Notes: added by Horstmann (2011a)

\section{Dusona stragifex (Förster, 1868)}

Nomenclature:

Campoplex stragifex Förster, 1868

delusor misident.

adjuncta (Förster, 1868, Campoplex)

areolata (Brauns, 1895, Campoplex)

Distribution: England, Scotland, Isle of Man

\section{Dusona stygia (Förster, 1868)}

Nomenclature:

Campoplex stygius Förster, 1868

Distribution: England

Notes: added by Horstmann (2011a)

Dusona subimpressa (Förster, 1868)

Nomenclature: 
Campoplex subimpressus Förster, 1868 transitoria (Kiss, 1924, Campoplex)

Distribution: England

Notes: added by Horstmann (2011a)

Dusona tenuis (Förster, 1868)

Nomenclature:

Campoplex tenuis Förster, 1868 agnata (Förster, 1868, Campoplex) anxia (Förster, 1868, Campoplex) peraffinis (Förster, 1868, Campoplex) proxima (Förster, 1868, Campoplex)

Distribution: England, Scotland

Dusona terebrator (Förster, 1868)

Nomenclature:

Campoplex terebrator Förster, 1868 added by

Distribution: England, Scotland, Wales, Ireland, Isle of Man

Notes: added by Horstmann (2011a)

Dusona thomsoni Hinz, 1966

Distribution: England

Notes: added by Horstmann (2011a)

Dusona vigilator (Förster, 1868)

Nomenclature:

Campoplex vigilator Förster, 1868

Distribution: England, Scotland 
Dusona xenocampta (Förster, 1868)

Nomenclature:

Campoplex xenocamptus Förster, 1868

polyxantha (Strobl, 1904, Campoplex)

baltica (Habermehl, 1926, Campoplex)

Distribution: Scotland

Genus Echthronomas Förster, 1869

Echthronomas facialis (Thomson, 1887)

Nomenclature:

Anilasta facialis Thomson, 1887

Distribution: England

Notes: Horniman, det. Shaw, added here

Echthronomas ochrostoma (Holmgren, 1860)

Nomenclature:

Casinaria ochrostoma Holmgren, 1860

Notes: BMNH, added here

Echthronomas tricincta (Gravenhorst, 1829)

Nomenclature:

Campoplex tricinctus Gravenhorst, 1829

Genus Enytus Cameron, 1905

Nomenclature:

IOCTES Förster, 1869

NAREOLATA Ellinger \& Sachtleben, 1928

Enytus apostata (Gravenhorst, 1829)

Nomenclature:

Campoplex apostata Gravenhorst, 1829

exareolata (Ratzeburg, 1852, Campoplex)

reticulata (Bridgman, 1884, Limneria) 
Distribution: England, Scotland, Ireland

Notes: distribution data from Shaw (1981)

Enytus appositor (Aubert, 1970)

Nomenclature:

Diadegma appositor Aubert, 1970

Distribution: England, Scotland, Wales, Isle of Man

Notes: NMS, BMNH, det. Horstmann, added here

Enytus crataegellae (Thomson, 1887)

Nomenclature:

Angitia crataegellae Thomson, 1887

Distribution: England

Notes: NMS, det. Horstmann, added here

Enytus neoapostata (Horstmann, 1969)

Nomenclature:

Diadegma neoapostata Horstmann, 1969

neapostatus misspelling

Distribution: England, Scotland, Ireland

Enytus parvicanda (Thomson, 1887)

Nomenclature:

Angitia parvicanda Thomson, 1887

parvicauda misspelling

Enytus styriacus (Horstmann, 1980)

Nomenclature:

Diadegma styriacum Horstmann, 1980

Distribution: Scotland

Notes: NMS, det. Horstmann, added here 
Genus Eriborus Förster, 1869

Nomenclature:

ZAPORUS Förster, 1869

Notes: Distribution data for Eriborus species from Horstmann (1987) and the collections of BMNH, NMS and UM.

Eriborus braccatus (Gmelin, 1790)

Nomenclature:

Ichneumon braccatus Gmelin, 1790

jocator (Fabricius, 1793, Ichneumon)

nigriventris (Habermehl, 1922, Anilasta)

Distribution: England

Eriborus dorsalis (Gravenhorst, 1829)

Nomenclature:

Campoplex dorsalis Gravenhorst, 1829

micorocephalus (Gravenhorst, 1829, Ischnoceros)

Distribution: England, Scotland

Notes: Listed twice by Fitton (1978), under Meloboris and Eriborus.

Eriborus perfidus (Gravenhorst, 1829)

Nomenclature:

Campoplex perfidus Gravenhorst, 1829

aberrans (Gravenhorst, 1829, Campoplex)

obscuriventris Kiss, 1926

Distribution: England

\section{Genus Gonotypus Förster, 1869}

Nomenclature:

GONOTYPA Thomson, 1887

Gonotypus melanostoma (Thomson, 1887)

Nomenclature: 
Gonotypa melanostoma Thomson, 1887

Distribution: England, Wales, Ireland, Isle of Man

\section{Genus Hyposoter Förster, 1869}

Nomenclature:

AMELOCTONUS Förster, 1869

ISCHNOSCOPUS Förster, 1869

RHYTHMONOTUS Förster, 1869

Notes: species of Hyposoter excluded from the British and Irish list:

[anglicanus (Habermehl, 1923, Anilasta)] Only included on the British list on the basis that it was described from Carr material, of doubtful origin (see Perkins 1953, Shaw 2003).

Hyposoter albonotatus (Bridgman, 1889)

Nomenclature:

Limneria albonotata Bridgman, 1889

melaleucus (Schmiedeknecht, 1909, Anilastus)

Distribution: England, Scotland

Hyposoter alienus (Brischke, 1880)

Nomenclature:

Limneria aliena Brischke, 1880

Distribution: Ireland

Hyposoter barrettii (Bridgman, 1881)

Nomenclature:

Limneria barrettii Bridgman, 1881

teucrii (Bridgman, 1889, Limneria)

Distribution: England

Hyposoter boops (Thomson, 1887)

Nomenclature:

Anilasta boops Thomson, 1887 
Distribution: England

Notes: Added by Horstmann (2013)and raised from synonymy with brischkei.

Hyposoter brischkei (Bridgman, 1882)

Nomenclature:

Limneria brischkei Bridgman, 1882

Distribution: England, Scotland, Ireland

Hyposoter caedator (Gravenhorst, 1829)

Nomenclature:

Campoplex caedator Gravenhorst, 1829

henscheli (Smits van Burgst, 1910, Anilastus)

persimilis (Szépligeti, 1916, Anilastus)

parvulus (Kiss, 1926, Anilastus)

Distribution: England

Hyposoter carbonarius (Ratzeburg, 1844)

Nomenclature:

Campoplex carbonaria Ratzeburg, 1844

Distribution: England, Scotland

Hyposoter clausus (Brischke, 1880)

Nomenclature:

Limneria clausa Brischke, 1880

Distribution: England, Scotland

Notes: Many specimens in BMNH are only doubtfully identified; material in NMS has been recently identified by $\mathrm{K}$. Horstmann.

Hyposoter coxator (Thomson, 1887)

Nomenclature:

Anilasta coxator Thomson, 1887

Distribution: England

Notes: BMNH, NMS, det. Horstmann, added here 
Hyposoter didymator (Thunberg, 1824)

Nomenclature:

Ichneumon didymator Thunberg, 1824

rotundator (Thunberg, 1824, Ichneumon)

ruficinctus (Gravenhorst, 1829, Campoplex)

schmiedeknechti (Smits van Burgst, 1913, Anilastus)

Distribution: England, Scotland, Wales, Ireland

Hyposoter discedens (Schmiedeknecht, 1909)

Nomenclature:

Anilastus discedens Schmiedeknecht, 1909

Distribution: Ireland

\section{Hyposoter dolosus (Gravenhorst, 1829)}

Nomenclature:

Campoplex dolosus Gravenhorst, 1829

rufimanus (Gravenhorst, 1829, Campoplex) synonymy by Horstmann (2000d) oculatus (Tschek, 1871, Limneria)

Distribution: England, Scotland

\section{Hyposoter fitchii (Bridgman, 1881)}

Nomenclature:

Limneria fitchii Bridgman, 1881

Hyposoter inquinatus (Holmgren, 1860)

Nomenclature:

Limneria inquinata Holmgren, 1860

Distribution: Scotland

Notes: One British specimen (no locality) in BMNH but pointing the other direction, implying erroneous identification (by J.F. Perkins?). Carried over from the 1978 checklist and on the basis of material in UM, det. J.P. Brock. 
Hyposoter leucomerus (Thomson, 1887)

Nomenclature:

Anilasta leucomera Thomson, 1887

tricinctus (Holmgren, 1858, Limneria) unavailable

Distribution: England, Ireland

Notes: NMS, det. Horstmann, added here

Hyposoter longulus (Thomson, 1887)

Nomenclature:

Anilasta longula Thomson, 1887

Distribution: England, Scotland, Wales

Hyposoter neglectus (Holmgren, 1860)

Nomenclature:

Limneria neglecta Holmgren, 1860

varicoxa (Thomson, 1887, Anilasta)

variicoxus (Dalla Torre, 1901, Anilastus)

Distribution: Scotland

Notes: NMS, det. Horstmann, added here

Hyposoter notatus (Gravenhorst, 1829)

Nomenclature:

Campoplex notatus Gravenhorst, 1829

Distribution: England, Scotland, Wales, Ireland

Hyposoter obscurellus (Holmgren, 1860)

Nomenclature:

Limneria obscurella Holmgren, 1860

Hyposoter orbator (Gravenhorst, 1829)

Nomenclature:

Campoplex orbator Gravenhorst, 1829

rufus (Bridgman, 1882, Limneria) 
Hyposoter placidus (Desvignes, 1856)

Nomenclature:

Campoplex placidus Desvignes, 1856

Distribution: England

Hyposoter rhodocerae (Rondani, 1877)

Nomenclature:

Tryphon rhodocerae Rondani, 1877

ebeninus misident.

Distribution: England, Ireland

Hyposoter ruficrus (Thomson, 1887)

Nomenclature:

Anilasta ruficrus Thomson, 1887

Distribution: England

Notes: NMS, det. Horstmann, added here

Hyposoter thuringiacus (Schmiedeknecht, 1909)

Nomenclature:

Anilastus thuringiacus Schmiedeknecht, 1909

Distribution: England, Ireland

Hyposoter tricolor (Ratzeburg, 1844)

Nomenclature:

Campoplex tricolor Ratzeburg, 1844

vidua misident.

henaultii (Desvignes, 1856, Campoplex)

Distribution: England, Scotland 
Hyposoter virginalis (Gravenhorst, 1829)

Nomenclature:

Campoplex virginalis Gravenhorst, 1829

Distribution: England

Notes: distribution data from Horstmann (2000d)

Hyposoter vividus (Holmgren, 1860)

Nomenclature:

Limneria vivida Holmgren, 1860

albicrus (Thomson, 1877, Anilasta)

Distribution: England

Notes: BMNH, added here

Genus Lathroplex Förster, 1869

Lathroplex clypearis Thomson, 1887

Distribution: England

Notes: NMS, det. Horstmann, added here

Genus Lathrostizus Förster, 1869

Nomenclature:

LATHROSTIZA Thomson, 1887

Lathrostizus clypeatus (Brischke, 1880)

Nomenclature:

Limneria clypeata Brischke, 1880

sternocerus (Thomson, 1887, Lathrostiza) synonymy by Horstmann (2004b)

Distribution: Scotland

Lathrostizus lugens (Gravenhorst, 1829)

Nomenclature:

Campoplex lugens Gravenhorst, 1829

vestigialis (Ratzeburg, 1852, Campoplex) 
Distribution: England, Ireland, Isle of Man

Genus Lemophagus Townes, 1965

Nomenclature:

HOLOCREMNODES Aubert, 1986 synonymy by Horstmann (2004b)

Lemophagus curtus Townes, 1965

Distribution: England, Wales

Notes: added by Cox (2007); specimens in BMNH; NMS

Lemophagus errabundus (Gravenhorst, 1829)

Nomenclature:

Campoplex errabundus Gravenhorst, 1829

Distribution: England

Notes: added by Salisbury (2003)

Genus Leptocampoplex Horstmann, 1970

Leptocampoplex cremastoides (Holmgren, 1860)

Nomenclature:

Nemeritis cremastoides Holmgren, 1860

Distribution: England, Scotland

Notes: NMS, BMNH, added here; Horstmann (1999a) removed punctulatus (Ratze.) to a synonym of Porizon moderator (L.).

Genus Macrus Gravenhorst, 1829

Macrus parvulus (Gravenhorst, 1829)

Nomenclature:

Campoplex parvulus Gravenhorst, 1829

fusicornis (Roman, 1923, Lathroplex)

Distribution: England, Scotland 
Genus Melanoplex Horstmann, 1987

Melanoplex bucculentus (Holmgren, 1860)

Nomenclature:

Limneria bucculentus Holmgren, 1860

Distribution: England

Notes: BMNH, det. Horstmann, added here

Genus Meloboris Holmgren, 1859

Nomenclature:

ASINAMORA Förster, 1869

NEPIERA Förster, 1869

PSEUDOCYMODUSA Habermehl, 1922

ANOIXIS Townes, 1970

Meloboris alternans (Gravenhorst, 1829)

Nomenclature:

Campoplex alternans Gravenhorst, 1829

elachistae (Brischke, 1880, Cymodusa)

ruficornis (Bridgman, 1884, Limneria) preocc.

Distribution: England, Scotland, Ireland

Meloboris cingulata Horstmann, 2004

Distribution: Scotland

Notes: added by Horstmann (2004b)

Meloboris collector (Thunberg, 1824)

Nomenclature:

Ichneumon collector Thunberg, 1824

concinna (Holmgren, 1860, Limneria)

signata (Szépligeti, 1916, Omorgus)

foersteri (Kiss, 1924, Idechthis)

albicincta (Seyrig, 1927, Angitia) 
Distribution: England, Scotland, Wales, Ireland, Isle of Man

Meloboris gracilis Holmgren, 1859

Nomenclature:

monticolana (Bridgman, 1881, Limneria)

Distribution: England, Scotland, Ireland

Meloboris neglecta (Habermehl, 1923)

Nomenclature:

Pseudocymodusa neglecta Habermehl, 1923

Meloboris proxima (Perkins, 1942)

Nomenclature:

Nepiera proxima Perkins, 1942

Distribution: England, Scotland, Ireland

Notes: BMNH, NMS, det. Horstmann, added here

Genus Nemeritis Holmgren, 1860

Nomenclature:

PSEUDONEMERITIS Szépligeti, 1916

Notes: Distribution data from Horstmann (1994a).

Nemeritis breviventris Horstmann, 1975

Distribution: England

Notes: BMNH, det. Horstmann, added here

Nemeritis caudatula Thomson, 1887

Nomenclature:

rhaphidiae Kriechbaumer, 1892

raphidiae Dalla Torre, 1901

monticola Habermehl, 1922

Distribution: England 
Nemeritis cingulata Horstmann, 1980

Distribution: England

Nemeritis fallax (Gravenhorst, 1829)

Nomenclature:

Campoplex fallax Gravenhorst, 1829

crassiceps Habermehl, 1922

Distribution: England

Notes: NMS, BMNH, det. Horstmann, added here

Nemeritis lativentris Thomson, 1887

Distribution: England

Nemeritis macrocentra (Gravenhorst, 1829)

Nomenclature:

Campoplex macrocentrus Gravenhorst, 1829

sordida (Gravenhorst, 1829, Campoplex)

varipes (Gravenhorst, 1829, Campoplex)

antennalis (Szépligeti, 1916, Angitia)

caudata (Szépligeti, 1916, Omorgus) preocc.

transsylvanica (Szépligeti, 1916, Canidia)

caudata (Gregor, 1940, Idechthis)

Distribution: England, Ireland

Nemeritis silvicola Horstmann, 1973

Distribution: England

Nemeritis stenura Thomson, 1887

Notes: One English specimen in NMS tentatively identified by K. Horstmann; carried over from Fitton (1978). 


\section{Genus Nepiesta Förster, 1869}

Nepiesta mandibularis (Holmgren, 1860)

Nomenclature:

Limneria mandibularis Holmgren, 1860

aberrans misident.

umbrata (Brischke, 1880, Canidia)

nigra Szépligeti, 1901

Distribution: England, Scotland, Ireland

Nepiesta subclavata Thomson, 1887

Distribution: England

Notes: NMS, det Horstmann, added here

\section{Nepiesta tricingulata Horstmann, 1973}

Distribution: England

Notes: NMS, det Horstmann, added here

\section{Genus Olesicampe Förster, 1869}

Nomenclature:

LIMNERIA Förster, 1859

HOLOCREMNUS Förster, 1869

OMOBORUS Förster, 1869

HOLOCREMNA Thomson, 1887

OLESICAMPA Thomson, 1887

Notes: Species of Olesicampe excluded from the British and Irish list:

[monticola (Hedwig, 1938, Holocremna)] Welsh specimens in BMNH, ex Cephalcia alpina (Hymenoptera: Pamphiliidae) (not a British native), det. K. Horstmann and I.D. Gauld, presumably brought over for biocontrol purposes.

[ratzeburgi (Tschek, 1871, Limneria)] Released in Wales for biocontrol purposes but with no evidence of successful establishment (Billany et al. 1983).

Doubtfully placed species of Olesicampe: 
[affinis (Parfitt, 1882, Limneria) nom. dub.]

[alienata (Gravenhorst, 1829, Campoplex) nom. dub.]

Olesicampe alboplica (Thomson, 1887)

Nomenclature:

Olesicampa alboplica Thomson, 1887

simplex (Thomson, 1887, Olesicampa)

Distribution: England, Ireland

Olesicampe argentata (Gravenhorst, 1829)

Nomenclature:

Campoplex argentatus Gravenhorst, 1829

Distribution: Scotland

Olesicampe auctor (Gravenhorst, 1829)

Nomenclature:

Campoplex auctor Gravenhorst, 1829

limbata (Gravenhorst, 1829, Campoplex)

auctrix (Schulz, 1906, Olesicampa)

Distribution: England

Olesicampe binotata (Thomson, 1887)

Nomenclature:

Olesicampa binotata Thomson, 1887

Distribution: England, Scotland

Notes: BMNH, det. Perkins, added here

Olesicampe buccata (Thomson, 1887)

Nomenclature:

Holocremna buccata Thomson, 1887

Distribution: Ireland 
Olesicampe canaliculata (Gravenhorst, 1829)

Nomenclature:

Campoplex canaliculatus Gravenhorst, 1829

Distribution: Isle of Man

Notes: NMS, det. Horstmann, added here

Olesicampe cavigena (Thomson, 1887)

Nomenclature:

Olesicampa cavigena Thomson, 1887

Olesicampe clandestina (Holmgren, 1860)

Nomenclature:

Limneria clandestina Holmgren, 1860

Distribution: England, Ireland

Notes: distribution data from Shaw (1999)

Olesicampe crassitarsis (Thomson, 1887)

Nomenclature:

Olesicampa crassitarsis Thomson, 1887

Distribution: Scotland, Ireland

Olesicampe erythropyga (Holmgren, 1860)

Nomenclature:

Limneria erythropyga Holmgren, 1860

Distribution: Ireland

Olesicampe femorella (Thomson, 1887)

Nomenclature:

Olesicampa femorella Thomson, 1887

Distribution: England

Notes: BMNH, det. Perkins, added here 
Olesicampe forticostata (Schmiedeknecht, 1909)

Nomenclature:

Anilastus forticostatus Schmiedeknecht, 1909

Distribution: England

Olesicampe fulcrans (Thomson, 1887)

Nomenclature:

Olesicampa fulcrans Thomson, 1887

Distribution: England, Scotland, Ireland

Olesicampe fulviventris (Gmelin, 1790)

Nomenclature:

Ichneumon fulviventris Gmelin, 1790

Distribution: England, Ireland

Olesicampe geniculella (Thomson, 1887)

Nomenclature:

Olesicampa geniculella Thomson, 1887

Distribution: Scotland, Ireland

Olesicampe gracilipes (Thomson, 1887)

Nomenclature:

Olesicampa gracilipes Thomson, 1887

Distribution: Ireland

Olesicampe longipes (Müller, 1776)

Nomenclature:

Ichneumon longipes Müller, 1776

canescens (Gmelin, 1790, Ichneumon)

Distribution: Ireland 
Olesicampe macellator (Thunberg, 1824)

Nomenclature:

Ichneumon macellator Thunberg, 1824

retecta (Hartig, 1838, Campoplex)

cothurnata (Holmgren, 1860, Limneria)

frutetorum (Thomson, 1887, Holocremna)

Distribution: England, Scotland

Olesicampe nigroplica (Thomson, 1887)

Nomenclature:

Olesicampa nigroplica Thomson, 1887

Distribution: England, Scotland

Olesicampe pagana (Holmgren, 1860)

Nomenclature:

Limneria pagana Holmgren, 1860

Olesicampe paludicola (Holmgren, 1860)

Nomenclature:

Limneria paludicola Holmgren, 1860

inculcator misident.

?sagittaria (Müller, 1776, Ichneumon)

Distribution: England, Ireland

Olesicampe patellana (Thomson, 1887)

Nomenclature:

Olesicampa patellana Thomson, 1887

Distribution: Scotland, Ireland

Notes: BMNH, det. Perkins, added here

Olesicampe praecox (Holmgren, 1860)

Nomenclature:

Limneria praecox Holmgren, 1860 
Distribution: Ireland

Olesicampe proterva (Brischke, 1880)

Nomenclature:

Limneria proterva Brischke, 1880

luteipes (Thomson, 1887, Olesicampa)

subcallosa (Thomson, 1887, Olesicampa)

Distribution: Ireland

Olesicampe pubescens (Ratzeburg, 1844)

Nomenclature:

Campoplex pubescens Ratzeburg, 1844

hyalinata (Holmgren, 1860, Limneria)

Distribution: Ireland

Olesicampe retusa (Thomson, 1887)

Nomenclature:

Olesicampa retusa Thomson, 1887

Olesicampe sericea (Holmgren, 1856)

Nomenclature:

Campoplex sericeus Holmgren, 1856

Distribution: England, Ireland

\section{Olesicampe sinuata (Thomson, 1887)}

Nomenclature:

Holocremna sinuata Thomson, 1887

Distribution: England, Scotland

Notes: BMNH, det. Perkins, UM, det. Brock, added here

\section{Olesicampe transiens (Ratzeburg, 1848)}

Nomenclature:

Campoplex transiens Ratzeburg, 1848 
incrassator (Holmgren, 1856, Campoplex) synonymy by Horstmann (2007a)

Distribution: England, Scotland, Wales, Ireland

Olesicampe vexata (Holmgren, 1860)

Nomenclature:

Limneria vexata Holmgren, 1860

Olesicampe vitripennis (Holmgren, 1860)

Nomenclature:

Limneria vitripennis Holmgren, 1860

Genus Phobocampe Förster, 1869

Nomenclature:

HYPOTHEREUTES Förster, 1869

PHOBOCAMPA Thomson, 1887

Phobocampe alticollis (Thomson, 1887)

Nomenclature:

Phobocampa alticollis Thomson, 1887

Distribution: England

Notes: added by Horstmann (2006a)

Phobocampe bicingulata (Gravenhorst, 1829)

Nomenclature:

Campoplex bicingulatus Gravenhorst, 1829

Distribution: England, Scotland, Wales

Phobocampe brumatae Horstmann, 2009

Distribution: England, Scotland

Notes: added by Horstmann (2006a) 
Phobocampe confusa (Thomson, 1887)

Nomenclature:

Phobocampa confusa Thomson, 1887

Distribution: England, Scotland, Ireland

Notes: Omitted by Fitton (1978) as it had previously only been recorded from Ireland

(Stelfox 1929), although it was also discussed as a British species by Shaw and Askew (1976).

Phobocampe coniferella (Roman, 1914)

Nomenclature:

Phobocampa coniferella Roman, 1914

facialis (Szépligeti, 1916, Holocremnus)

Distribution: England

Notes: added by Šedivý (2004); Britain given as a locality by Šedivý (2004) but the specimens in BMNH are destroyed, leaving only the parasitoid cocoons; however there are English specimens in NMS, det. K. Horstmann.

Phobocampe crassiuscula (Gravenhorst, 1829)

Nomenclature:

Campoplex crassiusculus Gravenhorst, 1829

Distribution: England, Scotland

Phobocampe croceipes (Marshall, 1876)

Nomenclature:

Limneria croceipes Marshall, 1876

albitarsis Szépligeti, 1916

Distribution: England, Scotland

Phobocampe flavicincta (Thomson, 1887)

Nomenclature:

Phobocampa flavicincta Thomson, 1887

Distribution: Scotland

Notes: BMNH, det. Perkins, added here 
Phobocampe horstmanni Šedivý, 2004

Distribution: England, Scotland

Notes: NMS, det. Horstmann, added here

Phobocampe lymantriae Gupta, 1983

Distribution: England

Notes: NMS, det. Horstmann, added here

Phobocampe neglecta (Holmgren, 1860)

Nomenclature:

Limneria neglecta Holmgren, 1860 varicoxa (Thomson, 1887, Anilasta) variicoxa (Dalla Torre, 1901, Anilastus)

Distribution: England, Scotland

Phobocampe nigra Šedivý, 2004

Distribution: England

Notes: BMNH, det. Horstmann, added here

Phobocampe pulchella (Thomson, 1887)

Nomenclature:

Phobocampa pulchella Thomson, 1887

Distribution: England, Scotland

Notes: NMS, det. Horstmann, added here

Phobocampe quercus Horstmann, 2008

Distribution: England

Notes: added by Horstmann (2008b) 
Phobocampe tempestiva (Holmgren, 1860)

Nomenclature:

Limneria tempestiva Holmgren, 1860

Distribution: England, Scotland

Notes: NMS, det. Horstmann, added here

Phobocampe unicincta (Gravenhorst, 1829)

Nomenclature:

Campoplex unicinctus Gravenhorst, 1829

disparis (Viereck, 1911, Hyoposoter)

Distribution: England

Phobocampe variabilis Šedivý, 2004

Distribution: England

Notes: NMS, det. Horstmann, added here

Genus Porizon Fallén, 1813

Nomenclature:

PHAEDROCTONUS Förster, 1869 synonymy by Horstmann (2004b)

Porizon humuli (Horstmann, 1987)

Nomenclature:

Phaedroctonus humuli Horstmann, 1987

Distribution: England

Notes: NMS, det. Horstmann, added here

\section{Porizon moderator (Linnaeus, 1758)}

Nomenclature:

Ichneumon moderator Linnaeus, 1758

strobinellae (Christ, 1791, Cynipsichneumon)

flaviventris (Ratzeburg, 1844, Campoplex)

punctulatus (Ratzeburg, 1844, Cremastus) synonymy by Horstmann (1999a)

ensifer (Brischke, 1880, Limneria) 
Distribution: England, Scotland

Porizon transfuga (Gravenhorst, 1829)

Nomenclature:

Campoplex transfuga Gravenhorst, 1829

syringellae Hedwig, 1944

Distribution: England, Scotland, Wales, Ireland, Isle of Man

Genus Pyracmon Holmgren, 1859

Pyracmon fumipennis (Zetterstedt, 1838)

Nomenclature:

Porizon fumipennis Zetterstedt, 1838

Distribution: Ireland

Pyracmon sepiellus (Holmgren, 1860)

Nomenclature:

Limneria sepiella Holmgren, 1860

Distribution: England

Notes: BMNH, det. Horstmann, added here

Genus Rhimphoctona Förster, 1869

Nomenclature:

PARAPYRACMON Clément, 1924

Subgenus Xylophylax Kriechbaumer, 1878

Rhimphoctona (Xylophylax) megacephalus (Gravenhorst, 1829)

Nomenclature:

Campoplex megacephalus Gravenhorst, 1829

corvina (Gravenhorst, 1829, Phytodietus)

austriaca (Tschek, 1871, Pyracmon)

megalocephalus (Schulz, 1906, Campoplex)

rufipes (Lange, 1911, Pyracmon) preocc. 
hungarica (Kiss, 1926, Pyracmon)

Distribution: England

Rhimphoctona (Xylophylax) melanura (Holmgren, 1860)

Nomenclature:

Pyracmon melanurus Holmgren, 1860

signata (Habermehl, 1922, Pyracmon)

Rhimphoctona (Xylophylax) obscuripes (Holmgren, 1860)

Nomenclature:

Pyracmon obscuripes Holmgren, 1860

alpina (Strobl, 1904, Pyracmon)

Distribution: Ireland

Rhimphoctona (Xylophylax) xoridiformis (Holmgren, 1860)

Nomenclature:

Pyracmon xoridiformis Holmgren, 1860

nigerrima (Kiss, 1924, Eclytus)

\section{Genus Scirtetes Hartig, 1838}

Nomenclature:

SPUDASTICA Förster, 1869

Scirtetes robustus (Woldstedt, 1874)

Nomenclature:

Limneria robusta Woldstedt, 1874

kriechbaumeri (Bridgman, 1882, Limneria)

petiolaris (Thomson, 1887, Spudastica)

Distribution: England, Scotland, Ireland

\section{Genus Sinophorus Förster, 1869}

Nomenclature:

EULIMNERIA Schmiedeknecht, 1907

Notes: doubtfully placed species of Sinophorus: 
[paniscus (Gravenhorst, 1829, Campoplex) nom. dub.] Morley (1915) identified Sinophorus paniscus as British but this name is now a synonym of Macrus filiventris Gravenhorst, 1829 (Horstmann 1978a), a rarely collected southern European species; the identity of Morley's specimens is not known.

Sinophorus albidus (Gmelin, 1790)

Nomenclature:

Ichneumon albidus Gmelin, 1790

hungaricus (Szépligeti, 1916, Omorgus)

Distribution: England, Scotland

Sinophorus bridgmanii (Dalla Torre, 1901)

Nomenclature:

Limnerium bridgmanii Dalla Torre, 1901

distinctus (Bridgman, 1887, Limneria) preocc.

renominatus (Morley, 1915, Limnerium)

Distribution: England

Sinophorus costalis (Thomson, 1887)

Nomenclature:

Limneria costalis Thomson, 1887

Distribution: Ireland

Sinophorus crassifemur (Thomson, 1887)

Nomenclature:

Limneria crassifemur Thomson, 1887

Distribution: Ireland

Sinophorus fuscicarpus (Thomson, 1887)

Nomenclature:

Limneria fuscicarpus Thomson, 1887

Distribution: England

Notes: added by Sanborne (1984) 
Sinophorus geniculatus (Gravenhorst, 1829)

Nomenclature:

Campoplex geniculatus Gravenhorst, 1829

nigritellus (Thomson, 1887, Limneria)

argentator (Aubert, 1960, Campoplex)

Distribution: Ireland

Sinophorus juniperinus (Holmgren, 1856)

Nomenclature:

Campoplex juniperinus Holmgren, 1856

ornatus (Gregor, 1941, Omorgus)

Distribution: England, Ireland

Notes: added by Sanborne (1984)

Sinophorus pleuralis (Thomson, 1887)

Nomenclature:

Limneria pleuralis Thomson, 1887

Distribution: England

Notes: added by Sanborne (1984)

\section{Sinophorus turionum (Ratzeburg, 1844)}

Nomenclature:

Campoplex turionum Ratzeburg, 1844

spectabilis (Rudow, 1883, Limneria)

planiscapus (Thomson, 1887, Limneria)

rufifemur (Thomson, 1887, Limneria)

nigrotibialis (Kiss, 1926, Eulimneria)

alkae (Ellinger \& Sachtleben, 1928, Limnerium)

Distribution: England, Scotland, Ireland

Notes: some distribution data from Sanborne (1984) 
Sinophorus xanthostomus (Gravenhorst, 1829)

Nomenclature:

Campoplex xanthostomus Gravenhorst, 1829

pineticola (Thomson, 1887, Limneria)

deserticola (Tosquinet, 1896, Campoplex)

Genus Synetaeris Förster, 1869

Synetaeris heteropus Thomson, 1887

Distribution: Scotland

Genus Tranosema Förster, 1869

Tranosema carbonellum (Thomson, 1887)

Nomenclature:

Synetaeris carbonella Thomson, 1887

aterrimum (Strobl, 1904, Pyracmon)

rossicum (Szépligeti, 1916, Canidia)

Distribution: England, Scotland

Notes: BMNH, det. Broad, Perkins, added here

Tranosema exoletum (Thomson, 1887)

Nomenclature:

Omorga exoleta Thomson, 1887

geniculatum (Ulbricht, 1910, Omorgus) unavailable

Distribution: England

Notes: BMNH, det. Perkins, UM, added here

Tranosema hyperboreum (Thomson, 1887)

Nomenclature:

Limneria hyperborea Thomson, 1887

Distribution: England

Notes: NMS, det. Horstmann, added here 
Tranosema intermedium (Szépligeti, 1916)

Nomenclature:

Gonotypus intermedius Szépligeti, 1916

majus (Szépligeti, 1916, Gonotypus)

minus (Szépligeti, 1916, Gonotypus)

Distribution: England

Notes: NMS, det. Horstmann, added here

Tranosema latiusculum (Thomson, 1887)

Nomenclature:

Omorga latiuscula Thomson, 1887

Distribution: England, Scotland

Notes: NMS, BMNH, det. Horstmann, added here

Tranosema nigridens (Thomson, 1887)

Nomenclature:

Omorga nigridens Thomson, 1887

striolatum (Thomson, 1887, Omorga)

alpinator Aubert, 1966

Distribution: England

Notes: added by Horstmann (1978a)

Tranosema rostrale (Brischke, 1880)

Nomenclature:

Limneria rostralis Brischke, 1880

arenicola Thomson, 1887

thuringiacum (Schmiedeknecht, 1907, Sinophorus)

Distribution: England, Scotland, Wales 


\section{Genus Tranosemella Horstmann, 1978}

Tranosemella citrofrontalis (Hedwig, 1939)

Nomenclature:

Anilasta citrofrontalis Hedwig, 1939

Distribution: England, Scotland, Wales, Ireland

Notes: BMNH, NMS, det. Horstmann, Shaw, Broad, added here

\section{Tranosemella coxalis (Brischke, 1880)}

Nomenclature:

Limneria coxalis Brischke, 1880

Distribution: England

Notes: One British specimen in BMNH is probably coxalis; another British specimen has been labelled 'robusta Wold.' (=Scirtetes) and an English specimen has been labelled as 'interruptus'. Locality data from reared specimens in NMS, det. K. Horstmann.

\section{Tranosemella praerogator (Linnaeus, 1758)}

Nomenclature:

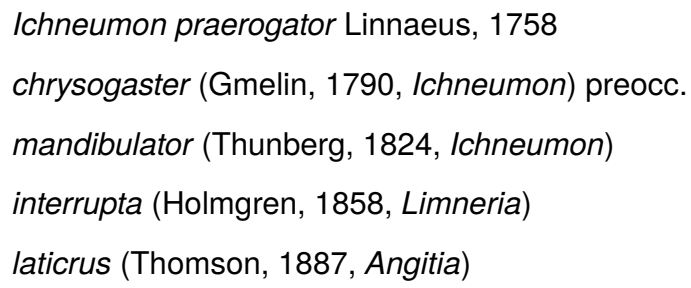

Distribution: England, Scotland, Wales, Ireland, Isle of Man

Notes: some distribution data from Shaw (1981)

\section{Genus Venturia Schrottky, 1902}

Nomenclature:

IDECHTHIS Förster, 1869

DEVORGILLA Cameron, 1907

NEMERITIS misident. 
Venturia canescens (Gravenhorst, 1829)

Nomenclature:

Campoplex canescens Gravenhorst, 1829

frumentaria (Rondani, 1874, Campoplex)

orientalis (Schmiedeknecht, 1909, Omorgus)

compressa (Hedwig, 1962, Angitia)

Distribution: England, Scotland, Ireland

\section{Collyriinae}

Subfamily COLLYRIINAE Cushman, 1924

Notes: Distribution data from Fitton (1984) and the collections of NMS.

\section{Genus Collyria Schiødte, 1839}

Nomenclature:

PACHYMERUS Gravenhorst, 1829

\section{Collyria coxator (Villers, 1789)}

Nomenclature:

Ichneumon coxator Villers, 1789

?falcata (Geoffroy, 1785, Ichneumon)

?arcuata (Olivier, 1792, Ichneumon)

calcitrator (Gravenhorst, 1807, Bassus)

puncticeps (Thomson, 1877, Pachymerus)

calcitratrix Schulz, 1906

Distribution: England

Collyria trichophthalma (Thomson, 1877)

Nomenclature:

Pachymerus trichophthalmus Thomson, 1877

Distribution: England 


\section{Cremastinae}

\section{Subfamily CREMASTINAE Förster, 1869}

Notes: Distribution data from Fitton and Gauld (1980) and the collections of NMS and $\mathrm{BMNH}$.

\section{Genus Cremastus Gravenhorst, 1829}

Notes: species excluded from the British and Irish list by Fitton and Gauld (1980):

[crassicornis Thomson, 1890]

\section{Cremastus bellicosus Gravenhorst, 1829}

Nomenclature:

partitus Szépligeti, 1899

meridionator Aubert, 1960

Distribution: England

Cremastus cephalotes Šedivý, 1970

Nomenclature:

ponticus Kolarov, 1982

Distribution: England

Cremastus geminus Gravenhorst, 1829

Nomenclature:

areolaris Strand, 1918

Distribution: England, Scotland, Wales, Ireland

Cremastus infirmus Gravenhorst, 1829

Nomenclature:

filicaudis Szépligeti, 1905

Distribution: England, Ireland 
Cremastus kratochvili Šedivý, 1970

Distribution: England

Notes: added by Fitton and Gauld (1980)

Cremastus pungens Gravenhorst, 1829

Nomenclature:

laeviusculus Thomson, 1890

Distribution: England, Ireland

Cremastus spectator Gravenhorst, 1829

Nomenclature:

binotatus Gravenhorst, 1829

melanarius Szépligeti, 1901

Distribution: England, Wales, Ireland

\section{Genus Dimophora Förster, 1869}

Nomenclature:

DIMOPHORUS Thomson, 1889

Dimophora nitens (Gravenhorst, 1829)

Nomenclature:

Campoplex nitens Gravenhorst, 1829

robusta Brischke, 1880

similis Brischke, 1880

arenicola (Thomson, 1890, Dimophorus)

Distribution: England

Genus Pristomerus Curtis, 1836

Pristomerus armatus (Lucas, 1849)

Nomenclature:

Collyria armata Lucas, 1849

glandarius (Rondani, 1877, Odontomerus) 
gratiosus Tosquinet, 1896

cingulatus Szépligeti, 1905

Distribution: England

Notes: NMS, det. Shaw \& Narolsky, added here

Pristomerus horribilis Narolsky, 1987

Distribution: England

Notes: BMNH, det. Broad, added here

Pristomerus vulnerator (Panzer, 1799)

Nomenclature:

Ichneumon vulnerator Panzer, 1799

marginalis Habermehl, 1923

stigmaticus Hellén, 1949

Distribution: England, Ireland

\section{Genus Temelucha Förster, 1869}

Nomenclature:

PARACREMASTUS Szépligeti, 1899

Notes: species excluded from the British and Irish list by Fitton and Gauld (1980):

[decorata (Gravenhorst, 1829, Cremastus)]

[subnasuta (Thomson, 1890, Cremastus)]

Temelucha arenosa (Szépligeti, 1899)

Nomenclature:

Cremastus arenosus Szépligeti, 1899

Distribution: England, Ireland

Notes: added by Fitton and Gauld (1980)

Temelucha interruptor (Gravenhorst, 1829)

Nomenclature:

Cremastus interruptor Gravenhorst, 1829 
buoliana (Curtis, 1854, Cremastus)

Distribution: England

Temelucha ophthalmica (Holmgren, 1860)

Nomenclature:

Cremastus ophthalmicus Holmgren, 1860

Distribution: England

Notes: added by Fitton and Gauld (1980)

Temelucha signata (Holmgren, 1860)

Nomenclature:

Cremastus signatus Holmgren, 1860

Distribution: England, Ireland

Notes: added by Fitton and Gauld (1980)

\section{Cryptinae}

Subfamily CRYPTINAE Kirby, 1837

Nomenclature:

PHYGADEUONTINAE Förster, 1869

GELINAE Viereck, 1918

HEMITELINAE Förster, 1869

\section{Tribe CRYPTINI Kirby, 1837}

Nomenclature:

MESOSTENINI Ashmead, 1900

ECHTHRINI Narayanan \& Kundanlal, 1958

Notes: Unless noted otherwise, distribution data from Schwarz and Shaw (1998) and the collections of BMNH and UM, with further references given.

Genus Acroricnus Ratzeburg, 1852

Nomenclature: 
MACROBATUS Holmgren, 1856

LINOCERAS Taschenberg, 1865

LEPTOBATIDES Buysson, 1896

\section{Acroricnus stylator (Thunberg, 1824)}

Nomenclature:

Ichneumon stylator Thunberg, 1824

macrobatus (Gravenhorst, 1829, Cryptus)

schaumii Ratzeburg, 1852

clavator (Holmgren, 1856, Macrobatus)

exannulatus (Kriechbaumer, 1894, Linoceras) unavailable

Distribution: England, Ireland

\section{Genus Agrothereutes Förster, 1850}

Nomenclature:

SPILOCRYPTUS Thomson, 1873

\section{Agrothereutes abbreviatus (Fabricius, 1794)}

\section{Nomenclature:}

Ichneumon abbreviatus Fabricius, 1794

abbreviator (Fabricius, 1793, Ichneumon) misident.

abbreviator (Fabricius, 1798, Ichneumon) preocc.

breviator (Thunberg, 1824, Ichneumon)

marginellus (Gravenhorst, 1829, Cryptus) synonymy by Horstmann (2001b)

pygoleucus (Gravenhorst, 1829, Cryptus)

tibiator (Gravenhorst, 1829, Cryptus)

ocellator (Zetterstedt, 1838, Cryptus)

evanescens (Ratzeburg, 1852, Cryptus)

leucomerus (Ratzeburg, 1852, Cryptus)

dispar (Thomson, 1873, Spilocryptus)

destitutus Vollenhoven, 1879

spectabilis (Rudow, 1886, Aptesis)

tricolor (Rudow, 1886, Aptesis)

brevipennis (Kriechbaumer, 1893, Spilocryptus) preocc. 


\author{
spectabilis (Rudow, 1914, Aptesis) preocc. \\ atratus (Rudow, 1917, Stibeutes) preocc. \\ livonensis (Rudow, 1917, Aptesis) \\ spectabilis (Rudow, 1917, Aptesis) preocc. \\ tricolor (Rudow, 1917, Aptesis) \\ cingulatus (Kiss, 1924, Gambrus) \\ variegatus (Kiss, 1924, Gambrus) preocc. \\ alpium Heinrich, 1951 synonymy by Schwarz (2005)
}

Distribution: England, Wales, Ireland, Isle of Man

Notes: Schwarz and Shaw (1998) separately list the distributions of three forms of abbreviatus, f. brevipennis (Marshall, 1867, Cryptus) (syn. batavus Vollenhoven, 1873), f. hopei (Gravenhorst, 1829, Pezomachus) and f. incubitor (Gravenhorst, 1829, Cryptus ).

\title{
Agrothereutes adustus (Gravenhorst, 1829)
}

Nomenclature:

Cryptus adustus Gravenhorst, 1829

albolineatus (Gravenhorst, 1829, Cryptus)

nubeculatus (Gravenhorst, 1829, Cryptus)

opisoleucus (Gravenhorst, 1829, Cryptus)

leucostictus (Hartig, 1838, Cryptus)

melanocerus (Ulbricht, 1916, Spilocryptus) unavailable

\section{Agrothereutes aterrimus (Gravenhorst, 1829)}

\section{Nomenclature:}

Cryptus aterrimus Gravenhorst, 1829

bicingulatus (Gravenhorst, 1829, Cryptus)

\section{Agrothereutes fumipennis (Gravenhorst, 1829)}

Nomenclature:

Cryptus fumipennis Gravenhorst, 1829

zygaenarum (Thomson, 1873, Spilocryptus)

hymotomadum (Rudow, 1883, Cryptus)

nigricans (Kiss, 1915, Spilocryptus) 
gracilentus (Habermehl, 1929, Spilocryptus)

Distribution: England, Wales

\section{Agrothereutes hospes (Tschek, 1871)}

Nomenclature:

Cryptus hospes Tschek, 1871

solitarius (Tschek, 1871, Cryptus)

intermedius (Verhoeff, 1890, Cryptus) preocc.

\section{Agrothereutes leucorhaeus (Donovan, 1810)}

Nomenclature:

Ichneumon leucorhaeus Donovan, 1810

migrator misident.

bombycis (Boudier, 1836, Cryptus)

?tibialis (Thomson, 1873, Spilocryptus)

temporalis (Szépligeti, 1916, Gambrus) synonymy by Schwarz (2005)

Distribution: England, Scotland

Notes: Spilocryptus tibialis was tentatively synonymised by Schwarz and Shaw (1998)

but treated as a valid species (occuring in England) by M. Schwarz in his identifications of BMNH specimens.

\section{Agrothereutes mandator (Linnaeus, 1758)}

Nomenclature:

Ichneumon mandator Linnaeus, 1758

ischioleucus (Gravenhorst, 1829, Cryptus) synonymy by Schwarz (2005)

cimbicis (Tschek, 1871, Cryptus)

Distribution: England, Scotland

\section{Agrothereutes mansuetor (Tschek, 1871)}

\section{Nomenclature:}

Cryptus mansuetor Tschek, 1871

nasutus (Thomson, 1873, Spilocryptus)

curiosus (Szépligeti, 1916, Gambrus) synonymy by Schwarz (2005) 
Distribution: Scotland

Notes: added by Schwarz and Shaw (1998)

Agrothereutes saturniae (Boie, 1855)

Nomenclature:

Cryptus saturniae Boie, 1855 pavoniae (Bauer, 1937, Spilocryptus)

Distribution: England, Scotland, Ireland

Genus Apsilops Förster, 1869

Nomenclature:

DAPANUS Förster, 1869

HETEROTYPUS Förster, 1869

SOBAS Förster, 1869

TRICHOCRYPTUS Thomson, 1873

Apsilops aquaticus (Thomson, 1874)

Nomenclature:

Trichocrytus aquaticus Thomson, 1874

napiformis (Rudow, 1882, Cryptus)

Distribution: England

Apsilops cinctorius (Fabricius, 1775)

Nomenclature:

Ichneumon cinctorius Fabricius, 1775

scirpi (Geoffroy, 1785, Ichneumon)

spinuosus (Rudow, 1886, Phygadeuon)

Distribution: England, Wales

Genus Aritranis Förster, 1869

Nomenclature:

PYCNOCRYPTUS Thomson, 1873 
Notes: Schwarz and Shaw (1998) clarified the uses of the generic names Aritranis, Hoplocryptus and Pycnocryptus.

\section{Aritranis director (Thunberg, 1824)}

Nomenclature:

Ichneumon director Thunberg, 1824

peregrinator misident.

Distribution: England, Scotland, Wales, Ireland, Isle of Man

\section{Aritranis nigripes (Gravenhorst, 1829)}

Nomenclature:

Cryptus nigripes Gravenhorst, 1829

fuscomarginatus (Gravenhorst, 1829, Cryptus)

insectator (Tschek, 1871, Cryptus)

jonicus (Tschek, 1872, Cryptus)

Distribution: England

\section{Aritranis occisor (Gravenhorst, 1829)}

Nomenclature:

Cryptus occisor Gravenhorst, 1829

gracilis (Taschenberg, 1865, Cryptus) preocc.

fuscicornis (Tschek, 1871, Cryptus)

notabilis (Habermehl, 1926, Hoplocryptus)

punguri (Kiss, 1915, Spilocryptus)

Notes: Listed as a valid species by Yu and Horstmann (1997), as a synonym of nigripes by Schwarz and Shaw (1998) and then taken out of synonymy with nigripes by Sawoniewicz (2003) and treated as a valid species, with revised synonymy of both species, by Schwarz (2005).

\section{Genus Ateleute Förster, 1869}

\section{Nomenclature:}


Ateleute linearis Förster, 1871

Nomenclature:

lissonotoides (Thomson, 1885, Hemiteles)

egregia (Schmiedeknecht, 1933, Hemiteles)

Distribution: England

Notes: added by Schwarz and Shaw (1998)

Genus Buathra Cameron, 1903

Buathra laborator (Thunberg, 1824)

Nomenclature:

Ichneumon laborator Thunberg, 1824

fabricii (Schiødte, 1839, Cryptus)

fulvipes (Magretti, 1884, Cryptus) synonymy by Horstmann (2004c)

fulvipes (Habermehl, 1902, Cryptus)

Distribution: England, Scotland, Wales

\section{Buathra tarsoleuca (Schrank, 1781)}

Nomenclature:

Ichneumon tarsoleucos Schrank, 1781

leucopus (Gmelin, 1790, Ichneumon)

leucotarsos (Gmelin, 1790, Ichneumon)

curvicauda (Thomson, 1896, Cryptus)

Distribution: England, Scotland

\section{Genus Caenocryptus Thomson, 1873}

\section{Caenocryptus rufiventris (Gravenhorst, 1829)}

Nomenclature:

Cryptus rufiventris Gravenhorst, 1829

eborinus (Ratzeburg, 1852, Cryptus)

collaris (Rudow, 1883, Cryptus) preocc.

Distribution: England, Scotland 
Notes: British specimens belong to the subspecies impunctatus Schwarz, 1991 (Schwarz and Shaw 1998).

\section{Genus Cryptus Fabricius, 1804}

Nomenclature:

EUCRYPTUS Haldeman, 1842

ITAMOPLEX Förster, 1869

\section{Cryptus apparitorius (Villers, 1789)}

\section{Nomenclature:}

Ichneumon apparitorius Villers, 1789

pungens Gravenhorst, 1829

gratiosus Tschek, 1871

histrionicus Rudow, 1882

\section{Cryptus arenicola Thomson, 1873}

Distribution: England

Notes: added by Schwarz (2005), as Cryptus macellus Tschek, 1871 with arenicola synonymised under macellus but later Schwarz (2015) raised arenicola from synonymy and confirmed that British specimens are arenicola.

\section{Cryptus armator Fabricius, 1804}

\section{Nomenclature:}

albatorius misident.

cunctator (Fabricius, 1793, Ichneumon) synonymy by Horstmann (2001a)

rusticator Zetterstedt, 1838

filicornis Rudow, 1886 preocc.

Distribution: England, Scotland, Wales

\section{Cryptus dianae Gravenhorst, 1829}

\section{Nomenclature:}

gracilicornis Gravenhorst, 1829

leucostomus Gravenhorst, 1829

stenogaster Gravenhorst, 1829 
seticornis (Ratzeburg, 1844, Ichneumon)

bolivari Kriechbaumer, 1898

solitarius Habermehl, 1909 preocc.

solitarius Habermehl, 1918 preocc.

Distribution: England

\section{Cryptus fibulatus Gravenhorst, 1829}

\section{Nomenclature:}

rhenanus Ulbricht, 1911

antennalis Szépligeti, 1916

Distribution: Scotland, Wales, Ireland

Notes: added by Schwarz and Shaw (1998)

\section{Cryptus inculcator (Linnaeus, 1758)}

Nomenclature:

Ichneumon inculcator Linnaeus, 1758

sponsor (Fabricius, 1793, Ichneumon)

regenerator (Panzer, 1804, Ichneumon)

quadrilineatus Gravenhorst, 1829

filicornis Ratzeburg, 1844

bicolor Rudow, 1882 preocc.

erythrostoma Rudow, 1882

lippensis Rudow, 1883

albopictus Seyrig, 1928 preocc.

\section{Cryptus minator Gravenhorst, 1829}

\section{Cryptus moschator (Fabricius, 1787)}

Nomenclature:

Ichneumon moschator Fabricius, 1787

polytropus Heinrich, 1951 synonymy by Schwarz (2005)

Distribution: England 


\section{Cryptus obscuripes Zetterstedt, 1838}

Nomenclature:

borealis Thomson, 1873 preocc.

carpathicus Szépligeti, 1916

Distribution: England

Notes: BMNH, det. Schwarz, added here

Cryptus spinosus Gravenhorst, 1829

Nomenclature:

armatorius misident.

leucostictus Gravenhorst, 1829 synonymy by Horstmann (2001b)

Distribution: England

Notes: Schwarz and Shaw (1998) list Ichneumon armatorius Fabricius, 1787, as a synonym of Cryptus spinosus but Horstmann (Horstmann 1982, Horstmann 2001a) treats it as a species of Hoplismenus (Ichneumoninae), tentatively as a synonym of albifrons (Gravenhorst) (as axillatorius (Thunberg))

\section{Cryptus spiralis (Geoffroy, 1785)}

Nomenclature:

Ichneumon spiralis Geoffroy, 1785

inconspicuus Gravenhorst, 1829

hispanicus Habermehl, 1918

\section{Cryptus titubator (Thunberg, 1824)}

Nomenclature:

Ichneumon titubator Thunberg, 1824

difficilis Tschek, 1871

infumatus Thomson, 1873

Distribution: England, Scotland, Wales, Ireland, Isle of Man

\section{Cryptus tuberculatus Gravenhorst, 1829}

\section{Nomenclature:}

investigator Tschek, 1871

solivagus Rossem, 1989 
Distribution: England

\section{Cryptus viduatorius Fabricius, 1804}

Nomenclature:

germari Taschenberg, 1865

Distribution: England, Scotland, Wales, Ireland

\section{Genus Echthrus Gravenhorst, 1829}

Nomenclature:

SPHAETES Bremi, 1849

Notes: Echthrus is placed in the Cryptini, following Laurenne et al. (2006), rather than in the Hemigastrini, where it was placed by Townes (1970). Townes and Townes (1960) had previously placed Echthrus in the Cryptini based on similarities to the genera that Townes (1970) classified as the subtribe Gabuniina. The molecular results of Laurenne et al. (2006) support this interpretation, in agreement with Townes and Townes (1962).

\section{Echthrus reluctator (Linnaeus, 1758)}

\section{Nomenclature:}

$$
\begin{aligned}
& \text { Ichneumon reluctator Linnaeus, } 1758 \\
& \text { usurpator (Scopoli, 1763, Ichneumon) } \\
& \text { obex (Müller, 1776, Ichneumon) } \\
& \text { rubiginosus (Christ, 1791, Ichneumon) } \\
& \text { carbonator (Thunberg, 1824, Ichneumon) } \\
& \text { chirothecator (Thunberg, 1824, Ichneumon) } \\
& \text { ternator (Thunberg, 1824, Ichneumon) } \\
& \text { crassicrus (Bremi, 1849, Sphaetes) } \\
& \text { corsicus (Marshall, 1901, Nyxeophilus) } \\
& \text { nigerrimus Strobl, 1902 }
\end{aligned}
$$

Distribution: England

\section{Genus Enclisis Townes, 1970}

\section{Enclisis alpicola (Habermehl, 1926)}

\section{Nomenclature:}


Distribution: England

Notes: Added by Schwarz (1989); omitted from the British list bySchwarz and Shaw (1998).

\section{Enclisis macilenta (Gravenhorst, 1829)}

\section{Nomenclature:}

Cryptus macilentus Gravenhorst, 1829

remex (Tschek, 1871, Cryptus)

inflata (Thomson, 1873, Caenocryptus)

gracilipes (Gravenhorst, 1829, Cryptus) synonymy by Sawoniewicz (2003)

antennata (Bridgman, 1881, Cryptus)

laticrus (Thomson, 1896, Caenocryptus)

exareolata (Strobl, 1901, Chaeretymma)

rubi (Habermehl, 1921, Microcryptus)

alboclypeata (Kiss, 1924, Hoplocryptus)

Distribution: England, Wales

\section{Enclisis ruficeps (Desvignes, 1856)}

Nomenclature:

Cryptus ruficeps Desvignes, 1856

pulchella Schwarz, 1989

\section{Enclisis vindex (Tschek, 1871)}

Nomenclature:

Cryptus vindex Tschek, 1871

pubiventris (Thomson, 1873, Caenocryptus)

tener (Thomson, 1873, Caenocryptus)

nubifer (Thomson, 1896, Caenocryptus)

striolata (Thomson, 1896, Caenocryptus)

nigriventris (Habermehl, 1919, Caenocryptus)

Distribution: England, Scotland 


\section{Genus Gambrus Förster, 1869}

Nomenclature:

KALTENBACHIA Förster, 1869

HYGROCRYPTUS Thomson, 1873

Notes: Synonymy follows Schwarz and Shaw (1998) and Schwarz (2005).

\section{Gambrus amoenus (Gravenhorst, 1829)}

Nomenclature:

Cryptus amoenus Gravenhorst, 1829

\section{Gambrus bipunctatus (Tschek, 1872)}

Nomenclature:

Cryptus bipunctatus Tschek, 1872

ornatus misident.; Schwarz and Shaw (1998)

maculatus Brischke, 1888 synonymy by Schwarz (2005)

Distribution: England, Scotland, Wales

Notes: Added by Schwarz and Shaw (1998)and transferred from Aritranis.

\section{Gambrus carnifex (Gravenhorst, 1829)}

Nomenclature:

Cryptus carnifex Gravenhorst, 1829

varicoxis (Taschenberg, 1865, Cryptus)

Distribution: England, Scotland, Wales

Gambrus incubitor (Linnaeus, 1758)

Nomenclature:

Ichneumon incubitor Linnaeus, 1758

vibex (Müller, 1776, Ichneumon)

upsaliensis (Geoffroy, 1785, Ichneumon)

superus Thomson, 1896

quadricinctus (Strobl, 1901, Spilocryptus)

incertus Habermehl, 1935 preocc.

Distribution: England, Scotland 
Notes: Schwarz and Shaw (1998) recorded Gambrus incubitor (including ornatus) from England, Scotland and Wales but, following Schwarz's (Schwarz 2005) separation of incubitor and ornatus, most specimens in NMS actually belong to ornatus (M. Schwarz, pers. comm.).

\section{Gambrus ornatus (Gravenhorst, 1829)}

Nomenclature:

Cryptus ornatus Gravenhorst, 1829

ornatulus (Thomson, 1873, Spilocryptus)

inferus Thomson, 1896

quadricinctus (Strobl, 1901, Spilocryptus)

ruficoxis Habermehl, 1919

meridionator (Aubert, 1965, Agrothereutes) preocc.

Distribution: England, Scotland, Wales, Ireland

Notes: added by Schwarz (2005); see note under incubitor. Irish occurrence confirmed by a specimen (coll. A. Anderson) identified by M. Schwarz. The species referred to as ornatus in older literature is now known to be bipunctatus (Schwarz and Shaw 1998).

\section{Gambrus tricolor (Gravenhorst, 1829)}

\section{Nomenclature:}

Cryptus tricolor Gravenhorst, 1829

subcinctus (Gravenhorst, 1829, Cryptus)

opacus Szépligeti, 1916

Distribution: England, Scotland, Wales

Notes: Listed as a species of Thrybius by Yu and Horstmann (1997), as a species of Gambrus by Schwarz and Shaw (1998).

\section{Genus Helcostizus Förster, 1869}

\section{Nomenclature:}

BRACHYCENTRUS Taschenberg, 1865 preocc.

CYRTOCRYPTUS Marshall, 1872

MESOCRYPTUS Thomson, 1873

HETEROCRYPTUS Woldstedt, 1874

CHENBERGUS Navás, 1930 
Notes: Helcostizus is transferred here from the Phygadeuontini, following the molecular phylogenetic results of Laurenne et al. (2006). The combination of morphological characters associated with parasitising wood-boring hosts had previously led Townes \& Townes (1962) to classify this genus in the Cryptini. Distribution data from Townes (1983) and Schwarz and Shaw (2010).

\section{Helcostizus restaurator (Fabricius, 1775)}

\section{Nomenclature:}

Ichneumon restaurator Fabricius, 1775

albator (Thunberg, 1824, Ichneumon)

brachycentrus (Gravenhorst, 1829, Cryptus)

crassipes (Hartig, 1847, Echthrus)

hercynianus (Hartig, 1847, Echthrus)

pimplarius (Taschenberg, 1865, Brachycentrus)

fuscitarsis (Haupt, 1917, Perosis)

turcicus (Fahringer, 1944, Brachycentrus)

serraticornis (Haupt, 1954, Perosis)

Distribution: England, Ireland

\section{Genus Hidryta Förster, 1869}

Nomenclature:

BRACHYCRYPTUS Thomson, 1873

EUTHYCRYPTUS Jussel, 1907

\section{Hidryta nigricoxa (Provancher, 1888)}

\section{Nomenclature:}

Cryptus nigricoxus Provancher, 1888

scrobiculifer (Jussel, 1907, Euthycryptus)

Distribution: Wales

Notes: added by Schwarz and Shaw (1998)

\section{Hidryta sordida (Tschek, 1871)}

Nomenclature:

Cryptus sordidus Tschek, 1871 
melanopus (Taschenberg, 1865, Cryptus) preocc., synonymy by Schwarz (2005)

erythrocera (Thomson, 1873, Brachycryptus)

sordidula (Thomson, 1873, Brachycryptus)

hueberi (Dalla Torre, 1901, Cryptus) synonymy by Schwarz (2005)

nigritarsis (Habermehl, 1918, Idiolispa)

pygmaea (Habermehl, 1918, Idiolispa)

henrichi (Kiss, 1924, Habrocryptus)

?atlantica Horstmann, 1990 tentative synonymy by Schwarz (2005)

Distribution: England, Scotland, Wales, Ireland

Notes: Added by Edgar (1971); overlooked by Fitton (1978).

Genus Hoplocryptus Thomson, 1873

Hoplocryptus bellosus (Curtis, 1837)

Nomenclature:

Cryptus bellosus Curtis, 1837

signatorius (Fabricius, 1793, Ichneumon) preocc.

pulcher Thomson, 1873

fuscipes (Tschek, 1871, Cryptus)

thoracicus (Brischke, 1881, Hygrocryptus)

macrophyiae (Rudow, 1911, Cryptus)

Distribution: England, Ireland

\section{Hoplocryptus bohemani (Holmgren, 1856)}

Nomenclature:

Cryptus bohemani Holmgren, 1856

rufoniger (Desvignes, 1856, Cryptus) synonymy by Horstmann (2000a)

mesoxanthus Thomson, 1873

Hoplocryptus confector (Gravenhorst, 1829)

Nomenclature:

Cryptus confector Gravenhorst, 1829

albus (Taschenberg, 1865, Cryptus) 
brachysoma (Taschenberg, 1865, Cryptus) synonymy by Schwarz (2005)

dubius (Taschenberg, 1865, Cryptus) synonymy by Schwarz and Shaw (1998)

elegans Thomson, 1873

thomsoni (Bridgman, 1881, Cryptus)

gladiator Kriechbaumer, 1899

caudatus Szépligeti, 1916

quadratus (Szépligeti, 1916, Gambrus) preocc.

gallicus (Habermehl, 1923, Gambrus)

enslini (Habermehl, 1923, Spilocryptus) preocc.

exannulatus Habermehl, 1926

hungaricus Habermehl, 1926 preocc.

Distribution: England

Hoplocryptus melanocephalus (Gravenhorst, 1829)

Nomenclature:

Cryptus melanocephalus Gravenhorst, 1829

Distribution: England

Notes: added by Schwarz (2007)

\section{Hoplocryptus murarius (Börner, 1782)}

\section{Nomenclature:}

Ichneumon murarius Börner, 1782

olitorius (Fabricius, 1793, Ichneumon) synonymy by Horstmann (2001a)

fugitivus (Gravenhorst, 1829, Cryptus) synonymy by Schwarz (2007)

gracilis (Gravenhorst, 1829, Cryptus)

binotatulus Thomson, 1873

pseudocryptus (Szépligeti, 1916, Gambrus)

tegularis (Szépligeti, 1916, Gambrus)

ignalinoensis Strand, 1918

ratzeburgi (Habermehl, 1919, Spilocryptus)

obscurata (Kiss, 1929, Habrocryptus) preocc.

Notes: Ichneumon murarius has priority over fugitivus (as used by Schwarz and Shaw 1998) or olitorius; Hoplocryptus binotatulus f. grandis Habermehl, 1926 was removed 
from synonymy and is a junior synonym of the extralimital $H$. besseianus (Seyrig, 1926) (Schwarz 2007).

\section{Hoplocryptus quadriguttatus (Gravenhorst, 1829)}

Nomenclature:

Cryptus quadriguttatus Gravenhorst, 1829

cognatus (Fonscolombe, 1850, Cryptus) synonymy by Schwarz (2005)

mallorcanus Kriechbaumer, 1894 synonymy by Schwarz (2007)

enslini Habermehl, 1921 synonymy by Schwarz (2007)

hebraicator (Aubert, 1970, Aritranis) synonymy by Schwarz (2005)

tiloidalis (Kolarov \& Beyarslan, 1994, Agrothereutes) synonymy by Schwarz (2005)

Distribution: England

\section{Genus Idiolispa Förster, 1869}

Nomenclature:

LIOCRYPTUS Thomson, 1873

PARACRYPTUS Szépligeti, 1916

Notes: species of Idiolispa deleted from the British and Irish list by Schwarz and Shaw (1998):

[obfuscator (Villers, 1789, Ichneumon)] Fitton (1978) listed obfuscator (Villers) as a doubtfully placed species of Trychosis but it was probably misidentified.

\section{Idiolispa analis (Gravenhorst, 1807)}

Nomenclature:

Bassus analis Gravenhorst, 1807

cursor (Thunberg, 1824, Ichneumon) preocc.

elevata (Zetterstedt, 1838, Cryptus)

dubiosa (Kiss, 1924, Spilocryptus)

Distribution: England, Scotland, Wales, Ireland

\section{Idiolispa ? hungarica (Szépligeti, 1916)}

\section{Nomenclature:}

Paracryptus hungaricus Szépligeti, 1916 
?grossa misident.; Schwarz and Shaw (1999)

meyeri (Habermehl, 1926, Spilocryptus)

Notes: Status as a British species (listed as Agrothereutes grossus (Grav.) by Fitton 1978) regarded as tentative by Schwarz and Shaw (1998).

\section{Idiolispa subalpina (Schmiedeknecht, 1904)}

Nomenclature:

Spilocryptus subalpinus Schmiedeknecht, 1904

heydeni (Habermehl, 1919, Spilocryptus)

Distribution: Scotland

Notes: added by Schwarz and Shaw (1998)

\section{Genus Ischnus Gravenhorst, 1829}

Nomenclature:

HABROCRYPTUS Thomson, 1873

\section{Ischnus agitator (Olivier, 1792)}

Nomenclature:

Ichneumon agitator Olivier, 1792

destructorius (Fabricius, 1793, Ichneumon) synonymy by Horstmann (2001a)

rubricator (Panzer, 1801, Ichneumon)

constrictor (Fabricius, 1804, Cryptus)

minutorius (Fabricius, 1804, Cryptus)

minor (Thunberg, 1824, Ichneumon)

pictor (Thunberg, 1824, Ichneumon)

dineurae (Rudow, 1882, Cryptus)

oriicus (De Stefani, 1886, Cryptus) synonymy by Horstmann (2000a)

\section{Ischnus alternator (Gravenhorst, 1829)}

Nomenclature:

Cryptus alternator Gravenhorst, 1829

unicinctus (Gravenhorst, 1829, Cryptus)

striatellus (Zetterstedt, 1838, Cryptus) 
annulipes (Taschenberg, 1865, Cryptus)

annulitarsis (Rudow, 1882, Cryptus)

Distribution: England, Scotland, Wales

\section{Ischnus inquisitorius (Müller, 1776)}

\section{Nomenclature:}

Ichneumon inquisitorius Müller, 1776

migrator misident.

dictator (Geoffroy, 1785, Ichneumon)

porrectorius (Fabricius, 1787, Ichneumon)

leucostictos (Gmelin, 1790, Ichneumon)

sanguinolentus (Gmelin, 1790, Ichneumon)

assertorius (Fabricius, 1793, Ichneumon)

zonator (Fabricius, 1793, Ichneumon)

triplicatorius (Thunberg, 1824, Ichneumon)

brachyurus (Gravenhorst, 1829, Cryptus)

geminus (Gravenhorst, 1829, Cryptus) synonymy by Sawoniewicz (2003)

sannio (Gravenhorst, 1829, Cryptus)

sedulus (Gravenhorst, 1829, Cryptus) synonymy by Sawoniewicz (2003)

flavopictus (Rudow, 1883, Cryptus)

alpinus (Strobl, 1901, Habrocryptus)

obscuratus (Kiss, 1924, Habrocryptus)

\section{Distribution: England}

Notes: Horstmann (1968) misidentified Fabricius's migrator, a mistake followed by Schwarz and Shaw (1998). Horstmann's subsequent (Horstmann 2001a) lectotype designations resulted in inquisitorius becoming the valid name for migrator sensu Horstmann (1968) and Schwarz and Shaw (1998) and migrator being the senior synonym for collaris. Sawoniewicz (2003) correctly applied the names (Horstmann, pers. comm.).

\section{Ischnus migrator (Fabricius, 1775)}

Nomenclature:

Ichneumon migrator Fabricius, 1775

collaris (Tschek, 1872, Cryptus) synonymy by Horstmann (2001a) 
punctiger (Thomson, 1896, Habrocryptus) synonymy by Schwarz and Shaw (1998)

insulanus (Krieger, 1897, Habrocryptus) synonymy by Schwarz and Shaw (1998)

helveticator Aubert, 1968 synonymy by Schwarz (2005)

Distribution: England, Scotland

Genus Listrognathus Tschek, 1871

Nomenclature:

MESOSTENIDEA Viereck, 1914

MESOSTENUS misident.

Listrognathus firmator (Fabricius, 1798)

Nomenclature:

Ichneumon firmator Fabricius, 1798

ligator (Gravenhorst, 1829, Mesostenus)

senilis (Rudow, 1882, Cryptus)

aculeatus (Rudow, 1883, Cryptus)

Distribution: England

Notes: The valid name according to Horstmann (2001a); listed as ligator by Schwarz and Shaw (1998). Distribution data from Horstmann (1990b).

\section{Listrognathus mactator (Thunberg, 1824)}

Nomenclature:

Ichneumon mactator Thunberg, 1824

niveatus (Gravenhorst, 1829, Mesostenus)

pygostolus (Gravenhorst, 1829, Mesostenus)

tricolor Tschek, 1872

intermedius (Szépligeti, 1916, Mesostenus)

\section{Listrognathus mengersseni Schmiedeknecht, 1905}

Distribution: England

Notes: added by Horstmann (1990b) 
Listrognathus obnoxius (Gravenhorst, 1829)

Nomenclature:

Mesostenus obnoxius Gravenhorst, 1829

zygaenarum (Ratzeburg, 1847, Cryptus) synonymy by Horstmann (1997)

subovalis (Thomson, 1873, Mesostenus)

robustus (Rudow, 1882, Cryptus)

subcircularis (Thomson, 1896, Mesostenus)

Distribution: England, Wales

\section{Genus Meringopus Förster, 1869}

Nomenclature:

GONIOCRYPTUS Thomson, 1873

\section{Meringopus attentorius (Panzer, 1804)}

Nomenclature:

Ichneumon attentorius Panzer, 1804

confiscator (Fabricius, 1804, Cryptus) synonymy by Horstmann (2001a)

alboannulatus (Szépligeti, 1916, Cryptus)

\section{Meringopus cyanator (Gravenhorst, 1829)}

Nomenclature:

Cryptus cyanator Gravenhorst, 1829

?fuscescens (Gmelin, 1790, Ichneumon)

?roeselii (Bechstein \& Scharfenberg, 1805, Ichneumon)

\section{Meringopus titillator (Linnaeus, 1758)}

\section{Nomenclature:}

Ichneumon titillator Linnaeus, 1758

recreator (Fabricius, 1804, Cryptus)

tornator (Panzer, 1804, Ichneumon)

pupurator (Thunberg, 1824, Ichneumon)

australis (Tschek, 1871, Cryptus) preocc.

latitarsis (Thomson, 1873, Cryptus)

pectinitarsis (Rudow, 1882, Cryptus) 
titillatrix (Schulz, 1906, Trychosis)

meridionalis (Szépligeti, 1916, Cryptus)

orientalis (Szépligeti, 1916, Cryptus)

nigripes (Seyrig, 1927, Cryptus)

Distribution: England

Genus Mesostenus Gravenhorst, 1829

Nomenclature:

STENARAEUS Thomson, 1896

Mesostenus transfuga Gravenhorst, 1829

Nomenclature:

gallarum (Rudow, 1881, Hemiteles)

gallarum (Rudow, 1882, Hemiteles) preocc.

ingenuus Tosquinet, 1896

niger Kiss, 1929 synonymy by Schwarz (2005)

Distribution: England

Genus Nematopodius Gravenhorst, 1829

Nomenclature:

PSEUDOPIMPLA Fahringer, 1935

Nematopodius debilis (Ratzeburg, 1852)

Nomenclature:

Mesostenus debilis Ratzeburg, 1852

formosus misident.

tricolor (Haupt, 1954, Mesostenus) preocc.

homonymator (Aubert, 1959, Mesostenus)

Distribution: England

\section{Genus Picardiella Lichtenstein, 1920}

Nomenclature:

BORCIELLA Constantineanu, 1929

NIPPORICNUS Uchida, 1931 
PARETHA Seyrig, 1952

Picardiella melanoleuca (Gravenhorst, 1829)

Nomenclature:

Cryptus melanoleucus Gravenhorst, 1829

argiola (Rudow, 1882, Mesostenus)

peregrina (Schmiedeknecht, 1905, Mesostenus)

crenulata (Constantineanu, 1929, Borciella)

tarsoleuca (Kiss, 1929, Habrocryptus)

Distribution: England

Notes: BMNH, det. Broad, added here

\section{Genus Polytribax Förster, 1869}

Nomenclature:

EPIPHOBUS Förster, 1869

NELEOPHRON Förster, 1869

PLESIGNATHUS Förster, 1869

Notes: Polytribax is transferred here from the Hemigastrini, following the molecular phylogenetic results of Laurenne et al. (2006). Unlike hemigastrine genera (where known), Polytribax attack Lepidoptera pupae.

\section{Polytribax arrogans (Gravenhorst, 1829)}

\section{Nomenclature:}

Cryptus arrogans Gravenhorst, 1829

sectator (Gravenhorst, 1829, Phygadeuon)

longipes (Hartig, 1838, Cryptus)

halensis (Taschenberg, 1865, Phygadeuon)

nigriventris (Habermehl, 1917, Microcryptus) preocc.

tricolor (Fahringer, 1935, Plectocryptus)

Distribution: England, Scotland, Wales, Ireland, Isle of Man

\section{Polytribax perspicillator (Gravenhorst, 1807)}

\section{Nomenclature:}




\author{
desertor (Gravenhorst, 1829, Phygadeuon) \\ obscuripes (Taschenberg, 1865, Phygadeuon) \\ errator (Marshall, 1868, Phygadeuon) \\ rufofemoratus (Strobl, 1901, Plectocryptus) \\ nigrifemur (Kiss, 1929, Cryptus) \\ mocsari (Györfi, 1944, Megaplectes)
}

Distribution: England, Scotland, Ireland

\title{
Polytribax picticornis (Ruthe, 1859)
}

Nomenclature:

Cryptus picticornis Ruthe, 1859

gravenhorstii (Thomson, 1883, Microcryptus)

Distribution: England, Scotland, Wales

Notes: NMS, BMNH, added here

\section{Polytribax rufipes (Gravenhorst, 1829)}

Nomenclature:

Cryptus rufipes Gravenhorst, 1829

curvus (Schrank, 1802, Ichneumon) preocc.

rufipes (Schrank, 1835, Ichneumon) preocc.

vexator (Pfankuch, 1921, Microcryptus)

Distribution: England, Ireland

\section{Genus Sphecophaga Westwood, 1840}

Nomenclature:

CHRYONOMON Desvignes, 1856

CACOTROPA Förster, 1869

\section{Sphecophaga vesparum (Curtis, 1828)}

Nomenclature:

Anomalon vesparum Curtis, 1828

striata (Zetterstedt, 1838, Bassus)

vesparum (Ratzeburg, 1852, Tryphon) preocc. 
sericea (Thomson, 1888, Cacotropa)

thuringiaca Schmiedeknecht, 1914

Distribution: England, Scotland, Ireland

Genus Thrybius Townes, 1965

Thrybius brevispina (Thomson, 1896)

Nomenclature:

Hygrocryptus brevispina Thomson, 1896

puhlmanni (Ulbricht, 1909, Hygrocryptus)

Distribution: England

Notes: Incorrectly listed as a synonym of praedator in Yu and Horstmann (1997).

\section{Thrybius praedator (Rossi, 1792)}

Nomenclature:

Ichneumon praedator Rossi, 1792

leucopygus (Gravenhorst, 1829, Hoplismenus)

praedator (Gravenhorst, 1829, Cryptus) preocc.

sanguinolentus (Gravenhorst, 1829, Cryptus)

elegans (Desvignes, 1856, Cryptus)

drewseni (Thomson, 1873, Hygrocryptus)

picticornis (Rudow, 1882, Cryptus) preocc.

praedatrix (Schulz, 1906, Aritranis)

puhlmanni (Ulbricht, 1909, Hygrocryptus)

continuus (Ulbricht, 1910, Hygrocryptus) unavailable

atrocoxatus (Ulbricht, 1916, Hygrocryptus) unavailable

Distribution: England, Wales

Genus Trychosis Förster, 1869

Trychosis ambigua (Tschek, 1871)

Nomenclature:

Cryptus ambiguus Tschek, 1871 
mesocastana (Tschek, 1871, Cryptus) synonymy by Horstmann (2005a)

molesta (Tschek, 1871, Cryptus)

annulicornis (Thomson, 1896, Goniocryptus)

trisculpta (Habermehl, 1929, Goniocryptus) synonymy by Schwarz (2005)

Distribution: England

Notes: BMNH, det. Schwarz, added here

\section{Trychosis atripes (Gravenhorst, 1829)}

Nomenclature:

Cryptus atripes Gravenhorst, 1829

castaniventris (Tschek, 1871, Cryptus)

curvipes (Tschek, 1871, Cryptus)

jugorum (Strobl, 1901, Idiolispa)

Distribution: England

Notes: BMNH, det. Schwarz, added here

\section{Trychosis ? ingrata (Tschek, 1871)}

Nomenclature:

Cryptus ingratus Tschek, 1871

macroura (Thomson, 1873, Goniocryptus)

Notes: added by Schwarz and Shaw (1998); tentative identification

Trychosis insularis Rossem, 1990

Distribution: England

Notes: added by Schwarz and Shaw (1998)

\section{Trychosis legator (Thunberg, 1824)}

\section{Nomenclature:}

Ichneumon legator Thunberg, 1824

mesocastana misident.

titillator misident.

bicolor (Lucas, 1849, Cryptus) 


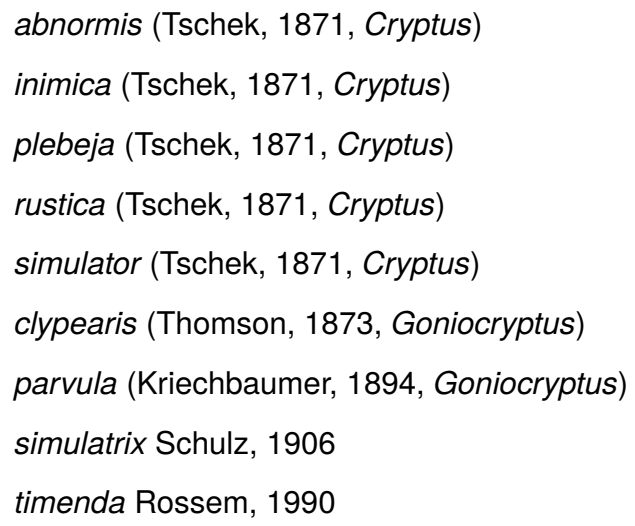

Distribution: England, Scotland, Wales, Ireland, Isle of Man

\section{Trychosis neglecta (Tschek, 1871)}

Nomenclature:

Cryptus neglectus Tschek, 1871

Distribution: England

Notes: BMNH, det. Schwarz, added here

\section{Trychosis picta (Thomson, 1873)}

Nomenclature:

Goniocryptus pictus Thomson, 1873

Distribution: England, Scotland

Notes: added by Schwarz and Shaw (1998) and removed from synonymy with legator

Trychosis tristator (Tschek, 1871)

Nomenclature:

Cryptus tristator Tschek, 1871

glabricula (Thomson, 1873, Goniocryptus)

pleuralis (Thomson, 1896, Goniocryptus)

tristatrix Schulz, 1906

Distribution: England

Notes: added by Schwarz and Shaw (1998); UM 


\section{Genus Xylophrurus Förster, 1869}

Nomenclature:

NYXEOPHILUS Förster, 1869

MACROCRYPTUS Thomson, 1873

NYXEOPHILUS Thomson, 1885

\section{Xylophrurus lancifer (Gravenhorst, 1829)}

Nomenclature:

$$
\begin{aligned}
& \text { Echthrus lancifer Gravenhorst, } 1829 \\
& \text { dispar (Thunberg, 1824, Ichneumon) } \\
& \text { ?nubeculatus (Gravenhorst, 1829, Echthrus) } \\
& \text { ?dentifer (Thomson, 1896, Caenocryptus) } \\
& \text { ?castaniventris (Habermehl, 1909, Kaltenbachia) } \\
& \text { ?rufescens (Ozolz, 1942, Kaltenbachia) }
\end{aligned}
$$

Distribution: England, Scotland

Notes: added by Schwarz and Shaw (1998); it is unclear whether several names are synonyms of lancifer or tumidus.

\section{Xylophrurus tumidus (Desvignes, 1856)}

Nomenclature:

Cryptus tumidus Desvignes, 1856

longiseta (Rudow, 1882, Cryptus)

Distribution: England

Notes: Separated from lancifer by Schwarz and Shaw (1998).

\section{Genus Stenarella Szépligeti, 1916}

Notes: Species of Stenarella excluded from the British and Irish list by Schwarz and Shaw (1998):

[domator (Poda, 1761, Ichneumon); syn. gladiator (Scopoli, 1763, Ichneumon)] 


\section{Tribe HEMIGASTRINI Ashmead, 1900}

\section{Nomenclature:}

APTESINI Smith \& Shenefelt, 1955

ECHTHRINI Narayanan \& Kundanlal, 1958

Notes: Following the molecular phylogenetic results of Laurenne et al. (2006), the genera Echthrus and Polytribax have been transferred to the Cryptini, together with Helcostizus from Phygadeuontini. 'Hemigastrini' is to be preferred over 'Hemigasterini' (Burks 2012). Distribution data from BMNH, NMS (mostly det. M. Schwarz and J. Sawoniewicz in the latter) and UM; Fitton (1976) also supplies some data on collection localities of type specimens.

\section{Genus Aconias Cameron, 1904}

\section{Aconias tarsatus (Bridgman, 1881)}

\section{Nomenclature:}

Phygadeuon tarsatus Bridgman, 1881

pectoralis (Thomson, 1896, Plectocryptus)

lateannulatus (Strobl, 1901, Chaeretymma)

nigrofemoratus (Strobl, 1901, Plectocryptus)

Distribution: England, Scotland, Ireland

\section{Genus Aptesis Förster, 1850}

Nomenclature:

PEZOPORUS Förster, 1869

CLYPEODIODON Aubert, 1968

Notes: doubtfully placed species of Aptesis:

[leucosticta (Gravenhorst, 1829, Cryptus) nom. dub.]

\section{Aptesis assimilis (Gravenhorst, 1829)}

\section{Nomenclature:}

Phygadeuon assimilis Gravenhorst, 1829

distans (Thomson, 1883, Microcryptus)

Distribution: Ireland, Isle of Man 
Aptesis cretata (Gravenhorst, 1829)

Nomenclature:

Phygadeuon cretatus Gravenhorst, 1829

Distribution: England

Aptesis femoralis (Thomson, 1883)

Nomenclature:

Microcryptus femoralis Thomson, 1883

zonata (Kriechbaumer, 1893, Microcryptus)

alpina (Strobl, 1901, Microcryptus)

Distribution: England, Ireland

\section{Aptesis flagitator (Rossius, 1794)}

Nomenclature:

Icheumon flagitator Rossius, 1794

pumilio (Gravenhorst, 1829, Phygadeuon)

tyranna (Gravenhorst, 1829, Phygadeuon) preocc.

hopei (Desvignes, 1856, Cryptus)

proximator (Costa, 1886, Phygadeuon)

tricolor (Kriechbaumer, 1894, Microcryptus)

hopei (Morley, 1907, Acanthocryptus) preocc.

feketei (Kiss, 1915, Acanthocryptus)

rufipes (Obrtel, 1953, Acanthocryptus)

Distribution: England, Ireland

\section{Aptesis improba (Gravenhorst, 1829)}

Nomenclature:

Phygadeuon improbus Gravenhorst, 1829

exigua (Habermehl, 1909, Microcryptus)

bisignata (Habermehl, 1919, Microcryptus)

Distribution: Ireland 
Aptesis jejunator (Gravenhorst, 1807)

Nomenclature:

Ichneumon jejunator Gravenhorst, 1807

abdominator (Gravenhorst, 1829, Phygadeuon)

nematorum (Rudow, 1886, Phygadeuon)

genalis (Kriechbaumer, 1895, Microcryptus)

albilarva (Speiser, 1908, Microcryptus)

brumatae (Silvestri, 1941, Microcryptus)

Distribution: England, Ireland

Aptesis nigricollis (Thomson, 1883)

Nomenclature:

Acanthocryptus nigricollis Thomson, 1883

Distribution: England, Ireland

Notes: Listed as a species of Rhembobius by Fitton (1978).

Aptesis nigritula (Thomson, 1885)

Nomenclature:

Microcryptus nigritulus Thomson, 1885

nigripes (Strobl, 1901, Stenocryptus)

Distribution: England, Ireland

\section{Aptesis nigrocincta (Gravenhorst, 1815)}

Nomenclature:

Ichneumon nigrocinctus Gravenhorst, 1815

?bimaculata (Christ, 1791, Ichneumon) preocc.

sudetica (Gravenhorst, 1815, Ichneumon)

duplicatoria (Thunberg, 1824, Ichneumon, Ichneumon)

flaveolata (Gravenhorst, 1829, Phygadeuon)

hostilis (Gravenhorst, 1829, Cryptus)

jucunda (Gravenhorst, 1829, Phygadeuon)

ephippia (Rudow, 1914, Agrothereutes)

fulvipes (Rudow, 1914, Agrothereutes) 
haemorrhoidalis (Rudow, 1914, Theroscopus)

analis (Rudow, 1917, Theroscopus)

borealis Rudow, 1917

clythrae (Rudow, 1917, Pezomachus)

ephippia (Rudow, 1917, Agrothereutes) preocc.

fulvipes (Rudow, 1917, Agrothereutes) preocc.

haemorhoidalis (Rudow, 1917, Theroscopus) preocc.

nigrocincta (Rudow, 1917, Pezomachus) preocc.

Distribution: England, Scotland, Wales, Ireland

\section{Aptesis orbitalis (Thomson, 1883)}

\section{Nomenclature:}

Microcryptus orbitalis Thomson, 1883

Notes: BMNH, added here; specimen labelled as from British Isles, Billups coll.

Aptesis scotica (Marshall, 1868)

Nomenclature:

Phygadeuon scoticus Marshall, 1868

Distribution: Scotland

Aptesis terminata (Gravenhorst, 1829)

Nomenclature:

Phygadeuon terminatus Gravenhorst, 1829

gilvipes (Gravenhorst, 1829, Phygadeuon)

ceilonota (Taschenberg, 1865, Phygadeuon)

Distribution: England

\section{Genus Colocnema Förster, 1869}

Nomenclature:

COELOCRYPTUS Thomson, 1873 
Colocnema rufina (Gravenhorst, 1829)

Nomenclature:

Phygadeuon rufinus Gravenhorst, 1829

romani (Pfankuch, 1914, Plectocryptus)

Distribution: England

Genus Cratocryptus Thomson, 1873

Cratocryptus ? furcator (Gravenhorst, 1829)

Nomenclature:

Cryptus ?furcator Gravenhorst, 1829

Distribution: Scotland

Notes: Material in UM, det. J.P. Brock, but see note under subpetiolatus.

Cratocryptus subpetiolatus (Gravenhorst, 1829)

Nomenclature:

Cryptus subpetiolatus Gravenhorst, 1829

Distribution: England

Notes: Transferred from Cubocephalus by Sawoniewicz and Wanat (2003). This species seems to have been confused with furcator and it is not clear if furcator occurs in Britain or Ireland.

\section{Genus Cubocephalus Ratzeburg, 1848}

Nomenclature:

CHAERETYMMA Förster, 1869

ECPORTHETOR Förster, 1869

PAMMACHUS Förster, 1869

MICROCRYPTUS Thomson, 1873

STENOCRYPTUS Thomson, 1873

PLANOCRYPTUS Heinrich, 1949

Cubocephalus anatorius (Gravenhorst, 1829)

Nomenclature:

Cryptus anatorius Gravenhorst, 1829 
?dumetorum (Geoffroy, 1785, Ichneumon)

stomaticus (Gravenhorst, 1829, Cryptus) synonymy by Sawoniewicz (2003)

exareolatus (Habermehl, 1917, Cratocryptus)

albopictus (Kiss, 1924, Plectocryptus)

Distribution: England, Wales, Ireland

\section{Cubocephalus associator (Thunberg, 1824)}

\section{Nomenclature:}

Ichneumon associator Thunberg, 1824

ruficoxis (Thomson, 1873, Cratocryptus)

Distribution: England

\section{Cubocephalus brevicornis (Taschenberg, 1865)}

\section{Nomenclature:}

Phygadeuon brevicornis Taschenberg, 1865

oviventris misident.

Distribution: England, Ireland

\section{Cubocephalus distinctor (Thunberg, 1824)}

Nomenclature:

Ichneumon distinctor Thunberg, 1824

fortipes misident.

Distribution: England, Scotland, Wales, Ireland

Notes: Sawoniewicz (2003) treats fortipes (Gravenhorst, 1829, Cryptus), with canaliculatus (Gravenhorst, 1829, Ichneumon) as a synonym, as distinct from distinctor . Despite Sawoniewicz's quote of Morley (1907) for the concept of fortipes, all the British material in BMNH is referrable to distinctor, based on Sawoniewicz's characters for separation of the species. NMS material has been identified by J. Sawoniewicz as distinctor.

\section{Cubocephalus femoralis (Thomson, 1873)}

\section{Nomenclature:}

Cratocryptus femoralis Thomson, 1873

kriegeri (Habermehl, 1911, Cratocryptus) 
Cubocephalus nigriventris (Thomson, 1874)

Nomenclature:

Stenocryptus nigriventris Thomson, 1874

Distribution: England, Scotland, Wales, Ireland

\section{Cubocephalus sperator (Müller, 1776)}

Nomenclature:

Ichneumon sperator Müller, 1776

bilineatus (Gravenhorst, 1829, Cryptus)

erythrinus (Gravenhorst, 1829, Cryptus) synonymy by Sawoniewicz (2003)

lacteator (Gravenhorst, 1829, Cryptus)

semiorbitalis (Gravenhorst, 1829, Phygadeuon)

cruentus (Kriechbaumer, 1891, Microcryptus)

Distribution: England, Scotland, Ireland

Notes: Listed as a species of Pleolophus in Yu and Horstmann (1997) but included in Cubocephalus by Sawoniewicz (2003).

\section{Genus Demopheles Förster, 1869}

\section{Demopheles corruptor (Taschenberg, 1865)}

Nomenclature:

Phygadeuon corruptor Taschenberg, 1865

Distribution: England, Scotland, Ireland

Notes: Phygadeuon caliginosus Grav. is listed as a synonym of $D$. corruptor by Fitton (1978) but is now treated as a synonym of Phygadeuon ovatus Grav.

\section{Genus Giraudia Förster, 1869}

\section{Nomenclature:}

CALOCRYPTUS Thomson, 1873

PSEUDOCRYPTUS Kriechbaumer, 1893 


\section{Giraudia grisescens (Gravenhorst, 1829)}

Nomenclature:

Cryptus grisescens Gravenhorst, 1829

scansor (Thomson, 1890, Plectocryptus)

nigritarsis (Ulbricht, 1910, Plectocryptus) unavailable

Distribution: Ireland

\section{Giraudia gyratoria (Thunberg, 1824)}

Nomenclature:

Ichneumon gyratorius Thunberg, 1824

congruens (Gravenhorst, 1829, Cryptus)

dimimilis (Kiss, 1924, Megaplectes)

Genus Javra Cameron, 1903

Nomenclature:

MONOCRYPTUS Hellén, 1957

Javra anomala (Morley, 1908)

Nomenclature:

Diadegma anomala Morley, 1908

hedwigi (Habermehl, 1929, Microcryptus)

Distribution: England, Scotland, Wales

Javra opaca (Thomson, 1873)

Nomenclature:

Cratocryptus opacus Thomson, 1873

gracilicornis (Kriechbaumer, 1891, Microcryptus)

jenneri (Heinrich, 1949, Microcryptus)

Distribution: Scotland

Javra tricincta (Gravenhorst, 1829)

Nomenclature:

Cryptus tricinctus Gravenhorst, 1829

areolaris (Thomson, 1883, Microcryptus) 
prominens (Schmiedeknecht, 1931, Microcryptus)

Distribution: England, Wales

Genus Listrocryptus Brauns, 1905

Listrocryptus spatulatus Brauns, 1905

Distribution: England

Notes: NMS (det. Schwarz), BMNH, added here

Genus Megaplectes Förster, 1869

Nomenclature:

IOCRYPTUS Thomson, 1873

MEGALOPLECTES Schulz, 1906

Megaplectes monticola (Gravenhorst, 1829)

Nomenclature:

Ichneumon monticola Gravenhorst, 1829

regius (Taschenberg, 1865, Phygadeuon)

andrei (Berthoumieu, 1897, Eurylabus)

lucens Torka, 1935

Genus Oresbius Marshall, 1867

Nomenclature:

OPIDNUS Förster, 1869

Oresbius arridens (Gravenhorst, 1829)

Nomenclature:

Phygadeuon arridens Gravenhorst, 1829

niveatus (Desvignes, 1856, Ichneumon)

rhombifer (Kriechbaumer, 1893, Microcryptus)

Distribution: England, Ireland

Oresbius castaneus Marshall, 1867

Nomenclature: 
terrestris (Roman, 1909, Microcryptus)

Distribution: Scotland

Oresbius funereus (Schmiedeknecht, 1905)

Nomenclature:

Microcryptus funereus Schmiedeknecht, 1905

Oresbius galactinus (Gravenhorst, 1829)

Nomenclature:

Phygadeuon galactinus Gravenhorst, 1829

fulgens (Taschenberg, 1865, Phygadeuon)

nigricans (Pfankuch, 1923, Microcryptus) unavailable

Distribution: Ireland

Oresbius leucopsis (Gravenhorst, 1829)

Nomenclature:

Cryptus leucopsis Gravenhorst, 1829

nycthemerus (Gravenhorst, 1829, Phygadeuon)

brumatae (Rudow, 1886, Phygadeuon)

nigriventris (Thomson, 1896, Mesocryptus)

victorovi Jonaitis, 1981

Distribution: England, Ireland

Oresbius nivalis (Zetterstedt, 1838)

Nomenclature:

Cryptus nivalis Zetterstedt, 1838

opacus (Taschenberg, 1865, Cryptus) preocc.

borealis (Thomson, 1883, Microcryptus)

exannulatus (Roman, 1909, Microcryptus) preocc.

\section{Oresbius subguttatus (Gravenhorst, 1829)}

\section{Nomenclature:}

Cryptus subguttatus Gravenhorst, 1829

contracta (Gravenhorst, 1829, Cryptus) 
punctata (Ratzeburg, 1844, Cryptus)

abscissa (Ratzeburg, 1852, Cryptus)

incerta (Ratzeburg, 1852, Cryptus)

silesiacus (Habermehl, 1920, Platylabus) synonymy by Horstmann (2006d)

discedens (Habermehl, 1929, Platylabus) synonymy by Horstmann (2006d)

Distribution: England, Ireland

Notes: Listed as a species of Aptesis by Yu and Horstmann (1997).

Genus Parmortha Townes, 1962

Parmortha parvula (Gravenhorst, 1829)

Nomenclature:

Cryptus parvulus Gravenhorst, 1829

erythropus (Gravenhorst, 1829, Cryptus)

Distribution: England, Scotland, Ireland

Parmortha pleuralis (Thomson, 1873)

Nomenclature:

Cratocryptus pleuralis Thomson, 1873

Distribution: England, Scotland, Ireland, Isle of Man

Genus Plectocryptus Thomson, 1873

Plectocryptus albulatorius (Gravenhorst, 1829)

Nomenclature:

Cryptus albulatorius Gravenhorst, 1829

hilarulus Schmiedeknecht, 1905

Plectocryptus digitatus (Gmelin, 1790)

Nomenclature:

Ichneumon digitatus Gmelin, 1790

bivinctus (Gravenhorst, 1829, Cryptus)

poecilopus (Rudow, 1883, Cryptus)

niger (Kiss, 1926, Habrocryptus) 
Distribution: England, Scotland

\title{
Plectocryptus effeminatus (Gravenhorst, 1829)
}

\section{Nomenclature:}

\author{
Cryptus effeminatus Gravenhorst, 1829 \\ flavopunctatus (Bridgman, 1889, Phygadeuon) \\ armatus (Kriechbaumer, 1893, Microcryptus) \\ clavatus (Kriechbaumer, 1893, Microcryptus) \\ sellatus Ulbricht, 1911 unavailable \\ lancifer (Roman, 1925, Acanthocryptus)
}

Distribution: England

Plectocryptus periculosus (Schmiedeknecht, 1905)

Nomenclature:

Microcryptus periculosus Schmiedeknecht, 1905

Distribution: England

Notes: NMS, det. Schwarz, added here

\section{Genus Pleolophus Townes, 1962}

\section{Pleolophus basizonus (Gravenhorst, 1829)}

Nomenclature:

Phygadeuon basizonus Gravenhorst, 1829

larvincola (Scharfenberg, 1805, Ichneumon) nom. ob.

varicolor (Gravenhorst, 1829, Cryptus)

pteronum (Hartig, 1838, Phygadeuon)

commutatus (Ratzeburg, 1848, Phygadeuon)

obscurus (Ulbricht, 1913, Microcryptus) unavailable

nigrinus (Fahringer, 1941, Spilocryptus)

Distribution: England, Scotland, Ireland

Notes: Sawoniewicz (2003) established that basizonus Grav. is a junior synonym of larvincola but under article 23.9 of the Code (ICZN 1999), bazizonus should be a protected name and larvincola a nomen oblitum (Horstmann 2006b). 
Pleolophus brachypterus (Gravenhorst, 1815)

\section{Nomenclature:}

Ichneumon brachypterus Gravenhorst, 1815

micropterus misident.

assimilis (Förster, 1850, Aptesis)

curtulus (Kriechbaumer, 1891, Microcryptus)

antennalis (Kiss, 1924, Habrocryptus)

hungaricus (Kis, 1924, Hemichneumon)

Distribution: England, Scotland, Wales, Ireland, Isle of Man

\section{Pleolophus sericans (Gravenhorst, 1829)}

Nomenclature:

Phygadeuon sericans Gravenhorst, 1829

pictus (Gmelin, 1790, Ichneumon) preocc.

eximius (Habermehl, 1935, Microcryptus)

Distribution: England, Ireland

Pleolophus vestigialis (Förster, 1850)

Nomenclature:

Aptesis vestigialis Förster, 1850

aphyopterus (Förster, 1850, Aptesis)

formosus (Förster, 1850, Aptesis)

unifasciatus (Schmiedeknecht, 1905, Microcryptus)

alpinus (Rudow, 1917, Aptesis)

triangularis (Kiss, 1924, Microcryptus)

angustipetiolatus (Ozols, 1934, Microcryptus)

piceus (Fahringer, 1935, Stibeutes)

\section{Genus Rhembobius Förster, 1869}

Nomenclature:

ULOTHYMUS Förster, 1869

ACANTHOCRYPTUS Thomson, 1873 
Notes: Since Townes (1970), Rhembobius has been placed in the Phygadeuontini, subtribe Ethelurgina, along with other parasitoids of syrphid (Diptera) larvae, but Quicke et al. (2009) always found Rhembobius to group with the Hemigastrini in their molecular phylogenetic analyses. Distribution data from Horstmann (2000e), Schwarz and Shaw (2010), BMNH, plus additional reference.

\section{Rhembobius bifrons (Gmelin, 1790)}

Nomenclature:

Ichneumon bifrons Gmelin, 1790

rufoniger (Bridgman, 1889, Phygadeuon)

minimus (Lange, 1911, Microcryptus)

Distribution: England, Scotland, Wales, Ireland

Notes: Listed as a species of Aptesis by Fitton (1978).

\section{Rhembobius perscrutator (Thunberg, 1824)}

Nomenclature:

Ichneumon perscrutator Thunberg, 1824

basalis (Smith, 1874, Cryptus)

Distribution: England, Scotland, Wales, Ireland

Notes: some distribution data from Godfrey and Whitehead (2001)

\section{Rhembobius quadrispinus (Gravenhorst, 1829)}

\section{Nomenclature:}

Phygadeuon quadrispinus Gravenhorst, 1829

ambiguus (Berthoumieu, 1914, Platylabus)

albicoxis (Kiss, 1915, Ryssolabus)

limnophilus (Smits van Burgst, 1920, Acanthocryptus) synonymy by Horstmann (2000e)

nigrobasicus (Kiss, 1924, Acanthocryptus)

Distribution: England, Scotland, Wales, Ireland, Isle of Man

\section{Genus Schenkia Förster, 1869}

Nomenclature: 
SCHENCKIA Dalla Torre, 1901

Schenkia graminicola (Gravenhorst, 1829)

Nomenclature:

Phygadeuon graminicola Gravenhorst, 1829

brevicornis (Gravenhorst, 1829, Cryptus)

humilis (Gravenhorst, 1829, Cryptus)

alta Jonaitis, 1981

Distribution: England, Scotland, Ireland

Schenkia labralis (Gravenhorst, 1829)

Nomenclature:

Phygadeuon labralis Gravenhorst, 1829

Distribution: Ireland

Schenkia spinolae (Gravenhorst, 1829)

Nomenclature:

Phygadeuon spinolae Gravenhorst, 1829

\section{Tribe PHYGADEUONTINI Förster, 1869}

Nomenclature:

HEMITELINI Förster, 1869

GELINI Viereck, 1918

Notes: Helcostizus is transferred here to the Cryptini, following the molecular phylogenetic results of Laurenne et al. (2006), which also accords better with its morphology, very aberrant within the Phygadeuontini. See notes under 'Cryptini'. Distribution data mainly taken from Schwarz and Shaw (1999), Schwarz and Shaw (2000), Schwarz and Shaw (2010), Schwarz and Shaw (2011), with some additional data from BMNH, in particular, also UM and some type localities from Fitton (1976); other sources are provided under the relevant genera/species.

\section{Genus Aclastus Förster, 1869}

Nomenclature:

DAETORA Förster, 1869

MICROPLEX Förster, 1869 
OPISTHOSTENUS Förster, 1869

FETIALIS Rossem, 1990 synonymy by anon. (2001), Broad (2004)

\section{Aclastus borealis (Boheman, 1866)}

Nomenclature:

Hemiteles borealis Boheman, 1866

septentrionalis (Holmgren, 1869, Hemiteles)

Distribution: Scotland

Notes: added by Horstmann (1980b)

\section{Aclastus eugracilis Horstmann, 1980}

Distribution: England, Scotland, Wales

Notes: added by Schwarz and Shaw (2000)

Aclastus flavipes Horstmann, 1980

Distribution: England

Notes: added by Horstmann (1980b)

\section{Aclastus gracilis (Thomson, 1884)}

Nomenclature:

Hemiteles gracilis Thomson, 1884

furcifer Hellén, 1967

Distribution: England, Scotland, Wales, Ireland, Isle of Man

\section{Aclastus micator (Gravenhorst, 1807)}

Nomenclature:

Ichneumon micator Gravenhorst, 1807

necator misident.

caudator Hellén, 1967

Distribution: England, Scotland, Wales

Notes: Listed, as necator (Fab.) (a name belonging to the Braconidae, Horstmann 2001a), as a doubtfully placed species of Hemiteles by Fitton (1978); Morley's (Morley 1907) redescription of necator refers partly to micator (Schwarz and Shaw 2000). 
Aclastus minutus (Bridgman, 1886)

Nomenclature:

Hemiteles minutus Bridgman, 1886

Distribution: England, Scotland, Ireland

Notes: Irish occurrence from Anderson et al. (2006)

Aclastus pilosus Horstmann, 1980

Distribution: England, Scotland, Wales, Ireland

Notes: added by Horstmann (1980b)

Aclastus solutus (Thomson, 1884)

Nomenclature:

Hemiteles solutus Thomson, 1884

Distribution: England, Scotland, Wales, Ireland

Aclastus transversalis Horstmann, 1980

Distribution: England, Wales

Notes: added by Horstmann (1980b)

Genus Acrolyta Förster, 1869

Nomenclature:

RHADINOCERA Förster, 1869

Acrolyta flagellator Schwarz \& Shaw, 2000

Distribution: Scotland

Notes: added by Schwarz and Shaw (2000)

Acrolyta marginata (Bridgman, 1883)

Nomenclature:

Hemiteles marginatus Bridgman, 1883

Distribution: England, Scotland 
Acrolyta nens (Hartig, 1838)

Nomenclature:

Hemiteles nens Hartig, 1838

submarginata (Bridgman, 1883, Hemiteles)

rufizonata (Schmiedeknecht, 1905, Hemiteles)

Distribution: England, Scotland, Wales, Ireland

Acrolyta okadai (Uchida, 1942)

Nomenclature:

Adiastola okadai Uchida, 1942

Notes: Added by Schwarz \& Shaw (2000)Schwarz and Shaw (2000)and transferred from Eudelus.

\section{Acrolyta pseudonens Schwarz \& Shaw, 2000}

Distribution: England, Scotland

Notes: added by Schwarz and Shaw (2000)

\section{Acrolyta rufocincta (Gravenhorst, 1829)}

Nomenclature:

Hemiteles rufocinctus Gravenhorst, 1829

distincta (Bridgman, 1883, Hemiteles)

capreolus (Thomson, 1884, Hemiteles)

quadrimaculata (Lange, 1911, Hemiteles)

obscurata (Kiss, 1924, Hemiteles)

unifasciata (Kiss, 1924, Hemiteles)

Distribution: England, Scotland, Wales

Notes: The name rufocincta was listed as a doubtfully placed species of Hemiteles by Fitton (1978) whilst the names distincta and capreola were listed as species of Acrolyta and Eudelus, respectively.

\section{Acrolyta semistrigosa (Schmiedeknecht, 1897)}

\section{Nomenclature:}


Distribution: England

Notes: added by Schwarz and Shaw (2000)

Genus Agasthenes Förster, 1869

Nomenclature:

ASTHENOPTERA Förster, 1869

Notes: Some distribution data from Townes (1983).

Agasthenes subarcticus (Jussila, 1965)

Nomenclature:

Hemiteles subarcticus Jussila, 1965

Distribution: Scotland, Ireland

Notes: added by Horstmann (1998a)

Agasthenes varitarsus (Gravenhorst, 1829)

Nomenclature:

Hemiteles varitarsus Gravenhorst, 1829

stagnalis (Thomson, 1884, Hemiteles)

Distribution: England, Wales, Ireland

\section{Genus Amphibulus Kriechbaumer, 1893}

Notes: Some dstribution data from Sawoniewicz (2003).

\section{Amphibulus gracilis Kriechbaumer, 1893}

Nomenclature:

bispinus (Thomson, 1894, Cratocryptus)

aertsi (Habermehl, 1926, Stylocryptus)

Distribution: England, Scotland, Ireland

Genus Arotrephes Townes, 1970

Notes: Some distribution data from Horstmann (1993c). 
Arotrephes laeviscutum Horstmann, 1993

Distribution: England

Notes: added by Horstmann (1993c)

Arotrephes parvipennis (Thomson, 1884)

Nomenclature:

Phygadeuon parvipennis Thomson, 1884

Distribution: England, Scotland

Notes: added by Horstmann (1993c)

Arotrephes perfusor (Gravenhorst, 1829)

Nomenclature:

Cryptus perfusor Gravenhorst, 1829

nitidus (Bridgman, 1889, Hemiteles)

Distribution: England, Scotland, Ireland

Notes: Listed by Fitton (1978) as Charitopes nitidus (Bridg.).

\section{Arotrephes speculator (Gravenhorst, 1829)}

Nomenclature:

Phygadeuon speculator Gravenhorst, 1829

Distribution: England, Scotland, Ireland

\section{Genus Atractodes Gravenhorst, 1829}

Notes: Distribution data from Jussila (Jussila 1979, Jussila 1983, Jussila 2001), and the collections of NMS (det. Jussila), with additional references given.

doubtfully placed species of Atractodes:

[dionaeus Haliday, 1837 nom. nud., from England, Ireland; Fitton (1976)]

[piceicornis Haliday, 1837 nom. nud., from Ireland; Fitton (1976)]

[salius Haliday, 1837 nom. nud., from Ireland; Fitton (1976)]

[vestalis Haliday, 1837 nom. nud.] 
Subgenus Asyncrita Förster, 1876

Atractodes (Asyncrita) acuminator Roman, 1909

Distribution: England

Notes: added byJussila (2001)

Atractodes (Asyncrita) albovinctus Haliday, 1837

Nomenclature:

mediatus (Förster, 1876, Asyncrita)

Distribution: Ireland

Notes: Treated as a nomen nudum by Fitton (1976) but was established as a senior synonym of mediatus by Jussila (1994a).

Atractodes (Asyncrita) ambiguus Ruthe, 1859

Nomenclature:

truncator Roman, 1909

Distribution: England

Atractodes (Asyncrita) angustipennis Förster, 1876

Nomenclature:

adversarius Förster, 1876

affinis Förster, 1876

cryptonastes Förster, 1876

gracilentus Förster, 1876

invalidus Förster, 1876

subdentatus Förster, 1876

vilis Förster, 1876

flavicoxa Thomson, 1884

thomsonii Dalla Torre, 1902 preocc.

Distribution: Scotland, Ireland 
Atractodes (Asyncrita) assimilis Förster, 1876

Nomenclature:

minusculus Förster, 1876

sordidus Förster, 1876

sponsus Förster, 1876

Distribution: Scotland

Notes: added by Jussila (2001)

Atractodes (Asyncrita) croceicornis Haliday, 1839

Nomenclature:

acceptus Förster, 1876

aemulator Förster, 1876

alticola Förster, 1876

atricornis Förster, 1876

contrarius Förster, 1876

designatus Förster, 1876

difficilis Förster, 1876

distinctus Förster, 1876

engadinus Förster, 1876

exosus Förster, 1876

expertus Förster, 1876

fatalis Förster, 1876

inclinans Förster, 1876

infestus Förster, 1876

intemperans Förster, 1876

laboriosus Förster, 1876

minax Förster, 1876

modestus Förster, 1876

nodifer Förster, 1876

obsoletus Förster, 1876

placidus Förster, 1876

praepotens Förster, 1876

progenitus Förster, 1876 


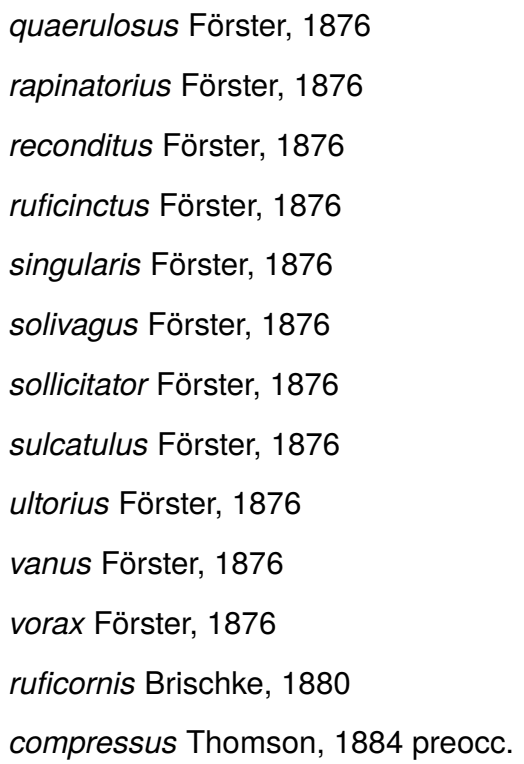

Distribution: England, Scotland, Ireland, Isle of Man

Atractodes (Asyncrita) cryptobius Förster, 1876

Nomenclature:

eryptobius misspelling

carinatus Förster, 1876

conspicuus Förster, 1876

custoditor Förster, 1876

fulvicornis Förster, 1876

parallelus Thomson, 1884

Distribution: Ireland

Notes: added by Jussila (1979)

Atractodes (Asyncrita) cultellator Haliday, 1839

Distribution: Ireland

Atractodes (Asyncrita) exilis Haliday, 1839

Nomenclature:

alpicola (Förster, 1876, Asyncrita)

angustulus Förster, 1876 
delicatulus Förster, 1876

dispar Förster, 1876

flavicoxis Förster, 1876

longiventris (Förster, 1876, Asyncrita)

suspicax Förster, 1876

xanthocarpus Förster, 1876

alpicola Strobl, 1901 preocc.

Distribution: England, Scotland, Ireland

Atractodes (Asyncrita) exitialis Förster, 1876

Nomenclature:

breviusculus Förster, 1876

callidus Förster, 1876

debilis Förster, 1876

difformis Förster, 1876

infimus Förster, 1876

parilis Förster, 1876

particeps Förster, 1876

perpusillus Förster, 1876

Distribution: Scotland

Notes: added by Jussila (2001)

Atractodes (Asyncrita) foveolatus Gravenhorst, 1829

Nomenclature:

canaliculatus (Hellén, 1944, Asyncrita)

Distribution: England

Atractodes (Asyncrita) picipes Holmgren, 1860

Nomenclature:

nigripes Förster, 1876 
Atractodes (Asyncrita) spiraculator Roman, 1918

Distribution: Scotland

Notes: added by Jussila (2001)

Subgenus Atractodes Gravenhorst, 1829

Nomenclature:

ZETESIMA Förster, 1876

Atractodes (Atractodes) alpestris Roman, 1918

Distribution: Ireland

Notes: added by Jussila (1979)

Atractodes (Atractodes) arator Haliday, 1839

Nomenclature:

rufiventris Strobl, 1901 preocc.

Distribution: Scotland, Ireland

Atractodes (Atractodes) bicolor Gravenhorst, 1829

Nomenclature:

pygmaeator (Zetterstedt, 1838, Ichneumon)

alpigradus Förster, 1876

analogus Förster, 1876

cultrarius Förster, 1876 preocc.

destructor Förster, 1876

incommodus Förster, 1876

indigena Förster, 1876

lepidus Förster, 1876

mesoxanthus Förster, 1876

montivagus Förster, 1876

tenax Förster, 1876

Distribution: Scotland 
Atractodes (Atractodes) citator Haliday, 1839

Distribution: Ireland

Atractodes (Atractodes) fumatus Haliday, 1839

Nomenclature:

abnormis Förster, 1876

ambifarius Förster, 1876

avidus Förster, 1876

castus Förster, 1876

discolor Förster, 1876

dissidens Förster, 1876

ecarinatus Förster, 1876

homologus Förster, 1876

incongruens Förster, 1876

isomorphus Förster, 1876

melanocerus Förster, 1876

melanostomus Förster, 1876

nigrocoxis Förster, 1876

proprius Förster, 1876

separatus Förster, 1876

subdolus Förster, 1876

tenuicinctus Förster, 1876

tenuis Förster, 1876

unicinctus Förster, 1876

Distribution: England, Scotland

Atractodes (Atractodes) gilvipes Holmgren, 1860

Atractodes (Atractodes) gravidus Gravenhorst, 1829

Nomenclature:

fraternus Förster, 1876

areolaris (Habermehl, 1909, Exolytus)

archangelicae Roman, 1913 
brevicornis Bauer, 1958

Distribution: England

Atractodes (Atractodes) magnus Jussila, 2001

Distribution: England

Notes: Ely coll., det Jussila, added here

Atractodes (Atractodes) obsoletor (Zetterstedt, 1838)

Nomenclature:

Porizon obsoletor Zetterstedt, 1838

agilis Förster, 1876

declinis Förster, 1876

neophytus Förster, 1876

niger Förster, 1876 preocc.

foersteri Dalla Torre, 1901

Distribution: England, Wales

Notes: added by Jussila (1979)

Atractodes (Atractodes) pauxillus Förster, 1876

Nomenclature:

montanus Förster, 1876

breviscapus Thomson, 1884

Distribution: England, Scotland, Ireland

Atractodes (Atractodes) pusillus Förster, 1876

Nomenclature:

calceatus Förster, 1876

linearis Förster, 1876

tenellus Förster, 1876

liogaster Förster, 1876

pernitens Kokujev, 1909

Distribution: England, Scotland, Ireland 
Atractodes (Atractodes) tenuipes Thomson, 1884

Distribution: England, Scotland

Notes: added by Bass and Cooling (1983)

Atractodes (Atractodes) townesi Jussila, 1983

Nomenclature:

thomsoni Jussila, 1979

Distribution: Scotland

Notes: added by Jussila (2001)

Subgenus Cyclaulatractodes Jussila, 1979

Atractodes (Cyclaulatractodes) helveticus Förster, 1876

Nomenclature:

aequilongus Förster, 1876

oreophilus Förster, 1876 synonymy by Jussila (2001)

Distribution: Ireland

Atractodes (Cyclaulatractodes) punctator Roman, 1909

Distribution: England, Scotland, Wales

Notes: added by Jussila (2001)

Subgenus Hadratractodes Jussila, 1979

Atractodes (Hadratractodes) vicinus Förster, 1876

Nomenclature:

absconditus Förster, 1876

cautior Förster, 1876

inquilinus Förster, 1876

intersectus Förster, 1876

lentus Förster, 1876

rufipes Förster, 1876 preocc.

sectator Förster, 1876 
venustulus Förster, 1876

crassicornis Förster, 1876

sarntheinii Dalla Torre, 1901

Distribution: Scotland

Notes: NMS, det. Jussila, added here

Subgenus Rugratractodes Jussila, 1979

Atractodes (Rugratractodes) alpinus Förster, 1876

Nomenclature:

inimicus Förster, 1876

Distribution: Scotland

Notes: added by Jussila (2001)

Atractodes (Rugratractodes) incrassator Roman, 1926

Distribution: Scotland

Notes: added by Jussila (2001)

\section{Genus Bathythrix Förster, 1869}

Nomenclature:

GAUSOCENTRUS Förster, 1869

ISCHNURGOPS Förster, 1869

PANARGYROPS Förster, 1869

STEGANOPS Förster, 1869

LEPTOCRYPTUS Thomson, 1873

Notes: Some distribution data from Sawoniewicz (1980).

Bathythrix aerea (Gravenhorst, 1829)

Nomenclature:

Cryptus aereus Gravenhorst, 1829

brevis (Thomson, 1884, Leptocryptus)

Distribution: England, Scotland, Wales, Ireland 
Bathythrix alter (Kerrich, 1942)

Nomenclature:

Panargyrops alter Kerrich, 1942

Distribution: England, Wales

Bathythrix argentata (Gravenhorst, 1829)

Nomenclature:

Hemiteles argentatus Gravenhorst, 1829

lacustris (Schmiedeknecht, 1905, Leptocryptus)

Distribution: England

Bathythrix claviger (Taschenberg, 1865)

Nomenclature:

Cryptus claviger Taschenberg, 1865

atra (Brischke, 1881, Cryptus)

Distribution: England, Scotland, Ireland

Bathythrix collaris (Thomson, 1896)

Nomenclature:

Leptocryptus collaris Thomson, 1896

Distribution: England, Scotland

Bathythrix decipiens (Gravenhorst, 1829)

Nomenclature:

Hemiteles decipiens Gravenhorst, 1829

gyrini (Parfitt, 1881, Hemiteles)

signata (Habermehl, 1919, Leptocryptus)

meridionator (Aubert, 1960, Panargyrops)

Distribution: England, Ireland, Isle of Man

Notes: Listed (twice, as decipiens and gyrini) as doubtfully placed species of Hemiteles in Fitton (1978). 
Bathythrix formosa (Desvignes, 1860)

Nomenclature:

Hemiteles formosus Desvignes, 1860

albomarginata (Kriechbaumer, 1892, Leptocryptus)

?grandimacula (Kriechbaumer, 1892, Leptocryptus) unavailable

geniculosa (Thomson, 1884, Leptocryptus)

Distribution: England

Notes: Bathythrix formosa was separated from fragilis by Horstmann (1998a) (although the name grandimacula was not mentioned).

\section{Bathythrix fragilis (Gravenhorst, 1829)}

Nomenclature:

Hemiteles fragilis Gravenhorst, 1829

bellula (Kriechbaumer, 1892, Leptocryptus)

urticarum (Habermehl, 1920, Leptocryptus)

Distribution: England, Scotland, Wales

Bathythrix lamina (Thomson, 1884)

Nomenclature:

Leptocryptus lamina Thomson, 1884

Distribution: England, Scotland, Wales, Ireland, Isle of Man

Notes: some distribution data from Kerrich (1942)

\section{Bathythrix linearis (Gravenhorst, 1829)}

Nomenclature:

Nematopodius linearis Gravenhorst, 1829

heteropus (Thomson, 1886, Leptocryptus)

Distribution: England

Bathythrix margaretae Sawoniewicz, 1980

Distribution: England

Notes: added by Schwarz and Shaw (2010) 
Bathythrix pellucidator (Gravenhorst, 1829)

Nomenclature:

Cryptus pellucidator Gravenhorst, 1829

ruficaudata (Bridgman, 1883, Hemiteles)

Distribution: England, Scotland, Wales, Ireland, Isle of Man

Bathythrix prominens (Strobl, 1901)

Nomenclature:

Leptocryptus prominens Strobl, 1901

Distribution: England, Scotland, Wales, Ireland, Isle of Man

Notes: added by Sawoniewicz (1980)

Bathythrix rugulosa (Thomson, 1884)

Nomenclature:

Leptocryptus rugulosus Thomson, 1884

Distribution: England, Scotland, Ireland

Notes: added by Sawoniewicz (1980)

Bathythrix spheginus (Gravenhorst, 1829)

Nomenclature:

Mesoleptus spheginus Gravenhorst, 1829

sphecinus (Schulz, 1906, Mesoleptus)

Distribution: England

Notes: added by Sawoniewicz (1980)

Bathythrix strigosa (Thomson, 1884)

Nomenclature:

Leptocryptus strigosus Thomson, 1884

ruficollis (Habermehl, 1919, Leptocryptus)

Distribution: England, Scotland

Notes: added by Schwarz and Shaw (2010) 
Bathythrix tenuis (Gravenhorst, 1829)

Nomenclature:

Cryptus tenuis Gravenhorst, 1829

rubens (Kriechbaumer, 1892, Leptocryptus)

Bathythrix thomsoni (Kerrich, 1942)

Nomenclature:

Thysiotorus thomsoni Kerrich, 1942

corsicator (Aubert, 1961, Panargyrops)

Distribution: England, Scotland, Wales, Ireland, Isle of Man

Genus Blapsidotes Förster, 1869

Blapsidotes vicinus (Gravenhorst, 1829)

Nomenclature:

Hemiteles vicinus Gravenhorst, 1829

melanarius (Gravenhorst, 1829, Hemiteles)

pimplarius (Berthoumieu, 1904, Platylabus)

Distribution: England, Scotland, Ireland

Notes: Listed (as melanarius) as a doubtfully placed species of Hemiteles by Fitton (1978).

Genus Cephalobaris Kryger, 1915

Cephalobaris eskelundi Kryger, 1915

Distribution: England

Notes: added by Schwarz and Shaw (2011)

Genus Ceratophygadeuon Viereck, 1924

Nomenclature:

EUROMONZIA Aubert, 1965 
Ceratophygadeuon bellus (Gravenhorst, 1829)

Nomenclature:

Ichneumon bellus Gravenhorst, 1829

longiceps (Thomson, 1884, Phygadeuon)

Notes: Not included in Fitton (1978) but described from a British specimen.

Ceratophygadeuon gracilicornis Horstmann, 1979

Distribution: England

Notes: added by Schwarz and Shaw (2011)

\section{Ceratophygadeuon ? parvicaudator (Aubert, 1965)}

Nomenclature:

Remonzia ?parvicaudator Aubert, 1965

Distribution: England

Notes: added by Schwarz and Shaw (2011); tentative identification

\section{Ceratophygadeuon varicornis (Thomson, 1885)}

Nomenclature:

Phygadeuon varicornis Thomson, 1885

maritimus Horstmann, 1979 synonymy by Horstmann (2001b)

Distribution: England

Notes: added by Horstmann (1993a)

\section{Genus Charitopes Förster, 1869}

Nomenclature:

ADIASTOLA Förster, 1869

Notes: Some distribution data from Townes (1983) and Horstmann (1998a).

\section{Charitopes areolaris (Thomson, 1884)}

Nomenclature:

Hemiteles areolaris Thomson, 1884

brunneus (Morley, 1907, Hemiteles) 
Distribution: England, Scotland

Notes: Koponen et al. (1999) treat brunneus as a species distinct from areolaris but Horstmann (1998a) and Townes (1983) retain it as a synonym.

\section{Charitopes carri (Roman, 1923)}

Nomenclature:

Cecidonomus carri Roman, 1923

londinensis (Morley, 1947, Phygadeuon)

hemerobii (Pfankuch, 1914, Hemiteles)

pusillus (Habermehl, 1920, Hemiteles) preocc.

Distribution: England, Scotland, Isle of Man

Notes: Horstmann (1998a) removed carri from synonymy with areolaris.

\section{Charitopes clausus (Thomson, 1888)}

Nomenclature:

Hemiteles clausus Thomson, 1888

Distribution: England, Scotland, Ireland, Isle of Man

Notes: added by Townes (1983)

\section{Charitopes gastricus (Holmgren, 1868)}

\section{Nomenclature:}

Hemiteles gastricus Holmgren, 1868

chrysopae (Brischke, 1890, Hemiteles)

flavigaster (Schmiedekecht, 1897, Hemiteles)

flavocinctus (Strobl, 1901, Hemiteles)

brunnescens (Schmiedekecht, 1905, Hemiteles)

sylvicola (Habermehl, 1920, Hemiteles)

Distribution: England, Scotland, Wales, Ireland

\section{Charitopes wesmaeliicida (Roman, 1934)}

\section{Nomenclature:}

Hemiteles wesmaeliicida Roman, 1934

Distribution: England, Scotland 


\section{Genus Chirotica Förster, 1869}

Nomenclature:

ALLOCOTA Förster, 1869 preocc.

DIAGLYPTA Förster, 1869

SPINOLIA Förster, 1869 preocc.

SYNECHES Förster, 1869 preocc.

DEUTEROSPINOLIA Dalla Torre, 1902

\section{Chirotica maculipennis (Gravenhorst, 1829)}

Nomenclature:

Hemiteles maculipennis Gravenhorst, 1829

excellens (Imhoff, 1850, Hemiteles)

mulsantii (Fonscolombe, 1852, Hemiteles)

glyptonota (Thomson, 1885, Hemiteles)

schiefereri (Strobl, 1904, Hemiteles)

Distribution: England

Notes: Listed as a doubtfully placed species of Hemiteles by Fitton (1978).

\section{Genus Clypeoteles Horstmann, 1974}

\section{Clypeoteles distans (Thomson, 1884)}

Nomenclature:

Hemiteles distans Thomson, 1884

rugifrons (Thomson, 1884, Hemiteles)

pseudorubiginosus (Strobl, 1901, Hemiteles)

xylonomoides (Morley, 1907, Cecidonomus)

fennicus (Hellén, 1967, Catalytus)

Distribution: England, Scotland

Notes: Listed as Acrolyta xylonomoides by Fitton (1978). 
Genus Cremnodes Förster, 1850

Nomenclature:

CAENOMERIS Förster, 1869

STYGERA Förster, 1869

CREMNIAS Roman, 1939

Cremnodes atricapillus (Gravenhorst, 1815)

Nomenclature:

Icheumon atricapillus Gravenhorst, 1815

combustus Förster, 1850

nanodes Förster, 1850

Distribution: England, Scotland, Wales, Ireland

Notes: some distribution data from Horstmann (1993c)

Cremnodes costalis Horstmann, 1992

Distribution: England, Scotland

Notes: added by Horstmann (1992b)

Cremnodes rufipes (Perkins, 1962)

Nomenclature:

Stygera rufipes Perkins, 1962

Distribution: England, Wales

Notes: some distribution data from Perkins (1962)

\section{Genus Diaglyptidea Viereck, 1913}

Diaglyptidea conformis (Gmelin, 1790)

Nomenclature:

Ichneumon conformis Gmelin, 1790

secernenda (Schmiedeknecht, 1897, Hemiteles)

Distribution: England, Scotland, Ireland, Isle of Man 
Genus Dichrogaster Doumerc, 1855

Nomenclature:

BRACHYCEPHALUS Förster, 1869

MICROTORUS Förster, 1869

OTACUSTES Förster, 1869

XENOBRACHYS Förster, 1869

Notes: Some distribution data from Townes (1983) and Horstmann (1992b).

Dichrogaster aestivalis (Gravenhorst, 1829)

Nomenclature:

Hemiteles aestivalis Gravenhorst, 1829

ruficollis (Gravenhorst, 1829, Hemiteles)

geniculata (Thomson, 1884, Hemiteles)

Distribution: England, Scotland, Wales, Ireland

Dichrogaster bischoffi (Schmiedeknecht, 1905)

Nomenclature:

Phygadeuon bischoffi Schmiedeknecht, 1905

rufovaria (Schmiedeknecht, 1905, Phygadeuon)

Distribution: England

Notes: added by Schwarz and Shaw (2000)

Dichrogaster genalis (Habermehl, 1925)

Nomenclature:

Phygadeuon genalis Habermehl, 1925

varsoviensis (Sawoniewicz, 1978, Ethelurgus)

Distribution: England, Scotland, Ireland

Notes: added by Townes (1983)

Dichrogaster heteropus (Thomson, 1896)

Nomenclature:

Phygadeuon heteropus Thomson, 1896 
rufithorax (Schmiedeknecht, 1932, Phygadeuon)

Distribution: England, Scotland

Notes: added by Horstmann (1992b)

Dichrogaster liostylus (Thomson, 1885)

Nomenclature:

Hemiteles liostylus Thomson, 1885

schaffneri (Schmiedeknecht, 1897, Hemiteles)

Distribution: England, Scotland, Ireland

Dichrogaster longicaudata (Thomson, 1884)

Nomenclature:

Hemiteles longicaudatus Thomson, 1884

diatropus Townes, 1983

Distribution: England, Scotland

Notes: NMS, det. Schwarz, added here

Dichrogaster mandibularis Horstmann, 1973

Distribution: England

Notes: added by Horstmann (1992b)

Dichrogaster modesta (Gravenhorst, 1829)

Nomenclature:

Hemiteles modestus Gravenhorst, 1829

brunnea (Kiss, 1924, Herpestomus)

Distribution: England, Scotland, Wales

Notes: added by Townes (1983)

Dichrogaster perlae (Doumerc, 1855)

Nomenclature:

Microgaster perlae Doumerc, 1855

Distribution: England 
Notes: added by Townes (1983)

Dichrogaster schimitscheki (Fahringer, 1935)

Nomenclature:

Phygadeuon schimitscheki Fahringer, 1935

nigrithorax Horstmann, 1976

Distribution: England

Notes: added by Townes (1983)

Genus Encrateola Strand, 1917

Nomenclature:

ENCRATES Förster, 1869

Encrateola glabra Horstmann, 1998

Distribution: England

Notes: added by Schwarz and Shaw (2000)

Encrateola laevigata (Ratzeburg, 1848)

Nomenclature:

Hemiteles laevigatus Ratzeburg, 1848

furcata (Taschenberg, 1865, Hemiteles)

subimpressa (Brischke, 1892, Hemiteles)

Distribution: England, Scotland, Wales, Ireland, Isle of Man

Notes: Listed as a doubtfully placed species of Hemiteles by Fitton (1978).

\section{Genus Endasys Förster, 1869}

Nomenclature:

BACHIA Förster, 1869 preocc.

SCINACOPUS Förster, 1869

STYLOCRYPTUS Thomson, 1873

BACHIANA Strand, 1929 
Notes: Distribution data from Sawoniewicz and Luhman (1992), supplemented by material in BMNH and NMS. Several species were listed under Glyphicnemis by Fitton (1978).

Endasys alutaceus (Habermehl, 1912)

Nomenclature:

Stylocryptus alutaceus Habermehl, 1912

nigriventris (Aerts, 1953, Stylocryptus)

Distribution: England

Notes: BMNH, det. Sawoniewicz \& Luhman, added here

Endasys analis (Thomson, 1883)

Nomenclature:

Stylocryptus analis Thomson, 1883

Distribution: England

Notes: BMNH, det. Sawoniewicz \& Luhman, added here

Endasys anglianus Sawoniewicz \& Luhman, 1992

Distribution: England, Scotland, Wales

Notes: added by Sawoniewicz and Luhman (1992)

Endasys brevis (Gravenhorst, 1829)

Nomenclature:

Phygadeuon brevis Gravenhorst, 1829

Distribution: England, Wales

Notes: added by Sawoniewicz and Luhman (1992)

Endasys brunnulus Sawoniewicz \& Luhman, 1992

Distribution: England

Notes: added by Sawoniewicz and Luhman (1992) 
Endasys erythrogaster (Gravenhorst, 1829)

Nomenclature:

Phygadeuon erythrogaster Gravenhorst, 1829

nigricoxis (Habermehl, 1912, Stylocryptus)

Endasys minutulus (Thomson, 1883)

Nomenclature:

Stylocryptus minutulus Thomson, 1883

nigripes (Strobl, 1904, Stylocryptus)

fusciventris (Habermehl, 1916, Stylocryptus)

Distribution: England, Wales, Ireland

Notes: added by Sawoniewicz and Luhman (1992)

\section{Endasys parviventris (Gravenhorst, 1829)}

Nomenclature:

Phygadeuon parviventris Gravenhorst, 1829

pictipes (Rudow, 1886, Phygadeuon)

tyrolensis (Schmiedeknecht, 1905, Stylocryptus)

Distribution: England

\section{Endasys petiolus Sawoniewicz \& Luhman, 1992}

Distribution: England

Notes: added by Sawoniewicz and Luhman (1992)

\section{Endasys plagiator (Gravenhorst, 1829)}

Nomenclature:

Phygadeuon plagiator Gravenhorst, 1829

braunsi (Lange, 1911, Acanthocryptus)

laetus (Habermehl, 1929, Stylocryptus)

Distribution: England, Scotland, Isle of Man

Notes: added by Sawoniewicz and Luhman (1992) 
Endasys proteuryopsis Sawoniewicz \& Luhman, 1992

Distribution: England

Notes: added by Sawoniewicz and Luhman (1992)

Endasys rusticus (Habermehl, 1912)

Nomenclature:

Stylocryptus rusticus Habermehl, 1912

Distribution: England

Endasys senilis (Gmelin, 1790)

Nomenclature:

Ichneumon senilis Gmelin, 1790

Distribution: England

Endasys striatus (Kiss, 1924)

Nomenclature:

Acanthocryptus striatus Kiss, 1924

Distribution: England

Notes: added bySawoniewicz and Luhman (1992)

Endasys talitzkii (Telenga, 1961)

Nomenclature:

Phygadaeuon talitzkii Telenga, 1961

Distribution: England

Notes: BMNH, det. Sawoniewicz \& Luhman, added here

Endasys testaceipes (Brischke, 1881)

Nomenclature:

Phygadeuon testaceipes Brischke, 1881

coxalis (Schmiedeknecht, 1905, Stylocryptus)

Distribution: England

Notes: added by Sawoniewicz and Luhman (1992) 
Endasys thunbergi Sawoniewicz \& Luhman, 1992

Nomenclature:

rubricator (Thunberg, 1824, Ichneumon)

Distribution: England

Endasys transverseareolatus (Strobl, 1901)

Nomenclature:

Stylocryptus transverseareolatus Strobl, 1901

Endasys triannulatus Sawoniewicz \& Luhman, 1992

Distribution: England

Notes: added by Sawoniewicz and Luhman (1992)

Endasys varipes (Gravenhorst, 1829)

Nomenclature:

Phygadeuon varipes Gravenhorst, 1829

Distribution: England, Isle of Man

\section{Genus Ethelurgus Förster, 1869}

Nomenclature:

NUNECHES Förster, 1869

TOLMERUS Förster, 1869

PLATYCRYPTUS Kriechbaumer, 1893

Notes: Some distribution and synonymic data from Horstmann (2000e).

\section{Ethelurgus sodalis (Taschenberg, 1865)}

Nomenclature:

Phygadeuon sodalis Taschenberg, 1865

pseudovulnerator (Strobl, 1901, Phygadeuon)

pici (Berthoumieu, 1908, Platylabus)

flavocinctus (Habermehl, 1909, Phygadeuon)

inermis (Habermehl, 1919, Phygadeuon)

niger (Pfankuch, 1824, Phygadeuon) preocc., unavailable 
Distribution: England, Scotland, Wales, Ireland, Isle of Man

\section{Ethelurgus vulnerator (Gravenhorst, 1829)}

Nomenclature:

Phygadeuon vulnerator Gravenhorst, 1829

Distribution: England, Scotland

Notes: Townes (1983) recorded vulnerator from Ireland but his interpretation of the species also included sodalis (Horstmann 2000e); I have seen an Irish specimen of sodalis coll. A. Anderson.

\section{Genus Eudelus Förster, 1869}

Nomenclature:

CALLIPHRURUS Förster, 1869

IDEMUM Förster, 1869

\section{Eudelus pallicarpus (Thomson, 1884)}

Nomenclature:

Hemiteles pallicarpus Thomson, 1884

pallidicarpus (Dalla Torre, 1902, Hemiteles)

crassiformis (Viereck, 1917, Hemiteles) synonymy by Schwarz and Shaw (2000)

Distribution: England

Notes: Raised from synonymy with simillimus by Schwarz and Shaw (2000).

\section{Eudelus scabriculus (Thomson, 1884)}

Nomenclature:

Hemiteles scabriculus Thomson, 1884

Distribution: England

Notes: Raised from synonymy with simillimus by Schwarz and Shaw (2000). 


\section{Eudelus simillimus (Taschenberg, 1865)}

Nomenclature:

Hemiteles simillimus Taschenberg, 1865

?sericeus (Rudow, 1886, Hemiteles)

?albidus (Pfankuch, 1925, Hemiteles)

?meridionator (Aubert, 1960, Astomaspis)

Distribution: England, Scotland, Ireland

Notes: Listed as a doubtfully placed species of Hemiteles by Fitton (1978). Eudelus nigricoxis (Kiss, 1924, Hemiteles) was treated as a valid species by Horstmann (2009c).

\section{Eudelus mediovittatus (Schmiedeknecht, 1897)}

Nomenclature:

Hemiteles mediovittatus Schmiedeknecht, 1897

Distribution: England

Notes: Listed as Encrateola mediovittatus by Fitton (1978), it was transferred to Acrolyta by Horstmann (1983) but left as possibly belonging to Eudelus but probably to a new genus by Schwarz and Shaw (2000); although its generic position is in doubt its status as a British species is not. Recent specimens from Worcestershire (coll. J. Rush, specimens in BMNH and Schwarz coll.) have been identified by M. Schwarz.

\section{Genus Fianoniella Horstmann, 1992}

Fianoniella punctiscutum (Horstmann, 1990)

Nomenclature:

Odontoneura punctiscutum Horstmann, 1990

Distribution: England

Notes: added by Horstmann (1990a)

\section{Genus Gelis Thunberg, 1827}

\section{Nomenclature:}

PEZOMACHUS Gravenhorst, 1829

PEZOLOCHUS Förster, 1850

CATALYTUS Förster, 1851 
HEMIMACHUS Ratzeburg, 1852

ALEGINA Förster, 1869

ASCHISTUS Förster, 1869

BARYDOTIRA Förster, 1869

ILAPINASTES Förster, 1869

PHILONYGMUS Förster, 1869

PLESIOMMA Förster, 1869 preocc.

RHADIURGUS Förster, 1869 preocc.

TERPIPHORA Förster, 1869

URITHEPTUS Förster, 1869

LEPTOGELIS Ceballos, 1925

FIANONIA Seyrig, 1952

HOLCOGELIS Aubert, 1957

ARCTODEUON Hellén, 1967

RHADIURGINUS Hellén, 1967

Notes: species of Gelis excluded from the British list by Schwarz and Shaw (1999)

[alpivagus (Strobl, 1901, Hemiteles) misident.]

[stevenii (Gravenhorst, 1829, Pezomachus) misident.]

[taschenbergii (Schmiedeknecht, 1897, Hemiteles) misident.] Listed as a doubtfully placed species of Hemiteles by Fitton (1978).

\section{Gelis acarorum (Linnaeus, 1758)}

\section{Nomenclature:}

Ichneumon acarorum Linnaeus, 1758

nigricornis (Retzius, 1783, Ichneumon)

audax (Förster, 1850, Pezomachus)

cautus (Förster, 1850, Pezomachus)

circumcinctus (Förster, 1850, Pezomachus)

fraudulentus (Förster, 1850, Pezomachus)

integer (Förster, 1850, Pezomachus)

providus (Förster, 1850, Pezomachus)

sericeus (Förster, 1850, Pezomachus) 
cruentatus (Rudow, 1917, Pezomachus)

fulvicornis (Rudow, 1917, Pezomachus)

unicinctus (Rudow, 1917, Pezomachus)

muscae Pisica \& Fabritius, 1986

\section{Gelis agilis (Fabricius, 1775)}

\section{Nomenclature:}

Ichneumon agilis Fabricius, 1775

cursor (Schrank, 1780, Ichneumon)

fuscicornis (Retzius, 1783, Ichneumon)

ruficornis (Retzius, 1783, Ichneumon) synonymy by Schwarz (2002)

apterus (Geoffroy, 1785, Ichneumon)

celer (Olivier, 1792, Ichneumon)

instabilis (Förster, 1850, Pezomachus)

mediocris (Förster, 1850, Pezomachus)

thoracicus (Brischke, 1878, Pezomachus)

breviceps (Thomson, 1884, Pezomachus)

alpigena (Strobl, 1901, Pezomachus)

rossicus (Szépligeti, 1901, Pezomachus)

albulae (Rudow, 1917, Pezomachus)

cuculliae (Rudow, 1917, Pezomachus)

eupitheciae (Rudow, 1917, Pezomachus)

intrans (Rudow, 1917, Pezomachus)

lineatus (Rudow, 1917, Pezomachus)

microrum (Rudow, 1917, Pezomachus)

monozonius (Rudow, 1917, Pezomachus) preocc.

nigerrimus (Rudow, 1917, Pezomachus) preocc.

rosarum (Rudow, 1917, Pezomachus)

rufostictus (Rudow, 1917, Pezomachus)

vanessae (Rudow, 1917, Pezomachus)

leucurus Ulbricht, 1926

laricellae (Fahringer, 1937, Pezomachus)

cephalotes Hellén, 1970 
Distribution: England, Scotland, Wales, Isle of Man

Gelis albicinctoides Schwarz, 1998

Distribution: England

Notes: added by Schwarz (1998)

Gelis albipalpus (Thomson, 1884)

Nomenclature:

Hemiteles albipalpus Thomson, 1884

austriacus (Fahringer, 1937, Hemiteles)

Distribution: England, Scotland, Ireland

\section{Gelis albopilosus Schwarz, 2002}

Distribution: England

Notes: added by Schwarz (2002)

Gelis anthracinus (Förster, 1850)

Nomenclature:

Pezomachus anthracinus Förster, 1850

linearis (Förster, 1851, Pezomachus)

gonatopinus (Thomson, 1884, Pezomachus)

Distribution: England, Scotland, Ireland

\section{Gelis areator (Panzer, 1804)}

Nomenclature:

Icneumon areator Panzer, 1804

aberrans (Gravenhorst, 1829, Pezomachus)

orbiculatus (Gravenhorst, 1829, Hemiteles)

pulchellus (Gravenhorst, 1829, Hemiteles)

coelebs (Ratzeburg, 1852, Hemiteles)

variabilis (Ratzeburg, 1852, Hemiteles) synonymy by Horstmann (2001b)

ephippium (Rudow, 1886, Hemimachus)

microgastri (Rudow, 1886, Hemiteles) 
ruficollis (Rudow, 1886, Hemiteles) preocc.

cognatus (Brischke, 1891, Hemiteles) synonymy by Horstmann (2001b)

minimus (Glowacki, 1967, Hemiteles)

Distribution: England, Scotland, Wales, Ireland

\section{Gelis avarus (Förster, 1850)}

Nomenclature:

Pezomachus avarus Förster, 1850

Distribution: England, Scotland, Wales, Ireland

Notes: added by Schwarz and Shaw (1999)

\section{Gelis balteatus (Thomson, 1885)}

Nomenclature:

Hemiteles balteatus Thomson, 1885

brevistylus (Hellén, 1967, Charitopes)

Distribution: England, Wales

Notes: Listed by Fitton (1978) as a doubtfully placed species of Hemiteles.

\section{Gelis bicolor (Villers, 1789)}

\section{Nomenclature:}

Ichneumon bicolor Villers, 1789

alacer (Förster, 1850, Pezomachus)

brachyurus (Förster, 1850, Pezomachus)

distinctus (Förster, 1850, Pezomachus)

furtivus (Förster, 1850, Pezomachus)

incertus (Förster, 1850, Pezomachus)

molestus (Förster, 1850, Pezomachus)

muelleri (Förster, 1850, Pezomachus)

petulans (Förster, 1850, Pezomachus)

sordidus (Förster, 1850, Pezomachus)

spadiceus (Förster, 1850, Pezomachus)

timidus (Förster, 1850, Pezomachus)

vicinus (Förster, 1850, Pezomachus) 
fusculus (Förster, 1851, Pezomachus)

rigii (De Stefani, 1884, Pezomachus)

facialis (Brishcke, 1891, Pezomachus)

riggioi (Schmiedeknecht, 1906, Pezomachus)

aphidicola (Rudow, 1917, Pezomachus)

formicarius (Rudow, 1917, Pezomachus) preocc.

?latus Jonaitis, 1981

Distribution: England, Scotland

Gelis brevis (Bridgman, 1883)

Nomenclature:

Pezomachus brevis Bridgman, 1883

\section{Gelis caudatulus Horstmann, 1997}

Nomenclature:

caudator Horstmann, 1986

Distribution: Scotland

Notes: added by Schwarz and Shaw (1999)

\section{Gelis cayennator (Thunberg, 1824)}

Nomenclature:

Ichneumon cayennator Thunberg, 1824

brassicae Horstmann, 1986 synonymy by Schwarz (2009)

sulcatus (Blunck, 1951, Hemiteles) preocc.

Distribution: England, Scotland, Wales

Notes: added by Schwarz and Shaw (1999)

\section{Gelis cinctus (Linnaeus, 1758)}

Nomenclature:

Ichneumon cinctus Linnaeus, 1758

cinctor (Thunberg, 1824, Ichneumon)

bicolorinus (Gravenhorst, 1829, Hemiteles)

Distribution: England, Scotland 
Gelis cursitans (Fabricius, 1775)

Nomenclature:

Ichneumon cursitans Fabricius, 1775

tuberculatus (Hartig, 1838, Pezomachus)

decipiens (Förster, 1850, Pezomachus)

peregrinator (Förster, 1850, Pezomachus)

alpinus (Rudow, 1917, Pezomachus) preocc.

braconidum (Rudow, 1917, Pezomachus)

helicis (Rudow, 1917, Pezomachus)

psychivorus (Rudow, 1917, Pezomachus)

\section{Gelis curvicauda Horstmann, 1993}

Distribution: England

Notes: added by Schwarz (1994)

\section{Gelis discedens (Förster, 1850)}

Nomenclature:

Pezomachus discedens Förster, 1850

vagans misident.

calvus (Förster, 1850, Pezomachus)

quaesitorius (Förster, 1850, Pezomachus)

collaris (Rudow, 1917, Pezomachus)

exareolatus (Rudow, 1917, Pezomachus) preocc.

potentillae (Rudow, 1917, Pezomachus)

psychidum (Rudow, 1917, Pezomachus)

nigrithorax (Habermehl, 1920, Pezomachus)

Distribution: England, Scotland, Wales

\section{Gelis divaricatus Horstmann, 1993}

Distribution: England, Wales

Notes: added by Horstmann (1993a) 
Gelis edentatus (Förster, 1850)

Nomenclature:

Pezomachus edentatus Förster, 1850

imbellis (Förster, 1850, Pezomachus)

modestus (Förster, 1850, Pezomachus)

vagantiformis (Bridgman, 1886, Pezomachus)

dusmeti Ceballos, 1925

Distribution: England

\section{Gelis exareolatus (Förster, 1850)}

Nomenclature:

Pezomachus exareolatus Förster, 1850

nigritus (Förster, 1850, Pezomachus)

simulans (Förster, 1850, Pezomachus)

micromelas (Kriechbaumer, 1894, Phygadeuon)

lapponicus Hellén, 1970

Distribution: England, Scotland

Gelis falcatus Horstmann, 1986

Distribution: Scotland

Notes: added by Horstmann (1986)

Gelis fallax (Förster, 1850)

Nomenclature:

Pezomachus fallax Förster, 1850

nigricornis (Förster, 1850, Pezomachus) preocc.

iglesiasi Ceballos, 1925

Distribution: Wales, Ireland

Gelis fasciitinctus (Dalla Torre, 1901)

Nomenclature:

Hemiteles fasciitinctus Dalla Torre, 1901

fasciipennis (Brischke, 1881, Hemiteles) preocc. 
Distribution: England, Scotland

Notes: added by Schwarz and Shaw (1999)

\section{Gelis festinans (Fabricius, 1798)}

Nomenclature:

Ichneumon festinans Fabricius, 1798

nanus (Förster, 1850, Pezomachus)

pothumus (Förster, 1850, Pezomachus)

pumilus (Förster, 1850, Pezomachus)

tener (Förster, 1850, Pezomachus)

anguinus (Förster, 1851, Pezomachus)

ocissimus (Förster, 1851, Pezomachus)

brunneus (Brischke, 1890, Pezomachus)

Distribution: England, Scotland, Wales, Ireland

\section{Gelis formicarius (Linnaeus, 1758)}

\section{Nomenclature:}

Mutilla formicaria Linnaeus, 1758

ratzeburgi (Förster, 1850, Pezomachus)

confusus (Bridgman, 1883, Hemimachus) synonymy by Schwarz and Shaw (1999)

verrucosus (Rudow, 1917, Pezomachus)

Distribution: England

\section{Gelis forticornis (Förster, 1850)}

\section{Nomenclature:}

Pezomachus forticornis Förster, 1850

manevali Seyrig, 1927

Distribution: England

Notes: added by Schwarz (1998) 


\section{Gelis fuscicornis (Retzius, 1783)}

\section{Nomenclature:}

Ichneumon fuscicornis Retzius, 1783

longulus (Zetterstedt, 1838, Cryptus) synonymy by Schwarz (2002)

Distribution: England, Scotland

Notes: added by Schwarz and Boriani (1994)

\section{Gelis hortensis (Christ, 1791)}

\section{Nomenclature:}

Ichneumon hortensis Christ, 1791

acarorum misident.

callidus (Förster, 1850, Pezomachus)

canaliculatus (Förster, 1850, Pezomachus)

gentilis (Förster, 1850, Pezomachus)

impotens (Förster, 1850, Pezomachus)

inermis (Förster, 1850, Pezomachus)

latrator (Förster, 1850, Pezomachus)

lepidus (Förster, 1850, Pezomachus)

xylochophilus (Förster, 1850, Pezomachus)

avidus (Förster, 1851, Pezomachus)

filicornis (Förster, 1851, Pezomachus)

nomas (Förster, 1851, Pezomachus)

subtilis (Förster, 1851, Pezomachus)

albipennis (Ratzeburg, 1852, Hemiteles)

Distribution: England, Scotland, Wales, Ireland

\section{Gelis intermedius (Förster, 1850)}

Nomenclature:

Pezomachus intermedius Förster, 1850

furax (Förster, 1850, Pezomachus)

Distribution: England

Notes: reinstated by Schwarz and Shaw (1999) 
Gelis kiesenwetteri (Förster, 1850)

Nomenclature:

Pezomachus kiesenwetteri Förster, 1850

bellicosus (Förster, 1850, Pezomachus)

debeyii (Förster, 1850, Pezomachus)

egregius (Förster, 1850, Pezomachus)

costatus (Bridgmanm, 1886, Pezomachus)

Distribution: England, Scotland, Ireland

\section{Gelis limbatus (Gravenhorst, 1829)}

Nomenclature:

Hemiteles limbatus Gravenhorst, 1829

Notes: Listed by Fitton (1978) as a doubtfully placed species of Hemiteles.

\section{Gelis liparae (Giraud, 1863)}

Nomenclature:

Hemiteles liparae Giraud, 1863

ilicicola (Seyrig, 1927, Hemiteles) synonymy by Schwarz and Shaw (1999)

ilicicolator Aubert, 1966 synonymy by Schwarz and Shaw (1999)

Distribution: England

Notes: added by Horstmann (1986)

Gelis longicauda (Thomson, 1884)

Nomenclature:

Hemiteles longicauda Thomson, 1884

Distribution: England, Scotland, Ireland

\section{Gelis lucidulus (Förster, 1850)}

Nomenclature:

Pezomachus lucidulus Förster, 1850

inquilinus (Förster, 1850, Pezomachus)

microstylus (Förster, 1851, Pezomachus) 


\section{Gelis mangeri (Gravenhorst, 1815)}

\section{Nomenclature:}

Ichneumon mangeri Gravenhorst, 1815

fulveolatus (Gravenhorst, 1829, Pezomachus)

longipennis (Gravenhorst, 1829, Pezomachus)

foersteri (Bridgman, 1882, Aptesis)

Distribution: England, Wales

\section{Gelis meigenii (Förster, 1850)}

Nomenclature:

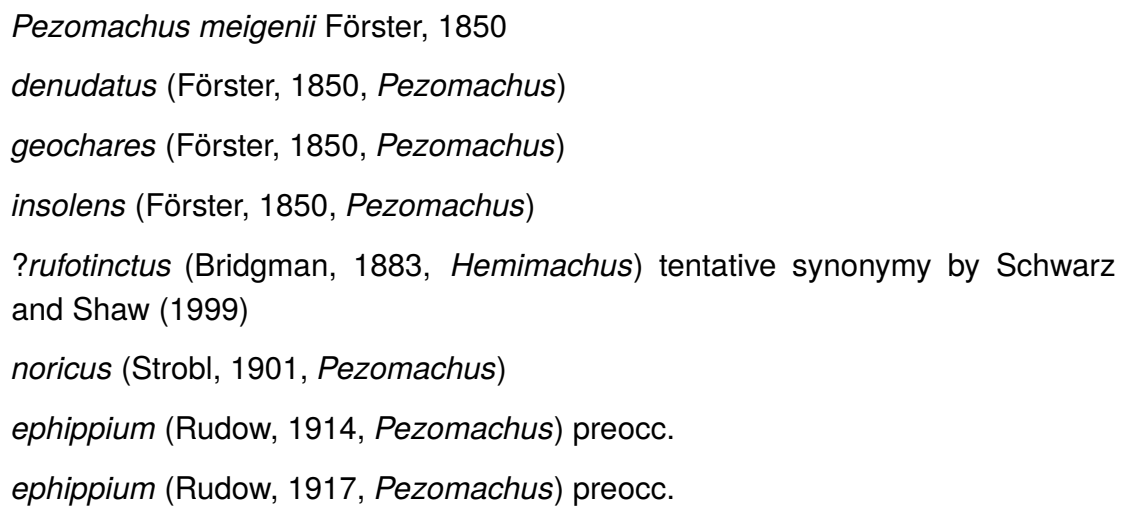

Distribution: England, Wales, Scotland

\section{Gelis melanocephalus (Schrank, 1781)}

Nomenclature:

Mutilla melanocephala Schrank, 1781

fasciatus (Fabricius, 1793, Ichneumon) preocc.

fasciatus (Ratzeburg, 1852, Hemiteles) preocc.

hercyniae (Rudow, 1917, Pezomachus)

Distribution: England, Scotland, Wales, Ireland, Isle of Man

\section{Gelis melanogaster (Thomson, 1884)}

Nomenclature:

Hemiteles melanogaster Thomson, 1884

Distribution: Wales 
Notes: Added by Schwarz and Shaw (1999), but it was overlooked that Fitton (1978) had treated this as a species of Charitopes.

\section{Gelis melanophorus (Förster, 1851)}

Nomenclature:

Pezomachus melanophorus Förster, 1851

fuscicornis (Förster, 1850, Pezomachus) synonymy by Schwarz and Shaw (1999)

foersteri (Bridgman, 1886, Pezomachus) synonymy by Schwarz and Shaw (1999)

Distribution: England, Scotland, Ireland

Notes: added by Schwarz and Shaw (1999)

\section{Gelis micrurus (Förster, 1850)}

Nomenclature:

Pezomachus micrurus Förster, 1850

pardosae (Giard, 1895, Hemiteles)

Distribution: England, Scotland, Wales, Ireland

\section{Gelis mitis Schwarz, 1994}

Distribution: England

Notes: added by Schwarz (1994)

\section{Gelis mutillatus (Gmelin, 1790)}

Nomenclature:

Ichneumon mutillatus Gmelin, 1790

mutillarius (Fabricius, 1787, Ichneumon) preocc.

vagans (Olivier, 1792, Ichneumon)

pedicularius (Fabricius, 1793, Ichneumon)

\section{Gelis nigritulus (Zetterstedt, 1838)}

\section{Nomenclature:}

Cryptus nigritulus Zetterstedt, 1838

terebrator (Ratzeburg, 1848, Pezomachus) 
Distribution: England, Scotland

Gelis nitidus Horstmann, 1986

Distribution: England

Notes: added by Horstmann (1986)

Gelis obscuripes Horstmann, 1986

Distribution: England, Scotland, Wales

Notes: added by Horstmann (1986)

\section{Gelis papaveris (Förster, 1856)}

Nomenclature:

Pezomachus papaveris Förster, 1856

hieracii (Bridgman, 1883, Pezomachus)

grandiceps (Thomson, 1884, Pezomachus)

Gelis problemator Aubert, 1989

Distribution: England, Scotland

Notes: added by Schwarz (1994)

Gelis proximus (Förster, 1850)

Nomenclature:

Pezomachus proximus Förster, 1850

analis (Förster, 1850, Pezomachus)

attentus (Förster, 1850, Pezomachus)

celer (Förster, 1850, Pezomachus)

consociatus (Förster, 1850, Pezomachus)

corruptor (Förster, 1850, Pezomachus)

derasus (Förster, 1850, Pezomachus)

dubitator (Förster, 1850, Pezomachus)

ephippiger (Förster, 1850, Pezomachus)

faunus (Förster, 1850, Pezomachus)

hostilis (Förster, 1850, Pezomachus) 
incubitor (Förster, 1850, Pezomachus)

latro (Förster, 1850, Pezomachus)

ochraceus (Förster, 1850, Pezomachus)

parvulus (Förster, 1850, Pezomachus)

sedulus (Förster, 1850, Pezomachus)

tonsus (Förster, 1850, Pezomachus)

vigil (Förster, 1850, Pezomachus)

vorax (Förster, 1850, Pezomachus)

xenoctonus (Förster, 1850, Pezomachus)

ageletes (Förster, 1851, Pezomachus)

ambulans (Förster, 1851, Pezomachus)

conveniens (Förster, 1851, Pezomachus)

decurtatus (Förster, 1851, Pezomachus)

dysalotus (Förster, 1851, Pezomachus)

elaphrus (Förster, 1851, Pezomachus)

erythropus (Förster, 1851, Pezomachus)

fugitivus (Förster, 1851, Pezomachus)

heydeni (Förster, 1851, Pezomachus)

histrio (Förster, 1851, Pezomachus)

imbecillus (Förster, 1851, Pezomachus)

indagator (Förster, 1851, Pezomachus)

indigator misspelling

insidiosus (Förster, 1851, Pezomachus)

inspector (Förster, 1851, Pezomachus)

lustrator (Förster, 1851, Pezomachus)

migrator (Förster, 1851, Pezomachus)

navus (Förster, 1851, Pezomachus)

procursorius (Förster, 1851, Pezomachus)

prudens (Förster, 1851, Pezomachus)

secretus (Förster, 1851, Pezomachus)

tentator (Förster, 1851, Pezomachus)

versatilis (Förster, 1851, Pezomachus)

violentus (Förster, 1851, Pezomachus) 


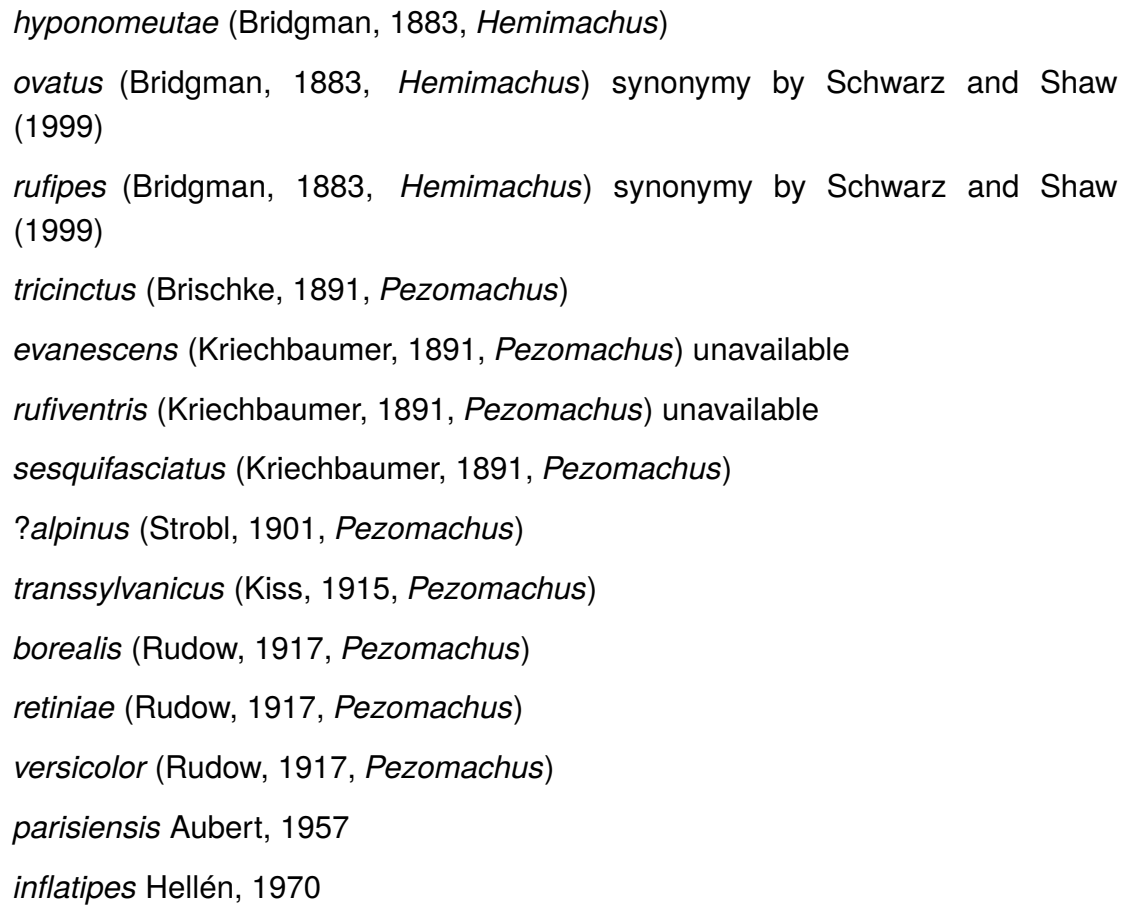

Distribution: England, Scotland, Wales, Ireland, Isle of Man

\section{Gelis pulicarius (Fabricius, 1793)}

Nomenclature:

Ichneumon pulicarius Fabricius, 1793

hoffmannseggii (Gravenhorst, 1815, Ichneumon)

\section{Gelis recens Schwarz, 2002}

Distribution: England

Notes: added by Schwarz (2002)

\section{Gelis rufipes (Förster, 1850)}

Nomenclature:

Pezolochus rufipes Förster, 1850

aries (Förster, 1850, Pezomachus)

ecarinatus (Förster, 1850, Pezomachus)

Distribution: England, Ireland 
Notes: added by Schwarz and Shaw (1999)

\section{Gelis rufogaster Thunberg, 1827}

\section{Nomenclature:}

aemulus (Förster, 1850, Pezomachus)

alienus (Förster, 1850, Pezomachus)

anceps (Förster, 1850, Pezomachus)

astutus (Förster, 1850, Pezomachus)

bicinctus (Förster, 1850, Pezomachus)

carnifex (Förster, 1850, Pezomachus)

consobrinus (Förster, 1850, Pezomachus)

currens (Förster, 1850, Pezomachus)

debilis (Förster, 1850, Pezomachus)

detritus (Förster, 1850, Pezomachus)

emarcidus (Förster, 1850, Pezomachus)

flavipes (Förster, 1850, Pezomachus)

gracilis (Förster, 1850, Pezomachus)

helvolus (Förster, 1850, Pezomachus)

immaturus (Förster, 1850, Pezomachus)

insectator (Förster, 1850, Pezomachus)

juvenilis (Förster, 1850, Pezomachus)

languidus (Förster, 1850, Pezomachus)

lividus (Förster, 1850, Pezomachus)

lugubris (Förster, 1850, Pezomachus)

lutescens (Förster, 1850, Pezomachus)

puberulus (Förster, 1850, Pezomachus)

puerilis (Förster, 1850, Pezomachus)

pulcher (Förster, 1850, Pezomachus)

pulex (Förster, 1850, Pezomachus)

rufulus (Förster, 1850, Pezomachus)

scitulus (Förster, 1850, Pezomachus)

squalidus (Förster, 1850, Pezomachus)

unicolor (Förster, 1850, Pezomachus) 


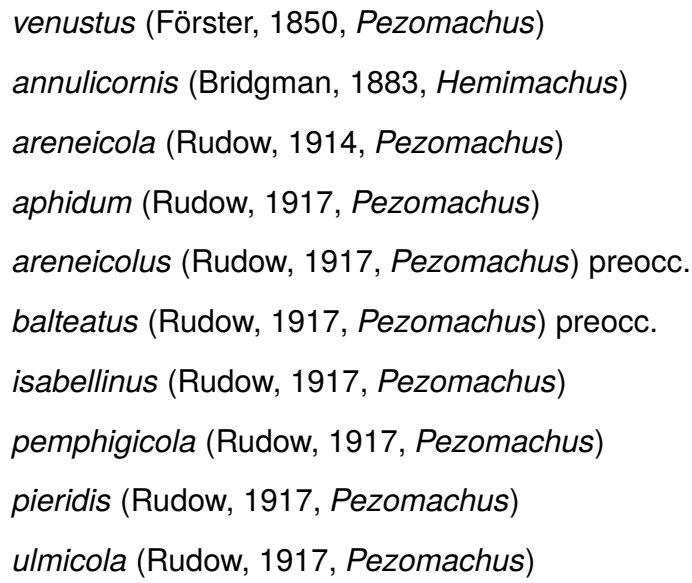

Distribution: England, Scotland, Wales, Ireland

Notes: Pezomachus annulicornis synonymised with rufulus (Förster), now a synonym of rufogaster, listed as a separate species in Yu and Horstmann (1997) but synonymy confirmed by Schwarz and Shaw (1999).

\section{Gelis rugifer (Thomson, 1884)}

Nomenclature:

Hemiteles rugifer Thomson, 1884

Distribution: England, Scotland, Wales

\section{Gelis seyrigi Ceballos, 1925}

Distribution: England

Notes: added by Schwarz (1998)

\section{Gelis spinula (Thomson, 1884)}

\section{Nomenclature:}

Pezomachus spinula Thomson, 1884

Distribution: England, Scotland, Ireland

\section{Gelis spurius (Förster, 1850)}

\section{Nomenclature:}

Pezomachus spurius Förster, 1850

ruficornis misident. in Schwarz and Shaw (1999)Schwarz (2002) 
Distribution: England, Scotland

Gelis terribilis Schwarz, 2002

Distribution: England, Scotland

Notes: added by Schwarz (2002)

\section{Gelis thomsoni (Schmiedeknecht, 1933)}

Nomenclature:

Hemiteles thomsoni Schmiedeknecht, 1933

dispar (Thomson, 1885, Hemiteles) preocc.

Distribution: England

Notes: Listed by Fitton (1978) as a doubtfully placed species of Hemiteles.

\section{Gelis trux (Förster, 1850)}

Nomenclature:

Pezomachus trux Förster, 1850

ruficornis Thunberg, 1827 preocc.

blandus (Förster, 1850, Pezomachus)

comes (Förster, 1850, Pezomachus)

transfuga (Förster, 1850, Pezomachus)

Distribution: England, Wales

\section{Gelis viduus (Förster, 1850)}

Nomenclature:

Pezomachus viduus Förster, 1850

congruus (Förster, 1850, Pezomachus)

doliopus (Förster, 1851, Pezomachus)

mandibularis (Thomson, 1884, Pezomachus)

Distribution: England, Scotland, Wales, Ireland 
Gelis vulnerans (Förster, 1850)

Nomenclature:

Pezomachus vulnerans Förster, 1850

affinis (Magretti, 1884, Pezomachus)

Gelis zeirapherator (Aubert, 1966)

Nomenclature:

Alegina zeirapherator Aubert, 1966

Distribution: Scotland

Notes: added by Schwarz and Shaw (1999)

\section{Genus Glyphicnemis Förster, 1869}

Nomenclature:

GNATHOCRYPTUS Thomson, 1873

Notes: Some distribution data from Fitton (1976) and Sawoniewicz (1985).

Glyphicnemis atrata (Strobl, 1901)

Nomenclature:

Stylocryptus atratus Strobl, 1901

alpina (Strobl, 1901, Stylocryptus)

suffolciensis Morley, 1907

Distribution: England, Scotland, Wales

Glyphicnemis clypealis (Thomson, 1883)

Nomenclature:

Stylocryptus clypealis Thomson, 1883

Distribution: England

\section{Glyphicnemis profligator (Fabricius, 1775)}

Nomenclature:

Ichneumon profligator Fabricius, 1775

abdominalis (Geoffroy, 1785, Ichneumon)

nigricornis (Gmelin, 1790, Ichneumon) preocc. 
textor (Thunberg, 1824, Ichneumon)

frequentoria (Zetterstedt, 1838, Ichneumon)

pygmaea (Habermehl, 1916, Stylocryptus)

ruficoxis (Habermehl, 1916, Stylocryptus) preocc.

Distribution: England, Scotland, Wales, Ireland, Isle of Man

\section{Glyphicnemis vagabunda (Gravenhorst, 1829)}

Nomenclature:

Phygadeuon vagabundus Gravenhorst, 1829

podagrica (Gravenhorst, 1829, Phygadeuon)

exannulata (Hedwig, 1956, Stylocryptus)

Distribution: England

\section{Genus Gnotus Förster, 1869}

\section{Gnotus chionops (Gravenhorst, 1829)}

Nomenclature:

Hemiteles chionops Gravenhorst, 1829

scutellator (Lange, 1911, Hemiteles)

Distribution: England, Scotland

\section{Gnotus macrurus (Thomson, 1884)}

Nomenclature:

Hemiteles macrurus Thomson, 1884

Distribution: Scotland

Notes: Listed as a doubtfully placed species of Hemiteles by Fitton (1978).

\section{Gnotus rugipectus (Thomson, 1886)}

Nomenclature:

Phygadeuon rugipectus Thomson, 1886

Notes: Transferred from Phygadeuon by Horstmann (1998a). 
Gnotus tenuipes (Gravenhorst, 1829)

Nomenclature:

Phygadeuon tenuipes Gravenhorst, 1829

tenuicornis (Gravenhorst, 1829, Hemiteles)

nebulosus (Rudow, 1886, Hemiteles)

cryptiformis (Kiss, 1924, Hemiteles)

nigripes (Bauer, 1958, Panargyrops)

Distribution: England

\section{Genus Gnypetomorpha Förster, 1869}

Nomenclature:

TRISACRA Förster, 1869

VICTOROVIA Tobias, 1963

Gnypetomorpha obscura (Bridgman, 1883)

Nomenclature:

Hemiteles obscurus Bridgman, 1883

aperta (Thomson, 1884, Hemiteles)

Distribution: England, Scotland

Gnypetomorpha tubertae Horstmann, 2012

Distribution: England

Notes: added by Horstmann (2012a)

Genus Grasseiteles Aubert, 1965

Nomenclature:

DIAGLYPTELLANA Horstmann, 1976 synonymy by Schwarz (2005)

Grasseiteles opaculus (Thomson, 1884)

Nomenclature:

Hemiteles opaculus Thomson, 1884

Distribution: England, Scotland 
Notes: Not mentioned by Schwarz and Shaw (2000); included in Fitton (1978) and recently identified by $\mathrm{M}$. Schwarz from material in NMS.

\section{Grasseiteles punctus (Holmgren, 1857)}

Nomenclature:

Adelognathus punctus Holmgren, 1857

sisyphii (Verhoeff, 1891, Hemiteles)

punctata Horstmann, 1986 synonymy by Schwarz and Shaw (2000)

Distribution: England, Scotland

Notes: added by Schwarz and Shaw (2000)

\section{Genus Hemiteles Gravenhorst, 1829}

Nomenclature:

OCYMORUS Förster, 1869

Notes: Many species listed as doubtfully placed species of Hemiteles by Fitton (1978) have since been transferred to other genera of Phygadeuontini. Hemiteles niger (Taschenberg) is now recognised to be a species of Thymaris (Tryphoninae).

doubtfully placed species of Hemiteles:

[liambus Thomson, 1885 nom. dub.]

[piceus (Bridgman, 1883, Hemimachus) nom. dub., from England; Fitton (1976)]

\section{Hemiteles bipunctator (Thunberg, 1824)}

Nomenclature:

Ichneumon bipunctator Thunberg, 1824

cingulator Gravenhorst, 1829

tristator Gravenhorst, 1829

rufipleuris Szépligeti, 1901

Distribution: England, Scotland, Ireland

\section{Hemiteles maricesca Schwarz \& Shaw, 2000}

Distribution: England, Scotland, Wales

Notes: added by Schwarz and Shaw (2000) 
Hemiteles rubropleuralis Kiss, 1929

Distribution: Scotland

Notes: added by Schwarz and Shaw (2000)

Hemiteles similis (Gmelin, 1790)

Nomenclature:

Ichneumon similis Gmelin, 1790

?debellator (Schrank, 1781, Ichneumon) preocc.

meridionalis Gravenhorst, 1829

unicolor Thomson, 1884

Distribution: England, Scotland, Wales, Ireland, Isle of Man

Genus Holcomastrus Horstmann, 2012

Holcomastrus bituberculatus (Schmiedeknecht, 1905)

Nomenclature:

Hemiteles bituberculatus Schmiedeknecht, 1905

Distribution: England

Notes: BMNH, det. Broad, added here

\section{Genus Isadelphus Förster, 1869}

Nomenclature:

PEROSIS Förster, 1869

CECIDONOMUS Bridgman, 1880

Notes: See note for Mastrus.

Isadelphus armatus (Gravenhorst, 1829)

Nomenclature:

Echthrus armatus Gravenhorst, 1829

mandibulator (Dufour \& Perris, 1840, Anomalon)

bidentulus (Thomson, 1844, Hemiteles)

Distribution: England, Wales 
Isadelphus coriarius (Taschenberg, 1865)

Nomenclature:

Hemiteles coriarius Taschenberg, 1865

rixator (Woldstedt, 1877, Polyblastus)

trochanteratus (Strobl, 1903, Erromenus) synonymy by Horstmann (1999a)

carbonarius (Schmiedeknecht, 1905, Hemiteles)

Isadelphus gallicola (Bridgman, 1880)

Nomenclature:

Cecidonomus gallicola Bridgman, 1880

nigriventris (Thomson, 1884, Hemiteles)

carpathicus (Kiss, 1924, Lissonota)

Distribution: England, Scotland

Isadelphus inimicus (Gravenhorst, 1829)

Nomenclature:

Hemiteles inimicus Gravenhorst, 1829

rufus (Bridgman, 1880, Cecidonomus)

obscuripes (Thomson, 1884, Hemiteles)

Distribution: England, Scotland, Wales, Ireland, Isle of Man

Isadelphus longisetosus (Schmiedeknecht, 1897)

Nomenclature:

Hemiteles longisetosus Schmiedeknecht, 1897

added by

Distribution: England, Scotland

Notes: added by Horstmann (2009b); treated as a separate species by Horstmann (2009b), rather than as a synonym of inimicus, as listed in Yu and Horstmann (1997).

Isadelphus minutus Horstmann, 2009

Distribution: England, Scotland

Notes: added by Horstmann (2009b) 
Genus Leptocryptoides Horstmann, 1976

Leptocryptoides clavipes (Thomson, 1888)

Nomenclature:

Leptocryptus clavipes Thomson, 1888

Distribution: England, Ireland

Notes: added by Schwarz and Shaw (2011)

Genus Lochetica Kriechbaumer, 1892

Notes: Some distribution data from Townes (1983).

Lochetica westoni (Bridgman, 1880)

Nomenclature:

Cecidonomus westoni Bridgman, 1880

pimplaria (Thomson, 1884, Phygadeuon) synonymy by Townes (1983)

Distribution: England, Scotland

Genus Lysibia Förster, 1869

Nomenclature:

PEMON Förster, 1869

STIBOSCOPUS Förster, 1869

HAPLASPIS Townes, 1944

Notes: Some distribution data from Perkins (1962) and Townes (1983).

Lysibia ceylonensis (Kerrich, 1956)

Nomenclature:

Haplaspis ceylonensis Kerrich, 1956

proxima (Perkins, 1962, Pemon)

Distribution: England

Lysibia nanus (Gravenhorst, 1829)

Nomenclature:

Tryphon nanus Gravenhorst, 1829 
fulvipes (Gravenhorst, 1829, Hemiteles)

socialis (Ratzeburg, 1844, Hemiteles)

populnea (Boie, 1855, Hemiteles)

Distribution: England, Scotland, Wales, Ireland, Isle of Man

Lysibia tenax Townes, 1983

Distribution: Scotland, Ireland

Notes: added by Townes (1983)

Genus Mastrulus Horstmann, 1978

Mastrulus marshalli (Bridgman \& Fitch, 1882)

Nomenclature:

Phygadeuon marshalli Bridgman \& Fitch, 1882

marshalli (Bridgman, 1883, Phygadeuon) preocc.

capra (Thomson, 1884, Hemiteles)

disputabilis (Schmiedeknecht, 1897, Hemiteles)

Distribution: England, Scotland

Notes: Listed as a species of Theroscopus by Fitton (1978).

\section{Genus Mastrus Förster, 1869}

Nomenclature:

AENOPLEX Förster, 1869

DAICTES Förster, 1869

Notes: Mastrus sensu Townes (1970) was split by Horstmann (1978b) into Isadelphus, Mastrus, Micromonodon, Odontoneura and Zoophthorus (and subsequently Fianoniella and Odontomastrus).

species of Mastrus excluded from the British and Irish list:

[pictipes (Gravenhorst, 1829, Hemiteles)] Listed as a doubtfully placed species of Hemiteles by Fitton (1978). Specimens under the name pictipes in the BMNH have now been identified as albobasalis and longicauda and there is no evidence that pictipes has ever been found here. 
Mastrus albobasalis (Schmiedeknecht, 1933)

Nomenclature:

Hemiteles albobasalis Schmiedeknecht, 1933

Distribution: England

Notes: added by Schwarz and Shaw (2010)

Mastrus boreaphilus (Roman, 1939)

Nomenclature:

Cecidonomus boreaphilus Roman, 1939

Distribution: Scotland

Notes: added bySchwarz and Shaw (2010)

Mastrus costalis (Thomson, 1884)

Nomenclature:

Hemiteles costalis Thomson, 1884

Distribution: England, Scotland

Notes: added by Schwarz and Shaw (2010)

Mastrus deminuens (Hartig, 1838)

Nomenclature:

Hemiteles deminuens Hartig, 1838

castaneus (Taschenberg, 1865, Hemiteles)

bredensis (Smits $\$$ )

ripicola (Habermehl, 1920, Hemiteles)

Distribution: England, Scotland, Wales, Ireland

Mastrus fumipennis (Thomson, 1884)

Nomenclature:

Hemiteles fumipennis Thomson, 1884

Distribution: Ireland

Notes: Listed as a doubtfully placed species of Hemiteles by Fitton (1978). 
Mastrus longicauda Horstmann, 1990

Distribution: England

Notes: BMNH, det. Broad \& Horstmann, added here

Mastrus longulus Horstmann, 1990

Distribution: England

Notes: added by Horstmann (1990a)

Mastrus mandibularis Horstmann, 1990

Distribution: England

Notes: added by Horstmann (1990a)

Mastrus parviceps (Hellén, 1967)

Nomenclature:

Isadelphus parviceps Hellén, 1967

Distribution: Scotland

Notes: added by Schwarz and Shaw (2010)

Mastrus ridibundus (Gravenhorst, 1829)

Nomenclature:

Hemiteles ridibundus Gravenhorst, 1829

Distribution: England, Ireland

Notes: Listed as a doubtfully placed species of Hemiteles by Fitton (1978).

\section{Mastrus ? rufobasalis (Habermehl, 1920)}

Nomenclature:

Hemiteles ?rufobasalis Habermehl, 1920

Distribution: Isle of Man

Notes: added by Schwarz and Shaw (2010); tentative identification by K. Horstmann. 
Mastrus rufulus (Thomson, 1884)

Nomenclature:

Hemiteles rufulus Thomson, 1884

nigrobasalis (Schmiedeknecht, 1905, Hemiteles)

rusticus (Habermehl, 1920, Hemiteles)

nigricoxis (Hedwig, 1959, Phygadeuon) unavailable

Distribution: England, Ireland

Notes: Listed as a doubtfully placed species of Hemiteles by Fitton (1978).

Mastrus silbernageli (Kiss, 1929)

Nomenclature:

Hemiteles silbernageli Kiss, 1929

leptocryptoides (Schmiedeknecht, 1933, Hemiteles)

Distribution: England, Scotland, Wales

Notes: added by Schwarz and Shaw (2010)

Mastrus sordipes (Gravenhorst, 1829)

Nomenclature:

Hemiteles sordipes Gravenhorst, 1829

karpinskii (Glowacki, 1967, Hemiteles)

Distribution: England, Scotland

Notes: Listed as a doubtfully placed species of Hemiteles by Fitton (1978).

Mastrus tenuicosta (Thomson, 1884)

Nomenclature:

Phygadeuon tenuicosta Thomson, 1884

Distribution: England, Scotland

Notes: added by Schwarz and Shaw (2010)

\section{Mastrus varicoxis (Taschenberg, 1865)}

Nomenclature:

Hemiteles varicoxis Taschenberg, 1865 
?coactus (Ratzeburg, 1852, Hemiteles)

tricoloripes (Schmiedeknecht, 1932, Hemiteles)

Distribution: England, Scotland, Ireland

Notes: Listed as a doubtfully placed species of Hemiteles by Fitton (1978).

\title{
Genus Medophron Förster, 1869
}

Nomenclature:

\author{
BARYNTICA Förster, 1869 \\ HEDYLUS Förster, 1869 \\ SUBHEMITELES Horstmann, 1976
}

Notes: Some distribution data from Horstmann (1998a). Townes (1983), whilst noting that crassicornis Ashmead 'might be segregated in a separate genus', retained crassicornis as a species of Medophron, whilst Yu and Horstmann (1997) follow in treating Hedylus as a separate genus. Schwarz and Shaw (2010) follow Townes (1983), noting that separate of Hedylus is inconsistent with the synonymy of Subhemiteles with Medophron.

\section{Medophron afflictor (Gravenhorst, 1829)}

Nomenclature:

Phygadeuon afflictor Gravenhorst, 1829

nigritus (Gravenhorst, 1829, Phygadeuon)

niger Brischke, 1881

Distribution: Wales

\section{Medophron armatulus (Thomson, 1888)}

Nomenclature:

Phygadeuon armatulus Thomson, 1888

Distribution: England, Scotland, Isle of Man

Notes: added by Schwarz and Shaw (2010)

\section{Medophron crassicornis (Gravenhorst, 1829)}

Nomenclature: 
Medophron mixtus (Bridgman, 1883)

Nomenclature:

Hemiteles mixtus Bridgman, 1883

flavipes (Thomson, 1888, Phygadeuon)

mandibularis (Brischke, 1891, Phygadeuon)

flavitarsis (Dalla Torre, 1901, Phygadeuon)

Distribution: England, Ireland

Medophron nigriceps (Thomson, 1883)

Nomenclature:

Acanthocryptus nigriceps Thomson, 1883

elegans (Schmiedeknecht, 1932, Stylocryptus)

Distribution: England

Medophron nitidus (Horstmann, 1976)

Nomenclature:

Subhemiteles nitidus Horstmann, 1976

Distribution: England

Notes: added by Horstmann (1998a)

Medophron recurvus (Thomson, 1884)

Nomenclature:

Phygadeuon recurvus Thomson, 1884

Distribution: England, Scotland

Notes: added by Schwarz and Shaw (2010)

\section{Medophron setosus (Hellén, 1967)}

Nomenclature:

Aclastus setosus Hellén, 1967

crassicornis Ashmead, 1899

Distribution: England, Scotland

Notes: added by Townes (1983) 


\section{Genus Megacara Townes, 1970}

Notes: Some distribution data from Townes (1983).

\section{Megacara hortulana (Gravenhorst, 1829)}

Nomenclature:

Cryptus hortulanus Gravenhorst, 1829

postica (Wollaston, 1858, Hemiteles)

rusticellae (Bridgman, 1886, Phygadeuon)

hispanator (Aubert, 1968, Phygadeuon)

Distribution: England, Scotland, Wales, Ireland

\section{Megacara vagans (Gravenhorst, 1829)}

\section{Nomenclature:}

Phygadeuon vagans Gravenhorst, 1829

apicalis (Gravenhorst, 1829, Cryptus)

alteareolata (Schmiedeknecht, 1905, Phygadeuon)

nova (Kiss, 1929, Acanthocryptus)

Distribution: England, Scotland, Wales, Ireland

\section{Genus Mesoleptus Gravenhorst, 1829}

\section{Nomenclature:}

EXOLYTUS Holmgren, 1859

Notes: Distribution and syonymic data from Jussila et al. (2010) and the collections of BMNH and NMS (det. Jussila). Jussila et al. (2010) placed a large number of Förster names in synonymy; we have not repeated all of the synonymy here, just listing those names that have appeared in the British literature.

doubtfully placed species of Mesoleptus:

[coarctatus (Gravenhorst, 1829, Cryptus) nom. dub.] Listed as a doubtfully placed species of Trychosis by Fitton (1978).

[mirabilis Stephens, 1835 nom. dub, from England; Fitton (1976)]

[speciosus Curtis, 1837 nom. dub.]

[splendens Gravenhorst, 1829 nom. dub.; Jussila et al. (2010)] 
[subcompressus Stephens, 1835 nom. dub., from England; Fitton (1976)]

Mesoleptus congener (Förster, 1876)

Nomenclature:

Exolytus congener Förster, 1876

Distribution: England, Scotland, Wales, Ireland

Notes: added by Jussila et al. (2010)

Mesoleptus devotus (Förster, 1876)

Nomenclature:

Exolytus devotus Förster, 1876

fulvipes (Förster, 1876, Exolytus)

secretus (Förster, 1876, Exolytus)

sollicitus (Förster, 1876, Exolytus)

Distribution: England

\section{Mesoleptus distinctus (Förster, 1876)}

Nomenclature:

Exolytus distinctus Förster, 1876

flavipes (Thomson, 1884, Atractodes)

Distribution: England, Scotland, Wales

Notes: added by Jussila et al. (2010)

\section{Mesoleptus incessor (Haliday, 1839)}

Nomenclature:

Atractodes incessor Haliday, 1839

scrutator (Haliday, 1839, Atractodes)

ambiguus (Förster, 1876, Exolytus)

incertus (Förster, 1876, Exolytus)

speculum (Förster, 1876, Exolytus)

marginatus (Thomson, 1884, Atractodes)

petiolaris (Thomson, 1884, Atractodes)

Distribution: England, Scotland, Ireland 
Mesoleptus laevigatus (Gravenhorst, 1820)

Nomenclature:

Ichneumon laevigatus Gravenhorst, 1820

transversor (Thunberg, 1824, Ichneumon)

aequalis (Förster, 1876, Exolytus)

transsylvanicus (Kiss, 1924, Exolytus)

Distribution: England

Mesoleptus laticinctus (Walker, 1874)

Nomenclature:

Mesostenus laticinctus Walker, 1874

angustulus (Förster, 1876, Exolytus)

ruficoxatus (Förster, 1876, Exolytus)

filicornis (Thomson, 1884, Atractodes)

Distribution: England, Scotland, Wales

Notes: added by Jussila et al. (2010); if ruficoxatus is raised to species level, this should also be on the list, based on specimens from England and Scotland in the NMS, det. Jussila. Mesoleptus laticinctus s.s. has not been recorded from Scotland.

Mesoleptus pronus (Förster, 1876)

Nomenclature:

Exolytus pronus Förster, 1876

consortius (Förster, 1876, Exolytus)

Distribution: England, Scotland, Wales

Notes: added by Jussila et al. (2010)

Mesoleptus vigilatorius (Förster, 1876)

Nomenclature:

Exolytus vigilatorius Förster, 1876

ripicola (Thomson, 1884, Atractodes)

Distribution: England, Scotland, Wales 


\section{Genus Micromonodon Förster, 1869}

Nomenclature:

HEMICRYPTUS Kriechbaumer, 1893

Notes: See note for Mastrus.

Micromonodon tener (Kriechbaumer, 1893)

Nomenclature:

Hemicryptus tener Kriechbaumer, 1893

Distribution: England

Notes: added by Laurenne et al. (2006)

Genus Neopimpla Ashmead, 1900

Neopimpla aleiodesi Schwarz \& Shaw, 2000

Distribution: England

Notes: added by Schwarz and Shaw (2000)

Genus Obisiphaga Morley, 1907

Obisiphaga stenoptera (Marshall, 1868)

Nomenclature:

Aptesis stenoptera Marshall, 1868

longicauda (Vollenhoven, 1873, Aptesis)

similis (Brischke, 1891, Thysiotorus) invalid

dimidiatipennis (Schmiedeknecht, 1905, Hemiteles)

ineptipennis (Speiser, 1908, Hemiteles)

Distribution: Scotland, Wales, Ireland

Genus Odontoneura Förster, 1869

Notes: See note for Mastrus. 
Odontoneura annulicornis (Thomson, 1884)

Nomenclature:

Phygadeuon annulicornis Thomson, 1884

csikii (Szépligeti, 1901, Phygadeuon)

formosa (Pfankuch, 1921, Phygadeuon)

Distribution: England, Scotland

Notes: Listed as a species of Theroscopus by Fitton (1978).

\section{Odontoneura sp. A}

Distribution: Scotland

Notes: added by Schwarz and Shaw (2010); to be described by the late K. Horstmann (in prep.).

\section{Genus Oecotelma Townes, 1970}

Notes: Townes (1970) refers to an undetermined species from Ireland.

\section{Genus Orthizema Förster, 1869}

Nomenclature:

NAETES Förster, 1869

PHYZELUS Förster, 1869

Notes: Some distribution data from Fitton (1976) and Horstmann (1993c).

\section{Orthizema amabile (Hedwig, 1939)}

Nomenclature:

Hemiteles amabilis Hedwig, 1939

Distribution: England

Notes: added by Horstmann (1993c)

\section{Orthizema francescae Schwarz \& Shaw, 2011}

Distribution: England, Scotland, Wales

Notes: added by Schwarz and Shaw (2011) 
Orthizema graviceps (Marshall, 1868)

Nomenclature:

Aptesis graviceps Marshall, 1868

Distribution: England, Wales

Orthizema hadrocerum (Thomson, 1884)

Nomenclature:

Hemiteles hadrocerus Thomson, 1884

fasciatum (Brischke, 1888, Phyzelus)

rufum (Brischke, 1892, Hemiteles)

Distribution: England, Scotland

Orthizema obscurum Horstmann, 1993

Notes: added by Horstmann (1993c)

Orthizema subannulatum (Bridgman, 1883)

Nomenclature:

Hemiteles subannulatus Bridgman, 1883

maculipennis (Rudow, 1886, Hemiteles)

ornatum (Brischke, 1890, Hemiteles)

Distribution: England, Scotland

Orthizema triannulatum (Thomson, 1884)

Nomenclature:

Hemiteles triannulatus Thomson, 1884

Distribution: England, Scotland

Notes: Listed as a doubtfully placed species of Hemiteles by Fitton (1978).

Genus Phygadeuon Gravenhorst, 1829

Nomenclature:

APTEROPHYGAS Förster, 1869

BATHYMETIS Förster, 1869

ERNOCTONA Förster, 1869 
GUNOPACHES Förster, 1869

HABROMMA Förster, 1869

HOMELYS Förster, 1869

ISELIX Förster, 1869

ISOCHRESTA Förster, 1869a

PANTOLISPA Förster, 1869

ZAPHLEGES Förster, 1869

ISCHNOCRYPTUS Kriechbaumer, 1892

Notes: Distribution data from Fitton (1976), Horstmann (1993a), Horstmann (2001d) and Schwarz and Shaw (2011).

Phygadeuon acutipennis Thomson, 1884

Distribution: England, Scotland

Phygadeuon atropos Kriechbaumer, 1892

Distribution: England

Notes: added by Horstmann (2001d); listed as a synonym of forticornis by $\mathrm{Yu}$ and Horstmann (1997).

Phygadeuon brachyurus Thomson, 1884

Phygadeuon brevitarsis Thomson, 1884

Distribution: England

Notes: added by Horstmann (2001d); listed as a synonym of hercynicus by Yu and Horstmann (1997).

Phygadeuon canaliculatus Thomson, 1889

Phygadeuon cephalotes Gravenhorst, 1829

Nomenclature:

transfuga (Gravenhorst, 1829, Ichneumon) 
Phygadeuon clotho Kriechbaumer, 1892

Nomenclature:

grossae Horstmann, 1981

Distribution: England, Scotland, Wales

Notes: added by Horstmann (1981b)

Phygadeuon clypearis Strobl, 1901

Distribution: England

Notes: added by Horstmann (2012a)

Phygadeuon cubiceps Thomson, 1884

Phygadeuon cylindraceus Ruthe, 1859

Nomenclature:

sudvoldensis Morley, 1947

Distribution: England, Scotland

Phygadeuon detestator (Thunberg, 1824)

Nomenclature:

Ichneumon detestator Thunberg, 1824

Phygadeuon devonensis Morley, 1947

Distribution: England

Notes: Phygadeuon neoflavicans Horstmann, 1967 was removed from synonymy by Horstmann (2008a).

Phygadeuon dimidiatus Thomson, 1884

Nomenclature:

cylindricus Brischke, 1891

Phygadeuon ? dromicus (Gravenhorst, 1815)

Nomenclature:

Ichneumon ?dromicus Gravenhorst, 1815 
Notes: Listed as a doubtfully placed species of Hemiteles by Fitton (1978). Male specimens in BMNH identified as dromicus may not be conspecific with Horstmann's (Horstmann 1993c) female neotype.

Phygadeuon dubius (Gravenhorst, 1829)

Nomenclature:

Hemiteles dubius Gravenhorst, 1829

scaposus Thomson, 1884

Phygadeuon elegans (Förster, 1850)

Nomenclature:

Theroscopus elegans Förster, 1850

cingulatus (Förster, 1850, Theroscopus)

confusus Hedwig, 1959 preocc.

Distribution: England, Scotland, Wales, Ireland

Notes: added by Horstmann (1993c)

Phygadeuon elliotti Morley, 1947

Distribution: Scotland

Phygadeuon exiguus Gravenhorst, 1829

Nomenclature:

gallevensis Morley, 1947

Distribution: England

Phygadeuon flavimanus Gravenhorst, 1829

Distribution: England

Phygadeuon forticornis Kriechbaumer, 1892

Distribution: England

Phygadeuon fraternae Horstmann, 2001

Distribution: Scotland

Notes: added by Horstmann (2001d) 
Phygadeuon fumator Gravenhorst, 1829

Nomenclature:

lycaenae Rudow, 1886

britannicus Habermehl, 1923

ragensis Morley, 1947

Distribution: England, Scotland

Phygadeuon geniculatus Kriechbaumer, 1892

Phygadeuon gracilentus Horstmann, 1997

Nomenclature:

gracilicornis Horstmann, 1993 preocc.

Distribution: England, Scotland

Notes: added by Horstmann (1993c)

Phygadeuon hercynicus Gravenhorst, 1829

Distribution: England

Phygadeuon infelix Dalla Torre, 1901

Nomenclature:

inflatus Thomson, 1884 invalid

Phygadeuon laeviventris Thomson, 1884

Nomenclature:

compactus Morley, 1947

Distribution: England

Phygadeuon leucostigmus Gravenhorst, 1829

Nomenclature:

punctigena Thomson, 1884

Distribution: England

Phygadeuon liosternus Thomson, 1886 
Phygadeuon magnicornis (Thomson, 1884)

Nomenclature:

Hemiteles magnicornis Thomson, 1884

Notes: Listed as a doubtfully placed species of Hemiteles by Fitton (1978).

Phygadeuon melanopygus (Gravenhorst, 1829)

Nomenclature:

Hemiteles melanopygus Gravenhorst, 1829

validicornis (Thomson, 1884, Hemiteles)

semicroceus (Schmiedeknecht, 1897, Hemiteles)

Distribution: England, Scotland, Isle of Man

Notes: Listed as a doubtfully placed species of Hemiteles by Fitton (1978), as a species of Theroscopus in Yu and Horstmann (1997) and transferred to Phygadeuon by Schwarz and Shaw (2011).

\section{Phygadeuon nanus (Gravenhorst, 1829)}

Nomenclature:

Cryptus nanus Gravenhorst, 1829

Phygadeuon nigrifemur Horstmann, 2001

Notes: added by Horstmann (2001d)

Phygadeuon nitidus Gravenhorst, 1829

Phygadeuon ovaliformis Dalla Torre, 1901

Nomenclature:

ovalis Thomson, 1884 preocc.

\section{Phygadeuon ovatus Gravenhorst, 1829}

Nomenclature:

caliginosus Gravenhorst, 1829

montanus (Lange, 1911, Stylocryptus) 
Phygadeuon pallicarpus Thomson, 1884

Nomenclature:

pallidicarpus Dalla Torre, 1902

Distribution: Scotland, Ireland

Phygadeuon palus Schwarz \& Shaw, 2011

Distribution: England, Wales

Notes: added by Schwarz and Shaw (2011)

Phygadeuon paradoxus (Bridgman, 1889)

Nomenclature:

Apterophygas paradoxus Bridgman, 1889

hungaricus (Kiss, 1915, Phygadeuon)

insulanus Hedwig, 1939

Distribution: England

Phygadeuon pegomyiae Habermehl, 1928

Phygadeuon punctiventris Thomson, 1884

Phygadeuon rotundipennis Thomson, 1884

Nomenclature:

differens Hedwig, 1938

Distribution: England, Scotland, Wales, Isle of Man

Phygadeuon rubricaudus Morley, 1947

Distribution: England

Phygadeuon rugulosus Gravenhorst, 1829

Nomenclature:

semipolitus Taschenberg, 1865 
Phygadeuon subtilis Gravenhorst, 1829

Nomenclature:

flavicans Thomson, 1884

oppositus Thomson, 1884

subalpinus Roman, 1909

lincolniae Morley, 1947

Distribution: England

Phygadeuon surriensis Morley, 1947

Distribution: England

Phygadeuon tenuiscapus Thomson, 1884

Phygadeuon thomsoni Roman, 1925

Distribution: England

Notes: added by Horstmann (2001d)

Phygadeuon trichops Thomson, 1884

Nomenclature:

ocularis Thomson, 1889

Distribution: Scotland

Phygadeuon troglodytes Gravenhorst, 1829

Nomenclature:

anthracinus Kriechbaumer, 1894

Phygadeuon variabilis Gravenhorst, 1829

Nomenclature:

confinis Smits

Phygadeuon varicornis (Gravenhorst, 1829)

Nomenclature:

Hemiteles varicornis Gravenhorst, 1829

Notes: Listed as a doubtfully placed species of Hemiteles by Fitton (1978). 
Phygadeuon vexator (Thunberg, 1824)

Nomenclature:

Ichneumon vexator Thunberg, 1824

patellator (Thunberg, 1824, Ichneumon)

diaphanus Gravenhorst, 1829

minor Fonscolombe, 1851

nigripes Aubert, 1959

Distribution: England

Notes: Phygadeuon domesticae Horstmann, 1986 was removed from synonymy by Horstmann (2008a).

\section{Genus Platyrhabdus Townes, 1970}

Notes: Some distribution data from Horstmann (1998a).

\section{Platyrhabdus clypeatus Horstmann, 1998}

Distribution: England, Scotland, Isle of Man

Notes: added by Horstmann (1998a)

\section{Platyrhabdus inflatus (Thomson, 1884)}

Nomenclature:

Hemiteles inflatus Thomson, 1884

rufus (Morley, 1907, Aritranis)

elongatus (Smits van Burgst, 1913, Hemiteles) preocc.

tunisiae (Morley, 1926, Hemiteles)

tunetanus (Schmiedeknecht, 1932, Hemiteles)

Distribution: England, Scotland, Ireland, Isle of Man

\section{Platyrhabdus monodon (Thomson, 1884)}

Nomenclature:

Hemiteles monodon Thomson, 1884

graciliventris (Schmiedeknecht, 1933, Hemiteles)

Distribution: England 
Platyrhabdus nervellator Horstmann, 1998

Distribution: England

Notes: added by Horstmann (1998a)

Genus Pleurogyrus Townes, 1970

Pleurogyrus persector (Parfitt, 1882)

Nomenclature:

Hemiteles persector Parfitt, 1882

Distribution: England

Notes: distribution data from Fitton (1976)

\section{Pleurogyrus pumilus (Hellén, 1967)}

Nomenclature:

Uchidella pumila Hellén, 1967

Distribution: England

Notes: added by Horstmann (1995)

\section{Genus Polyaulon Förster, 1869}

Nomenclature:

THAUMATOTYPUS Förster, 1869 synonymy by Horstmann (1998a)

THAUMATOTYPIDEA Viereck, 1912

RHACODOPTERON Čapek, 1956

Polyaulon paradoxus (Zetterstedt, 1838)

Nomenclature:

Cryptus paradoxus Zetterstedt, 1838

billupsi (Bridgman, 1882, Thaumatotypus)

evertsi (Smits van Burgst, 1912, Thaumatotypus)

Distribution: England, Scotland, Wales 
Polyaulon stiavnicensis (Čapek, 1956)

Nomenclature:

Rhacodopteron stiavnicense Čapek, 1956

Distribution: England

Notes: added by Schwarz and Shaw (2000)

Genus Pygocryptus Roman, 1925

Pygocryptus brevicornis (Brischke, 1881)

Nomenclature:

Macrocryptus brevicornis Brischke, 1881

grandis (Thomson, 1884, Phygadeuon) synonymy by Sawoniewicz (2003)

Distribution: England

Notes: added by Townes (1983)

\section{Genus Stibeutes Förster, 1850}

Nomenclature:

CHAMAEZELUS Förster, 1869

SCHIZOPLEURON Aubert, 1968

Notes: Some distribution data from Horstmann (1993c), Horstmann (2010b).

Stibeutes blandi Schwarz \& Shaw, 2011

Distribution: Scotland

Notes: added by Schwarz and Shaw (2011)

Stibeutes breviareolatus (Thomson, 1884)

Nomenclature:

Hemiteles breviareolatus Thomson, 1884

rugiventris (Strobl, 1901, Acanthocryptus) synonymy by Horstmann (2000e)

Notes: Listed as a doubtfully placed species of Hemiteles by Fitton (1978). 
Stibeutes brevicornis (Lange, 1911)

Nomenclature:

Stilpnus brevicornis Lange, 1911

Distribution: England

Notes: added by Horstmann (2010b)

Stibeutes calderonae Bordera \& Hernández-Rodríguez, 2004

Distribution: England

Notes: added by Horstmann (2010b)

Stibeutes curvispina (Thomson, 1884)

Nomenclature:

Phygadeuon curvispina Thomson, 1884

Distribution: England, Scotland, Wales

Stibeutes gravenhorstii Förster, 1850

Distribution: England

Stibeutes heinemanni Förster, 1850

Distribution: England, Scotland

Stibeutes heterogaster (Thomson, 1885)

Nomenclature:

Phygadeuon heterogaster Thomson, 1885

Distribution: England, Scotland, Wales, Isle of Man

Notes: added by Horstmann (2010b)

Stibeutes intermedius Horstmann, 2010

Distribution: England

Notes: added by Horstmann (2010b) 
Stibeutes nigrinus Horstmann, 2010

Distribution: England

Notes: added by Horstmann (2010b)

Stibeutes rozsypali (Gregor, 1941)

Nomenclature:

Phygadeuon rozsypali Gregor, 1941

Distribution: England

Notes: added by Horstmann (2010b)

\section{Genus Stilpnus Gravenhorst, 1829}

Notes: Distribution data from Jussila (1987), Jussila (1999) and BMNH and NMS (det. Jussila).

\section{Subgenus Polyrhembia Förster, 1869}

\section{Stilpnus (Polyrhembia) tenebricosus (Gravenhorst, 1829)}

\section{Nomenclature:}

Hemiteles tenebricosus Gravenhorst, 1829

nitidulator (Zetterstedt, 1838, Ichneumon)

vestalis (Haliday, 1839, Atractodes)

albicinctus (Förster, 1876, Polyrhembia)

anthracinus (Förster, 1876, Polyrhembia)

canaliculatus (Förster, 1876, Polyrhembia) preocc.

carbonarius (Förster, 1876, Polyrhembia)

corvinus (Förster, 1876, Polyrhembia)

discoloripes (Förster, 1876, Atractodes)

nigratus (Förster, 1876, Polyrhembia)

nigripes (Förster, 1876, Polyrhembia)

procerulus (Förster, 1876, Polyrhembia)

splendidus (Förster, 1876, Polyrhembia)

stygius (Förster, 1876, Polyrhembia)

rodnensis Kiss, 1924 synonymy by Horstmann (2011b) 
Distribution: England, Scotland, Wales, Ireland

Subgenus Stilpnus Gravenhorst, 1829

Stilpnus (Stilpnus) blandus Gravenhorst, 1829

Nomenclature:

assimilis Förster, 1876

callens Förster, 1876

cyclogaster Förster, 1876

fuscicornis Förster, 1876

pellucens Förster, 1876

Distribution: England, Scotland, Wales, Ireland

Stilpnus (Stilpnus) crassicornis Thomson, 1884

Distribution: England, Scotland, Ireland

Stilpnus (Stilpnus) deplanatus Gravenhorst, 1829

Distribution: England

Notes: distribution data from UM

Stilpnus (Stilpnus) gagates (Gravenhorst, 1807)

Nomenclature:

Ichneumon gagates Gravenhorst, 1807

aequilongus Förster, 1876

cyclodes Förster, 1876

denticulatus Förster, 1876

diffinis Förster, 1876

dimidiatus Förster, 1876

elimatus Förster, 1876

eurygaster Förster, 1876

fulvicornis Förster, 1876

gallicus Förster, 1876

robinsoni Roman, 1920 
Distribution: England, Scotland, Wales, Ireland

Stilpnus (Stilpnus) parvulus Förster, 1876

Nomenclature:

inaequalis Förster, 1876

Distribution: England

Notes: added by Jussila (1987)

Stilpnus (Stilpnus) pavoniae (Scopoli, 1763)

Nomenclature:

Ichneumon pavoniae Scopoli, 1763

agilis Förster, 1876

ambulatorius Förster, 1876

arridens Förster, 1876

conformatus Förster, 1876

declinis Förster, 1876

morionellus Förster, 1876

neglectus Förster, 1876

nigricoxis Förster, 1876

politus Förster, 1876 preocc.

retritus Förster, 1876

subtilis Förster, 1876

tersus Förster, 1876

trivialis Förster, 1876

unctus Förster, 1876

xanthopus Förster, 1876

angustatus Thomson, 1884

Distribution: England, Scotland, Wales, Ireland

Stilpnus (Stilpnus) subzonulus Förster, 1876

Nomenclature:

canaliculatus Förster, 1876

diversus Förster, 1876 
latens Förster, 1876

placitus Förster, 1876

tenuipes Thomson, 1884

Distribution: England, Scotland, Wales, Ireland

Subgenus Xestophyes Förster, 1869

Nomenclature:

XESTOPHYA Förster, 1876

Stilpnus (Xestophyes) dryadum Curtis, 1832

Distribution: England, Ireland

Notes: Listed as a synonym of Adelognathus dorsalis in Yu and Horstmann (1997) but according to Jussila (1987), whilst a paralectotype is referable to dorsalis, the lectotype specimen is a species of Stilpnus.

\section{Stilpnus (Xestophyes) fallax (Förster, 1876)}

Nomenclature:

Xestophya fallax Förster, 1876

Distribution: England

Notes: NMS, det. Jussila, added here

\section{Genus Sulcarius Townes, 1970}

Notes: Some distribution data from Townes (1983).

\section{Sulcarius biannulatus (Gravenhorst, 1829)}

Nomenclature:

Hemiteles biannulatus Gravenhorst, 1829

Distribution: Scotland

\section{Sulcarius bispinosus (Rudow, 1886)}

Nomenclature:

Phygadeuon bispinosus Rudow, 1886

hellbachi (Schmiedeknecht, 1905, Hemiteles) 
Distribution: England

Notes: added by Townes (1983)

Sulcarius fontinalis (Ruschka, 1926)

Nomenclature:

Hemiteles fontinalis Ruschka, 1926

Distribution: England, Scotland

Notes: added by Schwarz and Shaw (2011)

Sulcarius laevipleuris Horstmann, 1992

Distribution: England

Notes: added by Horstmann (1992b)

Sulcarius nigricornis (Thomson, 1884)

Nomenclature:

Hemiteles nigricornis Thomson, 1884

homocerus (Thomson, 1885, Hemiteles)

Distribution: England, Scotland, Wales, Ireland

Notes: Listed (as homocerus) as a doubtfully placed species of Hemiteles by Fitton (1978).

Sulcarius nigridens Horstmann, 1992

Distribution: England, Scotland

Notes: added by Horstmann (1992b)

\section{Sulcarius sp. A}

Distribution: England, Scotland

Notes: added by Schwarz and Shaw (2011); to be described by the late K. Horstmann.

\section{Genus Thaumatogelis Schwarz, 1995}

Nomenclature: 
CRYPTOGELIS Hellén, 1944 nom. nud.

Notes: species of Thaumatogelis excluded from the British and Irish list by Schwarz (1995):

[pilosus (Capron, 1888, Pezomachus)] The type of pilosus was ostensibly British but Schwarz (1995) has excluded this southern European species from the British list.

\section{Thaumatogelis audax (Olivier, 1792)}

Nomenclature:

Ichneumon audax Olivier, 1792

arnearum (Geoffroy, 1785, Ichneumon) preocc.

zonatus (Förster, 1850, Pezomachus)

Distribution: England, Wales

Thaumatogelis innoxius Schwarz, 2001

Nomenclature:

mingetshauricus misident.

Distribution: England

Notes: Added by Schwarz (2001); tentative identification as mingetshauricus (Bogačev, 1946, Gelis) by Schwarz and Shaw (2000) was a misidentification.

\section{Thaumatogelis lichtensteini (Pfankuch, 1913)}

\section{Nomenclature:}

Thaumatotypidea lichtensteini Pfankuch, 1913

cabrerai (Duchaussoy, 1915, Thaumatotypidea)

graecus (Rudow, 1917, Pezomachus)

siculus (Rudow, 1917, Pezomachus)

?maroccanus (Ceballos, 1925, Gelis) synonymy by Schwarz (2001)

dentatus (Seyrig, 1926, Thaumatotypidea)

longicornis (Seyrig, 1926, Thaumatotypidea)

medianus (Seyrig, 1926, Thaumatotypidea)

micariae (Seyrig, 1926, Thaumatotypidea) preocc.

muticus (Seyrig, 1926, Thaumatotypidea)

nigripes (Seyrig, 1926, Thaumatotypidea) 
Distribution: England

Notes: added by Schwarz and Shaw (2000)

Thaumatogelis neesii (Förster, 1850)

Nomenclature:

Pezomachus neesii Förster, 1850

quadrifasciatus (Kriechbaumer, 1899, Pezomachus) unavailable

Distribution: England, Scotland

Notes: added by Schwarz and Shaw (2000)

\section{Thaumatogelis sylvicola (Förster, 1850)}

Nomenclature:

Pezomachus sylvicola Förster, 1850

luceus (Seyrig, 1928, Gelis)

Distribution: England

Thaumatogelis vulpinus (Gravenhorst, 1815)

Nomenclature:

Ichneumon vulpinus Gravenhorst, 1815

aquisgranensis (Förster, 1850, Pezomachus)

Distribution: England, Scotland, Wales

\section{Genus Theroscopus Förster, 1850}

Nomenclature:

CHAMERPES Förster, 1869

ERIPLANUS Förster, 1869

PHYRTUS Förster, 1869

THYSIOTORUS Förster, 1869

Notes: Some distribution data from Horstmann (1993c) and Fitton (1976).

Theroscopus bonelli (Gravenhorst, 1815)

Nomenclature:

Ichneumon bonelli Gravenhorst, 1815 
ingrediens Förster, 1850

rufiventris (Rudow, 1917, Agrothereutes)

Distribution: England, Wales

Notes: added by Horstmann (1993c)

Theroscopus coriaceus Horstmann, 1993

Distribution: England

Notes: added by Horstmann (1993c)

Theroscopus esenbeckii (Gravenhorst, 1815)

Nomenclature:

Ichneumon esenbeckii Gravenhorst, 1815

subzonatus (Gravenhorst, 1815, Ichneumon)

gravenhorstii (Ratzeburg, 1844, Pezomachus)

inaequalis Förster, 1850

transsylvanicus (Kiss, 1929, Hemiteles)

Distribution: England, Scotland, Wales, Ireland

Notes: Theroscopus esenbeckii, subzonatus and inaequalis are all listed as doubtfully placed species of Hemiteles by Fitton (1978).

Theroscopus fasciatulus Horstmann, 1979

Nomenclature:

fasciatus (Thomson, 1884, Hemiteles) preocc.

Distribution: England

Notes: Listed as a doubtfully placed species of Hemiteles (as fasciatus) by Fitton (1978).

\section{Theroscopus hemipteron (Riche, 1791)}

Nomenclature:

Ichneumon hemipteron Riche, 1791

hemipterus (Fabricius, 1793, Ichneumon)

hemipterator (Thunberg, 1824, Ichneumon)

dissimilis (Gravenhorst, 1829, Hemiteles) 


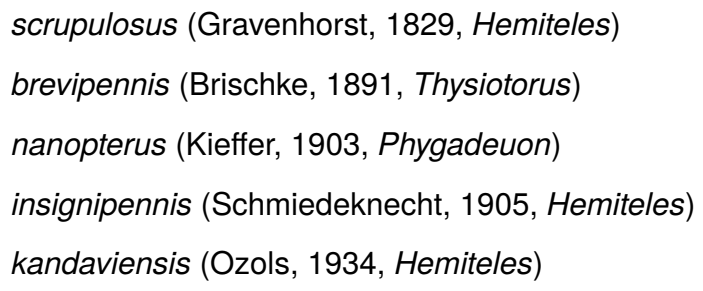

Distribution: England, Scotland, Wales, Ireland

Theroscopus horsfieldi Schwarz \& Shaw, 2011

Distribution: Scotland

Notes: added by Schwarz and Shaw (2011)

Theroscopus mariae Schwarz \& Shaw, 2011

Distribution: England, Scotland

Notes: added by Schwarz and Shaw (2011)

Theroscopus megacentrus (Schiødte, 1839)

Nomenclature:

Cryptus megacentrus Schiødte, 1839

ornaticornis (Schmiedeknecht, 1897, Hemiteles) synonymy by Horstmann (2004a)

occisor (Habermehl, 1923, Phygadeuon) synonymy by Horstmann (2000c)

Distribution: England, Scotland

Theroscopus naninae Schwarz \& Shaw, 2011

Distribution: Scotland

Notes: added by Schwarz and Shaw (2011)

Theroscopus ochrogaster (Thomson, 1888)

Nomenclature:

Phygadeuon ochrogaster Thomson, 1888

rotundator Aubert, 1989 synonymy by Schwarz and Shaw (2011)

Distribution: England, Scotland, Isle of Man 
Notes: added by Schwarz and Shaw (2011)

Theroscopus opacinotum (Hellén, 1967)

Nomenclature:

Aclastus opacinotum Hellén, 1967

Notes: Added by Schwarz and Shaw (2011) and transferred from Orthizema.

Theroscopus pedestris (Fabricius, 1775)

Nomenclature:

Ichneumon pedestris Fabricius, 1775

pilosellus (Rudow, 1917, Pezomachus)

\section{Theroscopus pullator (Gravenhorst, 1829)}

\section{Nomenclature:}

Cryptus pullator Gravenhorst, 1829

notaulium (Morley, 1947, Phygadeuon)

Distribution: England, Scotland, Ireland

Notes: Listed as Stiboscopus notaulius (Morley) (with pullator as a doubtfully placed species of Hemiteles) by Fitton (1978); listed as Orthizema pullator by $\mathrm{Yu}$ and Horstmann (1997), transferred to Theroscopus by Schwarz and Shaw (2011).

\section{Theroscopus rufulus (Gmelin, 1790)}

Nomenclature:

Ichneumon rufulus Gmelin, 1790

micator misident.

luteiventris (Gravenhorst, 1829, Hemiteles)

oxyphymus (Gravenhorst, 1829, Hemiteles)

rufulus (Gravenhorst, 1829, Hemiteles) preocc.

litoreus (Parfitt, 1882, Hemiteles)

politus (Bridgman, 1883, Hemiteles)

silesiacus (Habermehl, 1919, Phygadeuon)

Distribution: England, Scotland, Wales, Ireland 
Theroscopus ungularis (Thomson, 1884)

Nomenclature:

Phygadeuon ungularis Thomson, 1884

ungularis (Thomson, 1884, Hemiteles)

heteroneurus (Schmiedeknecht, 1933, Hemiteles)

Distribution: England

Notes: Listed as a doubtfully placed species of Hemiteles by Fitton (1978).

Genus Tricholinum Förster, 1869

Nomenclature:

STIBOSCOPELLUS Roman, 1930

Tricholinum ischnocerum (Thomson, 1888)

Nomenclature:

Hemiteles ischnocerus Thomson, 1888

pimploides (Roman, 1930, Stiboscopellus)

Distribution: England, Scotland, Wales

Notes: added by Schwarz and Shaw (2011)

\section{Genus Tropistes Gravenhorst, 1829}

Nomenclature:

PSEUDOLIMERODES Strobl, 1902

BOLESLAWIA Sawoniewicz, 1996 synonymy by Schwarz and Shaw (2011)

Tropistes falcatus (Thomson, 1884)

Nomenclature:

Hemiteles falcatus Thomson, 1884

nigriventris Kriechbaumer, 1894 preocc.

rufipes Kriechbaumer, 1894

compressiventris (Strobl, 1902, Pseudolimerodes)

Distribution: England

Notes: added by Schwarz and Shaw (2011) 
Tropistes nitidipennis Gravenhorst, 1829

Nomenclature:

fuscipes Kriechbaumer, 1894 unavailable

nigriventris Kriechbaumer, 1894 unavailable

Distribution: England, Scotland

Tropistes scoticus Schwarz \& Shaw, 2011

Distribution: England, Scotland

Notes: added by Schwarz and Shaw (2011); English distribution from specimen in $\mathrm{BMNH}$, det. Broad

Genus Uchidella Townes, 1957

Nomenclature:

ITAMUS Förster, 1869

Uchidella brevicauda Horstmann, 1993

Distribution: England, Scotland

Notes: added by Horstmann (1993b)

Uchidella flavilabris Horstmann, 1993

Distribution: England, Scotland

Notes: added by Schwarz and Shaw (2011)

Uchidella longicaudata Horstmann, 1997

Nomenclature:

longicauda Horstmann, 1993 preocc.

Distribution: England, Scotland, Ireland

Notes: added by Schwarz and Shaw (2011)

\section{Genus Xenolytus Förster, 1869}

Nomenclature:

STERNOCRYPTUS Roman, 1925 
Notes: Some distribution data from Townes (1983).

Xenolytus bitinctus (Gmelin, 1790)

Nomenclature:

Ichneumon bitinctus Gmelin, 1790

expulsor (Thunberg, 1824, Ichneumon)

Distribution: England, Scotland

Xenolytus substriatus Townes, 1983

Distribution: England

Notes: added by Townes (1983)

\section{Genus Xiphulcus Townes, 1970}

Nomenclature:

NOTOSTILBUS Townes, 1983

\section{Xiphulcus floricolator (Gravenhorst, 1807)}

Nomenclature:

Ichneumon floricolator Gravenhorst, 1807

imbecillus (Gravenhorst, 1829, Hemiteles)

longiventris (Schiødte, 1839, Hemiteles) synonymy by Horstmann (2004a)

longulus (Thomson, 1884, Hemiteles)

lucidus (Szépligeti, 1901, Phygadeuon)

muelleri (Kiss, 1924, Hemiteles)

ramellosus (Kiss, 1924, Leptocryptus)

longicauda Hellén, 1967, Uchidella)

Distribution: England, Scotland

Notes: Recorded by Kerrich (1935); English record from A.C. Galsworthy coll.

\section{Genus Zoophthorus Förster, 1869}

Nomenclature:

CHAETOMASTRUS Hellén, 1967 
Notes: See note for Mastrus. Some distribution data from Fitton (1976).

\section{Zoophthorus anglicanus (Morley, 1907)}

Nomenclature:

Hemiteles anglicanus Morley, 1907

Distribution: England, Isle of Man

Zoophthorus bridgmani (Schmiedeknecht, 1897)

Nomenclature:

Hemiteles bridgmani Schmiedeknecht, 1897

niger (Bridgman, 1883, Theroscopus) invalid

pfankuchi (Smits van Burgst, 1913, Hemiteles)

Distribution: England, Scotland

Zoophthorus cynipinus (Thomson, 1884)

Nomenclature:

Hemiteles cynipinus Thomson, 1884

Distribution: England, Scotland

Zoophthorus dodecellae (Obrtel \& Šedivý, 1960)

Nomenclature:

Hemiteles dodecellae Obrtel \& Šedivý, 1960

added by

Distribution: England, Scotland

Notes: added by Schwarz and Shaw (2010)

Zoophthorus graculus (Gravenhorst, 1829)

Nomenclature:

Bassus graculus Gravenhorst, 1829

auriculatus (Thomson, 1884, Hemiteles)

albomarginatus (Bridgman, 1887, Hemiteles)

Distribution: England, Isle of Man 
Zoophthorus infirmus (Gravenhorst, 1829)

Nomenclature:

Hemiteles infirmus Gravenhorst, 1829

tenerrimus (Gravenhorst, 1829, Hemiteles)

Notes: Transferred from Eudelus by Schwarz and Shaw (2000).

Zoophthorus notaticrus (Thomson, 1888)

Nomenclature:

Hemiteles notaticrus Thomson, 1888

Distribution: England, Scotland

Notes: added by Schwarz and Shaw (2010)

Zoophthorus palpator (Müller, 1776)

Nomenclature:

Ichneumon palpator Müller, 1776

incisus (Bridgman, 1883, Hemiteles)

hilarellus (Schmiedeknecht, 1905, Hemiteles)

Distribution: England, Scotland, Wales, Isle of Man

Zoophthorus plumbeus (Thomson, 1884)

Nomenclature:

Hemiteles plumbeus Thomson, 1884

Distribution: England

Notes: added by Schwarz and Shaw (2010)

\section{Zoophthorus sp. A}

Distribution: England

Notes: added by Schwarz and Shaw (2010); to be described by the late K. Horstmann (in prep.) 


\section{Ctenopelmatinae}

\section{Subfamily CTENOPELMATINAE Förster, 1869}

\section{Nomenclature:}

SCOLOBATINAE Schmiedeknecht, 1911

Notes: Parts of the NHM collection have relatively recently been determined by $\mathrm{J}$. Barron, R. Hinz, K. Horstmann, M. Idar and J.-F. Aubert, parts of the NMS have been determined by D.R. Kasparyan. the catalogue of Aubert (2000) frequently has different dates of publication and endings of specific names to those in $\mathrm{Yu}$ and Horstmann (1997), the latter is followed. Unless stated otherwise, distribution data from Aubert (2000), Fitton (1976), for type localities, and the collections of NHM, NMS and UM.

\section{Tribe CHRIONOTINI Uchida, 1957}

\section{Nomenclature:}

OLETHRODOTINI Townes, 1970

Notes: Distribution data from Shaw and Kasparyan (2002). Note that Olethrodotini has generally been used as the name for this small tribe but Chrionotini has precedence.

\section{Genus Olethrodotis Förster, 1869}

\section{Nomenclature:}

TASCHENBERGIA Schmiedeknecht, 1888

\section{Olethrodotis modestus (Gravenhorst, 1829)}

\section{Nomenclature:}

Mesoleptus modestus Gravenhorst, 1829

evolans (Gravenhorst, 1829, Tryphon)

microtamia (Gravenhorst, 1829, Phytodietus)

Distribution: England, Scotland

\section{Tribe CTENOPELMATINI Förster, 1869}

Notes: The checklist of British Ctenopelmatini was revised by Shaw et al. (2003), including nomenclatural changes and distribution data. 


\section{Genus Ctenopelma Holmgren, 1857}

\section{Nomenclature:}

DIEDRUS Förster, 1869

ERYMA Förster, 1869

XANIOPELMA Tschek, 1869

ZACHRESTA Förster, 1869

HOLMGRENIA Kriechbaumer, 1877

KRIECHBAUMERIA Dalla Torre, 1885

POLYOMORUS Kriechbaumer, 1894

POLYHOMORUS Schulz, 1906

PSEUDOBANCHUS Szépligeti, 1911

\section{Ctenopelma ruficorne Holmgren, 1857}

\section{Nomenclature:}

Ctenopelma ruficornis Holmgren, 1857

Distribution: Scotland

Notes: added by Shaw et al. (2003)

\section{Ctenopelma tomentosum (Desvignes, 1856)}

Nomenclature:

Campoplex tomentosus Desvignes, 1856

nigrum misident.

lucifer misident.

luteum Holmgren, 1857

xanthostigma Holmgren, 1857; synonymy by Kasparyan (2004a)

variabile Tschek, 1869

gagatinum (Kriechbaumer, 1894, Polyomorus)

athimi Kriechbaumer, 1896; synonymy by Kasparyan (2004a)

pulchrum (Kriechbaumer, 1877, Holmgrenia)

braunsii Pfankuch, 1904

dispar Ulbricht, 1916

Distribution: England 
Notes: Although Aubert (2000) placed tomentosum in synonymy with luteum, Holmgren's publication actually dates from 1857 , not 1855 , so tomentosum is the valid name (Shaw et al. 2003).

\section{Genus Homaspis Förster, 1869}

Nomenclature:

NEOHOMASPSIS Heinrich, 1949

\section{Homaspis analis (Holmgren, 1857)}

Nomenclature:

Notopygus analis Holmgren, 1857

subalpina misident.

defectivus (Tschek, 1869, Ctenopelma); synonymy by Kasparyan (2004a)

pectator Aubert, 1989; synonymy by Kasparyan (2004a)

Distribution: England

Notes: Listed as a synonym of $H$. narrator (Gravenhorst, 1829, Mesoleptus) by $Y u$ and Horstmann (1997) but this was not accepted by Shaw et al. (2003).

Genus Notopygus Holmgren, 1857

Nomenclature:

ANTIPYGUS Tschek, 1869

Notopygus emarginatus Holmgren, 1857

Nomenclature:

sinifer Ulbricht, 1922

Distribution: England, Scotland

Genus Xenoschesis Förster, 1869

Subgenus Xenoschesis Förster, 1869

Nomenclature:

HOMOBIA Förster, 1869

GLYPTOCENTRUS Kriechbaumer, 1894 
Xenoschesis (Xenoschesis) fulvipes (Gravenhorst, 1829)

Nomenclature:

Exetastes fulvipes Gravenhorst, 1829

ruficornis (Rudow, 1883, Exetastes)

ruficornis (Rudow, 1886, Exetastes)

varicoxa Heinrich, 1949; synonymy by Aubert (2000)

Distribution: England, Scotland

Subgenus Polycinetis Förster, 1869

Nomenclature:

ERIGLOEA Förster, 1869

PROSMORUS Förster, 1869

POLYCINETUS Thomson, 1893

Xenoschesis (Polycinetis) ustulata (Desvignes, 1856)

Nomenclature:

Tryphon ustulatus Desvignes, 1856

resplendens (Holmgren, 1857, Notopygus); synonymy by Shaw et al. (2003)

polita (Kriechbaumer, 1891, Erigloea)

montana (Habermehl, 1922, Hadrodactylus)

Distribution: England, Scotland

Notes: Horstmann (2006b) removed fulvicornis (Kriechbeumer, 1891, Erigloea) from synonymy.

Tribe EURYPROCTINI Thomson, 1883

Genus Anisotacrus Schmiedeknecht, 1913

Anisotacrus bipunctatus (Gravenhorst, 1829)

Nomenclature:

Mesoleptus bipunctatus Gravenhorst, 1829

Distribution: England 
Anisotacrus tenellus (Holmgren, 1857)

Nomenclature:

Mesoleius tenellus Holmgren, 1857

Distribution: England, Scotland

Notes: BMNH, NMS added here; treated as a synonym of bipunctatus by Aubert (2000) but as a valid species in Yu and Horstmann (1997).

Anisotacrus xanthostigma (Gravenhorst, 1829)

Nomenclature:

Mesoleptus xanthostigma Gravenhorst, 1829

vividus (Woldstedt, 1874, Mesoleptus)

Distribution: England

Genus Euryproctus Holmgren, 1857

Nomenclature:

HYPOCRYPTUS Förster, 1869

SYCHNOLETER Förster, 1869

XENONASTES Förster, 1869

Euryproctus alpinus Holmgren, 1857

Nomenclature:

exareolatus Thomson, 1889

Distribution: England

Euryproctus annulatus (Gravenhorst, 1829)

Nomenclature:

Mesoleptus annulatus Gravenhorst, 1829

annulator (Stephens, 1835, Mesoleptus)

Distribution: England, Scotland

Euryproctus bivinctus Holmgren, 1857

Distribution: England, Wales, Scotland

Notes: added by Aubert (2000) 
Euryproctus crassicornis Thomson, 1889

Distribution: England

Euryproctus geniculosus (Gravenhorst, 1829)

Nomenclature:

Mesoleptus geniculosus Gravenhorst, 1829

Distribution: England, Scotland, Wales, Isle of Man

Euryproctus holmgreni Kerrich, 1942

Distribution: England

Euryproctus inferus Thomson, 1889

Distribution: England, Scotland

Euryproctus luteicornis (Gravenhorst, 1829)

Nomenclature:

Tryphon luteicornis Gravenhorst, 1829

Distribution: England

Notes: added by Aubert (2000)

Euryproctus mundus (Gravenhorst, 1820)

Nomenclature:

Ichneumon mundus Gravenhorst, 1820

aberrans Woldstedt, 1877

testaceicornis (Brischke, 1892, Mesoleptus)

strandi (Gregor, 1937, Mesoleptus); synonymy by Horstmann (2002b)

Distribution: England, Scotland

Euryproctus nemoralis (Geoffroy, 1785)

Nomenclature:

Ichneumon nemoralis Geoffroy, 1785

digitator (Thunberg, 1824, Ichneumon)

?suborbitalis (Stephens, 1835, Mesoleptus) 
affinis (Holmgren, 1856, Mesoleptus)

vafer Woldstedt, 1874

foersteri Kriechbaumer, 1897; synonymy by Horstmann (2002c)

Distribution: England, Scotland, Isle of Man

\section{Euryproctus plantator (Thunberg, 1824)}

Nomenclature:

Ichneumon plantator Thunberg, 1824

albipes Holmgren, 1857

tuberculatus Holmgren, 1857

exareolatus Thomson, 1889

Distribution: England

Notes: added by Aubert (2000)

\section{Euryproctus ratzeburgi (Gorski, 1852)}

Nomenclature:

Tryphon ratzeburgi Gorski, 1852

sinister Brischke, 1871; synonymy by Horstmann (1998b)

nitidulus Thomson, 1889

phygadeuontoides (Kriechbaumer, 1896, Polyblastus)

pictus Habermehl, 1925

Distribution: England

Genus Gunomeria Schmiedeknecht, 1907

Gunomeria macrodactylus (Holmgren, 1856)

Nomenclature:

Mesoleptus macrodactylus Holmgren, 1856

scutellata (Bridgman, 1886, Mesoleptus)

Distribution: England, Scotland, Wales, Ireland

Notes: Treated as a synonym of sordida in Yu and Horstmann (1997), as a separate species by Aubert (2000) and then by Horstmann (2008a). 


\section{Gunomeria sordida (Gravenhorst, 1829)}

Nomenclature:

Mesoleptus sordidus Gravenhorst, 1829

Distribution: England, Scotland, Wales, Isle of Man

\section{Genus Hadrodactylus Förster, 1869}

\section{Nomenclature:}

DIZEMON Förster, 1869

NARCOPOEA Förster, 1869

ZEMIODES Förster, 1869

MEROPACHES Schmiedeknecht, 1913

Notes: Distribution data from Idar (1974), Idar (1975), Idar (1981), Aubert (2000), Kasparyan and Shaw (2009) and BMNH.

Species of Hadrodactylus excluded from the British and Irish list

[bidentulus Thomson, 1883] Kasparyan and Shaw (2009) could not find any British or Irish specimens; those in BMNH under bidentulus were misidentified.

[larvatus Kriechbaumer, 1891] Erroneously listed as occurring in the British Isles by Kasparyan (2011).

\section{Hadrodactylus confusus (Holmgren, 1858)}

Nomenclature:

Mesoleptus confusus Holmgren, 1858

albicoxa Thomson, 1883

Distribution: England

Notes: Listed, presumably erroneously, as occurring in Scotland by Kasparyan (2011).

\section{Hadrodactylus faciator (Thunberg, 1824)}

Nomenclature:

Ichneumon faciator Thunberg, 1824

gracilis (Holmgren, 1856, Mesoleptus)

curtus (Holmgren, 1857, Mesoleptus)

Distribution: England, Scotland, Isle of Man 
Hadrodactylus femoralis (Holmgren, 1857)

Nomenclature:

Mesoleptus femoralis Holmgren, 1857

intrepidus Kriechbaumer, 1891; synonymy by Horstmann (2000c)

nigricoxa (Thomson, 1893, Mesoleptus)

thomsoni Schmiedeknecht, 1913

Distribution: England, Scotland, Ireland

Notes: Added by Idar (1975); omitted by Fitton (1978).

Hadrodactylus flavofacialis Horstmann, 2000

Nomenclature:

flavifrontator misident.

Distribution: England, Scotland

Notes: Added by Idar (1981), some distribution data from Horstmann (2000b) and UM; listed as flavifrontator (Thunberg, 1824, Ichneumon) in Aubert (2000) (Horstmann 2000b).

\section{Hadrodactylus fugax (Gravenhorst, 1829)}

Nomenclature:

Mesoleptus fugax Gravenhorst, 1829

ventralis (Curtis, 1837, Mesoleptus)

marginatus (Bridgman, 1886, Mesoleptus)

alticola (Strobl, 1903, Mesoleptus)

branderi Jussila, 1967

Distribution: England, Scotland, Wales, Ireland

Hadrodactylus genalis Thomson, 1883

Nomenclature:

pygmaeus Habermehl

Distribution: England

Notes: NHM, det. Broad, added here 
Hadrodactylus gracilipes Thomson, 1883

Nomenclature:

meridionator Villemant, 1982; synonymy by Kasparyan (2011)

Distribution: England, Scotland

Hadrodactylus gracilis (Stephens, 1835)

Nomenclature:

Mesoleptus gracilis Stephens, 1835

Distribution: England

Hadrodactylus graminicola Idar, 1979

Distribution: England, Scotland

Notes: added by Kasparyan and Shaw (2009)

Hadrodactylus idari Kasparyan \& Shaw, 2009

Nomenclature:

gracilipes misident.

Distribution: England, Scotland

Notes: added by Kasparyan and Shaw (2009)

Hadrodactylus indefessus (Gravenhorst, 1820)

Nomenclature:

Ichneumon indefessus Gravenhorst, 1820

tarsator Thomson, 1883

Distribution: England, Scotland, Ireland

Notes: added by Kasparyan and Shaw (2009)

Hadrodactylus insignis Kriechbaumer, 1891

Nomenclature:

varicoxa (Thomson, 1893, Mesoleptus)

variicoxa Dalla Torre, 1901

Distribution: England, Scotland 
Hadrodactylus nigrifemur Thomson, 1883

Distribution: England, Scotland, Wales, Ireland

Hadrodactylus paludicola (Holmgren, 1856)

Nomenclature:

Mesoleptus paludicola Holmgren, 1856

subalpinus Schmiedeknecht, 1913

Distribution: England, Scotland, Ireland, Isle of Man

Hadrodactylus semirufus (Holmgren, 1858)

Nomenclature:

Mesoleptus semirufus Holmgren, 1858

erythropus Kriechbaumer, 1891; synonymy by Horstmann (2000c)

pubescens Ulbricht, 1922

Distribution: England, Scotland, Wales

Notes: added by Aubert (2000)

Hadrodactylus spiraculator Idar, 1979

Distribution: England, Scotland

Notes: added by Idar (1981)

Hadrodactylus tiphae (Geoffroy, 1785)

Nomenclature:

Ichneumon tiphae Geoffroy, 1785

luteolus (Gmelin, 1790, Ichneumon)

laticeps Thomson, 1883

erythropus Kriechbaumer, 1891

Distribution: England, Scotland, Ireland

Hadrodactylus villosulus Thomson, 1883

Distribution: England, Isle of Man 
Hadrodactylus vulneratus (Zetterstedt, 1838)

Nomenclature:

Tryphon vulneratus Zetterstedt, 1838

Distribution: England, Scotland

Notes: added by Aubert (2000)

Genus Hypamblys Förster, 1869

Nomenclature:

APYSTUS Förster, 1869

LATHROPHAGUS Förster, 1869

Hypamblys albopictus (Gravenhorst, 1829)

Nomenclature:

Tryphon albopictus Gravenhorst, 1829

transfuga (Holmgren, 1857, Mesoleius)

instabilis (Ruthe, 1859, Tryphon)

Distribution: England, Scotland

Genus Hypsantyx Pfankuch, 1906

Hypsantyx lituratorius (Linnaeus, 1761)

Nomenclature:

Ichneumon lituratorius Linnaeus, 1761

tenthredinum (Scharfenberg, 1805, Ichneumon)

impressus (Gravenhorst, 1829, Tryphon)

crassicornis (Zetterstedt, 1838, Pimpla)

Genus Mesoleptidea Viereck, 1912

Nomenclature:

GNATHONOPHORUS Schmiedeknecht, 1912

Mesoleptidea cingulata (Gravenhorst, 1829)

Nomenclature:

Mesoleptus cingulatus Gravenhorst, 1829 
bidens (Fabricius, 1798, Ichneumon) nom. ob.: Horstmann (2001c)

bidentor (Thunberg, 1824, Ichneumon) nom. ob.: Horstmann (2006b)

pectoralis (Gravenhorst, 1829, Mesoleptus)

submarginata (Stephens, 1835, Mesoleptus)

undecimnotata (Desvignes, 1856, Mesoleptus)

Distribution: England, Scotland

Mesoleptidea hilaris (Gravenhorst, 1829)

Nomenclature:

Mesoleptus hilaris Gravenhorst, 1829

Distribution: England

Mesoleptidea prosoleuca (Gravenhorst, 1820)

Nomenclature:

Ichneumon prosoleucus Gravenhorst, 1820

tricolor (Fabricius, 1793, Ophion) nom. ob.: Horstmann (2001c)

neglecta (Holmgren, 1857, Mesoleptus)

glacialis (Woldstedt, 1874, Mesoleptus)

similis (Brischke, 1878, Mesoleptus)

holmgreni (Thomson, 1893, Mesoleptus)

nigriventris (Habermehl, 1925, Mesoleptus)

Distribution: England, Scotland, Ireland

Mesoleptidea stallii (Holmgren, 1858)

Nomenclature:

Mesoleptus stallii Holmgren, 1858

Genus Occapes Townes, 1970

Occapes selandriae (Brischke, 1878)

Nomenclature:

Polyblastus selandriae Brischke, 1878

Distribution: England, Wales 
Notes: BMNH, det. Broad, added here

Genus Pantorhaestes Förster, 1869

Nomenclature:

TROPHOCTONUS Förster, 1869

Pantorhaestes xanthostomus (Gravenhorst, 1829)

Nomenclature:

Tryphon xanthostomus Gravenhorst, 1829

ochrostomus (Gravenhorst, 1829, Tryphon)

rufocinctus (Gravenhorst, 1829, Mesoleptus)

hilarellus (Holmgren, 1858, Euryproctus)

intensicolor (Heinrich, 1953, Dialges)

Distribution: England, Scotland, Wales, Isle of Man

\section{Genus Phobetes Förster, 1869}

Nomenclature:

IPOCTONUS Förster, 1869

PHILOTYMMA Förster, 1869

PHOBETUS Thomson, 1889

GRIPHODES Kriechbaumer, 1894

IPOCTONINUS Hincks, 1944

PHOBETELLUS Hincks, 1944

Phobetes atomator (Muiller, 1776)

Nomenclature:

Ichneumon atomator Müller, 1776

croatica (Kiss, 1926, Brischkea); synonymy by Horstmann (2007b)

Distribution: England, Wales, Isle of Man

Phobetes cerinostomus (Gravenhorst, 1829)

Nomenclature:

Mesoleptus cerinostomus Gravenhorst, 1829

Distribution: England 
Notes: NMS, det. Kasparyan, added here

Phobetes chrysostomus (Gravenhorst, 1820)

Nomenclature:

Ichneumon chrysostomus Gravenhorst, 1820

Distribution: England

Phobetes femorator (Thomson, 1893)

Nomenclature:

Phobetus femorator Thomson, 1893

subalpinus (Strobl, 1903, Euryproctus)

Distribution: England

Phobetes fuscicornis (Holmgren, 1856)

Nomenclature:

Tryphon fuscicornis Holmgren, 1856

fulviventris (Thomson, 1893, Phobetus)

Phobetes leptocerus (Gravenhorst, 1820)

Nomenclature:

Ichneumon leptocerus Gravenhorst, 1820

stigmaticus (Brischke, 1878, Mesoleptus)

schmiedeknechti (Lange, 1911, Hadrodactylus)

aigneri (Kiss, 1926, Brischkea); synonymy by Horstmann (2007b)

Distribution: England, Scotland, Wales

Phobetes liopleuris (Thomson, 1889)

Nomenclature:

Euryproctus liopleuris Thomson, 1889

Distribution: England

Notes: Added by Aubert (2000); treated as a separate species by Aubert (2000), as a synonym of leptocerus in Yu and Horstmann (1997). 
Phobetes nigriceps (Gravenhorst, 1829)

Nomenclature:

Tryphon nigriceps Gravenhorst, 1829

praetermissus (Woldstedt, 1874, Mesoleptus)

Distribution: England, Scotland

Notes: Eclytus transsylvanicus Kiss, 1924 removed from synonymy with nigriceps and placed in synonymy with Phobetes latipes (Thomson, 1895, Phobetus) by Horstmann (2007b); latipes and nigriceps were differentiated by Horstmann (2007b).

\section{Genus Syndipnus Förster, 1869}

Nomenclature:

POLYPYSTIS Förster, 1869

TLEMON Förster, 1869

DICKSONIA Holmgren, 1880

NEASTUS Holmgren, 1883

\section{Syndipnus alutaceus (Holmgren, 1857)}

Nomenclature:

Trematopygus alutaceus Holmgren, 1857

alutaceus (Woldstedt, 1874, Mesoleius)

Distribution: Scotland

Notes: added by Aubert (2000)

\section{Syndipnus decipiens (Woldstedt, 1877)}

Nomenclature:

Mesoleius decipiens Woldstedt, 1877

subscaber Thomson, 1893

Distribution: England

Notes: NMS, added here

Syndipnus lateralis (Gravenhorst, 1829)

Nomenclature:

Tryphon lateralis Gravenhorst, 1829 
punctiscuta Thomson, 1894

Distribution: England, Scotland

\section{Syndipnus macrocerus (Thomson, 1883)}

Nomenclature:

Euryproctus macrocerus Thomson, 1883

Distribution: England

Notes: NMS, added here

\section{Genus Synodites Förster, 1869}

\section{Nomenclature:}

CAMPONASTES Förster, 1869

LISTROTA Förster, 1869

POLYTERUS Förster, 1869

SARCORYCHUS Förster, 1869

SYCHNOPORTUS Förster, 1869

ZOOTREPHES Förster, 1869

ZOOTREPHUS Thomson, 1890

SYNODYTES Thomson, 1893

ANAGLYMMUS Roman, 1914

Notes: species of Synodites excluded from the British and Irish list:

[breviventris (Gravenhorst, 1829, Hemiteles)] Listed as a species of Dichrogaster (Cryptinae: Phygadeuontini) in Fitton (1978), there are no identified specimens in BMNH and Aubert (2000) does not list it as occurring in Britain or Ireland.

\section{Synodites breviusculus (Fonscolombe, 1849)}

\section{Nomenclature:}

Tryphon breviusculus Fonscolombe, 1849

delicatus (Fonscolombe, 1849, Tryphon)

buccatus (Holmgren, 1857, Mesoleius)

Distribution: England

Notes: Placed in Hypamblys in Yu and Horstmann (1997), in Synodites by Aubert (2000). 


\section{Synodites carinatus (Holmgren, 1857)}

Nomenclature:

Mesoleius carinatus Holmgren, 1857

Distribution: England

Notes: added by Aubert (2000)

\section{Synodites erosus (Holmgren, 1857)}

Nomenclature:

Trematopygus erosus Holmgren, 1857

Distribution: Scotland

Notes: Added by Aubert (2000); one specimen in BMNH from 'Wissant', which could not be located in a Gazetteer. 'England' is given in Aubert (2000) but all other BMNH specimens are from Scotland.

\section{Synodites facialis (Thomson, 1893)}

Nomenclature:

Spudaeus facialis Thomson, 1893

Distribution: England

Notes: In Yu and Horstmann (1997) as a synonym of discolor (Holmgren, 1857, Trematopygus) (placed in Syndipnus in Aubert 2000); the two species were differentiated by Horstmann (2011b).

\section{Synodites hilaris (Woldstedt, 1880)}

Nomenclature:

Bassus hilaris Woldstedt, 1880

Distribution: Scotland

Notes: NMS, added here

\section{Synodites lineiger (Thomson, 1893)}

Nomenclature:

Syndipnus lineiger Thomson, 1893

Distribution: England

Notes: added by Aubert (2000) 
Synodites notatus (Gravenhorst, 1829)

Nomenclature:

Tryphon notatus Gravenhorst, 1829

bimaculatus (Desvignes, 1856, Tryphon)

assimilis (Holmgren, 1858, Tryphon)

aberrans (Brischke, 1871, Polyblastus)

hungaricus (Kiss, 1924, Ipoctonus); synonymy by Horstmann (2007b)

Distribution: England, Scotland, Wales

Synodites parviceps (Thomson, 1894)

Nomenclature:

Syndipnus parviceps Thomson, 1894

Distribution: Scotland

Notes: NMS, det. Kasparyan, added here

\section{Genus Synomelix Förster, 1869}

Notes: Distribution data from Idar (1983).

Synomelix albipes (Gravenhorst, 1829)

Nomenclature:

Tryphon albipes Gravenhorst, 1829

sieboldii Kriechbaumer, 1897

kriechbaumeri Schmiedeknecht, 1913

Distribution: England, Scotland

Synomelix faciator Idar, 1983

Distribution: England, Scotland

Notes: added by Idar (1983)

\section{Synomelix perfida (Woldstedt, 1874)}

Nomenclature:

Tryphon perfidus Woldstedt, 1874

curvula (Thomson, 1895, Syndipnus) 
Distribution: Scotland

Genus Zemiophora Förster, 1869

Nomenclature:

ZEMIOPHORUS Thomson, 1893

\section{Zemiophora scutulata (Hartig, 1838)}

Nomenclature:

Tryphon scutulatus Hartig, 1838

brischkei (Holmgren, 1871, Mesoleius)

nobilis (Habermehl, 1909, Otlophorus)

Distribution: England

Tribe MESOLEIINI Thomson, 1883

Genus Alexeter Förster, 1869

Nomenclature:

ADRANES Förster, 1869

ZEMIOPHRON Förster, 1869

Notes: Distribution data from Gauld and Mitchell (1977a), Aubert (2000) and BMNH.

Species of Alexeter excluded from the British and Irish list:

[attenuatus (Bridgman, 1887, Mesoleius)] will be synonymised by Broad \& Rose (in prep.)

\section{Alexeter clavator (Müller, 1776)}

Nomenclature:

Ichneumon clavator Müller, 1776

testaceator misident.

testaceus misident.

venosus (Gmelin, 1790, Ichneumon)

Distribution: England 
Alexeter coxalis (Brischke, 1871)

Nomenclature:

Mesoleptus coxalis Brischke, 1871

inconspicuus Schiedeknecht, 1914

rufus Kiss, 1926

Distribution: Scotland, Ireland

Notes: BMNH, det. Aubert and Broad, added here

Alexeter fallax (Holmgren, 1857)

Nomenclature:

Mesoleius fallax Holmgren, 1857

Distribution: England, Scotland

Alexeter multicolor (Gravenhorst, 1829)

Nomenclature:

Tryphon multicolor Gravenhorst, 1829

dives (Holmgren, 1857, Mesoleius)

napaeus (Holmgren, 1857, Mesoleius)

Distribution: England, Scotland, Wales, Isle of Man

Alexeter nebulator (Thunberg, 1824)

Nomenclature:

Ichneumon nebulator Thunberg, 1824

melanocephalus (Gravenhorst, 1829, Mesoleptus)

gracilipes (Curtis, 1837, Mesoleptus)

paludicola Habermehl, 1922

Distribution: England, Scotland

Alexeter niger (Gravenhorst, 1829)

Nomenclature:

Tryphon niger Gravenhorst, 1829

Distribution: England, Ireland 
Alexeter rapinator (Gravenhorst, 1829)

Nomenclature:

Tryphon rapinator Gravenhorst, 1829

laevissimus (Strobl, 1903, Mesoleius)

Distribution: England

\section{Alexeter segmentarius (Fabricius, 1787)}

Nomenclature:

Ichneumon segmentarius Fabricius, 1787

sectator (Thunberg, 1824, Ichneumon); synonymy by Horstmann (2001c)

fraternarius (Thunberg, 1824, Ichneumon)

maxillarius (Thunberg, 1824, Ichneumon)

ruficornis (Gravenhorst, 1829, Mesoleptus)

lugubris (Woldstedt, 1874, Mesoleptus)

sibiricus Kiss, 1926

rufopetiolaris Kiss, 1933

Distribution: England, Scotland

\section{Genus Anoncus Townes, 1970}

Notes: Distribution data from Shaw and Kasparyan (2003).

Species of Anoncus excluded from the British and Irish list:

[linitus (Holmgren, 1857, Mesoleius)] Apparently only recorded as British by Carr (1924) and thus should have been deleted from the British list (see note under Lissonota funebris)

\section{Anoncus femorator (Thomson, 1893)}

Nomenclature:

Mesoleius femorator Thomson, 1893

Distribution: Scotland

Notes: added by Shaw and Kasparyan (2003) 
Anoncus gracilicornis (Holmgren, 1857)

Nomenclature:

Mesoleius gracilicornis Holmgren, 1857

Distribution: England

Genus Arbelus Townes, 1970

Arbelus athaliaeperda (Curtis, 1860)

Nomenclature:

Bassus athaliaeperda Curtis, 1860

athaliiperdus (Marshall, 1872, Bassus)

Distribution: England, Ireland

\section{Genus Azelus Förster, 1869}

Notes: Distribution data from Shaw and Kasparyan (2003).

Azelus erythropalpus (Gmelin, 1790)

Nomenclature:

Ichneumon erythropalpus Gmelin, 1790

laterator (Thunberg, 1824, Ichneumon)

triangulatus (Bridgman, 1886, Perilissus)

bipunctatus (Szépligeti, 1901, Mesoleius)

csikii (Kiss, 1926, Barytarbes); synonymy by Horstmann (2007b)

Distribution: England, Wales, Scotland

\section{Genus Barytarbes Förster, 1869}

Nomenclature:

HYBRISTES Förster, 1869

ISODIAETA Förster, 1869

POLYTRERA Förster, 1869

BARYTARBUS Thomson, 1883

POLYTRERES Thomson, 1892

APHOLIUM Townes, 1970; synonymy by Aubert (2000) 
Barytarbes colon (Gravenhorst, 1829)

Nomenclature:

Tryphon colon Gravenhorst, 1829

ventosus (Holmgren, 1876, Mesoleius)

Distribution: England

Barytarbes flavicornis (Thomson, 1892)

Nomenclature:

Mesoleius flavicornis Thomson, 1892

segmentarius (Perkins, 1962, Isodiaeta); unavailable (Horstmann 2004b, Horstmann 2005a)

Distribution: England

Notes: This species has often been referred to as Barytarbes segmentarius (Fabricius, 1787) sensu Gravenhorst, but that taxon is actually a species of Alexeter (Horstmann 2001c).

Barytarbes flavoscutellatus (Thomson, 1892)

Nomenclature:

Mesoleius flavoscutellatus Thomson, 1892

Distribution: England

Barytarbes laeviusculus (Thomson, 1883)

Nomenclature:

Mesoleius laeviusculus Thomson, 1883

Distribution: England

Genus Campodorus Förster, 1869

Nomenclature:

PHAGESORUS Förster, 1869

CUBOSCOPESIS Heinrich, 1952

Notes: Distribution data for species of Mesoleius and Campodorus, unless stated otherwise, are taken from Shaw and Kasparyan (2003). 
Campodorus alticola (Holmgren, 1857)

Nomenclature:

Mesoleius alticola Holmgren, 1857

Distribution: Scotland

Notes: added by Shaw and Kasparyan (2003)

Campodorus amictus (Holmgren, 1857)

Nomenclature:

Mesoleius amictus Holmgren, 1857

Distribution: England, Scotland

Campodorus astutus (Holmgren, 1876)

Nomenclature:

Mesoleius astutus Holmgren, 1876

Distribution: England, Scotland

Campodorus caligatus (Gravenhorst, 1829)

Nomenclature:

Tryphon caligatus Gravenhorst, 1829

nemati (Ratzeburg, 1852, Tryphon)

Distribution: England, Scotland

Campodorus ciliatus (Holmgren, 1857)

Nomenclature:

Mesoleius ciliatus Holmgren, 1857

Distribution: Scotland

Notes: added by Shaw and Kasparyan (2003)

Campodorus commotus (Holmgren, 1876)

Nomenclature:

Mesoleius commotus Holmgren, 1876

perturbatus (Holmgren, 1876, Mesoleius) 
Distribution: Scotland

Notes: added by Shaw and Kasparyan (2003)

Campodorus corrugatus (Holmgren, 1876)

Nomenclature:

Mesoleius corrugatus Holmgren, 1876

Campodorus difformis (Holmgren, 1876)

Nomenclature:

Mesoleius difformis Holmgren, 1876

Distribution: England, Scotland, Wales

Notes: added by Shaw and Kasparyan (2003)

Campodorus dorsalis (Gravenhorst, 1829)

Nomenclature:

Tryphon dorsalis Gravenhorst, 1829

Distribution: England, Scotland, Wales, Isle of Man

Campodorus efferus (Holmgren, 1876)

Nomenclature:

Mesoleius efferus Holmgren, 1876

Distribution: England, Scotland

Notes: added by Shaw and Kasparyan (2003)

Campodorus elegans (Parfitt, 1882)

Nomenclature:

Mesoleius elegans Parfitt, 1882

Distribution: England

Notes: Transferred from Mesoleius by Shaw and Kasparyan (2003).

Campodorus flavescens Kasparyan, 2003

Distribution: Scotland 
Notes: NMS, det. Kasparyan, added here

Campodorus gallicus (Thomson, 1893)

Nomenclature:

Mesoleius gallicus Thomson, 1893

Distribution: Scotland

Notes: added by Shaw and Kasparyan (2003)

Campodorus haematodes (Gravenhorst, 1829)

Nomenclature:

Tryphon haematodes Gravenhorst, 1829

alni (Woldstedt, 1874, Mesoleius)

Distribution: England, Ireland

\section{Campodorus hamulus (Gravenhorst, 1829)}

Nomenclature:

Tryphon hamulus Gravenhorst, 1829

nobilis (Holmgren, 1857, Mesoleius)

Distribution: England

Campodorus ignavus (Holmgren, 1857)

Nomenclature:

Mesoleius ignavus Holmgren, 1857

Campodorus immarginatus (Thomson, 1893)

Nomenclature:

Mesoleius immarginatus Thomson, 1893

Distribution: Scotland

Notes: Transferred from Mesoleius by Shaw and Kasparyan (2003).

Campodorus incidens (Thomson, 1893)

Nomenclature:

Mesoleius incidens Thomson, 1893 
Distribution: England, Scotland

Campodorus liosternus (Thomson, 1893)

Nomenclature:

Mesoleius liosternus Thomson, 1893

Campodorus luctuosus (Holmgren, 1857)

Nomenclature:

Mesoleius luctuosus Holmgren, 1857

Campodorus maculicollis (Stephens, 1835)

Nomenclature:

Tryphon maculicollis Stephens, 1835

vigens (Holmgren, 1857, Mesoleius)

Distribution: England, Scotland

Campodorus marginalis (Geoffroy, 1785)

Nomenclature:

Ichneumon marginalis Geoffroy, 1785

limbarius (Olivier, 1792, Ichneumon)

histrio (Fabricius, 1793, Ichneumon)

formosus (Gravenhorst, 1829, Tryphon)

limbarius (Fonscolombe, 1854, Lissonota)

lepidus (Giraud, 1872, Mesoleius); synonymy by Horstmann (2008c)

ornatus (Habermehl, 1925, Mesoleius); synonymy by Horstmann (2008c)

Distribution: England

\section{Campodorus mediosanguineus (Heinrich, 1950)}

Nomenclature:

Mesoleius mediosanguineus Heinrich, 1950

Distribution: Ireland

Notes: BMNH, det. Broad, added here 
Campodorus melanogaster (Holmgren, 1857)

Nomenclature:

Mesoleius melanogaster Holmgren, 1857

Distribution: England, Scotland

Notes: added by Shaw and Kasparyan (2003)

Campodorus mixtus (Holmgren, 1857)

Nomenclature:

Mesoleius mixtus Holmgren, 1857

Campodorus molestus (Holmgren, 1857)

Nomenclature:

Mesoleius molestus Holmgren, 1857

Campodorus nigridens (Thomson, 1893)

Nomenclature:

Spudaeus nigridens Thomson, 1893

Distribution: Scotland

Campodorus patagiatus (Holmgren, 1876)

Nomenclature:

Mesoleius patagiatus Holmgren, 1876

modestus (Holmgren, 1876, Mesoleius)

Campodorus pectinator Kasparyan, 2003

Distribution: England

Notes: added by Kasparyan (2003)

Campodorus ? pictipes (Habermehl, 1923)

Nomenclature:

Mesoleius pictipes Habermehl, 1923 
Notes: Described from a Carr specimen, this species should be deleted from the list if there are no other British or Irish specimens (see note under Lissonota funebris). Transferred from Mesoleius by Horstmann (2000e).

\section{Campodorus scapularis (Stephens, 1835)}

Nomenclature:

Tryphon scapularis Stephens, 1835

humerellus (Thomson, 1893, Mesoleius); synonymy by Kasparyan (2003)

Distribution: England, Scotland

\section{Campodorus tristis (Holmgren, 1857)}

Nomenclature:

Mesoleius tristis Holmgren, 1857

Campodorus variegatus (Jurine, 1807)

Nomenclature:

Anomalon variegatum Jurine, 1807

sanguinicollis (Gravenhorst, 1829, Tryphon)

Distribution: England, Scotland

Campodorus viduus (Holmgren, 1857)

Nomenclature:

Mesoleius viduus Holmgren, 1857

annulatus (Brischke, 1878, Trematopygus)

Distribution: England

Notes: distribution data from UM

\section{Campodorus vitosaensis (Gregor, 1933)}

Nomenclature:

Mesoleius vitosaensis Gregor, 1933

trochanteratus (Kriechbaumer, 1896, Mesoleius) preocc.

Notes: Mesoleius trochanteratus Kriechbaumer is listed as a subspecies of vitosaensis in Yu and Horstmann (1997), but is preoccupied by Mesoleius trochanteratus Brischke, 1871. The value of subspecific names in such little-known species is doubtful anyway. 


\section{Genus Himerta Förster, 1869}

Nomenclature:

CLEPSIPORTHUS Förster, 1869

DOLIOCTONUS Förster, 1869

ENOECETIS Förster, 1869

ITHAGENES Förster, 1869

HIMERTUS Thomson, 1883

Notes: Distribution data from Horstmann (2002a) who also provides synonymy, and $\mathrm{BMNH}$.

Himerta bisannulata (Thomson, 1883)

Nomenclature:

Euryproctus bisannulatus Thomson, 1883

pfeifferi (Bauer, 1939, Himertus)

Notes: Added by Horstmann (2002a)and taken out of synonymy with defectiva; one specimen in BMNH, 'British Isles, Desvignes coll.'

\section{Himerta defectiva (Gravenhorst, 1820)}

Nomenclature:

Ichneumon defectivus Gravenhorst, 1820

varicornis (Gravenhorst, 1829, Tryphon)

biannulata (Ulbricht, 1922, Barytarbes)

ihsseni (Bauer, 1939, Himertus)

Distribution: England, Scotland

\section{Himerta scutellaris (Kriechbaumer, 1897)}

Nomenclature:

Enoecetis scutellaris Kriechbaumer, 1897

NHM, det. Broad, added

Distribution: England

Notes: BMNH, det. Broad, added here 
Himerta sepulchralis (Holmgren, 1876)

Nomenclature:

Mesoleius sepulchralis Holmgren, 1876

sexannulatus Kriechbaumer, 1891

alboannulata (Strobl, 1903, Euryproctus)

Distribution: England, Scotland

\section{Genus Hyperbatus Förster, 1869}

Notes: Distribution data from Shaw and Kasparyan (2003).

Hyperbatus orbitalis (Thomson, 1893)

Nomenclature:

Mesoleius orbitalis Thomson, 1893

Distribution: England, Scotland

Notes: Added by Shaw and Kasparyan (2003); removed from synonymy with segmentator by Kasparyan (1998).

Hyperbatus segmentator (Holmgren, 1857)

Nomenclature:

Mesoleius segmentator Holmgren, 1857

solitarius (Holmgren, 1876, Mesoleius)

Distribution: Scotland

Hyperbatus sternoxanthus (Gravenhorst, 1829)

Nomenclature:

Tryphon sternoxanthus Gravenhorst, 1829

pulchellus (Holmgren, 1857, Mesoleius)

Distribution: England

\section{Genus Lagarotis Förster, 1869}

Nomenclature:

DASPLETIS Förster, 1869

DYSANTES Förster, 1869 
NYTHOPHONA Förster, 1869

ONEISTA Förster, 1869

LAGAROTUS Thomson, 1892

\section{Lagarotis debitor (Thunberg, 1824)}

Nomenclature:

Ichneumon debitor Thunberg, 1824

insolens (Gravenhorst, 1829, Tryphon)

Distribution: England

\section{Lagarotis erythrocera (Gravenhorst, 1829)}

Nomenclature:

Tryphon erythrocerus Gravenhorst, 1829

Distribution: England

Notes: Placed in Lagarotis in Yu and Horstmann (1997) and by Horstmann (2011b), although Aubert (2000) treated this as a species of Alexeter.

\section{Lagarotis semicaligata (Gravenhorst, 1820)}

Nomenclature:

Ichneumon semicaligatus Gravenhorst, 1820

longicornis (Woldstedt, 1874, Mesoleius)

Distribution: England, Scotland

\section{Genus Lamachus Förster, 1869}

Nomenclature:

ADEXIOMA Förster, 1869

ZAPHTHORA Förster, 1869

BATHYGLYPTUS Schmiedeknecht, 1913

TOROCAMPUS Schmiedeknecht, 1913

\section{Lamachus coalitorius (Thunberg, 1824)}

Nomenclature:

Ichneumon coalitorius Thunberg, 1824

variabilis (Ratzeburg, 1844, Tryphon); synonymy by Horstmann (2004b) 
ophthalmicus (Holmgren, 1857, Mesoleius)

marginatus (Brischke, 1871, Mesoleius)

spectabilis (Holmgren, 1876, Mesoleius)

Distribution: England, Wales

Notes: Also released, for biocontrol (Billany et al. 1983).

\section{Lamachus eques (Hartig, 1838)}

Nomenclature:

Tryphon eques Hartig, 1838

silvarum (Holmgren, 1876, Mesoleius)

aterrimus (Scönwiese, 1934, Torocampus)

Distribution: England, Scotland

Notes: Distribution data from Shaw and Kasparyan (2003)

Lamachus frutetorum (Hartig, 1838)

Nomenclature:

Tryphon frutetorum Hartig, 1838

lophyrum (Hartig, 1838, Tryphon)

nigrescens Kiss, 1926

altipeta Heinrich, 1953

Distribution: Scotland

Notes: added by Aubert (2000)

Lamachus pini (Bridgman, 1882)

Nomenclature:

Mesoleius pini Bridgman, 1882

caledonicus Laidlaw, 1933

Distribution: Scotland

Lamachus virgultorum (Gravenhorst, 1829)

Nomenclature:

Tryphon virgultorum Gravenhorst, 1829 
flavoscutellatus (Strobl, 1903, Notopygus)

Distribution: England

\section{Genus Mesoleius Holmgren, 1856}

Nomenclature:

ALLOCRITUS Förster, 1869

ALFKENIA Pfankuch, 1906

MESOLIUS Pfankuch, 1906

HABRODEMUS Schmiedeknecht, 1913

Notes: Distribution data principally from Shaw and Kasparyan (2003), plus Fitton (1976).

doubtfully placed species of Mesoleius:

[brachyacanthus Parfitt, 1881 nom. dub., from England]

\section{Mesoleius aceris Kasparyan \& Shaw, 2003}

Distribution: England, Scotland, Isle of Man

Notes: added by Shaw and Kasparyan (2003)

\section{Mesoleius armillatorius (Gravenhorst, 1807)}

Nomenclature:

Ichneumon armillatorius Gravenhorst, 1807

luteifrons (Gravenhorst, 1829, Tryphon)

flavipes Brischke, 1871; synonymy by Horstmann (2012b)

similis Brischke, 1892

bilineolatus Strobl, 1903; synonymy by Horstmann (2012b)

chyzeri (Kiss, 1926, Perilissus); synonymy by Horstmann (2007b)

Distribution: England, Scotland, Wales, Isle of Man

\section{Mesoleius aulicus (Gravenhorst, 1829)}

Nomenclature:

Tryphon aulicus Gravenhorst, 1829

Distribution: England, Scotland 
Mesoleius axillaris (Stephens, 1835)

Nomenclature:

Tryphon axillaris Stephens, 1835

amabilis Holmgren, 1857

leptogaster Holmgren, 1857

tenuiventris Holmgren, 1858

erythrogaster Holmgren, 1876

Distribution: England, Scotland, Wales Isle of Man

Notes: Transferred from Campodorus by Kasparyan (2000), who provides synonymy.

Mesoleius brevipalpis Thomson, 1893

Distribution: England

Notes: added by Shaw and Kasparyan (2003)

Mesoleius caninae Bridgman, 1886

Distribution: England

Notes: Transferred from Otlophorus (where it is listed as a synonym of $O$. congruens (Holmgren) by Yu and Horstmann 1997) by Kasparyan (2000).

Mesoleius dubius Holmgren, 1857

Distribution: England

Mesoleius filicornis Holmgren, 1876

Distribution: England, Scotland, Wales, Isle of Man

Mesoleius flavopictus (Gravenhorst, 1829)

Nomenclature:

Mesoleptus flavopictus Gravenhorst, 1829

trimaculatus (Stephens, 1835, Mesoleptus)

Distribution: England, Scotland

Mesoleius frenalis Thomson, 1893 
Mesoleius furax Holmgren, 1857

Mesoleius fuscipes Holmgren, 1857

Distribution: Scotland, Isle of Man

Notes: Transferred from Campodorus by Kasparyan (2000).

Mesoleius geniculatus Holmgren, 1857

Distribution: Wales, Scotland

Notes: added by Shaw and Kasparyan (2003)

Mesoleius intermedius (Gravenhorst, 1829)

Nomenclature:

Tryphon intermedius Gravenhorst, 1829

sinuatus Thomson, 1893; synonymy by Kasparyan (2000)

Distribution: England, Wales

Notes: Added by Shaw and Kasparyan (2003); transferred from Campodorus by Kasparyan (2000).

Mesoleius laricis Teunissen, 1953

Distribution: Scotland

Notes: added by Shaw and Kasparyan (2003)

Mesoleius lindemansi Teunissen, 1953

Nomenclature:

antennator Kasparyan, 2000; synonymy by Kasparyan (2004b)

Distribution: England, Scotland

Notes: added by Shaw and Kasparyan (2003)

Mesoleius melanoleucus (Gravenhorst, 1829)

Nomenclature:

Tryphon melanoleucus Gravenhorst, 1829

Distribution: England, Scotland 
Mesoleius nivalis Holmgren, 1857

Nomenclature:

aemulus (Ruthe, 1859, Tryphon)

Distribution: England, Scotland, Wales, Isle of Man

Mesoleius opticus (Gravenhorst, 1829)

Nomenclature:

Tryphon opticus Gravenhorst, 1829

Distribution: England, Scotland, Ireland

Mesoleius peronatus (Marshall, 1876)

Nomenclature:

Bassus peronatus Marshall, 1876

Distribution: Ireland

Notes: Transferred from Campodorus by Kasparyan (2000).

Mesoleius phyllotomae Cushman, 1933

Distribution: Scotland

Notes: added by Shaw and Kasparyan (2003)

Mesoleius placidus Holmgren, 1857

Mesoleius pyriformis (Ratzeburg, 1852)

Nomenclature:

Tryphon pyriformis Ratzeburg, 1852

unifasciatus Holmgren, 1857

Distribution: England, Scotland

Mesoleius ribesii Bauer, 1961

Distribution: England, Scotland

Notes: added by Shaw \& Kasparyan (2003) 
Mesoleius roepkii Teunissen, 1945

Distribution: Wales, Scotland

Notes: added by Shaw and Kasparyan (2003)

Mesoleius tenthredinis Morley, 1912

Nomenclature:

romani Teunissen, 1945

Distribution: England

Notes: distribution data from UM

Mesoleius varicoxa Thomson, 1893

Genus Otlophorus Förster, 1869

Nomenclature:

AEOLOMETIS Förster, 1869

DIALGES Förster, 1869

HOLMGRENIA Förster, 1869

NEALES Förster, 1869

TACHYPORTHUS Förster, 1869

AELOMETIS Thomson, 1893

OTLOPHORINUS Hincks, 1944

Otlophorus anceps (Holmgren, 1857)

Nomenclature:

Mesoleius anceps Holmgren, 1857

Distribution: England

Notes: added by Aubert (2000)

Otlophorus congruens (Holmgren, 1858)

Nomenclature:

Mesoleius congruens Holmgren, 1858

Distribution: England 
Notes: added by Aubert (2000)

Otlophorus italicus (Gravenhorst, 1829)

Nomenclature:

Scolobates italicus Gravenhorst, 1829

corallinus (Vollenhoven, 1873, Scolobates)

Distribution: England

Otlophorus pulverulentus (Holmgren, 1857)

Nomenclature:

Mesoleius pulverulentus Holmgren, 1857

minutus (Rudow, 1881, Meniscus)

Distribution: England

Otlophorus senilis (Holmgren, 1876)

Nomenclature:

Mesoleius senilis Holmgren, 1876

Distribution: England

Notes: BMNH, det. Aubert, added here

Otlophorus vepretorum (Gravenhorst, 1829)

Nomenclature:

Tryphon vepretorum Gravenhorst, 1829

verpetorum misspelling

Distribution: England

Genus Perispuda Förster, 1869

Nomenclature:

GENARCHES Förster, 1869

ZAPLETHIS Förster, 1869

PERISPUDUS Thomson, 1888 
Perispuda bignellii (Bridgman, 1881)

Nomenclature:

Mesoleius bignellii Bridgman, 1881

flavitarsis (Thomson, 1893, Mesoleius)

sulphuripes (Strobl, 1902, Procinetus)

Distribution: England, Scotland, Ireland

Notes: As a synonym of sulphurata in Yu and Horstmann (1997) but treated as a separate species by Aubert (2000).

Perispuda facialis (Gravenhorst, 1829)

Nomenclature:

Mesoleptus facialis Gravenhorst, 1829

Distribution: England

Perispuda sulphurata (Gravenhorst, 1807)

Nomenclature:

Ichneumon sulphuratus Gravenhorst, 1807

Distribution: England, Ireland

Genus Protarchus Förster, 1869

Nomenclature:

ZACALLES Förster, 1869

Protarchus melanurus (Thomson, 1893)

Nomenclature:

Mesoleius melanurus Thomson, 1893

rufus misident.

Distribution: England, Scotland

Notes: Added by Aubert (2000); also specimens in UM

Protarchus testatorius (Thunberg, 1824)

Nomenclature:

Ichneumon testatorius Thunberg, 1824 
binarius (Thunberg, 1824, Ichneumon) preocc.

decorius (Thunberg, 1824, Ichneumon)

rufus (Gravenhorst, 1829, Tryphon)

conspicuus (Stephens, 1835, Tryphon)

rufulus (Stephens, 1835, Tryphon)

Distribution: England, Scotland

\section{Genus Rhinotorus Förster, 1869}

Nomenclature:

SPUDAEA Förster, 1869

SPUDAEUS Thomson, 1883

PROSPUDAEA Hincks, 1944

\section{Rhinotorus compactor (Thunberg, 1824)}

Nomenclature:

Ichneumon compactor Thunberg, 1824

atratus (Holmgren, 1857, Trematopygus)

quadriguttatus (Vollenhoven, 1873, Bassus)

albotrochanteratus (Strobl, 1913, Polyblastus)

Distribution: England

Rhinotorus leucostomus (Gravenhorst, 1829)

Nomenclature:

Tryphon leucostomus Gravenhorst, 1829

impressus (Brischke, 1871, Mesoleius)

subimpressus (Thomson, 1873, Spudaeus)

Distribution: England

Rhinotorus longicornis (Schmiedeknecht, 1914)

Nomenclature:

Spudaea longicornis Schmiedeknecht, 1914

Distribution: England 
Rhinotorus mesocastanus (Thomson, 1892)

Nomenclature:

Spudaeus mesocastanus Thomson, 1892

Distribution: England

Notes: BMNH, det. Reshchikov, added here

Rhinotorus nasutus (Gravenhorst, 1829)

Nomenclature:

Tryphon nasutus Gravenhorst, 1829

confusus (Thomson, 1883, Mesoleius)

Distribution: England

Notes: BMNH, det. Perkins, added here

Rhinotorus similis (Brischke, 1892)

Nomenclature:

Mesoleius similis Brischke, 1892

Genus Saotis Förster, 1869

Nomenclature:

SAOTUS Thomson, 1883

ISKARUS Kolarov, 1987; synonymy by Kasparyan and Shaw (2003)

Notes: Distribution data taken from Kasparyan and Shaw (2003), Shaw and Kasparyan (2003), Kasparyan and Khalaim (2007a) and Fitton (1976).

Saotis albionis Kasparyan, 2007

Distribution: England

Notes: added by Kasparyan and Khalaim (2007a)

Saotis albiventris Kasparyan, 2007

Distribution: England, Scotland

Notes: added by Kasparyan and Khalaim (2007a) 
Saotis compressiuscula (Thomson, 1883)

Nomenclature:

Mesoleius compressiusculus Thomson, 1883

Distribution: England

Saotis morleyi Fitton, 1976

Nomenclature:

emarginata (Morley, 1911, Homocidus)

Distribution: England, Scotland

Notes: Listed as a synonym of $S$. liopleuris (Thomson, 1888, Mesoleius) in $Y u$ and Horstmann (1997) but Kasparyan and Shaw (2003) treat the two as separate species.

Saotis nigriscuta (Thomson, 1888)

Nomenclature:

Mesoleius nigriscuta Thomson, 1888

Distribution: England

Notes: added by Kasparyan and Shaw (2003)

Saotis renovata (Morley, 1911)

Nomenclature:

Mesoleius renovatus Morley, 1911

Distribution: England

Notes: This species was not included in the key by Kasparyan and Shaw (2003) but they did include notes on its British occurrence.

Saotis varicoxa (Thomson, 1893)

Nomenclature:

Saotus varicoxa Thomson, 1893

Distribution: England, Scotland

Genus Scopesis Förster, 1869

Nomenclature:

SCOPARCHES Förster, 1869 
SCOPESUS Thomson, 1893

Scopesis bicolor (Gravenhorst, 1829)

Nomenclature:

Tryphon bicolor Gravenhorst, 1829

praecatoria (Holmgren, 1876, Mesoleius)

longigena (Thomson, 1893, Mesoleius); synonymy by Horstmann (2006a)

Distribution: England

Scopesis depressa (Thomson, 1893)

Nomenclature:

Mesoleius depressus Thomson, 1893

Distribution: England

Scopesis fraterna (Holmgren, 1857)

Nomenclature:

Mesoleius fraternus Holmgren, 1857

Distribution: England

Scopesis frontator (Thunberg, 1824)

Nomenclature:

Ichneumon frontator Thunberg, 1824

rufolabris (Zetterstedt, 1838, Bassus)

Distribution: England, Scotland

Notes: Aubert (2000) gives guttiger (Holmgren, 1857, Mesoleius) as a junior synonym but Horstmann (2006d) differentiates the taxa.

\section{Scopesis gesticulator (Thunberg, 1824)}

Nomenclature:

Ichneumon gesticulator Thunberg, 1824

longipes (Gravenhorst, 1829, Tryphon)

nigricollis (Gravenhorst, 1829, Tryphon)

Distribution: England 
Scopesis macropus (Thomson, 1893)

Nomenclature:

Mesoleius macropus Thomson, 1893

Distribution: England

Scopesis obscura (Holmgren, 1857)

Nomenclature:

Mesoleius obscurus Holmgren, 1857

Scopesis rufonotata (Holmgren, 1876)

Nomenclature:

Mesoleius rufonotatus Holmgren, 1876

thomsoni (Habermehl, 1925, Scopesus)

Distribution: England

Scopesis tegularis (Thomson, 1893)

Nomenclature:

Mesoleius tegularis Thomson, 1893

Distribution: England

Notes: distribution data from UM

Genus Semimesoleius Ozols, 1963

Semimesoleius exophthalmicus Ozols, 1963

Distribution: Scotland

Notes: added by Shaw and Kasparyan (2003)

Genus Smicrolius Thomson, 1893

Smicrolius parvicalcar (Thomson, 1895)

Nomenclature:

Syndipnus parvicalcar Thomson, 1895 parumpictus (Roman, 1909, Mesoleius); synonymy by Kasparyan (1998) 
Distribution: England, Scotland

Notes: added by Shaw and Kasparyan (2003)

Tribe PERILISSINI Thomson, 1883

Genus Absyrtus Holmgren, 1859

Nomenclature:

ECZETESIS Förster, 1869

\section{Absyrtus vernalis Bauer, 1961}

Distribution: England

Notes: added by Aubert (2000)

Absyrtus vicinator (Thunberg, 1824)

Nomenclature:

Ichneumon vicinator Thunberg, 1824

luteus Holmgren, 1859

exareolatus Ulbricht, 1926 unavailable

Distribution: England, Scotland, Wales, Ireland

\section{Genus Lathiponus Förster, 1869}

Nomenclature:

POLYSELASMUS Schmiedeknecht, 1912

CERATOSAOTIS Gregor, 1939

\section{Lathiponus semiluctuosus (Vollenhoven, 1878)}

Nomenclature:

Eclytus semiluctuosus Vollenhoven, 1878

frigidus (Woldstedt, 1874, Perilissus) preocc.

bicolor (Brischke, 1878, Perilissus)

pulcherrimus (Thomson, 1888, Mesoleius)

ornatus (Gregor, 1939, Ceratosaotis)

Distribution: England 
Notes: BMNH, NMS, det. Perkins, Kasparyan, Broad, added here

\section{Genus Lathrolestes Förster, 1869}

Nomenclature:

CAMPORYCHUS Förster, 1869

ECCLINOPS Förster, 1869

HOMALOMMA Förster, 1869

LAPHYROSCOPUS Förster, 1869

POLYONCUS Förster, 1869

LATHROLESTUS Thomson, 1883

LUPHYROSCOPUS Thomson, 1883

TRYPHONOPSIS Brauns, 1898

RITZEMABOSIA Smits

CULMINA Benoit, 1955

Lathrolestes bipunctatus (Bridgman, 1886)

Nomenclature:

Grypocentrus bipunctatus Bridgman, 1886

Distribution: England, Scotland

Lathrolestes buccinator (Holmgren, 1857)

Nomenclature:

Perilissus buccinator Holmgren, 1857

vollenhoveni (Gribodo, 1880, Perilissus); synonymy by Horstmann (2003a)

Distribution: England

Notes: Treated as a species of Perilissus by Horstmann (2003a) but as a species of

Lathrolestes by Aubert (2000) and then by Horstmann (2004a).

Lathrolestes caudatus (Thomson, 1883)

Nomenclature:

Lathrolestus caudatus Thomson, 1883

Distribution: England, Scotland 
Notes: BMNH, NMS, added here

\section{Lathrolestes citreus (Brischke, 1878)}

Nomenclature:

Perilissus citreus Brischke, 1878

Distribution: England

Notes: BMNH, added here

Lathrolestes clypeatus (Zetterstedt, 1838)

Nomenclature:

Tryphon clypeatus Zetterstedt, 1838

Distribution: England, Scotland

Notes: Added by Heath (1961); overlooked by Fitton (1978); distribution data from

Reshchikov (2015) and specimens in BMNH and UM.

\section{Lathrolestes ensator (Brauns, 1898)}

Nomenclature:

Tryphonopsis ensator Brauns, 1898

dilatatus (Nordenström, 1905, Lathrolestus)

ensatrix (Schulz, 1906, Tryphonopsis)

Distribution: England

Lathrolestes erythrocephalus (Gravenhorst, 1829)

Nomenclature:

Tryphon erythrocephalus Gravenhorst, 1829

Distribution: England

Notes: Transferred from Perilissus by Aubert (2000).

Lathrolestes lucidulus (Holmgren, 1857)

Nomenclature:

Perilissus lucidulus Holmgren, 1857

Distribution: England 
Notes: American Entomological Institute, det. Reshchikov, added here

Lathrolestes luteolator (Gravenhorst, 1829)

Nomenclature:

Mesoleptus luteolator Gravenhorst, 1829

gorskii (Ratzeburg, 1852, Tryphon)

Distribution: England

Lathrolestes macropygus (Holmgren, 1857)

Nomenclature:

Perilissus macropygus Holmgren, 1857

Distribution: England, Scotland

Lathrolestes moravicus (Habermehl, 1923)

Nomenclature:

Perilissus moravicus Habermehl, 1923

Distribution: England

Notes: BMNH, NMS, added here

Lathrolestes nigricollis (Thomson, 1883)

Nomenclature:

Perilissus nigricollis Thomson, 1883

minutus (Bridgman, 1887, Perilissus)

Distribution: England

Lathrolestes orbitalis (Gravenhorst, 1829)

Nomenclature:

Tryphon orbitalis Gravenhorst, 1829

bucculentus (Holmgren, 1857, Perilissus)

Distribution: England, Scotland, Wales 


\section{Lathrolestes pictilis (Holmgren, 1857)}

Nomenclature:

Perilissus pictilis Holmgren, 1857

Distribution: England, Scotland, Ireland

Notes: Irish record based on a specimen in American Entomological Institute, det. A. Reshchikov.

\section{Lathrolestes pleuralis (Thomson, 1883)}

Nomenclature:

Lathrolestus pleuralis Thomson, 1883

Distribution: England

\section{Lathrolestes soperi Reshchikov, 2010}

Distribution: England

Notes: added by Reshchikov (2015)

\section{Lathrolestes tripunctor (Thunberg, 1824)}

Nomenclature:

$$
\begin{aligned}
& \text { Ichneumon tripunctor Thunberg, } 1824 \\
& \text { distichor (Thunberg, 1824, Ichneumon) } \\
& \text { Iongicornis (Brischke, 1871, Perilissus) } \\
& \text { luteocephalus (Giraud, 1872, Perilissus) } \\
& \text { singularis (Vollenhoven, 1878, Perilissus) } \\
& \text { grandiceps (Thomson, 1883, Perilissus) }
\end{aligned}
$$

Distribution: England

Notes: BMNH, added here; transferred from Perilissus by Aubert (2000).

\section{Lathrolestes ungularis (Thomson, 1883)}

Nomenclature:

Lathrolestus ungularis Thomson, 1883

citrofrontalis Schmiedeknecht, 1912; synonymy by Reshchikov (2011)

Distribution: England 
Lathrolestes verticalis (Brischke, 1871)

Nomenclature:

Perilissus verticalis Brischke, 1871

abdominalis (Brischke, 1878, Perilissus); synonymy by Horstmann (2006a)

marginatus (Thomson, 1883, Lathrolestus)

Distribution: England, Scotland, Ireland

Genus Lophyroplectus Thomson, 1883

Notes: Some distribution data from Huddleston and Gauld (1988).

Lophyroplectus oblongopunctatus (Hartig, 1838)

Nomenclature:

Paniscus oblongopunctatus Hartig, 1838

luteator (Thunberg, 1824, Ichneumon)

Distribution: England

\section{Genus Oetophorus Förster, 1869}

Nomenclature:

SYMPHOBUS Förster, 1869

Oetophorus naevius (Gmelin, 1790)

Nomenclature:

Ichneumon naevius Gmelin, 1790

dilector (Thunberg, 1824, Ichneumon)

limitaris (Gravenhorst, 1829, Mesoleptus)

Distribution: England, Scotland, Ireland, Isle of Man

\section{Genus Opheltes Holmgren, 1859}

Notes: Some distribution data from Huddleston and Gauld (1988).

Opheltes glaucopterus (Linnaeus, 1758)

Nomenclature:

Ichneumon glaucopterus Linnaeus, 1758 
pteromelas (Villers, 1789, Ichneumon)

Distribution: England, Scotland, Ireland

Genus Perilissus Holmgren, 1857

Nomenclature:

EXACRODUS Förster, 1869

ICHNAEOPS Förster, 1869

SPANOTECNUS Förster, 1869

UDENIA Förster, 1869

DAUGNA Seyrig, 1935

PSEUDOCHORUS Rao, 1953

Perilissus albitarsis Thomson, 1883

Nomenclature:

emarginatus Thomson, 1883

Distribution: England, Scotland

Notes: BMNH, NMS, UM, added here

Perilissus compressus Thomson, 1883

Distribution: England

Notes: BMNH, added here

Perilissus coxalis Thomson, 1883

Distribution: England

Notes: BMNH, det. Broad, added here

Perilissus lutescens Holmgren, 1857

Distribution: England

Perilissus pallidus (Gravenhorst, 1829)

Nomenclature:

Mesoleptus pallidus Gravenhorst, 1829

holmgreni Habermehl, 1925 
Distribution: England

Perilissus rufoniger (Gravenhorst, 1820)

Nomenclature:

Ichneumon rufoniger Gravenhorst, 1820

vernalis (Gravenhorst, 1820, Ichneumon)

petulans (Gravenhorst, 1829, Tryphon)

herrichii Kriechbaumer, 1892

Distribution: England

Perilissus sericeus (Gravenhorst, 1829)

Nomenclature:

Mesoleptus sericeus Gravenhorst, 1829

spiniger Thomson, 1883

\section{Perilissus spilonotus (Stephens, 1835)}

Nomenclature:

Mesoleptus spilonotus Stephens, 1835

subcinctus Holmgren, 1857

stigmaticus Woldstedt, 1874

dissimilis Woldstedt, 1878

thuringiacus Schmiedeknecht, 1912

alpinus Habermehl, 1935

Distribution: England, Scotland, Wales

\section{Perilissus variator (Müller, 1776)}

Nomenclature:

Ichneumon variator Müller, 1776

filicornis (Gravenhorst, 1820, Ichneumon)

interruptor (Thunberg, 1824, Ichneumon)

seminiger (Gravenhorst, 1829, Mesoleptus)

Distribution: England, Scotland, Wales, Ireland, Isle of Man

Notes: As filicornis in Aubert (2000). 
Genus Priopoda Holmgren, 1856

Nomenclature:

PRIONOPODA misspelling

Priopoda apicaria (Geoffroy, 1785)

Nomenclature:

Ichneumon apicarius Geoffroy, 1785

stictica misident.

luteolus (Thunberg, 1789, Ichneumon); synonymy by Horstmann (1999b)

glabrator (Thunberg, 1824, Ichneumon)

Distribution: England, Scotland

Notes: Some distribution data from Gauld (1970).

Priopoda xanthopsana (Gravenhorst, 1829)

Nomenclature:

xanthospana misspelling

Distribution: England

\section{Genus Synoecetes Förster, 1869}

Nomenclature:

POLYRHYSIA Förster, 1869

SYNAGRYPNUS Förster, 1869

POLYRHYSIUS Thomson, 1893

Synoecetes anterior (Thomson, 1893)

Nomenclature:

Syndipnus anterior Thomson, 1893

Distribution: England, Scotland, Ireland

Notes: BMNH, NMS, UM, added here 
Genus Trematopygodes Aubert, 1968

Trematopygodes aprilinus (Giraud, 1872)

Nomenclature:

Trematopygus aprilinus Giraud, 1872

blancoburgensis (Schmiedeknecht, 1912, Lathrolestes)

Distribution: England

Notes: added by Hinz and Horstmann (1998)

Trematopygodes rarus Horstmann, 1990

Distribution: England

Notes: NMS, det. Kasparyan, added here

Genus Zaplethocornia Schmiedeknecht, 1912

Zaplethocornia exstinctor Aubert, 1985

Distribution: England

Notes: added by Aubert (1985)

Tribe PIONINI Smith \& Shenefelt, 1955

Genus Asthenara Förster, 1869

Nomenclature:

ASTHENARUS Thomson, 1889

Asthenara scabricula (Thomson, 1893)

Nomenclature:

Catoglyptus scabriculus Thomson, 1893

Distribution: England, Scotland, Wales, Ireland

Notes: BMNH, NMS, UM, added here 
Asthenara socia (Holmgren, 1857)

Nomenclature:

Euryproctus socius Holmgren, 1857

crassifemur (Thomson, 1889, Asthenarus)

Distribution: England, Scotland, Ireland

Notes: BMNH, NMS, added here

Genus Glyptorhaestus Thomson, 1894

Nomenclature:

LOXONEURUS Schmiedeknecht, 1913

Glyptorhaestus boschmai Teunissen, 1953

Distribution: England

Notes: BMNH, NMS, added here

Glyptorhaestus periclistor Hinz, 1975

Distribution: England

Notes: NMS, added here

Glyptorhaestus punctatus (Thomson, 1890)

Nomenclature:

Rhaestus punctatus Thomson, 1890

Distribution: England

Notes: NMS, BMNH, added here

Glyptorhaestus punctulatus (Woldstedt, 1877)

Nomenclature:

Mesoleius punctulatus Woldstedt, 1877

wuestneii (Thomson, 1893, Rhaestus)

thuringiacus (Schmiedeknecht, 1913, Loxoneurus)

Distribution: England 
Glyptorhaestus selandrivorus (Giraud, 1872)

Nomenclature:

Trematopygus selandrivorus Giraud, 1872

Distribution: England

Notes: BMNH, added here

Genus Labrossyta Förster, 1869

Nomenclature:

LABROSSYTUS Thomson, 1893

LIOTRYPHON Strobl, 1903

Labrossyta scotoptera (Gravenhorst, 1820)

Nomenclature:

Ichneumon scotopterus Gravenhorst, 1820

fumata (Bridgman, 1880, Perilissus)

Distribution: England

Genus Lethades Davis, 1897

Lethades cingulator Hinz, 1976

Distribution: England, Scotland

Notes: BMNH, NMS, added here

Lethades curvispina (Thomson, 1883)

Nomenclature:

Trematopygus curvispina Thomson, 1883

alpinus (Zetterstedt, 1838, Tryphon) nom. ob.

flavifrons (Zetterstedt, 1838, Tryphon) nom. ob.

Distribution: Scotland

Notes: BMNH, NMS, added here; Aubert (2000) uses alpinus as the valid name but this is considered a nomen oblitum, as is flavifrons (Yu and Horstmann 1997). 
Lethades facialis (Brischke, 1871)

Nomenclature:

Trematopygus facialis Brischke, 1871

Distribution: England, Scotland, Wales

Notes: BMNH, det. Hinz \& Horstmann, added here; material in BMNH has been determined by K. Horstmann as this species and curvispina; D.R. Kasparyan has determined Scottish material (Cairngorms) in NMS as facialis. Aubert (2000) treats facialis as a syonym of alpinus Zett. but it is recognised as a valid species by Hinz (1996).

Lethades imperfecti Hinz, 1996

Distribution: England

Notes: added by Hinz (1996)

Lethades subcoriaceus (Strobl, 1903)

Nomenclature:

Mesoleius subcoriaceus Strobl, 1903

laricis Hinz, 1976; synonymy by Horstmann (2011b)

Distribution: England, Scotland

Notes: NMS, det. Kasparyan, added here

Genus Phaestus Förster, 1869

Phaestus anomalus (Brischke, 1871)

Nomenclature:

Grypocentrus anomalus Brischke, 1871

heterocerus (Thomson, 1893, Rhaestus)

Distribution: England

\section{Genus Pion Schiødte, 1839}

Nomenclature:

CATOGLYPTUS Förster, 1855 
Pion nigripes Schiødte, 1839

Nomenclature:

crassipes (Holmgren, 1857, Catoglyptus); synonymy by Horstmann (2004a)

Distribution: England, Scotland

Notes: BMNH, NMS, added here

Pion fortipes (Gravenhorst, 1829)

Nomenclature:

Mesoleptus fortipes Gravenhorst, 1829

pictus (Pfankuch, 1924, Catoglyptus)

transsylvanicus (Kiss, 1924, Mesoleptus); synonymy by Horstmann (2007b)

clarus (Kiss, 1933, Brischkea); synonymy by Horstmann (2007b)

Distribution: England, Scotland, Ireland, Isle of Man

Genus Rhaestus Thomson, 1883

Nomenclature:

RHAESTES Förster, 1869

\section{Rhaestus lativentris (Holmgren, 1858)}

Nomenclature:

Grypocentrus lativentris Holmgren, 1858

Distribution: England, Scotland

Rhaestus rufipes (Holmgren, 1857)

Nomenclature:

Grypocentrus rufipes Holmgren, 1857

assimilis (Holmgren, 1858, Trematopygus)

femoralis Thomson, 1893

Distribution: England, Scotland, Ireland

Notes: added by Aubert (2000) 


\section{Genus Rhorus Förster, 1869}

Nomenclature:

DOLICHOBLASTUS Strobl, 1903

Notes: Species excluded from the British and Irish list:

[neustriae (Schrank, 1802, Ichneumon)] Ichneumon neustriae has traditionally been treated as a species of Rhorus but the species involved has been in doubt (Aubert 1988) and Horstmann (2006a) tentatively transferred the species to Cotesia (Braconidae: Microgastrinae). According to Horstmann (2006a)neustriae auctt. is referable to austriator Aubert, 1988.

Rhorus anglicator Aubert, 1988

Distribution: England, Scotland, Wales

Notes: added by Aubert (1988)

Rhorus binotatus (Kriechbaumer, 1897)

Nomenclature:

Polyblastus binotatus Kriechbaumer, 1897

Distribution: England

Notes: added by Aubert (2000)

Rhorus brunnifemur Kasparyan, 2015

Distribution: England, Scotland

Notes: added by Kasparyan 2015

Rhorus chrysopus (Gmelin, 1790)

Nomenclature:

Ichneumon chrysopus Gmelin, 1790

caproni (Bridgman, 1882, Monoblastus)

capronii misspelling

Distribution: England, Scotland 
Rhorus chrysopygus (Roman, 1909)

Nomenclature:

Monoblastus chrysopyga Roman, 1909

Distribution: England

Rhorus exstirpatorius (Gravenhorst, 1829)

Nomenclature:

Tryphon exstirpatorius Gravenhorst, 1829

laevigatus (Holmgren,1856, Polyblastus)

levigatus (Dalla Torre, 1901, Monoblastus)

Distribution: England, Scotland

Notes: added by Aubert (2000)

Rhorus fasciatus (Gravenhorst, 1829)

Nomenclature:

Tryphon fasciatus Gravenhorst, 1829

Distribution: England

Rhorus flavopictus (Strobl, 1903)

Nomenclature:

Monoblastus flavopictus Strobl, 1903

braunsi Habermehl, 1903

Distribution: England

Notes: BMNH, added here

Rhorus gauldi Kasparyan, 2014

Distribution: England

Notes: added by Kasparyan (2014)

Rhorus lapponicus (Roman, 1909)

Nomenclature:

Monoblastus lapponicus Roman, 1909 
Distribution: England, Scotland

Rhorus laricis Kasparyan, 2014

Distribution: Scotland

Notes: added by Kasparyan (2014)

Rhorus longicornis (Holmgren, 1858)

Nomenclature:

Monoblastus longicornis Holmgren, 1858

glaber (Bridgman, 1886, Prionopoda)

flavomaculatus (Strobl, 1903, Ischyrocnemis); synonymy by Horstmann (2012b) vitosaensis (Gregor, 1933, Monoblastus)

Distribution: England, Scotland

Rhorus longigena (Thomson, 1883)

Nomenclature:

Monoblastus longigena Thomson, 1883

Distribution: Scotland

Rhorus neuter Aubert, 1988

Distribution: England

Notes: added by Aubert (1988)

Rhorus nigrifrons (Holmgren, 1883)

Nomenclature:

Polyblastus nigrifrons Holmgren, 1883

Distribution: Scotland

Notes: added by Kasparyan (2014)

Rhorus palustris (Holmgren, 1857)

Nomenclature:

Polyblastus palustris Holmgren, 1857

Distribution: England, Scotland 


\section{Rhorus punctus (Gravenhorst, 1829)}

Nomenclature:

Tryphon punctus Gravenhorst, 1829

mesoxanthus (Gravenhorst, 1829, Tryphon)

scoticus (Desvignes, 1856, Tryphon)

conspicuus Kriechbaumer, 1891; synonymy by Horstmann (2001a)

spectabilis Kriechbaumer, 1891; synonymy by Horstmann (2001a)

Distribution: England

Notes: Treated as a synonym of mesoxanthus in Aubert (2000).

Rhorus romani Kasparyan, 2014

Distribution: England

Notes: added by Kasparyan (2014)

Rhorus subfasciatus (Stephens, 1835)

Nomenclature:

Tryphon subfasciatus Stephens, 1835

Distribution: England

Rhorus versator Aubert, 1994

Distribution: Scotland

Notes: added by Aubert (1994)

\section{Genus Sympherta Förster, 1869}

Nomenclature:

ATRESTES Förster, 1869

CAMPOGENES Förster, 1869

STIPHROSOMUS Förster, 1869

TRAPEZOCORA Förster, 1869

EUSTIPHROSOMUS Hincks, 1944 


\section{Sympherta antilope (Gravenhorst, 1829)}

\section{Nomenclature:}

Mesoleptus antilope Gravenhorst, 1829

irata (Gravenhorst, 1829, Tryphon)

pulchricornis (Holmgren, 1857, Catoglyptus)

scabra (Brischke, 1871, Catoglyptus)

Distribution: England, Wales

\section{Sympherta foveolator (Holmgren, 1856)}

Nomenclature:

Mesoleptus foveolator Holmgren, 1856

Distribution: England, Scotland

Notes: BMNH, Ely coll., det. Broad and W.A. Ely, added here

\section{Sympherta obligator (Thunberg, 1824)}

Nomenclature:

Ichneumon obligator Thunberg, 1824

fuscicornis (Gmelin, 1790, Ichneumon)

waltoni (Curtis, 1837, Mesoleptus)

Distribution: England, Scotland

\section{Sympherta splendens (Strobl, 1903)}

Nomenclature:

Catoglyptus splendens Strobl, 1903

Distribution: England

Notes: BMNH, added here

\section{Sympherta ? sulcata (Thomson, 1893)}

Nomenclature:

Catoglyptus sulcatus Thomson, 1893

Notes: Added by Aubert (2000), who refers to material in BMNH but the only specimen that could be found is one labelled as sulcatus or splendens det. Aubert. 


\section{Sympherta tenthredinarum Horstmann, 1999}

Nomenclature:

ambulator (Thunberg, 1824, Ichneumon)

Distribution: England, Scotland

Notes: Replacement name, ambulator preoccupied; treated as a synonym of jactator

Thunberg by Aubert (2000) but the two species were separated by Horstmann (1999b).

\section{Sympherta ullrichi (Tschek, 1869)}

Nomenclature:

Catoglyptus ullrichi Tschek, 1869

Distribution: England, Wales, Ireland

Notes: $\mathrm{BMNH}$, added here

\section{Genus Syntactus Förster, 1869}

Nomenclature:

TROMOPOEA Förster, 1869

BRISCHKEA Kriechbaumer, 1897

\section{Syntactus delusor (Linnaeus, 1758)}

Nomenclature:

Ichneumon delusor Linnaeus, 1758

trochantericus (Geoffroy, 1785, Ichneumon)

parvulus (Kriechbaumer, 1897, Brischkea)

Distribution: England, Scotland

Syntactus minor (Holmgren, 1857)

Nomenclature:

Catoglyptus minor Holmgren, 1857

Distribution: England

Syntactus minutus (Bridgman, 1886)

Nomenclature:

Euryproctus minutus Bridgman, 1886 
Distribution: England

Genus Trematopygus Holmgren, 1857

Nomenclature:

AMORPHOGNATHON Förster, 1869

ASELASMA Förster, 1869

CAMPOPORUS Förster, 1869

RHIGELUS Förster, 1869

Trematopygus horvathi (Kiss, 1926)

Nomenclature:

Polyblastus horvathi Kiss, 1926

Distribution: Scotland

Notes: BMNH, det. Horstmann, added here; treated as a subspecies of vellicans by Hinz (1986), elevated to full species by Horstmann (2007b).

Trematopygus melanocerus (Gravenhorst, 1829)

Nomenclature:

Tryphon melanocerus Gravenhorst, 1829

kriechbaumeri Thomson, 1893

thalhammeri Strobl, 1901

romani Heinrich, 1929

Distribution: England, Scotland, Wales

Notes: BMNH, added here

Trematopygus nigricornis Holmgren, 1857

Nomenclature:

dictator (Thunberg, 1824, Ichneumon)

Distribution: England

Trematopygus ? rufator Hinz, 1986

Distribution: England, Scotland

Notes: BMNH, det. Horstmann, added here; tentative identification of males. 
Trematopygus spiniger Hinz, 1976

Distribution: England

Trematopygus vellicans (Gravenhorst, 1829)

Nomenclature:

Tryphon vellicans Gravenhorst, 1829

bicolor (Zetterstedt, 1838, Bassus)

ruficornis Holmgren, 1857

Distribution: England, Scotland

Tribe SCOLOBATINI Schmiedeknecht, 1911

Genus Scolobates Gravenhorst, 1829

Nomenclature:

AGLYPHUS Giraud, 1872

PARABRACONIA Schmiedeknecht, 1914

Scolobates auriculatus (Fabricius, 1804)

Nomenclature:

Ichneumon auriculatus Fabricius, 1804

auriculator (Thunberg, 1824, Ichneumon)

elevator (Thunberg, 1824, Ichneumon)

crassitarsus Gravenhorst, 1829

hylotomae Kriechbaumer, 1897

niger Roman, 1917

nigrifacies Teunissen, 1953

Distribution: England, Scotland, Wales, Ireland 


\section{Cylloceriinae}

Subfamily CYLLOCERIINAE Wahl, 1990

Genus Allomacrus Förster, 1869

Nomenclature:

SIBIRIAKOFFIA Holmgren, 1880

KENTROTRYPHON Strobl, 1903 synonymy by Schwarz (2003)

\section{Allomacrus arcticus (Holmgren, 1880)}

Nomenclature:

Sibiriakoffia arctica Holmgren, 1880

pimplarius Thomson, 1888

Distribution: England, Scotland, Ireland

Notes: BMNH, NMS, UM, added here

\section{Genus Cylloceria Schiødte, 1838}

Nomenclature:

CHALINOCERUS Ratzeburg, 1852

ASPHRAGIS Förster, 1869

Notes: Most distribution data from BMNH and NMS, some from Fitton (1976) and Rossem (1981).

\section{Cylloceria caligata (Gravenhorst, 1829)}

Nomenclature:

Phytodietus caligatus Gravenhorst, 1829

nunciator misident.

nigra (Gravenhorst, 1829, Phytodietus)

crenicornis (Curtis, 1832, Lampronota)

nuntiator (Zetterstedt, 1838, Bassus)

manca (Ruthe, 1855, Chalinocerus)

Distribution: England, Ireland 
Notes: Raised from synonymy (Dasch 1992, Yu and Horstmann 1997) by Humala (2002). Humala (2002) did not report synonymy, which therefore mostly follows Rossem (1981).

\section{Cylloceria melancholica (Gravenhorst, 1820)}

Nomenclature:

Ichneumon melancholicus Gravenhorst, 1820

accusator misidentification

defectiva (Gravenhorst, 1829, Lissonota)

affinis (Zetterstedt, 1838, Bassus)

marginator Schiødte, 1838

denticornis (Haliday, 1839, Lampronota)

fracticornis (Haliday, 1839, Lampronota)

longicornis (Ratzeburg, 1852, Chalinocerus)

marginatrix (Schulz, 1906, Lampronota)

rugulosa (Haupt, 1917, Tropistes)

altior (Heinrich, 1953, Chalinocerus)

Distribution: England, Scotland, Ireland

\section{Cylloceria sylvestris (Gravenhorst, 1829)}

Nomenclature:

Tryphon sylvestris Gravenhorst, 1829

striolata (Hellén, 1915, Lampronota)

Distribution: England

Notes: BMNH, NMS, det. Broad, added here

\section{Genus Hyperacmus Holmgren, 1858}

\section{Nomenclature:}

CUSHMANIA Dasch, 1992 synonymy by Wahl and Gauld (1998)

Notes: Hyperacmus has sometimes been included in the Microleptinae sensu stricto (e.g. Humala 1997, Yu and Horstmann 1997) but the Microleptinae is restricted to the single genus, Microleptes (Broad 2004). Wahl and Gauld (1998) argued that Hyperacmus is better placed in the Orthocentrinae but, on the basis of phylogenetic studies (Quicke et al. 2009), Hyperacmus is placed here in the Cylloceriinae. 
Hyperacmus crassicornis (Gravenhorst, 1829)

Nomenclature:

Exochus crassicornis Gravenhorst, 1829

brunniventris (Rudow, 1883, Exochus)

suerinensis (Brauns, 1905, Lampronota) synonymy by Humala (2002)

Distribution: England, Scotland, Ireland

\section{Diacritinae}

\section{Subfamily DIACRITINAE Townes, 1965}

Notes: Treated by Fitton et al. (1988), who give distribution data, as a tribe of the Pimplinae.

\section{Genus Diacritus Förster, 1869}

Nomenclature:

PHIDIAS Vollenhoven, 1878 preocc.

STENOLABIS Kriechbaumer, 1894

PHOSPHORUS Rossem, 1981 preocc.

PHOSPHORIANA Rossem, 1987 synonymy by Humala (2007)

\section{Diacritus aciculatus (Vollenhoven, 1878)}

Nomenclature:

Phidias aciculatus Vollenhoven, 1878

cingulatus (Kriechbaumer, 1894, Stenolabis)

rugosissima (Strobl, 1904, Entypoma) synonymy by Humala (2007)

Distribution: England, Scotland, Ireland

\section{Diplazontinae}

\section{Subfamily DIPLAZONTINAE Viereck, 1918}

Notes: Unless noted otherwise, distribution data from Beirne (1941), Kerrich (1949), Cowin (1953), Klopfstein (2014) and the collections of BMNH, NMS and UM, with data on type localities from Fitton (1976). Material collected by Fraser et al. (2007) is 
deposited in NMS. Additional references are given. Taxonomy, including synonymy, follows Klopfstein (2014).

\section{Genus Bioblapsis Förster, 1869}

Nomenclature:

TRICHOMASTIX Vollenhoven, 1878

Bioblapsis cultiformis (Davis, 1897)

Nomenclature:

Otoblastus cultiformis Davis, 1897

mallochi Rotheray, 1990

Distribution: England, Scotland

Notes: added by Rotheray (1990)

Bioblapsis polita (Vollenhoven, 1878)

Nomenclature:

Trichomastix polita Vollenhoven, 1878

flavipes (Holmgren, 1858, Bassus) preocc.

tibialis (Bridgman, 1883, Bassus) preocc.

Distribution: England, Scotland

Genus Campocraspedon Uchida, 1957

Campocraspedon annulitarsis (Hedwig, 1838)

Nomenclature:

Homocidus annulitarsis Hedwig, 1838

arcanus (Stelfox, 1941, Homocidus)

Distribution: England, Scotland, Wales, Ireland

Campocraspedon caudatus (Thomson, 1890)

Nomenclature:

Homotropus caudatus Thomson, 1890

Distribution: England, Scotland, Wales, Ireland, Isle of Man 


\section{Genus Diplazon Nees, 1819}

Nomenclature:

BASSUS misident.

\section{Diplazon albotibialis Dasch, 1964}

Nomenclature:

alpinus (Holmgren, 1858, Bassus) preocc.

neoalpinus Zwakhals, 1979

Distribution: England, Scotland, Ireland

\section{Diplazon annulatus (Gravenhorst, 1829)}

Nomenclature:

Bassus annulatus Gravenhorst, 1829

lapponicus (Zetterstedt, 1838, Bassus)

Distribution: England, Scotland, Wales, Ireland, Isle of Man

Notes: Diplazon multicolor (Gravenhorst, 1829, Bassus) was removed from synonymy by Klopfstein (2011).

\section{Diplazon deletus (Thomson, 1890)}

Nomenclature:

Bassus deletus Thomson, 1890

rufigaster Dasch, 1964

Distribution: England, Scotland, Wales, Ireland

\section{Diplazon laetatorius (Fabricius, 1781)}

\section{Nomenclature:}

Ichneumon laetatorius Fabricius, 1781

dichrous (Schrank, 1781, Ichneumon)

albovarius (Wollaston, 1858, Bassus)

cinctipes (Holmgren, 1868, Bassus)

varipes (Smith, 1878, Scolobates)

venustulus (Saussure, 1892, Bassus)

balearicus (Kriechbaumer, 1894, Bassus) 
Distribution: England, Scotland, Wales, Ireland, Isle of Man

Diplazon pectoratorius (Thunberg, 1824)

Nomenclature:

Ichneumon pectoratorius Thunberg, 1824

angustorius Thunberg, 1824, Ichneumon)

pectoratorius (Gravenhorst, 1829, Bassus) preocc.

nigrithorax (Strobl, 1902, Homotropus)

akaashii (Uchida, 1931, Homocidus)

urupensis (Uchida, 1935, Bassus)

Distribution: England, Scotland, Wales, Ireland, Isle of Man

Diplazon scutatorius Teunissen, 1943

Nomenclature:

pilosus Uchida, 1957

tetragonopsis Uchida, 1957

Distribution: England

Notes: Added by Thirion (1987); Diplazon tetragonopsis is listed as a synonym of tetragonus by Yu and Horstmann (1997) but Diller (1982) and Klopfstein (2014) treat it as a synonym of scutatorius.

\section{Diplazon tetragonus (Thunberg, 1824)}

\section{Nomenclature:}

Ichneumon tetragonus Thunberg, 1824

hortorius (Thunberg, 1824, Ichneumon)

ustorius (Thunberg, 1824, Ichneumon)

tricinctus (Gravenhorst, 1829, Bassus)

nemoralis (Holmgren, 1858, Bassus)

Distribution: England, Scotland, Wales, Ireland, Isle of Man

\section{Diplazon tibiatorius (Thunberg, 1824)}

Nomenclature:

Ichneumon tibiatorius Thunberg, 1824

albosignatus (Gravenhorst, 1829, Bassus) 
Distribution: England, Scotland, Wales, Ireland, Isle of Man

Notes: some distribution data from Askew (2000)

Diplazon varicoxa (Thomson, 1890)

Nomenclature:

Bassus varicoxa Thomson, 1890

Distribution: England, Scotland, Ireland, Isle of Man

Genus Enizemum Förster, 1869

Enizemum nigricorne (Thomson, 1890)

Nomenclature:

Homotropus nigricornis Thomson, 1890

Distribution: Ireland

Notes: Fitton and Rotheray (1982) doubted whether the few British specimens identified as nigricorne were distinct from ornatum. One apparently British specimen in BMNH (Capron coll., no other details) is nigricorne (det. Broad and Klopfstein). Beirne (1941) records nigricorne from Ireland.

\section{Enizemum ornatum (Gravenhorst, 1829)}

Nomenclature:

Bassus ornatus Gravenhorst, 1829

deplanatum (Gravenhorst, 1829, Bassus)

carinulatum (Ruthe, 1859, Bassus)

frenator (Desvignes, 1862, Bassus)

sumptuosum (Schmiedeknecht, 1926, Homocidus)

Distribution: England, Scotland, Wales, Ireland, Isle of Man

\section{Enizemum scutellare (Lange, 1911)}

Nomenclature:

Homotropus scutellaris Lange, 1911

albopictum (Lange, 1911, Homotropus) preocc.

rubiginosum (Schmiedeknecht, 1926, Homocidus)

Distribution: Wales 
Notes: added by Klopfstein (2014)

Enizemum tridentatum Dasch, 1964

Distribution: Ireland

Notes: BMNH, det. Klopfstein, Broad, added here

Genus Eurytyloides Nakanishi, 1978

Eurytyloides umbrinus Klopfstein, 2014

Distribution: England

Notes: added by Klopfstein (2014)

Genus Fossatyloides Klopfstein, Quicke, Kropf \& Frick, 2011

Fossatyloides gracilentus (Holmgren, 1858)

Nomenclature:

Bassus gracilentus Holmgren, 1858

pulcher (Holmgren, 1858, Bassus) preocc.

Distribution: England, Scotland, Wales

\section{Genus Homotropus Förster, 1869}

Nomenclature:

HOMOCIDUS Morley, 1911

Notes: Homotropus species have generally been included in Syrphoctonus; generic combinations and species-level taxonomy follow Klopfstein (2014).

species incertae sedis within Homotropus:

[impolitus (Stelfox, 1941, Homocidus), from Scotland] Known only from males, Klopfstein (2014) was unable to place this taxon but suggested that it may be a colour variant of pallipes. 
Homotropus collinus (Stelfox, 1941)

Nomenclature:

Homocidus collinus Stelfox, 1941

simulans (Stelfox, 1941, Homocidus)

Distribution: England, Scotland, Ireland

Homotropus crassicornis Thomson, 1890

Nomenclature:

brevicornis Thomson, 1890

asyntactus (Schmiedeknecht, 1926, Homocidus)

Distribution: England, Scotland, Wales, Isle of Man

Homotropus dimidiatus (Schrank, 1802)

Nomenclature:

Ichneumon dimidiatus Schrank, 1802

planus (Desvignes, 1862, Bassus)

crassicrus Thomson, 1890

Distribution: England, Scotland

Homotropus elegans (Gravenhorst, 1829)

Nomenclature:

Bassus elegans Gravenhorst, 1829

rufonotatus (Holmgren, 1858, Bassus)

affinis Szépligeti, 1898

Distribution: England, Scotland, Wales, Isle of Man

Homotropus frontorius (Thunberg, 1824)

Nomenclature:

Ichneumon frontorius Thunberg, 1824

subopacus (Stelfox, 1941, Homocidus)

Distribution: England, Scotland, Wales, Ireland 
Homotropus haemorrhoidalis Szépligeti, 1898

Nomenclature:

rhenanus (Habermehl, 1930, Homocidus)

struvei (Hedwig, 1939, Homocidus)

tricolor (Stelfox, 1941, Homocidus)

lipothrix Momoi, 1973

Distribution: England, Scotland, Wales, Ireland

Homotropus longiventris Thomson, 1890

Distribution: England, Scotland, Ireland

Homotropus megaspis Thomson, 1890

Nomenclature:

megalaspis Schulz, 1906

Distribution: England, Scotland, Ireland

Homotropus melanogaster (Holmgren, 1872)

Nomenclature:

Bassus melanogaster Holmgren, 1872

Distribution: England

Notes: added by Klopfstein (2014)

Homotropus nigritarsus (Gravenhorst, 1829)

Nomenclature:

Bassus nigritarsus Gravenhorst, 1829

picitans (Desvignes, 1862, Bassus)

groenlandicus (Holmgren, 1872, Bassus)

Distribution: England, Scotland, Wales, Ireland, Isle of Man

Homotropus pallipes (Gravenhorst, 1829)

Nomenclature:

Bassus pallipes Gravenhorst, 1829

pectoralis (Gravenhorst, 1829, Lissonota) 
pallidipes (Marshall, 1872, Bassus)

pallidipennis Dalla Torre, 1901

pallidipes Dalla Torre, 1901

Distribution: England, Scotland, Ireland, Isle of Man

\section{Homotropus pectoralis (Provancher, 1874)}

Nomenclature:

Bassus pectoralis Provancher, 1874

incisus Thomson, 1890

reflexus Morley, 1906

Distribution: England, Scotland, Wales

\section{Homotropus pictus (Gravenhorst, 1829)}

Nomenclature:

Bassus pictus Gravenhorst, 1829

nigricornis (Zetterstedt, 1838, Tryphon)

pumilus (Holmgren, 1858, Bassus)

thoracicus (Desvignes, 1862, Bassus)

brevis (Hedwig, 1938, Homocidus)

Distribution: England, Scotland, Wales, Ireland, Isle of Man

\section{Homotropus signatus (Gravenhorst, 1829)}

Nomenclature:

Bassus signatus Gravenhorst, 1829

hygrobius Thomson, 1890

bifoveolatus Kriechbaumer, 1894

Distribution: England, Scotland, Ireland, Isle of Man

\section{Homotropus strigator (Fabricius, 1793)}

Nomenclature:

Ichneumon strigator Fabricius, 1793

ruficornis (Holmgren, 1858, Bassus) preocc.

Distribution: England, Wales 
Homotropus sundevalli (Holmgren, 1858)

Nomenclature:

Bassus sundevalli Holmgren, 1858

scabrosus (Desvignes, 1862, Bassus)

Distribution: England, Scotland, Wales

Notes: some distribution data from Rotheray (1986)

Genus Phthorima Förster, 1869

Nomenclature:

PHTHORIMUS Thomson, 1890

Notes: Distribution data from Fitton and Boston (1988).

\section{Phthorima compressa (Desvignes, 1856)}

Nomenclature:

Bassus compressus Desvignes, 1856

ibalioidis (Kriechbaumer, 1878, Bassus)

nigra (Morley, 1906, Homotropus)

Distribution: England, Scotland, Wales, Ireland

Phthorima picta (Habermehl, 1925)

Nomenclature:

Phthorimus picta Habermehl, 1925

gaullei Seyrig, 1928

Distribution: England, Ireland

Notes: added by Fitton and Boston (1988)

Phthorima xanthaspis (Thomson, 1890)

Nomenclature:

Homotropus xanthaspis Thomson, 1890

Distribution: England

Notes: added by Fitton and Boston (1988) 


\section{Genus Promethes Förster, 1869}

Nomenclature:

LIOPSIS Förster, 1869

PROMETHUS Thomson, 1890

\section{Promethes bridgmani Fitton, 1976}

Nomenclature:

scutellaris (Bridgman, 1886, Bassus) preocc.

Distribution: England, Scotland, Wales, Ireland

Promethes sulcator (Gravenhorst, 1829)

Nomenclature:

Bassus sulcator Gravenhorst, 1829

areolatus (Holmgren, 1859, Bassus)

anomalus (Taschenberg, 1865, Orthopelma)

dodsi (Morley, 1906, Promethus)

Distribution: England, Scotland, Wales, Ireland, Isle of Man

Genus Sussaba Cameron, 1909

Sussaba cognata (Holmgren, 1858)

Nomenclature:

Bassus cognatus Holmgren, 1858

albicoxa (Thomson, 1890, Promethus)

Distribution: England, Scotland, Wales, Ireland, Isle of Man

Sussaba dorsalis (Holmgren, 1858)

Nomenclature:

Bassus dorsalis Holmgren, 1858

maculata (Desvignes, 1862, Bassus)

Distribution: England, Scotland, Wales, Ireland 


\section{Sussaba erigator (Fabricius, 1793)}

Nomenclature:

Ichneumon erigator Fabricius, 1793

festiva (Fabricius, 1798, Ichneumon)

festivator (Fabricius, 1804, Ophion)

Distribution: England

Notes: The only English specimens listed by Kerrich (1949) (and possibly the same as those listed by Beirne 1941) were supposedly from the Lichfield district (Carr (1924)) and are thus inadmissable (Perkins 1953, Shaw 2003). There are specimens in BMNH recently identified by $\mathrm{S}$. Klopfstein.

\section{Sussaba flavipes (Lucas, 1849)}

\section{Nomenclature:}

Bassus flavipes Lucas, 1849

pulchella misident.

neopulchella Diller, 1980

coriacea Dasch, 1964

Distribution: England, Scotland, Wales, Ireland

Notes: Sometimes identified as coriacea, but this is now classified as the Nearctic subspecies of flavipes (Yu and Horstmann 1997).

\section{Sussaba placita Dasch, 1964}

\section{Nomenclature:}

punctiventris misident.

\section{Distribution: England}

Notes: The only British specimens labelled as punctiventris in BMNH were misidentified flavipes, and Beirne (1941) did not mention any British or Irish specimens. According to S. Klopfstein (pers. comm.), specimens identified as punctiventris (Thomson, 1890, Homotropus) by Fraser et al. (2007) are actually placita and the species was subsequently recorded as placita by Mayhew et al. (2009).

\section{Sussaba pulchella (Holmgren, 1858)}

\section{Nomenclature:}




$$
\begin{aligned}
& \text { elongata (Provancher, 1874, Bassus) } \\
& \text { monticola (Vollenhoven, 1880, Bassus) } \\
& \text { laticarpus (Thomson, 1890, Promethus) } \\
& \text { ruthei (Roman, 1931, Promethes) }
\end{aligned}
$$

Distribution: England, Scotland, Wales, Ireland, Isle of Man

Notes: The name pulchella has frequently been applied to flavipes.

\section{Genus Syrphoctonus Förster, 1869}

Notes: Klopfstein et al. (2011) reassigned several species to Homotropus and Fossatyloides with other remaining species formally transferred by Klopfstein (2014).

\section{Syrphoctonus desvignesii (Marshall, 1870)}

\section{Nomenclature:}

Bassus desvignesii Marshall, 1870

pulcher misident.

pulchellus (Desvignes, 1862, Bassus) preocc.

neopulcher Horstmann, 1968

Distribution: England, Scotland, Wales, Ireland, Isle of Man

\section{Syrphoctonus fissorius (Gravenhorst, 1829)}

Nomenclature:

$$
\begin{aligned}
& \text { Bassus fissorius Gravenhorst, } 1829 \\
& \text { punctatus (Bridgman, 1887, Bassus) } \\
& \text { similis (Lange, 1911, Homotropus) }
\end{aligned}
$$

Distribution: England, Scotland, Wales, Ireland, Isle of Man

\section{Syrphoctonus tarsatorius (Panzer, 1809)}

\section{Nomenclature:}

Bassus tarsatorius Panzer, 1809

exsultans (Gravenhorst, 1829, Bassus)

insignis (Gravenhorst, 1829, Bassus)

flavus (Desvignes, 1862, Bassus)

indicus (Cameron, 1909, Bassus) 
eximius (Habermehl, 1922, Homotropus)

flavitrochanterus (Uchida, 1957, Homotropus)

Distribution: England, Scotland, Wales, Ireland, Isle of Man

Genus Syrphophilus Dasch, 1964

Syrphophilus bizonarius (Gravenhorst, 1829)

Nomenclature:

Bassus bizonarius Gravenhorst, 1829

cingulatus (Holmgren, 1858, Bassus)

frontalis (Brischke, 1878, Bassus) preocc.

iwatensis (Uchida, 1930, Homocidus)

satoi (Uchida, 1930, Homocidus)

Distribution: England, Scotland, Wales, Ireland

\section{Syrphophilus tricinctorius (Thunberg, 1824)}

Nomenclature:

Ichneumon tricinctorius Thunberg, 1824

cinctus (Gravenhorst, 1829, Bassus)

lateralis (Gravenhorst, 1829, Bassus)

albicinctus (Desvignes, 1862, Bassus)

takaozanus (Uchida, 1930, Homocidus)

Distribution: England, Scotland, Wales, Ireland, Isle of Man

Notes: some distribution data from Askew (2000)

\section{Genus Tymmophorus Schmiedeknecht, 1913}

Nomenclature:

ZOOTREPHES misident.

\section{Tymmophorus erythrozonus (Förster, 1850)}

\section{Nomenclature:}

Tryphon erythrozonus Förster, 1850

rufiventris (Gravenhorst, 1829, Bassus) preocc. (Horstmann 2006c) 
holmgreni (Bridgman, 1882, Bassus)

lacustris Schmiedeknecht, 1913

Distribution: England

Tymmophorus obscuripes (Holmgren, 1858)

Nomenclature:

Bassus obscuripes Holmgren, 1858

graculus misident.

rufocinctus (Desvignes, 1862, Bassus)

arcticus (Holmgren, 1869, Bassus)

luctuosus (Schmiedeknecht, 1926, Promethes)

Distribution: England, Scotland, Wales, Ireland, Isle of Man

Notes: Bassus graculus Gravenhorst, 1829, is a species of Zoophthorus (Cryptinae).

Tymmophorus suspiciosus (Brischke, 1871)

Nomenclature:

Bassus suspiciosus Brischke, 1871

Distribution: England, Scotland, Ireland

Notes: Raised from synonymy with erythrozonus by Klopfstein (2014); only country-

level data that can be directly attributable to each of these species are included here.

\section{Genus Woldstedtius Carlson, 1979}

Nomenclature:

SYRPHOCTONUS misident.

Woldstedtius bauri Klopfstein, 2014

Distribution: England

Notes: added by Klopfstein (2014)

Woldstedtius biguttatus (Gravenhorst, 1829)

Nomenclature:

Bassus biguttatus Gravenhorst, 1829

rufipes (Gravenhorst, 1829, Bassus) 
confusus (Woldstedt, 1874, Bassus)

Distribution: England, Scotland, Wales, Ireland

Woldstedtius citropectoralis (Schmiedeknecht, 1926)

Nomenclature:

Homocidus citropectoralis Schmiedeknecht, 1926

abdominator (Bridgman, 1886, Bassus) preocc.

Distribution: England, Scotland, Ireland

Woldstedtius flavolineatus (Gravenhorst, 1829)

Nomenclature:

Bassus flavolineatus Gravenhorst, 1829

bimaculatus (Holmgren, 1858, Bassus)

interruptus (Holmgren, 1858, Bassus)

Distribution: England, Scotland, Wales

Woldstedtius holarcticus (Diller, 1969)

Nomenclature:

Syrphoctonus holarcticus Diller, 1969

Distribution: England

Notes: added by Klopfstein (2014)

Genus Xestopelta Dasch, 1964

Xestopelta gracillima (Schmiedeknecht, 1926)

Nomenclature:

Promethes gracillimus Schmiedeknecht, 1926

amabilis (Habermehl, 1935, Homocidus)

Distribution: England

Notes: added by Fitton and Rotheray (1982) 


\section{Eucerotinae}

\section{Subfamily EUCEROTINAE Viereck, 1919}

Notes: Distribution data from Fitton (1984) and the collections of NMS.

\section{Genus Euceros Gravenhorst, 1829}

Nomenclature:

EUMESIUS Westwood, 1840

OMALOCEROS Giraud, 1857

TAUTOZELUS Förster, 1869

Euceros albitarsus Curtis, 1837

Nomenclature:

dimidiatus Brullé, 1846

Distribution: England, Ireland

Euceros pruinosus (Gravenhorst, 1829)

Nomenclature:

Tryphon pruinosus Gravenhorst, 1829

crassicornis Gravenhorst, 1829

morionellus Holmgren, 1857

unifasciatus Vollenhoven, 1878

castaneus (Pfankuch, 1906, Eumesius) unavailable

Distribution: England, Scotland

Notes: Horstmann (2006a) removed superbus Kriechbeumer, 1888 from synonymy.

\section{Euceros serricornis Haliday, 1839}

Nomenclature:

egregius Holmgren, 1857

grandicornis Holmgren, 1857

Distribution: England, Scotland, Ireland 


\section{Hybrizontinae}

Subfamily HYBRIZONTINAE Blanchard, 1845

Nomenclature:

PAXYLLOMATINAE Förster, 1862

Notes: Usually referred to as Paxyllomatinae (e.g. Fitton 1978, Mason 1981, Yu and Horstmann 1997), but Hybrizontinae has priority (Wharton and Achterberg 2000). Distribution data from Donisthorpe (1927), Achterberg (1999) and the collections of BMNH and NMS.

Genus Ghilaromma Tobias, 1988

Ghilaromma fuliginosi (Donisthorpe \& Wilkinson, 1930)

Nomenclature:

Paxylomma fuliginosi Donisthorpe \& Wilkinson, 1930

Distribution: England

\section{Genus Hybrizon Fallén, 1813}

Nomenclature:

PAXYLLOMA Latreille, 1817

PLANCUS Curtis, 1833

PACHYLOMMA Ratzeburg, 1848

Hybrizon buccatus (de Brébisson, 1825)

Nomenclature:

Paxylomma buccata de Brébisson, 1825

apicalis (Curtis, 1833, Plancus)

latebricola Nees, 1834

Distribution: England, Wales

Notes: Hybrizon pubicornis Zetterstedt, 1838, is listed as a synonym of buccatus in Yu and Horstmann (1997) but is actually a species of Anteon (Dryinidae) (Achterberg 1999). 


\section{Ichneumoninae}

Subfamily ICHNEUMONINAE Latreille, 1802

Notes: Distribution data from Perkins (1953), Perkins (1959), Perkins (1960), Fitton (1976), Boston and Nash (1989) and the collections of BMNH and NMS, except where noted. Additional distribution references are given.

Tribe EURYLABINI Heinrich, 1934

\section{Genus Eurylabus Wesmael, 1845}

Nomenclature:

MISCHOPHORUS Kriechbaumer, 1894

\section{Eurylabus larvatus (Christ, 1791)}

Nomenclature:

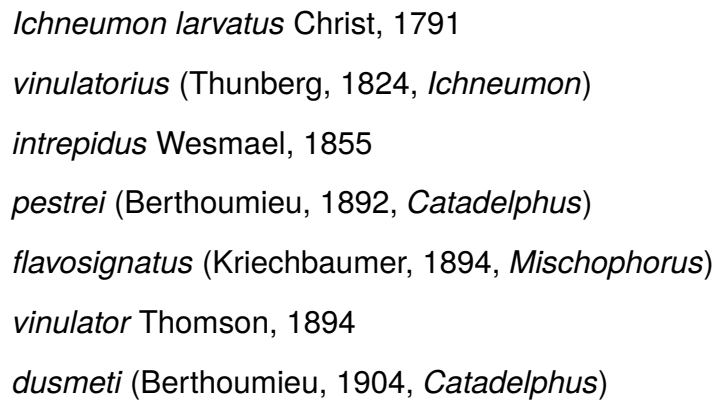

Distribution: England

Eurylabus torvus Wesmael, 1845

Distribution: England, Wales, Ireland

\section{Eurylabus tristis (Gravenhorst, 1829)}

Nomenclature:

Ichneumon tristis Gravenhorst, 1829

corvinus Wesmael, 1845

Distribution: England, Wales, Ireland 
Tribe GOEDARTIINI Townes, 1961

Genus Goedartia Boie, 1841

Nomenclature:

AUTOMALUS Wesmael, 1845

Goedartia alboguttata (Gravenhorst, 1829)

Nomenclature:

Trogus alboguttatus Gravenhorst, 1829

affinis (Boie, 1841, Trogus)

baltica (Ratzeburg, 1844, Ichneumon)

dimidiativentris (Rudow, 1888, Amblyteles)

Distribution: England, Wales, Ireland

Notes: Welsh specimens in World Museum Liverpool, det. T. Hunter.

Tribe HERESIARCHINI Ashmead, 1900

Nomenclature:

PROTICHNEUMONINI Heinrich, 1934

CALLAJOPPINI Heinrich, 1962 synonymy by Sime and Wahl (2002)

TROGINI Förster, 1869 synonymy by Sime and Wahl (2002)

Genus Amblyjoppa Cameron, 1902

Amblyjoppa fuscipennis (Wesmael, 1845)

Nomenclature:

Amblyteles fuscipennis Wesmael, 1845

Distribution: England, Wales, Ireland

Amblyjoppa proteus (Christ, 1791)

Nomenclature:

Ichneumon proteus Christ, 1791

laminatoria (Fabricius, 1798, Ichneumon)

nigratoria (Fabricius, 1798, Ichneumon) preocc.

bilineator (Donovan, 1810, Ichneumon) synonymy by Horstmann (1997) 
nigriculus (Walkley, 1958, Ichneumon)

Distribution: England, Scotland, Ireland

\section{Genus Callajoppa Cameron, 1903}

\section{Callajoppa cirrogaster (Schrank, 1781)}

Nomenclature:

Ichneumon cirrogaster Schrank, 1781

cirrogastra misspelling

nigrocaudata (Retzius, 1783, Ichneumon)

crocata (Geoffroy, 1785, Ichneumon)

Iutoria (Fabricius, 1787, Ichneumon)

rubricornuta (Christ, 1791, Ichneumon)

dessinator (Olivier, 1792, Ichneumon)

scutellaris (Olivier, 1792, Ichneumon)

imperatoria (Panzer, 1804, Ichneumon)

obscuratoria (Gravenhorst, 1807, Ichneumon)

atrocaudata (Stephens, 1835, Trogus)

excellens (Tischbein, 1882, Trogus)

Distribution: England, Ireland

\section{Callajoppa exaltatoria (Panzer, 1804)}

\section{Nomenclature:}

Ichneumon exaltatorius Panzer, 1804

latoria (Thunberg, 1824, Ichneumon)

atropos (Curtis, 1828, Ichneumon)

Distribution: England

\section{Genus Coelichneumon Thomson, 1893}

Notes: species of Coelichneumon excluded from the British and Irish list

[eximius (Stephens, 1835, Ichneumon)] Listed in error by Fitton (1978), this species is North American (Perkins 1953). 
Coelichneumon anthrax (Dalla Torre, 1901)

Nomenclature:

Ichneumon anthrax Dalla Torre, 1901

anthracinus (Holmgren, 1864, Ichneumon) preocc.

Distribution: England

Notes: added by Riedel (2012)

Coelichneumon biannulatus (Gravenhorst, 1820)

Nomenclature:

Ichneumon biannulatus Gravenhorst, 1820

auspex misident.

fasciatus (Gmelin, 1790, Ichneumon) preocc.

leucopis (Berthoumieu, 1894, Ichneumon) preocc., unavailable

Distribution: England, Wales

Notes: Coelichneumon funebrator Horstmann, 2006, (= funebris (Holmgren, 1864, Ichneumon) preocc.) was removed from synonymy by Horstmann (2006c).

\section{Coelichneumon biguttorius (Thunberg, 1789)}

Nomenclature:

Ichneumon biguttorius Thunberg, 1789

microstictus misident. (Horstmann 2002c)

serenus (Gravenhorst, 1829, Ichneumon) synonymy by Riedel (2012)

restaurator (Fabricius, 1793, Ichneumon) preocc.

restritutor (Thunberg, 1824, Ichneumon)

laticeps (Rudow, 1888, Amblyteles)

rufiapicalis (Pic, 1914, Ichneumon)

transsylvanicus (Kiss, 1924, Ichneumon) synonymy by Riedel (2012)

concolor Heinrich, 1949 synonymy by Riedel (2012)

Distribution: England

Coelichneumon bilineatus (Gmelin, 1790)

Nomenclature:

Ichneumon bilineatus Gmelin, 1790 
pulsator (Panzer, 1804, Ichneumon)

Distribution: England, Ireland

\section{Coelichneumon comitator (Linnaeus, 1758)}

Nomenclature:

Ichneumon comitator Linnaeus, 1758

auspex (Müller, 1776, Ichneumon)

biguttatus (Thunberg, 1784, Ichneumon)

tripunctorius (Thunberg, 1789, Ichneumon)

nigrator (Fabricius, 1793, Ichneumon) preocc.

narrator (Fabricius, 1804, Ichneumon)

restaurator (Gravenhorst, 1820, Ichneumon) preocc., synonymy by Horstmann (1998b)

fuscatorius (Thunberg, 1824, Ichneumon)

ferreus (Gravenhorst, 1829, Ichneumon)

coerulescens (Tischbein, 1879, Ichneumon)

purpurissatus Perkins, 1953 synonymy by Horstmann (2000b)

Distribution: England, Ireland

\section{Coelichneumon consimilis (Wesmael, 1845)}

Nomenclature:

Ichneumon consimilis Wesmael, 1845

caelareator (Tischbein, 1881, Ichneumon)

nigripes (Kriechbaumer, 1894, Ichneumon) preocc., unavailable

Distribution: England, Wales, Ireland

\section{Coelichneumon cyaniventris (Wesmael, 1859)}

Nomenclature:

Ichneumon cyaniventris Wesmael, 1859

biobliteratus (Pic, 1923, Ichneumon)

multialbonotatus (Pic, 1923, Ichneumon)

Distribution: England, Wales, Ireland

Notes: Irish occurrence from O'Connor (2004b) 
Coelichneumon desinatorius (Thunberg, 1824)

Nomenclature:

Ichneumon desinatorius Thunberg, 1824

fuscipes (Gmelin, 1790, Ichneumon) preocc.

subguttatus (Gravenhorst, 1829, Ichneumon)

Distribution: England, Scotland

\section{Coelichneumon falsificus (Wesmael, 1845)}

Nomenclature:

Ichneumon falsificus Wesmael, 1845

specularis (Tischbein, 1881, Ichneumon) synonymy by Riedel (2012)

chevrieri (Pic, 1902, Ichneumon)

Distribution: England

\section{Coelichneumon haemorrhoidalis (Gravenhorst, 1820)}

\section{Nomenclature:}

Ichneumon haemorrhoidalis Gravenhorst, 1820

castaniventris (Gravenhorst, 1829, Ichneumon) synonymy by Riedel (2012)

castanicauda (Tischbein, 1881, Ichneumon)

truncatulus (Thomson, 1886,)

secretus (Berthoumieu, 1894, Ichneumon) unavailable

subniger (Berthoumieu, 1894, Ichneumon) unavailable

strandi (Berthoumieu, 1910, Ichneumon)

binigronotatus (Pic, 1925, Ichneumon)

vulcanius (Pic, 1925, Ichneumon)

bipunctatus (Schmiedeknecht, 1928, Ichneumon) preocc.

Distribution: England, Ireland

\section{Coelichneumon leucocerus (Gravenhorst, 1820)}

\section{Nomenclature:}

Ichneumon leucocerus Gravenhorst, 1820

solitarius (Thunberg, 1824, Ichneumon)

ligeris (Pic, 1923, Ichneumon) 
Distribution: England, Ireland

\section{Coelichneumon litoralis Horstmann, 2000}

Nomenclature:

purpurissatus misident. (Horstmann 2000b)

Distribution: England

Notes: added by Horstmann (2000b)

\section{Coelichneumon nigerrimus (Stephens, 1835)}

Nomenclature:

Ichneumon nigerrimus Stephens, 1835

derasus (Wesmael, 1845, Ichneumon)

carbonator (Tischhbein, 1874, Amblyteles)

percussor (Tischbein, 1876, Ichneumon) synonymy by Riedel (2012)

minor (Kriechbaumer, 1894, Ichneumon) preocc., unavailable

annulatus Heinrich, 1929

Distribution: England, Scotland, Wales, Ireland

\section{Coelichneumon oltenensis Constantineanu, Pîrvescu \& Mihalache, 1979}

Nomenclature:

serenus misident.

Notes: Added by Horstmann (2002c)from 'British Isles, Stephens Coll.'.

\section{Coelichneumon orbitator (Thunberg, 1824)}

Nomenclature:

Ichneumon orbitator Thunberg, 1824

microstictus (Gravenhorst, 1829, Ichneumon) synonymy by Horstmann (2002c)

melanopyrrhus (Stephens, 1835, Ichneumon)

separator (Fonscolombe, 1847, Ichneumon) synonymy by Riedel (2012)

liocnemis (Thomson, 1888, Ichneumon)

Distribution: England 
Coelichneumon ruficauda (Wesmael, 1845)

Nomenclature:

Ichneumon ruficauda Wesmael, 1845

Distribution: England

Coelichneumon validus (Berthoumieu, 1894)

Nomenclature:

Ichneumon validus Berthoumieu, 1894

nigricornis (Wesmael, 1845, Ichneumon) preocc.

Distribution: England, Scotland

Genus Coelichneumonops Heinrich, 1958

Coelichneumonops solutus (Holmgren, 1864)

Nomenclature:

Ichneumon solutus Holmgren, 1864

chrysostomus (Thomson, 1896, Ichneumon) synonymy by Horstmann (1999a)

pictus (Roman, 1904, Ichneumon) preocc.

Distribution: Scotland

Genus Heresiarches Wesmael, 1859

Heresiarches eudoxius (Wesmael, 1845)

Nomenclature:

Hepiopelmus eudoxius Wesmael, 1845

Distribution: England

Genus Lymantrichneumon Heinrich, 1978

Lymantrichneumon disparis (Poda, 1761)

Nomenclature:

Sphex disparis Poda, 1761

Distribution: England

Notes: added by Broad and Davis (2015) 
Genus Protichneumon Thomson, 1893

Protichneumon pisorius (Linnaeus, 1758)

Nomenclature:

Ichneumon pisorius Linnaeus, 1758

fusorius misident.

lentorius (Panzer, 1799, Ichneumon)

fugatorius (Panzer, 1804, Ichneumon)

mediofulvus (Berthoumieu, 1894, Ichneumon) unavailable

dorsoniger Roman, 1910

Distribution: England, Ireland

Protichneumon similatorius (Fabricius, 1798)

Nomenclature:

Ichneumon similatorius Fabricius, 1798

?exspectorius (Fabricius, 1794, Ichneumon)

erythrogaster (Stephens, 1835, Ichneumon) preocc.

coqueberti (Wesmael, 1848, Ichneumon)

dorsoniger (Berthoumieu, 1894, Ichneumon)

Distribution: England

Notes: Amblyteles gigantorius Holmgren, 1871 was removed from synonymy as it was

found to be a junior synonym of Protichneumon fusorius (Linnaeus, 1761) (Horstmann 2008a).

Genus Psilomastax Tischbein, 1868

Nomenclature:

CERCODINOTOMUS Uchida, 1940

Psilomastax pyramidalis Tischbein, 1868

Nomenclature:

pictus Kriechbaumer, 1882

Distribution: England 


\section{Genus Syspasis Townes, 1965}

Syspasis carinator (Fabricius, 1798)

Nomenclature:

Ichneumon carinator Fabricius, 1798

helleri (Holmgren, 1878, Ichneumon)

rufipes (Strobl, 1901, Ichneumon) preocc.

tenuidens (Berthoumieu, 1904, Ichneumon)

Distribution: England

Notes: NMS, det. Riedel, added here

\section{Syspasis lineator (Fabricius, 1781)}

\section{Nomenclature:}

Ichneumon lineator Fabricius, 1781

trilineata (Gmelin, 1790, Ichneumon)

umbraculosa (Gravenhorst, 1829, Ichneumon)

binotata (Stephens, 1835, Ichneumon)

brischkii (Ratzeburg, 1852, Ichneumon)

adulator (Tischbein, 1881, Ichneumon)

calculosa (Berthoumieu, 1903, Ichneumon)

Distribution: England, Scotland, Ireland, Isle of Man

\section{Syspasis rufina (Gravenhorst, 1820)}

Nomenclature:

Ichneumon rufinus Gravenhorst, 1820

?judex (Müller, 1776, Ichneumon)

Distribution: England

\section{Syspasis scutellator (Gravenhorst, 1829)}

Nomenclature:

Ichneumon scutellator Gravenhorst, 1829

rufescens (Berthoumieu, 1894, Ichneumon) preocc., unavailable

Distribution: England 
Genus Trogus Panzer, 1806

Nomenclature:

DINOTOMUS Förster, 1869

\section{Trogus lapidator (Fabricius, 1787)}

Nomenclature:

Ichneumon lapidator Fabricius, 1787

anthracinus (Scopoli, 1763, Sphex) nom. ob. (Horstmann 2001a)

coerulator (Weber, 1795, Ichneumon)

coerulator (Fabricius, 1804, Ichneumon) preocc.

saxator (Thunberg, 1824, Ichneumon)

fuscipennis Gravenhorst, 1829

violaceus (Mocsáry,1883, Psilomastax)

cyaneipennis Costa, 1886

cyaneus (Kriechbaumer,1892, Psilomastax)

romani Uchida,1942

brevicaudae Heinrich,1975

panzeri Carlson,1975

Distribution: England

Notes: Distribution data from Shaw (1978); the English population has been treated as belonging to the subspecies panzeri, synonymised by Wahl and Sime (2006), along with many other names.

\section{Tribe ICHNEUMONINI Latreille, 1802}

Nomenclature:

JOPPINI Kriechbaumer, 1898

Genus Achaius Cameron, 1903

Achaius margineguttatus (Gravenhorst, 1829)

Nomenclature:

Ichneumon margineguttatus Gravenhorst, 1829

novitius (Wesmael, 1854, Amblyteles)

luteosignatus (Pic, 1914, Amblyteles) 
Distribution: England, Scotland

Achaius oratorius (Fabricius, 1793)

Nomenclature:

Ichneumon oratorius Fabricius, 1793

?dealbatus (Gmelin, 1790, Ichneumon)

cingulatorius (Weber, 1801, Ichneumon) synonymy by Horstmann (1997)

atramentarius (Gravenhorst, 1829, Ichneumon)

cingulipes (Stephens, 1835, Ichneumon)

bipunctus (Berthoumieu, 1896, Amblyteles) unavailable

theresae (Pic, 1897, Amblyteles)

albocingulatus (Strobl, 1901, Ichneumon)

marginalis (Habermehl, 1903, Amblyteles)

bellus (Habermehl, 1917, Spiloteles)

Distribution: England, Scotland, Wales, Ireland, Isle of Man

\section{Genus Acolobus Wesmael, 1845}

\section{Acolobus albimanus (Gravenhorst, 1829)}

Nomenclature:

Ichneumon albimanus Gravenhorst, 1829

buyssoni (Berthoumieu, 1892, Ichneumon)

\section{Acolobus sericeus Wesmael, 1845}

Distribution: England

\section{Genus Amblyteles Wesmael, 1845}

Notes: Many species previously placed in Amblyteles (Perkins 1960) are now classified in Achaius, Diphyus, Eutanyacra, Limerodops, Obtusodonta, Spilothyrateles and Triptognathus. 
Amblyteles armatorius (Forster, 1771)

Nomenclature:

Ichneumon armatorius Forster, 1771

fasciatorius (Fabricius, 1775, Ichneumon)

notatorius (Villers, 1789, Ichneumon)

dimicatorius (Gmelin, 1790, Ichneumon)

signatorius (Olivier, 1792, Ichneumon)

diversorius (Stephens, 1835, Ichneumon)

regius Tischbein, 1868

Distribution: England, Scotland, Ireland, Isle of Man

Genus Aoplus Tischbein, 1874

Aoplus altercator (Wesmael, 1855)

Nomenclature:

Ichneumon altercator Wesmael, 1855

Distribution: England, Scotland, Ireland

\section{Aoplus castaneus (Gravenhorst, 1820)}

\section{Nomenclature:}

Ichneumon castaneus Gravenhorst, 1820

rufoniger (Tischbein, 1881, Exephanes)

mesopyrrhus (Kriechbaumer, 1893, Ichneumon) synonymy by Hinz and Horstmann (2000)

subniger (Berthoumieu, 1894, Ichneumon) unavailable

fieschensis (Pic, 1926, Ichneumon)

royatensis (Pic, 1926, Ichneumon)

Distribution: England, Scotland, Ireland

\section{Aoplus defraudator (Wesmael, 1845)}

Nomenclature:

Ichneumon defraudator Wesmael, 1845

angustus (Tischbein, 1863, Ichneumon) 
jemilleri (Kriechbaumer, 1893, Ichneumon) synonymy by Hinz and Horstmann (2000) sabaudus (Berthoumieu, 1904, Ichneumon)

Distribution: Scotland, Ireland

Aoplus ochropis (Gmelin, 1790)

Nomenclature:

Ichneumon ochropis Gmelin, 1790

ephippium (Rudow, 1886, Cryptus)

Distribution: England, Scotland, Ireland

\section{Aoplus rubricosus (Holmgren, 1864)}

Nomenclature:

Ichneumon rubricosus Holmgren, 1864

Distribution: England

\section{Aoplus ruficeps (Gravenhorst, 1829)}

\section{Nomenclature:}

Ichneumon ruficeps Gravenhorst, 1829

leucocrepis (Wesmael, 1857, Ichneumon)

maximorufus (Pic, 1927, Ichneumon)

Distribution: England, Scotland, Ireland

Genus Baranisobas Heinrich, 1972

\section{Baranisobas ridibundus (Gravenhorst, 1829)}

Nomenclature:

Ichneumon ridibundus Gravenhorst, 1829

hassicus (Ratzeburg, 1848, Ichneumon)

variegator (Tischbein, 1881, Exephanes) synonymy by Hinz and Horstmann (2000)

polystictus (Kriechbaumer, 1887, Ichneumon)

instabilis (Berthoumieu, 1897, Ichneumon) preocc.

evianensis (Pic, 1902, Ichneumon) 
insperatus (Dalla Torre, 1902, Ichneumon)

fallaciosus (Berthoumieu, 1903, Ichneumon)

bulsanensis (Smits\$)

Distribution: England, Ireland

\section{Genus Barichneumon Thomson, 1893}

Notes: Many species previously placed in Barichneumon (Perkins 1960) are now classified in Baranisobas, Stenobarichneumon, Virgichneumon and Vulgichneumon.

\section{Barichneumon anator (Fabricius, 1793)}

Nomenclature:

Ichneumon anator Fabricius, 1793

?biscutatus (Gmelin, 1790, Ichneumon)

bulimorius (Thunberg, 1824, Ichneumon)

dealbator (Thunberg, 1824, Ichneumon)

femoratorius (Thunberg, 1824, Ichneumon)

retusorius (Thunberg, 1824, Ichneumon)

henschi (Schmiedeknecht, 1929, Ichneumon)

Distribution: England, Ireland

\section{Barichneumon bilunulatus (Gravenhorst, 1829)}

\section{Nomenclature:}

Ichneumon bilunulatus Gravenhorst, 1829

sexlineatus (Gravenhorst, 1829, Ichneumon)

piniperdae (Hartig, 1838, Phygadeuon)

troscheli (Ratzeburg, 1844, Ichneumon)

imitator (Kriechbaumer, 1882, Ichneumon) preocc.

moraguesi (Kriechbaumer, 1894, Ichneumon)

Distribution: England, Ireland

\section{Barichneumon chionomus (Wesmael, 1845)}

\section{Nomenclature:}

Ichneumon chionomus Wesmael, 1845 
Distribution: England, Scotland, Ireland

\section{Barichneumon derogator (Wesmael, 1845)}

Nomenclature:

Ichneumon derogator Wesmael, 1845

Distribution: England

Barichneumon gemellus (Gravenhorst, 1829)

Nomenclature:

Ichneumon gemellus Gravenhorst, 1829

inversus (Kriechbaumer, 1893, Ichneumon) preocc.

carri Habermehl, 1923

controversus (Schmiedeknecht, 1928, Ichneumon)

rubricans (Schmiedeknecht, 1929, Ichneumon)

semirufus (Schmiedeknecht, 1929, Ichneumon) preocc.

constantineanui (Heinrich, 1972, Stenobarichneumon)

Distribution: England, Scotland, Ireland, Isle of Man

Barichneumon heracliana (Bridgman, 1884)

Nomenclature:

Ichneumon heracliana Bridgman, 1884

Distribution: England, Isle of Man

Barichneumon peregrinator (Linnaeus, 1758)

Nomenclature:

Ichneumon peregrinator Linnaeus, 1758

scriptorius (Thunberg, 1824, Ichneumon)

vacillatorius (Gravenhorst, 1829, Ichneumon) preocc.

Distribution: England, Scotland, Ireland, Isle of Man

\section{Barichneumon plagiarius (Wesmael, 1848)}

Nomenclature:

Ichneumon plagiarius Wesmael, 1848 
merkli (Kiss, 1915, Plectocryptus) synonymy by

\section{Barichneumon praeceptor (Thunberg, 1824)}

Nomenclature:

Ichneumon praeceptor Thunberg, 1824

procerus (Gravenhorst, 1829, Ichneumon)

derivator (Wesmael, 1845, Ichneumon)

Iunuliger (Kriechbaumer, 1890, Ichneumon) synonymy by Horstmann (2006a)

kervillei (Berthoumieu, 1903, Ichneumon)

cenisiensis (Berthoumieu, 1906, Ichneumon)

atricornis (Pic, 1926, Ichneumon)

Distribution: England, Scotland

\section{Genus Chasmias Ashmead, 1900}

Nomenclature:

CHASMODES Wesmael, 1845

\section{Chasmias motatorius (Fabricius, 1775)}

Nomenclature:

Ichneumon motatorius Fabricius, 1775

importunus (Tischbein, 1874, Ichneumon)

transitorius (Berthoumieu, 1894, Chasmodes) unavailable

atronotatus Pic, 1917

berthoumieui Pic, 1917

bicoloripes Pic, 1917

diversipes Pic, 1917

rufonotatus Pic, 1917

Distribution: England, Ireland, Isle of Man

\section{Chasmias paludator (Desvignes, 1854)}

\section{Nomenclature:}

Ichneumon paludator Desvignes, 1854

paludicola (Wesmael, 1857, Chasmodes)

dissimulator (Tischbein, 1881, Ichneumon) 
Distribution: England

Genus Cratichneumon Thomson, 1893

\section{Cratichneumon albifrons (Stephens, 1835)}

Nomenclature:

Ichneumon albifrons Stephens, 1835

gravenhorstii (Fonscolombe, 1847, Ichneumon)

grandiceps (Thomson, 1887, Ichneumon)

Distribution: England, Ireland

\section{Cratichneumon coruscator (Linnaeus, 1758)}

Nomenclature:

Ichneumon coruscator Linnaeus, 1758

corruscator misspelling

ambulator (Müller, 1774, Ichneumon)

alacer (Gravenhorst, 1829, Ichneumon)

luridus (Gravenhorst, 1829, Ichneumon)

gasterator (Stephens, 1835, Ichneumon)

metaxanthus (Hartig, 1838, Ichneumon)

binotatus (Desvignes, 1856, Ichneumon) preocc.

pyrenaeus (Tischbein, 1882, Ichneumon)

Distribution: England, Wales, Ireland

\section{Cratichneumon culex (Müller, 1776)}

\section{Nomenclature:}

Ichneumon culex Müller, 1776

tibialis (Geoffroy, 1785, Ichneumon)

clavipes (Gmelin, 1790, Ichneumon)

leucostoma (Gmelin, 1790, Ichneumon)

quadricolor (Gmelin, 1790, Ichneumon)

versicolor (Gmelin, 1790, Ichneumon)

annulator (Fabricius, 1793, Ichneumon) preocc.

fabricator (Fabricius, 1793, Ichneumon) synonymy by Horstmann (2001a) 


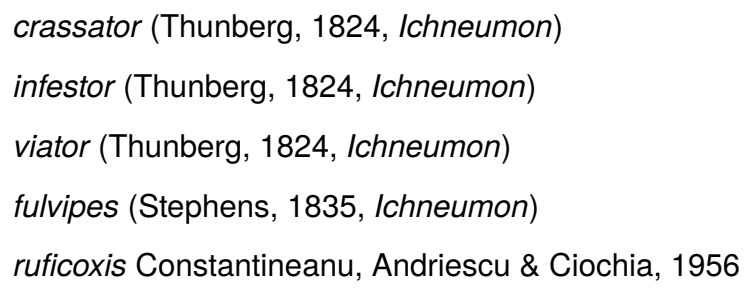

Distribution: England, Scotland, Wales, Ireland

\section{Cratichneumon flavifrons (Schrank, 1781)}

\section{Nomenclature:}

Ichneumon flavifrons Schrank, 1781

fabricator misident. (Horstmann 2001a)

frontalis (Geoffroy, 1785, Ichneumon)

tricolor (Razoumowsky, 1789, Ichneumon) preocc.

generator (Olivier, 1792, Ichneumon)

maculifrons (Stephens, 1835, Ichneumon)

pyrrhopus (Stephens, 1835, Ichneumon)

extinctus (Ratzeburg, 1844, Ichneumon)

hartigii (Ratzeburg, 1844, Ichneumon)

impugnator (Wesmael, 1845, Ichneumon) preocc.

spiracularis (Tischbein, 1881, Ichneumon)

baudyi (Pic, 1902, Ichneumon)

Distribution: England, Scotland, Ireland

\section{Cratichneumon fugitivus (Gravenhorst, 1829)}

Nomenclature:

Ichneumon fugitivus Gravenhorst, 1829

rutilus (Holmgren, 1864, Ichneumon) preocc.

capreolus (Berthoumieu, 1899, Ichneumon)

Distribution: England, Ireland

\section{Cratichneumon infidus (Wesmael, 1848)}

\section{Nomenclature:}

Ichneumon infidus Wesmael, 1848 
liostylus (Thomson, 1897, Ichneumon)

Distribution: England, Ireland

\section{Cratichneumon jocularis (Wesmael, 1848)}

Nomenclature:

Ichneumon jocularis Wesmael, 1848

punctifrons (Holmgren, 1864, Ichneumon)

semiannulatus (Kriechbaumer, 1895, Ichneumon) preocc., synonymy by Horstmann (2002a)

angusteannulatus (Strobl, 1901, Ichneumon)

Distribution: England, Scotland, Ireland

Notes: Horstmann (2006a) removed parvulus (Kriechbaumer, 1887, Ichneumon) from synonymy.

\section{Cratichneumon luteiventris (Gravenhorst, 1820)}

Nomenclature:

Ichneumon luteiventris Gravenhorst, 1820

indictus (Tischbein, 1874, Ichneumon)

\section{Cratichneumon pallitarsis (Thomson, 1887)}

\section{Nomenclature:}

Ichneumon pallitarsis Thomson, 1887

palliditarsis (Berthoumieu, 1895, Ichneumon)

\section{Distribution: Scotland}

Notes: NMS, det. Riedel, added here; it is unclear whether J.F. Perkins overlooked earlier records of this species from Scotland or whether these were based on misidentifications.

\section{Cratichneumon rufifrons (Gravenhorst, 1829)}

\section{Nomenclature:}

Ichneumon rufifrons Gravenhorst, 1829

frontatorius (Fabricius, 1793, Ichneumon) nom. ob., synonymy by Horstmann (2001a)

pallidiatorius (Gravenhorst, 1829, Ichneumon) 
Distribution: England, Scotland, Ireland

\section{Cratichneumon semirufus (Gravenhorst, 1820)}

Nomenclature:

Ichneumon semirufus Gravenhorst, 1820

nigroscutatus (Berthoumieu, 1895, Ichneumon)

Distribution: England, Ireland

\section{Cratichneumon sicarius (Gravenhorst, 1829)}

Nomenclature:

Ichneumon sicarius Gravenhorst, 1829

nigratorius (Panzer, 1800, Ichneumon) preocc.

ingratorius (Gravenhorst, 1829, Ichneumon)

jugatus (Gravenhorst, 1829, Ichneumon)

alboannulatus (Strobl, 1901, Ichneumon)

atrocellaris (Pic, 1927, Ichneumon)

forticornis (Hedwig, 1956, Hoplismenus)

Distribution: England, Ireland

\section{Cratichneumon versator (Thunberg, 1824)}

Nomenclature:

Ichneumon versator Thunberg, 1824

pallifrons (Gravenhorst, 1829, Ichneumon)

pallidifrons (Marshall, 1872, Ichneumon)

anotylus (Thomson, 1893, Ichneumon) synonymy by Riedel (2014)

Distribution: England, Scotland, Ireland

\section{Cratichneumon viator (Scopoli, 1763)}

Nomenclature:

Ichneumon viator Scopoli, 1763

nigritarius (Gravenhorst, 1820, Ichneumon)

obfuscator (Thunberg, 1824, Ichneumon) preocc.

aethiops (Gravenhorst, 1829, Ichneumon) 
pinetorum (Ratzeburg, 1852, Ichneumon)

parviscopa (Thomson, 1893, Ichneumon)

brischkei (Berthoumieu, 1895, Ichneumon) preocc., unavailable

nuperus (Berthoumieu, 1910, Ichneumon)

charadensis (Pic, 1924, Ichneumon)

atrifemur (Fahringer, 1943, Ichneumon)

rufipes Constantineanu, 1954

Distribution: England, Scotland, Wales, Ireland

\section{Cratichneumon vulpecula (Kriechbaumer, 1875)}

Nomenclature:

Ichneumon vulpecula Kriechbaumer, 1875

pseudogracilentus (Strobl, 1901, Ichneumon)

hemerythrus Heinrich, 1949

Distribution: Scotland

Notes: NMS, det. Hilpert, added here

\section{Genus Crypteffigies Heinrich, 1961}

\section{Crypteffigies albilarvatus (Gravenhorst, 1820)}

Nomenclature:

Ichneumon albilarvatus Gravenhorst, 1820

obscurior (Berthoumieu, 1895, Ichneumon) preocc., unavailable deubeli (Kiss, 1924, Megaplectes)

Distribution: England, Scotland, Wales, Ireland

\section{Crypteffigies lanius (Gravenhorst, 1829)}

Nomenclature:

Ichneumon lanius Gravenhorst, 1829

aberrans (Taschenberg, 1865, Phygadeuon)

muelleri (Kiss, 1929, Plectocryptus)

Distribution: England, Scotland, Ireland 


\section{Crypteffigies pseudocryptus (Wesmael, 1857)}

Nomenclature:

Ichneumon pseudocryptus Wesmael, 1857

punctulatus (Kriechbaumer, 1891, Microcryptus)

Distribution: England

\section{Genus Crytea Cameron, 1906}

\section{Crytea sanguinator (Rossi, 1794)}

Nomenclature:

Ichneumon sanguinator Rossi, 1794

ruficollis (Stephens, 1835, Ichneumon) preocc.

discrepator (Wesmael, 1845, Ichneumon)

sanguinator (Desvignes, 1856, Cryptus) preocc.

multifarius (Berthoumieu, 1897, Ichneumon)

Distribution: England, Scotland, Ireland

\section{Genus Ctenichneumon Thomson, 1894}

Nomenclature:

DOCHYTELES Berthoumieu, 1904

\section{Ctenichneumon castigator (Fabricius, 1793)}

Nomenclature:

Ichneumon castigator Fabricius, 1793

?certator (Müller, 1776, Ichneumon)

?abrogator (Schrank, 1781, Ichneumon)

?cardui (Schrank, 1786, Ichneumon)

?adustus (Gmelin, 1790, Ichneumon)

?ruficingulus (Schrank, 1802, Ichneumon)

Distribution: England, Ireland

\section{Ctenichneumon devylderi (Holmgren, 1871)}

Nomenclature:

Amblyteles devylderi Holmgren, 1871 
ineptus (Holmgren, 1871, Amblyteles)

tischbeini (Berthoumieu, 1896, Amblyteles) synonymy by Horstmann (2004c)

Distribution: England

\section{Ctenichneumon divisorius (Gravenhorst, 1820)}

\section{Nomenclature:}

Ichneumon divisorius Gravenhorst, 1820

obsoletorius (Fabricius, 1793, Ichneumon) nom. ob., synonymy by Horstmann (2001a)

baeticus (Spinola, 1843, Ichneumon)

clipeator (Habermehl, 1917, Dochyteles)

Distribution: England, Scotland

\section{Ctenichneumon edictorius (Linnaeus, 1758)}

\section{Nomenclature:}

Ichneumon edictorius Linnaeus, 1758

gladiatorius (Müller, 1776, Ichneumon)

fuscipes (Geoffroy, 1785, Ichneumon)

trichrous (Gmelin, 1790, Ichneumon)

erectorius (Fabricius, 1798, Ichneumon)

calceatorius (Panzer, 1801, Ichneumon)

amputatorius (Panzer, 1804, Ichneumon)

fossorius (Gravenhorst, 1820, Ichneumon) synonymy by Horstmann (2000b)

pallipes (Gravenhorst, 1820, Ichneumon)

depressorius (Thunberg, 1824, Ichneumon)

incertorius (Thunberg, 1824, Ichneumon)

perileucus (Gravenhorst, 1829, Ichneumon)

cognatus (Stephens, 1833, Ichneumon) synonymy by Horstmann (2000b)

nigricornis (Spinola, 1843, Ichneumon)

lotharingicus (Rudow, 1888, Amblyteles)

nigroscutellatus (Kriechbaumer, 1894, Amblyteles) unavailable

pallidipes (Dalla Torre, 1902, Amblyteles)

Distribution: England, Ireland 
Ctenichneumon funereus (Geoffroy, 1785)

Nomenclature:

Ichneumon funereus Geoffroy, 1785

funerarius (Olivier, 1792, Ichneumon)

Distribution: England, Wales

Ctenichneumon inspector (Wesmael, 1845)

Nomenclature:

Amblyteles inspector Wesmael, 1845

nigriventris (Berthoumieu, 1896, Amblyteles)

brunnicans (Constantineanu, 1956, Amblyteles)

Distribution: England

Ctenichneumon melanocastanus (Gravenhorst, 1820)

Nomenclature:

Ichneumon melanocastanus Gravenhorst, 1820

rubroater (Ratzeburg, 1852, Ichneumon)

erythropygus (Rudow, 1888, Amblyteles) preocc.

Distribution: England

Ctenichneumon messorius (Gravenhorst, 1820)

Nomenclature:

Ichneumon messorius Gravenhorst, 1820

montivagus (Giraud, 1877, Amblyteles)

Distribution: England

\section{Ctenichneumon nitens (Christ, 1791)}

Nomenclature:

Ichneumon nitens Christ, 1791

?glabratorius (Müller, 1776, Ichneumon)

vespertinus (Christ, 1791, Ichneumon)

mesocastanus (Gravenhorst, 1820, Ichneumon)

nigrocastaneus (Berthoumieu, 1896, Amblyteles) unavailable 
Distribution: England

\section{Ctenichneumon panzeri (Wesmael, 1845)}

Nomenclature:

Amblyteles panzeri Wesmael, 1845

flavocinctus (Desvignes, 1856, Ichneumon)

vexillarius (Tischbein, 1874, Amblyteles)

rufescens Morley, 1903

denticornis (Strobl, 1904, Amblyteles) synonymy by Horstmann (1999a)

styriacus (Strobl, 1904, Amblyteles) synonymy by Horstmann (1999a)

wormatiensis (Habermehl, 1909, Amblyteles)

nigrifemur (Ulbricht, 1926, Amblyteles) unavailable

rufifemur (Ulbricht, 1926, Amblyteles) unavailable

Distribution: England, Ireland, Isle of Man

\section{Genus Ctenochares Förster, 1869}

\section{Ctenochares bicolorus (Linnaeus, 1767)}

Nomenclature:

Ichneumon bicolorus Linnaeus, 1767

instructor (Fabricius, 1793, Ichneumon)

deustor (Thunberg, 1824, Ichneumon)

rufator (Thunberg, 1824, Ichneumon)

apicalis (Wiedemann, 1824, Ichneumon)

apicalis (Brullé, 1846, Joppa)

xanthomelas (Brullé, 1846, Ichneumon)

Notes: Added by Jones (2001); possibly inadvertently introduced, as this is a widespread species in the Old World tropics, but it is known from as far north as Spain (M.R. Shaw, pers. comm.). 
Genus Deuterolabops Heinrich, 1975

Deuterolabops eupitheciae (Brischke, 1878)

Nomenclature:

Ichneumon eupitheciae Brischke, 1878

pulchellatus (Bridgman, 1889, Ichneumon)

Distribution: England, Scotland

Genus Diphyus Kriechbaumer, 1890

Nomenclature:

PHYSCOTELES Berthoumieu, 1904

\section{Diphyus amatorius (Müller, 1776)}

Nomenclature:

Ichneumon amatorius Müller, 1776

laboratorius (Fabricius, 1793, Ichneumon) preocc.

nigronotatus (Pic, 1908, Amblyteles)

Distribution: England, Scotland, Ireland

\section{Diphyus castanopyga (Stephens, 1835)}

Nomenclature:

Ichneumon castanopyga Stephens, 1835

rubriventris (Wesmael, 1845, Amblyteles)

bicristatus (Strobl, 1901, Ichneumon)

Distribution: England, Scotland, Ireland

\section{Diphyus gradatorius (Thunberg, 1824)}

Nomenclature:

Ichneumon gradatorius Thunberg, 1824

egregius (Gravenhorst, 1829, Ichneumon)

sibiricus (Mocsáry, 1878, Amblyteles)

illustris (Kriechbaumer, 1894, Ichneumon) synonymy by Horstmann (2006a)

carlsbadensis (Pic, 1914, Ichneumon)

rufotriangularis (Pic, 1914, Ichneumon) 
Distribution: Scotland

Diphyus longigena (Thomson, 1888)

Nomenclature:

Amblyteles longigena Thomson, 1888

inermis (Berthoumieu, 1892, Amblyteles)

Distribution: England, Scotland, Wales

Diphyus luctatorius (Linnaeus, 1758)

Nomenclature:

Ichneumon luctatorius Linnaeus, 1758

erratorius (Thunberg, 1824, Ichneumon)

litigiosus (Wesmael, 1854, Amblyteles)

oblongatus (Tischbein, 1873, Ichneumon)

Distribution: England, Scotland, Ireland

\section{Diphyus mercatorius (Fabricius, 1793)}

Nomenclature:

Ichneumon mercatorius Fabricius, 1793

nugatorius (Fabricius, 1794, Ichneumon) synonymy by Horstmann (2001a)

nigricaudus (Berthoumieu, 1896, Amblyteles) preocc., unavailable

Distribution: England, Ireland

Notes: English record from P. Whitehead (pers. comm.)

\section{Diphyus monitorius (Panzer, 1801)}

Nomenclature:

Ichneumon monitorius Panzer, 1801

quadrimaculatus (Schrank, 1802, Ichneumon) preocc.

interruptorius (Fabricius, 1804, Ichneumon)

Diphyus ochromelas (Gmelin, 1790)

Nomenclature:

Ichneumon ochromelas Gmelin, 1790 
pulchellus (Christ, 1791, Ichneumon)

negatorius (Fabricius, 1793, Ichneumon)

ornatorius (Panzer, 1800, Ichneumon)

umbratorius (Thunberg, 1824, Ichneumon)

sartorius (Gravenhorst, 1829, Ichneumon)

canaliculatus (Saussure, 1892, Ichneumon) preocc.

nigripes (Seyrig, 1928, Spiloteles)

trialbatus (Constantineanu, 1954, Amblyteles)

Distribution: England

\section{Diphyus palliatorius (Gravenhorst, 1829)}

\section{Nomenclature:}

Ichneumon palliatorius Gravenhorst, 1829

?defensorius (Villers, 1789, Ichneumon)

erythropygus (Gravenhorst, 1829, Ichneumon)

spoliator (Wesmael, 1845, Amblyteles)

ancipiterus (Desvignes, 1856, Ichneumon)

dubitatus (Desvignes, 1856, Ichneumon)

ochraceus (Tischbein, 1873, Ichneumon)

aequivocus (Tischbein, 1879, Ichneumon)

infinitus (Tischbein, 1879, Ichneumon)

gemmatus (Tischbein, 1881, Ichneumon)

laetus (Tischbein, 1881, Ichneumon) preocc.

brunneonotatus (Pic, 1898, Amblyteles)

atratus (Berthoumieu, 1901, Amblyteles)

rufotriangularis (Pic, 1915, Amblyteles) preocc.

subniger (Habermehl, 1929, Amblyteles) preocc.

Distribution: England, Scotland, Wales, Ireland

\section{Diphyus quadripunctorius (Müller, 1776)}

\section{Nomenclature:}

Ichneumon quadripunctorius Müller, 1776

constellatus (Geoffroy, 1785, Ichneumon) 
citreus (Christ, 1791, Ichneumon) synonymy by Horstmann (2001a) intratorius (Fabricius, 1793, Ichneumon) synonymy by Horstmann (2001a) jubilatorius (Müller, 1776, Ichneumon) synonymy by Horstmann (2001a) pedatorius (Fabricius, 1793, Ichneumon) synonymy by Horstmann (2001a) natatorius (Fabricius, 1798, Ichneumon) mediatorius (Panzer, 1801, Ichneumon) bipunctatus (Schrank, 1802, Ichneumon) preocc. desertorius (Panzer, 1806, Ichneumon) xanthozosmus (Gravenhorst, 1820, Ichneumon) natator (Zetterstedt, 1838, Ichneumon) infestorius (Fonscolombe, 1847, Ichneumon) notatorius (Marshall, 1872, Amblyteles) bipunctatus (Rudow, 1888, Amblyteles) preocc. schrammi (Pic, 1827, Amblyteles) preocc.

Distribution: England, Wales, Ireland

\section{Diphyus raptorius (Linnaeus, 1758)}

Nomenclature:

Ichneumon raptorius Linnaeus, 1758

quadriguttorius (Thunberg, 1824, Ichneumon)

gravenhorstii (Wesmael, 1836, Ichneumon) preocc.

flavaginis (Schiødte, 1839, Ichneumon) synonymy by Horstmann (2004a)

flavolaetus (Berthoumieu, 1896, Amblyteles) preocc., unavailable quercus (Pic, 1917, Amblyteles)

Distribution: England

\section{Diphyus salicatorius (Gravenhorst, 1820)}

\section{Nomenclature:}

Ichneumon salicatorius Gravenhorst, 1820

cinctorius (Stephens, 1835, Ichneumon) preocc.

indocilis (Wesmael, 1845, Amblyteles) synonymy by Horstmann (1998b)

relucens (Desvignes, 1856, Ichneumon)

inaciculatus (Pic, 1927, Amblyteles) 
nigrobinotatus (Pic, 1927, Amblyteles)

Distribution: England, Scotland, Wales

Diphyus septemguttatus (Gravenhorst, 1829)

Nomenclature:

Ichneumon septemguttatus Gravenhorst, 1829

wesmaeli (Tischbein, 1868, Amblyteles)

triplicatus (Thomson, 1894, Amblyteles)

Distribution: England, Ireland

Diphyus trifasciatus (Gravenhorst, 1829)

Nomenclature:

Ichneumon trifasciatus Gravenhorst, 1829

triangulator (Stephens, 1835, Ichneumon)

daguini (Pic, 1920, Amblyteles)

Distribution: England, Scotland, Ireland

\section{Genus Eristicus Wesmael, 1845}

Eristicus clarigator (Wesmael, 1845)

Nomenclature:

Ichneumon clarigator Wesmael, 1845

pachycephalus (Rudow, 1886, Phygadeuon)

cephalotes (Berthoumieu, 1906, Amblyteles)

Distribution: England

Eristicus clericus (Gravenhorst, 1829)

Nomenclature:

Ichneumon clericus Gravenhorst, 1829

eucephalus (Wesmael, 1848, Ichneumon)

Distribution: England 


\section{Genus Eupalamus Wesmael, 1845}

\section{Eupalamus lacteator (Gravenhorst, 1829)}

Nomenclature:

Ichneumon lacteator Gravenhorst, 1829

fenestrator (Zetterstedt, 1838, Ichneumon)

depexus (Wesmael, 1845, Ichneumon)

albatus (Tischbein, 1879, Ichneumon)

Distribution: England

\section{Eupalamus wesmaeli (Thomson, 1886)}

Nomenclature:

Ichneumon wesmaeli Thomson, 1886

Distribution: England

Genus Eutanyacra Cameron, 1903

Eutanyacra crispatoria (Linnaeus, 1758)

Nomenclature:

Ichneumon crispatorius Linnaeus, 1758

limbatoria (Thunberg, 1824, Ichneumon)

rufatoria (Gravenhorst, 1829, Ichneumon) preocc.

nemoralis (Tischbein, 1876, Ichneumon) preocc.

laticincta (Rudow, 1888, Amblyteles)

bicuspis (Berthoumieu, 1892, Amblyteles)

pallidior (Pic, 1898, Amblyteles)

Distribution: England, Ireland

\section{Eutanyacra glaucatoria (Fabricius, 1793)}

Nomenclature:

Ichneumon glaucatorius Fabricius, 1793

?albiventris (Gmelin, 1790, Ichneumon)

hungarica (Tischbein, 1868, Amblyteles)

sicula (Rudow, 1888, Amblyteles) 


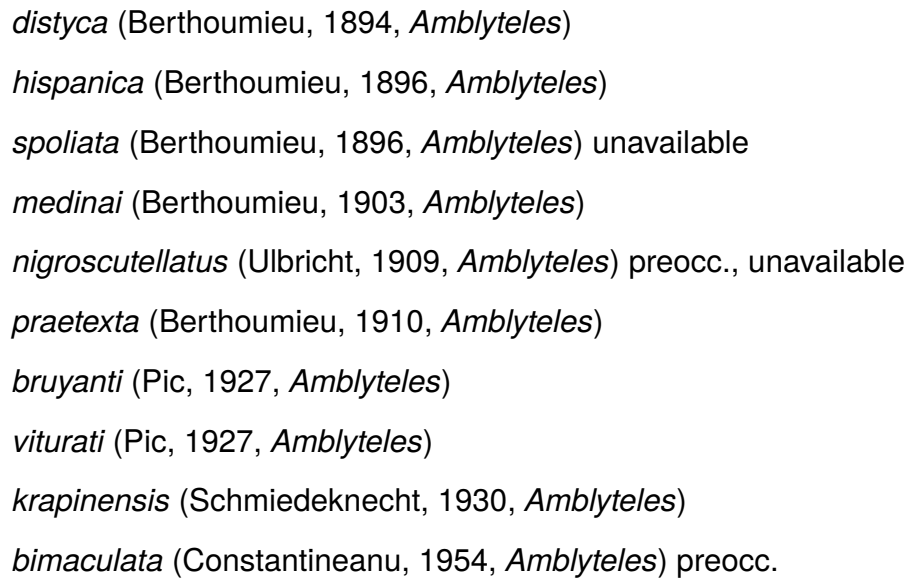

Distribution: England, Ireland

Notes: Eutanyacra ruficornis (Berthoumieu, 1894, Eurylabus) was removed from synonymy by Horstmann (2006c).

\section{Eutanyacra pallidicornis (Gravenhorst, 1829)}

\section{Nomenclature:}

Ichneumon pallidicornis Gravenhorst, 1829

dimidiata (Stephens, 1835, Ichneumon) preocc.

Distribution: England, Scotland

\section{Eutanyacra picta (Schrank, 1776)}

\section{Nomenclature:}

Ichneumon pictus Schrank, 1776

laboratoria (Müller, 1776, Ichneumon)

sanguinea (Christ, 1791, Ichneumon)

vadatoria (Illiger, 1807, Ichneumon)

affirmatoria (Thunberg, 1824, Ichneumon)

concinnus (Stephens, 1829, Ichneumon) synonymy by Horstmann (2000b)

Distribution: England 


\section{Genus Exephanes Wesmael, 1845}

Nomenclature:

OCTATOMUS Tischbein, 1881

Notes: Distribution data and synonymy from Hinz and Horstmann (2000) and BMNH.

Exephanes fulvescens Vollenhoven, 1875

Nomenclature:

ulbrichti Hinz, 1957

Distribution: England, Ireland

Exephanes ischioxanthus (Gravenhorst, 1829)

Nomenclature:

Ichneumon ischioxanthus Gravenhorst, 1829

exulans (Gravenhorst, 1829, Ichneumon)

hilaris (Gravenhorst, 1829, Ichneumon)

subnudus Tischbein, 1881

Distribution: England, Ireland

\section{Exephanes occupator (Gravenhorst, 1829)}

\section{Nomenclature:}

Ichneumon occupator Gravenhorst, 1829

contaminatus (Gravenhorst, 1829, Ichneumon)

munki (Kriechbaumer, 1893, Ichneumon) synonymy by Horstmann (2006a)

munki Kriechbaumer, 1895 preocc.

uniguttatus Kriechbaumer, 1895

unipunctatus Strobl, 1901

Distribution: England, Wales, Ireland

\section{Exephanes riesei (Habermehl, 1916)}

\section{Nomenclature:}

Ichneumon riesei Habermehl, 1916

hoerhammeri Heinrich, 1949

amabilis Kreichbaumer, 1895 preocc. 
Distribution: England, Ireland

Notes: Transferred to Exephanes (from Ichneumon) by Hinz and Horstmann (2000).

\section{Exephanes venustus (Tischbein, 1876)}

Nomenclature:

Ichneumon venustus Tischbein, 1876

insidiator (Tischbein, 1876, Ichneumon)

caelebs Kreichbaumer, 1890

Distribution: Ireland

\section{Genus Gareila Heinrich, 1980}

\section{Gareila tenebrosa (Wesmael, 1845)}

Nomenclature:

Ichneumon tenebrosus Wesmael, 1845

nigricornis (Schmiedeknecht, 1930, Ichneumon) preocc.

Distribution: Scotland

Notes: NMS, det. Riedel, added here

\section{Genus Hepiopelmus Wesmael, 1845}

Nomenclature:

EPIOPELMUS Dalla Torre, 1902

\section{Hepiopelmus melanogaster (Gmelin, 1790)}

\section{Nomenclature:}

Ichneumon melanogaster Gmelin, 1790

leucostigmus (Gravenhorst, 1820, Ichneumon)

maculiventris (Desvignes, 1856, Ichneumon)

aureosericeus Taschenberg, 1866

incorruptus (Holmgren, 1871, Amblyteles)

palliventris (Rudow, 1888, Amblyteles)

annulitarsis (Pic, 1914, Acolobus)

maculipes Hellén, 1951 
Distribution: England, Scotland, Wales, Ireland, Isle of Man

\section{Hepiopelmus variegatorius (Panzer, 1800)}

Nomenclature:

Ichneumon variegatorius Panzer, 1800

notatorius (Panzer, 1801, Ichneumon) preocc.

flavoguttatus (Gravenhorst, 1829, Ichneumon)

Distribution: England, Wales, Ireland

\section{Genus Homotherus Förster, 1869}

\section{Homotherus locutor (Thunberg, 1824)}

Nomenclature:

Ichneumon locutor Thunberg, 1824

labiatorius (Thunberg, 1824, Ichneumon)

albicinctus (Gravenhorst, 1829, Ichneumon)

albiceps (Hartig, 1838, Phygadeuon)

festinatorius (Zetterstedt, 1838, Ichneumon)

lautus (Tischbein, 1868, Ichneumon)

ruber (Kiss, 1924, Proscus)

Distribution: England, Scotland, Wales, Ireland

\section{Homotherus magus (Wesmael, 1855)}

Nomenclature:

Ichneumon magus Wesmael, 1855

clavipes (Möller, 1883, Ichneumon) preocc.

nitidus (Bridgman, 1886, Phaeogenes)

Distribution: England, Ireland

\section{Homotherus varipes (Gravenhorst, 1829)}

\section{Nomenclature:}

Ichneumon varipes Gravenhorst, 1829

costator (Donovan, 1810, Ichneumon) preocc., synonymy by Horstmann (2002b) 
decimator (Gravenhorst, 1829, Ichneumon)

laevis (Ratzeburg, 1844, Ichneumon)

pictipes (Holmgren, 1864, Ichneumon)

fallax (Habermehl, 1923, Cratichneumon) invalid

anglicanus (Schmiedeknecht, 1928, Ichneumon)

Distribution: England, Scotland, Wales, Ireland

\section{Genus Hoplismenus Gravenhorst, 1829}

Nomenclature:

PERITAENIUS Förster, 1869

TAENIASPIS Clément, 1927

\section{Hoplismenus albifrons Gravenhorst, 1829}

\section{Nomenclature:}

axillatorius misident.

?armatorius (Fabricius, 1787, Ichneumon) preocc.

albifrons Gravenhorst, 1829

perniciosus Gravenhorst, 1829

crassicornis (Rudow, 1883, Cryptus) preocc.

bellicosus (De Stefani, 1885, Ichneumon)

Distribution: England, Scotland, Ireland

Notes: Usually known as $H$. axillatorius (Thunberg) but Ichneumon axillatorius Thunberg, 1824 was found to be a senior synonym of Cyclolabus pactor (Wesmael) by Riedel (2014).

\section{Hoplismenus bidentatus (Gmelin, 1790)}

\section{Nomenclature:}

Ichneumon bidentatus Gmelin, 1790

moestus Gravenhorst, 1829

maurus (Marshall, 1873, Mesostenus)

ichneumonoides (Rudow, 1883, Cryptus)

berthoumieui Pic, 1897

spinosus (Morley, 1903, Dinotomus)

alpinus (Clément, 1927, Peritaenius) 
bavaricus (Clément, 1927, Peritaenius)

Distribution: England, Wales, Ireland

Notes: some distribution data from Whitehead (2003)

Hoplismenus bispinatorius (Thunberg, 1824)

Nomenclature:

Ichneumon bispinatorius Thunberg, 1824

annulatus Berthoumieu, 1894

nigripes Seyrig, 1927

rufitarsis Constantineanu, Andriescu \& Ciochia, 1956

Distribution: England, Scotland

Notes: NMS, det. Riedel, added here

\section{Genus Ichneumon Linnaeus, 1758}

Nomenclature:

BRACHYPTERUS Gravenhorst, 1829 preocc.

PTEROCORMUS Förster, 1850

Notes: Distribution data from Hilpert (1992), BMNH and NMS.

doubtfully placed species of Ichneumon:

[femorator Kirby, 1802 preocc., nom. dub.]

\section{Ichneumon albiger Wesmael, 1845}

Nomenclature:

tempestivus Holmgren, 1864

Distribution: England, Scotland, Ireland

\section{Ichneumon alius Tischbein, 1879}

Nomenclature:

eurycerus Thomson, 1890

dubiosus Habermehl, 1926

petrophilus Heinrich, 1951

Distribution: England, Scotland 
Ichneumon ? alpestris Holmgren, 1864

Notes: Added by Hilpert (1992); uncertain identification, based on female specimen.

Ichneumon analis Gravenhorst, 1829

Nomenclature:

nigroscutellatus Habermehl, 1916 preocc.

Ichneumon aquilonius Perkins, 1953

Distribution: England, Scotland, Ireland

Ichneumon bellipes Wesmael, 1845

Nomenclature:

medialis Wesmael, 1855

divergens Holmgren, 1864

strangulator Tischbein, 1876

evanidus Berthoumieu, 1892

orbitalis Kriechbaumer, 1894 preocc., unavailable

rasnitsyni Heinrich, 1978

Distribution: Scotland

Ichneumon bucculentus Wesmael, 1845

Nomenclature:

glaucus Tischbein, 1876

umbilicatus Valemberg, 1975

Distribution: England, Wales, Ireland

Ichneumon caloscelis Wesmael, 1845

Nomenclature:

caloscelus Marshall, 1872

decens (Berthoumieu, 1910, Amblyteles)

Distribution: England, Ireland 
Ichneumon cessator Müller, 1776

Nomenclature:

custodiator Fabricius, 1793

compunctor Stephens, 1835 preocc.

Distribution: England, Ireland

Ichneumon computatorius Müller, 1776

Nomenclature:

croceipes Wesmael, 1848

bicoloripes Tischbein, 1868

insolitus Berthoumieu, 1895 preocc., unavailable

Distribution: Ireland

Ichneumon confusor Gravenhorst, 1820

Nomenclature:

confusorius Gravenhorst, 1829

crassicornis Tischbein, 1873 preocc.

retectus Tischbein, 1873

atronotatus Pic, 1917

Distribution: England, Scotland, Wales, Ireland

Ichneumon crassifemur Thomson, 1886

Nomenclature:

sulphuratus Kriechbaumer, 1894 preocc.

Ichneumon deliratorius Linnaeus, 1758

Nomenclature:

alternatus Schrank, 1776

?fabricatorius Müller, 1776

palmarius Geoffroy, 1785

inflictorius Rossi, 1792

multiannulatus Gravenhorst, 1829

delirator Zetterstedt, 1838 
gmuendensis Pfeffer, 1913

schimitscheki Fahringer, 1943

Distribution: England, Scotland, Wales, Ireland

Notes: Heinrich transferred deliratorius, which is anomalous within Ichneumon, to Coelichneumon, which has been followed by most authors, but where deliratorius was also anomalous; Riedel (2012) transferred deliratorius back to Ichneumon, which is where, for example, Perkins (1960) classified the species; this result was supported by molecular phylogenetic evidence (Tschopp et al. 2013).

Ichneumon didymus Gravenhorst, 1829

Nomenclature:

bisignatus Gravenhorst, 1829

dissimulator (Stephens, 1835, Trogus)

crassorius Desvignes, 1856

Distribution: England, Scotland, Wales

Ichneumon emancipatus Wesmael, 1845

Nomenclature:

propinquus (Taschenberg, 1870, Exephanes)

rugosus Tischbein, 1873 preocc.

hostificus Tischbein, 1881

ramiformis Tischbein, 1881

alpinus Strobl, 1901 preocc.

vogesus Habermehl, 1916

circalpinus Heinrich, 1949

Distribution: England, Ireland

\section{Ichneumon exilicornis Wesmael, 1857}

\section{Nomenclature:}

hircinus Holmgren, 1864

rufolineatus Holmgren, 1864

caproni Perkins, 1953

Distribution: England 
Ichneumon extensorius Linnaeus, 1758

\section{Nomenclature:}

compressus Geoffroy, 1785 preocc.

tripunctatus Geoffroy, 1785

auratus Gmelin, 1790

lusorius Gravenhorst, 1807

vexatorius Gravenhorst, 1807

retractus Tischbein, 1873

longareolatus Thomson, 1886

atropunctum Pic, 1917

quercus Pic, 1917

cassonensis Pic, 1919

luteorufus Pic, 1919

polonicus (Heinrich, 1929, Euichneumon)

clypeonigro Constantineanu, 1954

transitorius Constantineanu, Suciu, Andriescu \& Ciochia, 1957

Distribution: England, Scotland, Wales, Ireland, Isle of Man

\section{Ichneumon formosus Gravenhorst, 1829}

\section{Nomenclature:}

obsessor Wesmael, 1845

obessor misspelling

batis Holmgren, 1880

brunneosparsus Strobl, 1901

schachti Heinrich, 1980

Distribution: England, Scotland, Ireland

Notes: Two subspecies, I. f. formosus and I. formosus microcephalus Stephens, 1835, have been recorded from Britain (Hilpert 1992).

Ichneumon fuscatus Gmelin, 1790

Distribution: England

Notes: Preoccupied by Ichneumon fuscatus Fabricius, 1781. 


\section{Ichneumon gracilentus Wesmael, 1845}

\section{Nomenclature:}

gratiosus Wesmael, 1845

vicinus Holmgren, 1864 preocc.

adscendens Tischbein, 1881

improbus Tischbein, 1881

quadrilineatus Tischbein, 1881

wuestneii Kriechbaumer, 1890

bioculatus Kriechbaumer, 1894 preocc., unavailable

trioculatus Habermehl, 1903

helveticus Habermehl, 1916 preocc.

Distribution: England, Scotland, Ireland

\section{Ichneumon gracilicornis Gravenhorst, 1829}

\section{Nomenclature:}

iocerus Gravenhorst, 1829

quadrinotatus Stephens, 1835

propinquus Taschenberg, 1870 synonymy by Hinz and Horstmann (2000)

longisectus Berthoumieu, 1895

nigricaudus Berthoumieu, 1895 preocc., unavailable

nigroscutellatus Berthoumieu, 1895 unavailable

quadrimaculatus Habermehl, 1916

daphne Bauer, 1985

Distribution: England, Ireland

\section{Ichneumon haemorrhoicus Kriechbaumer, 1887}

Nomenclature:

albicollis Wesmael, 1857 preocc.

nigrifemur Constantineanu, Andriescu \& Ciochia, 1956 preocc.

Distribution: England, Wales, Ireland 


\section{Ichneumon ignobilis Wesmael, 1855}

Nomenclature:

filatus (Tischbein, 1879, Amblyteles) synonymy by Riedel (2014)

debilis (Kriechbaumer, 1886, Amblyteles) synonymy by Horstmann (2006a)

isenschmidii (Kriechbaumer, 1887, Amblyteles)

ambifarius Berthoumieu, 1904

baueri Habermehl, 1935

Distribution: Scotland

\section{Ichneumon insidiosus Wesmael, 1845}

\section{Nomenclature:}

argali Kriechbaumer, 1882

corfitzi Thomson, 1890

jesperi Thomson, 1893 preocc.

gansuanus Kokujev, 1904

scanicus Schmiedeknecht, 1929 preocc.

Distribution: England, Scotland, Ireland

\section{Ichneumon languidus Wesmael, 1845}

\section{Nomenclature:}

immisericors Tischbein, 1876

malignus Tischbein, 1881

nigrocastaneus Tischbein, 1881

luteoannulatus Pic, 1915

Distribution: England

Notes: Added by Hilpert (1992); one specimen (Chobham, Surrey) in BMNH identified

by K. Horstmann as 'var. immisericors Tischbein, 1876'.

\section{Ichneumon lautatorius Desvignes, 1856}

\section{Nomenclature:}

amabilis Giraud, 1863

bizonatus (Rudow, 1888, Amblyteles)

cingulatus Berthoumieu, 1895 unavailable 
mutabilis Berthoumieu, 1895 preocc., unavailable

gynandra Habermehl, 1903

nigropunctatus Habermehl, 1903

trimaculatus Habermehl, 1903 preocc.

Distribution: England

\section{Ichneumon ligatorius Thunberg, 1824}

\section{Nomenclature:}

gradarius Wesmael, 1848

refractarius Wesmael, 1855

velatus Wesmael, 1855

firmipes Wesmael, 1857

thulensis Ruthe, 1859

faroensis Schmiedeknecht, 1938

plautus Hilpert, 1992 synonymy by Riedel (2014)

Distribution: England, Scotland, Ireland

\section{Ichneumon lugens Gravenhorst, 1829}

Nomenclature:

napaeus Holmgren, 1880

Distribution: England, Wales, Ireland

Notes: Transferred from Chasmias by Tschopp et al. (2013).

\section{Ichneumon megapodius Heinrich, 1949}

Nomenclature:

nigroscutellatus Kriechbaumer, 1897 unavailable

alpinus Habermehl, 1913 preocc.

megapodiops Bauer, 1985

Distribution: England, Scotland

Notes: The British population is referable to the subspecies fennicola Heinrich, 1951 (Hilpert 1992, Horstmann 2006c).

oblongus 
Ichneumon melanotis Holmgren, 1864

Nomenclature:

macrocerus Thomson, 1886

discolor Berthoumieu, 1895 preocc., unavailable

macrocerophorus Dalla Torre, 1901

Distribution: England, Scotland, Ireland

Ichneumon memorator Wesmael, 1845

Nomenclature:

incomptus Holmgren, 1864

Distribution: England, Wales, Ireland

Ichneumon minutorius Desvignes, 1856

Nomenclature:

guttatus Tischbein, 1873 synonymy by Horstmann (2003b)

captorius Thomson, 1887 preocc.

xanthognathus Thomson, 1887

flavipetiolatus Habermehl, 1903

Distribution: England, Scotland

Ichneumon molitorius Linnaeus, 1761

Nomenclature:

molitor Zetterstedt, 1838

holsaticus Tischebin, 1873

intrudens Smith, 1874

croceiventris (Rudow, 1888, Amblyteles)

montanus Habermehl, 1903

corsicator Aubert, 1961

Distribution: England, Scotland, Ireland

Ichneumon mordax Kriechbaumer, 1875

Notes: Added by Hilpert (1992); although Hilpert (1992) did not state the country of occurrence there is a specimen in NMS from Perthshire. 
Ichneumon oblongus Schrank, 1802

Nomenclature:

latrator misident. (Horstmann 2001a)

crassipes Gmelin, 1790, preocc.

geniculator Gravenhorst, 1807

elegans Gravenhorst, 1829

means (Gravenhorst, 1829, Brachypterus)

Distribution: England, Scotland, Ireland

\section{Ichneumon primatorius Forster, 1771}

Nomenclature:

bicinctus Christ, 1791 preocc.

grossorius Fabricius, 1793

gemellitorius Thunberg, 1824

flavolineatus Gravenhorst, 1829

monetierensis Pic, 1914

Distribution: England, Scotland, Wales, Ireland, Isle of Man

Notes: Manx occurrence from Cowin and Williamson (1940)

\section{Ichneumon rufidorsatus Bridgman, 1887}

Distribution: Scotland

\section{Ichneumon sarcitorius Linnaeus, 1758}

Nomenclature:

vaginatorius Linnaeus, 1758

curvatorius Müller, 1776

bipartitus Geoffroy, 1785

flavatus Gmelin, 1790 preocc.

farctor Gravenhorst, 1807

zaydamensis Kokujev, 1909

funereus Schmiedeknecht, 1928 preocc.

niger Constantineanu, 1954 preocc.

Distribution: England, Scotland, Wales, Ireland, Isle of Man 
Ichneumon sculpturatus Holmgren, 1864

Nomenclature:

nereni Thomson, 1887

albicaudus Berthoumieu, 1895 unavailable

flavocingulatus Habermehl, 1916

Distribution: England

Ichneumon simulans Tischbein, 1873

Nomenclature:

variolosus Holmgren, 1878

subquadratus Thomson, 1887

obscuratus Habermehl, 1916

Distribution: England, Scotland, Ireland

Ichneumon spurius Wesmael, 1848

Nomenclature:

sieberti Habermehl, 1929

Distribution: England, Ireland

Ichneumon stigmatorius Zetterstedt, 1838

Nomenclature:

cursorius Zetterstedt, 1838

walkeri Wesmael, 1848

rubedinis Desvignes, 1856

polyonomus Wesmael, 1859

kamtschaticus Roman, 1927

modestus Habermehl, 1935 preocc.

Distribution: Scotland, Ireland

Ichneumon stramentarius Gravenhorst, 1820

Nomenclature:

clitellarius Holmgren, 1880

rhaeticus (Habermehl, 1917, Dochyteles) 
scelestus Perkins, 1952 preocc.

atrifemur Perkins, 1953 preocc.

circumscriptor Valemberg, 1975

medianus Berthoumieu, 1910

Distribution: England, Scotland, Wales, Ireland, Isle of Man

Notes: According to Hilpert (1992) the subspecies stramentarius Grav. is found in England, boreomaritimus Hilpert, 1992 in Scotland and septentrionalis Holmgren, 1864 in England, Scotland, Wales and Ireland (additional data from Perkins 1952).

\section{Ichneumon stramentor Rasnitsyn, 1981}

\section{Nomenclature:}

stramentarius misident.

Distribution: England, Wales

Notes: Added by Hilpert (1992) Perkins's (Perkins 1960) stramentarius is properly called stramentor, the name stramentarius actually being a senior synonym of Perkins's septentrionalis (with the latter name valid as a subspecies).

\section{Ichneumon suspiciosus Wesmael, 1845}

\section{Nomenclature:}

mellinurus Wesmael, 1848

trispilus Thomson, 1888

rufonotatus $\mathrm{Pic}, 1929$

Distribution: England, Scotland, Ireland, Isle of Man

Notes: Manx occurrence from Cowin and Williamson (1940)

\section{Ichneumon terminatorius Gravenhorst, 1820}

\section{Nomenclature:}

concinnatorius Stephens, 1835

fulvoscutellatus Stephens, 1835

Distribution: England, Ireland 
Ichneumon tuberculipes Wesmael, 1848

Nomenclature:

cuneatus Tischbein, 1876

limbatus Tischbein, 1879

piceatus Tischbein, 1879

mediorufus Schmiedeknecht, 1930

Distribution: England, Ireland

Ichneumon vafer Tischbein, 1876

Nomenclature:

conjugalis Holmgren, 1878

brevicornis Tischbein, 1881 preocc.

rogenhoferi Kriechbaumer, 1888

quartanus Perkins, 1953

Distribution: England, Scotland

Ichneumon validicornis Holmgren, 1864

Nomenclature:

vivacior Tischbein, 1873

pseudoconfusor Heinrich, 1980

Distribution: England, Scotland, Ireland, Isle of Man

Ichneumon ventus Hilpert, 1992

Distribution: Scotland

Notes: added by Hilpert (1992)

Ichneumon vulneratorius Zetterstedt, 1838

Nomenclature:

dahlbomi Wesmael, 1857

versutus Holmgren, 1864

Distribution: Scotland, Ireland 
Ichneumon xanthorius Forster, 1771

Nomenclature:

flaviniger Gravenhorst, 1820

nassavicus (Habermehl, 1917, Physcoteles)

bimactulatus (Habermehl, 1917, Physcoteles)

Distribution: England, Scotland, Ireland

Genus Limerodes Wesmael, 1845

Limerodes arctiventris (Schiødte, 1839)

Nomenclature:

Ichneumon arctiventris Schiødte, 1839

arctiventris (Boie, 1841, Ichneumon)

ophioniventris Wesmael, 1845

Distribution: England, Scotland, Ireland

Genus Limerodops Heinrich, 1949

Nomenclature:

OXYSOMA Kriechbaumer, 1875 preocc.

Limerodops elongatus (Brischke, 1865)

Nomenclature:

Eurylabus elongatus Brischke, 1865

fluvipes (Matsumura, 1911, Hoplismenus)

Distribution: England, Scotland, Ireland

Limerodops subsericans (Gravenhorst, 1820)

Nomenclature:

Ichneumon subsericans Gravenhorst, 1820

pedestrinus (Gravenhorst, 1820, Ichneumon)

cognatus (Stephens, 1835, Ichneumon)

Distribution: England, Scotland, Ireland, Isle of Man 
Genus Melanichneumon Thomson, 1893

Melanichneumon leucocheilus (Wesmael, 1845)

Nomenclature:

Ichneumon leucocheilus Wesmael, 1845

arieticornis (Berthoumieu, 1906, Ichneumon)

Distribution: England

Genus Obtusodonta Heinrich, 1962

Obtusodonta equitatoria (Panzer, 1786)

Nomenclature:

Ichneumon equitatorius Panzer, 1786

antennatoria (Panzer, 1800, Ichneumon)

mediatoria (Fabricius, 1804, Ichneumon) preocc.

cingulatoria (Thunberg, 1824, Ichneumon) preocc.

haemorrhoidaria (Thunberg, 1824, Ichneumon)

rufa (De Stefani, 1885, Amblyteles)

nigricauda (Berthoumieu, 1896, Amblyteles) unavailable

Genus Platylabops Heinrich, 1950

Platylabops apricus (Gravenhorst, 1820)

Nomenclature:

Ichneumon apricus Gravenhorst, 1820

intersector (Wesmael, 1854, Amblyteles)

semirufus (Desvignes, 1856, Hoplismenus) preocc.

delphinas (Berthoumieu, 1892, Ichneumon)

solitarius (Habermehl, 1929, Ichneumon) synonymy by Horstmann (2000b)

Distribution: England, Ireland

Platylabops humilis (Wesmael, 1857)

Nomenclature:

Ichneumon humilis Wesmael, 1857 
rufipes (Strobl, 1901, Ichneumon) preocc.

Distribution: England

Platylabops lariciatae (Kreichbaumer, 1890)

Nomenclature:

Platylabus lariciatae Kreichbaumer, 1890

atrithorax (Berthoumieu, 1910, Ischnogaster)

Distribution: England, Scotland, Ireland

Platylabops speciosus (Wesmael, 1845)

Nomenclature:

Amblyteles speciosus Wesmael, 1845

castaneusimilis Heinrich, 1930, Aoplus)

Distribution: Wales

Notes: Recorded from Wales by Perkins (1960) but not listed by Fitton (1978).

\section{Platylabops virginalis (Wesmael, 1845)}

Nomenclature:

Ichneumon virginalis Wesmael, 1845

albicoxatus (Pfeffer, 1913, Ichneumon)

Distribution: England, Ireland

\section{Genus Probolus Wesmael, 1845}

Notes: synonymy from Horstmann (2000a)

\section{Probolus crassulus Horstmann, 2000}

Nomenclature:

concinnus misident.

crassicornis (Stephens, 1835, Ichneumon)

Distribution: England, Ireland 
Probolus culpatorius (Linnaeus, 1758)

Nomenclature:

Ichneumon culpatorius Linnaeus, 1758

alticola (Gravenhorst, 1820, Ichneumon)

trucidator (Gravenhorst, 1829, Ichneumon)

femorator (Stephens, 1835, Ichneumon) preocc.

fossorius Wesmael, 1845

Distribution: England, Scotland, Wales

Notes: distribution data from Horstmann (2000a)

Genus Rictichneumon Heinrich, 1961

Rictichneumon pachymerus (Hartig, 1838)

Nomenclature:

Phygadeuon pachymerus Hartig, 1838

trucidus (Wesmael, 1845, Ichneumon)

aciculator (Ratzeburg, 1852, Ichneumon)

steinii (Ratzeburg, 1852, Ichneumon)

septimus (Berthoumieu, 1910, Ichneumon)

Distribution: England

Notes: NMS, det. Riedel, added here

Genus Spilichneumon Thomson, 1894

Nomenclature:

SPILOTELES Berthoumieu, 1904

PSEUDICHNEUMON Kokujev, 1909

Spilichneumon ammonius (Gravenhorst, 1820)

Nomenclature:

Ichneumon ammonius Gravenhorst, 1820

nonagriae (Holmgren, 1871, Amblyteles)

stagnicola (Thomson, 1888, Amblyteles)

Distribution: England, Scotland 


\section{Spilichneumon celenae Perkins, 1953}

Distribution: Scotland, Wales, Ireland

\section{Spilichneumon johansoni (Holmgren, 1871)}

Nomenclature:

Amblyteles johansoni Holmgren, 1871

subalnotatus (Pic, 1914, Amblyteles)

Distribution: England, Ireland

\section{Spilichneumon occisorius (Fabricius, 1793)}

\section{Nomenclature:}

Ichneumon occisorius Fabricius, 1793

sanguinatorius (Gravenhorst, 1829, Ichneumon)

nigrinus (Berthoumieu, 1896, Ambyteles) preocc., unavailable

rufinus (Berthoumieu, 1896, Ambyteles) preocc., unavailable

plicatus (Morley, 1903, Ctenichneumon)

morvandicus (Pic, 1925, Amblyteles)

Distribution: England, Scotland, Ireland, Isle of Man

\section{Genus Spilothyrateles Heinrich, 1967}

\section{Spilothyrateles nuptatorius (Fabricius, 1793)}

\section{Nomenclature:}

Ichneumon nuptatorius Fabricius, 1793

fabricii (Schrank, 1802, Ichneumon) synonymy by Horstmann (2001a)

terminator (Panzer, 1804, Ichneumon)

insidiator (Fonscolombe, 1847, Ichneumon)

melanocerus (Wesmael, 1845, Ichneumon)

cubicularis (Desvignes, 1856, Ichneumon)

truncicola (Thomson, 1888, Amblyteles)

frustrator (Berthoumieu, 1892, Amblyteles)

paganus (Berthoumieu, 1892, Ichneumon)

australis (Habermehl, 1917, Anisobas) synonymy by Horstmann (1997) 
Distribution: Ireland

Spilothyrateles punctus (Gravenhorst, 1829)

Nomenclature:

Ichneumon punctus Gravenhorst, 1829

obscuripes (Holmgren, 1864, Ichneumon)

erraticus (Berthoumieu, 1892, Ichneumon)

nigriventris (Berthoumieu, 1895, Ichneumon) unavailable

lateobscurus (Pic, 1902, Amblyteles)

pillichi (Kiss, 1929, Ichneumon) synonymy by

Distribution: England

Genus Stenaoplus Heinrich, 1938

Stenaoplus pictus (Gravenhorst, 1829)

Nomenclature:

Hoplismenus pictus Gravenhorst, 1829

rufescens (Stephens, 1835, Ichneumon) preocc.

ratzeburgii (Hartig, 1838, Cryptus)

exornatus (Wesmael, 1845, Ichneumon)

obscurior (Pic, 1898, Ichneumon) preocc.

Distribution: England, Scotland, Ireland

\section{Genus Stenichneumon Thomson, 1893}

\section{Stenichneumon culpator (Schrank, 1802)}

Nomenclature:

Ichneumon culpator Schrank, 1802

?ani (Geoffroy, 1785, Ichneumon)

ater (Berthoumieu, 1894, Ichneumon) preocc., unavailable corsicator Aubert, 1960

Distribution: England, Scotland, Wales, Ireland, Isle of Man 
Stenichneumon militarius (Thunberg, 1824)

Nomenclature:

Ichneumon militarius Thunberg, 1824

pistorius (Gravenhorst, 1829, Ichneumon)

pistor (Zetterstedt, 1838, Ichneumon)

sexannularis (Berthoumieu, 1894, Ichneumon)

Distribution: England, Scotland

Genus Stenobarichneumon Heinrich, 1961

Stenobarichneumon basalis (Perkins, 1960)

Nomenclature:

Barichneumon basalis Perkins, 1960

Distribution: England, Scotland

Stenobarichneumon basiglyptus (Kriechbaumer, 1890)

Nomenclature:

Ichneumon basiglyptus Kriechbaumer, 1890

bifossatus (Berthoumieu, 1892, Ichneumon)

coxiglyptus (Heinrich, 1951, Barichneumon)

Distribution: England, Ireland

Stenobarichneumon citator (Thunberg, 1824)

Nomenclature:

Ichneumon citator Thunberg, 1824

incubitor misident.

Distribution: England

Genus Sycaonia Cameron, 1903

Sycaonia foersteri (Wesmael, 1848)

Nomenclature:

Ichneumon foersteri Wesmael, 1848

boreosicaria (Roman, 1913, Cratichneumon) 
Distribution: England, Scotland, Ireland

Genus Thyrateles Perkins, 1953

\section{Thyrateles camelinus (Wesmael, 1845)}

\section{Nomenclature:}

Amblyteles camelinus Wesmael, 1845

certator (Müller, 1776, Ichneumon) nom. ob., synonymy by Horstmann (2001a)

cardui (Schrank, 1786, Ichneumon) nom. ob., synonymy by Horstmann (2001a)

adustus (Gmelin, 1790, Ichneumon) nom. ob., synonymy by Horstmann (2001a)

malignus (Tischbein, 1868, Amblyteles)

brunnipes (Tischbein, 1879, Ichneumon)

rufomaculatus (Kriechbaumer, 1894, Amblyteles) unavailable

alticola (Habermehl, 1920, Ichneumon) preocc.

oisanensis (Pic, 1927, Ichneumon)

Distribution: England, Scotland

Thyrateles haereticus (Wesmael, 1854)

Nomenclature:

Amblyteles haereticus Wesmael, 1854

urticarum (Holmgren, 1880, Ichneumon)

binotatus (Kriechbaumer, 1894, Amblyteles) preocc., unavailable

?cinctor (Kriechbaumer, 1894, Amblyteles) preocc.

pyraeneus (Pic, 1914, Ichneumon) preocc.

\section{Genus Tricholabus Thomson, 1894}

\section{Tricholabus strigatorius (Gravenhorst, 1829)}

Nomenclature:

Ichneumon strigatorius Gravenhorst, 1829

quittardi (Pic, 1904, Amblyteles)

berthoumieui Pic, 1927

Distribution: England, Scotland, Ireland, Isle of Man 
Genus Triptognathus Berthoumieu, 1904

Triptognathus atripes (Gravenhorst, 1820)

Nomenclature:

Ichneumon atripes Gravenhorst, 1820

goedarti (Gravenhorst, 1829, Ichneumon)

interruptus (Gravenhorst, 1829, Ichneumon)

pratensis (Gravenhorst, 1829, Ichneumon)

quadricingulatus (Gravenhorst, 1829, Ichneumon)

uniguttatus (Gravenhorst, 1829, Ichneumon)

ignotus (Fonscolombe, 1847, Ichneumon)

praedator (Fonscolombe, 1847, Ichneumon) preocc.

flavifemur (Tischbein, 1873, Ichneumon)

interjectus (Tischbein, 1879, Amblyteles)

subfasciatus (Tischbein, 1879, Amblyteles)

stephani (Pic, 1903, Amblyteles)

taiyudongus (Uchida, 1926, Spilichneumon)

Distribution: England

Notes: NMS (from Santon Downham), det. Riedel, added here; tentative identification as the genus is in need of revision, but certainly in the atripes group (M. Riedel, pers. comm.)

Triptognathus sibilans (Gravenhorst, 1829)

Nomenclature:

Ichneumon sibilans Gravenhorst, 1829

propinquus (Perkins, 1953, Amblyteles)

Distribution: England

Genus Virgichneumon Heinrich, 1977

Virgichneumon albilineatus (Gravenhorst, 1820)

Nomenclature:

Ichneumon albilineatus Gravenhorst, 1820

albolineatus misspelling 
leucomelas (Gmelin, 1790, Ichneumon) preocc.

nigratorius (Panzer, 1804, Ichneumon) preocc.

?bilineator (Donovan, 1810, Ichneumon)

bipunctorius (Stephens, 1835, Ichneumon) preocc.

Distribution: England

\section{Virgichneumon albosignatus (Gravenhorst, 1829)}

Nomenclature:

Ichneumon albosignatus Gravenhorst, 1829

mesostilpnus (Thomson, 1888, Ichneumon)

punctus (Berthoumieu, 1895, Ichneumon) preocc., unavailable

nigricollis (Constantineanu, 1954, Melanichneumon)

Distribution: England

Virgichneumon callicerus (Gravenhorst, 1820)

Nomenclature:

Ichneumon callicerus Gravenhorst, 1820

plurialbatus (Wesmael, 1855, Ichneumon)

eremita (Kokujev, 1909, Ichneumon)

Distribution: England

Virgichneumon digrammus (Gravenhorst, 1820)

Nomenclature:

Ichneumon digrammus Gravenhorst, 1820

nudicoxa (Thomson, 1888, Ichneumon)

balearicus (Kriechbaumer, 1894, Ichneumon)

Distribution: England, Ireland

\section{Virgichneumon dumeticola (Gravenhorst, 1829)}

Nomenclature:

Ichneumon dumeticola Gravenhorst, 1829

Distribution: England 
Virgichneumon faunus (Gravenhorst, 1829)

Nomenclature:

Ichneumon faunus Gravenhorst, 1829

leucopygus (Gravenhorst, 1829, Ichneumon)

Distribution: England, Ireland

\section{Virgichneumon maculicauda (Perkins, 1953)}

Nomenclature:

Barichneumon maculicauda Perkins, 1953

perscrutator (Wesmael, 1845, Ichneumon) preocc.

Distribution: England, Scotland, Ireland

\section{Virgichneumon monostagon (Gravenhorst, 1820)}

Nomenclature:

Ichneumon monostagon Gravenhorst, 1820

luctuosus (Gravenhorst, 1820, Ichneumon)

indagator (Wesmael, 1845, Ichneumon) preocc.

redimitus (Tischbein, 1874, Ichneumon)

explorator (Tischbein, 1876, Ichneumon)

minor (Kriechbaumer, 1894, Ichneumon) preocc., unavailable

hexaleucus (Kriechbaumer, 1899, Ichneumon) synonymy by Horstmann (2006a)

annulicornis (Schmiedeknecht, 1928, Ichneumon) preocc.

Distribution: England, Ireland

\section{Virgichneumon tergenus (Gravenhorst, 1820)}

Nomenclature:

Ichneumon tergenus Gravenhorst, 1820

octoguttatus (Gravenhorst, 1829, Ichneumon)

Distribution: England, Wales 
Genus Vulgichneumon Heinrich, 1961

Vulgichneumon bimaculatus (Schrank, 1776)

Nomenclature:

Ichneumon bimaculatus Schrank, 1776

bimaculatorius (Panzer, 1801, Ichneumon)

Distribution: England, Ireland

Vulgichneumon deceptor (Scopoli, 1763)

Nomenclature:

Ichneumon deceptor Scopoli, 1763

deceptorius (Thunberg, 1824, Ichneumon)

deceptorius (Zetterstedt, 1838, Ichneumon) preocc.

vestigator (Wesmael, 1845, Ichneumon) preocc.

completus (Berthoumieu, 1894, Ichneumon)

obscurior (Berthoumieu, 1895, Ichneumon) unavailable

Distribution: England, Ireland

Vulgichneumon saturatorius (Linnaeus, 1758)

Nomenclature:

Ichneumon saturatorius Linnaeus, 1758

nigratorius (Pontoppidan, 1763, Ichneumon)

carnifex (Müller, 1776, Ichneumon)

clavatorius (Müller, 1776, Ichneumon)

fuscocastaneus (Gravenhorst, 1829, Ichneumon)

saturator (Zetterstedt, 1838, Ichneumon)

albotrochanteratus (Ulbricht, 1926, Ichneumon)

Distribution: England, Scotland, Wales, Ireland, Isle of Man

Vulgichneumon suavis (Gravenhorst, 1820)

Nomenclature:

Ichneumon suavis Gravenhorst, 1820

fallax (Gravenhorst, 1829, Ichneumon) 
lepidus (Gravenhorst, 1829, Ichneumon)

Distribution: England, Scotland, Wales, Ireland

Tribe LISTRODROMINI Förster, 1869

Genus Anisobas Wesmael, 1845

Nomenclature:

LYCAENIPHILOS Heinrich, 1934

Anisobas cingulatellus Horstmann, 1997

Nomenclature:

cingulatorius (Gravenhorst, 1820, Ichneumon) preocc.

Distribution: England, Wales

Notes: Replacement name for Ichneumon cingulatorius Gravenhorst, 1820, preoccupied by cingulatorius Weber, 1801. Listed as a subspecies of australis Habermehl, 1917, in Yu and Horstmann (1997) but the latter is a junior synonym of Spilothyrateles nuptatorius (Horstmann 1997).

Anisobas platystylus Thomson, 1888

Distribution: England

Genus Listrodromus Wesmael, 1845

Listrodromus nycthemerus (Gravenhorst, 1820)

Nomenclature:

Ichneumon nycthemerus Gravenhorst, 1820

quinqueguttatus (Gravenhorst, 1829, Ichneumon)

Distribution: England, Ireland

Genus Neotypus Förster, 1869

Neotypus nobilitator (Gravenhorst, 1807)

Nomenclature:

Ichneumon nobilitator Gravenhorst, 1807

erythronotus (Rudow, 1882, Cryptus) 
Distribution: England

Tribe OEDICEPHALINI Heinrich, 1934

Nomenclature:

NOTOSEMINI Townes, 1961

Genus Notosemus Förster, 1869

Nomenclature:

ISCHNIDIUM Kriechbaumer, 1890

ISCHNOGASTER Kriechbaumer, 1890 preocc.

Notosemus bohemani (Wesmael, 1855)

Nomenclature:

Phaeogenes bohemani Wesmael, 1855

dives Brischke, 1887

albibucca (Kriechbaumer, 1890, Ischnogaster)

gaullei (Berthoumieu, 1900, Ischnus)

atriventris (Pic, 1915, Ischnogaster)

Distribution: England

Tribe PHAEOGENINI Förster, 1869

Notes: Wahl and Mason (1995) included Alomya and relatives in this tribe, with the tribe taking the name Alomyini. With the removal of Alomya to a separate subfamily (see notes under Alomyinae) the tribe takes the name Phaeogenini. Some distribution data from Diller and Shaw (2014).

Genus Aethecerus Wesmael, 1845

Aethecerus discolor Wesmael, 1845

Nomenclature:

styriacus Strobl, 1901

Distribution: England, Scotland, Ireland 
Aethecerus dispar Wesmael, 1845

Nomenclature:

frontatus Wesmael, 1845

albipictus Berthoumieu, 1897 unavailable

rufipes Strobl, 1901

Distribution: England, Scotland

Aethecerus foveolatus Gregor, 1940

Nomenclature:

exilis (Berthoumieu, 1899, Diadromus) preocc.

Distribution: England, Scotland

Notes: added by Diller and Shaw (2014)

Aethecerus horstmanni Diller \& Shaw, 2014

Distribution: England, Scotland

Notes: added by Diller and Shaw (2014)

Aethecerus longulus Wesmael, 1845

Nomenclature:

formosus (Bridgman, 1881, Phaeogenes)

Distribution: England

Aethecerus nitidus Wesmael, 1845

Nomenclature:

corcyriensis (Berthoumieu, 1901, Phaeogenes)

Distribution: England, Scotland

Aethecerus placidus Wesmael, 1845

Nomenclature:

nigricoxatus Strobl, 1901

Distribution: England, Scotland, Ireland 
Aethecerus porcellus Holmgren, 1890

Distribution: England, Scotland, Wales

Notes: added by Diller and Shaw (2014)

Aethecerus ruberpedatus Diller \& Shaw, 2014E

Distribution: England, Scotland

Notes: added by Diller and Shaw (2014)

Aethecerus rugifrons Holmgren, 1890

Distribution: England, Scotland

Notes: added by Diller and Shaw (2014)

Aethecerus subuliferus (Holmgren, 1890)

Nomenclature:

Phaeogenes subuliferus Holmgren, 1890

Distribution: England

Notes: Added by Diller and Shaw (2014); a species of Aethecerus, not Phaeogenes, where it is listed by Yu et al. (2012) (Diller \& Shaw, 2014Diller and Shaw 2014).

Genus Baeosemus Förster, 1869

Baeosemus mitigosus (Gravenhorst, 1829)

Nomenclature:

Ichneumon mitigosus Gravenhorst, 1829

phaeocerus (Wesmael, 1845, Herpestomus)

vulpecula Holmgren, 1890

Distribution: England, Scotland

Notes: added by Diller and Shaw (2014) 


\section{Genus Centeterus Wesmael, 1845}

\section{Centeterus confector (Gravenhorst, 1829)}

Nomenclature:

Ichneumon confector Gravenhorst, 1829

picticollis Wesmael, 1845

nigridentis Constantineanu, 1951

Distribution: England, Ireland

\section{Centeterus rubiginosus (Gmelin, 1790)}

Nomenclature:

Ichneumon rubiginosus Gmelin, 1790

opprimator (Gravenhorst, 1820, Ichneumon)

rufipes (Brischke, 1891, Phaeogenes)

Distribution: England, Scotland, Wales, Ireland, Isle of Man

\section{Genus Colpognathus Wesmael, 1845}

\section{Colpognathus celerator (Gravenhorst, 1807)}

Nomenclature:

Ichneumon celerator Gravenhorst, 1807

procerus (Gravenhorst, 1829, Phygadeuon)

femorator (Stephens, 1835, Ichneumon) preocc.

celeratorius (Zetterstedt, 1838, Ichneumon)

armatus Thomson, 1891 synonymy by Diller and Schönitzer (2003)

atricornis Pic, 1914 synonymy by Diller and Schönitzer (2003)

femoralis Habermehl, 1917

petiolaris Constantineanu, 1954

nigroscaposus Aubert, 1959

Distribution: England, Scotland, Wales, Ireland, Isle of Man

Notes: Manx occurrence from Shaw and Bennett (2001) 
Colpognathus divisus Thomson, 1891

Nomenclature:

atricornis Pic, 1914

rufifemur Constantineanu, 1959

Distribution: England, Scotland, Wales, Ireland

Genus Diadromus Wesmael, 1845

Nomenclature:

THYRAEELLA Holmgren, 1890

Diadromus albinotatus (Gravenhorst, 1829)

Nomenclature:

Ichneumon albinotatus Gravenhorst, 1829

Distribution: England

Diadromus arrisor Wesmael, 1845

Distribution: England

Notes: BMNH, Ely coll., det. Diller, added here

Diadromus candidatus (Gravenhorst, 1829)

Nomenclature:

Ichneumon candidatus Gravenhorst, 1829

guttulatus (Gravenhorst, 1829, Ichneumon)

aries (Brischke, 1887, Phaegoenes)

decolor Holmgren, 1890

Diadromus collaris (Gravenhorst, 1829)

Nomenclature:

Ischnus collaris Gravenhorst, 1829

similis (Bridgman, 1881, Phaeogenes)

bellulus (Kriechbaumer, 1894, Phaeogenes)

brischkei Berthoumieu, 1897 unavailable

punicus Berthoumieu, 1898 
rufiscapus Pic, 1902

cabrerai Berthoumieu, 1903

hispanicus (Berthoumieu,1904, Heterischnus)

brevicauda (Hellén, 1949, Ischnopsidea)

Distribution: England, Scotland

Diadromus heteroneurus Holmgren, 1890

Nomenclature:

quadriguttatus misident.

prosopius Holmgren, 1890

nigroscutellatus Constantineanu, Suciu, Andriescu \& Ciochia, 1957

Distribution: England, Scotland

Diadromus nitidigaster Diller \& Shaw, 2014

Distribution: England

Notes: added by Diller and Shaw (2014)

\section{Diadromus pulchellus Wesmael, 1845}

Distribution: England

Notes: added by Diller and Shaw (2014)

Diadromus subtilicornis (Gravenhorst, 1829)

Nomenclature:

Ichneumon subtilicornis Gravenhorst, 1829

imbellis Wesmael, 1845

dolosus Berthoumieu, 1899

nigrinus (Berthoumieu, 1901, Phaeogenes)

Distribution: England, Scotland

Diadromus tenax Wesmael, 1845

Distribution: England 
Diadromus troglodytes (Gravenhorst, 1829)

Nomenclature:

Ichneumon troglodytes Gravenhorst, 1829

abdominator (Stephens, 1835, Ichneumon)

scobinatus Holmgren, 1890

Distribution: England, Scotland, Wales, Ireland

Diadromus varicolor Wesmael, 1845

Distribution: England, Scotland, Wales, Isle of Man

Genus Dicaelotus Wesmael, 1845

Nomenclature:

DELOGLYPTUS Förster, 1869

HOLOCREPIS Förster, 1869

LEPTODEMAS Förster, 1869

CINXAELOTUS Holmgren, 1890

EURYPTILUS Holmgren, 1890

Dicaelotus cameroni Bridgman, 1881

Nomenclature:

minutulus Kokujev, 1909

nigroclypeatus Constantineanu, 1959

Distribution: England, Scotland

Dicaelotus erythrostoma Wesmael, 1845

Distribution: England, Ireland

Dicaelotus inflexus Thomson, 1891

Distribution: England, Scotland, Wales, Ireland

Dicaelotus orbitalis Thomson, 1891

Distribution: England, Ireland 
Dicaelotus parvulus (Gravenhorst, 1829)

Nomenclature:

Ichneumon parvulus Gravenhorst, 1829

alpigenus Strobl, 1901

Distribution: England

Dicaelotus pictus (Schmiedeknecht, 1903)

Nomenclature:

Deloglyptus pictus Schmiedeknecht, 1903

lugens Berthoumieu, 1906

Distribution: England

Dicaelotus pudibundus (Wesmael, 1845)

Nomenclature:

Herpestomus pudibundus Wesmael, 1845

alboscutatus Berthoumieu, 1900

gaullei Berthoumieu, 1903

Distribution: England

Dicaelotus pumilus (Gravenhorst, 1829)

Nomenclature:

Ichneumon pumilus Gravenhorst, 1829

morosus Wesmael, 1855

analis Berthoumieu, 1901 unavailable

Distribution: England, Scotland, Wales, Ireland

Dicaelotus punctiventris (Thomson, 1891)

Nomenclature:

Deloglyptus punctiventris Thomson, 1891

punicus Berthoumieu, 1901 unavailable

Distribution: England, Scotland, Ireland 
Dicaelotus pusillator (Gravenhorst, 1807)

Nomenclature:

Ichneumon pusillator Gravenhorst, 1807

?pallidus (Gmelin, 1790, Ichneumon)

notator (Gravenhorst, 1807, Ichneumon)

Distribution: Scotland

Notes: added by Diller and Shaw (2014)

Dicaelotus pusillus Holmgren, 1890

Distribution: England

Notes: added by Diller and Shaw (2014)

Dicaelotus resplendens Holmgren, 1890

Nomenclature:

fitchi Perkins, 1953

Distribution: England, Ireland

Dicaelotus ruficoxatus (Gravenhorst, 1829)

Nomenclature:

Ichneumon ruficoxatus Gravenhorst, 1829

unipunctatus Wesmael, 1845

nigrescens Constantineanu, 1959

Distribution: England, Scotland, Ireland

Dicaelotus rufoniger Berthoumieu, 1897

Distribution: England, Scotland, Wales, Ireland

Dicaelotus schmiedeknechti Diller \& Shaw, 2014

Nomenclature:

ruficornis (Schmiedeknecht, 1903, Eparces) preocc.

Distribution: England

Notes: added by Diller and Shaw (2014) 
Dicaelotus suspectus Perkins, 1953

Distribution: England

Genus Dilleritomus Aubert, 1979

Dilleritomus apertor Aubert, 1979

Distribution: England

Notes: added by Diller and Shaw (2014)

Dilleritomus filiformis (Strobl, 1901)

Nomenclature:

Herpestomus filiformis Strobl, 1901

Distribution: England, Wales

Notes: added by Diller and Shaw (2014)

Genus Dirophanes Förster, 1869

Dirophanes callopus (Wesmael, 1845)

Nomenclature:

Phaeogenes callopus Wesmael, 1845

tibiator (Thunberg, 1824, Ichneumon) preocc.

palliditarsis (Berthoumieu, 1900, Diadromus)

Distribution: England, Scotland

Dirophanes foveolatus (Perkins, 1953)

Nomenclature:

Phaeogenes foveolatus Perkins, 1953

Distribution: England, Scotland, Ireland

Notes: Transferred from Phaeogenes by Schönitzer et al. (2006). 


\section{Dirophanes fulvitarsis (Wesmael, 1845)}

\section{Nomenclature:}

Phaeogenes fulvitarsis Wesmael, 1845

limatus (Wesmael, 1845, Phaeogenes)

hyperboreus (Holmgren, 1890, Phaeogenes)

nitidiventris (Holmgren, 1890, Phaeogenes)

ruficoxa (Thomson, 1891, Phaeogenes)

Distribution: England, Scotland, Ireland, Isle of Man

\section{Dirophanes invisor (Thunberg, 1824)}

\section{Nomenclature:}

Ichneumon invisor Thunberg, 1824

stimulator (Gravenhorst, 1829, Ichneumon)

homochlorus (Wesmael, 1845, Phaeogenes)

kabylianus (Pic, 1897, Phaeogenes)

Distribution: England, Scotland, Wales

\section{Dirophanes maculicornis (Stephens, 1835)}

Nomenclature:

Ichneumon maculicornis Stephens, 1835

scutellaris (Wesmael, 1845, Phaeogenes)

bisignatus (Holmgren, 1890, Phaeogenes)

dentatus (Pic, 1923, Phaeogenes)

nigroscutellatus (Habermehl, 1929, Phaeogenes)

murinanae (Fahringer, 1936, Microcryptus)

gigas (Fahringer, 1943, Phaeogenes)

dinianae (Fahringer, 1948, Phaeogenes)

ruficoxis (Constantineanu, 1959, Phaeogenes) preocc.

Distribution: England, Scotland

\section{Dirophanes mysticus (Wesmael, 1855)}

Nomenclature:

Phaeogenes mysticus Wesmael, 1855 
tetricus Wesmael, 1855

Distribution: England, Scotland

Notes: Transferred from Phaeogenes by Schönitzer et al. (2006).

Dirophanes regenerator (Fabricius, 1804)

Nomenclature:

Cryptus regenerator Fabricius, 1804

rusticatus (Wesmael, 1845, Phaeogenes) synonymy by Horstmann (2001a)

Distribution: England, Scotland, Wales, Ireland, Isle of Man

Genus Eparces Förster, 1869

Eparces grandiceps (Thomson, 1891)

Nomenclature:

Centeterus grandiceps Thomson, 1891

Distribution: England

\section{Genus Epitomus Förster, 1869}

Epitomus infuscatus (Gravenhorst, 1829)

Nomenclature:

Hemiteles infuscatus Gravenhorst, 1829

pygmaeus (Brischke, 1890, Hemiteles) preocc.

parvus Thomson, 1891

laeviareolatus Schmiedeknecht, 1904

Distribution: England, Scotland, Wales, Ireland, Isle of Man

\section{Epitomus proximus Perkins, 1953}

Distribution: England, Scotland, Wales, Ireland

\section{Genus Eriplatys Förster, 1869}

Nomenclature:

ANOPIESTA Förster, 1869

MELANOMICRUS Morley, 1903 


\section{Eriplatys ardeicollis (Wesmael, 1845)}

Nomenclature:

Herpestomus ardeicollis Wesmael, 1845

elliotti (Morley, 1903, Melanomicrus)

neirae (Ceballos, 1958, Herpestomus)

Distribution: England, Scotland, Ireland

Eriplatys sawoniewiczi Diller, 1993

Distribution: England, Scotland

Notes: added by Diller and Shaw (2014)

\section{Genus Hemichneumon Wesmael, 1857}

Hemichneumon subdolus Wesmael, 1857

Nomenclature:

elongatus (Ratzeburg, 1852, Hemiteles) preocc.

suspectus Wesmael, 1857

varians (Taschenberg, 1865, Cryptus) synonymy by Schwarz (2005)

tineidarum (Giraud, 1872, Ischnus)

Distribution: England, Ireland

\section{Genus Herpestomus Wesmael, 1845}

Herpestomus arridens (Gravenhorst, 1829)

Nomenclature:

Ichneumon arridens Gravenhorst, 1829

facialis (Gravenhorst, 1829, Ichneumon)

xanthops (Gravenhorst, 1829, Ichneumon)

erubescens (Berthoumieu, 1899, Diadromus)

subatriceps (Pic, 1914, Phaeogenes)

transsylvanicus Kiss, 1924

rufifrons (Aerts, 1957, Rhexidermus)

meridionator Aubert, 1960 synonymy by Diller and Tereshkin (2005) 
Distribution: England, Scotland, Wales

Herpestomus brunnicornis (Gravenhorst, 1829)

Nomenclature:

Ichneumon brunnicornis Gravenhorst, 1829

padella (Goureau, 1847, Ichneumon)

bruneicornis Dalla Torre, 1902

bisignatus Habermehl, 1917

nigriventris Constantineanu, 1944

Distribution: England, Scotland, Ireland

Herpestomus minimus (Berthoumieu, 1901)

Nomenclature:

Phaeogenes minimus Berthoumieu, 1901

Distribution: England, Scotland

Notes: Added by Diller and Shaw (2014); previously recorded as British by Carr (1924)

but these records are now discounted (Perkins 1953, Shaw 2003).

Herpestomus nasutus Wesmael, 1845

Nomenclature:

furunculus Wesmael, 1845

intermedius Wesmael, 1845

Distribution: England, Scotland, Ireland

Herpestomus wesmaeli Perkins, 1953

Distribution: England, Scotland, Ireland

Genus Heterischnus Wesmael, 1859

Nomenclature:

RHEXIDERMUS Förster, 1869

ISCHNOPSIDEA Viereck, 1914 
Heterischnus nigricollis (Wesmael, 1845)

Nomenclature:

Ischnus nigricollis Wesmael, 1845

rufipes (Wesmael, 1848, Ischnus)

Distribution: England, Scotland, Wales

Heterischnus pulex (Müller, 1776)

Nomenclature:

Ichneumon pulex Müller, 1776

murex (Müller, 1776, Ichneumon)

brevicornis (Gravenhorst, 1829, Ichneumon)

\section{Heterischnus truncator (Fabricius, 1798)}

Nomenclature:

Ichneumon truncator Fabricius, 1798

?colorator (Villers, 1789, Ichneumon)

filiformis (Gravenhorst, 1829, Ischnus)

thoracicus (Gravenhorst, 1829, Ischnus) synonymy by Horstmann (2001a)

elegans (Tischbein, 1868, Ischnus)

montanus (Berthoumieu, 1897, Ischnus)

moravicus (Gregor, 1939, Ischnus) synonymy by Horstmann (2002b)

nigrinus (Constantineanu, 1959, Ischnus) preocc.

Distribution: England, Scotland

Genus Mevesia Holmgren, 1890

\section{Mevesia arguta (Wesmael, 1845)}

Nomenclature:

Phaeogenes argutus Wesmael, 1845

tenuis (Berthoumieu, 1899, Phaeogenes)

albifemur Constantineanu, 1959

Distribution: England, Scotland, Wales, Ireland 
Mevesia guttata Perkins, 1953

Distribution: England

Genus Misetus Wesmael, 1845

Misetus oculatus Wesmael, 1845

Nomenclature:

obscurus Berthoumieu, 1897 unavailable

Distribution: England, Scotland, Ireland

Genus Nematomicrus Wesmael, 1845

Nematomicrus tenellus Wesmael, 1845

Distribution: England, Scotland, Ireland

Genus Oiorhinus Wesmael, 1845

Oiorhinus pallipalpis Wesmael, 1845

Nomenclature:

striatus (Bridgman, 1881, Herpestomus)

pallidipalpis Dalla Torre, 1902

Distribution: England, Scotland, Wales, Ireland

Notes: some distribution data from Shaw (1984)

Genus Oronotus Wesmael, 1845

Nomenclature:

ORONTUS misspelling

Oronotus binotatus (Gravenhorst, 1829)

Nomenclature:

Phygadeuon binotatus Gravenhorst, 1829

coarctatus Wesmael, 1845

Distribution: Ireland 
Genus Orotylus Holmgren, 1890

Orotylus mitis (Wesmael, 1848)

Nomenclature:

Diadromus mitis Wesmael, 1848

Distribution: England

Notes: The only British specimens seen by Perkins (1959) were from the Bridgman collection, apparently lacking locality data. There are recent specimens in BMNH and NMS.

Genus Paraethecerus Perkins, 1953

Paraethecerus elongatus Perkins, 1953

Distribution: England

\section{Genus Phaeogenes Wesmael, 1845}

Notes: Many species previously placed in the genus Phaeogenes (Perkins 1959, Fitton 1978) are now classified in Dirophanes and Tycherus.

doubtfully placed species of Phaeogenes:

[picipes (Stephens, 1835, Ichneumon) nom. dub., from England]

Phaeogenes curator (Thunberg, 1824)

Nomenclature:

Ichneumon curator Thunberg, 1824

crassidens Thomson, 1891

Distribution: England, Wales

Notes: some distribution data from Ely (2002)

Phaeogenes distinctus (Bridgman, 1887)

Nomenclature:

Herpestomus distinctus Bridgman, 1887

Distribution: England 
Phaeogenes heterogonus Holmgren, 1890

Distribution: Wales, Ireland

Phaeogenes melanogonos (Gmelin, 1790)

Nomenclature:

Ichneumon melanogonos Gmelin, 1790

protervus Wesmael, 1855

nigripes Constantineanu, 1954

Distribution: England, Scotland

Phaeogenes nigridens (Wesmael, 1845)

Nomenclature:

Phaeogenes nigridens Wesmael, 1845

major (Berthoumieu, 1901, Phaeogenes)

Distribution: England

Notes: Added by Ely (2002); transferred back to Phaeogenes (from Tycherus) by Diller and Shaw (2014).

Phaeogenes planifrons Wesmael, 1845

Nomenclature:

compar Berthoumieu, 1904

hungaricus (Kiss, 1926, Orotylus) synonymy by

Distribution: Ireland

\section{Phaeogenes semivulpinus (Gravenhorst, 1829)}

Nomenclature:

Ichneumon semivulpinus Gravenhorst, 1829

mutabilis (Gravenhorst, 1829, Ichneumon)

rufator (Stephens, 1835, Ichneumon) preocc.

primarius Wesmael, 1845

Distribution: England, Scotland 
Phaeogenes trepidus Wesmael, 1845

Distribution: England

Genus Stenodontus Berthoumieu, 1897

Nomenclature:

GNATHOXYS Wesmael, 1845

Stenodontus marginellus (Gravenhorst, 1829)

Nomenclature:

Ichneumon marginellus Gravenhorst, 1829

albicoxis Habermehl, 1917

Distribution: England, Scotland, Wales, Ireland

Genus Trachyarus Thomson, 1891

Notes: Gokhman (2007) states that a series of males from Hampshire, in BMNH, probably belong to $T$. prominulus Diller, 1989. I have also seen a female from the site and cannot differentiate these specimens from others that V. Gokhman has identified as corvinus.

Trachyarus corvinus Thomson, 1891

Nomenclature:

atratus (Berthoumieu, 1901, Phaeogenes)

Distribution: England

Genus Tycherus Förster, 1869

Nomenclature:

MICROPE Förster, 1869

PROSCUS Holmgren, 1890

MICROPA Schulz, 1906

GLYPTICHNEUMON Habermehl, 1917

Tycherus amaenus (Wesmael, 1845)

Nomenclature:

Phaeogenes amaenus Wesmael, 1845 
flavoclypeatus (Strobl, 1901, Phaeogenes)

Distribution: Wales

Notes: Added by Diller and Shaw (2014); transferred from Phaeogenes by Sebald et al. (2000).

\section{Tycherus bellicornis (Wesmael, 1845)}

Nomenclature:

Phaeogenes bellicornis Wesmael, 1845

rugulosus (Constantineanu, 1959, Phaeogenes)

Distribution: England, Scotland, Wales, Ireland

Tycherus brunneus (Kiss, 1924)

Nomenclature:

Eriplatys brunneus Kiss, 1924

Distribution: England

Notes: added by Diller and Shaw (2014)

Tycherus capitosus (Holmgren, 1890)

Nomenclature:

Phaeogenes capitosus Holmgren, 1890

Distribution: Scotland

Notes: added by Diller and Shaw (2014)

Tycherus cephalotes (Wesmael, 1845)

Nomenclature:

Phaeogenes cephalotes Wesmael, 1845

Distribution: England

Tycherus coriaceus (Perkins, 1953)

Nomenclature:

Phaeogenes coriaceus Perkins, 1953

Distribution: England, Scotland 
Tycherus dodecellae Ranin, 1983

Distribution: Scotland

Notes: added by Diller and Shaw (2014)

Tycherus elongatus (Thomson, 1891)

Nomenclature:

Phaeogenes elongatus Thomson, 1891

Distribution: England

Tycherus eques (Wesmael, 1845)

Nomenclature:

Phaeogenes eques Wesmael, 1845

Distribution: England, Scotland

Tycherus flavidens (Wesmael, 1845)

Nomenclature:

Phaeogenes flavidens Wesmael, 1845

flavoclypeatus (Strobl, 1901, Herpestomus)

Distribution: England

Tycherus fuscibucca (Berthoumieu, 1901)

Nomenclature:

Ischnogaster fuscibucca Berthoumieu, 1901

kratochvili (Gregor, 1943, Eriplatys)

Distribution: Scotland

Notes: added by Diller and Shaw (2014)

Tycherus fuscicornis (Wesmael, 1845)

Nomenclature:

Phaeogenes fuscicornis Wesmael, 1845

phaeogenoides (Habermehl, 1917, Glyptichneumon)

Distribution: England, Scotland, Ireland 
Tycherus helleni Ranin, 1983

Distribution: Scotland

Notes: added by Diller and Shaw (2014)

Tycherus histrio (Wesmael, 1848)

Nomenclature:

Phaeogenes histrio Wesmael, 1848

Notes: Removed from synonymy with ischiomelinus by Diller and Shaw (2014); only British specimen lacking locality data.

Tycherus impiger (Wesmael, 1845)

Nomenclature:

Phaeogenes impiger Wesmael, 1845

ruficoxis (Constantineanu, 1951, Phaeogenes)

Distribution: England, Scotland

Notes: Transferred from Phaeogenes by Bauer (2001).

Tycherus improcerus Ranin, 1983

Distribution: England

Notes: added by Diller and Shaw (2014)

Tycherus infimus (Wesmael, 1845)

Nomenclature:

Phaeogenes infimus Wesmael, 1845

minutus (Wesmael, 1845, Phaeogenes)

Distribution: England, Scotland, Ireland

Tycherus ischiomelinus (Gravenhorst, 1829)

Nomenclature:

Ichneumon ischiomelinus Gravenhorst, 1829

eximius (Wesmael, 1845, Phaeogenes)

Distribution: England, Scotland, Ireland 
Tycherus jucundus (Wesmael, 1845)

Nomenclature:

Phaeogenes jucundus Wesmael, 1845

Distribution: England

Notes: BMNH, added here; transferred from Phaeogenes by Diller and Schönitzer (2003). Has also been treated as a species of Colpognathus, including by J.F. Perkins in his curation of the collections of BMNH.

\section{Tycherus macilentus (Wesmael, 1845)}

Nomenclature:

Phaeogenes macilentus Wesmael, 1845

Distribution: Ireland

\section{Tycherus modestus (Wesmael, 1845)}

Nomenclature:

Phaeogenes modestus Wesmael, 1845

grammostoma (Kriechbaumer, 1887, Phaeogenes)

Distribution: England, Scotland

\section{Tycherus ophtalmicus (Wesmael, 1845)}

Nomenclature:

Phaeogenes ophtalmicus Wesmael, 1845

ophthalmicus misspelling

hybridus (Wesmael, 1845, Phaeogenes)

pulchricornis (Brischke, 1891, Phaeogenes)

palliventris (Berthoumieu, 1910, Phaeogenes)

Distribution: England, Scotland, Wales, Ireland

\section{Tycherus osculator (Thunberg, 1824)}

\section{Nomenclature:}

Ichneumon osculator Thunberg, 1824

nanus (Wesmael, 1845, Phaeogenes)

lascivus (Wesmael, 1855, Phaeogenes) 
socius (Holmgren, 1890, Phaeogenes)

inanis (Berthoumieu, 1901, Phaeogenes)

tristis (Berthoumieu, 1904, Phaeogenes)

strandi (Berthoumieu, 1910, Dicaelotus)

nigroclypeatus (Constantineanu, 1942, Phaeogenes)

Distribution: England, Scotland, Wales, Ireland

Tycherus planipectus (Holmgren, 1890)

Nomenclature:

Phaeogenes planipectus Holmgren, 1890

Distribution: Scotland

Notes: added by Diller and Shaw (2014)

Tycherus socialis (Ratzeburg, 1852)

Nomenclature:

Ichneumon socialis Ratzeburg, 1852

discoidalis (Ratzeburg, 1852, Ichneumon)

clypearis (Brischke, 1878, Phaeogenes)

martialis (Pic, 1899, Phaeogenes)

Distribution: England

Notes: added by Diller and Shaw (2014)

\section{Tycherus stipator (Wesmael, 1855)}

Nomenclature:

Phaeogenes stipator Wesmael, 1855

cambriensis (Desvignes, 1867, Ichneumon)

cicutellus (Brischke, 1878, Phaeogenes)

basirufus (Constantineanu, 1951, Phaeogenes)

fuscitarsis (Constantineanu, 1951, Phaeogenes)

Distribution: England, Scotland, Wales, Ireland 
Tycherus suspicax (Wesmael, 1845)

Nomenclature:

Phaeogenes suspicax Wesmael, 1845

crassiceps (Habermehl, 1917, Proscus)

Distribution: England, Scotland, Wales, Ireland

Tycherus teres (Berthoumieu, 1906)

Nomenclature:

Phaeogenes teres Berthoumieu, 1906

Distribution: England

Notes: added by Diller and Shaw (2014)

Tycherus vagus (Berthoumieu, 1899)

Nomenclature:

Phaeogenes vagus Berthoumieu, 1899

Distribution: England

Notes: added by Diller and Shaw (2014)

Tycherus verecundus Ranin, 1983

Distribution: England

Notes: added by Ranin (1983)

Tribe PLATYLABINI Berthoumieu, 1904

Nomenclature:

PRISTICEROTINI Townes, 1961

Genus Apaeleticus Wesmael, 1845

Apaeleticus bellicosus Wesmael, 1845

Nomenclature:

inclytus Wesmael, 1853

cautus (Berthoumieu, 1898, Diadromus)

rufipes Constantineanu, 1951 
Distribution: England

Apaeleticus inimicus (Gravenhorst, 1820)

Nomenclature:

Ichneumon inimicus Gravenhorst, 1820

haematodus (Gravenhorst, 1829, Cryptus) synonymy by Sawoniewicz and Wanat (2003)

flammeolus Wesmael, 1845

balearicus Kriechbaumer, 1894

amoenus Habermehl, 1917

muelleri (Kiss, 1929, Microcryptus)

Distribution: England

Genus Asthenolabus Heinrich, 1951

Nomenclature:

STENOLABUS Heinrich, 1936 preocc.

Asthenolabus latiscapus (Thomson, 1894)

Nomenclature:

Platylabus latiscapus Thomson, 1894

Distribution: England

\section{Asthenolabus vitratorius (Gravenhorst, 1829)}

Nomenclature:

Mesoleptus vitratorius Gravenhorst, 1829

albinus (Gravenhorst, 1829, Hoplismenus)

errabundus (Gravenhorst, 1829, Hoplismenus)

tinctorius (Gravenhorst, 1829, Cryptus)

coxalis (Habermehl, 1917, Platylabus)

Distribution: England, Wales 
Genus Cyclolabus Heinrich, 1936

Cyclolabus axillatorius (Thunberg, 1824)

Nomenclature:

Ichneumon axillatorius Thunberg, 1824

pactor (Wesmael, 1845, Platylabus) synonymy by Riedel (2014)

pici (Berthoumieu, 1910, Anisobas)

septentrionalis (Berthoumieu, 1910, Dicaelotus)

Distribution: England, Scotland, Ireland

Cyclolabus dubiosus Perkins, 1953

Distribution: England

Cyclolabus nigricollis (Wesmael, 1845)

Nomenclature:

Platylabus nigricollis Wesmael, 1845

Distribution: England, Wales

Genus Dentilabus Heinrich, 1974

Dentilabus variegatus (Wesmael, 1845)

Nomenclature:

Platylabus variegatus Wesmael, 1845

Distribution: England, Scotland

Genus Ectopius Wesmael, 1859

Ectopius rubellus (Gmelin, 1790)

Nomenclature:

Ichneumon rubellus Gmelin, 1790

thedenii (Holmgren, 1871, Platylabus)

Distribution: Ireland 
Genus Hypomecus Wesmael, 1845

Hypomecus quadriannulatus (Gravenhorst, 1829)

Nomenclature:

Mesoleptus quadriannulatus Gravenhorst, 1829

albitarsis Wesmael, 1845

submarginatus (Magretti, 1896, Platylabus) synonymy by Riedel (2008)

carens Berthoumieu, 1897

Distribution: England, Scotland

Genus Linycus Cameron, 1903

Linycus exhortator (Fabricius, 1787)

Nomenclature:

Ichneumon exhortator Fabricius, 1787

dimidiatus (Gravenhorst, 1829, Hoplismenus)

discedens (Gravenhorst, 1829, Phygadeuon)

latior (Pic, 1902, Platylabus)

tricolor (Berthoumieu, 1904, Platylabus)

balearicus (Hedwig, 1939, Platylabus) synonymy by Riedel (2008)

Distribution: England, Scotland, Wales, Ireland

Linycus flavitarsis (Heinrich, 1937)

Nomenclature:

Ectopius flavitarsis Heinrich, 1937

priesneri Heinrich, 1972

Distribution: Scotland

Notes: NMS, det. Riedel, added here

\section{Genus Platylabus Wesmael, 1845}

Notes: synonymy follows Riedel (2008) 
Platylabus concinnus Thomson, 1888

Distribution: England

Platylabus curtorius (Thunberg, 1824)

Nomenclature:

Ichneumon curtorius Thunberg, 1824

eurygaster Holmgren, 1871 synonymy by Riedel (2008)

punctifrons Thomson, 1888 synonymy by Riedel (2007)

Distribution: England, Scotland, Wales

Platylabus daemon Wesmael, 1845

Distribution: Wales

Notes: Added by Riedel (2008) and transferred from Asthenolabus.

Platylabus dolorosus (Gravenhorst, 1829)

Nomenclature:

Ichneumon dolorosus Gravenhorst, 1829

sollicitus Wesmael, 1845

viturati (Pic, 1902, Ichneumon)

Distribution: England, Scotland

Platylabus fugator (Gravenhorst, 1807)

Nomenclature:

Ichneumon fugator Gravenhorst, 1807

atricornis Pic, 1926 synonymy by Riedel (2007)

Distribution: Scotland

Notes: added by Riedel (2008)

Platylabus gigas Kriechbaumer, 1886

Distribution: England 
Platylabus heteromallus (Berthoumieu, 1910)

Nomenclature:

Amblyteles heteromallus Berthoumieu, 1910

pedatorius misident. (Horstmann 2001a)

rhenana Habermehl, 1917

Distribution: England, Scotland, Wales, Ireland, Isle of Man

Notes: Removed from synonymy with curtorius by Riedel (2008), who found that curtorius is a senior synonym of punctifrons and eurygaster.

\section{Platylabus histrio Wesmael, 1855}

Nomenclature:

varipedulis Wesmael, 1857

erberi Tischbein, 1868

Distribution: England, Scotland

Platylabus intermedius Holmgren, 1871

Nomenclature:

polonicus Heinrich, 1937

Distribution: England, Scotland

Platylabus iridipennis (Gravenhorst, 1829)

Nomenclature:

Ichneumon iridipennis Gravenhorst, 1829

helensis (Brischke, 1888, Ichneumon)

fornicatus Kriechbaumer, 1890 synonymy by Horstmann (2006a)

calidus Berthoumieu, 1904 synonymy by Riedel (2008)

novellus Berthoumieu, 1910

Distribution: England, Scotland

Platylabus judaicus Berthoumieu, 1900

Nomenclature:

stolidus Perkins, 1953 synonymy by Riedel (2007)

Distribution: England 
Platylabus neglectus (Fonscolombe, 1847)

Nomenclature:

Ichneumon neglectus Fonscolombe, 1847

decipiens Wesmael, 1848 synonymy by Riedel (2007)

minai (De Stefani, 1885, Ischnus) synonymy by Riedel (2008)

Distribution: England

Platylabus nigrocyaneus (Gravenhorst, 1829)

Nomenclature:

Ichneumon nigrocyaneus Gravenhorst, 1829

armatus Wesmael, 1845

Platylabus obator (Desvignes, 1856)

Nomenclature:

Ichneumon obator Desvignes, 1856

Distribution: England, Scotland

Platylabus odiosus Perkins, 1953

Distribution: England, Scotland

Platylabus opaculus Thomson, 1888

Distribution: England, Ireland

Platylabus orbitalis (Gravenhorst, 1829)

Nomenclature:

Ichneumon orbitalis Gravenhorst, 1829

vibratorius misident. (Riedel 2008)

persecutor (Gravenhorst, 1829, Ichneumon)

subalbellus (Gravenhorst, 1829, Ichneumon)

volubilis (Gravenhorst, 1829, Ichneumon) synonymy by Riedel (2008)

suborbitalis Kriechbaumer, 1894 synonymy by Horstmann (2006a)

muticus Thomson, 1894 synonymy by Riedel (2007)

Distribution: England, Scotland, Wales 
Platylabus perexiguus Heinrich, 1973

Distribution: England

Notes: NMS, det. Riedel, added here

Platylabus pseudopumilio Riedel, 2008

Distribution: England, Scotland, Wales

Notes: added by Riedel (2008)

Platylabus pumilio Holmgren, 1871

Distribution: England

Platylabus rufus Wesmael, 1845

Nomenclature:

pictus Vollenhoven, 1878

rubeus Valemberg, 1976 synonymy by Valemberg (2001)

Distribution: England, Scotland, Wales, Ireland

Platylabus sternoleucus Wesmael, 1853

Distribution: England, Scotland, Wales

Notes: Added by Riedel (2008) and transferred from Asthenolabus.

Platylabus tenuicornis (Gravenhorst, 1829)

Nomenclature:

Ichneumon tenuicornis Gravenhorst, 1829

niger Wesmael, 1845

Distribution: England

Platylabus transversus Bridgman, 1889

Nomenclature:

lativentris Thomson, 1894

Distribution: England 
Platylabus tricingulatus (Gravenhorst, 1820)

Nomenclature:

Ichneumon tricingulatus Gravenhorst, 1820

maurus Berthoumieu, 1900

berthoumieui Pic, 1923

zagoriensis Heinrich, 1930 synonymy by Riedel (2008)

Distribution: England

Platylabus vibratorius (Thunberg, 1824)

Nomenclature:

Ichneumon vibratorius Thunberg, 1824

wienkeri (Ratzeburg, 1844, Ichneumon) synonymy by Riedel (2008)

rufiventris Wesmael, 1845 synonymy by Horstmann (2000b)

Distribution: England

\section{Genus Platymischos Tischbein, 1868}

Nomenclature:

RYSSOLABUS Berthoumieu, 1894

Platymischos atriventris (Pic, 1914)

Nomenclature:

Ryssolabus atriventris Pic, 1914

arcticus (Hellén, 1942, Ryssolabus)

montanus (Heinrich, 1951, Ryssolabus)

Distribution: Scotland

Genus Poecilostictus Ratzeburg, 1852

Nomenclature:

IDIOSTOLIS Förster, 1869

NEOPLATYLABUS Heinrich, 1936 
Poecilostictus cothurnatus (Gravenhorst, 1829)

Nomenclature:

Hoplismenus cothurnatus Gravenhorst, 1829

orbitatus (Gravenhorst, 1829, Hoplismenus)

octopunctatus Ratzeburg, 1852

ratzeburgi Kawall, 1868 synonymy by Horstmann (1997)

apicalis (Brischke, 1892, Hepiopelmus)

geometrae (Berthoumieu, 1894, Platylabus)

saxonicus (Hedwig, 1939, Platylabus)

Distribution: England, Wales

Genus Pristicerops Heinrich, 1961

Pristicerops infractorius (Linnaeus, 1761)

Nomenclature:

Ichneumon infractorius Linnaeus, 1761

phaleratus (Haliday, 1839, Ichneumon)

leucogrammus (Wesmael, 1853, Platylabus)

Distribution: England, Scotland, Wales, Ireland, Isle of Man

Genus Pristiceros Gravenhorst, 1829

Pristiceros serrarius Gravenhorst, 1829

Distribution: England

Tribe ZIMMERIINI Heinrich, 1934

Genus Cotiheresiarches Telenga, 1929

Nomenclature:

ZIMMERIA Heinrich, 1934

Cotiheresiarches dirus (Wesmael, 1853)

Nomenclature:

Eurylabus dirus Wesmael, 1853 
niger Telenga, 1929

Notes: Specimens in BMNH lack locality data but reliably recorded as British by Perkins (1959).

\section{Lycorininae}

Subfamily LYCORININAE Cushman \& Rohwer, 1920

Nomenclature:

LYCORINAE misspelling

Genus Lycorina Holmgren, 1859

Nomenclature:

AMYS Schiødte, 1839 nom. ob., synonymy by Horstmann (2004a)

TOXOPHOROIDES Cresson, 1873

CHLOROLYCORINA Cushman, 1920

GONIOGLYPHUS Seyrig, 1932

\section{Lycorina triangulifera Holmgren, 1859}

Nomenclature:

flavilabris (Schiødte, 1839, Amys) nom. ob., synonymy by Horstmann (2004a)

lycorinoides (Costa, 1886, Glypta)

sardoa (Costa, 1886, Glypta)

Distribution: England, Scotland, Ireland

Notes: some distribution data from Shaw (2004)

\section{Mesochorinae}

\section{Subfamily MESOCHORINAE Förster, 1869}

Notes: Schwenke (1999) reinstated various synonyms of Astiphromma and Mesochorus as valid genera. This treatment is not followed here as Wahl (1993) gave good reasons for treating these as synonyms. Looking at material from across the globe, there are no clear-cut differences between, for example, Stictopisthus and Mesochorus. An exception is Dolichochorus, q.v. Distribution data, unless noted otherwise, are taken from Schwenke (1999), NMS, BMNH and UM. 


\section{Genus Astiphromma Förster, 1869}

\section{Nomenclature:}

ASTIPHROMMUS Thomson, 1886

MESOCHORELLA Szépligeti, 1911

PSEUDACOENITUS Kiss, 1924

DEMOPHORELLUS Hedwig, 1955

Notes: Some distribution data and much synonymy from Riedel (2015), with most identifications by M. Riedel and GRB. J.F. Perkins, A. Roman and W. Schwenke had also identified a few interesting specimens in $\mathrm{BMNH}$.

species of Astiphromma excluded from the British and Irish list:

[dorsale (Holmgren, 1860, Mesochorus)] No British or Irish specimens have been seen (Riedel 2015). The previously synonymous name hirsutum, described from British specimens, is a senior synonym of granigerum.

[striatum (Brischke, 1880, Mesochorus); syn. mandibulare (Thomson, 1886, Mesochorus)] All British specimens under the name mandibulare (synonymised under striatum by Riedel 2015) in BMNH have proved to be misidentifications.

\section{Astiphromma aggressor (Fabricius, 1804)}

\section{Nomenclature:}

Ophion aggressor Fabricius, 1804

marginellum (Holmgren, 1860, Mesochorus)

alpinum (Roman, 1909, Mesochorus)

barbatum Schwenke, 1999

caecum Schwenke, 1999

Distribution: England

Notes: added by Riedel (2015)

\section{Astiphromma albitarse (Brischke, 1880)}

\section{Nomenclature:}

Mesochorus albitarsis Brischke, 1880

nigrum Pfankuch, 1921

heydeni Habermehl, 1923

transsylvanicum (Kiss, 1924, Pseudacoenitus) 
Distribution: England, Scotland

Notes: added by Riedel (2015)

Astiphromma alpinum Roman, 1909

Nomenclature:

dispersum Schwenke, 1999

laricis Schwenke, 1999

Distribution: England

Notes: added by Riedel (2015)

Astiphromma anale (Holmgren, 1860)

Nomenclature:

Mesochorus analis Holmgren, 1860

Distribution: England, Scotland

Notes: added by Riedel (2015)

Astiphromma buccatum (Thomson, 1886)

Nomenclature:

Mesochorus buccatus Thomson, 1886

hamulum (Thomson, 1886, Mesochorus)

consertum Schwenke, 1999

Distribution: England

Astiphromma hirsutum (Bridgman, 1883)

Nomenclature:

Mesochorus hirsutus Bridgman, 1883

granigerum (Thomson, 1886, Mesochorus)

Distribution: England, Scotland, Ireland 
Astiphromma italicum Schwenke, 1999

Nomenclature:

contum Schwenke, 1999

Distribution: England

Notes: added by Riedel (2015)

Astiphromma leucogrammum (Holmgren, 1860)

Nomenclature:

Mesochorus leucogrammus Holmgren, 1860

Distribution: England, Scotland

Notes: NMS, added here

Astiphromma nigrocoxatum (Strobl, 1904)

Nomenclature:

Mesochorus nigrocoxatus Strobl, 1904

mimulum (Hedwig, 1955, Demophorellus)

Distribution: England

Notes: added by Riedel (2015)

Astiphromma pictum (Brischke, 1880)

Nomenclature:

Mesochorus pictus Brischke, 1880

incidens (Thomson, 1886, Mesochorus)

Distribution: England

Astiphromma scutellatum (Gravenhorst, 1829)

Nomenclature:

Mesochorus scutellatus Gravenhorst, 1829

festivum (Holmgren, 1860, Mesochorus)

Distribution: England 
Astiphromma splenium (Curtis, 1833)

Nomenclature:

Mesochorus splenium Curtis, 1833

sericans (Curtis, 1833, Mesochorus)

strenuum (Holmgren, 1860, Mesochorus)

plagiatum (Thomson, 1886, Mesochorus) synonymy by Schwenke (1999)

Distribution: England, Scotland, Ireland

Notes: Astiphromma sericans was reinstated as a valid species by Horstmann (2006b)

but synonymised under splenium again by Riedel (2015).

Astiphromma tenuicorne (Thomson, 1886)

Nomenclature:

Mesochorus tenuicornis Thomson, 1886

Distribution: England, Scotland

Astiphromma trimaculosum Schwenke, 2004

Distribution: England

Notes: added by Schwenke (2004)

Astiphromma uliginosum Schwenke, 1999

Distribution: England, Wales, Ireland

Notes: added by Riedel (2015)

Astiphromma varipes (Holmgren, 1860)

Nomenclature:

Mesochorus varipes Holmgren, 1860

variipes Dalla Torre, 1901

Distribution: England, Scotland

Notes: added by Riedel (2015) 


\section{Genus Cidaphus Förster, 1869}

Nomenclature:

PLESIOPHTHALMUS Förster, 1869

MATER Schluz, 1911

TETRAGONALYS Morley, 1913

OPHTHALMOCHORUS Roman, 1925

Notes: Distribution data for Cidaphus species is taken from Fitton (1985b) and NMS. Note that Schwenke (1999) used an out-of-date taxonomy for Cidaphus species. Fitton (1985b) and Horstmann (2002b) are followed here.

\section{Cidaphus alarius (Gravenhorst, 1829)}

Nomenclature:

Mesochorus alarius Gravenhorst, 1829

thuringiacus Brauns, 1889

Distribution: England, Wales

\section{Cidaphus areolatus (Boie, 1850)}

Nomenclature:

Paniscus areolatus Boie, 1850

gigas (Kriechbaumer, 1897, Meoschorus) synonymy by Horstmann (2002b)

brischkei (Szépligeti, 1911, Plesiophthalmus) synonymy by Horstmann (2002b)

Distribution: England, Scotland

Notes: Added by Fitton (1985b); listed as a synonym of alarius in Yu and Horstmann (1997), with brischkei as the valid name for this species.

\section{Cidaphus atricillus (Haliday, 1838)}

\section{Nomenclature:}

Cryptus atricillus Haliday, 1838

potanini Kokujev, 1906

melanocephalus (Habermehl, 1909, Plesiophthalmus)

Distribution: England, Scotland, Wales 


\section{Genus Dolichochorus Strobl, 1904}

Notes: Mesochorus longiceps is the type species of Dolichochorus Strobl, 1904. This genus was synonymised under Astiphromma by Townes et al. (1965) but reinstated as a valid genus by Schwenke (1999). Wahl (1993) listed Dolichochorus as a synonym of Astiphromma but did not include longiceps in his cladistic analysis. Broad \& Watanabe (in prep.) have found that Dolichochorus is a rather basal member of the Mesochorinae, and distinct from Astiphromma.

\section{Dolichochorus longiceps (Strobl, 1904)}

Nomenclature:

Mesochorus longiceps Strobl, 1904

Distribution: England

Notes: Added by Schwenke (1999); it is not known on what basis Schwenke (1999) recorded this as a British species but its occurrence in Britain is confirmed by Riedel (2015) and Broad \& Watanabe (in prep.).

\section{Genus Mesochorus Gravenhorst, 1829}

\section{Nomenclature:}

STICTOPISTHUS Thomson, 1886

Notes: Horstmann (2006b) substantially revised the taxonomy of various species of Mesochorus as W. Schwenke had misinterpreted many names.

species of Mesochorus excluded from the British and Irish list:

[nuncupator (Panzer, 1800, Ichneumon)] Horstmann (2006b)

\section{Mesochorus aggestus Schwenke, 2002}

Nomenclature:

sulcatus Schwenke, 1999

Distribution: England

Notes: added by Schwenke (1999)

\section{Mesochorus albionis Schwenke, 1999}

Distribution: England

Notes: added by Schwenke (1999) 
Mesochorus alpigenus Strobl, 1904

Nomenclature:

compactus Schwenke, 1999 synonymy by Horstmann (2001b)

Distribution: Scotland

Mesochorus anglicus Schwenke, 1999

Distribution: England

Notes: added by Schwenke (1999)

Mesochorus angustatus Thomson, 1886

Mesochorus anomalus Holmgren, 1860

Mesochorus arenarius (Haliday, 1839)

Nomenclature:

Cryptus arenarius Haliday, 1839

nigripes Ratzeburg, 1852 Horstmann (2006b)

melas Fonscolombe, 1852

gibbulus Holmgren, 1856

Distribution: England, Scotland, Ireland

Mesochorus atriventris Cresson, 1872

Nomenclature:

sylvarum (Haliday, 1839, Cryptus) synonymy by Horstmann (2006b)

Distribution: England, Scotland, Ireland, Isle of Man

Notes: some distribution data from Shaw (1993), Horstmann (2006b)

Mesochorus basalis Curtis, 1833

Distribution: England

Mesochorus bracatus Schwenke, 1999

Distribution: Ireland

Notes: added by Schwenke (1999) 
Mesochorus brevipetiolatus Ratzeburg, 1844

Mesochorus britannicus Schwenke, 1999

Distribution: England

Notes: added by Schwenke (1999)

Mesochorus carinatus Schwenke, 1999

Distribution: England

Notes: added by Schwenke (1999)

Mesochorus cimbicis Ratzeburg, 1844

Nomenclature:

confusus Holmgren, 1860 synonymy by Horstmann (2006b)

longicauda Thomson, 1886 synonymy by Schwenke (1999)

gallicator Aubert, 1963 synonymy by Schwenke (1999)

Distribution: England, Scotland

Mesochorus dimidiator Aubert, 1970

Distribution: England

Notes: added by Horstmann (2006b)

Mesochorus discitergus (Say, 1835)

Nomenclature:

Cryptus discitergus Say, 1835

facialis Bridgman, 1884

baueri Schwenke, 1999 synonymy by Horstmann (2003b)

Distribution: England

Notes: distribution data from Horstmann (2003b) 
Mesochorus discolor Schwenke, 1999

Distribution: England

Notes: added by Schwenke (1999)

Mesochorus dispar Brischke, 1880

Distribution: Ireland

Notes: added by Horstmann (2002c)

Mesochorus errabundus Hartig, 1838

Nomenclature:

politus misident.

Distribution: England, Scotland

Notes: BMNH, added here; this species was treated as a synonym of politus Gravenhorst, 1829 by Schwenke (1999) but Horstmann (2003b) established errabundus as a distinct species. Using the characters given by Horstmann for distinguishing the two species, both occur here.

Mesochorus extensator Schwenke, 2002

Distribution: England

Notes: added by Schwenke (2002)

Mesochorus flavescens Fonscolombe, 1852

Distribution: England

Notes: added by Schwenke (1999)

Mesochorus formosus Bridgman, 1882

Nomenclature:

convexicollis Thomson, 1886 synonymy by Schwenke (1999)

Distribution: England, Scotland

Mesochorus fulgurans Curtis, 1833

Nomenclature:

fulgurans (Haliday, 1839, Cryptus) preocc. 
pectinipes Thomson, 1886 synonymy by Horstmann (2006b)

fulvus Thomson, 1886 synonymy by Horstmann (2006b)

suecicus Dalla Torre, 1901 synonymy by Horstmann (2006b)

Distribution: England, Scotland, Ireland

Notes: some distribution data from Gauld (1970)

Mesochorus fulgurator Horstmann, 2006

Distribution: England

Notes: BMNH, det. Broad, added here

Mesochorus fuscicornis Brischke, 1880

Distribution: England, Scotland

Mesochorus fuscus Schwenke, 1999

Distribution: England

Notes: added by Schwenke (1999), Schwenke (2004)

Mesochorus gemellus Holmgren, 1860

Nomenclature:

tachypus Holmgren, 1860

brevicollis Thomson, 1886

Distribution: England

Mesochorus giberius (Thunberg, 1824)

Nomenclature:

Ichneumon giberius Thunberg, 1824

thoracicus Gravenhorst, 1829

sylvarum Curtis, 1833 synonymy by Horstmann (2006b)

marginatus Thomson, 1886 synonymy by Schwenke (1999)

Distribution: England, Scotland, Ireland 
Mesochorus globulator (Thunberg, 1824)

Nomenclature:

Ichneumon globulator Thunberg, 1824

crassimanus Holmgren, 1860

dimidiatus Holmgren, 1860

sericeus Brischke, 1880 synonymy by Horstmann (2006b)

Distribution: Scotland, Isle of Man

Notes: some distribution data from Horstmann (2004b), Horstmann (2006b)

Mesochorus gracilentus Brischke, 1880

Notes: reinstated by Horstmann (2006b)

Mesochorus iniquus Schwenke, 1999

Distribution: England, Scotland

Notes: added by Horstmann (2006b)

Mesochorus insularis Schwenke, 1999

Distribution: England

Notes: added by Schwenke (1999)

Mesochorus jenniferae Schwenke, 2002

Distribution: England

Notes: added by Schwenke (2002), Schwenke (2004)

Mesochorus laricis Hartig, 1838

Distribution: England

Notes: BMNH, det. Horstmann, added here

Mesochorus latus Schwenke, 1999

Distribution: England

Notes: added by Schwenke (1999) 
Mesochorus lilioceriphilus Schwenke, 2000

Distribution: England

Notes: added by Salisbury and Broad (2011)

Mesochorus liquidus Schwenke, 2002

Distribution: England

Notes: added by Schwenke (2002)

Mesochorus nematus Schwenke, 2004

Distribution: England

Notes: added by Schwenke (2004)

Mesochorus olerum Curtis, 1833

Nomenclature:

pectoralis Ratzeburg, 1844 synonymy by Horstmann (2006b)

rapae Schwenke, 1999 synonymy by Horstmann (2008a)

Distribution: England

Mesochorus orbitalis Holmgren, 1860

Distribution: England, Scotland

Mesochorus owenae Schwenke, 1999

Distribution: England

Notes: added by Schwenke (1999)

Mesochorus oxfordensis Schwenke, 1999

Distribution: England

Notes: added by Schwenke (1999)

Mesochorus pallipes Brischke, 1880

Nomenclature:

stigmaticus Brischke, 1880 synonymy by Horstmann (2006b) 
brunneus Brischke, 1880 synonymy by Horstmann (2006b)

rufipes Brischke, 1880 synonymy by Horstmann (2006b)

albipes Thomson, 1886 synonymy by Schwenke (1999)

crassicrus Thomson, 1886 synonymy by Horstmann (2006b)

Distribution: England, Scotland, Wales

Notes: Mesochorus pallipes sensu Schwenke (1999) is not this species (Horstmann 2006b).

Mesochorus pectinellus Horstmann, 2006

Distribution: England

Notes: BMNH, det. Horstmann, added here

Mesochorus pectinipes Bridgman, 1883

Distribution: England

Mesochorus perticatus Schwenke, 1999

Distribution: England

Notes: added by Schwenke (1999)

Mesochorus pictilis Holmgren, 1860

Mesochorus politus Gravenhorst, 1829

Distribution: England

Mesochorus pumilionis Schwenke, 1999

Distribution: England

Notes: added by Schwenke (1999), Schwenke (2004)

Mesochorus punctipleuris Thomson, 1886

Nomenclature:

nigriceps Thomson, 1886 preocc., synonymy by Horstmann (2001c)

thomsonii Dalla Torre, 1901

thomsoni Strobl, 1904 preocc. 
amplitudinis Schwenke, 1999 synonymy by Horstmann (2002c)

Distribution: England, Ireland

Notes: Added by Horstmann (2002c); listed as a synonym of M. agilis Cresson, 1865 in

Yu and Horstmann (1997).

Mesochorus quercus Schwenke, 2004

Distribution: England

Notes: added by Schwenke (2004)

Mesochorus rufoniger Brischke, 1880

Nomenclature:

brevigena Thomson, 1886

Distribution: England, Scotland

Notes: NMS, UM, det. Brock, added here

Mesochorus rutilus Schwenke, 2002

Distribution: England

Notes: added by Schwenke (2002)

Mesochorus scutellaris Schwenke, 2004

Distribution: England

Notes: added by Schwenke (2004)

Mesochorus semirufus Holmgren, 1860

Mesochorus stigmator (Thunberg, 1824)

Nomenclature:

Ichneumon stigmator Thunberg, 1824

splendidulus Gravenhorst, 1829 synonymy by Horstmann (2001b)

pallidus Brischke, 1880

stigmaticus Thomson, 1886 preocc.

orgyiae Dalla Torre, 1902 
Mesochorus temporalis Thomson, 1886

Distribution: England

Mesochorus tenuiscapus Thomson, 1886

Distribution: England, Scotland

Mesochorus testaceus Gravenhorst, 1829

Distribution: England

Mesochorus tetricus Holmgren, 1860

Nomenclature:

macrurus Thomson, 1886

Distribution: England

Mesochorus trifoveatus Schwenke, 2004

Distribution: England

Notes: added by Schwenke (2004)

Mesochorus unicinctor (Thunberg, 1824)

Nomenclature:

Ichneumon unicinctor Thunberg, 1824

complanatus (Haliday, 1839, Cryptus) synonymy by Schwenke (1999)

aciculatus Bridgman, 1881

laticeps Thomson, 1886

Distribution: England, Ireland

Mesochorus velox Holmgren, 1860

Mesochorus vittator (Zetterstedt, 1838)

Nomenclature:

Tryphon vittator Zetterstedt, 1838

Distribution: Isle of Man

Notes: added by Horstmann (2006b) 
Mesochorus vitticollis Holmgren, 1860

Nomenclature:

hungaricus Szépligeti, 1914 synonymy by Schwenke (1999)

Distribution: England, Scotland, Wales

Mesochorus windsorianus Schwenke, 2004

Distribution: England

Notes: added by Schwenke (2004)

\section{Metopiinae}

Subfamily METOPIINAE Förster, 1869

Genus Carria Schmiedeknecht, 1924

Carria paradoxa Schmiedeknecht, 1924

Distribution: England

Genus Chorinaeus Holmgren, 1858

Chorinaeus australis Thomson, 1887

Nomenclature:

flavifrons Schmiedeknecht, 1925 preocc.

xanthopsis (Townes, 1946, Trieces)

Distribution: England, Wales

Chorinaeus brevicalcar Thomson, 1887

Distribution: England, Scotland

Chorinaeus cristator (Gravenhorst, 1829)

Nomenclature:

Exochus cristator Gravenhorst, 1829

Distribution: England, Scotland, Wales 
Chorinaeus flavipes Bridgman, 1881

Distribution: England, Scotland

Chorinaeus funebris (Gravenhorst, 1829)

Nomenclature:

Exochus funebris Gravenhorst, 1829

femoratus Teunissen, 1948

Distribution: England, Scotland, Wales

Chorinaeus hastianae Aeschlimann, 1975

Distribution: England, Wales

Chorinaeus longicornis Thomson, 1887

Distribution: England, Scotland

Chorinaeus rhenanus Aeschlimann, 1981

Distribution: England

Notes: NMS, det. Aeschlimann, added here

Chorinaeus subcarinatus Holmgren, 1858

Nomenclature:

longicalcar Thomson, 1887

Distribution: England, Scotland

Chorinaeus talpa (Haliday, 1839)

Nomenclature:

Exochus talpa Haliday, 1839

Distribution: England, Scotland, Ireland

Genus Colpotrochia Holmgren, 1856

Colpotrochia cincta (Scopoli, 1763)

Nomenclature:

Sphex cincta Scopoli, 1763 


$$
\begin{aligned}
& \text { elegantula (Schrank, 1781, Ichneumon) } \\
& \text { mandator (Fabricius, 1787, Ichneumon) preocc. } \\
& \text { mundator (Thunberg, 1824, Ichneumon) } \\
& \text { affinis Vollenhoven, } 1875
\end{aligned}
$$

Distribution: England, Scotland

\section{Genus Exochus Gravenhorst, 1829}

\section{Nomenclature:}

Amesolytus Förster, 1869

Notes: doubtfully placed species of Exochus

[antiquus Haliday, 1839 nom. dub.]

\section{Exochus albicinctus Holmgren, 1873}

Nomenclature:

anospilus Thomson, 1887

nigricans (Szépligeti, 1898, Amesolytus)

Distribution: England, Scotland

Exochus alpinus (Zetterstedt, 1838)

Nomenclature:

Bassus alpinus Zetterstedt, 1838

Distribution: England

Exochus britannicus Morley, 1911

Distribution: England

Exochus carri Schmiedeknecht, 1924

Distribution: England

\section{Exochus citripes Thomson, 1877}

Distribution: England

Notes: BMNH, added here 
Exochus consimilis Holmgren, 1858

Nomenclature:

parvispina Thomson, 1887

decoloratus Schmiedeknecht, 1924

subalpinus Schmiedeknecht, 1924

Distribution: England

Notes: $\mathrm{BMNH}, \mathrm{UM}$, added here

Exochus erythronotus (Gravenhorst, 1820)

Nomenclature:

Ichneumon erythronotus Gravenhorst, 1820

concinnus Holmgren, 1858

pumilus Holmgren, 1873

rufidorsum (Szépligeti, 1898, Amesolytus)

ghigii Ferrière, 1929

Distribution: England

Exochus flavomarginatus Holmgren, 1856

Distribution: England, Scotland

Exochus fletcheri Bridgman, 1884

Nomenclature:

femoralis Pfankuch, 1925

Distribution: England, Scotland

Exochus frontellus Holmgren, 1858

Exochus gravipes (Gravenhorst, 1820)

Nomenclature:

Ichneumon gravipes Gravenhorst, 1820

Distribution: England, Scotland

Exochus gravis Gravenhorst, 1829 
Exochus intermedius Morley, 1911

Distribution: England

Exochus lentipes Gravenhorst, 1829

Nomenclature:

cylindricus Holmgen, 1858

Exochus lictor Haliday, 1839

Nomenclature:

pectoralis Haliday, 1839

decoratus Holmgren, 1873

Distribution: England, Scotland, Ireland

Exochus mitratus Gravenhorst, 1829

Nomenclature:

affninis Holmgren, 1858

australis Thomson, 1894

pseudaffinis Strobl, 1903

paradoxus Schmiedeknecht, 1924

punctifer Schmiedeknecht, 1924

Exochus nigripalpis Thomson, 1887

Distribution: England

Exochus notatus Holmgren, 1858

Nomenclature:

woldstedtii Holmgren, 1873

Distribution: England, Scotland

Exochus pictus Holmgren, 1858

Distribution: England, Scotland, Ireland 
Exochus prosopius Gravenhorst, 1829

Nomenclature:

maculatus Brischke, 1871

procerus Holmgren, 1873

dioszeghyi Kiss, 1926

Distribution: England, Scotland

Exochus ratzeburgi Holmgren, 1858

Notes: BMNH, added here

Exochus rubroater Schmiedeknecht, 1924

Distribution: England, Ireland

Exochus semilividus Vollenhoven, 1875

Nomenclature:

longicornis Thomson, 1887

Distribution: England

Notes: BMNH, added here

Exochus septentrionalis Holmgren, 1873

Exochus thomsoni Schmiedeknecht, 1924

Nomenclature:

crassicornis Thomson, 1894 preocc.

Distribution: England

Notes: BMNH, added here

Exochus tibialis Holmgren, 1858

Distribution: England, Scotland

Genus Hypsicera Latreille, 1829

Nomenclature:

METACOELUS Förster, 1869 
POLYCLISTUS Förster, 1869

Hypsicera britannica Tolkanitz, 2011

Nomenclature:

anglica (Schmiedeknecht, 1925, Metacoelus) preocc.

Distribution: England

Notes: NMS, det. Aeschlimann, added here; despite its original and replacement names, described from the Channel Islands.

Hypsicera curvator (Fabricius, 1793)

Nomenclature:

Ichneumon curvator Fabricius, 1793

mansuetor (Gravenhorst, 1807, Ichneumon)

affinis (Zetterstedt, 1838, Bassus)

Distribution: England, Scotland, Isle of Man

Hypsicera femoralis (Geoffroy, 1785)

Nomenclature:

Ichneumon femoralis Geoffroy, 1785

Distribution: England

Hypsicera flaviceps (Ratzeburg, 1852)

Nomenclature:

Exochus flaviceps Ratzeburg, 1852

Distribution: England, Scotland, Ireland

Hypsicera subtilitor Aubert, 1969

Distribution: England

Notes: added by Aeschlimann (1989)

Genus Ischyrocnemis Holmgren, 1858

Nomenclature:

TEROZOA Förster, 1869 
TERATOZOA Schulz, 1906

Ischyrocnemis goesi Holmgren, 1858

Distribution: England

Notes: added by Broad and Shaw (2005)

Genus Metopius Panzer, 1806

Subgenus Ceratopius Clément, 1927

Metopius (Ceratopius) citratus (Geoffroy, 1762)

Nomenclature:

Ichneumon citratus Geoffroy, 1762

dissectorius (Panzer, 1805, Ichneumon) synonymy by Horstmann (2006c)

sicarius Gravenhorst, 1829

zagoriensis Hensch, 1928

Distribution: England, Scotland, Ireland

Subgenus Metopius Panzer, 1806

Nomenclature:

PELTOPIUS Clément, 1927

Metopius (Metopius) anxius Wesmael, 1849

Nomenclature:

intermedius Förster, 1850

peltator Marshall, 1874

Distribution: England, Scotland, Wales, Ireland

Subgenus Peltastes Illiger, 1807

Nomenclature:

TYLOPIUS Townes, 1959 synonymy by Horstmann (2001a) 
Metopius (Peltastes) leiopygus Förster, 1850

Nomenclature:

marchandi Dominique, 1898

krapinensis Hensch, 1928

Distribution: England, Ireland

Metopius (Peltastes) pinatorius Brullé, 1846

Nomenclature:

meridionalis Hensch, 1928

gracilis Clément, 1930

Distribution: England, Scotland, Ireland

Subgenus Peltocarus Thomson, 1887

Nomenclature:

CLEMONTIA Michener, 1941

Metopius (Peltocarus) croceicornis Thomson, 1887

Nomenclature:

chrysopus (Lewin, 1797, Ichneumon) preocc.

Metopius (Peltocarus) dentatus (Fabricius, 1779)

Nomenclature:

Ichneumon dentatus Fabricius, 1779

?fasciatus (Geoffroy, 1785, Ichneumon)

?lunulatus (Villers, 1789, Ichneumon)

micratorius (Fabricius, 1804, Ichneumon)

denticularis (Thunberg, 1824, Ichneumon)

pini (Curtis, 1824, Peltastes)

incisus Clément, 1930

Distribution: England, Scotland, Ireland

Genus Periope Haliday, 1839

Nomenclature:

MONOPLECTRON Holmgren, 1856 
OLIGOPLECTRON Förster, 1869

MONOPLECTROCHUS Heinrich, 1949

\section{Periope auscultator Haliday, 1839}

Nomenclature:

zygaenator (Holmgren, 1856, Monoplectron)

Distribution: England, Scotland, Ireland

Genus Scolomus Townes, 1969

Nomenclature:

APOLOPHUS Townes, 1971 synonymy by Gauld and Wahl (2006)

Scolomus borealis (Townes, 1971)

Nomenclature:

Apolophus borealis Townes, 1971

Distribution: England, Scotland, Wales, Ireland

Notes: added by Owen et al. (1981); Broad and Shaw (2005)

Genus Stethoncus Townes, 1959

Stethoncus monopicida Broad \& Shaw, 2005

Nomenclature:

sulcator misident.

Distribution: England, Scotland

Notes: Added by Gauld and Sithole (2002); recorded (in the context of a host record) as Stethoncus sulcator Aubert, 1963.

Genus Synosis Townes, 1959

Synosis caesiellae Broad \& Shaw, 2005

Distribution: England, Scotland

Notes: added by Broad and Shaw (2005) 
Synosis fieldi Broad \& Shaw, 2005

Distribution: England

Notes: added by Broad and Shaw (2005)

Synosis parenthesellae Broad \& Shaw, 2005

Distribution: England, Scotland

Notes: added by Broad and Shaw (2005)

Genus Triclistus Förster, 1869

Triclistus aethiops (Gravenhorst, 1829)

Nomenclature:

Exochus aethiops Gravenhorst, 1829

Distribution: England, Scotland

Triclistus albicinctus Thomson, 1887

Triclistus anthophilae Aeschlimann, 1983

Distribution: England, Scotland, Wales

Notes: added by Aeschlimann (1983); Shaw (1984)

Triclistus areolatus Thomson, 1887

Distribution: England, Scotland

Triclistus congener (Holmgren, 1858)

Nomenclature:

Exochus congener Holmgren, 1858

meridionator Aubert, 1960

Distribution: England, Scotland

Triclistus epermeniae Shaw \& Aeschlimann, 1994

Distribution: England, Scotland

Notes: added by Shaw and Aeschliman (1994) 
Triclistus facialis Thomson, 1887

Triclistus globulipes (Desvignes, 1856)

Nomenclature:

Exochus globulipes Desvignes, 1856

holmgreni (Bohemani, 1863, Exochus)

Distribution: England, Scotland, Wales

Triclistus lativentris Thomson, 1887

Distribution: England

Triclistus longicalcar Thomson, 1887

Distribution: England, Scotland

Triclistus niger (Bridgman, 1883)

Nomenclature:

Exochus niger Bridgman, 1883

Distribution: England, Scotland

Triclistus pallipes Holmgren, 1873

Nomenclature:

nitifrons Thomson, 1887

pallidipes Dalla Torre, 1901

Distribution: England, Scotland

Notes: Triclistus nitifrons was regarded as a separate species by J.F. Perkins in his curation of the BMNH collection, with specimens from England and Scotland.

\section{Triclistus podagricus (Gravenhorst, 1829)}

Nomenclature:

Exochus podagricus Gravenhorst, 1829

nigritellus Holmgren, 1873

Distribution: England, Scotland, Ireland 
Triclistus pubiventris Thomson, 1887

Distribution: England, Scotland

Triclistus pygmaeus (Cresson, 1864)

Nomenclature:

Exochus pygmaeus Cresson, 1864

Distribution: England, Scotland, Wales

Triclistus spiracularis Thomson, 1887

Distribution: England

Triclistus squalidus (Holmgren, 1858)

Nomenclature:

Exochus squalidus Holmgren, 1858

Distribution: England, Wales

Triclistus yponomeutae Aeschlimann, 1973

Distribution: England, Scotland

Genus Trieces Townes, 1946

Trieces thuringiacus (Schmiedeknecht, 1925)

Nomenclature:

Chorinaeus thuringiacus Schmiedeknecht, 1925

Distribution: England

Notes: NMS, det. Aeschlimann, added here

Trieces tricarinatus (Holmgren, 1858)

Nomenclature:

Chorinaeus tricarinatus Holmgren, 1858

Distribution: England, Scotland, Ireland 


\section{Microleptinae}

\section{Subfamily MICROLEPTINAE Townes, 1958}

Notes: Microleptinae as treated here includes only the genus Microleptes (Broad 2004), contra Humala (1997). Dasch (1992) gives some distribution data.

\section{Genus Microleptes Gravenhorst, 1829}

Nomenclature:

MIOMERIS Förster, 1869

MIONOMERIS Schulz, 1906

GNATHONIELLA Schmiedeknecht, 1924

\section{Microleptes aquisgranensis (Förster, 1871)}

Nomenclature:

Miomeris aquisgranensis Förster, 1871

Distribution: England, Scotland, Wales

\section{Microleptes rectangulus (Thomson, 1888)}

Nomenclature:

Miomeris rectangulus Thomson, 1888

exareolatus (Strobl, 1903, Seleucus) synonymy by Horstmann (2011b)

egregius (Schmiedeknecht, 1924, Gnathoniella)

Distribution: England, Scotland, Wales, Ireland

\section{Microleptes splendidulus Gravenhorst, 1829}

\section{Nomenclature:}

$$
\text { glabriventris (Thomson, 1888, Miomeris) }
$$

Distribution: England, Scotland, Ireland 


\section{Neorhacodinae}

\section{Subfamily NEORHACODINAE Hedicke, 1922}

Notes: Although synonymised with Tersilochinae by Quicke et al. (2009) the Neorhacodinae are here treated as a separate subfamily again following the phylogenetic results of Bennett et al. (A.M.R. Bennett, pers. comm.) and in light of the different host associations.

\section{Genus Neorhacodes Hedicke, 1922}

\section{Nomenclature:}

RHACODES Ruschka, 1922

Notes: Distribution data from Fitton 1984, Boston 1986 and Notton and Shaw (1988).

Neorhacodes enslini (Ruschka, 1922)

Nomenclature:

Rhacodes enslini Ruschka, 1922

Distribution: England, Scotland, Ireland

\section{Ophioninae}

\section{Subfamily OPHIONINAE Shuckard, 1840}

Notes: Unless stated otherwise, all distribution data are taken from Brock (1982), Gauld (1973), Gauld (1974) and from the nocturnal Ichneumonoidea recording scheme (database maintained by GRB).

\section{Genus Enicospilus Stephens, 1835}

Nomenclature:

HENICOSPILUS Agassiz, 1846

ALLOCAMPTUS Förster, 1869

CYMATONEURA Kriechbaumer, 1901

CRYPTOCAMPTUS Brèthes, 1909

AMESOPHILUS Enderlein, 1914

Notes: Distribution and taxonomy follow Broad and Shaw (2016) 


\section{Enicospilus adustus (Haller, 1885)}

Nomenclature:

Ophion adustus Haller, 1885

merdarius misident.

Distribution: England, Scotland, Ireland

Notes: Enicospilus 'merdarius' auctt. has now been split into three species, none of which is actually merdarius (Broad and Shaw 2016); E. merdarius was regarded as a synonym of E. ramidulus by Gauld (1973) and listed as such in Fitton (1978) but regarded by most recent authors as a valid species, which is borne out by the lack of intermediate specimens and differences in distribution (Broad and Shaw 2016) (allowing for the fact that the name merdarius was misappplied).

\section{Enicospilus cerebrator Aubert, 1966}

Distribution: England

Notes: added by Broad and Shaw (2016)

\section{Enicospilus combustus (Gravenhorst, 1829)}

Nomenclature:

Ophion combustus Gravenhorst, 1829

Distribution: England, Wales

\section{Enicospilus inflexus (Ratzeburg, 1844)}

Nomenclature:

Ophion inflexus Ratzeburg, 1844

Distribution: England, Scotland, Wales

\section{Enicospilus merdarius (Gravenhorst, 1829)}

Nomenclature:

Ophion merdarius Gravenhorst, 1829

repentinus misident.

tournieri (Vollenhoven, 1879, Ophion) synonymy by Broad and Shaw (2016)

rossicus (Kokujev, 1907, Henicospilus)

contributus Shestakov, 1926

Distribution: England, Scotland 
Notes: see note under repentinus

Enicospilus myricae Broad \& Shaw, 2016

Distribution: England, Scotland, Wales

Notes: added by Broad and Shaw (2016)

Enicospilus ramidulus (Linnaeus, 1758)

Nomenclature:

Ichneumon ramidulus Linnaeus, 1758

truncatus (Poda, 1761, Sphex)

instabilis (Kokujev, 1907, Henicospilus)

Distribution: England, Scotland, Wales, Ireland

Enicospilus repentinus (Holmgren, 1860)

Nomenclature:

Ophion repentinus Holmgren, 1860

Distribution: England

Notes: Added by Broad and Shaw (2016); although previously recognised as British (Gauld 1973), this was in error and the specimens are merdarius (=tournieri auctt.), however, Broad and Shaw (2016) record the true repentinus from Britain.

Enicospilus undulatus (Gravenhorst, 1829)

Nomenclature:

Ophion undulatus Gravenhorst, 1829

arcuatus (Brullé, 1846, Ophion)

Distribution: England

\section{Genus Eremotylus Förster, 1869}

Nomenclature:

CAMPTONEURA Kriechbaumer, 1901

CAMPTONEUROIDES Strand, 1928

CLISTORAPHA Cushman, 1947 
Eremotylus curvinervis (Kriechbaumer, 1878)

Nomenclature:

Ophion curvinervis Kriechbaumer, 1878

hungaricus Szépligeti, 1905

dryobotae Seyrig, 1926

Distribution: England

Notes: added by Horstmann (1981b)

Eremotylus marginatus (Jurine, 1807)

Nomenclature:

Anomalon marginatum Jurine, 1807

Distribution: England

Notes: a distribution record from George (1957)

\section{Genus Ophion Fabricius, 1798}

Nomenclature:

PANISCUS Schrank, 1802

STENOPHTHALMUS Szépligeti, 1905

PACHYPROTOMA Kohl, 1906

APATOPHION Shestakov, 1926

PLATOPHION Hellén, 1926

Notes: The species areolaris and ocellaris have often been treated as comprising a separate genus, Platophion (e.g. Gauld 1973, Brock 1982) but are now generally treated as a species-group of Ophion (see Schwarzfeld et al. 2016).

Ophion areolaris Brauns, 1889

Distribution: Scotland

\section{Ophion brevicornis Morley, 1915}

Distribution: England, Scotland, Wales 
Ophion costatus Ratzeburg, 1848

Distribution: England, Scotland, Wales, Ireland

Ophion crassicornis Brock, 1982

Distribution: England, Scotland, Wales

Notes: added by Brock (1982)

Ophion forticornis Morley, 1915

Nomenclature:

baueri Habermehl, 1930

Distribution: England, Ireland

Ophion longigena Thomson, 1888

Distribution: England, Scotland

Ophion luteus (Linnaeus, 1758)

Nomenclature:

Ichneumon luteus Linnaeus, 1758

distans Thomson, 1888

slaviceki Kriechbaumer, 1892

pictus Kokujev, 1906

calcaratus Morley, 1915

Distribution: England, Scotland, Wales, Ireland, Isle of Man

Notes: slaviceki was re-synonymised by Brock (1982)

\section{Ophion minutus Kriechbaumer, 1879}

Nomenclature:

eremotyloides Ceballos, 1962

Distribution: England, Scotland, Wales

Ophion mocsaryi Brauns, 1889

Nomenclature:

fossulatus Hedwig, 1957 unavailable 
Distribution: England, Scotland, Wales, Ireland

Ophion obscuratus Fabricius, 1798

Nomenclature:

obscurus Fabricius, 1804

polyguttator (Thunberg, 1824, Ichneumon)

flavolineatus Brullé, 1846

variegatus Rudow, 1883

Distribution: England, Scotland, Wales, Ireland

Ophion ocellaris Ulbricht, 1926

Distribution: England, Scotland

Ophion parvulus Kriechbaumer, 1879

Distribution: England, Scotland, Wales, Ireland

Ophion perkinsi Brock, 1982

Distribution: England, Scotland, Wales

Notes: added by Brock (1982)

Ophion pteridis Kriechbaumer, 1879

Distribution: England, Scotland, Wales, Ireland

Ophion scutellaris Thomson, 1888

Nomenclature:

longicornis Brauns, 1889

stigmaticus Morley, 1915

Distribution: England, Scotland, Wales

Ophion ventricosus Gravenhorst, 1829

Nomenclature:

impressus (Thunberg, 1824, Ichneumon) preocc.

Distribution: England, Scotland, Wales, Ireland 
Genus Stauropoctonus Brauns, 1889

Nomenclature:

STAUROPODOCTONUS Morley, 1913

\section{Stauropoctonus bombycivorus (Gravenhorst, 1829)}

Nomenclature:

Ophion bombycivorus Gravenhorst, 1829

infuscatus (Taschenberg, 1875, Ophion)

Distribution: England

\section{Orthocentrinae}

\section{Subfamily ORTHOCENTRINAE Förster, 1869}

\section{Nomenclature:}

\section{HELICTINAE Gupta, 1987}

Notes: Traditionally, the Orthocentrinae comprised a tightly-knit group of genera related to Orthocentrus but now includes many genera of the former 'Microleptinae' sensu Townes (1971) (Wahl 1990). Distribution data taken from BMNH, NMS (det. J.F. Perkins and GRB), UM, Rossem (1982), Rossem (1983a), Rossem (1987), Rossem (1988) and Dasch (1992), with additional references given.

\section{Genus Aniseres Förster, 1871}

\section{Aniseres lapponicus Jussila, 1994}

Distribution: Scotland

Notes: NMS, added here; synonymised lapponicus under pallipes; I do not follow this as there are two clearly distinct species in Britain and one corresponds to Jussila's (Jussila 1994b) description of lapponicus. However, I have not yet examined type material.

\section{Aniseres pallipes Förster, 1871}

Nomenclature:

pallidipes Dalla Torre, 1901

Distribution: England 
Notes: NMS, added here

\section{Genus Aperileptus Förster, 1869}

\section{Aperileptus albipalpus (Gravenhorst, 1829)}

\section{Nomenclature:}

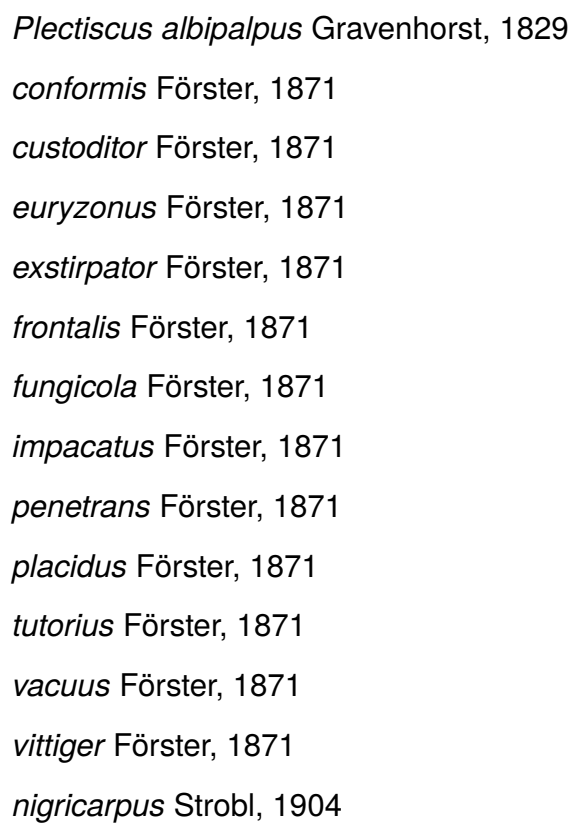

Distribution: England, Scotland

\section{Aperileptus impurus Förster, 1871}

\section{Nomenclature:}

electus Förster, 1871

filiventris Förster, 1871

immundus Förster, 1871

inamoenus Förster, 1871

inclinans Förster, 1871

labilis Förster, 1871

languidus Förster, 1871

notabilis Förster, 1871

secretus Förster, 1871

sternoxanthus Förster, 1871 
trivittatus Strobl, 1904

Distribution: England, Scotland

Aperileptus infuscatus Förster, 1871

Distribution: England

Notes: BMNH, added here

Aperileptus microspilus Förster, 1871

Nomenclature:

spoliator Förster, 1871

Distribution: England, Scotland

Notes: NMS, W.A. Ely coll., added here

Aperileptus vanus Förster, 1871

Nomenclature:

obliquus (Thomson, 1888, Plectiscus)

Distribution: England, Scotland, Wales, Ireland

Notes: added by Dasch (1992)

Genus Apoclima Förster, 1869

Apoclima signaticorne Förster, 1881

Distribution: Scotland

Notes: NMS, added here

Genus Batakomacrus Kolarov, 1986

Batakomacrus caudatus (Holmgren, 1858)

Nomenclature:

Orthocentrus caudatus Holmgren, 1858

crassicaudatus (Kolarov, 1986, Batakomacrus) synonymy by Broad (2010)

Distribution: England, Scotland, Wales 
Batakomacrus flaviceps (Gravenhorst, 1829)

Nomenclature:

Orthocentrus flaviceps Gravenhorst, 1829

Distribution: England

Batakomacrus noyesi Broad, 2010

Distribution: England, Scotland

Notes: added by Broad (2010)

Genus Catastenus Förster, 1871

Nomenclature:

CATATENUS misspelling

Catastenus femoralis Förster, 1871

Distribution: England, Scotland, Wales

Genus Dialipsis Förster, 1869

Nomenclature:

PARENTYPOMA Strobl, 1901

Dialipsis exilis Förster, 1871

Nomenclature:

conjuncta Förster, 1871

diversa Förster, 1871

intermedia Förster, 1871

mesomelana Förster, 1871

observatrix Förster, 1871

pallida Förster, 1871

crassipes (Thomson, 1888, Plectiscus)

femorata (Strobl, 1901, Parentypoma)

Distribution: England, Scotland, Ireland 
Genus Entypoma Förster, 1869

Nomenclature:

ENTELECHIA Förster, 1871

ENTELECHIUS Thomson, 1888

ENTYPOMUS Thomson, 1888

Entypoma robustator Aubert, 1968

Distribution: England

Notes: BMNH, added here

Entypoma robustum Förster, 1871

Distribution: England

Entypoma suspiciosum (Förster, 1871)

Nomenclature:

Entelechia suspiciosa Förster, 1871

remotum (Marshall, 1896, Bassus)

Distribution: England, Scotland

Genus Eusterinx Förster, 1869

Subgenus Divinatrix Rossem, 1987

Eusterinx (Divinatrix) inaequalis Rossem, 1981

Distribution: England

Notes: NMS, BMNH, det. Schwarz \& Broad, added here

Subgenus Eusterinx Förster, 1869

Eusterinx (Eusterinx) argutula Förster, 1871

Nomenclature:

ambigua Förster, 1871

divulgata Förster, 1871

intermedia Förster, 1871 
scitula Förster, 1871

subcincta Förster, 1871

Distribution: England

Eusterinx (Eusterinx) obscurella Förster, 1871

Nomenclature:

exigua Förster, 1871

Eusterinx (Eusterinx) oligomera Förster, 1871

Nomenclature:

fulvicincta Förster, 1871

fulvicornis Förster, 1871

moesta Förster, 1871

oreophila Förster, 1871

speculifera Förster, 1871

tenuis Förster, 1871

Distribution: England, Ireland

Notes: added by Dasch (1992)

Subgenus Holomeristus Förster, 1869

Eusterinx (Holomeristus) refractaria Rossem, 1982

Distribution: Isle of Man

Notes: added by Dasch (1992)

Eusterinx (Holomeristus) tenuicincta (Förster, 1871)

Nomenclature:

Holomeristus tenuicinctus Förster, 1871

Distribution: England, Scotland 
Subgenus Ischyracis Förster, 1869

Eusterinx (Ischyracis) bispinosa (Strobl, 1901)

Nomenclature:

Hemiteles bispinosus Strobl, 1901

alpigena (Strobl, 1904, Catomicrus)

Distribution: England

Notes: added by Dasch (1992)

\section{Genus Gnathochorisis Förster, 1869}

Nomenclature:

LAEPSERUS Förster, 1869

ACROBLAPTICUS Schmiedeknecht, 1911

BLAPTICUS misident.

Gnathochorisis crassula (Thomson, 1888)

Nomenclature:

Blapticus crassulus Thomson, 1888

Distribution: England, Scotland, Ireland

Notes: NMS, BMNH, added here

Gnathochorisis dentifer (Thomson, 1888)

Nomenclature:

Blapticus dentifer Thomson, 1888

debilis (Schmiedeknecht, 1911, Acroblapticus)

Distribution: England, Scotland

\section{Genus Helictes Haliday, 1837}

Nomenclature:

ENCOPIUS Schiødte, 1839

MYRIARTHRUS Förster, 1869 
Helictes borealis (Holmgren, 1857)

Nomenclature:

Megastylus borealis Holmgren, 1857

clypeatus (Förster, 1871, Idioxenus)

coxalis (Förster, 1871, Idioxenus)

invalidus (Förster, 1871, Idioxenus)

polymerus (Förster, 1871, Idioxenus)

propinquus (Förster, 1871, Idioxenus)

variator (Förster, 1871, Idioxenus)

pilicornis (Thomson, 1888, Megastylus)

Distribution: England, Scotland, Wales

Helictes erythrostoma (Gmelin, 1790)

Nomenclature:

Ichneumon erythrostoma Gmelin, 1790

mediator misident.

fulvicornis (Haliday, 1839, Cryptus)

conspicuus (Förster, 1871, Idioxenus)

inaequalis (Förster, 1871, Idioxenus)

inquilinus (Förster, 1871, Idioxenus)

intricator (Förster, 1871, Idioxenus)

tetraglyptus (Förster, 1871, Idioxenus)

nigricoxus Strobl, 1904

Distribution: England, Scotland, Wales, Ireland

Helictes varius (Haliday, 1839)

Nomenclature:

Cryptus varius Haliday, 1839

Distribution: Ireland 


\section{Genus Hemiphanes Förster, 1869}

Hemiphanes erratum Humala, 2007

Distribution: Scotland, Wales, Ireland

Notes: Added by Humala et al. (2007) on the basis of information from GRB. According to Humala et al. (2007), Hemiphanes flavipes Förster, 1871 sensu Rossem is a misidentification; Humala (2007) described this species as new.

\section{Hemiphanes gravator Förster, 1871}

Nomenclature:

laevithorax (Strobl, 1903, Trematopygus)

Distribution: Scotland

Notes: NMS, det. Brock, added here

Hemiphanes performidatum Rossem, 1988

Distribution: England, Scotland

Notes: Added by Humala et al. (2007) on the basis of information from GRB.

Genus Megastylus Schiødte, 1838

Nomenclature:

DICOLUS Förster, 1869

IDIOXENUS Förster, 1869

MEGALOSTYLUS Schulz, 1906

MIOMEROIDES Kiss, 1924

MYRIARTHRUS misident.

\section{Megastylus cruentator Schiødte, 1838}

\section{Nomenclature:}

mediator Schiødte, 1838

cruentatus (Haliday, 1839, Cryptus)

conformis Förster, 1871

fuscicornis Förster, 1871

nigriventris Förster, 1871 
pectoralis (Rudow, 1886, Hemiteles) preocc.

Distribution: England, Scotland, Wales, Ireland

Megastylus excubitor (Förster, 1871)

Nomenclature:

Dicolus excubitor Förster, 1871

Distribution: England, Scotland

Megastylus flavopictus (Gravenhorst, 1829)

Nomenclature:

Plectiscus flavopictus Gravenhorst, 1829

lineator Schiødte, 1838

cingulator (Förster, 1871, Myriarthrus)

Distribution: England, Scotland

Megastylus impressor Schiødte, 1838

Nomenclature:

insectator (Förster, 1871, Dicolus)

Distribution: England, Scotland, Wales

\section{Megastylus orbitator Schiødte, 1838}

Nomenclature:

maderensis (Wollaston, 1858, Mesoleptus)

leptoderus Förster, 1871

pauxillus Förster, 1871

pumilio Förster, 1871

retroligatus Förster, 1871

rufipleuris (Förster, 1871, Myriarthrus)

Distribution: England, Scotland, Wales, Ireland

Notes: added by Rossem (1983b) 
Megastylus pectoralis (Förster, 1871)

Nomenclature:

Dicolus pectoralis Förster, 1871

subtiliventris (Förster, 1871, Dicolus)

Distribution: England, Scotland, Ireland

Megastylus suecicus Rossem, 1983

Distribution: England, Scotland, Ireland

Notes: NMS, BMNH, det. Broad, added here

Genus Neurateles Ratzeburg, 1848

Neurateles falcatus (Thomson, 1897)

Nomenclature:

Orthocentrus falcatus Thomson, 1897

Distribution: Scotland

Notes: BMNH, added here

Neurateles papyraceus Ratzeburg, 1848

Nomenclature:

britteni (Waterson, 1929, Stenomacrus)

Distribution: England

Genus Orthocentrus Gravenhorst, 1829

Nomenclature:

ATMETUS Förster, 1869

PHAENOSEMUS Förster, 1869

TAPINOPS Förster, 1869

Orthocentrus asper (Gravenhorst, 1829)

Nomenclature:

Exochus asper Gravenhorst, 1829

discolor Holngren, 1858 
Distribution: England, Ireland

Orthocentrus attenuatus Holmgren, 1858

Distribution: England

Orthocentrus corrugatus Holmgren, 1858

Distribution: England

Orthocentrus frontator (Zetterstedt, 1838)

Nomenclature:

Tryphon frontator Zetterstedt, 1838

repentinus Holmgren, 1858

frontalis Brischke, 1871

pirasii Costa, 1886

Distribution: England, Scotland, Ireland

Orthocentrus fulvipes Gravenhorst, 1829

Nomenclature:

anomalus Gravenhorst, 1829

Distribution: England, Scotland, Ireland

Orthocentrus marginatus Holmgren, 1858

Distribution: England

Orthocentrus monilicornis Holmgren, 1858

Distribution: England, Ireland

Orthocentrus petiolaris Thomson, 1897

Distribution: England

Orthocentrus protervus Holmgren, 1858

Distribution: England 
Orthocentrus radialis Thomson, 1897

Distribution: England

Orthocentrus sannio Holmgren, 1858

Nomenclature:

histrio Holmgren, 1858

Distribution: England, Scotland

Orthocentrus spurius Gravenhorst, 1829

Nomenclature:

protuberans Holmgren, 1858

Distribution: England, Scotland

Orthocentrus winnertzii Förster, 1850

Nomenclature:

stigmaticus Holmgren, 1858 synonymy by Horstmann (2002c)

borealis Roman, 1915

meridionator Aubert, 1960

Distribution: England, Scotland

Genus Pantisarthrus Förster, 1871

Pantisarthrus dispar Rossem, 1981

Distribution: England

Notes: NMS, added here

Pantisarthrus lubricus (Förster, 1871)

Nomenclature:

Aniseres lubricus Förster, 1871

inaequalis Förster, 1871

ochropus Förster, 1871

pseudochropus Strobl, 1904

subalpinus (Strobl, 1904, Aniseres) 
Distribution: England, Scotland

\section{Pantisarthrus luridus Förster, 1871}

Distribution: England, Scotland, Ireland

\section{Genus Picrostigeus Förster, 1869}

Notes: Some distribution data from Horstmann (1994b).

Picrostigeus brevicauda Horstmann, 1994

Distribution: England

Notes: added by Horstmann (1994b)

Picrostigeus debilis (Gravenhorst, 1829)

Nomenclature:

Orthocentrus debilis Gravenhorst, 1829

Distribution: England, Scotland, Ireland

Picrostigeus obscurus Horstmann, 1994

Distribution: England, Scotland, Wales, Ireland

Notes: added by Horstmann (1994b)

Picrostigeus recticauda (Thomson, 1897)

Nomenclature:

Orthocentrus recticauda Thomson, 1897

Distribution: England, Scotland, Wales, Ireland

Picrostigeus setiger (Brischke, 1871)

Nomenclature:

Orthocentrus setiger Brischke, 1871

Distribution: England, Ireland

Notes: added by Horstmann (1994b) 
Genus Plectiscidea Viereck, 1914

Subgenus Fugatrix Rossem, 1987

Plectiscidea (Fugatrix) communis (Förster, 1871)

Nomenclature:

Plectiscus communis Förster, 1871

elumbis (Förster, 1871, Plectiscus)

gilva (Förster, 1871, Plectiscus)

infirma (Förster, 1871, Plectiscus)

nigrita (Förster, 1871, Plectiscus)

parviceps (Förster, 1871, Plectiscus)

tantilla (Förster, 1871, Plectiscus)

Distribution: England, Scotland, Ireland

\section{Subgenus Plectiscidea Viereck, 1914}

Nomenclature:

EPHALMATOR Rossem, 1981

Notes: species of Plectiscidea excluded from the British and Irish list:

[amicalis (Förster, 1871, Plectiscus); syn. sodalis (Förster, 1871, Plectiscus)] Listed as sodalis by Fitton (1978), presumaby either on the basis of specimens recorded by Carr, which are not now accepted as necessarily British (see note under Lissonota funebris), or on the basis of specimens in BMNH identified as sodalis var. moerens, which is now regarded as a separate species.

Plectiscidea (Plectiscidea) aquilonia Humala, 2003

Distribution: England, Wales

Notes: BMNH, det. Broad, added here

Plectiscidea (Plectiscidea) bistriata (Thomson, 1888)

Nomenclature:

Plectiscus bistriatus Thomson, 1888

Distribution: England 
Plectiscidea (Plectiscidea) canaliculata (Förster, 1871)

Nomenclature:

Plectiscus canaliculatus Förster, 1871

distincta (Förster, 1871, Plectiscus)

subcurvata (Förster, 1871, Plectiscus)

subtilis (Förster, 1871, Plectiscus)

Plectiscidea (Plectiscidea) collaris (Gravenhorst, 1829)

Nomenclature:

Plectiscus collaris Gravenhorst, 1829

binodula (Förster, 1871, Plectiscus)

Distribution: England, Scotland, Wales

Plectiscidea (Plectiscidea) conjuncta (Förster, 1871)

Nomenclature:

Plectiscus conjunctus Förster, 1871

flavicoxis (Förster, 1871, Plectiscus)

Distribution: England

Notes: NMS, added here

Plectiscidea (Plectiscidea) eurystigma (Thomson, 1888)

Nomenclature:

Plectiscus eurystigma Thomson, 1888

Plectiscidea (Plectiscidea) humeralis (Förster, 1871)

Nomenclature:

Plectiscus humeralis Förster, 1871

fulva (Förster, 1871, Plectiscus)

hostilis (Förster, 1871, Plectiscus)

Plectiscidea (Plectiscidea) ? hyperborea (Holmgren, 1869)

Nomenclature:

Plectiscus hyperboreus Holmgren, 1869 
Notes: The identification of specimens as hyperborea away from Svalbard is uncertain

(Horstmann 2003b), the identity of specimens recorded as this species is therefore unknown.

Plectiscidea (Plectiscidea) melanocera (Förster, 1871)

Nomenclature:

Plectiscus melanocerus Förster, 1871

proxima (Förster, 1871, Plectiscus)

Distribution: England

Plectiscidea (Plectiscidea) moerens (Förster, 1871)

Nomenclature:

Plectiscus moerens Förster, 1871

eversoria (Förster, 1871, Plectiscus)

flavizona (Förster, 1871, Plectiscus)

xanthoneuris (Förster, 1871, Plectiscus)

Distribution: England

Notes: BMNH, added here; see note under Plectiscidea.

Plectiscidea (Plectiscidea) subteres (Thomson, 1888)

Nomenclature:

Plectiscus subteres Thomson, 1888

Plectiscidea (Plectiscidea) tener (Förster, 1871)

Nomenclature:

Plectiscus tener Förster, 1871

incerta (Förster, 1871, Plectiscus)

Distribution: England

Notes: added by Rossem (1988)

Plectiscidea (Plectiscidea) tenuicornis (Förster, 1871)

Nomenclature:

Plectiscus tenuicornis Förster, 1871

brachyura (Förster, 1871, Plectiscus) 
Distribution: England

Plectiscidea (Plectiscidea) terebrator (Förster, 1871)

Nomenclature:

Plectiscus terebrator Förster, 1871

habilis (Förster, 1871, Plectiscus)

praeposita (Förster, 1871, Plectiscus)

Distribution: England

Plectiscidea (Plectiscidea) ventosa Rossem, 1987

Distribution: Scotland

Notes: NMS, added here

Plectiscidea (Plectiscidea) zonata (Gravenhorst, 1829)

Nomenclature:

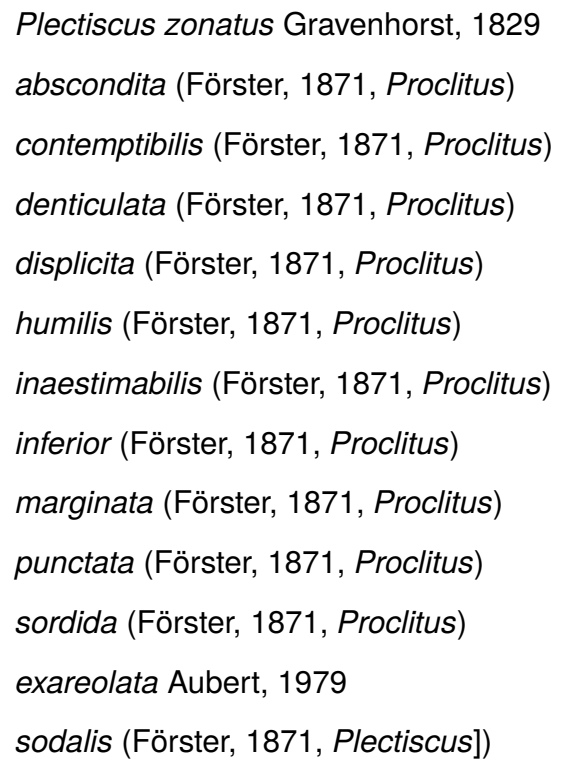

Distribution: England

Notes: BMNH, added here; transferred from Proclitus by Humala (2007). This is a typical species of Plectiscidea and there seems to be no reason why Rossem (1983a) transferred it to Proclitus. 
Genus Plectiscus Gravenhorst, 1829

Nomenclature:

BREPHOCTONUS Förster, 1869

LEIPAULUS Townes, 1945

Plectiscus agilis (Holmgren, 1858)

Nomenclature:

Orthocentrus agilis Holmgren, 1858

flavicornis (Thomson, 1897, Orthocentrus)

Distribution: Ireland

Plectiscus impurator Gravenhorst, 1829

Nomenclature:

ventralis (Holmgren, 1858, Orthocentrus)

vittatus (Holmgren, 1858, Orthocentrus)

Distribution: England, Scotland, Wales, Ireland

Plectiscus ridibundus (Gravenhorst, 1829)

Nomenclature:

Orthocentrus ridibundus Gravenhorst, 1829

exilis (Holmgren, 1858, Orthocentrus)

Distribution: England, Scotland, Wales, Ireland

\section{Genus Proclitus Förster, 1869}

Nomenclature:

CLEPTICUS Haliday, 1839 preocc.

ACLASTONEURA Kriechbaumer, 1896

\section{Proclitus attentus Förster, 1871}

Nomenclature:

fossulatus Förster, 1871

gracilentus Förster, 1871

leptosomus Förster, 1871

melanocephalus Förster, 1871 
mesoxanthus Förster, 1871

procerulus Förster, 1871

quaestorius Förster, 1871

sincerus Förster, 1871

stenogaster Förster, 1871

substriatus Förster, 1871

vallidus Förster, 1871

Distribution: England, Scotland

\section{Proclitus comes (Haliday, 1839)}

Nomenclature:

Cryptus comes Haliday, 1839

caudiger Förster, 1871

macrurus Förster, 1871

pallens Förster, 1871

perditorius Förster, 1871

Distribution: England, Scotland, Ireland

Proclitus edwardsi Roman, 1923

Distribution: England

\section{Proclitus fulvicornis Förster, 1871}

Nomenclature:

cupidus Förster, 1871

evacuator Förster, 1871

inquietus Förster, 1871

periculosus Förster, 1871

ruficaudator Aubert, 1963

Distribution: England, Ireland

Notes: Plectiscus heterocerus Thomson, 1888 was removed from synonymy by Humala (2007) and regarded as a valid species of Proclitus; it is not known if it occurs in Britain or Ireland. 


\section{Proclitus paganus (Haliday, 1839)}

Nomenclature:

Cryptus paganus Haliday, 1839

autumnalis Förster, 1871

clypearis Förster, 1871

conturbator Förster, 1871

curiosus Förster, 1871

dimidiatus Förster, 1871

instigator Förster, 1871

providus Förster, 1871

longitarsis (Thomson, 1888, Plectiscus)

Distribution: England, Scotland, Ireland

\section{Proclitus praetor (Haliday, 1839)}

Nomenclature:

Cryptus praetor Haliday, 1839

grandis Förster, 1871

Distribution: England, Scotland, Wales, Ireland

\section{Proclitus socius (Haliday, 1839)}

\section{Nomenclature:}

Cryptus socius Haliday, 1839

Distribution: Ireland

Notes: BMNH, added here; described from Ireland so not included in the 1978 British checklist, and Fitton (1976) considered the name to be a nomen dubium.

Genus Proeliator Rossem, 1982

Proeliator proprius Rossem, 1982

Distribution: England

Notes: BMNH, det. Broad, added here 


\section{Genus Stenomacrus Förster, 1869}

Notes: species of Stenomacrus excluded from the British and Irish list:

[affinis misident.] Stenomacrus affinis (Zetterstedt, 1838, Bassus) of authors was redescribed as $S$. affinitor Aubert, 1981 as the type of affinis is a species of Hypsicera (Metopiinae), a junior synonym of curvator (Fabricius). Old British records of ' Stenomacrus affinis' are not valid.

[exserens (Thomson, 1897, Orthocentrus)] Morley (1911) recorded Stenomacrus exserens as a British species but the only two specimens purportedly of this species in $\mathrm{BMNH}$, from C. Morley's collection, are misidentified Eusterinx. There is no other evidence that exserens is a British or Irish species.

Stenomacrus binotatus (Holmgren, 1858)

Nomenclature:

Orthocentrus binotatus Holmgren, 1858

Distribution: England

Stenomacrus carbonariae Roman, 1939

Distribution: England

Stenomacrus celer (Holmgren, 1858)

Nomenclature:

Orthocentrus celer Holmgren, 1858

Distribution: England, Scotland

Notes: BMNH, added here

Stenomacrus cognatus (Holmgren, 1858)

Nomenclature:

Orthocentrus cognatus Holmgren, 1858

confinis (Holmgren, 1858, Orthocentrus)

tristis (Holmgren, 1858, Orthocentrus)

Distribution: England 
Stenomacrus cubiceps (Thomson, 1897)

Nomenclature:

Orthocentrus cubiceps Thomson, 1897

Distribution: England, Ireland

Stenomacrus curvicaudatus (Brischke, 1871)

Nomenclature:

Orthocentrus curvicaudatus Brischke, 1871

Distribution: England

Stenomacrus curvulus (Thomson, 1897)

Nomenclature:

Orthocentrus curvulus Thomson, 1897

Distribution: England, Wales

Notes: BMNH, det. Perkins, Broad, added here

Stenomacrus deletus (Thomson, 1897)

Nomenclature:

Orthocentrus deletus Thomson, 1897

Stenomacrus holmgreni (Kirchner, 1867)

Nomenclature:

Orthocentrus holmgreni Kirchner, 1867

lapponicus Horstmann \& Yu, 1999 synonymy by Horstmann (2006a)

intermedius (Holmgren, 1858, Orthocentrus) preocc.

Distribution: England

Stenomacrus incisus (Gravenhorst, 1829)

Nomenclature:

Orthocentrus incisus Gravenhorst, 1829

Stenomacrus innotatus (Thomson, 1897)

Nomenclature: 
Orthocentrus innotatus Thomson, 1897

Stenomacrus laricis (Haliday, 1839)

Nomenclature:

Bassus laricis Haliday, 1839

concinnus (Holmgren, 1858, Orthocentrus)

fortipes (Thomson, 1897, Orthocentrus)

Distribution: England, Scotland, Ireland

Stenomacrus molestus (Holmgren, 1858)

Nomenclature:

Orthocentrus molestus Holmgren, 1858

Stenomacrus ochripes (Holmgren, 1858)

Nomenclature:

Orthocentrus ochripes Holmgren, 1858

Stenomacrus palustris (Holmgren, 1858)

Nomenclature:

Orthocentrus palustris Holmgren, 1858

Distribution: England

Stenomacrus pedestris (Holmgren, 1869)

Nomenclature:

Orthocentrus pedestris Holmgren, 1869

reptilis (Marshall, 1877, Orthocentrus)

Distribution: England

Stenomacrus pygmaeus Horstmann \& Yu, 1999

Nomenclature:

pusillus (Zetterstedt, 1838, Bassus)

Distribution: England

Notes: BMNH, added here 
Stenomacrus silvaticus (Holmgren, 1858)

Nomenclature:

Orthocentrus silvaticus Holmgren, 1858

Distribution: England, Scotland

Stenomacrus vafer (Holmgren, 1858)

Nomenclature:

Orthocentrus vafer Holmgren, 1858

Distribution: Scotland

Notes: BMNH, added here

\section{Genus Symplecis Förster, 1869}

Nomenclature:

Orthocentrus vafer Holmgren, 1858

\section{Symplecis bicingulata (Gravenhorst, 1829)}

Nomenclature:

Mesoleptus bicingulatus Gravenhorst, 1829

facialis Thomson, 1888

albicoxis (Kiss, 1924, Rhaestes) synonymy by Horstmann (2007b)

Distribution: England, Scotland, Ireland

Notes: Symplecis leucostoma (Förster, 1871, Blapticus) (with xanthostoma Förster, 1871 as a junior synonym) and alpicola Förster, 1871 (with zonaria Förster, 1871 and basalis Brischke, 1880 as junior synonyms) were removed from synonymy and treated as valid species by Humala (2007); it is not yet known whether leucostoma or alpicola occur in Britain or Ireland; a previous listing of leucostoma as a British species rested on a record by Carr (1924), which cannot be considered reliable (see note under Lissonota funebris). Irish record from a specimen in the Canadian National Collection (Ottawa), det. GRB.

\section{Symplecis breviuscula Roman, 1923}

Nomenclature:

breviscula misspelling

infavorabilis Rossem, 1981

Distribution: England 


\section{Orthopelmatinae}

Subfamily ORTHOPELMATINAE Schmiedeknecht, 1910

Genus Orthopelma Taschenberg, 1865

Nomenclature:

PROEDRUS Förster, 1869

Notes: Distribution data taken from Gauld and Mitchell (1977b) and BMNH.

Orthopelma brevicorne Morley, 1907

Distribution: England, Wales

Orthopelma mediator (Thunberg, 1824)

Nomenclature:

Ichneumon mediator Thunberg, 1824

bedeguaris (Geoffroy, 1785, Ichneumon) preocc.

luteolator (Gravenhorst, 1829, Hemiteles)

rufinum (Gravenhorst, 1829, Porizon)

pavoniae (Rondani, 1877, Hemiteles)

Distribution: England, Scotland, Wales, Ireland

\section{Oxytorinae}

\section{Subfamily OXYTORINAE Thomson, 1883}

Notes: The name Oxytorinae, prior to Wahl (1986), Wahl (1990), has been applied much more widely, encompassing the subfamilies Cylloceriinae, Microleptinae and Orthocentrinae.

\section{Genus Oxytorus Förster, 1869}

Nomenclature:

CALLIDIOTES Förster, 1869

DELOLYTUS Förster, 1869

PANTOPORTHUS Förster, 1869 
MESATRACTODES Morley, 1907

Oxytorus armatus Thomson, 1883

Distribution: England, Scotland, Wales

\section{Oxytorus luridator (Gravenhorst, 1820)}

Nomenclature:

Ichneumon luridator Gravenhorst, 1820

coxator (Gravenhorst, 1829, Mesoleptus)

ventrator (Gravenhorst, 1829, Mesoleptus)

properator (Haliday, 1839, Atractodes)

varicornis (Holmgren, 1860, Atractodes)

longicornis (Habermehl, 1909, Exolytus)

nigricoxa (Kiss, 1924, Callidiotes)

Distribution: England, Scotland, Wales

\section{Pimplinae}

\section{Subfamily PIMPLINAE Wesmael, 1845}

Nomenclature:

EPHIALTINAE Hellén, 1915

Notes: Distribution data from Fitton et al. (1988), O'Connor and Butler (1992) and Shaw (2006a), with further distribution data for the Polysphincta genus-group taken from Hudson (1988). Additional distribution references are given. Tribal classification follows Gauld et al. (2002).

\section{Tribe DELOMERISTINI Hellén, 1915}

\section{Nomenclature:}

PERITHOINI Wahl \& Gauld, 1998 synonymy by Gauld et al. (2002)

\section{Genus Delomerista Förster, 1869}

Notes: Phillips (1997) provides some Scottish records for novita and pfankuchi. 
Delomerista borealis Walkley, 1960

Distribution: England

Notes: BMNH, det. Broad, added here

Delomerista laevis (Gravenhorst, 1829)

Nomenclature:

Pimpla laevis Gravenhorst, 1829

suborbitalis (Gravenhorst, 1829, Lissonota) synonymy by Horstmann (2001b)

laevifrons (Thomson, 1877, Pimpla)

levifrons (Dalla Torre, 1901, Pimpla) preocc.

Distribution: England

Notes: Added by Horstmann (2001b); Fitton et al. (1988) excluded laevis from the British list as specimens identified as such were found to be novita; Horstmann (2001b) subsequently established that the holotype of Lissonota suborbitalis, supposedly taken in Netley, Shropshire, is the true laevis.

\section{Delomerista mandibularis (Gravenhorst, 1829)}

Nomenclature:

Pimpla mandibularis Gravenhorst, 1829

albicinctus (Desvignes, 1862, Ephialtes) preocc.

desvignesii (Marshall, 1870, Ephialtes)

Distribution: England

Notes: some distribution data from anon. (2001)

Delomerista novita (Cresson, 1870)

Nomenclature:

Pimpla novita Cresson, 1870

laevis misident.

europa Gupta, 1982

Distribution: England, Scotland, Ireland

Notes: Gupta (1982) separated European populations as the subspecies europa. 
Delomerista pfankuchi Brauns, 1905

Nomenclature:

unicolor (Hedwig, 1959, Troctocerus)

Distribution: England, Scotland

Notes: added by Fitton et al. (1988)

Genus Perithous Holmgren, 1859

Nomenclature:

HYBOMISCHOS Baltazar, 1961 synonymy by Wahl and Gauld (1998)

HYBOISCHOS misspelling

Perithous albicinctus (Gravenhorst, 1829)

Nomenclature:

Ephialtes albicinctus Gravenhorst, 1829

Distribution: England

Notes: added by Brock and Shaw (1997)

Perithous divinator (Rossi, 1790)

Nomenclature:

Ichneumon divinator Rossi, 1790

ephippiatorius (Dufour \& Perris, 1840, Pimpla)

amoenus (Rudow, 1881, Pimpla)

rubi (Habermehl, 1917, Itoplectis)

pimplarius Haupt, 1938

Distribution: England, Ireland

Notes: some distribution data from Field and Foster (1988)

Perithous scurra (Panzer, 1804)

Nomenclature:

Ichneumon scurra Panzer, 1804

mediator (Fabricius, 1804, Pimpla)

asilatorius (Thunberg, 1824, Ichneumon) 
modulator (Thunberg, 1824, Ichneumon)

senator (Haliday, 1839, Pimpla)

decoratus (Ratzeburg, 1848, Pimpla)

longiseta Haupt, 1954

moldavicus Constantineanu \& Constantineanu, 1968

Distribution: England, Scotland, Wales, Ireland

Notes: Welsh occurrence from Formstone (1999)

Perithous septemcinctorius (Thunberg, 1824)

Nomenclature:

Ichneumon septemcinctorius Thunberg, 1824

varius (Gravenhorst, 1829, Ephialtes)

marginellatorius (Dufour \& Perris, 1840, Pimpla)

brunnescens Koornneef, 1951

exiguus Haupt, 1954

meridionator Aubert, 1963

rufatus Constantineanu \& Constantineanu, 1968

Distribution: England, Ireland

Perithous speculator Haupt, 1954

Nomenclature:

transsylvanicus Constantineanu \& Constantineanu, 1968

Distribution: England

Notes: added by Shaw (2006a)

Tribe EPHIALTINI Hellén, 1915

Nomenclature:

POLYSPHINCTINI Hellén, 1915 synonymy by Wahl and Gauld (1998)

\section{Genus Acrodactyla Haliday, 1839}

Nomenclature:

BARYPUS Haliday, 1837 preocc.

COLPOMERIA Holmgren, 1859 
SYMPHYLUS Förster, 1869 preocc.

POLEMOPHTHORUS Schulz, 1911

PANTOMIMA Rossem, 1990 synonymy by Broad (2004)

Notes: O'Connor (2012) confirms the presence of carinator and quadrisculpta in Ireland.

\section{Acrodactyla carinator (Aubert, 1965)}

Nomenclature:

Colpomeria carinator Aubert, 1965

braconiformis Kolarov, 1990 synonymy by Zwakhals (2006)

Distribution: England, Wales, Ireland

Notes: added by Shaw (2006a)

\section{Acrodactyla degener (Haliday, 1839)}

Nomenclature:

Pimpla degener Haliday, 1839

hadrodactyla (Förster, 1871, Symphylus)

festata (Rossem, 1990, Pantomima) synonymy by Broad (2004)

Distribution: England, Scotland, Wales, Ireland, Isle of Man

\section{Acrodactyla quadrisculpta (Gravenhorst, 1820)}

Nomenclature:

Ichneumon quadrisculptus Gravenhorst, 1820

laevigata (Holmgren, 1859, Colpomeria)

Distribution: England, Scotland, Wales, Ireland, Isle of Man

\section{Acrodactyla similis Horstmann, 2011}

Distribution: England, Scotland, Wales

Notes: added by Horstmann (2011b)

\section{Genus Acropimpla Townes, 1960}

Nomenclature: 
Acropimpla didyma (Gravenhorst, 1829)

Nomenclature:

Pimpla didyma Gravenhorst, 1829

Distribution: England, Wales, Ireland

\section{Genus Clistopyga Gravenhorst, 1829}

Nomenclature:

HYMENOMACROPYGA Uchida, 1941

ICHNEUMONOGLYPTA Blanchard, 1941

\section{Clistopyga canadensis Provancher, 1880}

Nomenclature:

sauberi Brauns, 1898 synonymy by Bordera et al. (2014)

terebralis Shestakov, 1927

Distribution: England

\section{Clistopyga incitator (Fabricius, 1793)}

Nomenclature:

Ichneumon incitator Fabricius, 1793

haemorrhoidalis Gravenhorst, 1829

elegans (Ratzeburg, 1848, Polysphincta)

incitatrix Schulz, 1906

excavata (Telenga, 1930, Polysphincta)

temporalis Hellén, 1949

Distribution: England, Scotland, Wales, Ireland, Isle of Man

Clistopyga rufator Holmgren, 1856

Nomenclature:

rufatrix Schulz, 1906

Distribution: England, Wales

Genus Dolichomitus Smith, 1877

Nomenclature: 
CLOSTEROCERUS Hartig, 1847 preocc.

MESOEPHIALTES Schmiedeknecht, 1906

DICLOSTEROCERUS Viereck, 1914

EXERISTOIDEA Viereck, 1924

TUBERCULEPHIALTES Ozols, 1962

PAUCDOLICHOMITUS Constantineanu \& Pisica, 1970

Dolichomitus agnoscendus (Roman, 1939)

Nomenclature:

Ephialtes agnoscendus Roman, 1939

Distribution: England, Wales, Ireland

Dolichomitus diversicostae (Perkins, 1943)

Nomenclature:

Ephialtes diversicostae Perkins, 1943

Distribution: Scotland

Dolichomitus imperator (Kriechbaumer, 1854)

Nomenclature:

Ephialtes imperator Kriechbaumer, 1854

?adulterator (Villers, 1789, Ichneumon)

?gracilis (Gmelin, 1790, Ichneumon)

?melanopus (Gmelin, 1790, Ichneumon)

Distribution: England, Scotland

Dolichomitus mesocentrus (Gravenhorst, 1829)

Nomenclature:

Ephialtes mesocentrus Gravenhorst, 1829

rex (Kriechbaumer, 1854, Ephialtes)

insignis (Habermehl, 1903, Ephialtes)

krapinensis (Hensch, 1930, Ephialtes)

gaurottii (Gregor, 1941, Ephialtes)

Distribution: England, Wales 
Dolichomitus messor (Gravenhorst, 1829)

Nomenclature:

Ephialtes messor Gravenhorst, 1829

continuus (Ratzeburg, 1848, Ephialtes)

reissigii (Ratzeburg, 1848, Pimpla)

pusillus (Ratzeburg, 1852, Ephialtes)

heteropus (Thomson, 1888, Ephialtes)

simillimus (Hensch, 1930, Ephialtes)

zagoriensis (Hensch, 1930, Ephialtes)

Distribution: England

Dolichomitus populneus (Ratzeburg, 1848)

Nomenclature:

Ephialtes populneus Ratzeburg, 1848

abbreviatus (Thomson, 1877, Ephialtes)

Distribution: England

Dolichomitus pterelas (Say, 1829)

Nomenclature:

Ichneumon pterelas Say, 1829

discrepans (Hensch, 1929, Ephialtes)

Distribution: England, Ireland

Dolichomitus terebrans (Ratzeburg, 1844)

Nomenclature:

Pimpla terebrans Ratzeburg, 1844

planifrons (Thomson, 1877, Ephialtes)

borealis (Hellén, 1915, Ephialtes)

kangasi (Györfi, 1941, Pimpla)

Distribution: England, Scotland, Wales 
Dolichomitus tuberculatus (Geoffroy, 1785)

Nomenclature:

Ichneumon tuberculatus Geoffroy, 1785

hyalinus (Gmelin, 1790, Ichneumon)

leucopterus (Gmelin, 1790, Ichneumon)

crispus (Christ, 1791, Ichneumon)

fluctuans (Christ, 1791, Ichneumon)

parallelus (Thomson, 1888, Ephialtes)

dentiventris (Hellén, 1915, Ephialtes)

pfefferi (Habermehl, 1917, Ephialtes)

Distribution: England, Scotland, Wales, Ireland

\section{Genus Dreisbachia Townes, 1962}

Nomenclature:

LAUFEIA Tosquinet, 1903

Notes: Synonymised under Schizopyga by Gauld and Dubois (2006), Shaw (2006a)

disagrees with this synonymy, on the basis of substantial differences in biology.

Dreisbachia pictifrons (Thomson, 1877)

Nomenclature:

Pimpla pictifrons Thomson, 1877

bridgmani (Bignell, 1894, Pimpla)

Distribution: England, Scotland, Ireland

Genus Endromopoda Hellén, 1939

\section{Endromopoda arundinator (Fabricius, 1804)}

\section{Nomenclature:}

Pimpla arundinator Fabricius, 1804

melanopyga (Gravenhorst, 1829, Pimpla)

erythrosoma (Rudown, 1883, Pimpla)

arundinatrix (Schulz, 1906, Pimpla)

nigricans (Ulbricht, 1913, Pimpla) preocc.

culpator (Morley, 1914, Epiurus) 
crefeldensis (Strand, 1918, Pimpla)

Distribution: England, Ireland

Endromopoda detrita (Holmgren, 1860)

Nomenclature:

Pimpla detrita Holmgren, 1860

laevidorsum (Vollenhoven, 1873, Pimpla)

brunnea (Brischke, 1880, Pimpla)

punctator (Müller, 1766, Ichneumon) synonymy by Horstmann (2001b)

Distribution: England, Scotland, Wales, Ireland, Isle of Man

\section{Endromopoda nigricoxis (Ulbricht, 1910)}

Nomenclature:

Pimpla nigricoxis Ulbricht, 1910

melanopyga (Ulbricht, 1909, Pimpla) preocc.

ulbrichtiana (Strand, 1918, Pimpla)

Distribution: England, Scotland, Wales, Ireland, Isle of Man

Notes: added by Fitton et al. (1988)

Endromopoda nitida (Brauns, 1898)

Nomenclature:

Pimpla nitida Brauns, 1898

deplanata (Morley, 1908, Pimpla)

Distribution: England, Wales

Endromopoda phragmitidis (Perkins, 1957)

Nomenclature:

Ephialtes phragmitidis Perkins, 1957

rufipes (Aubert, 1963, Scambus)

Distribution: England, Wales 
Genus Ephialtes Gravenhorst, 1829

Nomenclature:

APECHTHIS misident.

Ephialtes duplicauda Heinrich, 1949

Nomenclature:

spatulata (Townes, 1960, Pimpla) synonymy by Horstmann (2008c)

Distribution: Ireland

Notes: added by Horstmann (2008c)

\section{Ephialtes manifestator (Linnaeus, 1758)}

Nomenclature:

Ichneumon manifestator Linnaeus, 1758

extricator (Villers, 1789, Ichneumon)

leucopalpus (Gmelin, 1790, Ichneumon)

carbonarius (Christ, 1791, Ichneumon) preocc.

gracilis (Schrank, 1802, Ichneumon) preocc.

nepotor (Thunberg, 1824, Ichneumon)

elongator (Zetterstedt, 1838, Pimpla)

Distribution: England, Wales

Notes: Irish occurrence needs to be confirmed since Horstmann's confirmation that duplicauda occurs there; there are no Irish specimens in BMNH or NMS.

\section{Genus Exeristes Förster, 1869}

Nomenclature:

EREMOCHILA Förster, 1869

\section{Exeristes ruficollis (Gravenhorst, 1829)}

Nomenclature:

Pimpla ruficollis Gravenhorst, 1829

variegatus (Ratzeburg, 1844, Pimpla)

Distribution: England, Scotland, Wales 


\section{Genus Flavopimpla Betrem, 1932}

Nomenclature:

AFREPHIALTES Benoit, 1953

Notes: treated Afrephialtes as a junior synonym of Flavopimpla whilst retained two separate genera. Fitton et al. (1988) and Yu et al. (2012) have followed but Gauld et al. (2002) found 'Flavopimpla' to be nested within 'Afrephialtes' in their morphological phylogeny, which result is followed here.

\section{Flavopimpla cicatricosa (Ratzeburg, 1848)}

Nomenclature:

Pimpla cicatricosa Ratzeburg, 1848

Distribution: England, Ireland

Notes: Irish occurrence from O'Connor and Shaw (2004) and Allen (2007) documents a recent English record.

Genus Fredegunda Fitton, Shaw \& Gauld, 1988

Fredegunda diluta (Ratzeburg, 1852)

Nomenclature:

Pimpla diluta Ratzeburg, 1852

nigriceps (Taschenberg, 1863, Pimpla) preocc.

media (Verhoeff, 1891, Pimpla)

taschenbergii (Dalla Torre, 1901, Pimpla)

Distribution: England, Wales

\section{Genus Gregopimpla Momoi, 1965}

Gregopimpla inquisitor (Scopoli, 1763)

Nomenclature:

Ichneumon inquisitor Scopoli, 1763

?visitator (Poda, 1761, Ichneumon)

?visitator (Scopoli, 1763, Ichneumon) preocc.

scanica (Geoffroy, 1785, Ichneumon)

albipes (Gmelin, 1790, Ichneumon) preocc. 


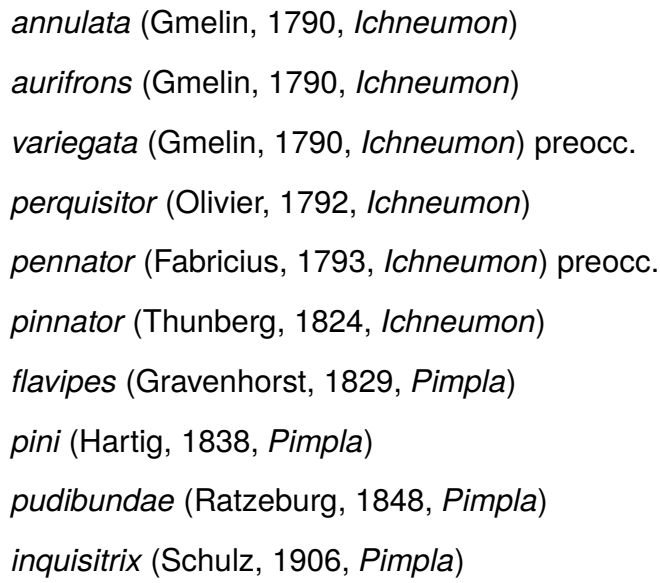

Distribution: England, Scotland, Ireland

\section{Genus Iseropus Förster, 1869}

Nomenclature:

CNEMOPIMPLA Cameron, 1903

\section{Iseropus stercorator (Fabricius, 1793)}

Nomenclature:

Ichneumon stercorator Fabricius, 1793

graminellae (Schrank, 1802, Ichneumon)

mussii (Hartig, 1838, Pimpla)

holmgreni (Schmiedeknecht, 1888, Pimpla)

Distribution: England, Scotland, Wales, Ireland

\section{Genus Liotryphon Ashmead, 1900}

\section{Nomenclature:}

LIOGASTER Kriechbaumer, 1890 preocc.

APISTES Seyrig, 1927 preocc.

APISTEPHIALTES Seyrig, 1928

NEOEPHIALTES Constantineanu \& Pisica, 1970

Notes: species of Liotryphon excluded from the British and Irish list:

[strobilellae (Linnaeus, 1758, Ichneumon); syn. resinosus (Retzius, 1783, Ichneumon); strobilator (Thunberg, 1824, Ichneumon); glabratus (Ratzeburg, 1852, Ephialtes); 
zhedenevensis (Shestakov, 1927, Ephialtes); discedens (Hensch, 1930, Ephialtes)] As discussed in Fitton et al. (1988), only one 19th century record, and not certainly of British or Irish specimens.

\section{Liotryphon ascaniae (Rudow, 1883)}

\section{Nomenclature:}

Ephialtes ascaniae Rudow, 1883

ruficollis (Desvignes, 1856, Ephialtes) invalid

sanguinicollis (Brauns, 1901, Ephialtes)

perversus (Seyrig, 1927, Apistes)

Distribution: England, Scotland

\section{Liotryphon caudatus (Ratzeburg, 1848)}

Nomenclature:

Pimpla caudata Ratzeburg, 1848

brevivalvis (Hensch, 1929, Ephialtes)

incertus (Hensch, 1929, Ephialtes)

foveolatus (Constantineanu \& Pisica, 1970, Neoephialtes) preocc.

Distribution: England, Scotland

\section{Liotryphon crassiseta (Thomson, 1877)}

Nomenclature:

Ephialtes crassiseta Thomson, 1877

pleuralis (Thomson, 1877, Ephialtes)

musculus (Kriechbaumer, 1889, Ephialtes)

albispiculus (Morley, 1908, Ephialtes)

taschenbergi (Ulbricht, 1909, Ephialtes) preocc., unavailable

sternoleucus (Lange, 1911, Ephialtes)

foveolatus (Ulbricht, 1912, Ephialtes)

taschenbergella (Strand, 1918, Pimpla)

rufipes (Hensch, 1930, Ephialtes)

Distribution: England, Ireland

Notes: some distribution data from Godfrey and Whitehead (2001) 
Liotryphon punctulatus (Ratzeburg, 1848)

Nomenclature:

Pimpla punctulata Ratzeburg, 1848

discolor (Brischke, 1880, Ephialtes)

macrurus (Förster, 1888, Epiurus)

longulus (Kriechbaumer, 1890, Liogaster)

tener (Hensch, 1929, Ephialtes)

vernalis (Hensch, 1929, Ephialtes)

gracilentus (Hensch, 1930, Ephialtes)

Distribution: England

Genus Megaetaira Gauld \& Dubois, 2006

Notes: The one included species was transferred from Acrodactyla by Gauld and Dubois (2006).

\section{Megaetaira madida (Haliday, 1839)}

Nomenclature:

Pimpla madida Haliday, 1839

clypeata (Holmgren, 1860, Polysphincta)

Distribution: England, Scotland, Ireland

\section{Genus Oxyrrhexis Förster, 1869}

\section{Oxyrrhexis carbonator (Gravenhorst, 1807)}

Nomenclature:

Cryptus carbonator Gravenhorst, 1807

velata (Hartig, 1838, Polysphincta)

pusilla (Fonscolombe, 1854, Polysphincta)

carbonatrix (Schulz, 1906, Acrodactyla)

Distribution: England

Notes: Added by Shaw (1998); previously excluded from the British list by Fitton et al. (1988): see Shaw (2006a). 
Genus Paraperithous Haupt, 1954

Nomenclature:

GNATHAULAX Townes, 1964

Paraperithous gnathaulax (Thomson, 1877)

Nomenclature:

Ephialtes gnathaulax Thomson, 1877

luteipes (Thomson, 1877, Ephialtes)

ruficollis (Rudow, 1881, Ephialtes) preocc.

aterrimus (Haupt, 1954, Perithous)

moldavicus Constantineanu \& Pisica, 1970

Distribution: England, Scotland

Genus Piogaster Perkins, 1958

Piogaster albina Perkins, 1958

Distribution: England

Piogaster punctulata Perkins, 1958

Distribution: England

Genus Polysphincta Gravenhorst, 1829

Polysphincta boops Tschek, 1869

Nomenclature:

eltshaninovi Shestakov, 1927

Distribution: England, Scotland

Polysphincta longa Kasparyan, 1976

Distribution: England, Wales

Notes: Added by Fritzén and Shaw (2014), from Wales, with an English specimen in BMNH subsequently identified by GRB. 
Polysphincta rufipes Gravenhorst, 1829

Nomenclature:

drewseni Holmgren, 1860

Distribution: England, Scotland, Wales, Ireland

Polysphincta tuberosa Gravenhorst, 1829

Nomenclature:

taschenbergi Woldstedt, 1877

sculpturata Roman, 1931

Distribution: England, Scotland, Wales, Ireland, Isle of Man

Polysphincta vexator Fitton, Shaw \& Gauld, 1988

Distribution: England, Wales, Ireland

Notes: added by Fitton et al. (1988)

Genus Reclinervellus He \& Ye, 1998

Reclinervellus nielseni (Roman, 1923)

Nomenclature:

Polysphincta nielseni Roman, 1923

Distribution: England

Notes: Added by Fitton et al. (1988); transferred from Polysphincta by Gauld and Dubois (2006).

Genus Scambus Hartig, 1838

Nomenclature:

EPIURUS Förster, 1869

TROMERA Förster, 1869

TROCTOCERUS Woldstedt, 1877

ATELEOPHADNUS Cameron, 1905

PSEUDOPOEMENIA Kiss, 1924

ERYTHROSCAMBUS Walley, 1930

LISSOSCAMBUS Walley, 1930 
Scambus brevicornis (Gravenhorst, 1829)

Nomenclature:

Pimpla brevicornis Gravenhorst, 1829

concolor (Ratzeburg, 1848, Pimpla) preocc.

nigriscaposus (Thomson, 1877, Pimpla)

punctiventris (Thomson, 1877, Pimpla)

agilis (Förster, 1888, Epiurus)

centaureae (Förster, 1888, Epiurus) synonymy by Horstmann (2010b)

depositor (Förster, 1888, Epiurus)

infestus (Förster, 1888, Epiurus)

anomalus (Morley, 1906, Phthorimus)

tibialis (Ulbricht, 1910, Pimpla) unavailable

puniceus (Schmiedeknecht, 1914, Pimpla)

pratensis (Pfankuch, 1921, Pimpla) unavailable

terrestris (Pfankuch, 1921, Pimpla) unavailable

ribesii (Hensch, 1929, Pimpla)

Distribution: England, Scotland, Wales, Ireland, Isle of Man

Notes: Horstmann (2010a) split up brevicornis but none of the additional species have yet been found in Britain or Ireland.

\section{Scambus buolianae (Hartig, 1838)}

Nomenclature:

Pimpla buolianae Hartig, 1838

triangularis (Verhoeff, 1890, Pimpla)

flavotrochanteratus (Pfeffer, 1913, Pimpla)

Distribution: England, Scotland

\section{Scambus calobatus (Gravenhorst, 1829)}

Nomenclature:

Pimpla calobata Gravenhorst, 1829

planatus (Hartig, 1838, Pimpla) synonymy by Shaw et al. (2011)

ghilianii (Spinola, 1843, Pimpla)

nucum (Ratzeburg, 1844, Pimpla) 


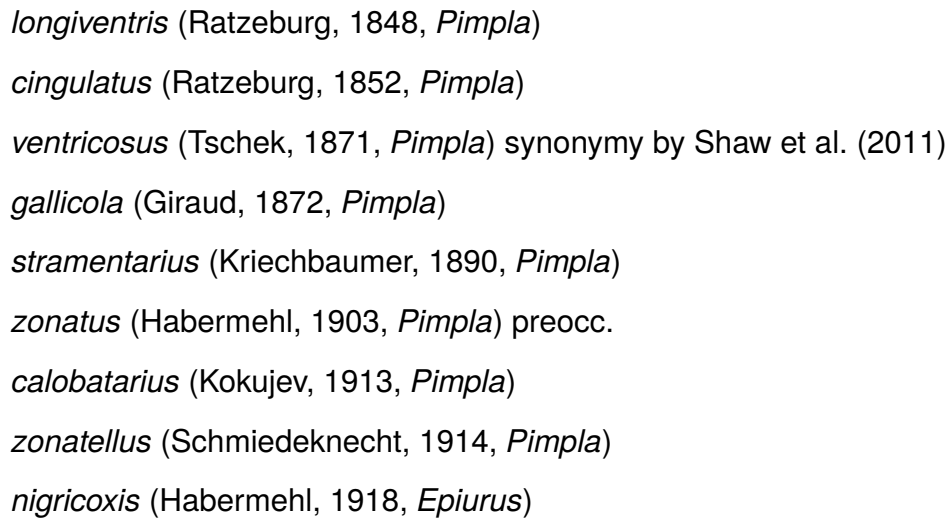

Notes: Horstmann (2009c) recognised ventricosus as a separate species, occurring in Britain, but Shaw et al. (2011) subsequently demonstrated that these individuals, together with the previously recognised species planatus, are seasonal forms of calobatus.

\section{Scambus cincticarpus (Kriechbaumer, 1895)}

\section{Nomenclature:}

Pimpla cincticarpus Kriechbaumer, 1895

affinis (Habermehl, 1903, Pimpla)

divergens (Hensch, 1929, Pimpla)

Distribution: England

Notes: added by Fitton et al. (1988)

\section{Scambus elegans (Woldstedt, 1877)}

\section{Nomenclature:}

Troctocerus elegans Woldstedt, 1877

albicrus (Rondani, 1877, Ephialtes)

cingulatellus (Costa, 1885, Pimpla)

erythronotus (Förster, 1888, Epiurus)

ulicicida (Morley, 1911, Pimpla)

cottei (Seyrig, 1926, Pimpla)

dumeticola (Hensch, 1929, Pimpla)

zagoriensis (Hensch, 1929, Troctocerus) 
Distribution: England, Wales, Ireland

\section{Scambus eucosmidarum (Perkins, 1957)}

Nomenclature:

Ephialtes eucosmidarum Perkins, 1957

Distribution: England, Scotland, Wales, Ireland

\section{Scambus foliae (Cushman, 1938)}

Nomenclature:

Epiurus foliae Cushman, 1938

Distribution: England, Scotland, Isle of Man

Notes: added by Fitton et al. (1988)

\section{Scambus inanis (Schrank, 1802)}

\section{Nomenclature:}

Ichneumon inanis Schrank, 1802

agilis (Förster, 1888, Epiurus)

depositor (Förster, 1888, Epiurus)

distinctus (Förster, 1888, Epiurus)

annulatus (Kiss, 1924, Pseudopoemenia)

lativentris (Ulbricht, 1926, Epiurus)

trilobatus (Keler, 1937, Pimpla)

Distribution: England, Scotland, Wales, Ireland

Notes: Added by Fitton et al. (1988); synonymy follows Horstmann (2005b).

\section{Scambus nigricans (Thomson, 1877)}

\section{Nomenclature:}

Pimpla nigricans Thomson, 1877

similis (Bridgman, 1884, Pimpla)

fulvus (Szépligeti, 1898, Pimpla)

lucens (Szépligeti, 1898, Pimpla)

interruptecallosus (Strobl, 1902, Pimpla)

kriechbaumeri (Habermehl, 1903, Pimpla) preocc. 


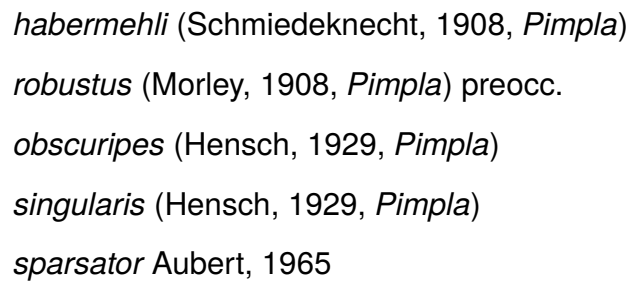

Distribution: England, Scotland, Wales, Ireland, Isle of Man

\section{Scambus pomorum (Ratzeburg, 1848)}

Nomenclature:

Pimpla pomorum Ratzeburg, 1848

Distribution: England, Scotland, Ireland

\section{Scambus sagax (Hartig, 1838)}

Nomenclature:

Pimpla sagax Hartig, 1838

linearis (Ratzeburg, 1844, Pimpla)

atrocoxatus (Pfeffer, 1913, Pimpla)

suecicus (Roman, 1917, Epiurus)

sanctacrucianus (Glowacki, 1967, Ephialtes)

Distribution: England, Scotland

\section{Scambus signatus (Pfeffer, 1913)}

Nomenclature:

Pimpla signata Pfeffer, 1913

Distribution: England, Scotland

Notes: Originally established as British by Perkins (1943) then excluded from the British list by Fitton et al. (1988); Horstmann (2005b) confirmed that some British specimens are indeed signatus; see Shaw (2006a).

\section{Scambus tenthredinum (Goeze, 1776)}

Nomenclature:

Ichneumon tenthredinum Goeze, 1776

Distribution: England, Scotland 
Notes: added by Horstmann (2005b); see Shaw (2006a)

\section{Scambus vesicarius (Ratzeburg, 1844)}

Nomenclature:

$$
\begin{aligned}
& \text { Pimpla vesicaria Ratzeburg, } 1844 \\
& \text { cryptocampi (Boie, 1857, Pimpla) } \\
& \text { gallicolus (Morley, 1908, Pimpla) preocc. } \\
& \text { ruficoxis (Ulbricht, 1909, Pimpla) unavailable } \\
& \text { rhenanus (Ulbricht, 1910, Pimpla) unavailable } \\
& \text { salicola (Hensch, 1929, Pimpla) } \\
& \text { morleyi (Schmiedeknecht, 1934, Pimpla) }
\end{aligned}
$$

Distribution: England, Scotland, Ireland

\section{Genus Schizopyga Gravenhorst, 1829}

Nomenclature:

AFROSPHINCTA Benoit, 1953

SCHIZOPYGOIDES Kasparyan, 1976

\section{Schizopyga circulator (Panzer, 1800)}

\section{Nomenclature:}

Ichneumon circulator Panzer, 1800

analis Gravenhorst, 1829

circulatrix Schulz, 1906

Distribution: England, Scotland, Wales, Ireland

\section{Schizopyga frigida Cresson, 1870}

Nomenclature:

atra Kriechbaumer, 1887

Distribution: England, Scotland, Wales, Ireland 
Schizopyga podagrica Gravenhorst, 1829

Nomenclature:

minuta Gravenhorst, 1829

silbernageli (Kiss, 1933, Polysphincta) preocc.

Distribution: England, Scotland, Ireland

\section{Schizopyga varipes Holmgren, 1856}

Distribution: Ireland

Notes: Added by Shaw (2006a); incorrectly listed as a synonym of flavifrons Holmgren, 1856 by Fitton et al. (1988) and as a synonym of podagrica by Yu and Horstmann (1997) (Shaw 2006a).

Genus Sinarachna Townes, 1960

Sinarachna nigricornis (Holmgren, 1860)

Nomenclature:

Polysphincta nigricornis Holmgren, 1860

caudata (Thomson, 1888, Polysphincta)

Distribution: England, Scotland

Notes: added by Fitton et al. (1988)

Sinarachna pallipes (Holmgren, 1860)

Nomenclature:

Polysphincta pallipes Holmgren, 1860

Distribution: England, Ireland

Notes: Irish occurrence from O'Connor (2004b)

Genus Townesia Ozols, 1962

Townesia tenuiventris (Holmgren, 1860)

Nomenclature:

Ephialtes tenuiventris Holmgren, 1860

geniculata (Brischke, 1865, Ephialtes) 
antefurcalis (Thomson, 1877, Ephialtes)

gracilis (Hensch, 1930, Ephialtes)

Distribution: England, Scotland, Ireland

\section{Genus Tromatobia Förster, 1869}

Nomenclature:

AUSTROPIMPLA Brèthes, 1913

Tromatobia forsiusi (Hellén, 1915)

Nomenclature:

Polysphincta forsiusi Hellén, 1915

Distribution: Scotland

Notes: added by Fitton et al. (1988)

Tromatobia lineatoria (Villers, 1789)

Nomenclature:

Ichneumon lineatorius Villers, 1789

oculatoria misident. (Horstmann 2001a)

tipulatoria (Thunberg, 1824, Ichneumon)

balanini (Rudow, 1883, Ephialtes)

multipicta (Kiss, 1924, Pimpla)

sanguinolenta (Kiss, 1924, Pimpla)

rufiventris Hellén, 1949

amoena (Haupt, 1954, Pimpla) preocc.

orbitalis (Haupt, 1954, Pimpla) preocc.

Distribution: England, Scotland, Wales, Ireland, Isle of Man

Notes: Although the name oculatoria (Fabricius, 1798, Ichneumon) has usually been used for this species (e.g. Fitton et al. 1988), the type of oculatoria is actually a species of Lissonota (Horstmann 2001a) so this species has to take the name lineatoria.

Tromatobia ornata (Gravenhorst, 1829)

Nomenclature:

Pimpla ornata Gravenhorst, 1829 
soror (Ratzeburg, 1848, Polysphincta)

arachnicida Förster, 1888 synonymy by Horstmann (2000c)

concors (Kriechbaumer, 1894, Pimpla)

semivaria (Kriechbaumer, 1894, Pimpla)

tricolor (Kriechbaumer, 1894, Pimpla) preocc.

kriechbaumeri (Dalla Torre, 1901, Pimpla)

Distribution: England

\section{Tromatobia ovivora (Boheman, 1821)}

\section{Nomenclature:}

Pimpla ovivora Boheman, 1821

armillatoria (Thunberg, 1824, Ichneumon)

vexatoria (Thunberg, 1824, Ichneumon)

angens (Gravenhorst, 1829, Pimpla)

parallela (Thomson, 1877, Pimpla)

rufipleura (Bignell, 1899, Pimpla)

albipes (Brischke, 1891, Pimpla) preocc.

contraria Förster, 1888 synonymy by Horstmann (2000c)

evacuans Förster, 1888 synonymy by Horstmann (2000c)

brischkei (Dalla Torre, 1901, Pimpla)

obscurata (Ulbricht, 1910, Pimpla) preocc., unavailable

rugulosa (Morley, 1914, Apechtis)

obscurascens (Strand, 1918, Pimpla)

simulans (Hensch, 1929, Pimpla)

Distribution: England, Scotland, Wales, Ireland, Isle of Man

\section{Tromatobia variabilis (Holmgren, 1856)}

\section{Nomenclature:}

Pimpla variabilis Holmgren, 1856

abdominalis (Brullé, 1846,) preocc.

epeirae (Bignell, 1893, Pimpla)

hibernica (Morley, 1908, Pimpla)

ruficoxa (Kokujev, 1913, Pimpla) 
inornata (Ulbricht, 1926, Pimpla)

Distribution: England, Wales, Ireland

\section{Genus Zaglyptus Förster, 1869}

\section{Zaglyptus multicolor (Gravenhorst, 1829)}

Nomenclature:

Polysphincta multicolor Gravenhorst, 1829

fairmairii (Laboulbene, 1858, Pimpla)

ephippium (Rudow, 1883, Pimpla) preocc.

moldavicus (Costantineanu, 1929, Pimpla)

rufus Aubert, 1959

Distribution: England

\section{Zaglyptus varipes (Gravenhorst, 1829)}

\section{Nomenclature:}

Polysphincta varipes Gravenhorst, 1829

tricingulatus (Gravenhorst, 1829, Schizopyga)

cingulatus (Kriechbaumer, 1894, Pimpla) preocc.

variipes Dalla Torre, 1901 preocc.

rufithorax (Habermehl, 1917, Polysphincta)

silbernageli (Kiss, 1926, Polysphincta)

Distribution: England, Scotland, Wales, Ireland

\section{Genus Zatypota Förster, 1869}

Nomenclature:

POLYSPHINCTOPSIS Habermehl, 1917

LYCORINOPSIS Haupt, 1954

Notes: species of Zatypota excluded from the British and Irish list by Fitton et al. (1988)

[anomala (Holmgren, 1860, Polysphincta); syn. minor (Kolarov, 1982, Sinarachna): Zwakhals (2006)] usually treated as a species of Sinarachna (e.g. Fitton et al. 1988, Yu and Horstmann 1997) but transferred to Zatypota by Zwakhals (2006) (and, independently, by Gauld and Dubois 2006). 


\section{Zatypota albicoxa (Walker, 1874)}

Nomenclature:

Glypta albicoxa Walker, 1874

colorata (Rudow, 1883, Pimpla)

eximia (Schmiedeknecht, 1907, Polysphincta)

nigriventris (Habermehl, 1917, Polysphinctopsis)

Distribution: England

Notes: added by Hudson (1985)

\section{Zatypota bohemani (Holmgren, 1860)}

\section{Nomenclature:}

Polysphincta bohemani Holmgren, 1860

Distribution: England, Scotland, Wales, Isle of Man

\section{Zatypota discolor (Holmgren, 1860)}

\section{Nomenclature:}

Polysphincta discolor Holmgren, 1860

thoracica (Brischke, 1864, Polysphincta)

Distribution: England, Scotland, Ireland

Notes: Irish occurrence from O'Connor (2004b)

\section{Zatypota percontatoria (Müller, 1776)}

Nomenclature:

Ichneumon percontatorius Müller, 1776

phoenicea (Haliday, 1839, Pimpla)

gracilis (Holmgren, 1860, Polysphincta) synonymy by Horstmann (2000c)

scutellaris (Holmgren, 1860, Polysphincta)

pulchrator (Thomson, 1877, Polysphincta)

pulchratrix (Schulz, 1906, Polysphincta)

decorata (Haupt, 1954, Lycorinopsis)

rhombifer (Haupt, 1954, Lycorinopsis)

Distribution: England, Scotland, Wales 


\section{Tribe PIMPLINI Wesmael, 1845}

Nomenclature:

THERONIINI Cushman \& Rohwer, 1920

\section{Genus Apechthis Förster, 1869}

Nomenclature:

EPHIALTES Schrank, 1802 nom. ob.

APECHTIS Thomson, 1889

PARAPECHTHIS Blanchard, 1936

TAIWATHERONIA Sonan, 1936

\section{Apechthis compunctor (Linnaeus, 1758)}

\section{Nomenclature:}

Ichneumon compunctor Linnaeus, 1758

brassicariae (Poda, 1761, Ichneumon)

cunctator (Scopoli, 1763, Ichneumon)

annulosa (Gmelin, 1790, Ichneumon)

cylindrica (Gmelin, 1790, Ichneumon) preocc.

melanoxantha (Gmelin, 1790, Ichneumon)

imminuitor (Christ, 1791, Ichneumon) synonymy by Horstmann (2000c)

vigilans (Christ, 1791, Ichneumon) synonymy by Horstmann (2000c)

varicornis (Fabricius, 1793, Ichneumon)

conjunctor (Panzer, 1804, Ichneumon)

varicator (Thunberg, 1824, Ichneumon)

lativentris (Rudow, 1881, Pimpla)

rufipes (Rudow, 1883, Pimpla)

Distribution: England, Wales

Apechthis quadridentata (Thomson, 1887)

Nomenclature:

Pimpla quadridentata Thomson, 1887

resinator misident.

Distribution: England, Scotland, Wales, Ireland 
Apechthis rufata (Gmelin, 1790)

Nomenclature:

Ichneumon rufatus Gmelin, 1790

flavonotata (Holmgren, 1860, Pimpla)

rufithorax (Strobl, 1902, Pimpla)

pectoralis (Ulbricht, 1909, Pimpla) unavailable

Distribution: England, Scotland, Wales, Ireland

Genus Itoplectis Förster, 1869

Nomenclature:

NESOPIMPLA Ashmead, 1906

EXERISTESOIDES Uchida, 1928

Itoplectis alternans (Gravenhorst, 1829)

Nomenclature:

Pimpla alternans Gravenhorst, 1829

examinanda (Ratzeburg, 1852, Pimpla)

tricolor (Ratzeburg, 1852, Pimpla) preocc.

tricincta (Thomson, 1877, Pimpla) preocc.

spiracularis (Morley, 1908, Pimpla)

ruficoxis (Ulbricht, 1916, Pimpla) unavailable

Distribution: England, Scotland, Wales, Ireland

\section{Itoplectis aterrima Jussila, 1965}

Nomenclature:

kolthoffi misident.

enslini (Ulbricht, 1916, Pimpla) preocc., unavailable

ultimator Aubert, 1966

Distribution: England, Scotland, Wales, Ireland

Notes: added by Fitton et al. (1988) 
Itoplectis clavicornis (Thomson, 1889)

Nomenclature:

Pimpla clavicornis Thomson, 1889

curticauda misident.

Distribution: England, Scotland, Ireland

Itoplectis enslini (Ulbricht, 1911)

Nomenclature:

Pimpla enslini Ulbricht, 1911

insignis misident. (Shaw 2006a)

ignalinoensis (Strand, 1918, Pimpla)

griseanae Perkins, 1957

Distribution: England, Scotland

Notes: added by Fitton et al. (1988)

Itoplectis maculator (Fabricius, 1775)

Nomenclature:

Ichneumon maculator Fabricius, 1775

arlequinata (Geoffroy, 1785, Ichneumon)

plaesseus (Geoffroy, 1785, Ichneumon)

scanica (Villers, 1789, Ichneumon) preocc.

laetatoria (Thunberg, 1824, Ichneumon)

vincta (Vollenhoven, 1873, Pimpla)

maculatrix (Schulz, 1906, Pimpla)

Distribution: England, Scotland, Wales, Ireland, Isle of Man

Itoplectis melanocephala (Gravenhorst, 1829)

Nomenclature:

Pimpla melanocephala Gravenhorst, 1829

ephippium (Brullé, 1846, Pimpla)

bicolor (Boie, 1855, Pimpla) preocc.

ragusae (De Stefani, 1885, Pimpla)

cleopatra (Schmiedeknecht, 1897, Pimpla) 
burtoni (Morley, 1946, Pimpla)

Distribution: England, Wales

Itoplectis viduata (Gravenhorst, 1829)

Nomenclature:

Pimpla viduata Gravenhorst, 1829

atrocoxalis (Cresson, 1870, Pimpla)

ovalis (Thomson, 1877, Pimpla)

meridionalis (Kriechbaumer, 1887, Pimpla)

annulata (Ulbricht, 1911, Pimpla) unavailable

Distribution: England

Notes: added by Shaw (2006a)

\section{Genus Pimpla Fabricius, 1804}

Nomenclature:

COCCYGOMIMUS Saussure, 1892

HABROPIMPLA Cameron, 1900

LISSOTHERONIA Cameron, 1905

PHYTODIAETOIDES Morley, 1913

PIMPLIDEA Viereck, 1914

COELOPIMPLA Brèthes, 1916

DIHYBOPLAX Enderlein, 1919

LIOTHERONIA Enderlein, 1919

NEOGABUNIA Brèthes, 1927

OPODACTYLA Seyrig, 1932

OXYPIMPLA Noskiewicz \& Chudoba, 1951

JAMAICAPIMPLA Mason, 1975

Notes: doubtfully placed species of Pimpla:

[cossivora (Curtis, 1826, Lissonota) nom. dub.] 
Pimpla aethiops Curtis, 1828

Nomenclature:

aterrima Gravenhorst, 1829

parnarae Viereck, 1912

Distribution: England

Pimpla arctica Zetterstedt, 1838

Nomenclature:

heraclii Boie, 1855

coxator Ruthe, 1859

heraclei Dalla Torre, 1901

coxatrix Schulz, 1906

Distribution: Scotland

\section{Pimpla contemplator (Müller, 1776)}

Nomenclature:

Ichneumon contemplator Müller, 1776

geniculata (Geoffroy, 1785, Ichneumon)

rufistigma Morley, 1908

rufitibia Morley, 1908

Distribution: England, Scotland, Wales, Ireland, Isle of Man

Pimpla flavicoxis Thomson, 1877

Distribution: England, Scotland, Wales, Ireland, Isle of Man

Notes: Pimpla aquilonia Cresson, 1870, is a possible senior synonym of flavicoxis (e.g. Oehlke 1964) but is currently classified as a separate species (e.g. Kasparyan 1974, Fitton et al. 1988).

\section{Pimpla insignatoria (Gravenhorst, 1807)}

Nomenclature:

Cryptus insignatorius Gravenhorst, 1807

mixta Ratzeburg, 1848

coxalis Habermehl, 1917 
scutellaris Habermehl, 1917 preocc.

conmixta Kiss, 1929

Distribution: England, Scotland, Wales, Ireland, Isle of Man

Notes: Added by Horstmann (2000c), who lists synonymy; Pimpla insignatoria has, until recently, been confused under flavicoxis in Britain (Shaw 2006a), although Kasparyan (1974) differentiated the two species (using the name conmixta for insignatoria).

\section{Pimpla melanacrias Perkins, 1941}

\section{Nomenclature:}

geniculata Hensch, 1929 preocc., invalid

Distribution: England, Scotland, Wales, Ireland, Isle of Man

\section{Pimpla rufipes (Miller, 1759)}

Nomenclature:

Ichneumon rufipes Miller, 1759

hypochondriaca (Retzius, 1783, Ichneumon) synonymy by Horstmann (1999a)

compunctor (Geoffroy, 1785, Ichneumon) preocc.

inguinalis (Geoffroy, 1785, Ichneumon)

instigator (Fabricius, 1793, Ichneumon) preocc.

intermedia Holmgren, 1860

aegyptiaca Schmiedeknecht, 1897

instigatrix Schulz, 1906

scutellaris Ulbricht, 1909 preocc., unavailable

sibirica Meyer, 1926

Distribution: England, Scotland, Wales, Ireland, Isle of Man

\section{Pimpla sodalis Ruthe, 1859}

\section{Nomenclature:}

cheloniae Giraud, 1869 synonymy by Horstmann (2001b)

nordenskioldii Holmgren, 1872

longiceps Thomson, 1877

Distribution: Scotland 


\title{
Pimpla spuria Gravenhorst, 1829
}

\section{Nomenclature:}

bilineata Brullé, 1846

strigipleuris Thomson, 1877 synonymy by Shaw (2006b)

dubitata Pérez, 1895

nilotica Schmiedeknecht, 1914

turionelloides Aubert, 1959

Distribution: England, Scotland, Wales, Ireland, Isle of Man

\section{Pimpla turionellae (Linnaeus, 1758)}

\section{Nomenclature:}

\author{
Ichneumon turionellae Linnaeus, 1758 \\ variegata (Schrank, 1785, Ichneumon) \\ leucogonos (Gmelin, 1790, Ichneumon) \\ rufescens (Gmelin, 1790, Ichneumon) preocc. \\ examinator (Fabricius, 1804, Cryptus) \\ cingulator (Thunberg, 1824, Ichneumon) \\ turionator (Thunberg, 1824, Ichneumon) \\ opacellata Desvignes, 1868 \\ examinatrix Schulz, 1906 \\ pubescens Hellén, 1915 \\ padellae Torka, 1918 \\ rufoannula Schmiedeknecht, 1934 \\ freyi Hellén, 1949 \\ variegata Constantineanu, 1954 preocc.
}

Distribution: England, Scotland, Wales, Ireland

\section{Pimpla wilchristi Fitton, Shaw \& Gauld, 1988}

Distribution: England, Scotland, Wales, Isle of Man

Notes: Added by Fitton et al. (1988); incorrectly synonymised with strigipleuris

Thomson (a junior synonym of spuria) by Hedström (1990) (Shaw 2006b). 


\section{Genus Theronia Holmgren, 1859}

\section{Nomenclature:}

PSEUDACOENITES Kriechbaumer, 1892

POECILOPIMPLA Cameron, 1903

ERYTHROTHERONIA Cameron, 1905

ORIENTOTHERONIA Morley, 1913

\section{Theronia atalantae (Poda, 1761)}

\section{Nomenclature:}

Ichneumon atalantae Poda, 1761

speculator (Scopoli, 1763, Ichneumon)

acuminator (Müller, 1776, Ichneumon)

melanops (Schrank, 1781, Ichneumon)

nigroculus (Schrank, 1781, Ichneumon)

quadripunctata (Schrank, 1781, Ichneumon)

vincta (Schrank, 1781, Ichneumon)

scutellata (Geoffroy, 1785, Ichneumon)

albiscutata (Gmelin, 1790, Ichneumon)

crassipes (Rossi, 1790, Ichneumon) preocc.

incisa (Gmelin, 1790, Ichneumon)

superba (Christ, 1791, Ichneumon) preocc.

vulpes (Christ, 1791, Ichneumon)

varia (Olivier, 1792, Ichneumon) preocc.

flavicans (Fabricius, 1793, Ichneumon)

varia (Fabricius, 1793, Ichneumon) preocc.

variatoria (Fabricius, 1804, Cryptus)

colonator (Thunberg, 1824, Ichneumon)

femoralis Benoit, 1953

Distribution: England 


\section{Poemeniinae}

Subfamily POEMENIINAE Narayanan \& Lal, 1953

Notes: Distribution data from Fitton et al. (1988), where treated as a tribe of Pimplinae, and Shaw (2006a). Further distribution references are given.

Tribe POEMENIINI Narayanan \& Lal, 1953

Genus Deuteroxorides Viereck, 1914

Deuteroxorides elevator (Panzer, 1799)

Nomenclature:

Ichneumon elevator Panzer, 1799

albitarsus (Gravenhorst, 1829, Xorides)

nigricornis Clément, 1938

nigritarsus Clément, 1938

Distribution: England, Ireland

Genus Podoschistus Townes, 1957

Podoschistus scutellaris (Desvignes, 1856)

Nomenclature:

Xorides scutellaris Desvignes, 1856

wahlbergi (Holmgren, 1860, Xorides)

erosus (Tschek, 1869, Xorides)

Distribution: England, Wales

Genus Poemenia Holmgren, 1859

Nomenclature:

OPHIODES Hartig, 1847

CALLICLISIS Förster, 1869

PHTHINODES Tschek, 1869

LISSONOTOPSIS Habermehl, 1917 


\section{Poemenia collaris (Haupt, 1917)}

Nomenclature:

Calliclisis collaris Haupt, 1917

picta (Haupt, 1938, Calliclisis)

Distribution: England

Notes: added by Fitton et al. (1988)

Poemenia hectica (Gravenhorst, 1829)

Nomenclature:

Ephialtes hecticus Gravenhorst, 1829

montana (Hartig, 1847, Ophiodes)

tipularia Holmgren, 1860

Distribution: England, Scotland, Ireland

Poemenia notata Holmgren, 1859

Nomenclature:

novakii Strobl, 1902

rufa (Habermehl, 1918, Lissonotopsis)

rufa (Habermehl, 1918, Xorides) preocc.

intermedia Constantineanu \& Constantineanu, 1969

moldavica Constantineanu \& Constantineanu, 1969

Distribution: England

Notes: added by Fitton et al. (1988)

Tribe PSEUDORHYSSINI Wahl \& Gauld, 1998

Genus Pseudorhyssa Merrill, 1915

Pseudorhyssa alpestris (Holmgren, 1860)

Nomenclature:

Rhyssa alpestris Holmgren, 1860

ruficoxis (Kriechbaumer, 1887, Rhyssa)

hungarica (Mocsáry, 1905, Rhyssa) 
Distribution: England, Wales

Notes: Welsh occurrence from Formstone (1999)

\section{Rhyssinae}

\section{Subfamily RHYSSINAE Morley, 1913}

Notes: Distribution data from Fitton et al. (1988), where treated as a tribe of Pimplinae. Further distribution references are given.

\section{Genus Rhyssa Gravenhorst, 1829}

Nomenclature:

CRYPTOCENTRUM Kirby, 1837

PARARHYSSA Walsh, 1873

\section{Rhyssa persuasoria (Linnaeus, 1758)}

Nomenclature:

Ichneumon persuasorius Linnaeus, 1758

marginalis Brullé, 1846

lineolata Kriechbaumer, 1887 preocc.

gloriosa Rudow, 1889

Notes: Some Scottish and Manx records from Hayes (1982) and Cowin and Williamson (1940).

\section{Genus Rhyssella Rohwer, 1920}

\section{Rhyssella approximator Fabricius, 1793}

Nomenclature:

Ichneumon approximator Fabricius, 1793

curvipes (Gravenhorst, 1829, Rhyssa)

rugicollis (Zetterstedt, 1838, Tryphon)

bellator (Schiødte, 1839, Rhyssa) synonymy by Horstmann (2004c)

approximatrix (Schulz, 1906, Rhyssa)

silbernageli (Kiss, 1926, Rhyssa) 


\section{Stilbopinae}

Subfamily STILBOPINAE Townes \& Townes, 1949

\section{Genus Panteles Förster, 1869}

Nomenclature:

BRACHYPIMPLA misident.

Notes: Panteles has frequently been classified as a genus of Banchinae but we follow Wahl (1988) in classifying it in the Stilbopinae.

\section{Panteles schuetzeanus (Roman, 1925)}

Nomenclature:

Brachypimpla schuetzeana Roman, 1925

schnetzeanus misspelling

Distribution: England, Scotland, Ireland

Notes: Quicke (2005) has published on the biology of schuetzeanus in Britain, unfortunately misspelling the name.

\section{Genus Stilbops Förster, 1869}

\section{Nomenclature:}

APHANOROPTRUM Förster, 1869

APHANOROPTRA Thomson, 1877

APHANORRHOPTRUM Dalla Torre, 1901

ERITRACHYNUS Schmiedeknecht, 1913

Notes: Distribution data from Fitton (1984), with additional data from Shaw (1989) and NMS.

\section{Stilbops asper (Schmiedeknecht, 1913)}

Nomenclature:

Eritrachynus asper Schmiedeknecht, 1913

Distribution: England, Scotland

Notes: added by Fitton (1984) 
Stilbops limneriaeformis (Schmiedeknecht, 1888)

Nomenclature:

Pimpla limneriaeformis Schmiedeknecht, 1888

Distribution: Scotland, Ireland

\section{Stilbops ruficornis (Gravenhorst, 1829)}

Nomenclature:

Lissonota ruficornis Gravenhorst, 1829

abdominalis (Gravenhorst, 1829, Pimpla)

nematorum (Rudow, 1881, Pimpla)

longiceps (Strobl, 1903, Polyblastus)

Distribution: England

\section{Stilbops vetula (Gravenhorst, 1829)}

Nomenclature:

Pimpla vetula Gravenhorst, 1829

chrysostomus (Gravenhorst, 1829, Phytodietus)

pallipes (Gravenhorst, 1829, Lissonota)

pallidipes (Marshall, 1872, Lissonota)

varicauda (Capron, 1888, Pimpla)

Distribution: England, Scotland, Wales, Ireland

\section{Tersilochinae}

\section{Subfamily TERSILOCHINAE Schmiedeknecht, 1910}

\section{Nomenclature:}

PHRUDINAE Townes \& Townes, 1949

Notes: Following the phylogenetic results of Quicke et al. (2009), the Tersilochinae now encompasses the former subfamily Phrudinae (containing the genera Astrenis, Phrudus and Pygmaeolus in Britain) (they also synonymised Neorhacodinae under Tersilochinae but I don't follow that decision). Distribution data for the 'tersilochine' genera from Horstmann (1971), Horstmann (1981a) and material in BMNH and NMS, mostly determined by K. Horstmann and, latterly, A. Khalaim; distribution data for the 
'phrudine' genera from Gauld and Fitton (1980) and Vikberg and Koponen (2000); additional references are given.

Genus Allophroides Horstmann, 1971

Allophroides boops (Gravenhorst, 1829)

Nomenclature:

Porizon boops Gravenhorst, 1829

italicus (Gravenhorst, 1829, Porizon)

breviventris (Hellén, 1958, Allophrys)

Genus Aneuclis Förster, 1869

Aneuclis melanaria (Holmgren, 1860)

Nomenclature:

Thersilochus melanarius Holmgren, 1860

diversa (Szépligeti, 1899, Isurgus)

petiolaris (Szépligeti, 1899, Isurgus)

Distribution: England, Ireland

Genus Astrenis Förster, 1869

Nomenclature:

MENGERSENIA Schmiedeknecht, 1907

HAMBERGIELLA Roman, 1909

Notes: Until recently, Astrenis has usually been regarded as a synonym of Phrudus.

Astrenis brunneofacies Vikberg, 2000

Distribution: England, Scotland

Notes: added by Vikberg and Koponen (2000)

Astrenis nigrifacies Vikberg, 2000

Distribution: England, Scotland

Notes: added by Vikberg and Koponen (2000) 
Astrenis paradoxus (Schmiedeknecht, 1907)

Nomenclature:

Mengersenia paradoxa Schmiedeknecht, 1907

Distribution: England

Notes: added by Gauld and Fitton (1980)

Astrenis sinuatus (Roman, 1909)

Nomenclature:

Hambergiella sinuata Roman, 1909

Distribution: England, Scotland, Ireland

\section{Genus Barycnemis Förster, 1869}

Nomenclature:

LEPTOPYGUS Förster, 1869

CRATOPHION Thomson, 1889

CYRTOPHION Thomson, 1889

Barycnemis agilis (Holmgren, 1860)

Nomenclature:

Porizon agilis Holmgren, 1860

Distribution: England, Scotland

Notes: BMNH, NMS, det. Khalaim \& Horstmann, added here

Barycnemis angustipennis (Holmgren, 1860)

Nomenclature:

Porizon angustipennis Holmgren, 1860

added by

Distribution: England, Scotland, Ireland

Notes: added by Horstmann (1981a) 
Barycnemis bellator (Müller, 1776)

Nomenclature:

Ichneumon bellator Müller, 1776

laeviceps (Thomson, 1889, Porizon)

leviceps Dalla Torre, 1901

pfankuchi Lange, 1911

Distribution: England, Scotland

Barycnemis blediator (Aubert, 1970)

Nomenclature:

Leptopygus blediator Aubert, 1970

Distribution: England, Wales

Notes: added by Wyatt and Foster (1989)

Barycnemis confusa Horstmann, 1981

Distribution: Scotland

Notes: BMNH, NMS, det. Broad \& Horstmann, added here

Barycnemis dissimilis (Gravenhorst, 1829)

Nomenclature:

Porizon dissimilis Gravenhorst, 1829

erythrura (Strobl, 1904, Porizon)

Distribution: England

Barycnemis exhaustator (Fabricius, 1798)

Nomenclature:

Ichneumon exhaustator Fabricius, 1798

obtusator (Panzer, 1809, Ophion)

Distribution: England

Barycnemis gravipes (Gravenhorst, 1829)

Nomenclature:

Porizon gravipes Gravenhorst, 1829 
hostilis (Gravenhorst, 1829, Porizon)

Distribution: England, Scotland, Ireland

Barycnemis guttulator (Thunberg, 1824)

Nomenclature:

Ichneumon guttulator Thunberg, 1824

caudatula (Thomson, 1889, Porizon)

Barycnemis harpura (Schrank, 1802)

Nomenclature:

Ichneumon harpurus Schrank, 1802

bedeguaris (Panzer, 1809, Ophion)

Distribution: England, Scotland, Wales, Ireland

Barycnemis punctifrons Horstmann, 1981

Distribution: England, Ireland

Notes: added by Horstmann (1981a)

Genus Diaparsis Förster, 1869

Subgenus Diaparsis Förster, 1869

Nomenclature:

DIAPARSUS Thomson, 1889

Diaparsis (Diaparsis) carinifer (Thomson, 1889)

Nomenclature:

Thersilochus carinifer Thomson, 1889

carinata (Bridgman, 1889, Thersilochus)

vernalis (Szépligeti, 1899, Thersilochus)

Distribution: England, Scotland, Wales, Ireland

Diaparsis (Diaparsis) multiplicator Aubert, 1969

Distribution: England 
Diaparsis (Diaparsis) nutritor (Fabricius, 1804)

Nomenclature:

Ophion nutritor Fabricius, 1804

gemina (Holmgren, 1860, Thersilochus)

genalis (Thomson, 1889, Thersilochus)

rugosa (Szépligeti, 1905, Temelucha)

nutritrix Schulz, 1906

Diaparsis (Diaparsis) punctipleuris Horstmann, 1981

Distribution: England

Notes: $\mathrm{BMNH}$, det. Khalaim, added here

Subgenus Ischnobatis Förster, 1869

Diaparsis (Ischnobatis) stramineipes (Brischke, 1880)

Nomenclature:

Thersilochus stramineipes Brischke, 1880

rufiventris (Brischke, 1880, Thersilochus)

flavicornis (Thomson, 1889, Thersilochus)

petiolata (Szépligeti, 1899, Thersilochus)

Distribution: England, Ireland

Subgenus Nanodiaparsis Horstmann, 1971

Diaparsis (Nanodiaparsis) aperta (Thomson, 1889)

Nomenclature:

Thersilochus apertus Thomson, 1889

Distribution: England

Notes: NMS, BMNH, det. Horstmann and Khalaim, added here

Diaparsis (Nanodiaparsis) frontella (Holmgren, 1860)

Nomenclature:

Thersilochus frontellus Holmgren, 1860 
Distribution: England

Subgenus Pseudaneuclis Horstmann, 1971

Diaparsis (Pseudaneuclis) rara (Horstmann, 1971)

Nomenclature:

Pseudaneuclis rarus Horstmann, 1971

Distribution: England

Notes: BMNH, det. Horstmann, added here

Genus Epistathmus Förster, 1869

Epistathmus crassicornis Horstmann, 1971

Distribution: England, Scotland, Ireland

Notes: added by Horstmann (1981a)

Genus Gelanes Horstmann, 1981

Gelanes fusculus (Holmgren, 1860)

Nomenclature:

Thersilochus fusculus Holmgren, 1860

Distribution: England, Scotland

Notes: NMS, det. Horstmann, BMNH, det. Khalaim, added here

Gelanes simillimus Horstmann, 1981

Distribution: England, Scotland, Ireland

Notes: added by Horstmann (1981a)

Genus Heterocola Förster, 1869

Subgenus Heterocoloides Horstmann, 1971

Heterocola (Heterocoloides) linguaria (Haliday, 1839)

Nomenclature: 
Porizon linguarius Haliday, 1839

punctulata (Szépligeti, 1899, Ischnobatis)

Distribution: England, Ireland

Notes: Two specimens in BMNH, from Cornwall \& Co. WX, have been identified as $H$. rufiventris Horstmann, 1971. There are no specimens of linguaria; their identity needs to be checked.

\section{Genus Phradis Förster, 1869}

Nomenclature:

EUTOMUS Förster, 1869

ISURGUS Förster, 1869

Phradis brevis (Brischke, 1880)

Nomenclature:

Thersilochus brevis Brischke, 1880

temporalis (Thomson, 1889, Thersilochus)

styriacus (Strobl, 1904, Thersilochus)

Distribution: England, Ireland

Notes: added by Horstmann (1981a)

Phradis interstitialis (Thomson, 1889)

Nomenclature:

Thersilochus interstitialis Thomson, 1889

brachygaster (Szépligeti, 1899, Isurgus)

Distribution: England, Scotland, Wales, Ireland

Phradis minutus (Bridgman, 1889)

Nomenclature:

Thersilochus minutus Bridgman, 1889

Distribution: England, Wales, Ireland

Phradis monticola Szépligeti, 1899

Distribution: England 
Notes: BMNH, det. Ely, added here

Phradis morionellus (Holmgren, 1860)

Nomenclature:

Thersilochus morionellus Holmgren, 1860

lanceolatus (Szépligeti, 1899, Isurgus)

oudesmani (Smits\$)

Distribution: England, Ireland

Phradis nigritulus (Gravenhorst, 1829)

Nomenclature:

Porizon nigritulus Gravenhorst, 1829

albipennis (Szépligeti, 1899, Isurgus)

Distribution: England

Phradis polonicus Horstmann, 1981

Distribution: England, Scotland

Notes: BMNH, NMS, added here

Phradis rufiventris Horstmann, 1981

Distribution: England

Notes: BMNH, det. Ely, added here

Phradis terebrator Horstmann, 1981

Distribution: England

Notes: BMNH, det. Ely, added here

Phradis thyridialis Horstmann, 1981

Distribution: England, Ireland

Notes: BMNH, det. Ely, added here 
Genus Phrudus Förster, 1869

Nomenclature:

PHRUDUS Bridgman, 1886 preocc.

KTENOSTILPNUS Strobl, 1901

VENDOLUS Roman, 1914

Phrudus badensis Hilpert, 1987

Distribution: England

Notes: added by Shaw (1991)

Phrudus defectus Stelfox, 1966

Distribution: England, Scotland, Ireland, Isle of Man

Phrudus monilicornis Bridgman, 1886

Nomenclature:

aequearticulatus (Strobl, 1901, Ktenostilpnus)

stilpninus (Roman, 1914, Vendolus)

Distribution: England, Scotland, Wales, Ireland

Genus Probles Förster, 1869

Subgenus Euporizon Horstmann, 1971

Probles (Euporizon) brevicauda Horstmann, 1981

Distribution: England

Notes: added by Horstmann (1981a)

Probles (Euporizon) exilis (Holmgren, 1860)

Nomenclature:

Thersilochus exilis Holmgren, 1860

Distribution: Scotland

Notes: NMS, det. Horstmann, added here 
Probles (Euporizon) gilvipes (Gravenhorst, 1829)

Nomenclature:

Porizon gilvipes Gravenhorst, 1829

pallipes (Holmgren, 1860, Thersilochus)

orchesiae (Morley, 1915, Thersilochus)

Distribution: England

Probles (Euporizon) longicaudator Aubert, 1972

Distribution: England, Ireland

Notes: added by Horstmann (1981a)

Probles (Euporizon) marginatus (Bridgman, 1886)

Nomenclature:

Thersilochus marginatus Bridgman, 1886

Distribution: England, Ireland

Probles (Euporizon) montanus Horstmann, 1971

Distribution: Scotland, Ireland

Notes: added by Horstmann (1981a)

Probles (Euporizon) nigriventris Horstmann, 1971

Distribution: Ireland

Notes: added by Horstmann (1981a)

Probles (Euporizon) rufipes (Holmgren, 1860)

Nomenclature:

Thersilochus rufipes Holmgren, 1860

flavigaster (Szépligeti, 1899, Ischnobatis)

Distribution: England, Ireland

Probles (Euporizon) truncorum (Holmgren, 1860)

Nomenclature:

Thersilochus truncorum Holmgren, 1860 
Distribution: England, Ireland

Subgenus Microdiaparsis Horstmann, 1971

Probles (Microdiaparsis) caudiculatus Khalaim, 2007

Distribution: England

Notes: added by Khalaim (2007)

Probles (Microdiaparsis) microcephalus (Gravenhorst, 1829)

Nomenclature:

Porizon microcephalus Gravenhorst, 1829

quercetorum (Szépligeti, 1899, Thersilochus)

ruficoxis (Seyrig, 1927, Diaparsis)

Distribution: England, Scotland, Ireland

Probles (Microdiaparsis) neoversutus (Horstmann, 1967)

Nomenclature:

Diaparsis neoversutus Horstmann, 1967

parviceps (Szépligeti, 1899, Thersilochus) preocc.

Distribution: England, Ireland

Probles (Microdiaparsis) versutus (Holmgren, 1860)

Nomenclature:

Thersilochus versutus Holmgren, 1860

parviceps (Thomson, 1899, Thersilochus)

Distribution: England

Subgenus Probles Förster, 1869

Probles (Probles) erythrostomus (Gravenhorst, 1829)

Nomenclature:

Porizon erythrostomus Gravenhorst, 1829

minator (Gravenhorst, 1829, Porizon)

melanarius Szépligeti, 1899 
Distribution: England, Scotland, Ireland

Probles (Probles) flavipes (Szépligeti, 1899)

Nomenclature:

Ischnobatis flavipes Szépligeti, 1899

Distribution: England, Ireland

Notes: added by Horstmann (1981a)

Subgenus Rugodiaparsis Horstmann, 1971

Probles (Rugodiaparsis) crassipes (Thomson, 1889)

Nomenclature:

Thersilochus crassipes Thomson, 1889

Distribution: England, Scotland, Wales

Notes: BMNH, NMS, det. Horstmann, added here

Genus Pygmaeolus Hellén, 1958

Pygmaeolus nitidus (Bridgman, 1889)

Nomenclature:

Thersilochus nitidus Bridgman, 1889

Distribution: England, Scotland, Ireland

Genus Sathropterus Förster, 1869

Sathropterus pumilus (Holmgren, 1860)

Nomenclature:

Thersilochus pumilus Holmgren, 1860

Distribution: England, Ireland

Notes: added by Horstmann (1981a) 
Genus Spinolochus Horstmann, 1971

Spinolochus laevifrons (Holmgren, 1860)

Nomenclature:

Thersilochus laevifrons Holmgren, 1860

levifrons (Dalla Torre, 1901, Cyrtophion) preocc.

Distribution: England, Scotland, Ireland

Notes: added by Horstmann (1981a)

Genus Tersilochus Holmgren, 1859

Subgenus Gonolochus Förster, 1869

Tersilochus (Gonolochus) caudatus (Holmgren, 1860)

Nomenclature:

Thersilochus caudatus Holmgren, 1860

pratensis (Szépligeti, 1899, Thersilochus)

salinus (Kiss, 1924, Temelucha)

Distribution: England, Ireland

Tersilochus (Gonolochus) rugulosus Horstmann, 1981

Distribution: England

Notes: BMNH, det. Khalaim, added here

Subgenus Pectinolochus Aubert, 1960

Nomenclature:

POLEMOLOCHUS Aubert, 1964

Tersilochus (Pectinolochus) intermedius Horstmann, 1981

Distribution: England

Notes: NMS, det. Horstmann, added here 
Tersilochus (Pectinolochus) lapponicus Hellén, 1958

Distribution: England, Wales, Ireland

Notes: added by Horstmann (1981a)

Tersilochus (Pectinolochus) spiracularis Horstmann, 1971

Distribution: England

Notes: BMNH, det. Broad and Khalaim, added here

Tersilochus (Pectinolochus) striola (Thomson, 1889)

Nomenclature:

Thersilochus striola Thomson, 1889

unguiculator (Aubert, 1960, Thersilochus)

Distribution: England, Scotland, Ireland

Notes: added by Horstmann (1981a)

Tersilochus (Pectinolochus) terebrator (Horstmann, 1971)

Nomenclature:

Pectinolochus terebrator Horstmann, 1971

Distribution: England, Ireland

Notes: added by Horstmann (1981a)

Subgenus Tersilochus Holmgren, 1859

Nomenclature:

THERSILOCHUS misspelling

Tersilochus (Tersilochus) cognatus (Holmgren, 1860)

Nomenclature:

Thersilochus cognatus Holmgren, 1860

jocator Holmgren, 1859 unavailable (Horstmann 2005a)

Distribution: England, Wales, Ireland 
Tersilochus (Tersilochus) curvator Horstmann, 1981

Nomenclature:

saltator misident.

Distribution: England, Ireland

Notes: added by Horstmann (1981a)

Tersilochus (Tersilochus) heterocerus (Thomson, 1889)

Nomenclature:

Thersilochus heterocerus Thomson, 1889

stanionyteus Jonaitis, 1974

vicinus Jonaitis, 1974

Distribution: England, Ireland

Tersilochus (Tersilochus) liopleuris (Thomson, 1889)

Nomenclature:

Thersilochus liopleuris Thomson, 1889

Distribution: England, Ireland

Tersilochus (Tersilochus) longicaudatus Horstmann, 1971

Distribution: England, Ireland

Notes: added by Horstmann (1981a)

Tersilochus (Tersilochus) longicornis (Thomson, 1889)

Nomenclature:

Thersilochus longicornis Thomson, 1889

Distribution: England, Scotland, Ireland

Notes: added by Horstmann (1981a)

Tersilochus (Tersilochus) microgaster (Szépligeti, 1899)

Nomenclature:

Isurgus microgaster Szépligeti, 1899

Distribution: England 
Notes: added by Barari et al. (2005)

Tersilochus (Tersilochus) nitidipleuris Horstmann, 1971

Distribution: England, Ireland

Notes: added by Horstmann (1981a)

Tersilochus (Tersilochus) obliquus (Thomson, 1889)

Nomenclature:

Thersilochus obliquus Thomson, 1889

Distribution: England, Ireland

Notes: added by Horstmann (1981a)

Tersilochus (Tersilochus) obscurator (Aubert, 1959)

Nomenclature:

Thersilochus obscurator Aubert, 1959

Distribution: England, Scotland, Ireland

Notes: added by Horstmann (1981a)

Tersilochus (Tersilochus) ruberi Horstmann, 1981

Distribution: Ireland

Notes: added by Horstmann (1981a)

Tersilochus (Tersilochus) triangularis (Gravenhorst, 1807)

Nomenclature:

Ophion triangulare Gravenhorst, 1807

minutus (Szépligeti, 1899, Isurgus)

Distribution: England

Tersilochus (Tersilochus) tripartitus (Brischke, 1880)

Nomenclature:

Thersilochus tripartitus Brischke, 1880

melanogaster (Thomson, 1889, Thersilochus) 
nigricans (Szépligeti, 1899, Thersilochus)

Distribution: England, Ireland

Notes: added by Horstmann (1981a)

\section{Tryphoninae}

Tribe ECLYTINI Townes \& Townes, 1945

Notes: Distribution data from Fitton and Ficken (1990) and Shaw and Kasparyan (2005).

Genus Eclytus Holmgren, 1857

Subgenus Anoplectes Kriechbaumer, 1896

Eclytus (Anoplectes) multicolor (Kriechbaumer, 1896)

Nomenclature:

Anoplectes multicolor Kriechbaumer, 1896

praeclerus Schmiedeknecht, 1912

Distribution: England, Scotland, Ireland

Notes: added by Fitton and Ficken (1990)

Subgenus Eclytus Holmgren, 1857

Nomenclature:

ZAPEDIAS Förster, 1869 synonymy by Bennett (2015)

Notes: species of Eclytus (Eclytus) excluded from the British and Irish list:

[ornatus Holmgren, 1857] Fitton and Ficken (1990) recorded Eclytus ornatus from England and Scotland but their identifications were not based on egg characters, used by Kasparyan (1977) to separate species, and they suggested that their records of ornatus might be based on misidentifications. Shaw and Kasparyan (2005) did not find any specimens of the true ornatus in NMS. 
Eclytus (Eclytus) difficilis Kasparyan, 1977

Distribution: Scotland

Notes: added by Shaw and Kasparyan (2005)

Eclytus (Eclytus) egregius Kasparyan, 1977

Distribution: Scotland

Notes: added by Shaw and Kasparyan (2005)

Eclytus (Eclytus) exornatus (Gravenhorst, 1829)

Nomenclature:

Mesoleptus exornatus Gravenhorst, 1829

Distribution: England, Scotland, Wales, Ireland

Eclytus (Eclytus) haustatorius Kasparyan, 1977

Distribution: England, Scotland

Notes: added by Shaw and Kasparyan (2005)

Tribe IDIOGRAMMATINI Cushman, 1942

Notes: Distribution data from Shaw and Kasparyan (2005).

Genus Idiogramma Förster, 1869

Nomenclature:

MACROCHASMUS Thomson, 1888

Idiogramma euryops Förster, 1869

Distribution: Scotland

Tribe OEDEMOPSINI Woldstedt, 1877

Nomenclature:

THYMARIDINI Schmiedeknecht, 1911

Notes: Distribution data from Fitton and Ficken (1990) and NMS, additional references given. 
Genus Cladeutes Townes, 1969

Cladeutes discedens (Woldstedt, 1874)

Nomenclature:

Perilissus discedens Woldstedt, 1874

haematothorax (Strobl, 1903, Eclytus)

lepidus Townes, 1969

Distribution: England, Ireland

Notes: added by Fitton and Ficken (1990)

Genus Hercus Townes, 1969

Hercus fontinalis (Holmgren, 1857)

Nomenclature:

Eclytus fontinalis Holmgren, 1857

frontalis (Zetterstedt, 1838, Bassus) nom. ob.

Distribution: England, Scotland, Wales, Ireland

Genus Neliopisthus Thomson, 1883

Neliopisthus elegans (Ruthe, 1855)

Nomenclature:

Phytodiaetus elegans Ruthe, 1855

ops (Morley, 1908, Oedematopsis)

Distribution: England

Genus Oedemopsis Tschek, 1869

Nomenclature:

CAMPOTHREPTUS Förster, 1869

HYBOPHANES Förster, 1869

OEDEMATOPSIS Morley, 1908

ODEMOPSIS misspelling 
Oedemopsis scabricula (Gravenhorst, 1829)

Nomenclature:

Tryphon scabriculus Gravenhorst, 1829

dorsata (Zetterstedt, 1838, Bassus)

pulchra (Zetterstedt, 1839, Bassus)

rogenhoferi Tschek, 1869

limbata Thomson, 1883

Distribution: England, Scotland, Ireland, Isle of Man

Notes: Irish distribution data from O'Connor (2003)

\section{Genus Thymaris Förster, 1869}

Nomenclature:

THYMARUS Thomson, 1883

\section{Thymaris niger (Taschenberg, 1865)}

Nomenclature:

Hemiteles niger Taschenberg, 1865

fenestralis Morley, 1908

modestus Schmiedeknecht, 1912

simplicicornis Kiss, 1924 synonymy by Horstmann (1998b)

tristrigator Aubert, 1960

Distribution: England, Scotland

Thymaris srikem Fitton \& Ficken, 1990

Distribution: England, Wales, Ireland

Notes: added by Fitton and Ficken (1990)

Thymaris tener (Gravenhorst, 1829)

Nomenclature:

Mesoleptus tener Gravenhorst, 1829

contaminatus (Gravenhorst, 1829, Hemiteles)

marchicus (Hartig, 1838, Ischnoceros)

pulchricornis Brischke, 1880 
compressus (Thomson, 1883, Thymarus)

Distribution: England, Scotland

Notes: Fitton and Ficken (1990) synonymised collaris (Thomson, 1883) with tener but Kasparyan (1993) treated it as a valid species and gave characters for their separation; no British specimens of collaris have been seen, it is best separated from tener by the all red pronotum and partly red first metasomal tergite.

Tribe PHYTODIETINI Hellén, 1915

Nomenclature:

NETELIINI Townes, 1938

\section{Genus Netelia Gray, 1860}

Nomenclature:

PANISCUS misident.

Notes: Distribution data from the nocturnal Ichneumonoidea recording scheme (run by G. Broad) and Shaw (2001), with additional references given.

\section{Subgenus Bessobates Townes, Townes \& Gupta, 1961}

Netelia (Bessobates) cristata (Thomson, 1888)

Nomenclature:

Parabatus cristatus Thomson, 1888

frankii (Brauns, 1889, Parabatus)

Distribution: England, Scotland, Wales, Ireland, Isle of Man

Notes: Parabatus frankii removed from synonymy by Horstmann (1998c) but resynonymised by Broad \& Shaw (in prep.).

Netelia (Bessobates) latungula (Thomson, 1888)

Nomenclature:

Parabatus latungulus Thomson, 1888

Distribution: England, Scotland, Ireland 
Netelia (Bessobates) pallescens (Schmiedeknecht, 1910)

Nomenclature:

Parabatus pallescens Schmiedeknecht, 1910

Distribution: England, Scotland, Ireland

Notes: added by Broad \& Shaw (in prep.)

Netelia (Bessobates) virgata (Geoffroy, 1785)

Nomenclature:

Ichneumon virgatus Geoffroy, 1785

Distribution: England, Scotland, Wales, Ireland, Isle of Man

Netelia (Bessobates) sp. R

Distribution: Scotland

Notes: added Broad \& Shaw (in prep.)

\section{Subgenus Netelia Gray, 1860}

Nomenclature:

NETELIA Schulz, 1906

Notes: Netelia fulvator Delrio, 1971, was recorded as British by Horstmann (1992b), on the basis that the paralectotype specimens of Paniscus testaceus belong to this species; however, Broad \& Shaw (in prep.) will synonymise fulvator under another name.

Netelia (Netelia) dilatata (Thomson, 1888)

Nomenclature:

Paniscus dilatatus Thomson, 1888

brachycera (Thomson, 1888, Paniscus)

capito (Kokujev, 1889, Paniscus)

genalis (Kokujev, 1889, Paniscus)

schirjajewi (Kokujev, 1889, Paniscus)

sibiricola (Kokujev, 1889, Paniscus)

nigricans (Kriechbaumer, 1898, Paniscus)

nigridorsum (Meyer, 1929, Paniscus) 
Distribution: England

Netelia (Netelia) fuscicarpus (Kokujev, 1899)

Nomenclature:

Paniscus fuscicarpus Kokujev, 1899

desertus (Kokujev, 1915, Paniscus)

maltractus (Roman, 1938, Paniscus)

ambiguator Aubert, 1969

Distribution: England, Wales

Notes: added by Broad \& Shaw (in prep.)

Netelia (Netelia) fuscicornis (Holmgren, 1860)

Nomenclature:

Paniscus fuscicornis Holmgren, 1860
gracilipes (Thomson, 1888, Paniscus)
intersita (Kokujev, 1889, Paniscus)
montana (Kokujev, 1889, Paniscus)
praetermissa (Kokujev, 1889, Paniscus)

Distribution: England, Scotland

Netelia (Netelia) infractor Delrio, 1971

Distribution: England, Scotland, Wales, Ireland

Notes: added by Broad \& Shaw (in prep.)

Netelia (Netelia) melanura (Thomson, 1888)

Nomenclature:

Paniscus melanurus Thomson, 1888

Distribution: England, Scotland

Netelia (Netelia) ocellaris (Thomson, 1888)

Nomenclature:

Paniscus ocellaris Thomson, 1888

Distribution: England 
Netelia (Netelia) opacula (Thomson, 1888)

Nomenclature:

Paniscus opaculus Thomson, 1888

Distribution: England

Netelia (Netelia) testacea (Gravenhorst, 1829)

Nomenclature:

Paniscus testaceus Gravenhorst, 1829

Distribution: England, Scotland

Notes: Added by Horstmann (1992b); this has been a much misunderstood name. Listed as a possible (senior) synonym of opacula in Fitton (1978), Horstmann (1992b) regarded it as a possible senior synonym of valvator Aubert, 1969. Broad \& Shaw (in prep.), following a dissection of the lectotype male's genitalia, offer a different interpretation of testacea. Tolkanitz (1974) and Kasparyan and Tolkanitz (2000) treated fuscicarpus, together with its associated synonyms, as a junior synonym of testacea. This is not followed here.

Netelia (Netelia) vinulae (Scopoli, 1763)

Nomenclature:

Ichneumon vinulae Scopoli, 1763

inquinata (Gravenhorst, 1829, Paniscus) synonymy by Horstmann (1998b)

vinulae (Stephens, 1829, Ophion) preocc., synonymy by Horstmann (2000c)

cephalotes (Holmgren, 1860, Paniscus)

Distribution: England, Scotland

Netelia (Netelia) sp. W

Distribution: England, Wales

Notes: added by Broad \& Shaw (in prep.)

\section{Subgenus Parabates Förster, 1869}

Nomenclature:

PARABATUS Thomson, 1888 
Netelia (Parabates) nigricarpa (Thomson, 1888)

Nomenclature:

Parabatus nigricarpus Thomson, 1888

semifusca (Strobl, 1904, Parabatus)

Distribution: England, Scotland

Subgenus Paropheltes Cameron, 1907

Netelia (Paropheltes) sp. C

Distribution: England

Notes: added by Broad \& Shaw (in prep.)

Netelia (Paropheltes) inedita (Kokujev, 1899)

Nomenclature:

Paniscus ineditus Kokujev, 1899

longipes misident.

ornata misident.

thomsonii misident.

Distribution: England, Scotland, Wales, Ireland

Notes: Added by Shaw (2001) who tentatively identified this widespread species as thomsoni (lapsus for Netelia thomsonii (Brauns, 1899, Paniscus), whilst noting that in Delrio (1975) specimens would key to inedita. Tolkanitz (1974) synonymised inedita under thomsonii but these two species are distinct (Broad \& Shaw, in prep.), with inedita occurring in Britain and Ireland. This species has also been referred to in the literature as ornata and longipes.

Netelia (Paropheltes) millieratae (Kriechbaumer, 1897)

Nomenclature:

Parabatus millieratae Kriechbaumer, 1897

added by

Distribution: England

Notes: added by Broad \& Shaw (in prep.) 
Netelia (Paropheltes) ornata (Vollenhoven, 1873)

Nomenclature:

Paniscus ornatus Vollenhoven, 1873

longipes (Brauns, 1889, Paniscus)

catagrapha (Kokujev, 1915, Paniscus)

ignobilis (Kokujev, 1915, Paniscus)

versicolor (Kokujev, 1915, Paniscus)

decorator (Seyrig, 1927, Paniscus)

Distribution: England

Notes: Previous records of ornata in Britain were probably based on misidentifications of inedita but there are specimens of the true ornata in BMNH (Broad \& Shaw, in prep.). Netelia longipes has been treated as a separate species by Tolkanitz (1974) and Kasparyan and Tolkanitz (2000) but as a synonym of ornata by Delrio (1975). The type of longipes is very similar to material corresponding to ornata (Broad \& Shaw, in press).

Netelia (Paropheltes) tarsata (Brischke, 1880)

Nomenclature:

Paniscus tarsatus Brischke, 1880

Distribution: England, Scotland, Wales, Ireland

Subgenus Prosthodocis Enderlein, 1912

Netelia (Prosthodocis) sp. A

Distribution: England, Ireland

Notes: added by Broad \& Shaw (in prep.)

Netelia (Prosthodocis) sp. B

Distribution: Scotland, Ireland

Notes: added by Broad \& Shaw (in prep.)

Genus Phytodietus Gravenhorst, 1829

Notes: Distribution data mainly from Kasparyan and Shaw (2008), also BMNH (det. A. Kostro-Ambroziak). 


\section{Subgenus Neuchorus Uchida, 1931}

Notes: species of Phytodietus (Neuchhorus) excluded from the British and Irish list:

[obscurus (Ratzeburg, 1852, Lissonota); syn. rufipes Holmgren, 1860 (Horstmann 1998b); orbitalis Ulbricht, 1911 unavailable] Kasparyan and Shaw (2008) do not list any British or Irish specimens of obscurus and English specimens in BMNH, det. J.F. Perkins, are actually elongator.

Phytodietus (Neuchorus) elongator Aubert, 1963

Nomenclature:

iassiensis Constantineanu, 1929 invalid

Distribution: England

Notes: added by Kasparyan and Shaw (2008)

Phytodietus (Neuchorus) maculator Kasparyan \& Shaw, 2008

Distribution: England

Notes: added by Kasparyan and Shaw (2008)

Subgenus Phytodietus Gravenhorst, 1829

Nomenclature:

PHYTODIAETUS Aggasiz, 1846

PHYTODIAETUS Morley, 1913

Phytodietus (Phytodietus) astutus Gravenhorst, 1829

Nomenclature:

obscurus Desvignes, 1856 preocc.

continuus Thomson, 1887 synonymy by Horstmann (1998b)

britannicus (Habermehl, 1923, Barytarbes)

Distribution: England, Scotland, Wales

Phytodietus (Phytodietus) basalis Kasparyan, 1993

Distribution: England, Scotland

Notes: added by Kasparyan and Shaw (2008) 
Phytodietus (Phytodietus) femoralis Holmgren, 1860

Distribution: Scotland

Notes: added by Kasparyan and Shaw (2008)

Phytodietus (Phytodietus) gelitorius (Thunberg, 1824)

Nomenclature:

Ichneumon gelitorius Thunberg, 1824

polyzonius (Thunberg, 1824, Ichneumon) preocc.

coryphaeus Gravenhorst, 1829

carinatus Hellén, 1915

coxator (Aubert, 1963, Lathrolestes)

Distribution: England, Scotland, Wales, Ireland

Phytodietus (Phytodietus) geniculatus Thomson, 1877

Distribution: England, Scotland, Wales, Ireland

Phytodietus (Phytodietus) griseanae Kerrich, 1962

Distribution: England, Scotland, Wales

Phytodietus (Phytodietus) montanus Tolkanitz, 1979

Distribution: England, Scotland, Wales, Ireland

Notes: added by Kasparyan and Shaw (2008)

Phytodietus (Phytodietus) ornatus Desvignes, 1856

Nomenclature:

rubricosus Thomson, 1877

rufipictus Brischke, 1880

pictus (Habermehl, 1923, Barytarbes) synonymy by Horstmann (2004b)

Distribution: England, Scotland, Wales, Ireland

Phytodietus (Phytodietus) polyzonias (Forster, 1771)

Nomenclature:

Ichneumon polyzonias Forster, 1771 
segmentator Gravenhorst, 1829

Distribution: England

Notes: some distribution data from Fitton (1976)

Phytodietus (Phytodietus) variegatus (Fonscolombe, 1854)

Nomenclature:

Lissonota variegata Fonscolombe, 1854

albipes Holmgren, 1856

Distribution: England, Scotland

Notes: added by Kasparyan and Shaw (2008)

Tribe SPHINCTINI Förster, 1869

Genus Sphinctus Gravenhorst, 1829

Sphinctus serotinus Gravenhorst, 1829

Distribution: England

Notes: Probably extinct in Britain (Shaw and Voogd 2016).

\section{Tribe TRYPHONINI Shuckard, 1840}

Nomenclature:

EXENTERINI Förster, 1869

CTENISCINI Thomson, 1883

GRYPOCENTRINI Townes \& Townes, 1949

Notes: Except where noted otherwise, distribution data from Kerrich (1952), Kasparyan (1973), Fitton (1976), Shaw and Kasparyan (2005) and the collections of the BMNH. Additional references are given. The Exenterini has usually been considered a separate tribe, the distinctive group of genera lacking hind tibial spurs (in Britain comprising Acrotomus, Cteniscus, Cycasis, Eridolius, Excavarus, Exenterus, Exyston, Kristotomus, Orthomiscus and Smicroplectus) were recognised as being a derived group of Tryphonini by Bennett (2015). 
Genus Acrotomus Holmgren, 1857

Nomenclature:

DELOTOMUS Förster, 1869

Acrotomus lucidulus (Gravenhorst, 1829)

Nomenclature:

Tryphon lucidulus Gravenhorst, 1829

sexcinctus (Gravenhorst, 1829, Tryphon)

auriculatus (Thomson, 1883, Delotomus)

Distribution: England, Scotland, Wales

Acrotomus succinctus (Gravenhorst, 1829)

Nomenclature:

Tryphon succinctus Gravenhorst, 1829

Distribution: England, Scotland, Wales, Ireland

Genus Cosmoconus Förster, 1869

Cosmoconus ceratophorus (Thomson, 1888)

Nomenclature:

Tryphon ceratophorus Thomson, 1888

Distribution: England, Scotland

Cosmoconus elongator (Fabricius, 1775)

Nomenclature:

Ichneumon elongator Fabricius, 1775

elliotti (Morley, 1911, Tryphon)

Distribution: England, Scotland, Ireland, Isle of Man

Cosmoconus meridionator Aubert, 1963

Distribution: England, Scotland

Notes: added by Shaw and Kasparyan (2005) 
Cosmoconus nigriventris Kasparyan, 1971

Distribution: England, Scotland

Notes: added by Shaw and Kasparyan (2005)

\section{Genus Cteniscus Haliday, 1832}

Nomenclature:

EUDIABORUS Kerrich, 1952

Cteniscus maculiventris (Ashmead, 1896)

Nomenclature:

Diaborus maculiventris Ashmead, 1896

Distribution: Scotland

Notes: Added by Kerrich (1952); British specimens belong to the subspecies boreoalpinus (Kerrich, 1952, Eudiaborus). Overlooked by Fitton (1978).

\section{Cteniscus nigrifrons (Thomson, 1883)}

Nomenclature:

Diaborus nigrifrons Thomson, 1883

Distribution: England

\section{Cteniscus pedatorius (Panzer, 1809)}

Nomenclature:

$$
\begin{aligned}
& \text { Bassus pedatorius Panzer, } 1809 \\
& \text { sexlituratus (Gravenhorst, 1829, Tryphon) } \\
& \text { filipalpis (Thomson, 1883, Diaborus) } \\
& \text { moravicus (Gregor, 1937, Diaborus) }
\end{aligned}
$$

Distribution: England, Scotland, Ireland

\section{Cteniscus scalaris (Gravenhorst, 1829)}

Nomenclature:

Tryphon scalaris Gravenhorst, 1829

pallitarsis (Thomson, 1883, Diaborus)

palliditarsis (Dalla Torre, 1901, Diaborus) 
Distribution: England, Scotland

Notes: some distribution data from Rahoo and Luff (1988)

\section{Genus Ctenochira Förster, 1855}

Nomenclature:

CTENACME Förster, 1869

GEMOPHAGA Förster, 1869

SCOPIORUS Förster, 1869

CTENACMUS Thomson, 1883

CTENACMA Schulz, 1906

EXOCHOBLASTUS Schmiedeknecht, 1912

SCOPIMENUS Roman, 1937

COELOPROSOPON Bauer, 1958

Notes: species of Ctenochira excluded from the British and Irish list:

[?breviseta (Ratzeburg, 1852, Pimpla); syn. aberrans (Ruthe, 1855, Tryphon) (Horstmann 2002a)] Although appearing on the last British list (Fitton 1978, as aberrans), this may have been on the basis of misidentifications of haemosterna ( $\mathrm{K}$. Horstmann, pers. comm.). Kasparyan and Tolkanitz (2000) interpreted aberrans as a synonym of haemosterna but K. Horstmann's interpretation is followed here, having revised the closely related species involved (Horstmann 2002a).

\section{Ctenochira angulata (Thomson, 1883)}

Nomenclature:

Polyblastus angulatus Thomson, 1883

Distribution: Scotland, Isle of Man

\section{Ctenochira angustata (Roman, 1909)}

Nomenclature:

Polyblastus angustatus Roman, 1909

\section{Ctenochira arcuata (Holmgren, 1857)}

\section{Nomenclature:}

Polyblastus arcuatus Holmgren, 1857

antennator Aubert, 1965 
Distribution: England

Notes: W. Ely has noted that many specimens under this name (including British?) are marginata sensu Kasparyan.

\section{Ctenochira ? gelida Kasparyan, 1973}

Distribution: Scotland

Notes: added by Shaw and Kasparyan (2005), who suggest that the British specimens, which differ slightly from Continental forms, may represent a separate species.

\section{Ctenochira genalis (Thomson, 1883)}

Nomenclature:

Polyblastus genalis Thomson, 1883

Distribution: England

Notes: Added by Kasparyan (1973); Kasparyan's inclusion of British specimens (coll. J.P. Brock) was overlooked by Fitton (1978) and by Shaw and Kasparyan (2005).

\section{Ctenochira gilvipes (Holmgren, 1857)}

Nomenclature:

albiventris (Brischke, 1892, Polyblastus)

Distribution: England, Scotland, Isle of Man

\section{Ctenochira grossa (Brischke, 1871)}

Nomenclature:

Polyblastus grossus Brischke, 1871

annulicornis (Giraud, 1872, Polyblastus)

Distribution: England, Scotland

Notes: Listed in Fitton (1978) (as a doubtfully placed species of Polyblastus) as annulicornis, it was subsequently recorded as Ctenochira grossa by Kasparyan and Tolkanitz (2000).

\section{Ctenochira haemosterna (Haliday, 1839)}

Nomenclature:

Tryphon haemosternus Haliday, 1839 senilis (Holmgren, 1857, Polyblastus) 
nigripalpis (Thomson, 1883, Polyblastus)

subrufa (Bridgman, 1887, Polysphincta) synonymy by Kasparyan and Tolkanitz (2000)

haematosterna (Dalla Torre, 1901, Polyblastus)

Distribution: England, Scotland, Ireland

Notes: Some distribution data from Horstmann (2002a); Shaw and Kasparyan (2005) listed aberrans as a junior synonym of haemosterna as they were unaware of Horstmann's (Horstmann 2002a) revised synonymy (see note under breviseta).

\section{Ctenochira marginata (Holmgren, 1857)}

Nomenclature:

Polyblastus marginatus Holmgren, 1857

fractigena (Heinrich, 1953, Scopiorus)

Distribution: England, Scotland, Wales, Isle of Man

\section{Ctenochira meridionator Aubert, 1969}

Distribution: England

Notes: Added by Shaw and Kasparyan (2005); listed as a subspecies of genalis in Yu and Horstmann (1997).

\section{Ctenochira pastoralis (Gravenhorst, 1829)}

Nomenclature:

Tryphon pastoralis Gravenhorst, 1829

mutabilis (Holmgren, 1857, Polyblastus)

nitidiventris (Holmgren, 1857, Polyblastus)

\section{Ctenochira pratensis (Gravenhorst, 1829)}

Nomenclature:

Tryphon pratensis Gravenhorst, 1829

Distribution: England 


\section{Ctenochira propinqua (Gravenhorst, 1829)}

Nomenclature:

Tryphon propinquus Gravenhorst, 1829

obscura (Stephens, 1835, Tryphon) synonymy by Kasparyan and Tolkanitz (2000)

caudata (Holmgren, 1856, Poyblastus)

Distribution: England, Scotland, Ireland

\section{Ctenochira romani (Pfankuch, 1925)}

Nomenclature:

Poyblastus romani Pfankuch, 1925

pygobarba (Roman, 1937, Scopimenus)

Distribution: England, Isle of Man

\section{Ctenochira rubranator Aubert, 1965}

Distribution: Scotland

Notes: Added by Shaw and Kasparyan (2005); listed as a subspecies of genalis in Yu and Horstmann (1997).

\section{Ctenochira rufipes (Gravenhorst, 1829)}

\section{Nomenclature:}

Tryphon rufipes Gravenhorst, 1829

anilis (Holmgren, 1857, Polyblastus)

glabella (Holmgren, 1857, Polyblastus)

limosa (Holmgren, 1857, Polyblastus)

mixta (Holmgren, 1857, Polyblastus)

nigella (Holmgren, 1857, Polyblastus)

praedator (Holmgren, 1857, Polyblastus)

holmgreni (Woldstedt, 1874, Polyblastus) preocc.

woldstedtii (Dalla Torre, 1901, Polyblastus)

Distribution: England, Scotland 


\section{Ctenochira sanguinatoria (Ratzeburg, 1852)}

Nomenclature:

Tryphon sanguinatorius Ratzeburg, 1852

Distribution: England

\section{Ctenochira sphaerocephala (Gravenhorst, 1829)}

Nomenclature:

Tryphon sphaerocephalus Gravenhorst, 1829

bisculpta (Gravenhorst, 1829, Tryphon)

trisculpta (Stephens, 1835, Tryphon)

bifasciata (Zetterstedt, 1838, Tryphon)

trisculpta (Holmgren, 1856, Polyblastus)

Distribution: England, Scotland, Isle of Man

\section{Ctenochira validicornis (Brischke, 1871)}

Nomenclature:

Polyblastus validicornis Brischke, 1871

added by

fusicornis (Thomson, 1883, Polyblastus)

insculpta (Habermehl, 1922, Polyblastus)

Distribution: England, Scotland, Ireland

Notes: Added by Shaw and Kasparyan (2005); listed as a subspecies of genalis in Yu and Horstmann (1997).

\section{Ctenochira xanthopyga (Holmgren, 1857)}

Nomenclature:

Polyblastus xanthopygus Holmgren, 1857

rivalis (Holmgren, 1857, Polyblastus)

Distribution: England, Scotland 


\section{Genus Cycasis Townes, 1965}

\section{Cycasis rubiginosa (Gravenhorst, 1829)}

Nomenclature:

Tryphon rubiginosus Gravenhorst, 1829

insidiator (Holmgren, 1857, Acrotomus)

morio (Holmgren, 1857, Exenterus)

binotata (Thomson, 1883, Delotomus)

parvula (Thomson, 1883, Delotomus)

Distribution: England, Scotland

\section{Genus Dyspetes Förster, 1869}

Nomenclature:

DYSPETUS Thomson, 1883

\section{Dyspetes arrogator Heinrich, 1949}

Nomenclature:

rufatus Gregor, 1929 unavailable (Horstmann 2005a)

Distribution: England, Ireland

\section{Dyspetes luteomarginatus Habermehl, 1925}

\section{Nomenclature:}

chrysogaster (Gmelin, 1790, Ichneumon) preocc., synonymy by Horstmann (2006a)

praerogator (Thomson, 1883, Dyspetus) unavailable (Horstmann 2005a)

fracticeps (Townes \& Townes, 1950, Dyspetus) synonymy by Horstmann (2006a)

Distribution: England, Scotland, Wales, Ireland, Isle of Man

Notes: NMS, det. Horstmann, BMNH, det. Broad, added here; specimens of Dyspetes in Britain and Ireland have usually been treated as one species (named praerogator or arrogator) but Horstmann (2006a) demonstrated that the commoner species is actually luteomarginatus. The name praerogator was used by Gravenhorst and Thomson, referring to Linnaeus's praerogator, which is actually a species of Tranosemella (Campopleginae). These usages of praerogator are unavailable names (Horstmann 2005a, Horstmann 2006a). 


\section{Genus Eridolius Förster, 1869}

Nomenclature:

ANISOCTENION Förster, 1869

Eridolius alacer (Gravenhorst, 1829)

Nomenclature:

Tryphon alacer Gravenhorst, 1829

xanthopus (Holmgren, 1857, Acrotomus)

Distribution: England, Scotland

\section{Eridolius aurifluus (Haliday, 1839)}

Nomenclature:

Tryphon aurifluus Haliday, 1839

geniculosus (Schiødte, 1839, Exenterus)

approximatus (Holmgren, 1857, Exenterus)

Distribution: England, Ireland

\section{Eridolius basalis (Stephens, 1835)}

Nomenclature:

Tryphon basalis Stephens, 1835

connatus (Holmgren, 1857, Exenterus)

flavilabris (Holmgren, 1857, Exenterus)

gracilis (Holmgren, 1857, Exenterus)

hostilis (Holmgren, 1857, Exenterus)

limbatellus (Holmgren, 1857, Exenterus)

umbellatarum (Woldstedt, 1874, Exenterus)

rufofasciatus (Strobl, 1903, Polyblastus) synonymy by Horstmann (2012a)

minutulus (Pfankuch, 1907, Cteniscus)

Distribution: England, Scotland, Wales, Ireland, Isle of Man 
Eridolius bimaculatus (Holmgren, 1856)

Nomenclature:

Exenterus bimaculatus Holmgren, 1856

zonellus (Holmgren, 1857, Exenterus)

alpinus (Roman, 1909, Cteniscus)

Distribution: England, Scotland, Wales, Ireland

Eridolius consobrinus (Holmgren, 1857)

Nomenclature:

Exenterus consobrinus Holmgren, 1857

Distribution: Ireland

Eridolius curtisii (Haliday, 1839)

Nomenclature:

Tryphon curtisii Haliday, 1839

Distribution: England, Scotland, Ireland

Eridolius dorsator (Thunberg, 1824)

Nomenclature:

Ichneumon dorsator Thunberg, 1824

mitigosus (Gravenhorst, 1829, Tryphon)

lineola (Stephens, 1835, Tryphon)

similatorius (Schiødte, 1839, Exenterus)

limbatus (Holmgren, 1856, Exenterus)

alpicola (Holmgren, 1857, Exenterus)

borealis (Holmgren, 1857, Exenterus)

frigidus (Holmgren, 1857, Exenterus)

brevigena (Thomson, 1883, Cteniscus)

punctipes (Thomson, 1883, Cteniscus)

punctipleuris (Thomson, 1883, Cteniscus)

signifer (Thomson, 1883, Cteniscus)

albicollis (Habermehl, 1925, Cteniscus)

Distribution: England, Ireland 
Eridolius elegans (Stephens, 1835)

Nomenclature:

Tryphon elegans Stephens, 1835

aulicus (Roman, 1914, Cteniscus)

Distribution: England, Scotland

Eridolius ermolenkoi Kasparyan, 1990

Distribution: England

Notes: added by Shaw and Kasparyan (2005)

Eridolius flavomaculatus (Gravenhorst, 1829)

Nomenclature:

Tryphon flavomaculatus Gravenhorst, 1829

praeustus (Holmgren, 1857, Exenterus)

pumilus (Holmgren, 1857, Exenterus)

ustulatus (Holmgren, 1857, Exenterus)

quadrinotatus (Thomson, 1883, Cteniscus)

t-nigrum (Thomson, 1883, Cteniscus)

facialis (Roman, 1913, Cteniscus)

Distribution: England, Scotland

Eridolius gnathoxanthus (Gravenhorst, 1829)

Nomenclature:

Tryphon gnathoxanthus Gravenhorst, 1829

hachfeldi (Ulbricht, 1926, Polyblastus)

Distribution: England, Scotland, Ireland

Eridolius hofferi (Gregor, 1937)

Nomenclature:

Cteniscus hofferi Gregor, 1937

Distribution: England, Ireland 
Eridolius lineiger (Thomson, 1883)

Nomenclature:

Cteniscus lineiger Thomson, 1883

nordstromi (Kerrich, 1952, Cteniscus)

Distribution: Scotland

Notes: added by Shaw and Kasparyan (2005)

Eridolius pachysoma (Stephens, 1835)

Nomenclature:

Tryphon pachysoma Stephens, 1835

colorator (Zetterstedt, 1838, Tryphon)

Distribution: England, Ireland

Eridolius pictus (Gravenhorst, 1829)

Nomenclature:

Tryphon pictus Gravenhorst, 1829

marginatus (Thomson, 1833, Cteniscus)

crassiceps (Szépligeti, 1901, Diaborus)

Distribution: England, Scotland, Wales, Ireland, Isle of Man

Eridolius romani (Kerrich, 1952)

Nomenclature:

Cteniscus romani Kerrich, 1952

Distribution: Ireland

Eridolius rufilabris (Holmgren, 1857)

Nomenclature:

Exenterus rufilabris Holmgren, 1857

genalis (Thomson, 1883, Cteniscus)

Distribution: England, Ireland

Notes: some distribution data from Kerrich (1962) 
Eridolius rufonotatus (Holmgren, 1857)

Nomenclature:

Exenterus rufonotatus Holmgren, 1857

breviventris (Thomson, 1883, Cteniscus)

fulvipes (Kriechbaumer, 1896, Exenterus)

Distribution: Scotland, Ireland

Eridolius similis (Holmgren, 1857)

Nomenclature:

Exenterus similis Holmgren, 1857

Distribution: England

Notes: added by Ely (2010)

Eridolius taigensis Kasparyan, 1985

Distribution: Scotland

Notes: added by Shaw and Kasparyan (2005)

Genus Erromenus Holmgren, 1857

Nomenclature:

ANIAROPHRON Förster, 1869

TRICHOCALYMMA Förster, 1869

TRICHOCALYMMUS Thomson, 1887

Erromenus analis Brischke, 1871

Distribution: England, Scotland

Erromenus bibulus Kasparyan, 1973

Distribution: England, Scotland, Wales

Erromenus brunnicans (Gravenhorst, 1829)

Nomenclature:

Tryphon brunnicans Gravenhorst, 1829

brunicans Dalla Torre, 1901 preocc. 
Distribution: England, Scotland

\section{Erromenus calcator (Müller, 1776)}

Nomenclature:

Ichneumon calcator Müller, 1776

erythropus (Gmelin, 1790, Ichneumon)

carinatus (Holmgren, 1857, Polyblastus)

oelandicus (Holmgren, 1857, Polyblastus)

scutellaris (Holmgren, 1857, Polyblastus)

Distribution: England, Scotland

\section{Erromenus junior (Thunberg, 1824)}

Nomenclature:

Ichneumon junior Thunberg, 1824

frenator (Gravenhorst, 1829, Exochus)

arenicola Thomson, 1883

Distribution: England, Scotland

Erromenus plebejus (Woldstedt, 1878)

Nomenclature:

Trichocalymma plebejus Woldstedt, 1878

bipunctatus (Woldstedt, 1878, Trichocalymma)

brevitarsis Thomson, 1883

Distribution: England

\section{Erromenus punctatus (Woldstedt, 1878)}

Nomenclature:

Trichocalymma punctatus Woldstedt, 1878

added by

simplex Thomson, 1883

defectivus Strobl, 1903

Distribution: Scotland, Wales

Notes: added by Shaw and Kasparyan (2005) 
Erromenus punctulatus Holmgren, 1857

Nomenclature:

niger (Szépligeti, 1901, Aniarophron)

Distribution: England, Scotland, Ireland

Erromenus zonarius (Gravenhorst, 1820)

Nomenclature:

Ichneumon zonarius Gravenhorst, 1820

obscuratus Habermehl, 1925

Distribution: England, Scotland, Wales

Genus Excavarus Davis, 1897

Excavarus apiarius (Gravenhorst, 1829)

Nomenclature:

Tryphon apiarius Gravenhorst, 1829

obscuratorius (Panzer, 1809, Ichneumon) preocc.

Distribution: England, Wales

\section{Genus Exenterus Hartig, 1837}

Nomenclature:

ACTENONYX Förster, 1869

PICROSCOPUS Förster, 1869

Notes: species of Exenterus excluded from the British and Irish list:

[confusus Kerrich, 1952]

[tricolor Roman, 1913]

[vellicatus Cushman, 1940]

Released into Wales from Austrian stock, no evidence of successful establishment (Billany et al. 1983).

Doubtfully placed species of Exenterus: 
[anceps (Stephens, 1835, Tryphon) nom. dub., from England] Listed as a doubtfully placed species of Tryphon by Fitton (1978), placed here in Exenterus following Yu and Horstmann (1997).

\section{Exenterus abruptorius (Thunberg, 1824)}

\section{Nomenclature:}

Ichneumon abruptorius Thunberg, 1824

cingulatorius Holmgren, 1857

Distribution: England, Scotland

\section{Exenterus adspersus Hartig, 1838}

Nomenclature:

lepidus Holmgren, 1857

laricinus Thomson, 1888

Distribution: England

\section{Exenterus amictorius (Panzer, 1801)}

Nomenclature:

Ichneumon amictorius Panzer, 1801

marginatorius (Fabricius, 1793, Ichneumon) preocc.

sulcatorius (Thunberg, 1824, Ichneumon)

claripennis Thomson, 1883

Distribution: England

\section{Exenterus ictericus (Gravenhorst, 1829)}

\section{Nomenclature:}

Tryphon ictericus Gravenhorst, 1829

Distribution: Scotland

Notes: added by Shaw and Kasparyan (2005)

\section{Exenterus oriolus Hartig, 1838}

\section{Nomenclature:}

flavellus Thomson, 1883

brunnescens Fahringer, 1941 
Distribution: England

Genus Exyston Schiødte, 1839

Nomenclature:

ANECPHYSIS Förster, 1869

DIABORUS Förster, 1869

TRICAMPTUS Förster, 1869

PAREXYSTON Kerrich, 1952

Exyston calcaratus Thomson, 1883

Distribution: England, Ireland

Exyston pratorum (Woldstedt, 1874)

Nomenclature:

Exenterus pratorum Woldstedt, 1874

sedulus (Woldstedt, 1878, Cteniscus)

brevipetiolatus Thomson, 1883

melanurus Ulbricht, 1926 unavailable

Distribution: England, Scotland, Ireland

Exyston sponsorius (Fabricius, 1781)

Nomenclature:

Ichneumon sponsorius Fabricius, 1781

cinctulus (Gravenhorst, 1820, Ichneumon)

conopsator (Thunberg, 1824, Ichneumon)

carinatus Thomson, 1883

Distribution: England, Scotland, Wales, Ireland

Exyston subnitidus (Gravenhorst, 1829)

Nomenclature:

Tryphon subnitidus Gravenhorst, 1829

phaeorrhaeus (Haliday, 1839, Tryphon)

Distribution: England, Ireland 
Genus Grypocentrus Ruthe, 1855

Nomenclature:

APIMELES Förster, 1869

Grypocentrus albipes Ruthe, 1855

Distribution: England, Scotland

Grypocentrus apicalis Thomson, 1883

Grypocentrus basalis Ruthe, 1855

Distribution: England, Scotland

Grypocentrus bilobus Kasparyan, 1976

Distribution: Scotland

Notes: added by Shaw and Kasparyan (2005)

Grypocentrus cinctellus Ruthe, 1855

Distribution: England

Grypocentrus incisulus Ruthe, 1855

Nomenclature:

erythrurus Ulbricht, 1926

Genus Kristotomus Mason, 1962

Kristotomus laetus (Gravenhorst, 1829)

Nomenclature:

Mesoleptus laetus Gravenhorst, 1829

cephalotes (Gravenhorst, 1829, Tryphon)

orbitatorius (Schiødte, 1839, Exenterus)

calcaratus (Thomson, 1883, Delotomus)

marginatus (Thomson, 1883, Delotomus)

dioszeghyi (Kiss, 1924, Cteniscus) synonymy by Horstmann (2009d)

Distribution: England, Ireland 
Kristotomus laticeps (Gravenhorst, 1829)

Nomenclature:

Tryphon laticeps Gravenhorst, 1829

Distribution: England

Kristotomus pumilio (Holmgren, 1857)

Nomenclature:

Exenterus pumilio Holmgren, 1857

Distribution: England

Kristotomus ridibundus (Gravenhorst, 1829)

Nomenclature:

Tryphon ridibundus Gravenhorst, 1829

Distribution: England

Kristotomus triangulatorius (Gravenhorst, 1829)

Nomenclature:

Tryphon triangulatorius Gravenhorst, 1829

mesoleptoides (Stephens, 1835, Tryphon)

coarctatus (Holmgren, 1857, Acrotomus)

Distribution: England, Scotland, Ireland

\section{Genus Monoblastus Hartig, 1837}

Nomenclature:

COELOCONUS Förster, 1869

XIPHURUS Kriechbaumer, 1896 preocc., synonymy by Horstmann (2002a)

IDOTHRICHUS Schmiedeknecht, 1907

PSEUDOPSILOSAGE Gregor, 1929

Monoblastus brachyacanthus (Gmelin, 1790)

Nomenclature:

Ichneumon brachyacanthus Gmelin, 1790

testaceus (Gmelin, 1790, Ichneumon) 
oraniensis (Schmiedeknecht, 1912, Psilosage)

Distribution: England, Scotland, Ireland

\section{Monoblastus caudatus (Hartig, 1837)}

Nomenclature:

Tryphon caudatus Hartig, 1837

lateralis (Giraud, 1872, Tryphon) preocc.

sericeus (Brischke, 1892, Phaestus)

lateralis Kriechbaumer, 1896 preocc.

Distribution: England

Notes: BMNH, det. Broad, added here

Monoblastus marginellus (Gravenhorst, 1829)

Nomenclature:

Lissonota marginella Gravenhorst, 1829

compunctor misident.

Distribution: England

Genus Neleges Förster, 1869

Nomenclature:

ANELPISTUS Brauns, 1898 preocc.

BRAUNSIANUS Berg, 1898

Neleges proditor (Gravenhorst, 1829)

Nomenclature:

Tryphon proditor Gravenhorst, 1829

bidentatus (Brauns, 1898, Anelpistus)

bidentatus (Strobl, 1903, Tryphon) preocc.

bimucronatus (Strobl, 1903, Erromenus)

Distribution: England 
Genus Orthomiscus Mason, 1955

Orthomiscus unicinctus (Holmgren, 1857)

Nomenclature:

Exenterus unicinctus Holmgren, 1857

macrocephalus (Holmgren, 1857, Exenterus)

Distribution: England

Genus Otoblastus Förster, 1869

Otoblastus luteomarginatus (Gravenhorst, 1829)

Nomenclature:

Tryphon luteomarginatus Gravenhorst, 1829

Distribution: England

\section{Genus Polyblastus Hartig, 1837}

Notes: doubtfully placed species of Polyblastus:

[bridgmani Parfitt, 1882 nom. dub., from England] Fitton (1976)

[parvulus (Gravenhorst, 1829, Tryphon) nom. dub.]

Subgenus Cophencus Townes \& Townes, 1949

Polyblastus (Cophencus) macrocentrus Thomson, 1888

Distribution: England, Scotland, Wales

Subgenus Labroctonus Förster, 1869

Nomenclature:

NEMIOBLASTUS Thomson, 1883

Polyblastus (Labroctonus) alternans Schiødte, 1838

Nomenclature:

albicoxa Thomson, 1883

Notes: British specimens without locality data in BMNH. 
Polyblastus (Labroctonus) melanostigmus Holmgren, 1857

Nomenclature:

grammicus Holmgren, 1857

lucidus Brischke, 1892

Distribution: England, Scotland, Isle of Man

Polyblastus (Labroctonus) nanus Kasparyan, 1973

Distribution: England

Notes: added by Shaw and Kasparyan (2005)

Polyblastus (Labroctonus) pallicoxa Thomson, 1888

Nomenclature:

pallidicoxa Dalla Torre, 1901

Distribution: England, Ireland, Isle of Man

Polyblastus (Labroctonus) stenocentrus Holmgren, 1857

Nomenclature:

albicoxa Thomson, 1883

Distribution: England, Scotland, Isle of Man

Notes: Shaw and Kasparyan (2005) treat this as species separate from alternans

Schiødte, 1838, of which it is listed as a subspecies by Yu and Horstmann (1997).

Polyblastus (Labroctonus) westringi Holmgren, 1857

Distribution: England, Scotland

Subgenus Polyblastus Hartig, 1837

Polyblastus (Polyblastus) cancer (Hartig, 1837)

Nomenclature:

Tryphon cancer Hartig, 1837

palaemon Schiødte, 1838

holosericeus (Ratzeburg, 1848, Tryphon)

pyramidatus Holmgren, 1857 
Distribution: England, Scotland, Isle of Man

Polyblastus (Polyblastus) cothurnatus (Gravenhorst, 1829)

Nomenclature:

Tryphon cothurnatus Gravenhorst, 1829

drewseni Schiødte, 1838

unicinctus Bridgman, 1889

intermedius Ulbricht, 1916 unavailable

hungaricus (Kiss, 1926, Trematopygus)

Distribution: England, Scotland

Polyblastus (Polyblastus) pedalis (Cresson, 1864)

Nomenclature:

Tryphon pedalis Cresson, 1864

carbonarius misident.

rhenanus Ulbricht, 1926 unavailable

rufifemur Hedwig, 1943 unavailable

Distribution: England, Scotland

Notes: British specimens belong to the subspecies carbonator Kasparyan, 1970.

Polyblastus (Polyblastus) pinguis (Gravenhorst, 1920)

Nomenclature:

Ichneumon pinguis Gravenhorst, 1920

petryi Schmiedeknecht, 1912

Polyblastus (Polyblastus) subalpinus Holmgren, 1857

Distribution: Scotland

Polyblastus (Polyblastus) tener Habermehl, 1909

Distribution: England, Scotland, Wales 
Polyblastus (Polyblastus) tuberculatus Teunissen, 1953

Distribution: England

Notes: added by Shaw and Kasparyan (2005)

Polyblastus (Polyblastus) varitarsus (Gravenhorst, 1829)

Nomenclature:

Tryphon varitarsus Gravenhorst, 1829

strobilator misident.

albovinctus (Gravenhorst, 1829, Tryphon)

affinis Woldstedt, 1874

subtilis Thomson, 1883

variitarsus Dalla Torre, 1901 preocc.

rufus Kiss, 1926

Distribution: England, Scotland, Wales, Ireland, Isle of Man

Polyblastus (Polyblastus) wahlbergi Holmgren, 1857

Nomenclature:

wesmaeli Holmgren, 1857

Distribution: England, Scotland, Ireland

Genus Smicroplectrus Thomson, 1883

Nomenclature:

MICROPLECTRON Förster, 1869 preocc.

Smicroplectrus bohemani (Holmgren, 1857)

Nomenclature:

Exenterus bohemani Holmgren, 1857

Distribution: England

Smicroplectrus erosus (Holmgren, 1857)

Nomenclature:

Exenterus erosus Holmgren, 1857

Distribution: England 
Smicroplectrus excisus Kerrich, 1952

Distribution: England

Notes: some distribution data from Kerrich (1962)

Smicroplectrus heinrichi Kerrich, 1952

Distribution: Scotland, Ireland

Smicroplectrus jucundus (Holmgren, 1857)

Nomenclature:

Exenterus jucundus Holmgren, 1857

Distribution: England

Smicroplectrus nigricornis Kasparyan, 1976

Distribution: England, Scotland

Notes: added by Shaw and Kasparyan (2005)

Smicroplectrus perkinsorum Kerrich, 1952

Distribution: England

Smicroplectrus quinquecinctus (Gravenhorst, 1820)

Nomenclature:

Ichneumon quinquecinctus Gravenhorst, 1820

trianguligena Kerrich, 1952

Distribution: England, Scotland

\section{Genus Tryphon Fallén, 1813}

Notes: Distribution data from Fitton (1975) and Shaw and Kasparyan (2005).

Doubtfully placed species of Tryphon (Fitton 1976):

[flavilabris Stephens, 1835 nom. dub., from England]

[thoracicus Stephens, 1835 nom. dub., from England]

[zonatus Stephens, 1835 nom. dub., from England] 
Subgenus Stenocrotaphon Kasparyan, 1969

Tryphon (Stenocrotaphon) obtusator (Thunberg, 1824)

Nomenclature:

Ichneumon obtusator Thunberg, 1824

?subrufus (Gmelin, 1790, Ichneumon)

consobrinus Holmgren, 1857

Distribution: England

Tryphon (Stenocrotaphon) subsulcatus Holmgren, 1857

Distribution: England

\section{Subgenus Symboethus Förster, 1869}

Tryphon (Symboethus) bidentatus Stephens, 1835

Nomenclature:

incestus Holmgren, 1857

incertus Brischke, 1871

tricolor Rudow, 1910 preocc.

abnormis Habermehl, 1925

rufescens (Kiss, 1926, Erromenus)

Distribution: England, Scotland, Wales

Notes: Shaw and Kasparyan (2005) list the vice counties separately for the two forms of bidentatus, the typical form and f. rufifemur Kasparyan, the latter only recorded from Scotland and possibly belonging to a separate species, T. hinzi (Heinrich, 1953, Symboethus) (but see the note regarding Tryphon duplicatus).

Tryphon (Symboethus) brunniventris Gravenhorst, 1829

Distribution: England, Scotland

Tryphon (Symboethus) duplicatus (Heinrich, 1953)

Nomenclature:

Symboethus duplicatus Heinrich, 1953

discedens (Heinrich, 1953, Symboethus) 
Distribution: England, Scotland

Notes: H.K. Townes (pers. comm. to M.G. Fitton) regarded duplicatus as a junior synonym of exclamationis, the correct name for the species here called duplicatus being Tryphon hinzi (Heinrich, 1953). Townes also regarded 'duplicatus' (=hinzi sensu Townes) as comprising two species, one with red hind femora and one with black hind femora, also differing in characteristics of the egg. However, Shaw and Kasparyan (2005) regard a form of bidentatus with red hind femora as possibly being synonymous with hinzi. Further clarification is needed.

\section{Tryphon (Symboethus) exclamationis Gravenhorst, 1829}

\section{Nomenclature:}

connectens Roman, 1909

Distribution: England

Tryphon (Symboethus) fulviventris Holmgren, 1857

Distribution: England, Scotland

Notes: Added by Kasparyan (1973), whose inclusion of British specimens was overlooked by Fitton (Fitton 1975, Fitton 1978).

Tryphon (Symboethus) heliophilus Gravenhorst, 1829

Nomenclature:

bicornutus Holmgren, 1856

confinis Holmgren, 1856

maculatus (Pfankuch, 1924, Symboethus) unavailable

Distribution: England

\section{Subgenus Tryphon Fallén, 1813}

Nomenclature:

OTITOCHILUS Förster, 1869

PSILOSAGE Förster, 1869

Tryphon (Tryphon) abditus Kasparyan, 1969

Nomenclature:

pleuralis Thomson, 1883 preocc.

Distribution: England, Scotland 
Tryphon (Tryphon) atriceps Stephens, 1835

Nomenclature:

ephippium Holmgren, 1857

Distribution: England, Wales

Tryphon (Tryphon) bidentulus Thomson, 1883

Nomenclature:

separandus Schmiedeknecht, 1912

Distribution: England

Tryphon (Tryphon) latrator (Fabricius, 1781)

Nomenclature:

Ichneumon latrator Fabricius, 1781

auricularis Thomson, 1883 synonymy by Horstmann (2001a)

Distribution: England, Scotland, Wales, Ireland, Isle of Man

Tryphon (Tryphon) nigripes Holmgren, 1857

Distribution: England

Tryphon (Tryphon) relator (Thunberg, 1824)

Nomenclature:

Ichneumon relator Thunberg, 1824

vulgaris Holmgren, 1857

erythrogaster Thomson, 1883

Distribution: England, Scotland

Tryphon (Tryphon) rutilator (Linnaeus, 1761)

Nomenclature:

Ichneumon rutilator Linnaeus, 1761

impraegnator (Schrank, 1781, Ichneumon)

cepae (Geoffroy, 1785, Ichneumon)

anodon (Schrank, 1802, Ichneumon)

ceparum (Schrank, 1802, Ichneumon) 
insultator (Gravenhorst, 1807, Ichneumon)

quadratus Stephens, 1835

Distribution: England, Scotland, Wales

Tryphon (Tryphon) signator Gravenhorst, 1829

Nomenclature:

facialis Stephens, 1835

nigrifacies Ulbricht, 1926 unavailable

Distribution: England, Scotland, Wales, Isle of Man

Tryphon (Tryphon) thomsoni Roman, 1939

Distribution: England, Scotland, Wales

Tryphon (Tryphon) trochanteratus Holmgren, 1857

Distribution: England, Scotland, Wales, Isle of Man

\section{Xoridinae}

Subfamily XORIDINAE Shuckard, 1840

Notes: Some distribution data from Gauld and Fitton (1981).

Genus Ischnoceros Gravenhorst, 1829

Nomenclature:

MITROBORIS Holmgren, 1859

Ischnoceros caligatus (Gravenhorst, 1829)

Nomenclature:

Xylonomus caligatus Gravenhorst, 1829

seticornis Kriechbaumer, 1879

Distribution: England, Scotland, Ireland 
Ischnoceros rusticus (Geoffroy, 1785)

Nomenclature:

Ichneumon rusticus Geoffroy, 1785

striatus (Brullé, 1846, Odontomerus)

cornutus (Ratzeburg, 1848, Xorides)

filicornis Kriechbaumer, 1879

caper (Hedwig, 1957, Eclytus)

Distribution: England, Scotland, Wales, Ireland

\section{Genus Odontocolon Cushman, 1942}

Nomenclature:

ODONTOMERUS Gravenhorst, 1829 preocc.

\section{Odontocolon dentipes (Gmelin, 1790)}

Nomenclature:

Ichneumon dentipes Gmelin, 1790

femoratum (Olivier, 1811, Ophion)

pinetorum (Thomson, 1877, Odontomerus)

Distribution: England, Scotland, Wales, Ireland

\section{Odontocolon quercinum (Thomson, 1877)}

Nomenclature:

Odontomerus quercinus Thomson, 1877

liogaster (Szépligeti, 1914, Odontomerus)

simile (Habermehl, 1920, Odontomerus)

brunneiventre (Telenga, 1930, Odontomerus)

Distribution: England

Genus Xorides Latreille, 1809

Nomenclature:

XYLONOMUS Gravenhorst, 1829

GONOPHONUS Förster, 1869

MOEROPHORA Förster, 1869 
RHADINA Förster, 1869 preocc.

SICHELIA Förster, 1869

STEROTRICHUS Förster, 1869

RHADINOPIMPLA Schulz, 1911

NEOXYLONOMUS Clément, 1938

XYLONOMINUS Clément, 1938

Notes: The British species have usually been separated into the subgenera Moerophora (containing rufipes and rusticus) and Xorides s. str. (containing the others) but Wahl (1997) has placed the subgenera of Xorides in synonymy.

\section{Xorides brachylabis (Kriechbaumer, 1889)}

\section{Nomenclature:}

Xylonomus brachylabis Kriechbaumer, 1889

brachylabris misspelling

Distribution: England

\section{Xorides csikii Clément, 1938}

Distribution: England

\section{Xorides fuligator (Thunberg, 1824)}

\section{Nomenclature:}

Ichneumon fuligator Thunberg, 1824

sordator (Thunberg, 1824, Ichneumon)

pilicornis (Gravenhorst, 1829, Xylonomus)

Distribution: England, Wales

\section{Xorides gravenhorstii (Curtis, 1831)}

\section{Nomenclature:}

Xylonomus gravenhorstii Curtis, 1831

securicornis (Holmgren, 1860, Xylonomus)

glyptus (Thomson, 1877, Xylonomus)

clavicornis (Kriechbaumer, 1879, Xylonomus)

distinguendus (Magretti, 1884, Xylonomus)

hungaricus (Szépligeti, 1899, Sichelia) 
seticornis (Strobl, 1902, Xylonomus)

rufoscutellatus (Habermehl, 1918, Xylonomus)

kokujevi (Meyer, 1922, Xylonomus)

rufus (Kiss, 1924, Xylonomus)

caucasicus (Shestakov, 1925, Xylonomus)

romani Clément, 1938

Distribution: England, Wales, Ireland

Xorides irrigator (Fabricius, 1793)

Nomenclature:

Ichneumon irrigator Fabricius, 1793

planus Šedivý, 1958

Distribution: England

\section{Xorides niger (Pfeffer, 1913)}

Nomenclature:

Xylonomus niger Pfeffer, 1913

bicolor Clément, 1938

Distribution: England

\section{Xorides praecatorius (Fabricius, 1793)}

Nomenclature:

Ichneumon praecatorius Fabricius, 1793

?articulatus (Geoffroy, 1785, Ichneumon)

?falsatorius (Olivier, 1792, Ichneumon)

parvulus (Gravenhorst, 1829, Xylonomus)

praecatorius (Marshall, 1872, Xylonomus) preocc.

rufopictus (Kiss, 1926, Xylonomus)

ruficoxis (Kiss, 1929, Xylonomus) preocc.

temporalis (Kiss, 1929, Xylonomus)

holsaticus Heinrich, 1951

Distribution: England, Scotland 


\section{Xorides rufipes (Gravenhorst, 1829)}

Nomenclature:

Xylonomus rufipes Gravenhorst, 1829

Distribution: England

\section{Xorides rusticus (Desvignes, 1856)}

\section{Nomenclature:}

Xylonomus rusticus Desvignes, 1856

Distribution: England

\section{Acknowledgements}

Jim Brock, Mike Fitton, the late Klaus Horstmann, Heinz Schnee and Mark Shaw have all been of great help in maintaining the accuracy of the list and in providing the encouragement to actually finish it. Klaus Horstmann and Kees Zwakhals bravely checked drafts of the entire list and provided many useful comments. Tony Hunter (World Museum Liverpool) kindly provided several unpublished Welsh records. Natalie Dale-Skey transformed the original Word files into spreadsheets for import. I am grateful to the photographers who were happy for me to use their images to brighten up the checklist.

\section{References}

- Achterberg Cv (1999) The West Palaearctic species of the subfamily Paxylommatinae (Hymenoptera: Ichneumonidae), with special reference to the genus Hybrizon Fallén. Zoologische Mededelingen, Leiden 73 (2): 11-26.

- Aeschlimann J-P (1983) Note sur les Métopiines ouest-palaéarctiques, avec description de deux espèces nouvelles (Hymenoptera, Ichneumonidae). Annales de la Société Entomologique de France 19 (1): 3-6.

- $\quad$ Aeschlimann J-P (1989) Révision des espèces ouest-paléarctiques du genre Hypsicera Latreille (Hymenoptera : Ichneumonidae). Annales de la Société Entomologique de France 25: 33-39.

- $\quad$ Allen AA (2007) A record of Afrephialtes cicatricosus (Ratzeburg) (Hymenoptera: Ichneumonidae) from South Devon. British Journal of Entomology and Natural History 20: 45.

- Anderson A, Broad G, Baur H (2006) New Irish records of Ichneumonidae, Braconidae and Pteromalidae (Hymenoptera) collected from agricultural grasslands. Irish Naturalists Journal 28 (6): 246-248.

- $\quad$ anon. (2001) 14 December 2000. British Journal of Entomology and Natural History 14: 125. 
- $\quad$ Ashmole NP, Nelson JM, Shaw MR, Garside A (1983) Insects and spiders on snowfields in the Cairngorms, Scotland. Journal of Natural History 17: 599-613. DOI: 1 $0.1080 / 00222938300770491$

- $\quad$ Askew RR (2000) An aggregation of Parasitic Hymenoptera . Entomologist's Monthly Magazine 136: 147.

- $\quad$ Aubert J (1978) Les ichneumonides ouest-palearctiques et leurs hôtes 2. Banchinae et Suppl. aux Pimplinae . Laboratoire d'Evolution des Etres Organises, Paris \& EDIFATOPIDA, Echauffour, Paris, 318 pp.

- $\quad$ Aubert J (1985) Ichneumonides Scolobatinae des collections suédoises (suite) et du Musée de Léningrad. Bulletin de la Société Entomologique de Mulhouse 1985: 49-58.

- Aubert J (1988) Troisème prélude à une révision des Ichneumonides Scolobatinae: les Rhorus Foerst., du groupe de neustriae Schrk. Bulletin de la Société Entomologique de Mulhouse 1988: 1-10.

- $\quad$ Aubert J (1994) Supplément aux Ichneumonides Rhorus Foerst. (1). Bulletin de la Société Entomologique de Mulhouse 1994: 61-64.

- $\quad$ Aubert J (2000) Les ichneumonides ouest-paléartiques et leurs hôtes. 3 Scolobatinae (= Ctenopelmatinae) et supplement aux volumes précédents. Littererae Zoologicae, Actes du Musée cantonal de Zoologie, Lausanne 5: 1-310.

- $\quad$ Azidah AA, Fitton MG, Quicke DL (2000) Identification of the Diadegma species (Hymenoptera: Ichneumonidae, Campopleginae) attacking the diamondback moth, Plutella xylostella (Lepidoptera : Plutellidae). Bulletin of Entomological Research 90: 375-389.

- $\quad$ Barari H, Ferguson AW, Piper RW, Smith E, Quicke DL, Williams IH (2005) The separation of two hymenopteran parasitoids, Tersilochus obscurator and Tersilochus microgaster (Ichneumonidae), of stem-mining pests of winter oilseed rape using DNA, morphometric and ecological data. Bulletin of Entomological Research 95 (4): 299-307.

- Bass JA, Cooling DA (1983) As association between a muscid, an ichneumonid and a simuliid recorded from a reservoir outfall in southern England. Entomologist's Gazette 34: 125-127.

- Bauer R (2001) Bemerkungen über die Ichneumoniden der Alpen mit einigen Neubeschreibungen Teil III (Hymenoptera, Ichenumonidae, Ichneumoninae). Entomofauna 22 (12): 245-269.

- Beirne BP (1941) British species of Diplazonini (Bassini auctt.) with a study of the genital and postgenital abdominal sclerites in the male (Hym.: Ichneum.). Transactions of the Royal Entomological Society of London 91 (13): 661-712. DOI: $10.1111 /$ j.1365-2311.1941.tb01042.x

- $\quad$ Bennett AM (2001) Phylogeny of the Agriotypinae (Hymenoptera: Ichneumonidae), with comments on the subfamily relationships of the basal Ichneumonidae. Systematic Entomology 26 (3): 329-356. DOI: 10.1046/j.0307-6970.2001.00155.x

- Bennett AM (2015) Revision of the world genera of Tryphoninae (Hymenoptera: Ichneumonidae). Memoirs of the American Entomological Institute 86: 1-387.

- Bennett FD, Askew RR, Shaw MR (2002) A second rearing of Telepsogina adelognathi Hedqvist, 1958 (Hym., Pteromalidae, Miscogastrinae). Entomologist's Monthly Magazine 138: 59-61.

- $\quad$ Billany DJ, Carter CI, Winter TG, Fielding NJ (1983) The effects of climate and parasites on Gilpinia hercyniae (Hartig) (Hymenoptera: Diprionidae) in Britain. Entomologist's Monthly Magazine 119: 117-120. 
- Bordera S, González-Moreno A, Khalaim Al, Sääksjärvi IE (2014) Revision of North American species of Clistopyga (Hymenoptera: Ichneumonidae: Pimplinae). Canadian Entomologist 146 (4): 355-414. DOI: 10.4039/tce.2013.74

- Boston MR (1986) First Irish record of Neorhacodes enslini (Ruschka) (Hymenoptera: Ichneumonidae). Irish Naturalists' Journal 22 (3): 116.

- Boston MR, Nash R (1989) Twenty-one species of Hymenoptera (Ichneumonidae: Ichneumoninae) new to Ireland, with a list of ichneumonine species in the Ulster Museum, Belfast. Irish Naturalists' Journal 23 (4): 134-137.

- $\quad$ Broad GR (2004) Generic synonymies affecting the Orthocentrinae (Hym., Ichneumonidae), with notes on the composition of the subfamily. Entomologist's Monthly Magazine 140: 297-299.

- Broad GR (2010) Status of Batakomacrus Kolarov (Hymenoptera: Ichneumonidae: Orthocentrinae), with new generic combinations and description of a new species. Zootaxa 2394: 51-68.

- Broad GR (2014) Checklist of British and Irish Hymenoptera - Introduction. Biodiversity Data Journal 2: e1113. DOI: 10.3897/BDJ.2.e1113

- $\quad$ Broad GR, Davis AM (2015) Lymantrichneumon disparis (Poda, 1761) (Hymenoptera: Ichneumonidae), a genus and species new to Britain. Entomologist's Gazette 66: 216-218.

- $\quad$ Broad GR, Livermore L (2014a) Checklist of British and Irish Hymenoptera Ceraphronoidea . Biodiversity Data Journal 2: e1167. DOI: 10.3897/BDJ.2.e1167

- Broad GR, Livermore L (2014b) Checklist of British and Irish Hymenoptera - Evanioidea . Biodiversity Data Journal 2: e1116. DOI: 10.3897/BDJ.2.e1116

- $\quad$ Broad GR, Shaw MR (2005) The species of four genera of Metopiinae (Hymenoptera: Ichneumonidae) in Britain, with new host records and descriptions of four new species. Journal of Natural History 39 (26): 2389-2407. DOI: 10.1080/00222930500102074

- $\quad$ Broad GR, Shaw MR (2016) The British species of Enicospilus (Hymenoptera: Ichneumonidae: Ophioninae). European Journal of Taxonomy 187: 1-31. DOI: http:// dx.doi.org/10.5852/ejt.2016.187

- Brock JP (1982) A systematic study of the genus Ophion in Britain (Hymenoptera, Ichneumonidae). Tijdschrift voor Entomologie 125: 57-97.

- Brock JP, Shaw MR (1997) Perithous albicinctus (Gravenhorst), a large pimpline ichneumon-wasp new to Britain (Hymenoptera: Ichneumonidae). Entomologist's Gazette 48: 49-50.

- $\quad$ Burks RA (2012) Formation of family group names using the stem of -gaster, with special reference to names based on Miscogaster and Sphegigaster (Hymenoptera: Chalcidoidea: Pteromalidae). Zootaxa 3389: 61-64.

- $\quad$ Carr LA (1924) The Ichneumonidae of the Lichfield district, Staffordshire. Transactions of the North Staffordshire Field Club 58 (Appendix): 1-70.

- $\quad$ Cowin WS (1953) Entomological Notes. The Peregrine 2: 22-23.

- Cowin WS, Williamson K (1940) Manx entomological notes. The Entomologist 73: 67-68.

- Cox ML (2007) Atlas of the Seed and Leaf Beetles of Britain and Ireland. Pisces Publications, Newbury, $344 \mathrm{pp}$.

- Dasch C (1992) The ichneumon-flies of America north of Mexico. Pt. 12. Subfamilies Microleptinae, Helictinae, Cylloceriinae and Oxytorinae (Hymenoptera: Ichneumonidae). Memoirs of the American Entomological Institute 52: 1-470. 
- Delrio G (1975) Révision des espèces ouest-paléarctiques du genre Netelia Gray (Hym., Ichneumonidae). Studi Sassaresi sez. III.- Annali della Facolta di Agraria dell'Università di Sassari 23: 1-126.

- Diller E (1981) Bemerkungen zur Systematik der Phaeogenini mit einem vorläufigen Katalog der Gattungen (Hymenoptera, Ichneumonidae). Entomofauna 2: 93-111.

- Diller E (1982) Untersuchungen über Arten der Gattungen Diplazon Viereck, 1914, und Sussaba Cameron, 1909 (Hymenoptera, Ichneumonidae, Diplazontinae). Entomofauna 3 (6): 65-80.

- Diller E, Schönitzer K (2003) Revision einiger westpaläarktischer und nearktischer Arten der Gattung Colpognathus Wesmael, [1845] (Hymenoptera, Ichneumonidae, Ichneumoninae, Alomyini). Entomofauna 24 (24): 333-344.

- Diller E, Shaw MR (2014) Western Palaearctic Oedicephalini and Phaeogenini (Hymenoptera: Ichneumonidae, Ichneumoninae) in the National Museums of Scotland, with distributional data including 28 species new to Britain, rearing records, and descriptions of two new species of Aethecerus Wesmael and one of Diadromus Wesmael. Entomologist's Gazette 65: 109-129.

- Diller E, Tereshkin AM (2005) Neue Erkenntnisse zur Gattung Herpestomus Wesmael, [1845] mit Beschreibung neuer Arten (Hymenoptera, Ichneumonidae, Ichneumoninae, Phaeogenini). Entomofauna 26 (17): 305-312.

- Donisthorpe HS (1927) The Guests of British Ants: their habits and life-histories. George Routledge and Sons Ltd, London, $244 \mathrm{pp}$.

- Edgar WD (1971) Aspects of the ecology and energetics of the egg sac parasites of the wolf spider Pardosa lugubris (Walckenaer). Oecologia 7: 155-163. DOI: $10.1007 /$ BF00346357

- $\quad$ Ely WA (2001) First UK record of Adelognathus stelfoxi Fitton, Gauld \& Shaw (Hym.: Ichneumonidae). The Entomologist's Record and Journal of Variation 113: 140.

- $\quad$ Ely WA (2002) Tycherus nigridens (Wesmael, 1845) (Hym.: Ichneumonidae) new to Britain. The Entomologist's Record and Journal of Variation 114: 109.

- Ely WA (2010) Eridolius similis (Holmgren) (Hymenoptera, Ichneumonidae, Tryphoninae) at Blacktoft Sands NR. Yorkshire Naturalists' Union Bulletin 54: 33.

- $\quad$ Field JP, Foster WA (1988) The bees and wasps of Scolt Head Island national nature reserve, Norfolk. British Journal of Entomology and Natural History 1: 79-83.

- $\quad$ Fitton MG (1975) A review of the British species of Tryphon Fallén (Hym.,Ichneumonidae). Entomologist's Monthly Magazine 110: 153-171.

- $\quad$ Fitton MG (1976) The western palaearctic Ichneumonidae (Hymenoptera) of British authors. Bulletin of the British Museum (Natural History), Entomology 32 (8): 301-373.

- Fitton MG (1978) 14. Ichneumonidae . In: Fitton MG, Graham MWdV, Boucek ZR, Fergusson ND, Huddleston T, Quinlan J, Richards OW (Eds) Kloet and Hincks. A check list of British insects. Part 4: Hymenoptera . Handbooks for the Identification of British Insects 11, ix + 159 pp., 34 pp.

- $\quad$ Fitton MG (1981) The British Acaenitinae (Hymenoptera: Ichneumonidae). Entomologist's Gazette 32: 185-192.

- Fitton MG (1984) A review of the British Collyriinae, Eucerotinae, Stilbopinae and Neorhacodinae (Hymenoptera: Ichneumonidae). Entomologist's Gazette 35: 185-195.

- Fitton MG (1985a) The British species of Cidaphus (Hymenoptera: Ichneumonidae). Entomologist's Gazette 36: 293-297. 
- $\quad$ Fitton MG (1985b) The ichneumon-fly genus Banchus (Hymenoptera) in the Old World. Bulletin of the British Museum (Natural History) (Entomology) 51: 1-60.

- Fitton MG (1987) A review of the Banchus-group of ichneumon-flies, with a revision of the Australian genus Philogalleria (Hymenoptera: Ichneumonidae). Systematic Entomology 12: 33-45. DOI: 10.1111/j.1365-3113.1987.tb00545.x

- $\quad$ Fitton MG, Boston M (1988) The British species of Phthorima (Hymenoptera: Ichneumonidae). Entomologist's Gazette 39: 165-170.

- $\quad$ Fitton MG, Ficken L (1990) British ichneumon-flies of the tribe Oedemopsini (Hymenoptera: Ichneumonidae). The Entomologist 109: 200-214.

- $\quad$ Fitton MG, Gauld ID (1980) A review of the British Cremastinae (Hymenoptera: Ichneumonidae), with keys to the species. Entomologist's Gazette 31: 63-71.

- $\quad$ Fitton MG, Rotheray GE (1982) A key to the European genera of diplazontine ichneumon-flies, with notes on the British fauna. Systematic Entomology 7: 311-320.

DOI: 10.1111/j.1365-3113.1982.tb00448.x

- $\quad$ Fitton MG, Gauld ID, Shaw MR (1982) The taxonomy and biology of the British Adelognathinae (Hymenoptera: Ichneumonidae). Journal of Natural History 16: 275-283. DOI: $10.1080 / 00222938200770221$

- Fitton MG, Shaw MR, Gauld ID (1988) Pimpline Ichneumon-flies. Hymenoptera, Ichneumonidae (Pimplinae). Handbooks for the Identification of British Insects 7: 1-110.

- $\quad$ Formstone JB (1999) Two first records for Wales of pimpline ichneumon-flies (Hymenoptera: Ichneumonidae: Pimplinae). Journal of the Lancashire and Cheshire Entomological Society 121-123: 95.

- $\quad$ Fraser SE, Dytham C, Mayhew PJ (2007) Determinants of parasitoid abundance and diversity in woodland habitats. Journal of Applied Ecology 44 (2): 352-361. DOI: 10.111 1/j.1365-2664.2006.01266.x

- $\quad$ Fritzén NR, Shaw MR (2014) On the spider parasitoids Polysphincta longa Kasparyan and $P$. boops Tschek (Hymenoptera, Ichneumonidae, Pimplinae), with the first host records of $P$. longa . Journal of Hymenoptera Research 39: 71-82. DOI: $10.3897 /$ JHR.39.7591

- Gauld ID (1970) Some records of Ichneumonoidea (Hym.) collected at light, during autumn 1969 in Perthshire and southern Inverness-shire, including a species new to Britain. Entomologist's Gazette 21: 282-284.

- Gauld ID (1973) Notes on the British Ophionini (Hym., Ichneumonidae) including a provisional key to species. Entomologist's Gazette 24: 55-65.

- Gauld ID (1974) Further notes on the British Ophionini (Hym., Ichneumonidae). Entomologist's Gazette 25: 147-148.

- Gauld ID (1976a) The classification of the Anomaloninae (Hymenoptera: Ichneumonidae). Bulletin of the British Museum (Natural History), Entomology 33: $1-135$.

- Gauld ID (1976b) The taxonomy of the genus Heteropelma Wesmael (Hymenoptera: Ichneumonidae). Bulletin of the British Museum (Natural History), Entomology 34: 153-219.

- Gauld ID (1997) The Ichneumonidae of Costa Rica, 2. Memoirs of the American Entomological Institute 57: 1-485.

- Gauld ID, Dubois J (2006) Phylogeny of the Polysphincta group of genera (Hymenoptera: Ichneumonidae; Pimplinae): a taxonomic revision of spider 
ectoparasitoids. Systematic Entomology 31 (3): 529-564. DOI: $10.1111 /$ j.1365-3113.2006.00334.x

- Gauld ID, Fitton MG (1980) The British species of Phrudinae (Hym., Ichneumonidae). Entomologist's Monthly Magazine 115: 197-199.

- $\quad$ Gauld ID, Fitton MG (1981) Keys to the British xoridine parasitoids of the wood-boring beetles (Hymenoptera: Ichneumonidae). Entomologist's Gazette 32: 259-267.

- Gauld ID, Mitchell PA (1977a) Nocturnal Ichneumonidae of the British Isles: the genus Alexeter Foerster. Entomologist's Gazette 28: 51-55.

- Gauld ID, Mitchell PA (1977b) Ichneumonidae. Orthopelmatinae and Anomaloninae . Handbooks for the Identification of British Insects VII (2): 1-32.

- Gauld ID, Sithole R (2002) Subfamily Metopiinae . In: Gauld ID, Godoy C, Sithole R, Ugalde Gómez JA (Eds) Ichneumonidae of Costa Rica, 4. Memoirs of the American Entomological Society, $251 \mathrm{pp}$.

- $\quad$ Gauld ID, Wahl DB (2006) The relationship and taxonomic position of the genera Apolophus and Scolomus (Hymenoptera: Ichneumonidae). Zootaxa 1130: 35-41.

- Gauld ID, Wahl DB, Broad G (2002) The suprageneric groups of the Pimplinae (Hymenoptera: Ichneumonidae): a cladistic re-evaluation and evolutionary biological study. Zoological Journal of the Linnean Society 136: 421-485. DOI: 10.1046/ j.1096-3642.2002.00031.x

- $\quad$ George AM (1978) A new Ichneumon parasite of the purple hair streak (Quercusia quercus L.). Entomologist's Record 90 (10): 275.

- $\quad$ George RS (1957) Eremotylus (Ophion) marginatus (Jurine 1807), (Hym.: Ichneumonidae) in Gloucestershire East. Proceedings C.N.F.C. 32 (2): 82-83.

- $\quad$ Godfrey A, Whitehead PF (2001) The Diptera, Coleoptera and other invertebrates recorded from oak sap-flows at Brayton Barff, North Yorkshire. British Journal of Entomology and Natural History 14: 65-84.

- Gokhman VE (2007) Revision of the genus Trachyarus Thomson (Insecta, Hymenoptera, Ichneumonidae, Alomyini). Spixiana 30: 65-83.

- Gupta VK (1982) A revision of the genus Delomerista (Hymenoptera: Ichneumonidae). Contributions of the American Entomological Institute 19 (1): 1-42.

- Hayes AJ (1982) A new record of Sirex cyaneus F. in south Scotland with notes on the Scottish Siricidae (Hym.). Entomologist's Monthly Magazine 118: 195-198.

- Heath J (1961) Some parasites of Eriocraniidae (Lep.). Entomologist's Monthly Magazine 92: 163.

- Hedström L (1990) [Swedish insect records - report 6]. Entomologisk Tidskrift 111 (4): 133-147.

- $\quad$ Hilpert H (1992) Zur Systematik der Gattung Ichneumon Linnaeus in der Westpalaearktis (Hymenoptera, Ichneumonidae, Ichneumoninae). Entomofauna Supplement 6: 1-389.

- Hinz R (1986) Die paläarktischen Arten der Gattung Trematopygus Holmgren (Hymenoptera, Ichneumonidae). Spixiana 8 [1985] (3): 265-276.

- Hinz R (1996) Übersicht über die europäischen Arten von Lethades Davis (Insecta, Hymenoptera, Ichneumonidae, Ctenopelmatinae). Spixiana 19 (3): 271-279.

- Hinz R, Horstmann K (1998) Holarctic species of Trematopygodes Aubert (Insecta Hymenoptera, Ichneumonidae, Ctenopelmatinae). Spixiana 21 (3): 241-251.

- Hinz R, Horstmann K (2000) Die westpaläarktischen Arten von Exephanes Wesmael (Insecta, Hymenoptera, Ichneumonidae, Ichneumoninae). Spixiana 23 (1): 15-32. 
- Hinz R, Horstmann K (2004) Revision of the eastern Palearctic species of Dusona Cameron (Insecta, Hymenoptera, Ichneumonidae, Campopleginae). Spixiana Supplement 29: 1-183.

- Horstmann K (1968) Revision einiger Arten der Gattungen Mesostenus Gravenhorst, Agrothereutes Förster und Ischnus Gravenhorst [Hymenoptera, Ichneumonidae]. Entomophaga 13: 121-133. DOI: 10.1007/BF02371782

- Horstmann K (1971) Revision der europäischen Tersilochinen 1 (Hymenoptera: Ichneumonidae). Veröffentlichungen der Zoologischen Staatsammlung (München) 15: 47-138.

- Horstmann K (1974) Revision der westpaläarktischen Arten der SchlupfwespenGattungen Bathyplectes und Biolysia (Hymenoptera: Ichneumonidae). Entomologica Germanica 1 (1): 58-81.

- Horstmann K (1978a) Revision der gattungen der Mastrina Townes (Hymenoptera, Ichneumonidae, Hemitelinae). Zeitschrift der Arbeitsgemenischaft Österreichischer Entomologen 30: 65-70.

- Horstmann K (1978b) Bemerkungen zur Systematik einiger Gattungen der Campopleginae II (Hymenoptera, Ichneumonidae). Mitteilungen der Münchner Entomologischen Gesellschaft 67[1977]: 65-83.

- Horstmann K (1980a) Neue westpaläarktische Campopleginen-Arten (Hymenoptera, Ichneumonidae). Mitteilungen der Münchner Entomologischen Gesellschaft 69: 117-132.

- Horstmann K (1980b) Revision der europäischen Arten der Gattung Aclastus Förster (Hymenoptera, Ichneumonidae). Polskie Pismo Entomologiczne 50: 133-158.

- Horstmann K (1981a) Revision der europäischen Tersilochinen II (Hymenoptera, Ichneumonidae). Spixiana Supplement 4 (1980): 1-76.

- Horstmann K (1981b) Die paläarktischen Arten der Gattungen Eremotylus Förster, 1869, und Simophion Cushman, 1947 (Hymenoptera, Ichneumonidae). Entomofauna 2 (29): 415-432.

- Horstmann K (1981c) Zwei neue Arten der Gattungen Phygadeuon Gravenhorst und Tersilochus Holmgren, die aus phytophagen Insekten an Disteln gezogen wurden (Hymenoptera, Ichneumonidae). Spixiana 4 (2): 153-158.

- Horstmann K (1982) Revision der von Panzer beschriebenen Ichneumoniden-Arten (Hymenoptera). Spixiana 5: 231-246.

- Horstmann K (1983) Typenrevision der von Schmiedeknecht beschriebenen HemitelesArten (Hymenoptera, Ichneumonidae). Mitteilungen der Münchner Entomologischen Gesellschaft 72: 147-158.

- Horstmann K (1985) Revision der mit difformis (Gmelin, 1790) verwandten westpaläarktischen Arten der Gattung Campoplex Gravenhorst, 1829 (Hymenoptera, Ichneumonidae). Entomofauna 6 (12): 129-163.

- Horstmann K (1986) Die westpaläarktischen Arten der Gattung Gelis Thunberg, 1827, mit macropteren oder brachypteren Weibchen (Hymenoptera, Ichneumonidae).

Entomofauna 7 (30): 389-424.

- Horstmann K (1987) Die europäischen Arten der Gattungen Echthronomas Förster und Eriborus Förster (Hymenoptera, Ichneumonidae). Nachrichtenblatt der Bayerischen Entomologen 36 (3): 57-67.

- Horstmann K (1990a) Neubeschreibungen einiger Schlupfwespen-Arten aus den Gattungen Mastrus Förster, Odontoneura Förster und Zoophthorus Förster 
(Hymenoptera, Ichneumonidae, Cryptinae). Zeitschrift der Arbeitsgemeinschaft Österreichischer Entomologen 42: 1-14.

- Horstmann K (1990b) Die westpaläarktischen Arten einiger Gattungen der Cryptini (Hymenoptera, Ichneumonidae). Mitteilungen der Münchner Entomologischen Gesellschaft 79[1989]: 65-89.

- Horstmann K (1992a) Revision einiger Gattungen und Arten der Phygadeuontini (Hymenoptera, Ichneumonidae). Mitteilungen der Münchner Entomologischen Gesellschaft 81[1991]: 229-254.

- Horstmann K (1992b) Revisionen einiger von Linnaeus, Gmelin, Fabricius, Gravenhorst und Förster beschriebener Arten der Ichneumonidae (Hymenoptera, Ichneumonidae). Mitteilungen der Münchner Entomologischen Gesellschaft 82: 21-33.

- Horstmann K (1993a) Nachträge zu Revisionen der Gattungen Aclastus Förster, Ceratophygadeuon Viereck, Chirotica Förster und Gelis Thunberg (Hymenoptera, Ichneumonidae, Cryptinae). Nachrichtenblatt der Bayerischen Entomologen 42 (1): 7-15.

- Horstmann K (1993b) Die europäischen Arten von Gnotus Förster und Uchidella Townes (Hymenoptera, Ichneumonidae, Cryptinae). Zeitschrift der Arbeitsgemeinschaft Österreichischer Entomologen 45: 35-45.

- Horstmann K (1993c) Revision der brachypteren Weibchen der wespaläarktischen Cryptinae (Hymenoptera, Ichneumonidae). Entomofauna 14 (7): 85-148.

- Horstmann K (1994a) Nachtrag zur Revision der wespaläarktischen Nemeritis-Arten (Hymenoptera, Ichneumonidae, Campopleginae). Mitteilungen der Münchner Entomologischen Gesellschaft 84: 79-90.

- Horstmann K (1994b) Die europäischen Arten von Picrostigeus Förster (Hymenoptera, Ichneumonidae, Orthocentrinae). Zeitschrift der Arbeitsgemenischaft Österreichischer Entomologen 46: 111-120.

- Horstmann K (1995) Die europäischen Arten von Arotrephes Townes, 1970 und Pleurogyrus Townes, 1970 (Hymenoptera, Ichneumonidae, Cryptinae). Entomofauna 16 (12): 261-275.

- Horstmann K (1997) Revision von Schlupfwespen-Arten (Hymenoptera: Ichneumonidae, Braconidae, Eulophidae, Torymidae). Mitteilungen der Münchner Entomologischen Gesellschaft 87: 109-119.

- Horstmann K (1998a) Übersicht über die von Sigismund Brauns beschriebenen europäischen Ichneumonidae (Hymenoptera). Nachrichtenblatt der Bayerischen Entomologen 47: 80-87.

- Horstmann K (1998b) Revisionen von Schlupfwespen-Arten II (Hymenoptera: Ichneumonidae, Braconidae). Mitteilungen der Münchner Entomologischen Gesellschaft 88: 3-12.

- Horstmann K (1998c) Revisionen einiger Gattungen und Arten der Phygadeuontini II (Hymenoptera, Ichneumonidae, Cryptinae). Entomofauna 19 (26): 433-460.

- Horstmann K (1999a) Zur Interpretation der von Thunberg in der Gattung Ichneumon Linnaeus beschriebenen oder benannten Arten (Hymenoptera). Zeitschrift der Arbeitsgemeinschaft Österreich Entomologen 51: 65-74.

- Horstmann K (1999b) Revisionen von Schlupfwespen-Arten III (Hymenoptera: Ichneumonidae). Mitteilungen der Münchner Entomologischen Gesellschaft 89: 47-57. 
- Horstmann K (2000a) Die westpaläarktischen Arten von Ethelurgus Förster, 1869 und Rhembobius Förster, 1869 (Hymenoptera, Ichneumonidae, Cryptinae). Entomofauna 21 (8): 65-76.

- Horstmann K (2000b) Typenrevisionen der von Gravenhorst beschriebenen oder gedeuteten Campoplex-Arten (Hymenoptera, Ichneumonidae). Linzer Biologische Beiträge 32 (2): 1203-1214.

- Horstmann K (2000c) Revisionen von Schlupfwespen-Arten IV (Hymenoptera: Ichneumonidae). Mitteilungen der Münchner Entomologischen Gesellschaft 90: 39-50.

- Horstmann K (2000d) Typenrevisionen einiger von Habermehl beschriebener Ichneumonidae (Hymenoptera). Nachrichtenblatt der Bayerischen Entomologen 49: 67-70.

- $\quad$ Horstmann K (2000e) Die europäischen Arten von Probolus Wesmael, 1845 (Hymenoptera: Ichneumonidae). Entomofauna 21 (25): 293-300.

- Horstmann K (2001a) Revision der bisher zu Iselix Förster gestellten westpaläarktischen Arten von Phygadeuon Gravenhorst (Insecta, Hymenoptera, Ichneumonidae, Cryptinae). SPIXIANA - Zeitschrift für Zoologie 24 (3): 207-229.

- $\quad H o r s t m a n n ~ K ~(2001 b)$ Revisionen von Schlupfwespen-Arten V (Hymenoptera: Ichneumonidae). Mitteilungen der Münchner Entomologischen Gesellschaft 91: 77-86.

- Horstmann K (2001c) Revision der von Johann Christian Fabricius beschriebenen Ichneumonidae (Hymenoptera). Beiträge zur Entomologie 51 (1): 7-50.

- Horstmann K (2001d) Typenrevisionen der von Thomson aus der Sammlung Lethierry beschriebenen Cryptinae (Hymenoptera, Ichneumonidae). Nachrichtenblatt der Bayerischen Entomologen 50: 78-81.

- Horstmann K (2002a) Über einige mit Coelichneumon orbitator (Thunberg, 1824) nah verwandte Arten (Hymenoptera, Ichneumonidae, Ichneumoninae). Entomofauna 23 (6): 73-84.

- Horstmann K (2002b) Revisionen der von Gregor beschriebenen Ichneumonidae (Hymenoptera). Linzer Biologische Beiträge 34 (1): 377-382.

- Horstmann K (2002c) Revisionen von Schlupfwespen-Arten VI. Mitteilungen der Münchner Entomologischen Gesellschaft 92: 79-91.

- Horstmann K (2003a) Revisionen von Schlupfwespen-Arten VII. Mitteilungen der Münchner Entomologischen Gesellschaft 93: 25-37.

- Horstmann K (2003b) Über die aus der Sammlung Degeer beschriebenen Ichneumonidae (Hymenoptera). Linzer Biologische Beiträge 35 (2): 877-887.

- Horstmann K (2004a) Bemerkungen zur Systematik einiger Gattungen der Campopleginae IV (Hymenoptera, Ichneumonidae). Zeitschrift der Arbeitsgemeinschaft Österreichischer Entomologen 56: 13-35.

- Horstmann K (2004b) Revision von Schulpfwespen-Arten VIII (Hymenoptera: Ichneumonidae). Mitteilungen der Münchner Entomologischen Gesellschaft 94: 55-62.

- Horstmann K (2004c) Übersicht über die von Schiødte (1839a) eingeführten Namen für Taxa der Ichneumonidae (Hymenoptera). Linzer Biologische Beiträge 36 (1): 253-263.

- Horstmann K (2005a) Über einige mit Scambus inanis (Schrank, 1802) nah verwandte Arten (Hymenoptera, Ichneumonidae, Pimplinae). Entomofauna 26 (7): 101-116.

- Horstmann K (2005b) Über einige Gattungen der Ichneumonidae mit fehlbestimmten Typusarten (Hymenoptera). Linzer Biologische Beiträge 37 (2): 1257-1275.

- Horstmann K (2006a) Revisionen von Schlupfwespen-Arten IX (Hymenoptera, Ichneumonidae). Mitteilungen der Münchner Entomologischen Gesellschaft 95: 75-86. 
- Horstmann K (2006b) Revision einiger europäischer Mesochorinae (Hymenoptera, Ichneumonidae). Linzer Biologische Beiträge 38 (2): 1449-1492.

- Horstmann K (2006c) Revisionen der von Kriechbaumer aus der Westpaläarktis und Zentralasien beschriebenen Ichneumonidae (Insecta, Hymenoptera). Spixiana 29 (1): 1-30.

- Horstmann K (2006d) Revision von Schlupfwespen-Arten X (Hymenoptera: Ichneumonidae, Braconidae). Mitteilungen der Münchner Entomologischen Gesellschaft 96: 5-16.

- Horstmann K (2007a) Typenrevisionen der von Kiss beschriebenen Taxa der Ctenopelmatinae (Hymenoptera, Ichneumonidae). Linzer Biologische Beiträge 39 (1): 313-322.

- Horstmann K (2007b) Revisionen von Schlupfwespen-Arten XI (Hymenoptera: Ichneumonidae). Mitteilungen der Münchner Entomologischen Gesellschaft 97: 73-80.

- Horstmann K (2008a) Revision der europäischen Arten von Ephialtes Gravenhorst, 1829, mit Bemerkungen zu weiteren holarktischen Arten (Hymenoptera, Ichneumonidae, Pimplinae). Entomofauna 29 (9): 145-168.

- Horstmann K (2008b) Revisionen von Schlupfwespen-Arten XII (Hymenoptera: Ichneumonidae). Mitteilungen der Münchner Entomologischen Gesellschaft 98: 21-29.

- Horstmann K (2008c) Neue westpaläarktische Arten der Campopleginae (Hymenoptera: Ichneumonidae). Zeitschrift der Arbeitsgemeinschaft Österreichischer Entomologen 60: 3-27.

- Horstmann K (2009a) Revision of the western Palearctic species of Dusona Cameron (Hymenoptera, Ichneumonidae, Campopleginae). Spixiana 32 (1): 45-110.

- Horstmann K (2009b) Revision der europäischen Arten von Isadelphus Förster, 1869 (Hymenoptera, Ichneumonidae, Cryptinae). Entomofauna 30 (28): 473-492.

- Horstmann K (2009c) Typenrevisionen der von Kiss beschriebenen Taxa der Ichneumonidae III. Verschiedene Unterfamilien (Hymenoptera, Ichneumonidae). Linzer Biologische Beiträge 41 (1): 673-689.

- Horstmann K (2009d) Revisionen von Schlupfwespen-Arten XIII (Hymenoptera: Ichneumonidae). Mitteilungen der Münchner Entomologischen Gesellschaft 99: 37-44.

- Horstmann K (2010a) Revision der europäischen Arten von Stibeutes Förster, 1850 (Hymenoptera, Ichneumonidae, Cryptinae). Entomofauna 31 (15): 229-264.

- Horstmann K (2010b) Revisionen von Schlupfwespen-Arten XIV (Hymenoptera: Ichneumonidae). Mitteilungen der Münchner Entomologischen Gesellschaft 100: 119-130.

- Horstmann K (2011a) Revisionen von Schlupfwespen-Arten XV (Hymenoptera: Ichneumonidae). Mitteilungen Münchner Entomologischen Gesellschaft 101: 5-13.

- Horstmann K (2011b) Verbreitung und Wirte der Dusona-Arten in der Westpalaearktis (Hymenoptera. Ichneumonidae, Campopleginae). [Distribution and hosts of Dusona species in the West Palaearctic (Hymenoptera. Ichneumonidae, Campopleginae).]. Linzer Biologische Beiträge 43 (2): 1295-1330.

- Horstmann K (2012a) Revisionen von Schlupfwespen-Arten XVI (Hymenoptera: Ichneumonidae). Mitteilungen der Münchner Entomologischen Gesellschaft 102: 105-115.

- Horstmann K (2012b) Typenrevision einiger von Strobl beschriebener Taxa der Ichneumonidae (Hymenoptera, Ichneumonidae). Linzer Biologische Beiträge 44 (2): 1137-1153. 
- Horstmann K (2012c) Revisionen einiger Gattungen und Arten der Phygadeuontini III (Hymenoptera, Ichneumonidae, Cryptinae). Entomofauna 33 (28): 397-422.

- Horstmann K (2013) Revisionen von Schlupfwespen-Arten XVII (Hymenoptera: Ichneumonidae). Mitteilungen der Münchner Entomologischen Gesellschaft 103: 1-14.

- Horstmann K, Shaw MR (1984) The taxonomy and biology of Diadegma chrysostictos (Gmelin) and Diadegma fabricianae sp.n. (Hymenoptera: Ichneumonidae). Systematic Entomology 9: 329-337. DOI: 10.1111/j.1365-3113.1984.tb00057.x

- Horstmann K, Yu DS (1999) Bemerkungen zur Taxonomie und Nomenklatur westpaläarktischer Ichneumonidae (Hymenoptera). Zeitschrift der Arbeitsgemeinschaft Österreich Entomologen 50[1998]: 77-84.

- Howe MA, Howe EA (2005) Some recent records of parasitic Hymenoptera in Wales. Entomologist's Monthly Magazine 141: 247-248.

- Huddleston T, Gauld ID (1988) Parasitic wasps (Ichneumonoidea) in British light-traps. The Entomologist 107 (2): 134-154.

- Hudson IR (1985) Notes on species of Ichneumonidae reared as ectoparasites of spiders. Proceedings and Transactions of the British Entomological and Natural History Society $18: 32-34$.

- Hudson IR (1988) Some further notes on species of Ichneumonidae reared as ectoparasites of spiders. British Journal of Entomology and Natural History 1: 77-78.

- Humala AE (1997) Oxytorinae from Karelia new to Russia with description of a new genus and two new species (Hymenoptera: Ichneumonidae). Zoosystematica Rossica 5: 297-300.

- Humala AE (2002) A Review of Parasitic Wasps of the Genera Cylloceria Schiodte, 1838 and Allomacrus Forster, 1868 (Hymenoptera, Ichneumonidae) of the Fauna of Russia. Entomological Review 82 (3): 301-313.

- Humala AE (2007) Orthocentrinae (Microleptinae + Orthocentrinae sensu Townes). In: Ler PA (Ed.) Key to the insects of Russian Far East. Vol. IV. Neuropteroidea, Mecoptera, Hymenoptera. Pt 5 [in Russian]. Dal'nauka, Vladivostok, 38 pp.

- Humala AE, Jussila R, Koponen M (2007) Ichneumonids (Hymenoptera, Ichneumonidae) new to Finland. Sahlbergia 12: 50-59.

- ICZN (1999) International Code of Zoological Nomenclature. The International Trust for Zoological Nomenclature c/o The Natural History Museum, London, 306 pp.

- Idar M (1974) Redescriptions of Hadrodactylus tarsator Ths., H. gracilipes Ths. and $H$. nigrifemur Ths., with notes on $H$. bidentulus Ths. and $H$. villosulus Ths. (Hym. Ichneumonidae). Entomologisk Tidskrift 95: 107-116.

- Idar M (1975) Redescriptions of Hadrodactylus paludicola (Hlgr.), H. femoralis (Hlgr.) and $H$. insignis (Krb.) (Hymenoptera, Ichneumonidae). Entomologica Scandinavica 6 (2): 182-190. DOI: 10.1163/187631275X00299

- Idar M (1981) Revision of the European species of the genus Hadrodactylus Förster (Hymenoptera: Ichneumonidae). Part 2. Entomologica Scandinavica 12 (2): 231-239. DOI: $10.1163 / 187631281794709917$

- Idar M (1983) Revision of European Synomelix Förster (Hymenoptera: Ichneumonidae) with description of $S$. faciator $n$. sp. Entomologica Scandinavica 14 (2): 168-172. DOI: 1 $0.1163 / 187631283 \times 00047$

- Johnson WF (1921) Irish Ichneumonidae and Braconidae in 1920. Irish Naturalist 30: 104-107. 
- Johnson WF (1929) Irish Ichneumonidae . Entomologist's Monthly Magazine 65: 134-137.

- Jones RA (2001) Ctenochares bicolorus (L.), an African ichneumonid (Hymenoptera) found in Britain. British Journal of Entomology and Natural History 14: 96-99.

- Jussila R (1979) A revision of the genus Atractodes (Hymenoptera, Ichneumonidae) in the Western Palaearctic region. Acta Entomologica Fennica 34: 1-44.

- Jussila R (1983) Addition to the revision of the genus Atractodes (Hymenoptera: Ichneumonidae) of the Western Palearctic region I. Contributions of the American Entomological Institute 20: 201-204.

- Jussila R (1987) Revision of the genus Stilpnus (Hymenoptera, Ichneumonidae) of the western Palaearctic Region. Annales Entomologici Fennici 53 (1): 1-16.

- Jussila R (1994a) Aperileptus rossemi sp. n., Aniseres lapponicus sp. n., and additions to descriptions of other Oxytorinae species (Hymenoptera, Ichneumonidae).

Entomologica Fennica 5: 115-118.

- Jussila R (1994b) Additions to the revision of the genus Atractodes (Hymenoptera, Ichneumonidae) of the Western Palaearctic region. II. Entomologica Fennica 5: 129-134.

- Jussila R (1999) Additions to the revisions of the genus Stilpnus (Hymenoptera, Ichneumonidae) of the Palaearctic Region. I. Entomologica Fennica 10 (2): 107-112.

- Jussila R (2001) Additions to the revision of the genus Atractodes (Hymenoptera: Ichneumonidae) of the Palaearctic Region. III. Entomologica Fennica 12 (4): 193-216.

- Jussila R, Sääksjärvi IE, Bordera S (2010) Revision of the western Palaearctic Mesoleptus (Hymenoptera: Ichneumonidae). Annales de la Société entomologique du France (n.s.) 46: 499-518. DOI: 10.1080/00379271.2010.10697687

- $\quad$ Kasparyan DR (1973) Fauna of the USSR Hymenoptera Vol.III Number 1. Ichneumonidae (Subfamily Tryphoninae) Tribe Tryphonini . Nauka Publishers [translated from Russian. Amerind Publishing Co. Ltd., New Delhi, 1981. 414pp.], Leningrad, 414 pp.

- Kasparyan DR (1974) A review of Palearctic species of the tribe Pimplini (Hymenoptera, Ichneumonidae). The genus Pimpla Fabricius. Entomologicheskoe Obozrenie 53: 382-403.

- Kasparyan DR (1977) A review of the Ichneumonflies of the genus Eclytus Holmgren (Hymenoptera, Ichneumonidae) [partial translation by Dr A. Woroncow]. Entomologicheskoye Obozreniye 56: 156-170.

- Kasparyan DR (1986) [Towards a revision of the ichneumonids genus Adelognathus Holmgren (Hymenoptera, Ichneumonidae)] (in Russian). Proceedings of the Zoological Institute, Leningrad 159: 38-56.

- Kasparyan DR (1990) [Fauna of USSR. Insecta Hymenoptera. Vol.III(2). Ichneumonidae. Subfamily Tryphoninae: Tribe Exenterini. Subfamily Adelognathinae]. Nauka Publishing House, Leningrad, 342 pp.

- $\quad$ Kasparyan DR (1993) [Revision of the genus Thymaris (Hymenoptera, 1. Ichneumonidae)]. Zoologichesky Zhurnal 72 (9): 105-117.

- Kasparyan DR (1998) Taxonomic notes on the species of Mesoleius s.l., Hyperbatus and Phaestus in the museums of Stokholm, Lund and Munich (Hymenoptera: Ichneumonidae, Ctenopelmatinae). Zoosystematica Rossica 7 (1): 181-183.

- Kasparyan DR (2000) Palaearctic ichneumonid wasps of the genus Mesoleius (s. str.) Holmgren (Hymenoptera, Ichneumonidae): I. Entomological Review 80 (2): 144-168. 
- Kasparyan DR (2003) [Palaearctic species of the ichneumonid-wasp genus Campodorus Foerster (s. str.) (Hymenoptera, Ichneumonidae) with pectinate claws]. Entomologicheskoe Obozrenie 82 (3): 758-766.

- Kasparyan DR (2004a) [A review of Palaearctic species of tribe Ctenopelmatini (Hymenoptera, Ichneumonidae). The genera Ctenopelma Holmgren and Homaspis Foerster]. Entomologicheskoe Obozrenie 83 (2): 437-467.

- Kasparyan DR (2004b) Nomenclatural notes on some Ctenopelmatinae from Dutch and Hungarian museums (Hymenoptera: Ichneumonidae). Zoosystematica Rossica 13: 47-48.

- Kasparyan DR (2011) A review of the Palaearctic species of the genus Hadrodactylus Förster (Hymenoptera, Ichneumonidae, Ctenopelmatinae) with a description of five new species. Entomological Review 91 (7): 866-888. DOI: 10.1134/S0013873811070086

- Kasparyan DR (2014) Review of the Western Palaearctic ichneumon-flies of the genus Rhorus Förster, 1869 (Hymenoptera, Ichneumonidae: Ctenopelmatinae). II. The species of the punctus, longicornis, chrysopygus, and substitutor groups, the species with the black metasoma and some others. Entomological Review 94 (5): 712-755. DOI: 10.113 4/S0013873814050078

- Kasparyan DR (2015) Review of the Western Palaearctic ichneumon-flies of the genus Rhorus Förster, 1869 (Hymenoptera, Ichneumonidae: Ctenopelmatinae). Part III. The species with the reddish metasoma and black face. Entomological Review 95 (9): 1257-1291. DOI: 10.1134/S0013873815080158

- Kasparyan DR, Khalaim AI (2007a) Subfamily Acaenitinae . In: Ler PA (Ed.) Key to the insects of Russian Far East. Vol. IV. Neuropteroidea, Mecoptera, Hymenoptera. Pt 5 [in Russian]. Dal'nauka, Vladivostok, 667-676 pp.

- Kasparyan DR, Khalaim Al (2007b) Subfamily Ctenopelmatinae (Scolobatinae). In: Ler PA (Ed.) Key to the insects of Russian Far East. Vol. IV. Neuropteroidea, Mecoptera, Hymenoptera. Pt 5 [in Russian]. Dal'nauka, Vladivostok, 474-559 pp.

- Kasparyan DR, Shaw MR (2003) A preliminary key to the European species of the genus Saotis Förster, 1869, with a list of British species (Ichneumonidae:

Ctenopelmatinae: Mesoleiini). Zoosystematica Rossica 11 (2): 351-355.

- Kasparyan DR, Shaw MR (2008) British and European Phytodietus Gravenhorst (Hymenoptera: Ichneumonidae,Tryphoninae) in the National Museums of Scotland, with a key to European species of the subgenus Neuchorus Uchida and descriptions of three new species. Entomologist's Gazette 59: 184-198.

- Kasparyan DR, Shaw MR (2009) A new species of Hadrodactylus Foerster (Hymenoptera: Ichneumonidae, Ctenopelmatinae, Euryproctini) from Britain and mainland Europe, with a review of material of the genus in the National Museums of Scotland. Entomologist's Gazette 60: 251-258.

- Kasparyan DR, Tolkanitz VI (2000) Fauna of Russia and Neighbouring Countries. Insecta Hymenoptera Volume III, 3. Ichneumonidae subfamily Tryphoninae: tribes Sphinctini, Phytodietini, Oedemopsini, Tryphonini (addendum), Townesioninae . Nauka, Saint Petersburg, 404 pp.

- Kerrich GJ (1935) Notes on Ichneumonidae (Hym.) new to or rare in Britain; with a brief review of British lists published since 1915. Transactions of the Society for British Entomology 2 (1): 33-46. 
- Kerrich GJ (1942) Second review of literature concerning British Ichneumonidae (Hym.), with notes on Palaearctic species. Transactions of the Society for British Entomology 8 (2): 43-77.

- Kerrich GJ (1949) Taxonomic and distributional notes on British Diplazoninae (Hym., Ichneumonidae). Journal of the Society for British Entomology 3 (2): 67-83.

- Kerrich GJ (1952) A review, and a revision in greater part, of the Cteniscini of the old world (Hym., Ichneumonidae). Bulletin of the British Museum (Natural History) (Entomology) 2 (6): 305-460.

- $\quad$ Kerrich GJ (1962) Systematic notes on tryphonine Ichneumonidae (Hym.). Opuscula Entomologica 27: 45-56.

- Khalaim Al (2007) Subfamily Tersilochinae . In: Ler PA (Ed.) Key to the insects of Russian Far East. Vol. IV. Neuropteroidea, Mecoptera, Hymenoptera. Pt 5 [in Russian]. Dal'nauka, Vladivostok, 566-597 pp.

- Klopfstein S (2011) A review of the Diplazontinae of Mongolia (Hymenoptera: Ichneumonidae). Zootaxa 2790: 35-53.

- Klopfstein S (2014) Revision of the Western Palaearctic Diplazontinae (Hymenoptera, Ichneumonidae). Zootaxa 3801 (1): 1-143. DOI: 10.11646/zootaxa.3801.1.1

- Klopfstein S, Quicke DL, Kropf C, Frick H (2011) Molecular and morphological phylogeny of Diplazontinae (Hymenoptera, Ichneumonidae). Zoologica Scripta 40: 379-402. DOI: 10.1111/j.1463-6409.2011.00481.x

- Koponen M, Jussila R, Vikberg V (1999) Suomen loispistiäisluettelo (Hymenoptera, Parasitica) Osa 3. heimo Ichneumonidae, alaheimo Cryptinae . Sahlbergia 4: 19-52.

- $\quad$ Laurenne NM, Broad GR, Quicke DLJ (2006) Direct optimization and multiple alignment of 28S D2-D3 rDNA sequences: problems with indels on the way to a molecular phylogeny of the cryptine ichneumon wasps (Insecta: Hymenoptera). Cladistics 22: 442-473. DOI: 10.1111/j.1096-0031.2006.00112.x

- $\quad$ Liston AD, Knight GT, Sheppard DA, Broad GR, Livermore L (2014) Checklist of British and Irish Hymenoptera - Sawflies, 'Symphyta'. Biodiversity Data Journal 2: e1168. DOI: 10.3897/BDJ.2.e1168

- Mason WR (1981) Paxylommatidae: the correct family-group name for Hybrizon Fallén (Hymenoptera: Ichneumonoidea), with figures of unusual antennal sensilla. Canadian Entomologist 113: 433-439.

- Mayhew PJ, Dytham C, Shaw MR, Fraser SE (2009) Collections of ichneumonids wasps (subfamilies Diacritinae, Diplazontinae, Pimplinae and Poemeniinae) from woodlands near York and their implications for conservation planning. Naturalist 134: 3-24.

- Morley C (1907) Ichneumonologica Brittanica, ii. The ichneumons of Great Britain. Cryptinae . 2. James H. Keys, Plymouth, $351 \mathrm{pp}$.

- Morley C (1908) Ichneumonologica Brittanica, iii. The ichneumons of Great Britain. Pimplinae . H. \& W. Brown, London, 328 pp.

- Morley C (1911) Ichneumonologia Britannica, iv. Tryphoninae . IV. H. \& W. Brown, London, 344 pp. pp.

- Morley C (1915) Ichneumonologia Britannica, V. The Ichneumons of Great Britain. Ophioninae. H. \& W. Brown, London [1914], 400 pp. pp.

- Notton D, Shaw M (1988) A review of the Palaearctic Neorhacodinae (Hymenoptera, Ichneumonidae) with Eremura Kasparyan, 1995 new to the west Palaearctic. Bulletin of the Natural History Museum (London) (Entomology) 67: 209-218. 
- O'Connor JP (2003) A second Irish record of Oedemopsis scabricula (Gravenhorst) (Hymenoptera: Ichneumonidae). Irish Naturalists' Journal 27 (7): 278-279.

- O'Connor JP (2004a) Coelichneumon cyaniventris (Wesmael) (Hym., Ichneumonidae) new to Ireland. Entomologist's Monthly Magazine 140: 26.

- O'Connor JP (2004b) Sinarachna pallipes (Holmgren) and Zatypota discolor (Holmgren) (Hym., Ichneumonidae, Pimplinae) new to Ireland. Entomologist's Monthly Magazine 140: 296.

- O'Connor JP (2012) Acrodactyla carinator (Aubert, 1965) new to Ireland and Acrodactyla quadrisculpta (Gravenhorst, 1820) (Hym., Ichneumonidae) confirmed as an Irish species. Entomologist's Monthly Magazine 147 (2011): 222.

- O'Connor JP, Butler FT (1992) Notes on some interesting Irish Pimplinae (Hym., Ichneumonidae). Entomologist's Monthly Magazine 128: 164.

- O'Connor JP, Shaw MR (2004) Afrephialtes cicatricosa (Ratzeburg) (Hymenoptera: Ichneumonidae, Pimplinae), an unexpected addition to the Irish fauna. Entomologist's Gazette 55: 119-120.

- O'Connor JP, Nash R, Fitton MG (2007) A catalogue of the Irish Ichneumonidae (Hymenoptera: Ichneumonidae). Occasional Publication of the Irish Biogeographical Society 10: 1-310.

- $\quad$ Oehlke J (1964) Übersicht und Bestimmungstabelle der palaearktischen Gattungen der ehemaligen Unterfamilie Pimplinae auct. (Hymenoptera, Ichneumonidae). Entomologische Abhandlungen 29: 533-590.

- Owen J, Townes HK, Townes M (1981) Species diversity of Ichneumonidae and Serphidae (Hymenoptera) in an English suburban garden. Biological Journal of the Linnean Society 16: 315-336. DOI: 10.1111/j.1095-8312.1981.tb01656.x

- Perkins JF (1943) Preliminary notes on the synonymy of the European species of the Ephialtes complex (Hym.), Ichneumonidae. Annals and Magazine of Natural History (11) 10: 249-273. DOI: 10.1080/03745481.1943.9728015

- $\quad$ Perkins JF (1952) On some British species of Ichneumon and Alomyia (Hym., Ichneumonidae). Bulletin of Entomological Research 43: 361-363. DOI: $10.1017 /$ S0007485300040530

- $\quad$ Perkins JF (1953) Notes on the British Ichneumoninae with descriptions of new species (Hym., Ichneumonidae). Bulletin of the British Museum (Natural History) (Entomology) 3 (4): 9-176.

- $\quad$ Perkins JF (1959) Hymenoptera. Ichneumonoidea. Ichneumonidae, key to subfamilies and Ichneumoninae - 1. Handbooks for the Identification of British Insects 7: 1-116.

- Perkins JF (1960) Hymenoptera. Ichneumonoidea. Ichneumonidae, subfamilies Ichneumoninae II, Alomyinae, Agriotypinae and Lycorininae. Handbooks for the Identification of British Insects 7: 117-213.

- $\quad$ Perkins JF (1962) On the type species of Foerster's genera (Hymenoptera: Ichneumonidae). Bulletin of the British Museum (Natural History) (Entomology) 11 (8): 385-483.

- $\quad$ Phillips DS (1997) Delomerista novita (Cresson) and D. pfankuchi (Brauns) (Hym., Ichneumonidae) new to Scotland. Entomologist's Monthly Magazine 133: 8.

- $\quad$ Quicke DL (2005) Biology and immature stages of Panteles schnetzeanus [sic] (Hymenoptera: Ichneumonidae), a parasitoid of Lampronia fuscatella (Lepidoptera: Incurvariidae). Journal of Natural History 39 (5): 431-443. DOI: 10.1080/002229304100 $\underline{01708669}$ 
- $\quad$ Quicke DL, Laurenne NM, Fitton MG, Broad GR (2009) A thousand and one wasps: a 28S rDNA and morphological phylogeny of the Ichneumonidae (Insecta: Hymenoptera) with an investigation into alignment parameter space and elision. Journal of Natural History 43: 1305-1421.

- $\quad$ Rahoo GM, Luff M (1988) Ichneumonid (Hym.) parasitoids of gooseberry sawflies (Hym., Tenthredinidae). Entomologist's Monthly Magazine 124: 161-164.

- $\quad$ Ranin O (1983) Über die Artengruppe Tycherus elongatus (Thomson) und nahe Verwandte (Hymenoptera, Ichneumonidae). Annales Entomologici Fennici 49 (2): 33-44.

- $\quad$ Reshchikov A (2011) Lathrolestes (Hymenoptera, Ichneumonidae) from Turkey with description of three new species and new synonymy. Journal of the Entomological Research Society 13 (1): 83-89.

- Reshchikov A (2015) Review of North European species of the genus Lathrolestes (Hymenoptera, Ichneumonidae) with description of one new species from Öland (Sweden). Zootaxa 4033 (3): 1-47. DOI: 10.11646/zootaxa.4033.1.1

- $\quad$ Riedel M (2007) Revision des in Stockholm, Lund und Paris vorhandenen Typenmaterials der Tribus Platylabini (Hymenoptera, Ichneumonidae, Ichneumoninae). Linzer Biologische Beiträge 39 (1): 521-530.

- $\quad$ Riedel M (2008) Revision der westpaläarktischen Platylabini 1. Die Gattung Platylabus Wesmael, 1845 (Hymenoptera, Ichneumonidae, Ichneumoninae). Spixiana 31 (1): 105-172.

- $\quad$ Riedel M (2012) Revision der westpaläarktischen Arten der Gattung Coelichneumon Thomson (Hymenoptera: Ichneumonidae: Ichneumoninae). Linzer Biologische Beiträge 44 (2): 1477-1611.

- $\quad$ Riedel M (2014) Zur Taxonomie der europäischen Ichneumoninae (Hymenoptera: Ichneumonidae). Mitteilungen der Münchner Entomologischen Gesellschaft 104: 103-108.

- Riedel M (2015) Revision of the European species of the genus Astiphromma Förster, 1869. Spixiana 38 (1): 85-132.

- Rossem Gv (1981) A revision of some western Palaearctic oxytorine genera (Hymenoptera, Ichneumonidae). Spixiana Supplement 4(1980): 79-135.

- Rossem Gv (1982) A revision of some western Palaearctic oxytorine genera. Part II. Genus Eusterinx (Hymenoptera, Ichneumonidae). Spixiana 5 (2): 149-170.

- Rossem Gv (1983a) A revision of Western Palearctic oxytorine genera. Part III. Genus Proclitus (Hymenoptera, Ichneumonidae). Contributions of the American Entomological Institute 20: 153-165.

- Rossem Gv (1983b) A revision of Western Palaearctic oxytorine genera. Part IV. Genus Megastylus . Entomofauna 4 (8): 121-132.

- Rossem Gv (1987) A revision of Western Palaearctic oxytorine genera. Part VI. (Hymenoptera, Ichneumonidae). Tijdschrift voor Entomologie 130: 49-108.

- Rossem Gv (1988) A revision of Palaearctic oxytorine genera. Part VII. (Hymenoptera, Ichneumonidae). Tijdschrift voor Entomologie 131: 103-112.

- $\quad$ Rotheray GE (1986) The larva and puparium of Epistrophe grossulariae (Meigen) (Dipt., Syrphidae) with a note on overwintering behaviour. Entomologist's Monthly Magazine 122: $215-218$. 
- Rotheray GE (1990) A new species of Bioblapsis (Hymenoptera: Ichneumonidae) from Scotland parasitising a mycophagous hoverfly, Cheilosia longula (Diptera: Syrphidae). Entomologica Scandinavica 21: 277-280. DOI: 10.1163/187631290X00193

- $\quad$ Salisbury A (2003) Two parasitoids of the lily beetle, Lilioceris lilii (Scopoli) (Coleoptera: Chrysomelidae), in Britain, including the first record of Lemophagus errabundus Gravenhorst (Hymenoptera: Ichneumonidae). British Journal of Entomology and Natural History 16 (2): 103-104.

- Salisbury A, Broad GR (2011) The hyperparasitoid Mesochorus lilioceriphilus (Hymenoptera: Ichneumonidae), an addition to the parasitoid complex of the lily beetle in Britain. British Journal of Entomology and Natural History 24: 95-99.

- Sanborne M (1984) A revision of the world species of Sinophorus (Ichneumonidae). Memoirs of the American Entomological Institute 38: 1-403.

- Sawoniewicz J (1980) Revision of European species of the genus Bathythrix Foerster (Hymenoptera, Ichneumonidae). Annales Zoologici 35: 1-47.

- Sawoniewicz J (1985) Revision of European species of the subtribe Endaseina (Hymenoptera, Ichneumonidae), I. Annales Zoologici 39: 131-145.

- Sawoniewicz J (2003) Zur Systematik und Faunistik europäischer Ichneumonidae II (Hymenoptera, Ichneumonidae). Entomofauna 24 (15): 209-228.

- Sawoniewicz J, Luhman JC (1992) Revision of European species of the subtribe Endaseina, III genus: Endasys Foerster, 1868. Entomofauna 13: 1-94.

- Sawoniewicz J, Wanat M (2003) Gravenhorst's types of Cryptus subgenus Cyptus in the Museum of Natural History, Wroclaw University (Hymenoptera, Ichneumonidae). Genus 14: $549-579$.

- $\quad$ Schnee H (1978) Revision der palaearktischen Arten der Gattung Perisphincter Townes (Hym. Ichneumonidae). Entomologische Nachrichten 22 (9): 129-143.

- Schnee H (2008) Die Anomaloninae der Sammlung Arnold Förster - Typenrevision und faunistische Anmerkungen (Hymenoptera, Ichneumonidae) [The Anomaloninae of the Arnold Förster collection - type revision and faunistic remarks (Hymenoptera, Ichneumonidae)]. Beiträge zur Entomologie 58: 249-266.

- $\quad$ Schönitzer K, Hower E, Melzer RR, Diller E (2006) Taxonomie und vergleichende Morphologie der Gattung Dirophanes Foerster, 1869 (Ichneumonidae, Ichneumoninae, Phaeogenini). Mitteilungen der Münchner Entomologischen Gesellschaft 95: 87-142.

- Schwarzfeld MD, Broad GR, Sperling FA (2016) Molecular phylogeny of the diverse parasitoid wasp genus Ophion Fabricius (Hymenoptera: Ichneumonidae: Ophioninae). Systematic Entomology 41 (1): 191-206. DOI: 10.1111/syen.12152

- Schwarz M (1989) Revision der Gattung Enclisis Townes (Ichneumonidae, Hymenoptera). Linzer Biologische Beiträge 21: 479-522.

- Schwarz M (1994) Beitrag zur Systematik und Taxonomie europäischer Gelis-Arten mit macropteren oder brachypteren Weibchen (Hymenoptera, Ichneumonidae). Linzer Biologische Beiträge 26 (1): 381-391.

- Schwarz M (1995) Revision der westpaläarktischen Arten der Gattungen Gelis Thunberg mit apteren Weibchen und Thaumatogelis Schmiedeknecht (Hymenoptera, Ichneumonidae). Linzer Biologische Beiträge 27: 5-105.

- Schwarz M (1998) Revision der westpaläarktischen Arten der Gattungen Gelis Thunberg mit apteren Weibchen und Thaumatogelis Schmiedeknecht (Hymenoptera, Ichneumonidae). Teil 2. Linzer Biologische Beiträge 30 (2): 629-704. 
- $\quad$ Schwarz M (2001) Revision der westpaläarktischen Arten der Gattungen Gelis Thunberg mit apteren Weibchen und Thaumatogelis Schwarz (Hymenoptera, Ichneumonidae). Teil 4. Linzer Biologische Beiträge 33: 1111-1155.

- Schwarz M (2002) Revision der westpaläarktischen Arten der Gattungen Gelis Thunberg mit apteren Weibchen und Thaumatogelis Schwarz (Hymenoptera, Ichneumonidae). Teil 3. Linzer Biologische Beiträge 34 (2): 1293-1392.

- Schwarz M (2003) Schlupfwespen (Insecta, Hymenoptera, Ichneumonidae) in den Hochlagen der Hohern Tauern (Österreich). Teil 2: Bemerkungen zu ausgewählten Arten einschließlich der Beschreibung neuer Arten. Linzer Biologische Beiträge 35 (2): 1097-1118.

- Schwarz M (2005) Revisionen und Neubeschreibungen von Cryptinae (Hymenoptera, Ichneumonidae) 1. Linzer Biologische Beiträge 37 (2): 1641-1710.

- Schwarz M (2007) Revision der westpaläarktischen Arten der Gattung Hoplocryptus Thomson (Hymenoptera, Ichneumonidae). Linzer Biologische Beiträge 39 (2): 1161-1219.

- Schwarz M (2009) Ostpaläarktische und orientalische Gelis-Arten (Hymenoptera, Ichneumonidae, Cryptinae) mit macropteren Weibchen. Linzer Biologische Beiträge 41 (2): 1103-1146.

- Schwarz M (2015) Zur Kenntnis paläarktischer Cryptus-Arten (Hymenoptera, Ichneumonidae, Cryptinae). Linzer Biologische Beiträge 47 (1): 749-896.

- Schwarz M, Boriani M (1994) Redescription of Gelis longulus (Hymenoptera: Ichneumonidae), a parasitoid of Ocnerostoma piniariellum (Lepidoptera: Yponomeutidae). European Journal of Entomology 91: 331-334.

- Schwarz M, Shaw MR (1998) Western Palaearctic Cryptinae (Hymenoptera: Ichneumonidae) in the National Museums of Scotland, with nomenclatural changes, taxonomic notes, rearing records and special reference to the British check list. Part 1. Tribe Cryptini . Entomologist's Gazette 49: 101-127.

- Schwarz M, Shaw MR (1999) Western Palaearctic Cryptinae (Hymenoptera: Ichneumonidae) in the National Museums of Scotland, with nomenclatural changes, taxonomic notes, rearing records and special reference to the British check list. Part 2. Genus Gelis Thunberg (Phygadeuontini: Gelina). Entomologist's Gazette 50: 117-142.

- Schwarz M, Shaw MR (2000) Western Palaearctic Cryptinae (Hymenoptera: Ichneumonidae) in the National Museums of Scotland, with nomenclatural changes, taxonomic notes, rearing records and special reference to the British check list. Part 3. Tribe Phygadeuontini, subtribes Chiroticina, Acrolytina, Hemitelina and Gelina (excluding Gelis), with descriptions of new species. Entomologist's Gazette 51: 147-186.

- $\quad$ Schwarz M, Shaw MR (2010) Western Palaearctic Cryptinae (Hymenoptera: Ichneumonidae) in the National Museums of Scotland, with nomenclatural changes, taxonomic notes, rearing records and special reference to the British check list. Part 4.Tribe Phygadeuontini, subtribes Mastrina, Ethelurgina, Endaseina (excluding Endasys ), Bathythrichina and Cremnodina . Entomologist's Gazette 61: 187-206.

- Schwarz M, Shaw MR (2011) Western Palaearctic Cryptinae (Hymenoptera: Ichneumonidae) in the National Museums of Scotland, with nomenclatural changes, taxonomic notes, rearing records and special reference to the British check list. Part 5. Tribe Phygadeuontini, subtribe Phygadeuontina, with descriptions of new species. Entomologist's Gazette 62: 175-210. 
- Schwenke W (1999) Revision der europäischen Mesochorinae (Hymenoptera, Ichneumonoidea, Ichneumonidae). Spixiana Supplement: 1-124.

- Schwenke W (2002) Neue europäische Mesochorinae-Arten (Hymenoptera, Ichneumonidae, Mesochorinae). Entomofauna 23 (7): 85-92.

- $\quad$ Schwenke W (2004) Eine neue Gattung und 19 neue Arten und Geschlechter europäischer Mesochorinae (Hymenoptera, Ichneumonidae). Entomofauna 25 (4): 81-88.

- $\quad$ Sebald H, Schönitzer K, Diller E (2000) Eine neue Art der Gattung Aethecerus Wesmael, [1845] aus der Ospaläarktis (Hymenoptera, Ichneumonidae, Alomyini). Entomofauna 21 (28): 313-320.

- S Šedivý J (2004) European species of the genus Phobocampe Förster (Hymenoptera: Ichneumonidae). Acta Universitatis Carolinae Biologica 48: 203-235.

- $\quad$ Selfa J, Diller E (1994) Illustrated key to the Western Palearctic genera of Phaeogenini (Hymenoptera, Ichneumonidae, Ichneumoninae). Entomofauna 15 (20): 237-251.

- Shaw MR (1978) The status of Trogus lapidator (F.) (Hymenoptera: Ichneumonidae) in Britain, a parasite of Papilio machaon L. Entomologist's Gazette 29: 287-288.

- $\quad$ Shaw MR (1981) Some parasites (Hymenoptera) of Epiphyas postvittana (Walker) (Lepidoptera: Tortricidae) in Cornwall. Entomologist's Gazette 32: 36-38.

- $\quad$ Shaw MR (1984) Foodplants and parasites (Hymenoptera: Ichneumonidae) of Choreutis pariana (Clerck) (Lepidoptera: Choreutidae) in Edinburgh. Entomologist's Gazette 35 (1): 41-44.

- Shaw MR (1986) Coleocentrus excitator (Poda) (Hymenoptera: Ichneumonidae) new to Britain. Entomologist's Gazette 37: 221-224.

- $\quad$ Shaw MR (1989) A host record for Stilbops limneriaeformis Schmiedeknecht (Hymenoptera: Ichneumonidae, Stilbopinae) in Scotland. Entomologist's Gazette 40: 5-6.

- $\quad$ Shaw MR (1991) Phrudus badensis Hilpert (Hym., Ichneumonidae) new to Britain. Entomologist's Monthly Magazine 127: 157-158.

- Shaw MR (1993) Species of Mesochorus (Hymenoptera: Ichneumonidae) reared as hyperparasitoids of Lepidoptera via koinobiont ectoparasitoid Tryphoninae (Ichneumonidae). Entomologist's Gazette 44: 181-182.

- Shaw MR (1998) British Steatoda watch. Newsletter of the British arachnological Society 83: 5.

- Shaw MR (1999) Gregarious development in endoparasitic koinobiont Ichneumonidae (Hymenoptera). Entomologist's Gazette 50: 55-56.

- Shaw MR (2001) Interactions between adults of some species of Netelia Gray (Hymenoptera: Ichneumonidae: Tryphoninae) and their caterpillar hosts (Lepidoptera). Journal of Hymenoptera Research 10 (1): 101-111.

- $\quad$ Shaw MR (2003) Adverse comment on the supposed British status of the web-spinning sawfly Cephalcia arvensis Panzer (Hymenoptera: Pamphiliidae). British Journal of Entomology and Natural History 16: 2.

- Shaw MR (2004) Notes on the biology of Lycorina triangulifera Holmgren (Hymenoptera: Ichneumonidae: Lycorininae). Journal of Hymenoptera Research 13 (2): 302-308.

- Shaw MR (2006a) Notes on British Pimplinae and Poemeniinae (Hymenoptera, Ichneumonidae), with additions to the British list. British Journal of Entomology and Natural History 19 (4): 217-238. 
- Shaw MR (2006b) Pimpla wilchristi Fitton, Shaw \& Gauld, 1988 stat. rev.: lectotype designation for Pimpla strigipleuris Thomson, 1877, and its identity as a junior subjective synonym of Pimpla spuria Gravenhorst, 1829 (Hymenoptera: Ichneumonidae, Pimplinae). Entomologist's Gazette 57: 147-149.

- Shaw MR (2008) Review. A Catalogue of the Irish Ichneumonidae (Hymenoptera: Ichneumonoidea) by J. P. O'Connor, R. Nash and M. G. Fitton. Entomologist's Monthly Magazine 144: 101-102.

- $\quad$ Shaw MR, Aeschliman J-P (1994) Host ranges of parasitoids (Hymenoptera: Braconidae and Ichneumonidae) reared from Epermenia chaerophyllella (Goeze) (Lepidoptera: Epermeniidae) in Britain, with description of a new species of Triclistus (ichneumonidae). Journal of Natural History 28: 619-629. DOI: 10.1080/002229394007 70281

- $\quad$ Shaw MR, Askew RR (1976) Ichneumonoidea (Hymenoptera) parasitic upon leafmining insects of the orders Lepidoptera, Hymenoptera and Coleoptera . Ecological Entomology 1: 127-133. DOI: 10.1111/i.1365-2311.1976.tb01213.x

- Shaw MR, Bennett FD (2001) Host prepupal mummification by Colpognathus Wesmael (Hymenoptera: Ichneumonidae, Ichneumoninae). Entomologist's Gazette 52: 201-203.

- Shaw MR, Horstmann K (1997) An analysis of host range in the Diadegma nanus group of parasitoids in Western Europe, with a key to species (Hymenoptera: Ichneumonidae: Campopleginae). Journal of Hymenoptera Research 6: 273-296.

- Shaw MR, Kasparyan DR (2002) Some British records of Olethrodotis modestus (Gravenhorst) (Hymenoptera: Ichneumonidae: Ctenopelmatinae). Entomologist's Record 114: 137-139.

- Shaw MR, Kasparyan DR (2003) Some genera of British and European Mesoleiini (Hym., Ichneumonidae: Ctenopelmatinae) in the National Museums of Scotland, including a new species of Mesoleius and a further twenty species new to Britain. Entomologist's Monthly Magazine 139: 17-28.

- $\quad$ Shaw MR, Kasparyan DR (2005) British and European Tryphonini, Exenterini, Eclytini and Idiogrammatini (Hym., Ichneumonidae: Tryphoninae) in the National Museums of Scotland, including 19 species new to Britain. Entomologist's Monthly Magazine 141: 1-14.

- Shaw MR, Voogd J (2016) Illustrated notes on the biology of Sphinctus serotinus Gravenhorst (Hymenoptera, Tryphoninae, Sphinctini). Journal of Hymenoptera Research 49: 81-93. DOI: 10.3897/JHR.49.7705

- Shaw MR, Wahl DB (1989) The biology, egg and larvae of Acaenitus dubitator (Panzer) (Hymenoptera, Ichneumonidae: Acaenitinae). Systematic Entomology 14: 117-125. DOI: $10.1111 /$ j.1365-3113.1989.tb00269.x

- Shaw MR, Wahl DB (2014) Biology, early stages and description of a new species of Adelognathus Holmgren (Hymenoptera: Ichneumonidae: Adelognathinae). Zootaxa 3884 (3): 235-252. DOI: 10.11646/zootaxa.3884.3.3

- Shaw MR, Jennings MT, Quicke DL (2011) The identity of Scambus planatus (Hartig, 1838) and Scambus ventricosus (Tschek, 1871) as seasonal forms of Scambus calobatus (Gravenhorst, 1829) in Europe (Hymenoptera, Ichneumonidae, Pimplinae, Ephialtini). Journal of Hymenoptera Research 23: 55-64. DOI: 10.3897/jhr.23.1974

- Shaw MR, Kasparyan DR, Fitton MG (2003) Revision of the British checklist of Ctenopelmatini (Hymenoptera: Ichneumonidae, Ctenopelmatinae). Entomologist's Gazette 54: 137-141. 
- $\quad$ Sime KR, Wahl DB (2002) The cladistics and biology of the Callajoppa genus-group (Hymenoptera: Ichneumonidae, Ichneumoninae). Zoological Journal of the Linnean Society 134 (1): 1-56. DOI: 10.1046/j.1096-3642.2002.00006.x

- Stelfox AW (1929) Phobocampa [sic] confusa Thoms. in Ireland. Entomologist's Monthly Magazine 65: 66-67.

- Stelfox AW (1932) Northern insects in Co. Wicklow, Ireland. Entomologist's Record and Journal of Variation 44: 76-77.

- Thirion C (1987) Diplazon scutatorius Teunissen, 1943 (Hymenoptera: Ichneumonidae) new to Britain. Entomologist's Gazette 38: 55-56.

- Tolkanitz VI (1974) [Revision of the Netelia (Hymenoptera, Ichneumonidae) and a review of the species of the fauna of the USSR.] (in Russian with English summary). Zoologicheskii Zhurnal 53: 376-393.

- Townes H (1971) The genera of Ichneumonidae, Part 4. Memoirs of the American Entomological Institute 17: 1-372.

- Townes H (1983) Revisions of twenty genera of Gelini (Ichneumonidae). Memoirs of the American Entomological Institute 35: 1-281.

- Townes HK (1970) The genera of Ichneumonidae, Part 2. Memoirs of the American Entomological Institute 12: 1-537.

- Townes HK, Townes M (1960) Ichneumon-flies of American north of Mexico: 2 Subfamily Ephialtinae, Xoridinae, Acaenitinae . United States National Museum Bulletin 216 (2): 1-676. DOI: 10.5479/si.03629236.216.1-2

- Townes HK, Townes M (1962) Ichneumon-flies of America north of Mexico: 3. Subfamily Gelinae, tribe Mesostenini . United States National Museum Bulletin 216 (3): 1-602. DOI: $10.5479 /$ si.03629236.216.1-3

- Townes HK, Momoi S, Townes M (1965) A catalogue and reclassification of the eastern Palearctic Ichneumonidae. Memoirs of the American Entomological Institute 5: 1-661.

- $\quad$ Tschopp A, Riedel M, Kropf C, Nentwig W, Klopfstein S (2013) The evolution of host associations in the parasitic wasp genus Ichneumon (Hymenoptera: Ichneumonidae): convergent adaptations to host pupation sites. BMC Evolutionary Biology 13 (74): 13 pp.. DOI: http://www.biomedcentral.com/1471-2148/13/74

- Valemberg J (2001) Nomenclature taxonomique et synonymique des formes valides, spécifiques et infraspécifiques, d'hym. Ichneumonidae Latr. de la zone paléarctiques. Bulletin de la Société Entomologique du Nord de la France Supplement 200: 1-356.

- Varga A (2013) A review of the subfamily Acaenitinae Förster, 1869 (Hymenoptera, Ichneumonidae) from Ukrainian Carpathians. Biodiversity Data Journal 1: 19 pp..

- Vikberg V, Koponen M (2000) On the taxonomy of Seleucus Holmgren and the European species of Phrudinae (Hymenoptera: Ichneumonidae). Entomologica Fennica 11(4) (4): 195-228.

- Wahl DB (1986) Larval structures of oxytorines and their significance for the higher classification of some Ichneumonidae (Hymenoptera). Systematic Entomology 11: 117-127. DOI: 10.1111/j.1365-3113.1986.tb00171.x

- Wahl DB (1988) A review of the mature larvae of the Banchini and their phylogenetic significance, with comments on the Stilbopinae (Hymenoptera: Ichneumonidae). In: Gupta VK (Ed.) Advances in Parasitic Hymenoptera Research. E.J.Brill, Leiden, New York, København, Köln, 14 pp.

- Wahl DB (1990) A review of the mature larvae of Diplazontinae, with notes on larvae of Acaenitinae and Orthocentrinae and proposal of two new subfamilies (Insecta: 
Hymenoptera, Ichneumonidae). Journal of Natural History 24: 27-52. DOI: 10.1080/002 22939000770041

- Wahl DB (1993) Cladistics of the genera of Mesochorinae (Hymenoptera: Ichneumonidae). Systematic Entomology 18: 371-387. DOI: 10.1111/ j.1365-3113.1993.tb00673.x

- Wahl DB (1997) The cladistics of the genera and subgenera of Xoridinae. Memoirs of the American Entomological Institute 57: 454-460.

- Wahl DB, Gauld ID (1998) The cladistics and higher classification of the Pimpliformes (Hymenoptera: Ichneumonidae). Systematic Entomology 23: 299-303.

- Wahl DB, Mason WR (1995) The family-group names of the Ichneumoninae (Hymenoptera: Ichneumonidae). Journal of Hymenoptera Research 4: 285-293.

- Wahl DB, Sime KR (2006) A revision of the genus Trogus (Hymenoptera: Ichneumonidae, Ichneumoninae). Systematic Entomology 31 (4): 584-610. DOI: 10.111 1/j.1365-3113.2006.00329.x

- Walker F (1872) Ichneumonidae of the Isle of Man. Entomologist 6: 431-432.

- Watanabe K, Maeto K (2013) Review of the subgenus Diblastomorpha Förster, 1869, of the genus Glypta Gravenhorst, 1829 (Hymenoptera, Ichneumonidae, Banchinae). Journal of Japanese Systematic Entomology 19 (1): 129-137.

- Wharton RA, Achterberg Cv (2000) Family group names in Braconidae (Hymenoptera: Ichneumonoidea). Journal of Hymenoptera Research 9 (2): 254-270.

- Whitehead PF (2003) Epicaelilius pilipennis (Lienhard, 1966) (Psocoptera, Caeciliusidae) new to Wales. Entomologist's Monthly Magazine 139: 237-239.

- Wyatt TD, Foster WA (1989) Parental care in the subsocial intertidal beetle, Bledius spectabilis, in relation to parasitism by the ichneumonid wasp, Barycnemis blediator . Behaviour 110: 76-92. DOI: 10.1163/156853989X00394

- Yu DS, Horstmann K (1997) A catalogue of world Ichneumonidae (Hymenoptera). Memoirs of the American Entomological Institute 58: 1-1558.

- $\quad$ Yu DS, Achterberg Cv, Horstmann K (2012) Taxapad 2012, Ichneumonoidea 2011. Database on flash-drive. www.taxapad.com, Ottawa, Ontario, Canada.

- Z Zwakhals CJ (2006) The European species of the genera Zatypota and Sinarachna (Hymenoptera: Ichneumonidae, Pimplinae, Polysphinctini). Entomologische Berichten 66 (2): 34-37.

\section{Supplementary materials}

\section{Suppl. material 1: Checklist of British and Irish Ichneumonidae}

Authors: Broad, G.R.

Data type: Excel spreadsheet

Brief description: Excel spreadsheet version of the British and Irish Ichneumonidae checklist

Filename: Ichneumonidae_checklist_spreadsheet_June_2016.xlsx - Download file (659.54 kb)

\section{Suppl. material 2: Checklist of British and Irish Ichneumonidae}

Authors: Broad, G.R.

Data type: Word document 
Brief description: text version of the British and Irish Ichneumonidae checklist

Filename: Hym_checklist_8_Ichneumonidae_June_2016_update.docx - Download file (685.65 $\mathrm{kb})$ 